\title{
Fundamentals of Mathematics
}

\author{
By: \\ Denny Burzynski \\ Wade Ellis
}





\title{
Fundamentals of Mathematics
}

\author{
By: \\ Denny Burzynski \\ Wade Ellis \\ Online: \\ < http://cnx.org/content/col10615/1.4/ >
}

\section{O N N EXI O N S}

Rice University, Houston, Texas 
This selection and arrangement of content as a collection is copyrighted by Denny Burzynski, Wade Ellis. It is licensed under the Creative Commons Attribution 2.0 license (http://creativecommons.org/licenses/by/2.0/).

Collection structure revised: August 18, 2010

PDF generated: July 29, 2013

For copyright and attribution information for the modules contained in this collection, see p. 699. 


\section{Table of Contents}

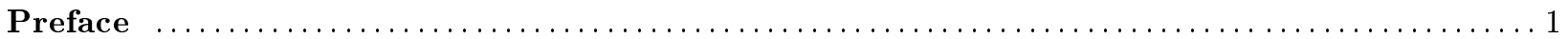

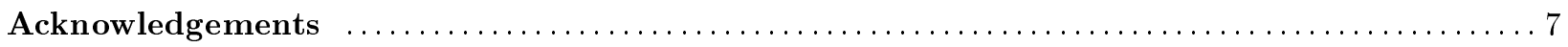

1 Addition and Subtraction of Whole Numbers

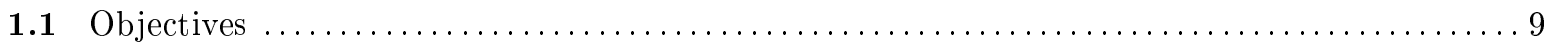

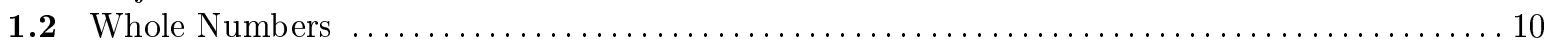

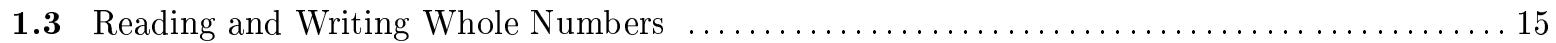

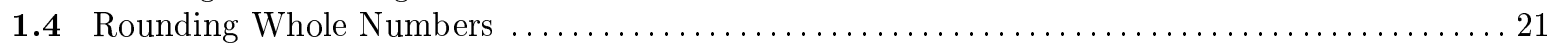

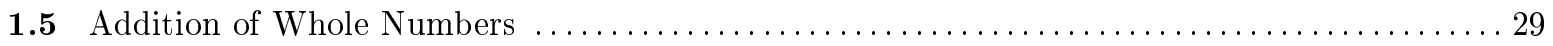

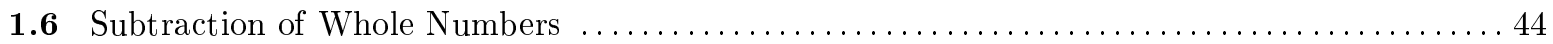

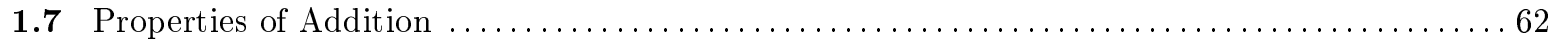

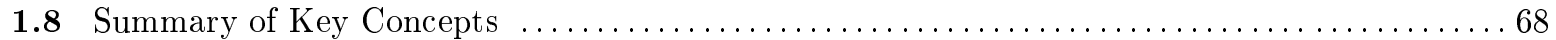

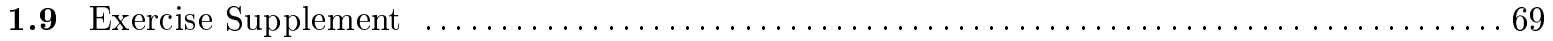

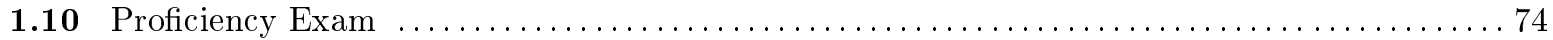

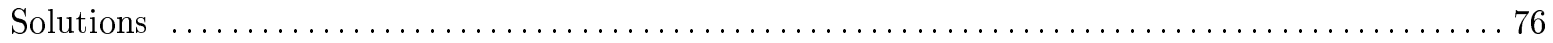

2 Multiplication and Division of Whole Numbers

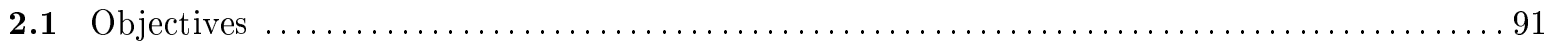

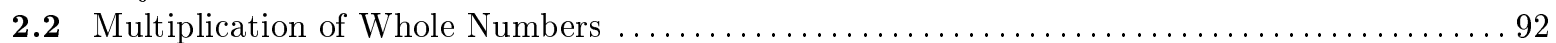

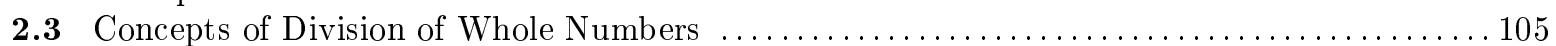

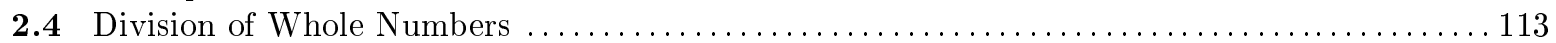

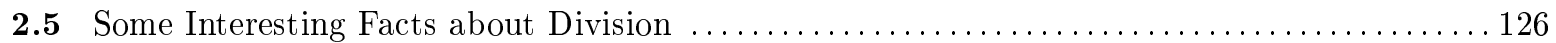

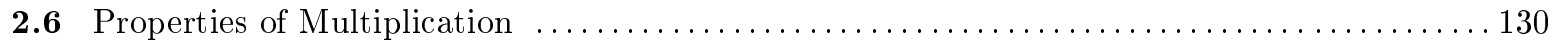

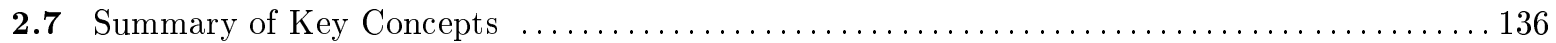

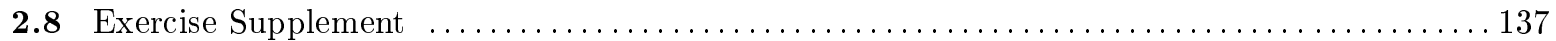

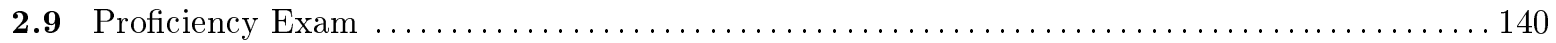

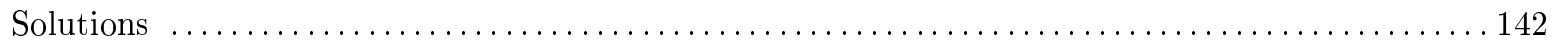

3 Exponents, Roots, and Factorization of Whole Numbers

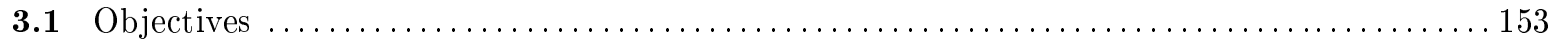

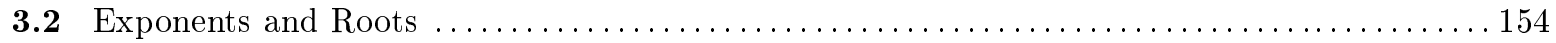

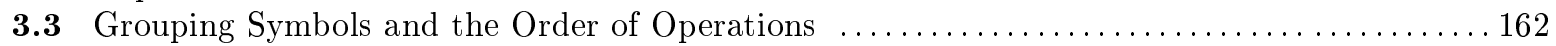

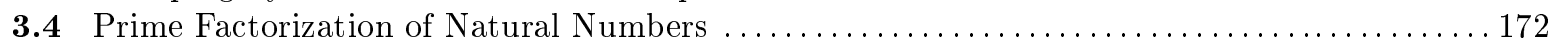

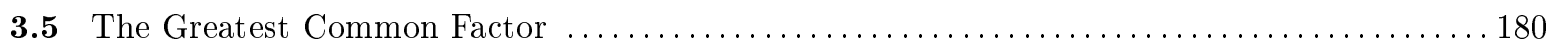

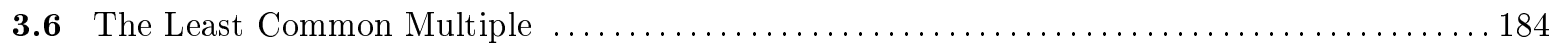

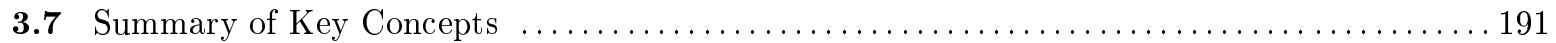

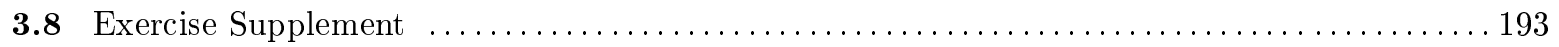

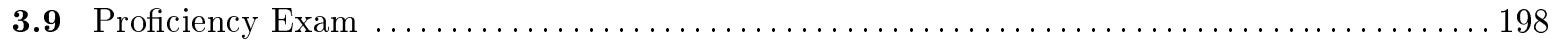

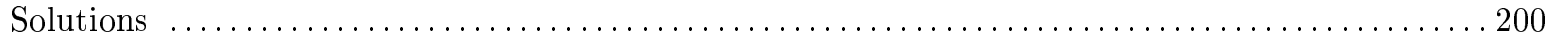

4 Introduction to Fractions and Multiplication and Division of Fractions

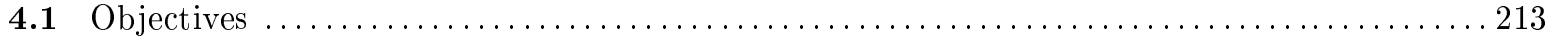

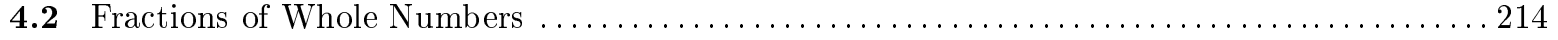

4.3 Proper Fractions, Improper Fractions, and Mixed Numbers $\ldots \ldots \ldots \ldots \ldots \ldots \ldots \ldots \ldots \ldots \ldots \ldots \ldots \ldots \ldots$

4.4 Equivalent Fractions, Reducing Fractions to Lowest Terms, and Raising Fractions

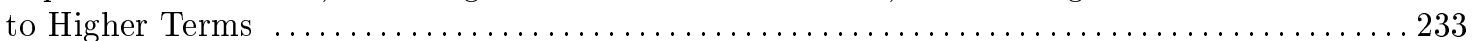

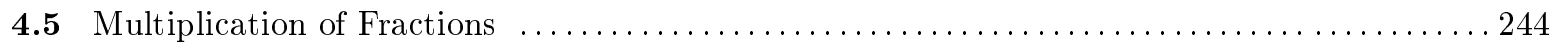

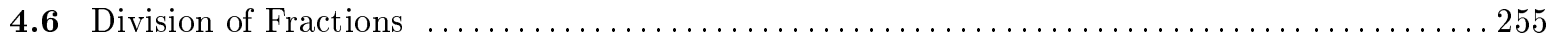

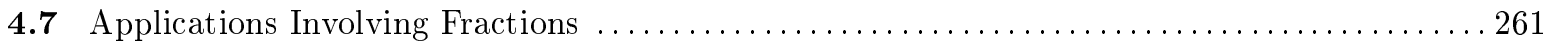

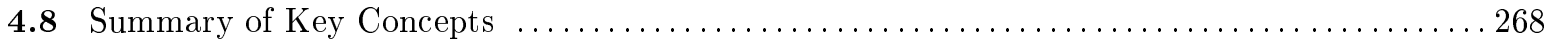

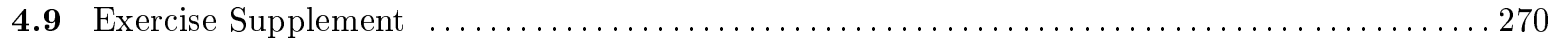




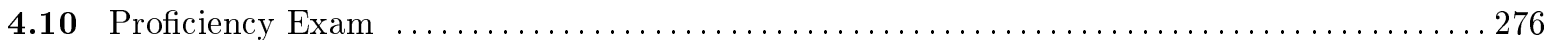

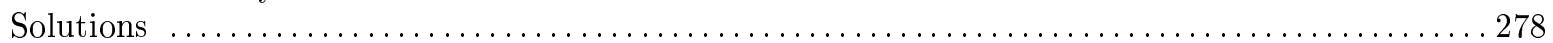

5 Addition and Subtraction of Fractions, Comparing Fractions, and Complex Fractions

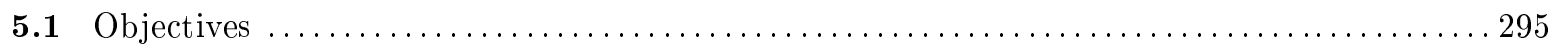

5.2 Addition and Subtraction of Fractions with Like Denominators $\ldots \ldots \ldots \ldots \ldots \ldots \ldots \ldots \ldots \ldots$

5.3 Addition and Subtraction of Fractions with Unlike Denominators $\ldots \ldots \ldots \ldots \ldots \ldots \ldots \ldots \ldots$

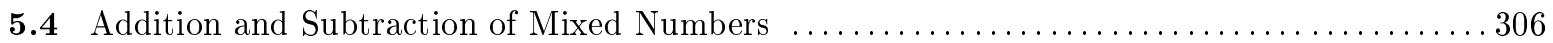

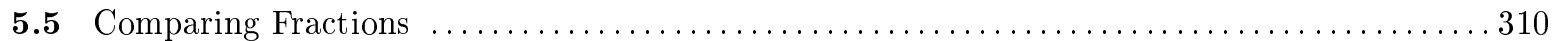

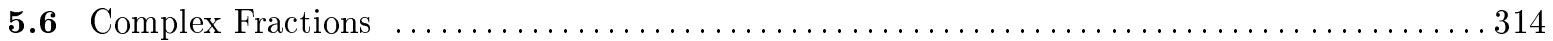

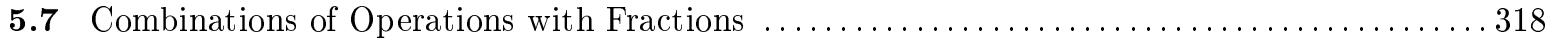

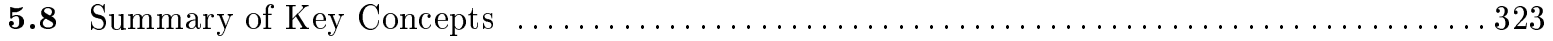

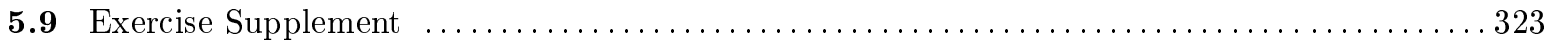

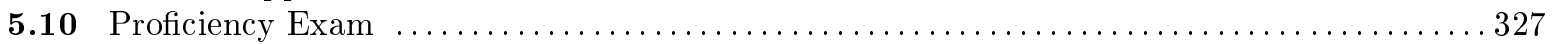

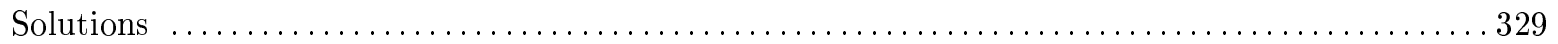

6 Decimals

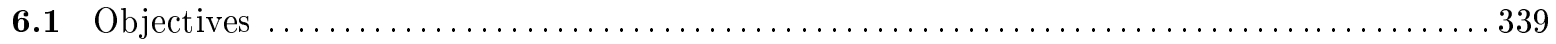

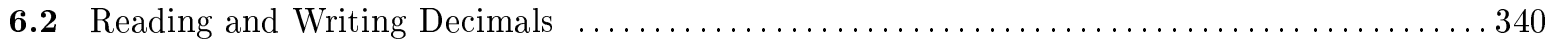

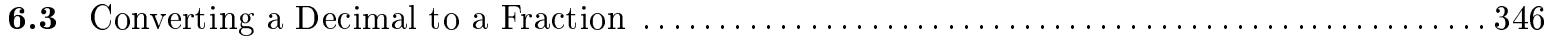

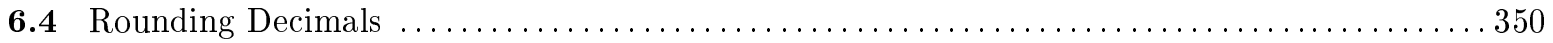

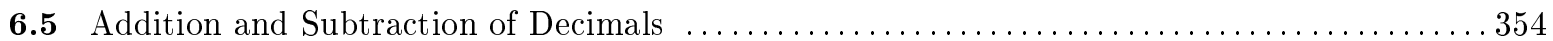

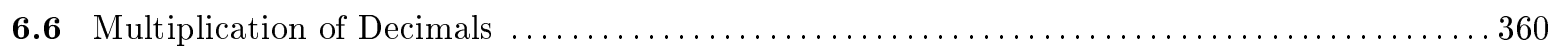

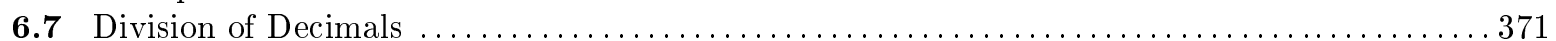

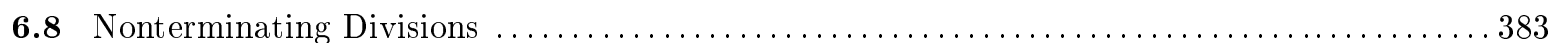

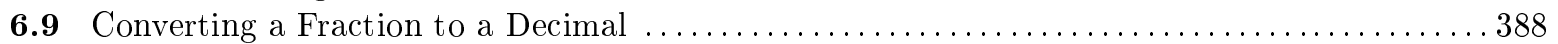

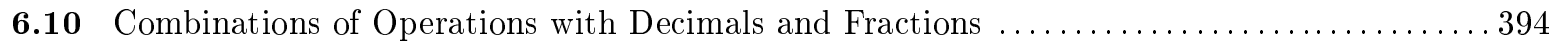

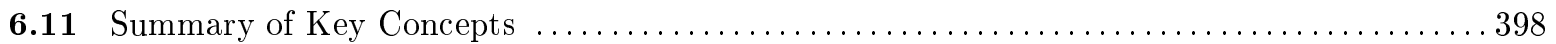

6.12 Exercise Supplement ................................................... 399

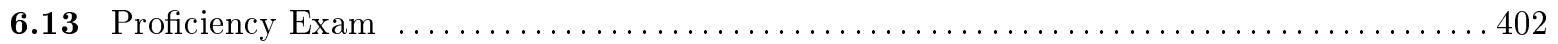

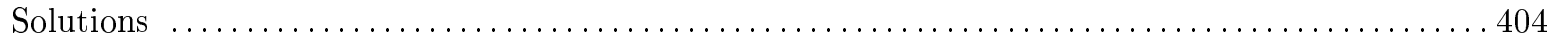

7 Ratios and Rates

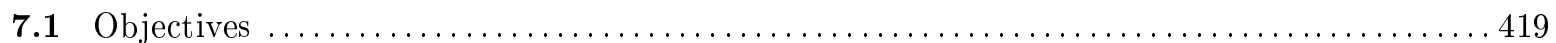

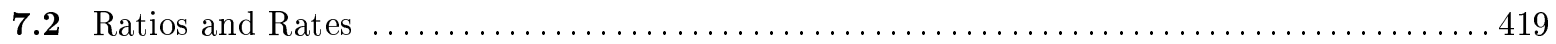

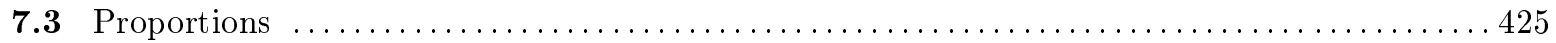

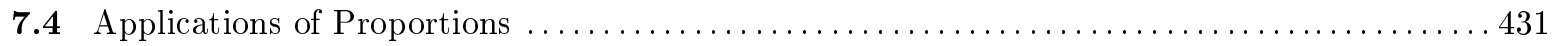

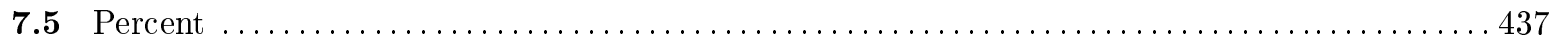

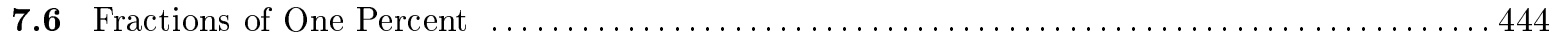

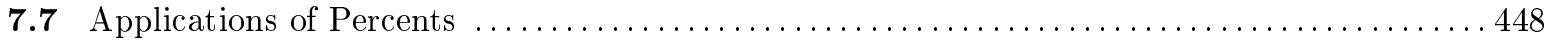

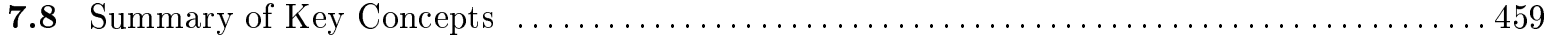

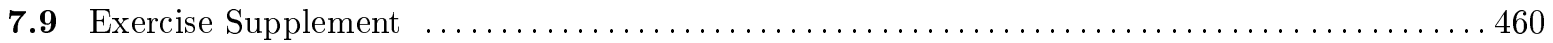

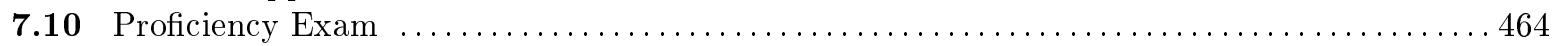

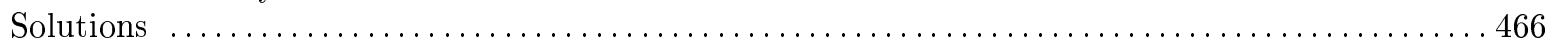

\section{Techniques of Estimation}

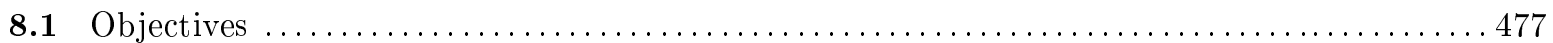

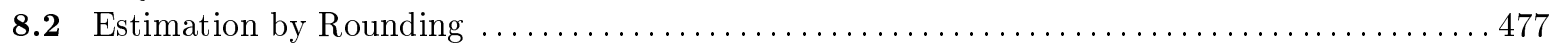

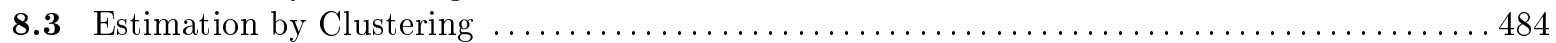

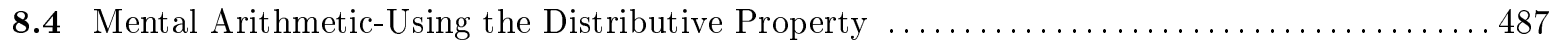

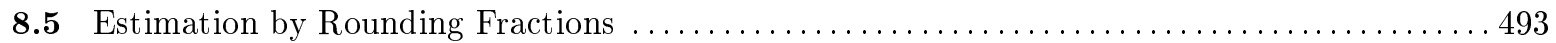

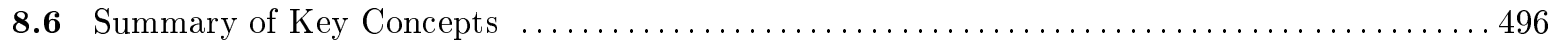

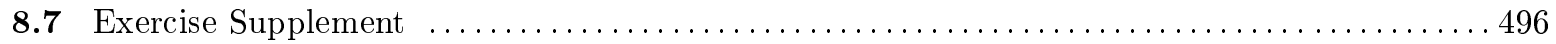

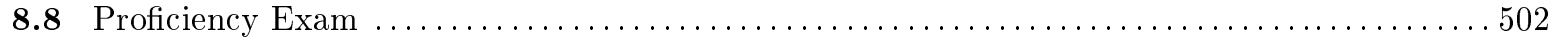




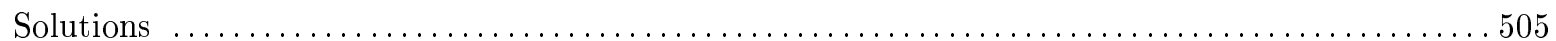

9 Measurement and Geometry

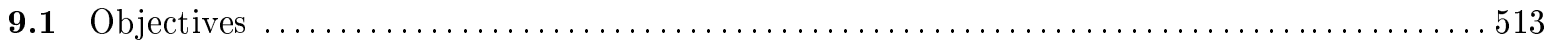

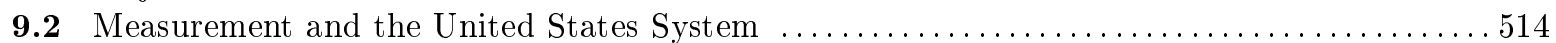

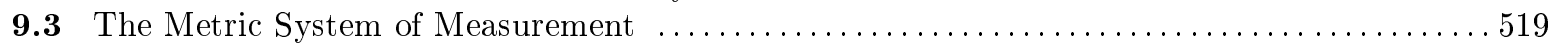

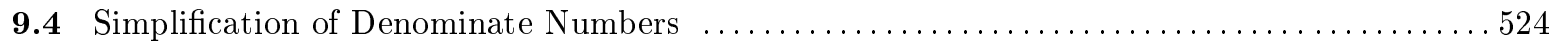

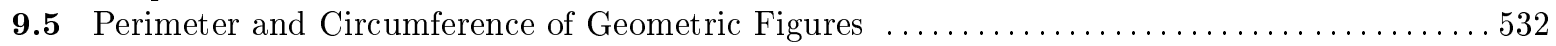

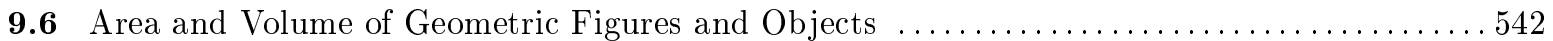

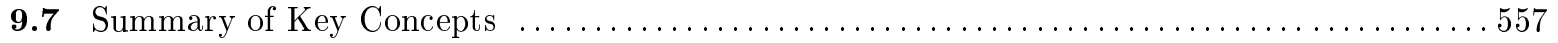

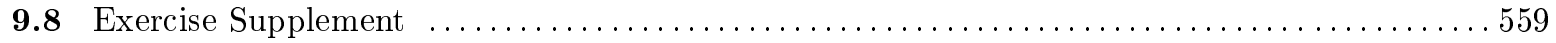

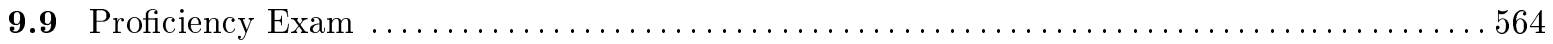

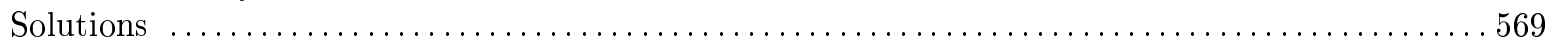

10 Signed Numbers

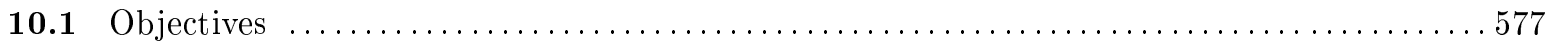

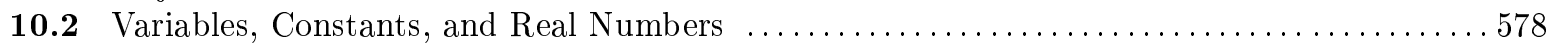

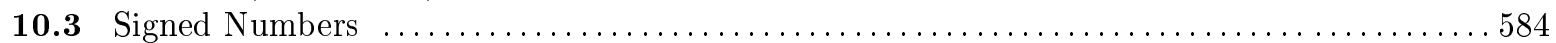

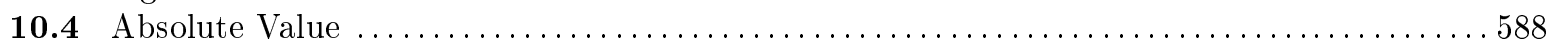

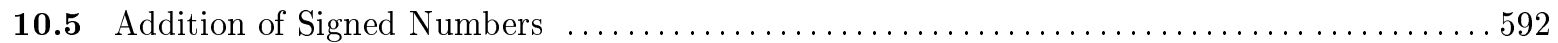

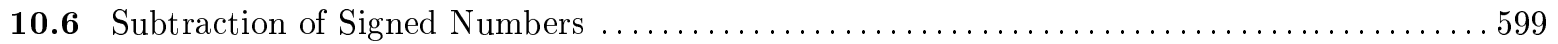

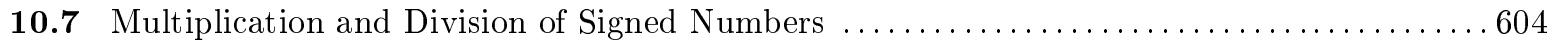

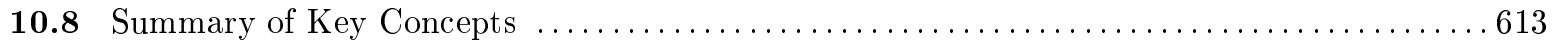

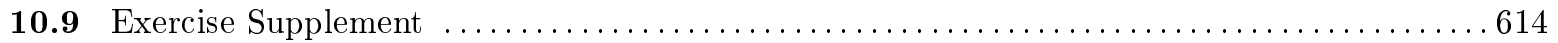

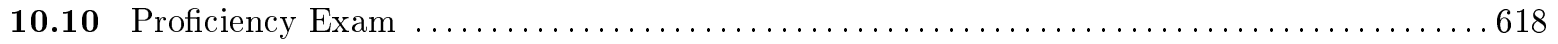

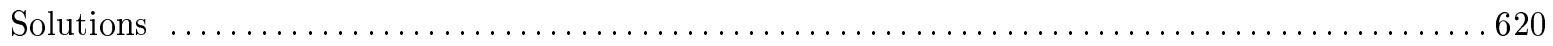

11 Algebraic Expressions and Equations

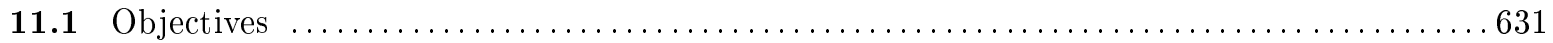

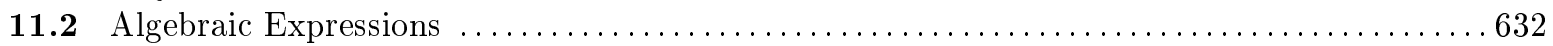

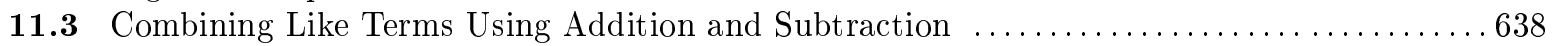

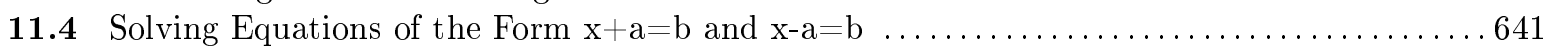

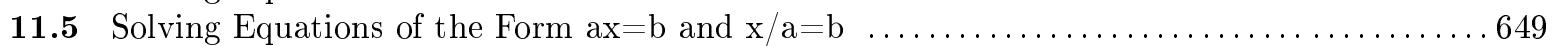

11.6 Applications I: Translating Words to Mathematical Symbols $\ldots \ldots \ldots \ldots \ldots \ldots \ldots \ldots \ldots \ldots 67$

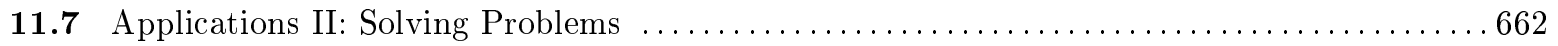

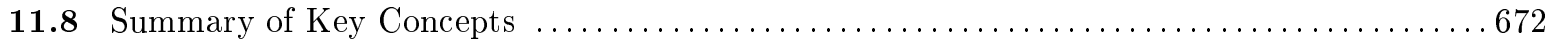

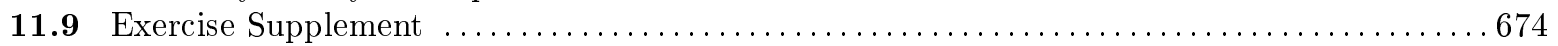

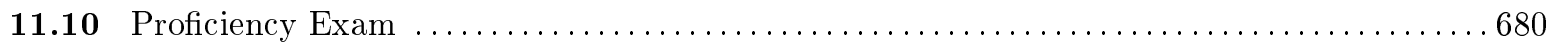

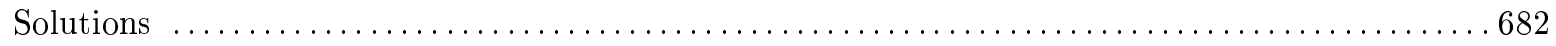

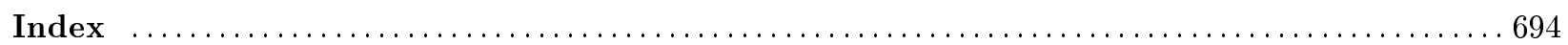

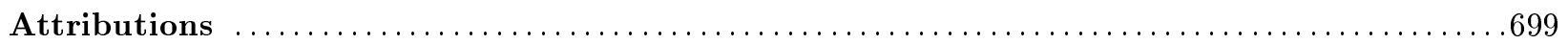


Available for free at Connexions <http://cnx.org/content/col10615/1.4> 


\section{Preface $^{1}$}

To the next generation of explorers: Kristi, BreAnne, Lindsey, Randi, Piper, Meghan, Wyatt, Lara, Mason, and Sheanna.

Fundamentals of Mathematics is a work text that covers the traditional topics studied in a modern prealgebra course, as well as the topics of estimation, elementary analytic geometry, and introductory algebra. It is intended for students who

1. have had a previous course in prealgebra,

2. wish to meet the prerequisite of a higher level course such as elementary algebra, and

3. need to review fundamental mathematical concepts and techniques.

This text will help the student develop the insight and intuition necessary to master arithmetic techniques and manipulative skills. It was written with the following main objectives:

1. to provide the student with an understandable and usable source of information,

2. to provide the student with the maximum opportunity to see that arithmetic concepts and techniques are logically based,

3. to instill in the student the understanding and intuitive skills necessary to know how and when to use particular arithmetic concepts in subsequent material, courses, and nonclassroom situations, and

4. to give the student the ability to correctly interpret arithmetically obtained results.

We have tried to meet these objectives by presenting material dynamically, much the way an instructor might present the material visually in a classroom. (See the development of the concept of addition and subtraction of fractions in Section 5.3, for example.) Intuition and understanding are some of the keys to creative thinking; we believe that the material presented in this text will help the student realize that mathematics is a creative subject.

This text can be used in standard lecture or self-paced classes. To help meet our objectives and to make the study of prealgebra a pleasant and rewarding experience, Fundamentals of Mathematics is organized as follows.

\section{Pedagogical Features}

The work text format gives the student space to practice mathematical skills with ready reference to sample problems. The chapters are divided into sections, and each section is a complete treatment of a particular topic, which includes the following features:

- Section Overview

- Sample Sets

- Practice Sets

${ }^{1}$ This content is available online at $<\mathrm{http}: / / \mathrm{cnx} . o r g /$ content $/ \mathrm{m} 18884 / 1.4 />$.

Available for free at Connexions <http://cnx.org/content/col10615/1.4> 
- Section Exercises

- Exercises for Review

- Answers to Practice Sets

The chapters begin with Objectives and end with a Summary of Key Concepts, an Exercise Supplement, and a Proficiency Exam.

\section{Objectives}

Each chapter begins with a set of objectives identifying the material to be covered. Each section begins with an overview that repeats the objectives for that particular section. Sections are divided into subsections that correspond to the section objectives, which makes for easier reading.

\section{Sample Sets}

Fundamentals of Mathematics contains examples that are set off in boxes for easy reference. The examples are referred to as Sample Sets for two reasons:

1. They serve as a representation to be imitated, which we believe will foster understanding of mathematical concepts and provide experience with mathematical techniques.

2. Sample Sets also serve as a preliminary representation of problem-solving techniques that may be used to solve more general and more complicated problems.

The examples have been carefully chosen to illustrate and develop concepts and techniques in the most instructive, easily remembered way. Concepts and techniques preceding the examples are introduced at a level below that normally used in similar texts and are thoroughly explained, assuming little previous knowledge.

\section{Practice Sets}

A parallel Practice Set follows each Sample Set, which reinforces the concepts just learned. There is adequate space for the student to work each problem directly on the page.

\section{Answers to Practice Sets}

The Answers to Practice Sets are given at the end of each section and can be easily located by referring to the page number, which appears after the last Practice Set in each section.

\section{Section Exercises}

The exercises at the end of each section are graded in terms of difficulty, although they are not grouped into categories. There is an ample number of problems, and after working through the exercises, the student will be capable of solving a variety of challenging problems.

The problems are paired so that the odd-numbered problems are equivalent in kind and difficulty to the even-numbered problems. Answers to the odd-numbered problems are provided at the back of the book.

\section{Exercises for Review}

This section consists of five problems that form a cumulative review of the material covered in the preceding sections of the text and is not limited to material in that chapter. The exercises are keyed by section for easy reference. Since these exercises are intended for review only, no work space is provided.

\section{Summary of Key Concepts}

A summary of the important ideas and formulas used throughout the chapter is included at the end of each chapter. More than just a list of terms, the summary is a valuable tool that reinforces concepts in preparation for the Proficiency Exam at the end of the chapter, as well as future exams. The summary keys each item to the section of the text where it is discussed. 


\section{Exercise Supplement}

In addition to numerous section exercises, each chapter includes approximately 100 supplemental problems, which are referenced by section. Answers to the odd-numbered problems are included in the back of the book.

\section{Proficiency Exam}

Each chapter ends with a Proficiency Exam that can serve as a chapter review or evaluation. The Proficiency Exam is keyed to sections, which enables the student to refer back to the text for assistance. Answers to all the problems are included in the Answer Section at the end of the book.

\section{Content}

The writing style used in Fundamentals of Mathematics is informal and friendly, offering a straightforward approach to prealgebra mathematics. We have made a deliberate effort not to write another text that minimizes the use of words because we believe that students can best study arithmetic concepts and understand arithmetic techniques by using words and symbols rather than symbols alone. It has been our experience that students at the prealgebra level are not nearly experienced enough with mathematics to understand symbolic explanations alone; they need literal explanations to guide them through the symbols.

We have taken great care to present concepts and techniques so they are understandable and easily remembered. After concepts have been developed, students are warned about common pitfalls. We have tried to make the text an information source accessible to prealgebra students.

\section{Addition and Subtraction of Whole Numbers}

This chapter includes the study of whole numbers, including a discussion of the Hindu-Arabic numeration and the base ten number systems. Rounding whole numbers is also presented, as are the commutative and associative properties of addition.

\section{Multiplication and Division of Whole Numbers}

The operations of multiplication and division of whole numbers are explained in this chapter. Multiplication is described as repeated addition. Viewing multiplication in this way may provide students with a visualization of the meaning of algebraic terms such as $8 x$ when they start learning algebra. The chapter also includes the commutative and associative properties of multiplication.

\section{Exponents, Roots, and Factorizations of Whole Numbers}

The concept and meaning of the word root is introduced in this chapter. A method of reading root notation and a method of determining some common roots, both mentally and by calculator, is then presented. We also present grouping symbols and the order of operations, prime factorization of whole numbers, and the greatest common factor and least common multiple of a collection of whole numbers.

\section{Introduction to Fractions and Multiplication and Division of Fractions}

We recognize that fractions constitute one of the foundations of problem solving. We have, therefore, given a detailed treatment of the operations of multiplication and division of fractions and the logic behind these operations. We believe that the logical treatment and many practice exercises will help students retain the information presented in this chapter and enable them to use it as a foundation for the study of rational expressions in an algebra course. 


\section{Addition and Subtraction of Fractions, Comparing Fractions, and Complex Fractions}

A detailed treatment of the operations of addition and subtraction of fractions and the logic behind these operations is given in this chapter. Again, we believe that the logical treatment and many practice exercises will help students retain the information, thus enabling them to use it in the study of rational expressions in an algebra course. We have tried to make explanations dynamic. A method for comparing fractions is introduced, which gives the student another way of understanding the relationship between the words denominator and denomination. This method serves to show the student that it is sometimes possible to compare two different types of quantities. We also study a method of simplifying complex fractions and of combining operations with fractions.

\section{Decimals}

The student is introduced to decimals in terms of the base ten number system, fractions, and digits occurring to the right of the units position. A method of converting a fraction to a decimal is discussed. The logic behind the standard methods of operating on decimals is presented and many examples of how to apply the methods are given. The word of as related to the operation of multiplication is discussed. Nonterminating divisions are examined, as are combinations of operations with decimals and fractions.

\section{Ratios and Rates}

We begin by defining and distinguishing the terms ratio and rate. The meaning of proportion and some applications of proportion problems are described. Proportion problems are solved using the "Five-Step Method." We hope that by using this method the student will discover the value of introducing a variable as a first step in problem solving and the power of organization. The chapter concludes with discussions of percent, fractions of one percent, and some applications of percent.

\section{Techniques of Estimation}

One of the most powerful problem-solving tools is a knowledge of estimation techniques. We feel that estimation is so important that we devote an entire chapter to its study. We examine three estimation techniques: estimation by rounding, estimation by clustering, and estimation by rounding fractions. We also include a section on the distributive property, an important algebraic property.

\section{Measurement and Geometry}

This chapter presents some of the techniques of measurement in both the United States system and the metric system. Conversion from one unit to another (in a system) is examined in terms of unit fractions. A discussion of the simplification of denominate numbers is also included. This discussion helps the student understand more clearly the association between pure numbers and dimensions. The chapter concludes with a study of perimeter and circumference of geometric figures and area and volume of geometric figures and objects.

\section{Signed Numbers}

A look at algebraic concepts and techniques is begun in this chapter. Basic to the study of algebra is a working knowledge of signed numbers. Definitions of variables, constants, and real numbers are introduced. We then distinguish between positive and negative numbers, learn how to read signed numbers, and examine the origin and use of the double-negative property of real numbers. The concept of absolute value is presented both geometrically (using the number line) and algebraically. The algebraic definition is followed by an interpretation of its meaning and several detailed examples of its use. Addition, subtraction, multiplication, and division of signed numbers are presented first using the number line, then with absolute value. 


\section{Algebraic Expressions and Equations}

The student is introduced to some elementary algebraic concepts and techniques in this final chapter. Algebraic expressions and the process of combining like terms are discussed in Section 11.2 and Section 11.3. The method of combining like terms in an algebraic expression is explained by using the interpretation of multiplication as a description of repeated addition (as in Section 2.1). 
Available for free at Connexions <http://cnx.org/content/col10615/1.4> 


\section{Acknowledgements ${ }^{2}$}

Many extraordinarily talented people are responsible for helping to create this text. We wish to acknowledge the efforts and skill of the following mathematicians. Their contributions have been invaluable.

Barbara Conway, Berkshire Community College

Bill Hajdukiewicz, Miami-Dade Community College

Virginia Hamilton, Shawnee State University

David Hares, El Centro College

Norman Lee, Ball State University

Ginger Y. Manchester, Hinds Junior College

John R. Martin, Tarrant County Junior College

Shelba Mormon, Northlake College

Lou Ann Pate, Pima Community College

Gus Pekara, Oklahoma City Community College

David Price, Tarrant County Junior College

David Schultz, Virginia Western Community College

Sue S. Watkins, Lorain County Community College

Elizabeth M. Wayt, Tennessee State University

Prentice E. Whitlock, Jersey City State College

Thomas E. Williamson, Montclair State College

Special thanks to the following individuals for their careful accuracy reviews of manuscript, galleys, and page proofs: Steve Blasberg, West Valley College; Wade Ellis, Sr., University of Michigan; John R. Martin, Tarrant County Junior College; and Jane Ellis. We would also like to thank Amy Miller and Guy Sanders, Branham High School.

Our sincere thanks to Debbie Wiedemann for her encouragement, suggestions concerning psychobiological examples, proofreading much of the manuscript, and typing many of the section exercises; Sandi Wiedemann for collating the annotated reviews, counting the examples and exercises, and untiring use of "white-out"; and Jane Ellis for solving and typing all of the exercise solutions.

We thank the following people for their excellent work on the various ancillary items that accompany Fundamentals of Mathematics: Steve Blasberg, West Valley College; Wade Ellis, Sr., University of Michigan; and Jane Ellis ( Instructor's Manual); John R. Martin, Tarrant County Junior College (Student Solutions Manual and Study Guide); Virginia Hamilton, Shawnee State University (Computerized Test Bank); Patricia Morgan, San Diego State University (Prepared Tests); and George W. Bergeman, Northern Virginia Community College (Maxis Interactive Software).

We also thank the talented people at Saunders College Publishing whose efforts made this text run smoothly and less painfully than we had imagined. Our particular thanks to Bob Stern, Mathematics Editor, Ellen Newman, Developmental Editor, and Janet Nuciforo, Project Editor. Their guidance, suggestions, open

\footnotetext{
${ }^{2}$ This content is available online at $<\mathrm{http}: / / \mathrm{cnx}$. org $/$ content $/ \mathrm{m} 34775 / 1.2 />$. 
minds to our suggestions and concerns, and encouragement have been extraordinarily helpful. Although there were times we thought we might be permanently damaged from rereading and rewriting, their efforts have improved this text immensely. It is a pleasure to work with such high-quality professionals.

Denny Burzynski

Wade Ellis, Jr.

San Jose, California

December 1988

I would like to thank Doug Campbell, Ed Lodi, and Guy Sanders for listening to my frustrations and encouraging me on. Thanks also go to my cousin, David Raffety, who long ago in Sequoia National Forest told me what a differential equation is.

Particular thanks go to each of my colleagues at West Valley College. Our everyday conversations regarding mathematics instruction have been of the utmost importance to the development of this text and to my teaching career.

D.B. 


\section{Chapter 1}

\section{Addition and Subtraction of Whole Numbers}

\subsection{Objectives ${ }^{1}$}

After completing this chapter, you should

Whole Numbers (Section 1.2)

- know the difference between numbers and numerals

- know why our number system is called the Hindu-Arabic numeration system

- understand the base ten positional number system

- be able to identify and graph whole numbers

\section{Reading and Writing Whole Numbers (Section 1.3)}

- be able to read and write a whole number

\section{Rounding Whole Numbers (Section 1.4)}

- understand that rounding is a method of approximation

- be able to round a whole number to a specified position

\section{Addition of Whole Numbers (Section 1.5)}

- understand the addition process

- be able to add whole numbers

- be able to use the calculator to add one whole number to another

\section{Subtraction of Whole Numbers (Section 1.6)}

- understand the subtraction process

- be able to subtract whole numbers

- be able to use a calculator to subtract one whole number from another whole number

\section{Properties of Addition (Section 1.7)}

- understand the commutative and associative properties of addition

- understand why 0 is the additive identity

\footnotetext{
${ }^{1}$ This content is available online at $<$ http://cnx.org/content/m18885/1.4/>.

Available for free at Connexions $<$ http://cnx.org/content/col10615/1.4 $>$
} 


\subsection{Whole Numbers ${ }^{2}$}

\subsubsection{Section Overview}

- Numbers and Numerals

- The Hindu-Arabic Numeration System

- The Base Ten Positional Number System

- Whole Numbers

- Graphing Whole Numbers

\subsubsection{Numbers and Numerals}

We begin our study of introductory mathematics by examining its most basic building block, the number.

\section{Number}

A number is a concept. It exists only in the mind.

The earliest concept of a number was a thought that allowed people to mentally picture the size of some collection of objects. To write down the number being conceptualized, a numeral is used.

\section{Numeral}

A numeral is a symbol that represents a number.

In common usage today we do not distinguish between a number and a numeral. In our study of introductory mathematics, we will follow this common usage.

\subsubsection{Sample Set A}

The following are numerals. In each case, the first represents the number four, the second represents the number one hundred twenty-three, and the third, the number one thousand five. These numbers are represented in different ways.

- Hindu-Arabic numerals

4, 123, 1005

- Roman numerals

IV, CXXIII, MV

- Egyptian numerals

I I | |,

Strokes
$9 \cap \cap 111$, Coiled rope, heel bones, and strokes

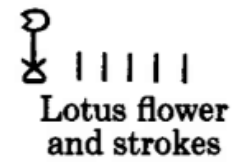

\subsubsection{Practice Set A}

Exercise 1.2.1

(Solution on p. 76.)

Do the phrases "four," "one hundred twenty-three," and "one thousand five" qualify as numerals?

Yes or no?

\footnotetext{
${ }^{2}$ This content is available online at $<$ http://cnx.org/content $/ \mathrm{m} 34795 / 1.5 />$.
} 


\subsubsection{The Hindu-Arabic Numeration System}

\section{Hindu-Arabic Numeration System}

Our society uses the Hindu-Arabic numeration system. This system of numeration began shortly before the third century when the Hindus invented the numerals

\section{9}

\section{Leonardo Fibonacci}

About a thousand years later, in the thirteenth century, a mathematician named Leonardo Fibonacci of Pisa introduced the system into Europe. It was then popularized by the Arabs. Thus, the name, Hindu-Arabic numeration system.

\subsubsection{The Base Ten Positional Number System}

\section{Digits}

The Hindu-Arabic numerals 0123456789 are called digits. We can form any number in the number system by selecting one or more digits and placing them in certain positions. Each position has a particular value. The Hindu mathematician who devised the system about A.D. 500 stated that "from place to place each is ten times the preceding."

\section{Base Ten Positional Systems}

It is for this reason that our number system is called a positional number system with base ten.

\section{Commas}

When numbers are composed of more than three digits, commas are sometimes used to separate the digits into groups of three.

\section{Periods}

These groups of three are called periods and they greatly simplify reading numbers.

In the Hindu-Arabic numeration system, a period has a value assigned to each or its three positions, and the values are the same for each period. The position values are

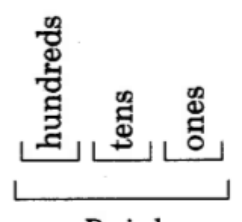

Period

Thus, each period contains a position for the values of one, ten, and hundred. Notice that, in looking from right to left, the value of each position is ten times the preceding. Each period has a particular name.

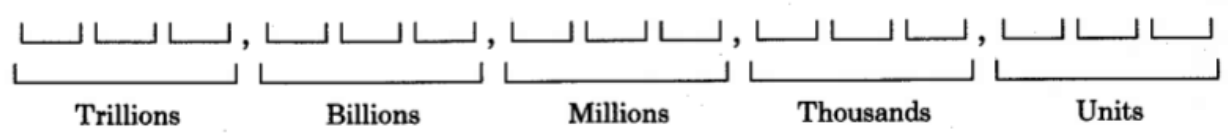

As we continue from right to left, there are more periods. The five periods listed above are the most common, and in our study of introductory mathematics, they are sufficient.

The following diagram illustrates our positional number system to trillions. (There are, to be sure, other periods.) 


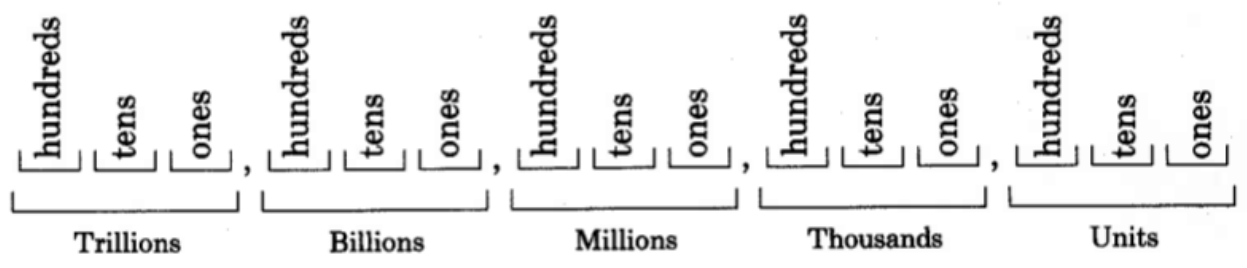

In our positional number system, the value of a digit is determined by its position in the number.

\subsubsection{Sample Set B}

\section{Example 1.1}

Find the value of 6 in the number 7,261 .

Since 6 is in the tens position of the units period, its value is 6 tens.

6 tens $=60$

\section{Example 1.2}

Find the value of 9 in the number $86,932,106,005$.

Since 9 is in the hundreds position of the millions period, its value is 9 hundred millions.

9 hundred millions $=9$ hundred million

\section{Example 1.3}

Find the value of 2 in the number 102,001.

Since 2 is in the ones position of the thousands period, its value is 2 one thousands.

2 one thousands $=2$ thousand

\subsubsection{Practice Set $B$}

Exercise 1.2.2

(Solution on p. 76.)

Find the value of 5 in the number 65,000 .

Exercise 1.2.3

(Solution on p. 76.)

Find the value of 4 in the number 439,997,007,010.

Exercise 1.2.4

(Solution on p. 76.)

Find the value of 0 in the number 108.

\subsubsection{Whole Numbers}

\section{Whole Numbers}

Numbers that are formed using only the digits

0123456789

are called whole numbers. They are

$0,1,2,3,4,5,6,7,8,9,10,11,12,13,14,15, \ldots$

The three dots at the end mean "and so on in this same pattern." 


\subsubsection{Graphing Whole Numbers}

\section{Number Line}

Whole numbers may be visualized by constructing a number line. To construct a number line, we simply draw a straight line and choose any point on the line and label it 0.

\section{Origin}

This point is called the origin. We then choose some convenient length, and moving to the right, mark off consecutive intervals (parts) along the line starting at 0 . We label each new interval endpoint with the next whole number.

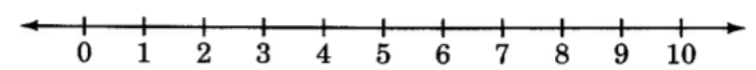

\section{Graphing}

We can visually display a whole number by drawing a closed circle at the point labeled with that whole number. Another phrase for visually displaying a whole number is graphing the whole number. The word graph means to "visually display."

\subsubsection{Sample Set C}

Example 1.4

Graph the following whole numbers: $3,5,9$.

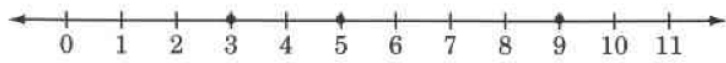

\section{Example 1.5}

Specify the whole numbers that are graphed on the following number line. The break in the number line indicates that we are aware of the whole numbers between 0 and 106, and 107 and 872, but we are not listing them due to space limitations.

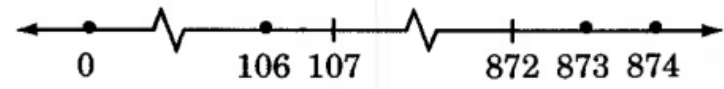

The numbers that have been graphed are

$0,106,873,874$

\subsubsection{Practice Set $\mathrm{C}$}

Exercise 1.2.5

(Solution on p. 76.)

Graph the following whole numbers: $46,47,48,325,327$.

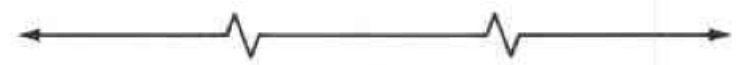

Exercise 1.2.6

(Solution on p. 76.)

Specify the whole numbers that are graphed on the following number line.

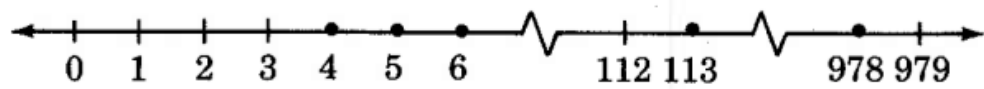

A line is composed of an endless number of points. Notice that we have labeled only some of them. As we proceed, we will discover new types of numbers and determine their location on the number line. 


\subsubsection{Exercises}

Exercise 1.2.7

(Solution on p. 76.)

What is a number?

Exercise 1.2.8

What is a numeral?

Exercise 1.2.9

Does the word "eleven" qualify as a numeral?

(Solution on p. 76.)

Exercise 1.2.10

How many different digits are there?

Exercise 1.2.11

(Solution on p. 76.)

Our number system, the Hindu-Arabic number system, is a number system with base

Exercise 1.2.12

Numbers composed of more than three digits are sometimes separated into groups of three by commas. These groups of three are called

Exercise 1.2.13

(Solution on p. 76.)

In our number system, each period has three values assigned to it. These values are the same for each period. From right to left, what are they?

Exercise 1.2.14

Each period has its own particular name. From right to left, what are the names of the first four?

Exercise 1.2.15

In the number 841 , how many tens are there?

(Solution on p. 76.)

Exercise 1.2.16

In the number 3,392 , how many ones are there?

Exercise 1.2.17

In the number 10,046 , how many thousands are there?

(Solution on p. 76.)

Exercise 1.2.18

In the number $779,844,205$, how many ten millions are there?

Exercise 1.2.19

In the number 65,021 , how many hundred thousands are there?

(Solution on p. 76.)

For following problems, give the value of the indicated digit in the given number.

Exercise 1.2.20

5 in 599

Exercise 1.2.21

1 in 310,406

Exercise 1.2.22

9 in 29,827

Exercise 1.2.23

6 in $52,561,001,100$

(Solution on p. 76.)

Exercise 1.2.24

Write a two-digit number that has an eight in the tens position.

Exercise 1.2.25

(Solution on p. 76.)

Write a four-digit number that has a one in the thousands position and a zero in the ones position.

Exercise 1.2.26

How many two-digit whole numbers are there? 
Exercise 1.2.27

(Solution on p. 76.)

How many three-digit whole numbers are there?

Exercise 1.2.28

How many four-digit whole numbers are there?

Exercise 1.2.29

(Solution on p. 76.)

Is there a smallest whole number? If so, what is it?

Exercise 1.2.30

Is there a largest whole number? If so, what is it?

Exercise 1.2.31

(Solution on p. 76.)

Another term for "visually displaying" is

Exercise 1.2.32

The whole numbers can be visually displayed on a

Exercise 1.2.33

(Solution on p. 76.)

Graph (visually display) the following whole numbers on the number line below: $0,1,31,34$.

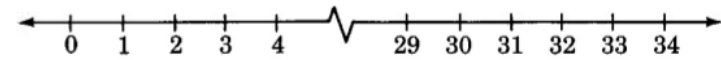

\section{Exercise 1.2.34}

Construct a number line in the space provided below and graph (visually display) the following whole numbers: 84, 85, 901, 1006, 1007.

Exercise 1.2.35

(Solution on p. 76.)

Specify, if any, the whole numbers that are graphed on the following number line.

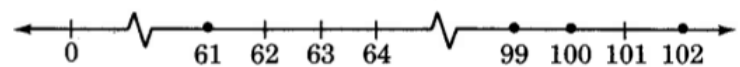

\section{Exercise 1.2.36}

Specify, if any, the whole numbers that are graphed on the following number line.

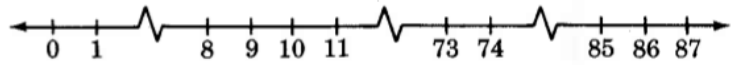

\subsection{Reading and Writing Whole Numbers ${ }^{3}$}

\subsubsection{Section Overview}

- Reading Whole Numbers

- Writing Whole Numbers

Because our number system is a positional number system, reading and writing whole numbers is quite simple.

\footnotetext{
${ }^{3}$ This content is available online at $<$ http://cnx.org/content $/ \mathrm{m} 34778 / 1.5 />$.
} 


\subsubsection{Reading Whole Numbers}

To convert a number that is formed by digits into a verbal phrase, use the following method:

1. Beginning at the right and working right to left, separate the number into distinct periods by inserting commas every three digits.

2. Beginning at the left, read each period individually, saying the period name.

\subsubsection{Sample Set A}

Write the following numbers as words.

\section{Example 1.6}

Read 42958.

1. Beginning at the right, we can separate this number into distinct periods by inserting a comma between the 2 and 9 .

42,958

2. Beginning at the left, we read each period individually:

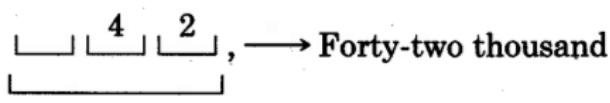

Thousands period

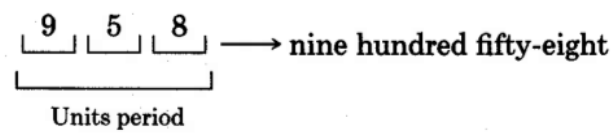

Forty-two thousand, nine hundred fifty-eight.

\section{Example 1.7}

Read 307991343

1. Beginning at the right, we can separate this number into distinct periods by placing commas between the 1 and 3 and the 7 and 9 .

$307,991,343$

2. Beginning at the left, we read each period individually.

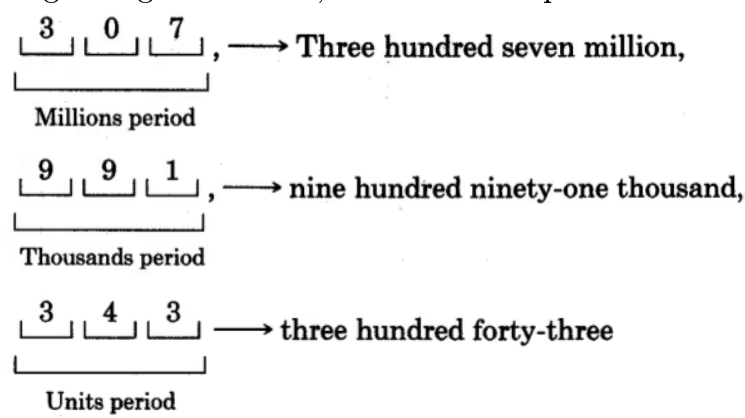

Three hundred seven million, nine hundred ninety-one thousand, three hundred forty-three.

\section{Example 1.8}

Read 36000000000001 .

1. Beginning at the right, we can separate this number into distinct periods by placing commas. $36,000,000,001$ 
2. Beginning at the left, we read each period individually.

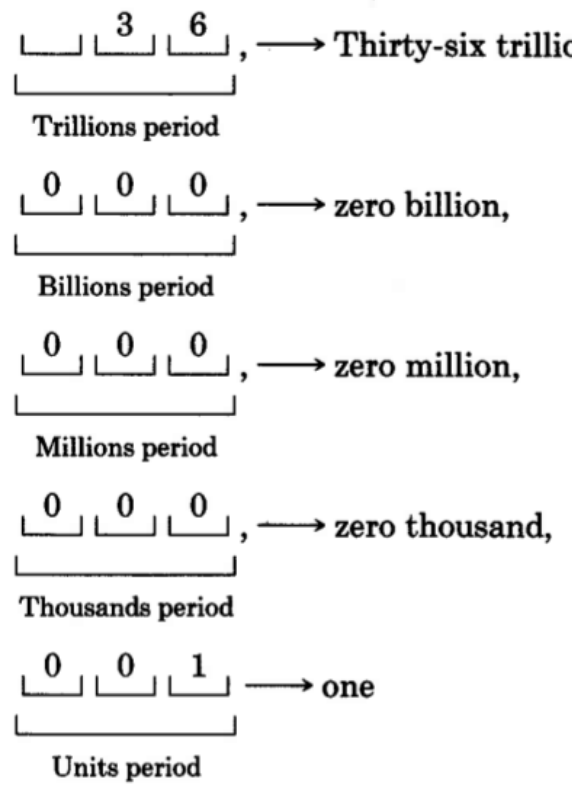

Thirty-six trillion, one.

\subsubsection{Practice Set A}

Write each number in words.

Exercise 1.3.1

(Solution on p. 76.)

12,542

Exercise 1.3.2

$101,074,003$

Exercise 1.3.3

$1,000,008$

(Solution on p. 76.)

(Solution on p. 76.)

\subsubsection{Writing Whole Numbers}

To express a number in digits that is expressed in words, use the following method:

1. Notice first that a number expressed as a verbal phrase will have its periods set off by commas.

2. Starting at the beginning of the phrase, write each period of numbers individually.

3. Using commas to separate periods, combine the periods to form one number.

\subsubsection{Sample Set B}

Write each number using digits.

Example 1.9

Seven thousand, ninety-two.

Using the comma as a period separator, we have 


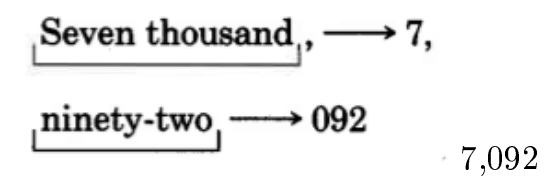

Example 1.10

Fifty billion, one million, two hundred thousand, fourteen.

Using the commas as period separators, we have

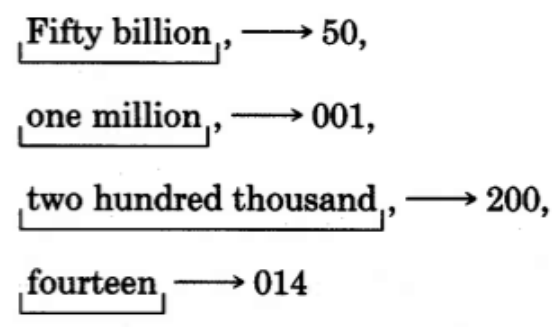

\section{Example 1.11}

Ten million, five hundred twelve.

The comma sets off the periods. We notice that there is no thousands period. We'll have to insert this ourselves.

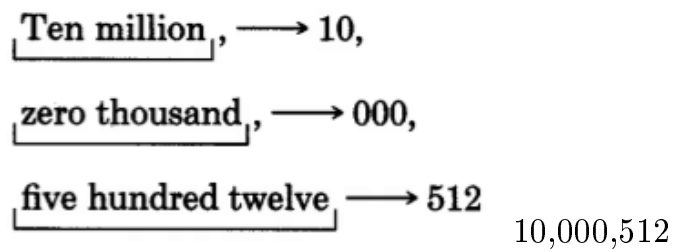

\subsubsection{Practice Set B}

Express each number using digits.

Exercise 1.3.4

(Solution on p. 77.)

One hundred three thousand, twenty-five.

Exercise 1.3.5

(Solution on p. 77.)

Six million, forty thousand, seven.

Exercise 1.3.6

(Solution on p. 77.)

Twenty trillion, three billion, eighty million, one hundred nine thousand, four hundred two.

Exercise 1.3.7

(Solution on p. 77.)

Eighty billion, thirty-five.

\subsubsection{Exercises}

For the following problems, write all numbers in words.

Exercise 1.3.8

(Solution on p. 77.)

912

Exercise 1.3.9

84 
Exercise 1.3.10

(Solution on p. 77.)

1491

Exercise 1.3.11

8601

Exercise 1.3.12

(Solution on p. 77.)

35,223

Exercise 1.3.13

71,006

Exercise 1.3.14

(Solution on p. 77.)

437,105

Exercise 1.3.15

201,040

Exercise 1.3.16

8,001,001

(Solution on p. 77.)

Exercise 1.3.17

$16,000,053$

Exercise 1.3.18

(Solution on p. 77.)

$770,311,101$

Exercise 1.3.19

$83,000,000,007$

Exercise 1.3.20

(Solution on p. 77.)

106,100,001,010

Exercise 1.3.21

$3,333,444,777$

Exercise 1.3.22

$800,000,800,000$

(Solution on p. 77.)

Exercise 1.3.23

A particular community college has 12,471 students enrolled.

Exercise 1.3.24

(Solution on p. 77.)

A person who watches 4 hours of television a day spends 1460 hours a year watching T.V.

Exercise 1.3.25

Astronomers believe that the age of the earth is about 4,500,000,000 years.

Exercise 1.3.26

(Solution on p. 77.)

Astronomers believe that the age of the universe is about 20,000,000,000 years.

Exercise 1.3.27

There are 9690 ways to choose four objects from a collection of 20 .

Exercise 1.3.28

(Solution on p. 77.)

If a 412 page book has about 52 sentences per page, it will contain about 21,424 sentences.

Exercise 1.3.29

In 1980 , in the United States, there was $\$ 1,761,000,000,000$ invested in life insurance.

Exercise 1.3.30

(Solution on p. 77.)

In 1979, there were 85,000 telephones in Alaska and 2,905,000 telephones in Indiana.

Exercise 1.3.31

In 1975, in the United States, it is estimated that 52,294,000 people drove to work alone.

Exercise 1.3.32

(Solution on p. 77.)

In 1980, there were 217 prisoners under death sentence that were divorced. 


\section{Exercise 1.3.33}

In 1979, the amount of money spent in the United States for regular-session college education was $\$ 50,721,000,000,000$.

Exercise 1.3.34

(Solution on p. 77.)

In 1981, there were 1,956,000 students majoring in business in U.S. colleges.

Exercise 1.3.35

In 1980, the average fee for initial and follow up visits to a medical doctors office was about $\$ 34$.

Exercise 1.3.36

(Solution on p. 77.)

In 1980, there were approximately 13,100 smugglers of aliens apprehended by the Immigration border patrol.

Exercise 1.3.37

In 1980, the state of West Virginia pumped 2,000,000 barrels of crude oil, whereas Texas pumped $975,000,000$ barrels.

Exercise 1.3.38

(Solution on p. 77.)

The 1981 population of Uganda was $12,630,000$ people.

Exercise 1.3.39

In 1981, the average monthly salary offered to a person with a Master's degree in mathematics was $\$ 1,685$.

For the following problems, write each number using digits.

Exercise 1.3.40

(Solution on p. 77.)

Six hundred eighty-one

Exercise 1.3.41

Four hundred ninety

Exercise 1.3.42

(Solution on p. 77.)

Seven thousand, two hundred one

Exercise 1.3.43

Nineteen thousand, sixty-five

Exercise 1.3.44

(Solution on p. 77.)

Five hundred twelve thousand, three

Exercise 1.3.45

Two million, one hundred thirty-three thousand, eight hundred fifty-nine

Exercise 1.3.46

(Solution on p. 77.)

Thirty-five million, seven thousand, one hundred one

Exercise 1.3.47

One hundred million, one thousand

Exercise 1.3.48

(Solution on p. 77.)

Sixteen billion, fifty-nine thousand, four

Exercise 1.3.49

Nine hundred twenty billion, four hundred seventeen million, twenty-one thousand

Exercise 1.3.50

(Solution on p. 78.)

Twenty-three billion

Exercise 1.3.51

Fifteen trillion, four billion, nineteen thousand, three hundred five

Exercise 1.3.52

(Solution on p. 78.)

One hundred trillion, one 


\subsubsection{Exercises for Review}

Exercise 1.3.53

(Section 1.2) How many digits are there?

Exercise 1.3.54

(Solution on p. 78.)

(Section 1.2) In the number 6,641, how many tens are there?

Exercise 1.3.55

(Section 1.2) What is the value of 7 in 44,763 ?

Exercise 1.3.56

(Section 1.2) Is there a smallest whole number? If so, what is it?

(Solution on p. 78.)

Exercise 1.3.57

(Section 1.2) Write a four-digit number with a 9 in the tens position.

\subsection{Rounding Whole Numbers ${ }^{4}$}

\subsubsection{Section Overview}

- Rounding as an Approximation

- The Method of Rounding Numbers

\subsubsection{Rounding as an Approximation}

A primary use of whole numbers is to keep count of how many objects there are in a collection. Sometimes we're only interested in the approximate number of objects in the collection rather than the precise number. For example, there are approximately 20 symbols in the collection below.

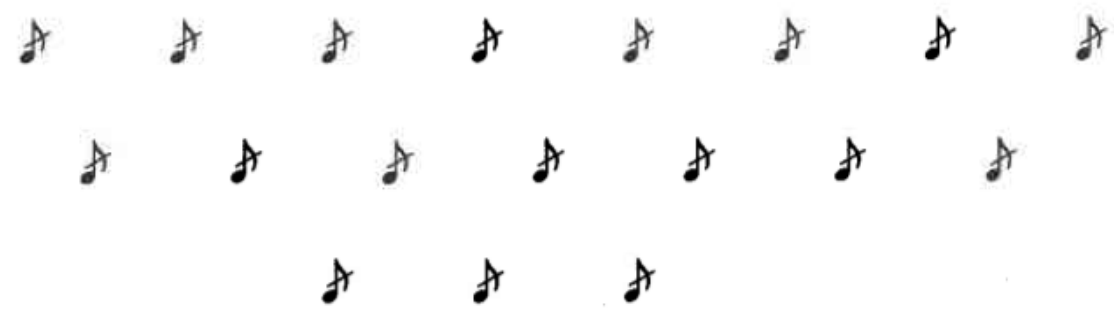

The precise number of symbols in the above collection is 18 .

\section{Rounding}

We often approximate the number of objects in a collection by mentally seeing the collection as occurring in groups of tens, hundreds, thousands, etc. This process of approximation is called rounding. Rounding is very useful in estimation. We will study estimation in Chapter 8.

When we think of a collection as occurring in groups of tens, we say we're rounding to the nearest ten. When we think of a collection as occurring in groups of hundreds, we say we're rounding to the nearest hundred. This idea of rounding continues through thousands, ten thousands, hundred thousands, millions, etc.

The process of rounding whole numbers is illustrated in the following examples.

\section{Example 1.12}

Round 67 to the nearest ten.

\footnotetext{
${ }^{4}$ This content is available online at $<$ http://cnx.org/content $/ \mathrm{m} 34780 / 1.3 />$.
} 
On the number line, 67 is more than halfway from 60 to 70 . The digit immediately to the right of the tens digit, the round-off digit, is the indicator for this.

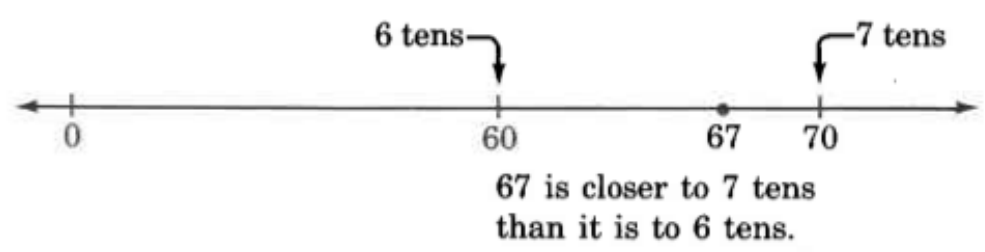

est ten, is 70 .

Thus, 67 , rounded to the near-

Example 1.13

Round 4,329 to the nearest hundred.

On the number line, 4,329 is less than halfway from 4,300 to 4,400 . The digit to the immediate right of the hundreds digit, the round-off digit, is the indicator.

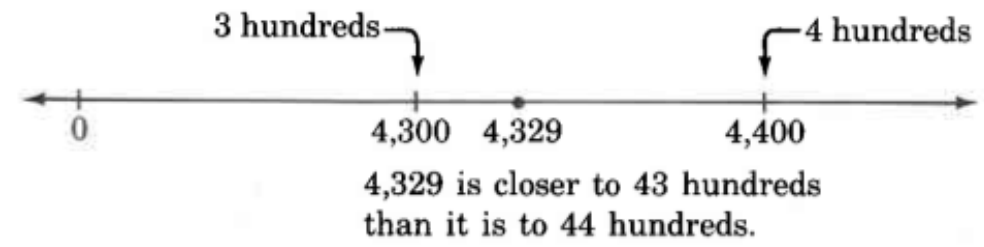

nearest hundred is 4,300 .

Thus, 4,329, rounded to the

\section{Example 1.14}

Round 16,500 to the nearest thousand.

On the number line, 16,500 is exactly halfway from 16,000 to 17,000 .

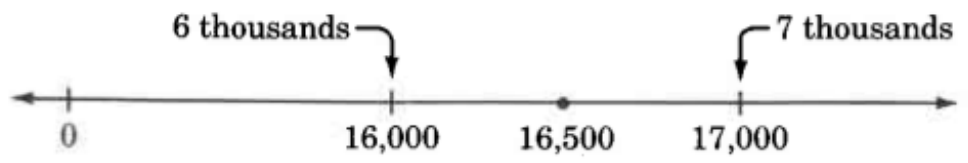

By convention, when the number to be rounded is exactly halfway between two numbers, it is rounded to the higher number.

Thus, 16,500 , rounded to the nearest thousand, is 17,000 .

\section{Example 1.15}

A person whose salary is $\$ 41,450$ per year might tell a friend that she makes $\$ 41,000$ per year. She has rounded 41,450 to the nearest thousand. The number 41,450 is closer to 41,000 than it is to 42,000 .

\subsubsection{The Method of Rounding Whole Numbers}

From the observations made in the preceding examples, we can use the following method to round a whole number to a particular position.

1. Mark the position of the round-off digit.

2. Note the digit to the immediate right of the round-off digit. 
a. If it is less than 5 , replace it and all the digits to its right with zeros. Leave the round-off digit unchanged.

b. If it is 5 or larger, replace it and all the digits to its right with zeros. Increase the round-off digit by 1 .

\subsubsection{Sample Set A}

Use the method of rounding whole numbers to solve the following problems.

\section{Example 1.16}

Round 3,426 to the nearest ten.

1. We are rounding to the tens position. Mark the digit in the tens position

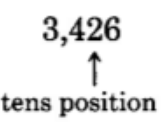

2. Observe the digit immediately to the right of the tens position. It is 6 . Since 6 is greater than 5 , we round up by replacing 6 with 0 and adding 1 to the digit in the tens position (the round-off position): $2+1=3$.

3,430

Thus, 3,426 rounded to the nearest ten is 3,430 .

\section{Example 1.17}

Round $9,614,018,007$ to the nearest ten million.

1. We are rounding to the nearest ten million.

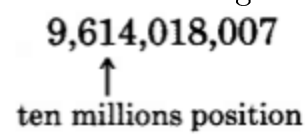

2. Observe the digit immediately to the right of the ten millions position. It is 4 . Since 4 is less than 5 , we round down by replacing 4 and all the digits to its right with zeros.

$9,610,000,000$

Thus, $9,614,018,007$ rounded to the nearest ten million is $9,610,000,000$.

\section{Example 1.18}

Round 148,422 to the nearest million.

1. Since we are rounding to the nearest million, we'll have to imagine a digit in the millions position. We'll write 148,422 as $0,148,422$.

\section{$0,148,422$$$
\uparrow
$$$$
\text { millions position }
$$

2. The digit immediately to the right is 1 . Since 1 is less than 5 , we'll round down by replacing it and all the digits to its right with zeros.

$0,000,000$

This number is 0 . 
Thus, 148,422 rounded to the nearest million is 0 .

Example 1.19

Round 397,000 to the nearest ten thousand.

1. We are rounding to the nearest ten thousand.

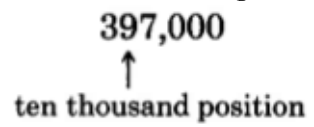

2. The digit immediately to the right of the ten thousand position is 7 . Since 7 is greater than 5, we round up by replacing 7 and all the digits to its right with zeros and adding 1 to the digit in the ten thousands position. But $9+1=10$ and we must carry the 1 to the next (the hundred thousands) position.

400,000

Thus, 397,000 rounded to the nearest ten thousand is 400,000 .

\subsubsection{Practice Set A}

Use the method of rounding whole numbers to solve each problem.

Exercise 1.4.1

(Solution on p. 78.)

Round 3387 to the nearest hundred.

Exercise 1.4.2

(Solution on p. 78.)

Round 26,515 to the nearest thousand.

Exercise 1.4.3

(Solution on p. 78.)

Round $30,852,900$ to the nearest million.

Exercise 1.4.4

(Solution on p. 78.)

Round 39 to the nearest hundred.

Exercise 1.4.5

(Solution on p. 78.)

Round 59,600 to the nearest thousand.

\subsubsection{Exercises}

For the following problems, complete the table by rounding each number to the indicated positions.

Exercise 1.4.6

(Solution on p. 78.) 1,642

\begin{tabular}{|l|l|l|l|}
\hline hundred & thousand & ten thousand & million \\
\hline & & & \\
\hline
\end{tabular}

Table 1.1

Exercise 1.4.7

5,221 


\begin{tabular}{|l|l|l|l|}
\hline hundred & thousand & ten thousand & million \\
\hline & & & \\
\hline
\end{tabular}

Table 1.2

Exercise 1.4.8

91,803

(Solution on p. 78.)

\begin{tabular}{|l|l|l|l|}
\hline Hundred & thousand & ten thousand & million \\
\hline & & & \\
\hline
\end{tabular}

Table 1.3

Exercise 1.4.9

106,007

\begin{tabular}{|l|l|l|l|}
\hline hundred & thousand & ten thousand & million \\
\hline & & & \\
\hline
\end{tabular}

Table 1.4

Exercise 1.4.10

208

(Solution on p. 78.)

\begin{tabular}{|l|l|l|l|}
\hline hundred & thousand & ten thousand & million \\
\hline & & & \\
\hline
\end{tabular}

Table 1.5

Exercise 1.4.11

199

\begin{tabular}{|l|l|l|l|}
\hline hundred & thousand & ten thousand & million \\
\hline & & & \\
\hline
\end{tabular}

Table 1.6

Exercise 1.4.12

863

(Solution on p. 78.)

\begin{tabular}{|l|l|l|l|}
\hline hundred & thousand & ten thousand & million \\
\hline & & & \\
\hline
\end{tabular}

Table 1.7

Available for free at Connexions $<$ http://cnx.org/content/col10615/1.4> 
Exercise 1.4.13 794

\begin{tabular}{|l|l|l|l|}
\hline hundred & thousand & ten thousand & million \\
\hline & & & \\
\hline
\end{tabular}

Table 1.8

Exercise 1.4.14 925

(Solution on p. 79.)

\begin{tabular}{|l|l|l|l|}
\hline hundred & thousand & ten thousand & million \\
\hline & & & \\
\hline
\end{tabular}

Table 1.9

Exercise 1.4.15

909

\begin{tabular}{|l|l|l|l|}
\hline hundred & thousand & ten thousand & million \\
\hline & & & \\
\hline
\end{tabular}

Table 1.10

Exercise 1.4.16 981

\begin{tabular}{|l|l|l|l|}
\hline hundred & thousand & ten thousand & million \\
\hline & & & \\
\hline
\end{tabular}

Table 1.11

Exercise 1.4.17 965

\begin{tabular}{|l|l|l|l|}
\hline hundred & thousand & ten thousand & million \\
\hline & & & \\
\hline
\end{tabular}

Table 1.12

Exercise 1.4.18 $551,061,285$

(Solution on p. 79.)

\begin{tabular}{|l|l|l|l|}
\hline hundred & thousand & ten thousand & million \\
\hline & & & \\
\hline
\end{tabular}

Available for free at Connexions $<$ http://cnx.org/content/col10615/1.4 $>$ 
Table 1.13

Exercise 1.4.19

$23,047,991,521$

\begin{tabular}{|l|l|l|l|}
\hline hundred & thousand & ten thousand & million \\
\hline & & & \\
\hline
\end{tabular}

Table 1.14

Exercise 1.4.20

(Solution on p. 79.)

$106,999,413,206$

\begin{tabular}{|l|l|l|l|}
\hline Hundred & thousand & ten thousand & million \\
\hline & & & \\
\hline
\end{tabular}

Table 1.15

Exercise 1.4.21

$5,000,000$

\begin{tabular}{|l|l|l|l|}
\hline hundred & thousand & ten thousand & million \\
\hline & & & \\
\hline
\end{tabular}

Table 1.16

Exercise 1.4.22

$8,006,001$

(Solution on p. 79.)

\begin{tabular}{|l|l|l|l|}
\hline hundred & thousand & ten thousand & million \\
\hline & & & \\
\hline
\end{tabular}

Table 1.17

Exercise 1.4.23

94,312

\begin{tabular}{|l|l|l|l|}
\hline hundred & thousand & ten thousand & million \\
\hline & & & \\
\hline
\end{tabular}

Table 1.18

Exercise 1.4.24

(Solution on p. 79.) 


\begin{tabular}{|l|l|l|l|}
\hline hundred & thousand & ten thousand & million \\
\hline & & & \\
\hline
\end{tabular}

Table 1.19

Exercise 1.4.25 560,669

\begin{tabular}{|l|l|l|l|}
\hline hundred & thousand & ten thousand & million \\
\hline & & & \\
\hline
\end{tabular}

Table 1.20

Exercise 1.4.26 388,551

(Solution on p. 80.)

\begin{tabular}{|l|l|l|l|}
\hline hundred & thousand & ten thousand & million \\
\hline & & & \\
\hline
\end{tabular}

Table 1.21

Exercise 1.4.27 4,752

\begin{tabular}{|l|l|l|l|}
\hline hundred & thousand & ten thousand & million \\
\hline & & & \\
\hline
\end{tabular}

Table 1.22

Exercise 1.4.28 8,209

(Solution on p. 80.)

\begin{tabular}{|l|l|l|l|}
\hline hundred & thousand & ten thousand & million \\
\hline & & & \\
\hline
\end{tabular}

Table 1.23

\section{Exercise 1.4.29}

In 1950 , there were 5,796 cases of diphtheria reported in the United States. Round to the nearest hundred.

Exercise 1.4.30

(Solution on p. 80.) In 1979, 19,309,000 people in the United States received federal food stamps. Round to the nearest ten thousand.

\section{Exercise 1.4.31}

In 1980 , there were $1,105,000$ people between 30 and 34 years old enrolled in school. Round to the nearest million. 
Exercise 1.4.32

(Solution on p. 80.)

In 1980 , there were $29,100,000$ reports of aggravated assaults in the United States. Round to the nearest million.

For the following problems, round the numbers to the position you think is most reasonable for the situation.

Exercise 1.4.33

In 1980, for a city of one million or more, the average annual salary of police and firefighters was $\$ 16,096$.

Exercise 1.4.34

(Solution on p. 80.)

The average percentage of possible sunshine in San Francisco, California, in June is $73 \%$.

Exercise 1.4.35

In 1980, in the state of Connecticut, $\$ 3,777,000,000$ in defense contract payroll was awarded.

Exercise 1.4.36

(Solution on p. 80.)

In 1980 , the federal government paid $\$ 5,463,000,000$ to Viet Nam veterans and dependants.

Exercise 1.4.37

In 1980, there were 3,377,000 salespeople employed in the United States.

Exercise 1.4.38

(Solution on p. 80.)

In 1948, in New Hampshire, 231,000 popular votes were cast for the president.

Exercise 1.4.39

In 1970 , the world production of cigarettes was $2,688,000,000,000$.

Exercise 1.4.40

(Solution on p. 80.)

In 1979, the total number of motor vehicle registrations in Florida was 5,395,000.

Exercise 1.4.41

In 1980, there were 1,302,000 registered nurses the United States.

\subsubsection{Exercises for Review}

Exercise 1.4.42

(Solution on p. 80.)

(Section 1.2) There is a term that describes the visual displaying of a number. What is the term?

Exercise 1.4.43

(Section 1.2) What is the value of 5 in $26,518,206$ ?

Exercise 1.4.44

(Solution on p. 80.)

(Section 1.3) Write 42,109 as you would read it.

Exercise 1.4.45

(Section 1.3) Write "six hundred twelve" using digits.

Exercise 1.4.46

(Section 1.3) Write "four billion eight" using digits.

(Solution on p. 80.)

\subsection{Addition of Whole Numbers ${ }^{5}$}

\subsubsection{Section Overview}

- Addition

- Addition Visualized on the Number Line

- The Addition Process

${ }^{5}$ This content is available online at $<$ http://cnx.org/content $/ \mathrm{m} 34786 / 1.5 />$. 
- Addition Involving Carrying

- Calculators

\subsubsection{Addition}

Suppose we have two collections of objects that we combine together to form a third collection. For example,

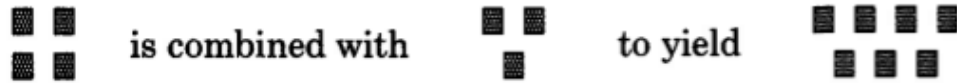

We are combining a collection of four objects with a collection of three objects to obtain a collection of seven objects.

\section{Addition}

The process of combining two or more objects (real or intuitive) to form a third, the total, is called addition.

In addition, the numbers being added are called addends or terms, and the total is called the sum. The plus symbol $(+)$ is used to indicate addition, and the equal symbol $(=)$ is used to represent the word "equal." For example, $4+3=7$ means "four added to three equals seven."

\subsubsection{Addition Visualized on the Number Line}

Addition is easily visualized on the number line. Let's visualize the addition of 4 and 3 using the number line.

To find $4+3$,

1. Start at 0 .

2. Move to the right 4 units. We are now located at 4 .

3. From 4, move to the right 3 units. We are now located at 7 .

Thus, $4+3=7$.

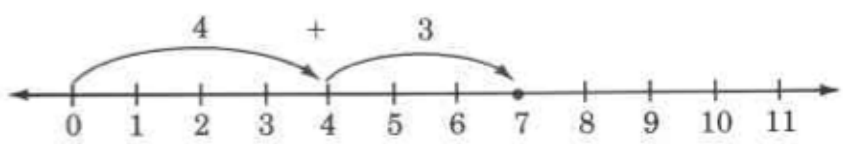

\subsubsection{The Addition Process}

We'll study the process of addition by considering the sum of 25 and 43 .

\begin{tabular}{rr} 
& 2 tens +5 ones \\
25 & +4 tens +3 ones \\
\hline & means 5 tens +8 ones
\end{tabular}

We write this as 68 .

We can suggest the following procedure for adding whole numbers using this example.

Example 1.20: The Process of Adding Whole Numbers

To add whole numbers,

The process: 
1. Write the numbers vertically, placing corresponding positions in the same column.

25

$+43$

2. Add the digits in each column. Start at the right (in the ones position) and move to the left, placing the sum at the bottom.

25

$+43$

68

Caution: Confusion and incorrect sums can occur when the numbers are not aligned in columns properly. Avoid writing such additions as

25

$+43$

25

$+43$

\subsubsection{Sample Set A}

Example 1.21

Add 276 and 103.

$\begin{array}{rl}276 & 6+3=9 . \\ +103 & 7+0=7 . \\ 379 & 2+1=3 .\end{array}$

Example 1.22

Add 1459 and 130

1459

$$
9+0=9 .
$$

$+130$ $5+3=8$.

1589

$$
4+1=5 \text {. }
$$

$$
1+0=1 .
$$

In each of these examples, each individual sum does not exceed 9. We will examine individual sums that exceed 9 in the next section.

\subsubsection{Practice Set A}

Perform each addition. Show the expanded form in problems 1 and 2.

Exercise 1.5.1 Add 63 and 25 .

(Solution on p. 80.)

Exercise 1.5.2

(Solution on p. 80.)

Add 4,026 and 1,501.

Exercise 1.5.3

(Solution on p. 80.)

Add 231,045 and 36,121. 


\subsubsection{Addition Involving Carrying}

It often happens in addition that the sum of the digits in a column will exceed 9. This happens when we add 18 and 34 . We show this in expanded form as follows.

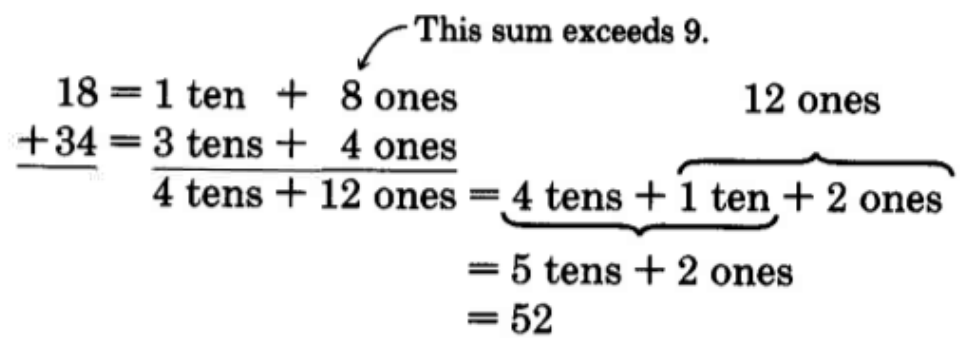

Notice that when we add the 8 ones to the 4 ones we get 12 ones. We then convert the 12 ones to 1 ten and 2 ones. In vertical addition, we show this conversion by carrying the ten to the tens column. We write a 1 at the top of the tens column to indicate the carry. This same example is shown in a shorter form as follows:

1

18

$+34$

$528+4=12$ Write 2 , carry 1 ten to the top of the next column to the left.

\subsubsection{Sample Set B}

Perform the following additions. Use the process of carrying when needed.

Example 1.23

Add 1875 and 358.

111

1875

$+358$

2233

$5+8=13 \quad$ Write 3 , carry 1 ten.

$1+7+5=13 \quad$ Write 3 , carry 1 hundred.

$1+8+3=12 \quad$ Write 2 , carry 1 thousand.

$1+1=2$

The sum is 2233 .

Example 1.24

Add 89,208 and 4,946.

111

89,208

$+4,946$

94,154 


$$
\begin{array}{cc}
8+6=14 & \text { Write } 4, \text { carry } 1 \text { ten. } \\
1+0+4=5 & \text { Write the } 5 \text { (nothing to carry). } \\
2+9=11 & \text { Write } 1, \text { carry one thousand. } \\
1+9+4=14 & \text { Write } 4, \text { carry one ten thousand. } \\
1+8=9 &
\end{array}
$$

The sum is 94,154 .

Example 1.25

Add 38 and 95 .

11

38

$\begin{array}{r}+95 \\ \hline 133\end{array}$

$$
\begin{array}{cc}
8+5=13 & \text { Write } 3, \text { carry } 1 \text { ten. } \\
1+3+9=13 & \text { Write } 3, \text { carry } 1 \text { hundred. } \\
1+0=1 &
\end{array}
$$

As you proceed with the addition, it is a good idea to keep in mind what is actually happening.

38 means
+95

\begin{tabular}{llr} 
& 3 tens & +8 ones \\
+9 tens & +5 ones \\
\hline 12 tens & +13 ones \\
$=$ & 12 tens +1 ten +3 ones \\
$=$ & 13 tens & +3 ones \\
$=1$ hundred +3 tens & +3 ones \\
$=133$ &
\end{tabular}

The sum is 133 .

\section{Example 1.26}

Find the sum 2648, 1359, and 861 .

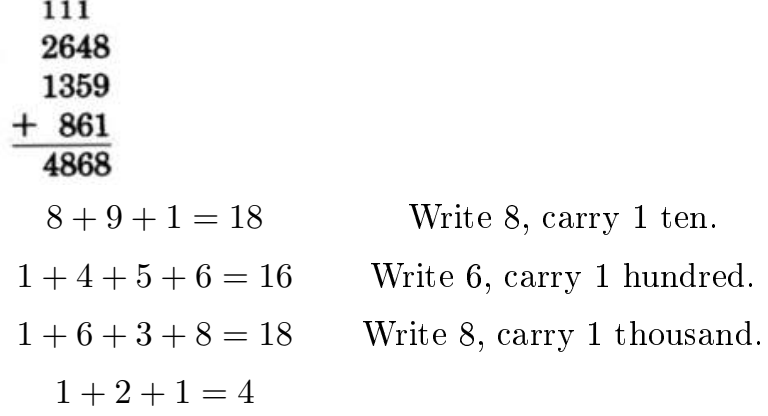

The sum is 4,868 .

Numbers other than 1 can be carried as illustrated in Example 1.27.

Example 1.27

Find the sum of the following numbers. 


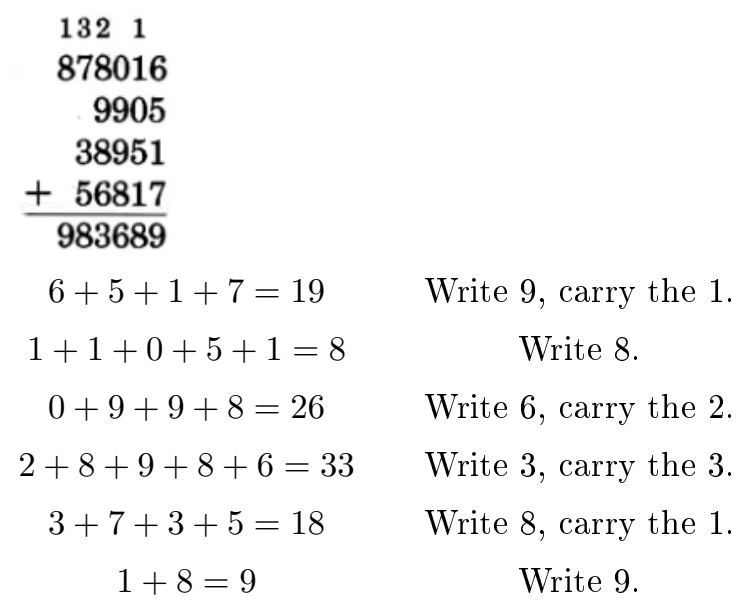

The sum is 983,689 .

\section{Example 1.28}

The number of students enrolled at Riemann College in the years 1984, 1985, 1986, and 1987 was $10,406,9,289,10,108$, and 11,412 , respectively. What was the total number of students enrolled at Riemann College in the years 1985, 1986, and 1987?

We can determine the total number of students enrolled by adding $9,289,10,108$, and 11,412 , the number of students enrolled in the years 1985, 1986, and 1987.

111

9,289

10,108

$+11,412$

30,809

The total number of students enrolled at Riemann College in the years 1985, 1986, and 1987 was 30,809 .

\subsubsection{Practice Set $B$}

Perform each addition. For the next three problems, show the expanded form.

Exercise 1.5.4

Add 58 and 29.

(Solution on p. 80.)

Exercise 1.5.5

(Solution on p. 81.)

Add 476 and 85 .

(Solution on p. 81.)

Add 27 and 88 .

(Solution on p. 81.)

Exercise 1.5.7

Add 67,898 and 85,627.

For the next three problems, find the sums.

Exercise 1.5.8

(Solution on p. 81.)

57

26

84 
Exercise 1.5.9

(Solution on p. 81.)

847

825

796

Exercise 1.5.10

(Solution on p. 81.)

16,945

8,472

387,721

21,059

629

\subsubsection{Calculators}

Calculators provide a very simple and quick way to find sums of whole numbers. For the two problems in Sample Set C, assume the use of a calculator that does not require the use of an ENTER key (such as many Hewlett-Packard calculators).

\subsubsection{Sample Set C}

Use a calculator to find each sum.

\section{Example 1.29}

\begin{tabular}{|c|l|l|}
\hline \multicolumn{2}{|c|}{$34+21$} & Display Reads \\
\hline Type & 34 & 34 \\
\hline Press & + & 34 \\
\hline Type & 21 & 21 \\
\hline Press & $=$ & 55 \\
\hline
\end{tabular}

Table 1.24

The sum is 55 .

Example 1.30 


\begin{tabular}{|l|l|l|l|}
\hline \multicolumn{2}{|c|}{$106+85+322+406$} & Display Reads & \\
\hline Type & 106 & 106 & The calculator keeps a running subtotal \\
\hline Press & + & 106 & \\
\hline Type & 85 & 85 & \\
\hline Press & $=$ & 191 & $\leftarrow 106+85$ \\
\hline Type & 322 & 322 & \\
\hline Press & + & 513 & $\leftarrow 191+322$ \\
\hline Type & 406 & 406 & \\
\hline Press & $=$ & 919 & $\leftarrow 513+406$ \\
\hline
\end{tabular}

Table 1.25

The sum is 919 .

\subsubsection{Practice Set C}

Use a calculator to find the following sums.

Exercise 1.5.11

(Solution on p. 81.)

$62+81+12$

Exercise 1.5.12

(Solution on p. 81.)

$9,261+8,543+884+1,062$

Exercise 1.5.13

(Solution on p. 81.)

$10,221+9,016+11,445$

\subsubsection{Exercises}

For the following problems, perform the additions. If you can, check each sum with a calculator.

Exercise 1.5.14

(Solution on p. 81.)

$14+5$

Exercise 1.5.15

$12+7$

Exercise 1.5.16

(Solution on p. 81.)

$46+2$

Exercise 1.5.17

$83+16$

Exercise 1.5.18

$77+21$

(Solution on p. 81.)

Exercise 1.5.19

321

$+42$ 
Exercise 1.5.20

(Solution on p. 82.)

916

$+62$

Exercise 1.5.21

104

$+561$

Exercise 1.5.22

(Solution on p. 82.)

265

$+103$

Exercise 1.5.23

$552+237$

Exercise 1.5.24

$8,521+4,256$

(Solution on p. 82.)

Exercise 1.5.25

16,408

$+3,101$

Exercise 1.5.26

(Solution on p. 82.)

16,515

$+42,223$

Exercise 1.5.27

$616,702+101,161$

Exercise 1.5.28

(Solution on p. 82.)

$43,156,219+2,013,520$

Exercise 1.5.29

$17+6$

Exercise 1.5.30

$25+8$

(Solution on p. 82.)

Exercise 1.5.31

84

$\begin{array}{r}+7 \\ \hline\end{array}$

Exercise 1.5.32

(Solution on p. 82.)

75

$+6$

Exercise 1.5.33

$36+48$

Exercise 1.5.34

(Solution on p. 82.)

$74+17$

Exercise 1.5.35

$486+58$

Exercise 1.5.36

$743+66$

(Solution on p. 82.)

Exercise 1.5.37

$381+88$ 
Exercise 1.5.38

(Solution on p. 82.)

687

$+175$

Exercise 1.5.39 931

$+853$

Exercise 1.5.40

(Solution on p. 82.)

$1,428+893$

Exercise 1.5.41

$12,898+11,925$

Exercise 1.5.42

(Solution on p. 82.)

631,464

$+509,740$

Exercise 1.5.43

805,996

$+98,516$

Exercise 1.5.44

(Solution on p. 82.)

$38,428,106$

$+522,936,005$

Exercise 1.5.45

$5,288,423,100+16,934,785,995$

Exercise 1.5.46

$98,876,678,521,402+843,425,685,685,658$

(Solution on p. 82.)

Exercise 1.5.47

$41+61+85+62$

Exercise 1.5.48

$21+85+104+9+15$

(Solution on p. 82.)

Exercise 1.5.49

116

27

110

110

$+8$

Exercise 1.5.50

(Solution on p. 82.)

75,206

4,152

$+16,007$ 
Exercise 1.5.51

8,226

143

92,015

8

487,553

5,218

Exercise 1.5.52

(Solution on p. 82.)

50,006

1,005

100, 300

20,008

1, 000, 009

800,800

Exercise 1.5.53

616

42,018

1,687

225

$8,623,418$

$12,506,508$

19

2,121

195,643

For the following problems, perform the additions and round to the nearest hundred.

Exercise 1.5.54

(Solution on p. 82.)

1,468

2,183

Exercise 1.5.55

928,725

15,685

Exercise 1.5.56

(Solution on p. 82.)

82,006

$3,019,528$

Exercise 1.5.57

18,621

5,059 
Exercise 1.5.58

(Solution on p. 82.)

92

48

Exercise 1.5.59

16

37

Exercise 1.5.60

(Solution on p. 82.)

21

16

Exercise 1.5.61

11,172

22,749

12,248

Exercise 1.5.62

(Solution on p. 82.)

240

280

210

310

Exercise 1.5.63

9,573

101, 279

122,581

For the next five problems, replace the letter $m$ with the whole number that will make the addition true.

Exercise 1.5.64

(Solution on p. 82.)

62

$+\quad m$

67

Exercise 1.5.65

106

$+\quad m$

113

Exercise 1.5.66

(Solution on p. 82.)

432

$+\quad m$
451

Exercise 1.5.67

803

$\begin{array}{r}+\quad m \\ \hline 830\end{array}$

Available for free at Connexions $<$ http://cnx.org/content/col10615/1.4 $>$ 


$$
\begin{array}{r}
1,893 \\
+\quad m \\
\hline 1,981
\end{array}
$$

\section{Exercise 1.5.69}

The number of nursing and related care facilities in the United States in 1971 was 22,004. In 1978, the number was 18,722. What was the total number of facilities for both 1971 and 1978 ?

\section{Exercise 1.5.70}

(Solution on p. 83.)

The number of persons on food stamps in 1975, 1979, and 1980 was 19,179,000, 19,309,000, and $22,023,000$, respectively. What was the total number of people on food stamps for the years 1975 , 1979 , and 1980?

\section{Exercise 1.5.71}

The enrollment in public and nonpublic schools in the years 1965, 1970, 1975, and 1984 was $54,394,000,59,899,000,61,063,000$, and $55,122,000$, respectively. What was the total enrollment for those years?

\section{Exercise 1.5.72}

(Solution on p. 83.)

The area of New England is 3,618,770 square miles. The area of the Mountain states is 863,563 square miles. The area of the South Atlantic is 278,926 square miles. The area of the Pacific states is 921,392 square miles. What is the total area of these regions?

\section{Exercise 1.5.73}

In 1960, the IRS received 1,188,000 corporate income tax returns. In 1965, 1,490,000 returns were received. In 1970, 1,747,000 returns were received. In $1972-1977,1,890,000 ; 1,981,000 ; 2,043,000$; $2,100,000 ; 2,159,000$; and 2,329,000 returns were received, respectively. What was the total number of corporate tax returns received by the IRS during the years 1960, 1965, 1970, $1972-1977$ ?

Exercise 1.5.74

(Solution on p. 83.)

Find the total number of scientists employed in 1974.

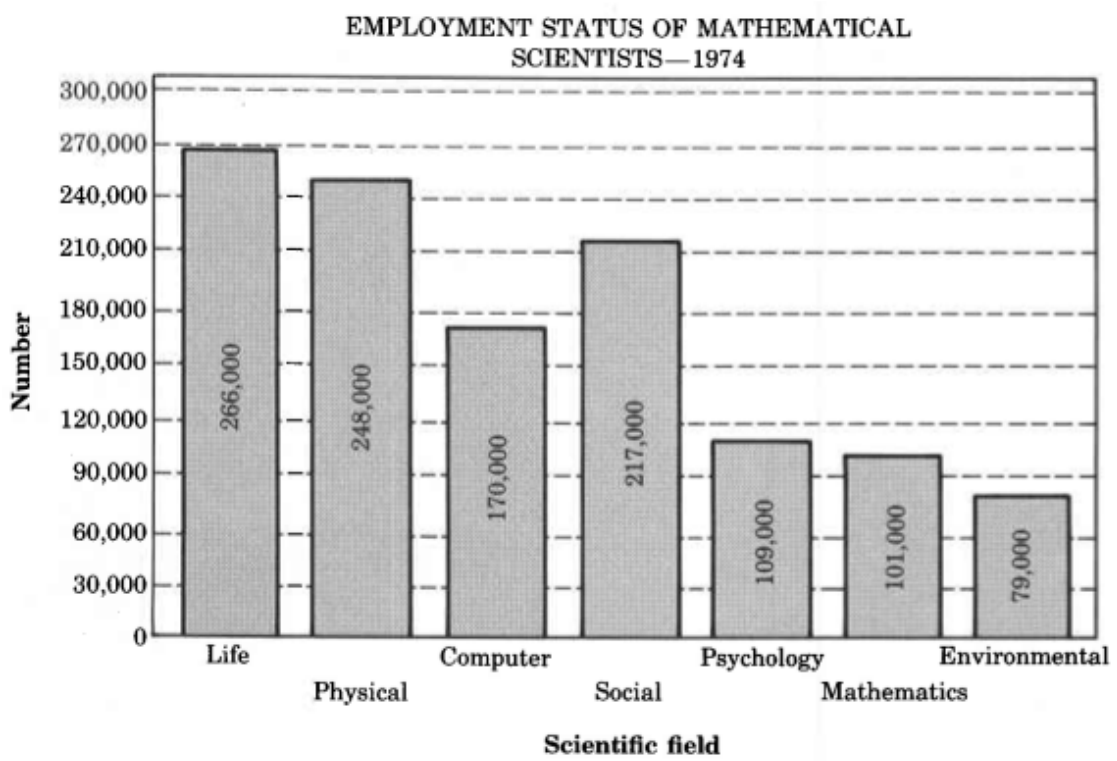

\section{Exercise 1.5.75}

Find the total number of sales for space vehicle systems for the years 1965-1980. 


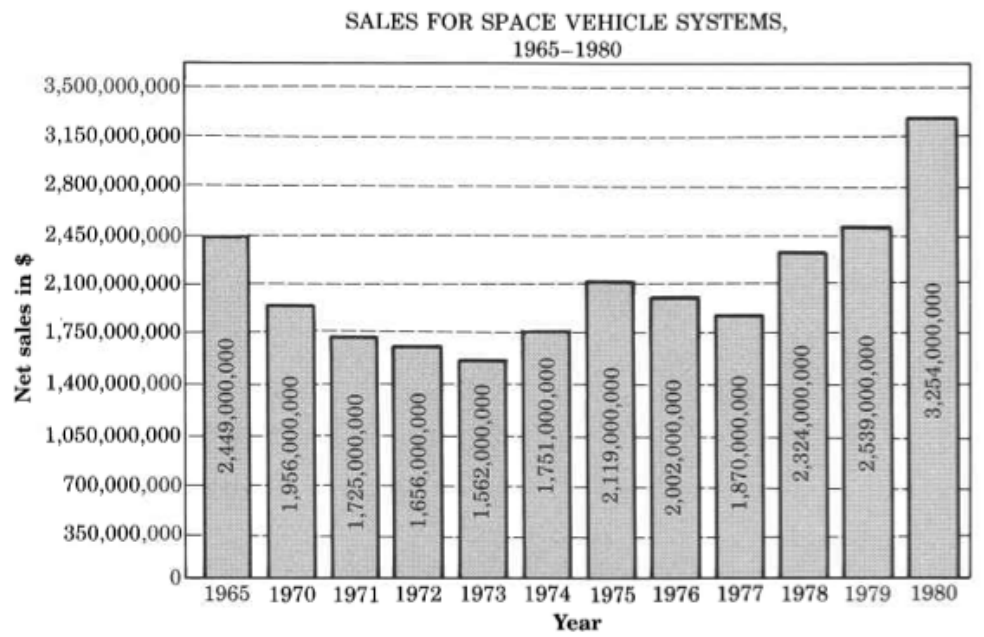

Exercise 1.5.76

(Solution on p. 83.)

Find the total baseball attendance for the years 1960-1980.

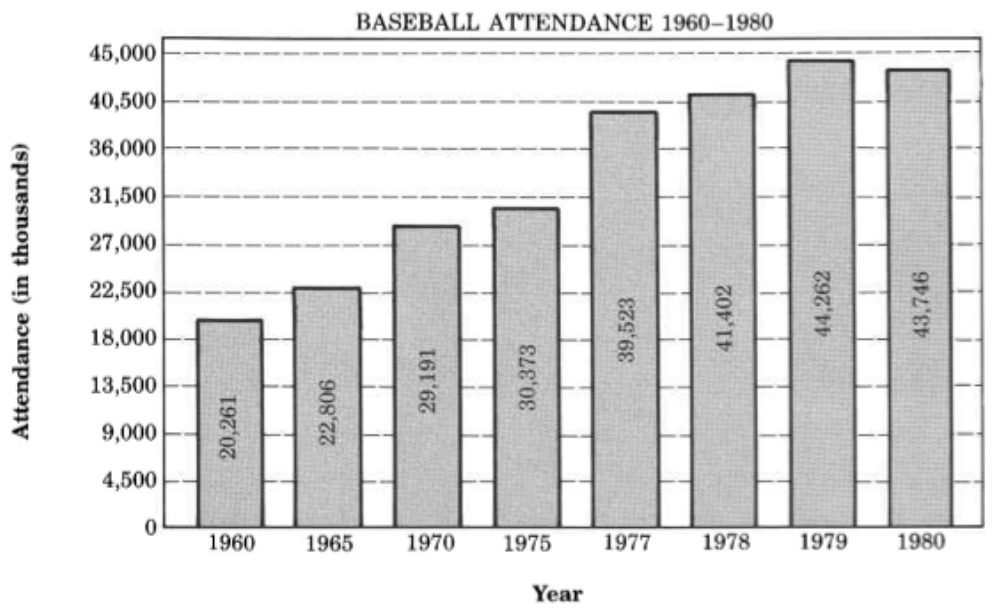

Exercise 1.5.77

Find the number of prosecutions of federal officials for 1970-1980.

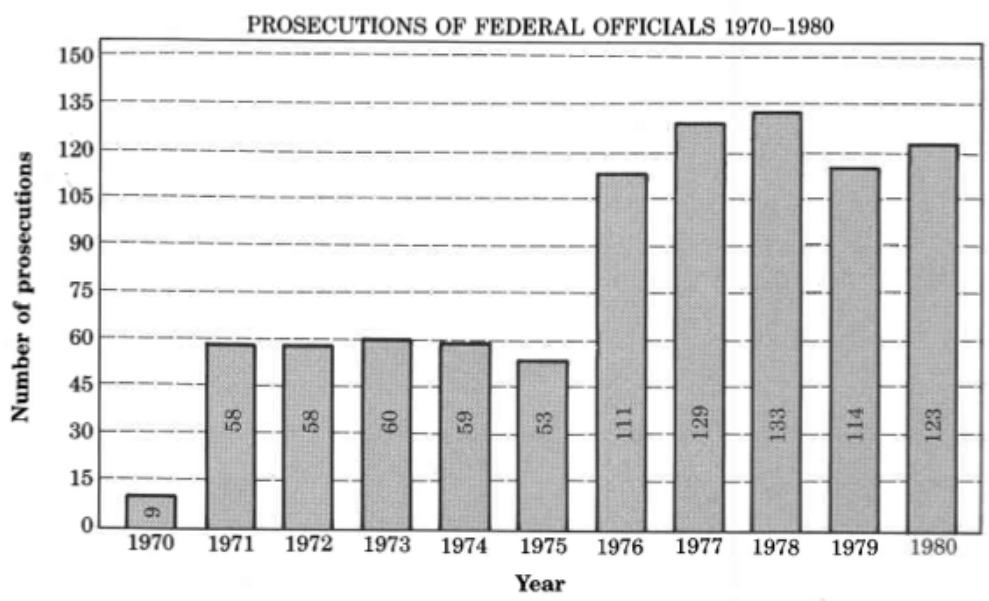

For the following problems, try to add the numbers mentally. 
Exercise 1.5.78

(Solution on p. 83.)

5

5

3

7

Exercise 1.5.79

8

2

6

4

Exercise 1.5.80

(Solution on p. 83.)

9

1

8

5

2

Exercise 1.5.81

5

2

5

8

3

7

Exercise 1.5.82

(Solution on p. 83.)

6

4

3

1

6

7

9

4

Exercise 1.5.83

20

30

Exercise 1.5.84

(Solution on p. 83.)

15

35

Available for free at Connexions $<$ http://cnx.org/content/col10615/1.4 $>$ 
Exercise 1.5.85

16

14

Exercise 1.5.86

(Solution on p. 83.)

23

27

Exercise 1.5.87

82

18

Exercise 1.5.88

(Solution on p. 83.)

36

14

\subsubsection{Exercises for Review}

Exercise 1.5.89

(Section 1.2) Each period of numbers has its own name. From right to left, what is the name of the fourth period?

Exercise 1.5.90

(Solution on p. 83.)

(Section 1.2) In the number 610,467, how many thousands are there?

Exercise 1.5.91

(Section 1.3) Write 8,840 as you would read it.

Exercise 1.5.92

(Solution on p. 83.)

(Section 1.4) Round 6,842 to the nearest hundred.

Exercise 1.5.93

(Section 1.4) Round 431,046 to the nearest million.

\subsection{Subtraction of Whole Numbers ${ }^{6}$}

\subsubsection{Section Overview}

- Subtraction

- Subtraction as the Opposite of Addition

- The Subtraction Process

- Subtraction Involving Borrowing

- Borrowing From Zero

- Calculators

\footnotetext{
${ }^{6}$ This content is available online at $<$ http://cnx.org/content/m34784/1.5/>.
} 


\subsubsection{Subtraction}

Subtraction

Subtraction is the process of determining the remainder when part of the total is removed.

Suppose the sum of two whole numbers is 11 , and from 11 we remove 4 . Using the number line to help our visualization, we see that if we are located at 11 and move 4 units to the left, and thus remove 4 units, we will be located at 7 . Thus, 7 units remain when we remove 4 units from 11 units.

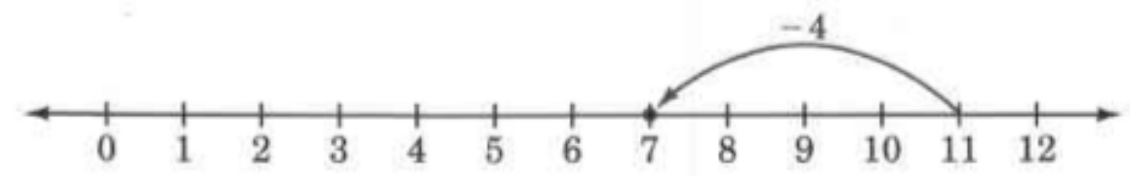

The Minus Symbol

The minus symbol (-) is used to indicate subtraction. For example, $11-4$ indicates that 4 is to be subtracted from 11.

\section{Minuend}

The number immediately in front of or the minus symbol is called the minuend, and it represents the original number of units.

\section{Subtrahend}

The number immediately following or below the minus symbol is called the subtrahend, and it represents the number of units to be removed.

\section{Difference}

The result of the subtraction is called the difference of the two numbers. For example, in $11-4=7,11$ is the minuend, 4 is the subtrahend, and 7 is the difference.

\subsubsection{Subtraction as the Opposite of Addition}

Subtraction can be thought of as the opposite of addition. We show this in the problems in Sample Set A.

\subsubsection{Sample Set A}

\section{Example 1.31}

$8-5=3$ since $3+5=8$.

Example 1.32

$9-3=6$ since $6+3=9$.

\subsubsection{Practice Set A}

Complete the following statements.

Exercise 1.6.1

$7-5=$ since $+5=7$

Exercise 1.6.2

$9-1=$ since $+1=9$.

Exercise 1.6.3 $17-8=$ since $+8=17$.
(Solution on p. 83.)

(Solution on p. 83.)

(Solution on p. 83.) 


\subsubsection{The Subtraction Process}

We'll study the process of the subtraction of two whole numbers by considering the difference between 48 and 35 .

\begin{tabular}{rrr}
48 & means & 4 tens +8 ones \\
-35 & & -3 tens -5 ones \\
\hline & & 1 ten +3 ones
\end{tabular}

which we write as 13 .

Example 1.33: The Process of Subtracting Whole Numbers

To subtract two whole numbers,

The process

1. Write the numbers vertically, placing corresponding positions in the same column.

48

$\underline{-35}$

2. Subtract the digits in each column. Start at the right, in the ones position, and move to the left, placing the difference at the bottom. 48

$\underline{-35}$

13

\subsubsection{Sample Set B}

Perform the following subtractions.

$$
\begin{aligned}
& \text { Example 1.34 } \\
& 275 \\
& \frac{-142}{133} \\
& 5-2=3 . \\
& 7-4=3 . \\
& 2-1=1 .
\end{aligned}
$$

Example 1.35

46, 042

$-1,031$

45,011

$2-1=1$.

$4-3=1$.

$0-0=0$.

$6-1=5$.

$4-0=4$. 


\section{Example 1.36}

Find the difference between 977 and 235 .

Write the numbers vertically, placing the larger number on top. Line up the columns properly.

$$
\begin{array}{r}
977 \\
-235 \\
\hline 742
\end{array}
$$

The difference between 977 and 235 is 742 .

\section{Example 1.37}

In Keys County in 1987, there were 809 cable television installations. In Flags County in 1987, there were 1,159 cable television installations. How many more cable television installations were there in Flags County than in Keys County in 1987?

We need to determine the difference between 1,159 and 809 .

$$
\begin{array}{r}
11 \\
1,159 \\
-\quad 809 \\
\hline 350
\end{array}
$$

There were 350 more cable television installations in Flags County than in Keys County in 1987.

\subsubsection{Practice Set B}

Perform the following subtractions.

Exercise 1.6.4

(Solution on p. 83.)

534

$-203$

Exercise 1.6.5

(Solution on p. 83.) 857

$-43$

Exercise 1.6.6

(Solution on p. 83.)

95,628

$-34,510$

Exercise 1.6.7

(Solution on p. 83.)

11,005

$-1,005$

Exercise 1.6.8

Find the difference between 88,526 and 26,412.

(Solution on p. 83.)

In each of these problems, each bottom digit is less than the corresponding top digit. This may not always be the case. We will examine the case where the bottom digit is greater than the corresponding top digit in the next section. 


\subsubsection{Subtraction Involving Borrowing}

Minuend and Subtrahend

It often happens in the subtraction of two whole numbers that a digit in the minuend (top number) will be less than the digit in the same position in the subtrahend (bottom number). This happens when we subtract 27 from 84 .

84

$\underline{-27}$

We do not have a name for $4-7$. We need to rename 84 in order to continue. We'll do so as follows:

$$
\begin{aligned}
& 84=8 \text { tens }+4 \text { ones } \\
&-27=2 \text { tens }+7 \text { ones } \\
& \hline
\end{aligned}
$$

\section{7 tens +1 ten +4 ones

2 tens $\quad+7$ ones

\section{7 tens +10 ones +4 ones}

$\underline{2 \text { tens } \quad+7 \text { ones }}$

Our new name for 84 is 7 tens +14 ones.

\section{7 tens +14 ones \\ 2 tens +7 ones \\ 5 tens +7 ones \\ $=57$}

Notice that we converted 8 tens to 7 tens +1 ten, and then we converted the 1 ten to 10 ones. We then had 14 ones and were able to perform the subtraction.

\section{Borrowing}

The process of borrowing (converting) is illustrated in the problems of Sample Set C.

\subsubsection{Sample Set C}

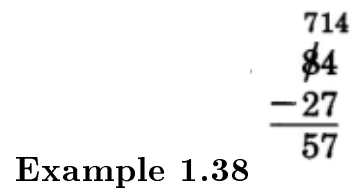

1. Borrow 1 ten from the 8 tens. This leaves 7 tens.

2. Convert the 1 ten to 10 ones.

3. Add 10 ones to 4 ones to get 14 ones.

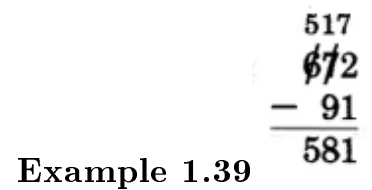

1. Borrow 1 hundred from the 6 hundreds. This leaves 5 hundreds. 
2. Convert the 1 hundred to 10 tens.

3. Add 10 tens to 7 tens to get 17 tens.

\subsubsection{Practice Set C}

Perform the following subtractions. Show the expanded form for the first three problems.

Exercise 1.6.9

(Solution on p. 83.)

53

$\underline{-35}$

Exercise 1.6.10

(Solution on p. 84.)

76

$\underline{-28}$

Exercise 1.6.11

(Solution on p. 84.)

872

$-565$

Exercise 1.6.12

(Solution on p. 84.)

441

$-356$

Exercise 1.6.13

(Solution on p. 84.)

775

$-66$

Exercise 1.6.14

(Solution on p. 84.)

5,663

$-2,559$

\section{Borrowing More Than Once}

Sometimes it is necessary to borrow more than once. This is shown in the problems in Section 1.6.5.3 (Sample Set D).

\subsubsection{Sample Set D}

Perform the Subtractions. Borrowing more than once if necessary

\section{Example 1.40}

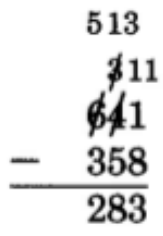

1. Borrow 1 ten from the 4 tens. This leaves 3 tens.

2. Convert the 1 ten to 10 ones.

3. Add 10 ones to 1 one to get 11 ones. We can now perform $11-8$.

4. Borrow 1 hundred from the 6 hundreds. This leaves 5 hundreds. 
5. Convert the 1 hundred to 10 tens.

6. Add 10 tens to 3 tens to get 13 tens.

7. Now $13-5=8$.

8. $5-3=2$.

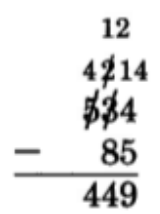

\section{Example 1.41}

1. Borrow 1 ten from the 3 tens. This leaves 2 tens.

2. Convert the 1 ten to 10 ones.

3. Add 10 ones to 4 ones to get 14 ones. We can now perform $14-5$.

4. Borrow 1 hundred from the 5 hundreds. This leaves 4 hundreds.

5. Convert the 1 hundred to 10 tens.

6. Add 10 tens to 2 tens to get 12 tens. We can now perform $12-8=4$.

7. Finally, $4-0=4$.

\section{Example 1.42}

71529

$\underline{-6952}$

After borrowing, we have

10

14

$6 \phi 412$

71529

6952

64577

\subsubsection{Practice Set D}

Perform the following subtractions.

Exercise 1.6.15

(Solution on p. 84.)

526

$-358$

Exercise 1.6.16

(Solution on p. 84.)

63,419

$-7,779$

Exercise 1.6.17

4,312

$\underline{-3,123}$ 


\subsubsection{Borrowing from Zero}

It often happens in a subtraction problem that we have to borrow from one or more zeros. This occurs in problems such as

1. 503

$$
-37
$$

and

2 .

5000

$-\quad 37$

We'll examine each case.

Example 1.43: Borrowing from a single zero.

Consider the problem 503

$-37$

Since we do not have a name for $3-7$, we must borrow from 0 .

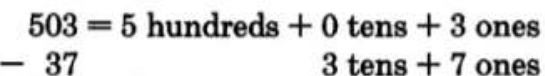

Since there are no tens to borrow, we must borrow 1 hundred. One hundred $=10$ tens.

\section{4 hundreds +10 tens +3 ones} 3 tens +7 ones

We can now borrow 1 ten from 10 tens (leaving 9 tens). One ten $=10$ ones and 10 ones +3 ones $=13$ ones.

4 hundreds +9 tens +13 ones
$\frac{3 \text { tens }+7 \text { ones }}{4 \text { hundreds }+6 \text { tens }+6 \text { ones }}=466$

Now we can suggest the following method for borrowing from a single zero.

\section{Borrowing from a Single Zero}

To borrow from a single zero,

1. Decrease the digit to the immediate left of zero by one.

2. Draw a line through the zero and make it a 10 .

3. Proceed to subtract as usual.

\subsubsection{Sample Set E}

Example 1.44

Perform this subtraction.

503

$-37$

The number 503 contains a single zero 
1. The number to the immediate left of 0 is 5 . Decrease 5 by 1 .

$$
\begin{gathered}
5-1=4 \\
410 \\
\not \phi \phi 3 \\
-\quad 37 \\
\hline
\end{gathered}
$$

2. Draw a line through the zero and make it a 10 .

3. Borrow from the 10 and proceed.

\section{9}

4,1013

$\$ \phi 3$

$\begin{array}{r}-\quad 37 \\ \hline 466\end{array}$

1 ten +10 ones

10 ones +3 ones $=13$ ones

\subsubsection{Practice Set E}

Perform each subtraction.

Exercise 1.6.18

(Solution on p. 84.)

906

$-18$

Exercise 1.6.19

(Solution on p. 84.)

5102

$-559$

Exercise 1.6.20

(Solution on p. 85.)

9055

$-386$

Example 1.45: Borrowing from a group of zeros

5000

Consider the problem

$-\quad 37$

In this case, we have a group of zeros.

$$
5000=5 \text { thousands }+0 \text { hundred }+0 \text { tens }+0 \text { ones }
$$

$-37=\quad 3$ tens +7 ones

Since we cannot borrow any tens or hundreds, we must borrow 1 thousand. One thousand $=10$ hundreds.

$$
\begin{array}{r}
4 \text { thousands }+10 \text { hundreds }+0 \text { tens }+0 \text { ones } \\
3 \text { tens }+7 \text { ones }
\end{array}
$$

We can now borrow 1 hundred from 10 hundreds. One hundred $=10$ tens. 


\section{4 thousands +9 hundreds +10 tens +0 ones \\ 3 tens +7 ones}

We can now borrow 1 ten from 10 tens. One ten $=10$ ones.

4 thousands +9 hundreds +9 tens +10 ones

3 tens +7 ones

4 thousands +9 hundreds +6 tens +3 ones $=4,963$

From observations made in this procedure we can suggest the following method for borrowing from

a group of zeros.

\section{Borrowing from a Group of zeros}

To borrow from a group of zeros,

1. Decrease the digit to the immediate left of the group of zeros by one.

2. Draw a line through each zero in the group and make it a 9 , except the rightmost zero, make it 10 .

3. Proceed to subtract as usual.

\subsubsection{Sample Set F}

Perform each subtraction.

\section{Example 1.46}

40,000

$-\quad 125$

The number 40,000 contains a group of zeros.

1. The number to the immediate left of the group is 4 . Decrease 4 by 1 .

$$
4-1=3
$$

2. Make each 0 , except the rightmost one, 9. Make the rightmost 0 a 10.

$$
\begin{array}{r}
399910 \\
4 \phi, \phi \phi \phi \\
-\quad 125 \\
\hline
\end{array}
$$

3. Subtract as usual.

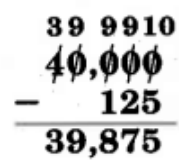

Example 1.47

$$
\text { 8, 000, } 006
$$$$
-\quad 41,107
$$

The number 8,000,006 contains a group of zeros.

1. The number to the immediate left of the group is 8 . Decrease 8 by $1.8-1=7$

2. Make each zero, except the rightmost one, 9 . Make the rightmost 0 a 10 . 


\section{0 $\phi, \phi \phi \phi, \phi \phi 6$ $-\quad 41,107$}

3. To perform the subtraction, we'll need to borrow from the ten.

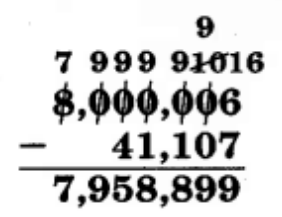

1 ten $=10$ ones

10 ones +6 ones $=16$ ones

\subsubsection{Practice Set $F$}

Perform each subtraction.

Exercise 1.6.21

(Solution on p. 85.)

21,007

$-4,873$

Exercise 1.6.22

(Solution on p. 85.)

10,004

$-5,165$

Exercise 1.6.23

(Solution on p. 85.)

$16,000,000$

$-\quad 201,060$

\subsubsection{Calculators}

In practice, calculators are used to find the difference between two whole numbers.

\subsubsection{Sample Set G}

Find the difference between 1006 and 284 .

\begin{tabular}{|c|l|l|}
\hline \multicolumn{3}{|c|}{ Display Reads } \\
\hline Type & 1006 & 1006 \\
\hline Press & - & 1006 \\
\hline Type & 284 & 284 \\
\hline Press & $=$ & 722 \\
\hline
\end{tabular}

Table 1.26 
The difference between 1006 and 284 is 722 .

(What happens if you type 284 first and then 1006? We'll study such numbers in Chapter 10.)

\subsubsection{Practice Set G}

Exercise 1.6.24

Use a calculator to find the difference between 7338 and 2809 .

Exercise 1.6.25

Use a calculator to find the difference between 31,060,001 and 8,591,774.
(Solution on p. 85.)

(Solution on p. 85.)

\subsubsection{Exercises}

For the following problems, perform the subtractions. You may check each difference with a calculator.

Exercise 1.6.26

(Solution on p. 85.)

15

$-8$

Exercise 1.6.27

19

$-8$

Exercise 1.6.28

(Solution on p. 85.)

11

$-5$

Exercise 1.6.29

14

$-6$

Exercise 1.6.30

(Solution on p. 85.)

12

$-9$

Exercise 1.6.31

56

$-12$

Exercise 1.6.32

(Solution on p. 85.)

74

$-33$

Exercise 1.6.33

80

$-61$

Exercise 1.6.34

(Solution on p. 85.)

350

$-141$ 
Exercise 1.6.35

800

$-650$

Exercise 1.6.36

(Solution on p. 85.)

35,002

$-14,001$

Exercise 1.6.37

$5,000,566$

$-2,441,326$

Exercise 1.6.38

(Solution on p. 85.)

400, 605

$-121,352$

Exercise 1.6.39

46,400

- 2,012

Exercise 1.6.40

(Solution on p. 85.)

77,893

- 421

Exercise 1.6.41

42

$-18$

Exercise 1.6.42

(Solution on p. 85.)

51

$-27$

Exercise 1.6.43

622

$-88$

Exercise 1.6.44

(Solution on p. 85.)

261

$-73$

Exercise 1.6.45

242

$-158$

Exercise 1.6.46

(Solution on p. 85.)

3,422

$-1,045$

Exercise 1.6.47

5,565

$-3,985$ 
Exercise 1.6.48

(Solution on p. 85.)

42,041

$-15,355$

Exercise 1.6.49

304, 056

$-20,008$

Exercise 1.6.50

(Solution on p. 85.)

64, 000, 002

- 856,743

Exercise 1.6.51

4, 109

$-856$

Exercise 1.6.52

(Solution on p. 85.)

10,113

$-\quad 2,079$

Exercise 1.6.53

605

$-\quad 77$

Exercise 1.6.54

(Solution on p. 85.)

59

$-26$

Exercise 1.6.55

36,107

$-8,314$

Exercise 1.6.56

(Solution on p. 85.)

$92,526,441,820$

$-59,914,805,253$

Exercise 1.6.57

1,605

$-881$

Exercise 1.6.58

(Solution on p. 85.)

30,000

$-26,062$

Exercise 1.6.59

600

$-216$

Exercise 1.6.60

(Solution on p. 85.)

$9,000,003$

- 726,048

For the following problems, perform each subtraction.

Available for free at Connexions $<$ http://cnx.org/content/col10615/1.4 $>$ 
Exercise 1.6.61

Subtract 63 from 92 .

Hint: The word "from" means "beginning at." Thus, 63 from 92 means beginning at 92, or $92-63$.

Exercise 1.6.62

(Solution on p. 85.)

Subtract 35 from 86 .

Exercise 1.6.63

Subtract 382 from 541 .

Exercise 1.6.64

(Solution on p. 86.)

Subtract 1,841 from 5,246.

Exercise 1.6.65

Subtract 26,082 from 35,040 .

Exercise 1.6.66

(Solution on p. 86.)

Find the difference between 47 and 21 .

Exercise 1.6.67

Find the difference between 1,005 and 314 .

Exercise 1.6.68

(Solution on p. 86.)

Find the difference between 72,085 and 16 .

Exercise 1.6.69

Find the difference between 7,214 and 2,049.

Exercise 1.6.70

(Solution on p. 86.)

Find the difference between 56,108 and 52,911.

Exercise 1.6.71

How much bigger is 92 than 47 ?

Exercise 1.6.72

(Solution on p. 86.)

How much bigger is 114 than 85 ?

Exercise 1.6.73

How much bigger is 3,006 than 1,918 ?

Exercise 1.6.74

How much bigger is 11,201 than 816 ?

(Solution on p. 86.)

Exercise 1.6.75

How much bigger is $3,080,020$ than $1,814,161$ ?

Exercise 1.6.76

(Solution on p. 86.)

In Wichita, Kansas, the sun shines about $74 \%$ of the time in July and about $59 \%$ of the time in November. How much more of the time (in percent) does the sun shine in July than in November?

\section{Exercise 1.6.77}

The lowest temperature on record in Concord, New Hampshire in May is $21^{\circ} \mathrm{F}$, and in July it is $35^{\circ} \mathrm{F}$. What is the difference in these lowest temperatures?

Exercise 1.6.78

(Solution on p. 86.)

In 1980, there were 83,000 people arrested for prostitution and commercialized vice and 11,330,000

people arrested for driving while intoxicated. How many more people were arrested for drunk driving than for prostitution?

Exercise 1.6.79

In 1980, a person with a bachelor's degree in accounting received a monthly salary offer of $\$ 1,293$, and a person with a marketing degree a monthly salary offer of $\$ 1,145$. How much more was offered to the person with an accounting degree than the person with a marketing degree? 
Exercise 1.6.80

(Solution on p. 86.)

In 1970, there were about 793 people per square mile living in Puerto Rico, and 357 people per square mile living in Guam. How many more people per square mile were there in Puerto Rico than Guam?

\section{Exercise 1.6.81}

The 1980 population of Singapore was 2,414,000 and the 1980 population of Sri Lanka was $14,850,000$. How many more people lived in Sri Lanka than in Singapore in 1980?

Exercise 1.6.82

(Solution on p. 86.)

In 1977, there were 7,234,000 hospitals in the United States and 64,421,000 in Mainland China. How many more hospitals were there in Mainland China than in the United States in 1977?

\section{Exercise 1.6.83}

In 1978, there were 3,095,000 telephones in use in Poland and 4,292,000 in Switzerland. How many more telephones were in use in Switzerland than in Poland in 1978?

For the following problems, use the corresponding graphs to solve the problems.

Exercise 1.6.84

(Solution on p. 86.)

How many more life scientists were there in 1974 than mathematicians? (this image)

\section{Exercise 1.6.85}

How many more social, psychological, mathematical, and environmental scientists were there than life, physical, and computer scientists? (this image)

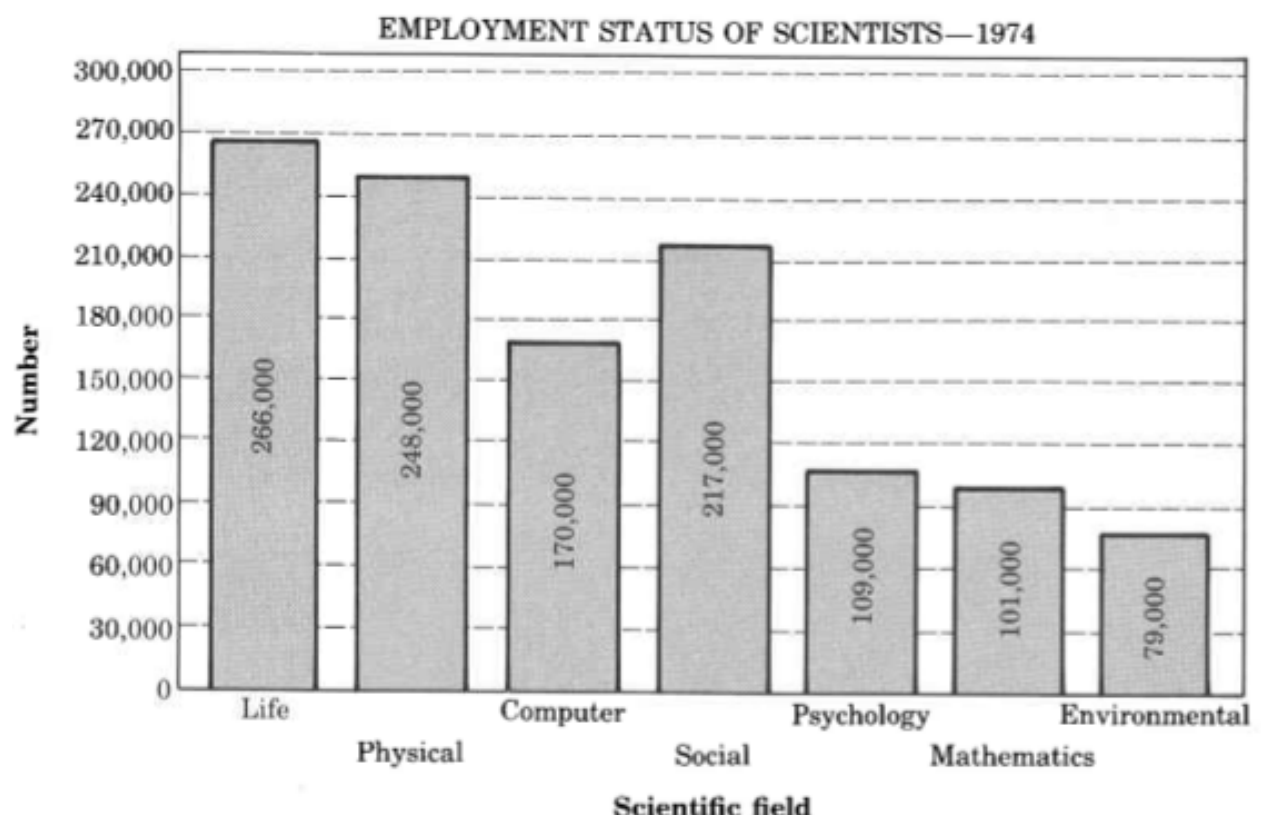

Exercise 1.6.86

(Solution on p. 86.)

How many more prosecutions were there in 1978 than in 1974? (this image)

Exercise 1.6.87

How many more prosecutions were there in 1976-1980 than in 1970-1975? (this image) 


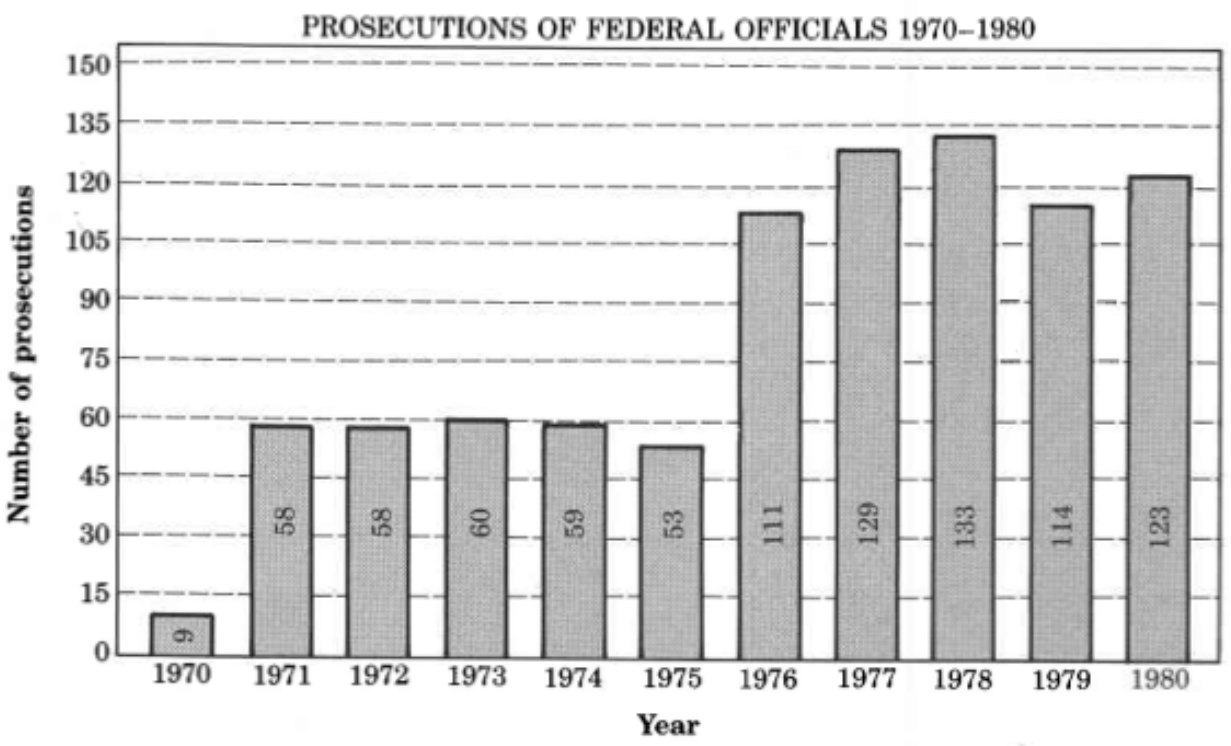

Exercise 1.6.88

(Solution on p. 86.)

How many more dry holes were drilled in 1960 than in 1975? (this image)

Exercise 1.6.89

How many more dry holes were drilled in 1960, 1965, and 1970 than in 1975, 1978 and 1979? (this image)

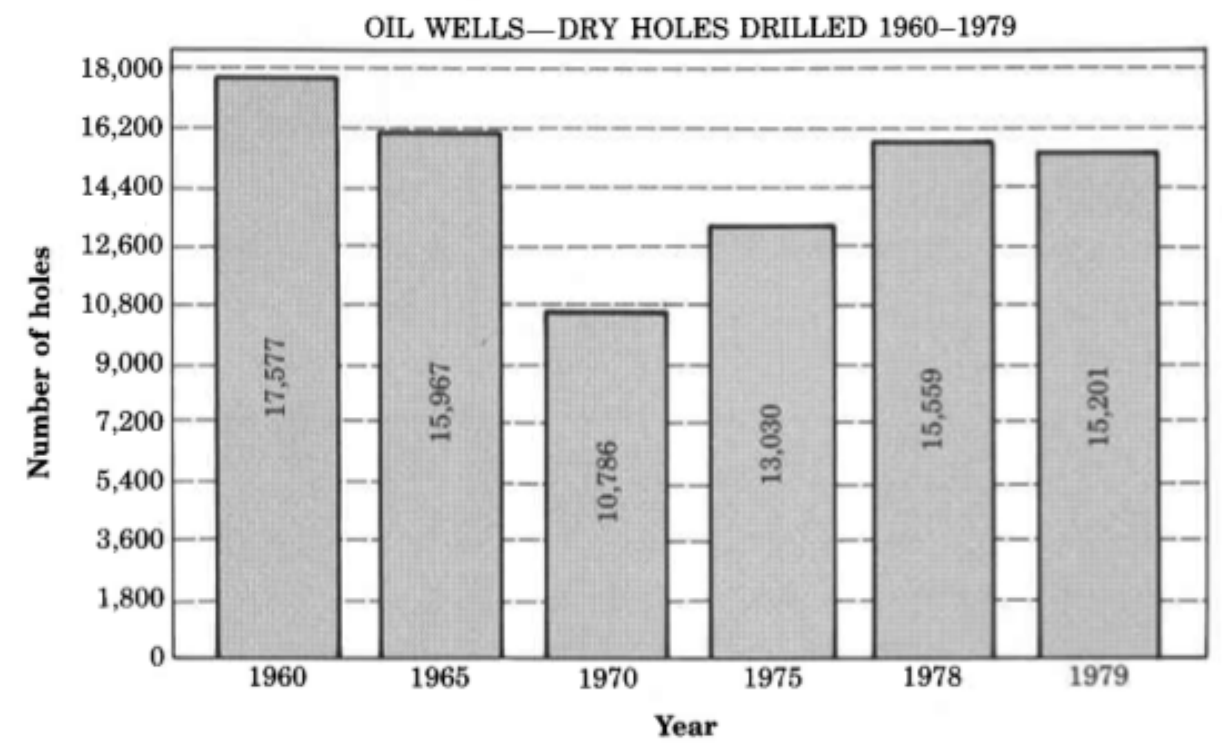

For the following problems, replace the [U+2610] with the whole number that will make the subtraction true.

Exercise 1.6.90

(Solution on p. 86.)

14

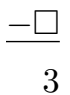

Available for free at Connexions $<$ http://cnx.org/content/col10615/1.4 $>$ 
Exercise 1.6.91

21

14

Exercise 1.6.92

(Solution on p. 86.)

35

25

Exercise 1.6.93

16

9

Exercise 1.6.94

(Solution on p. 86.)

28

16

For the following problems, find the solutions.

Exercise 1.6.95

Subtract 42 from the sum of 16 and 56 .

Exercise 1.6.96

(Solution on p. 86.)

Subtract 105 from the sum of 92 and 89 .

Exercise 1.6.97

Subtract 1,127 from the sum of 2,161 and 387 .

Exercise 1.6.98

(Solution on p. 86.)

Subtract 37 from the difference between 263 and 175 .

Exercise 1.6.99

Subtract 1,109 from the difference between 3,046 and 920 .

Exercise 1.6.100

(Solution on p. 86.)

Add the difference between 63 and 47 to the difference between 55 and 11 .

Exercise 1.6.101

Add the difference between 815 and 298 to the difference between 2,204 and 1,016.

Exercise 1.6.102

(Solution on p. 86.)

Subtract the difference between 78 and 43 from the sum of 111 and 89 .

Exercise 1.6.103

Subtract the difference between 18 and 7 from the sum of the differences between 42 and 13 , and 81 and 16.

Exercise 1.6.104

(Solution on p. 86.)

Find the difference between the differences of 343 and 96, and 521 and 488 . 


\subsubsection{Exercises for Review}

Exercise 1.6.105

(Section 1.2) In the number 21,206, how many hundreds are there?

Exercise 1.6.106 (Solution on p. 86.)

(Section 1.2) Write a three-digit number that has a zero in the ones position.

Exercise 1.6.107

(Section 1.2) How many three-digit whole numbers are there?

Exercise 1.6.108

(Solution on p. 86.)

(Section 1.4) Round 26,524,016 to the nearest million.

Exercise 1.6.109

(Section 1.5) Find the sum of $846+221+116$.

\subsection{Properties of Addition ${ }^{7}$}

\subsubsection{Section Overview}

- The Commutative Property of Addition

- The Associative Property of Addition

- The Additive Identity

We now consider three simple but very important properties of addition.

\subsubsection{The Commutative Property of Addition}

\section{Commutative Property of Addition}

If two whole numbers are added in any order, the sum will not change.

\subsubsection{Sample Set A}

Example 1.48

Add the whole numbers

8

5

$8+5=13$

$5+8=13$

The numbers 8 and 5 can be added in any order. Regardless of the order they are added, the sum is 13 .

\footnotetext{
${ }^{7}$ This content is available online at $<$ http://cnx.org/content $/ \mathrm{m} 34802 / 1.5 />$.
} 


\subsubsection{Practice Set A}

\section{Exercise 1.7.1}

(Solution on p. 86.) Use the commutative property of addition to find the sum of 12 and 41 in two different ways.

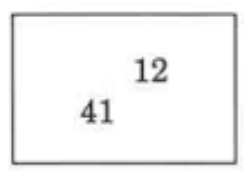

Exercise 1.7.2

(Solution on p. 86.)

Add the whole numbers

$$
837
$$

1,958

\subsubsection{The Associative Property of Addition}

\section{Associative Property of Addition}

If three whole numbers are to be added, the sum will be the same if the first two are added first, then that sum is added to the third, or, the second two are added first, and that sum is added to the first.

\section{Using Parentheses}

It is a common mathematical practice to use parentheses to show which pair of numbers we wish to combine first.

\subsubsection{Sample Set B}

Example 1.49

Add the whole numbers.

$$
\begin{gathered}
\mathbf{4 3} \text { and } 16 \text { are associated. } \\
(43+16)+27=59+27=86 \\
43+(16+27)=43+43=86 \\
16 \text { and } 27 \text { are associated. }
\end{gathered}
$$

\subsubsection{Practice Set B}

Exercise 1.7.3

(Solution on p. 87.) Use the associative property of addition to add the following whole numbers two different ways.

17 32 25

Exercise 1.7.4 (Solution on p. 87.)

1,629

806 429 


\subsubsection{The Additive Identity}

\section{Is the Additive Identity}

The whole number 0 is called the additive identity, since when it is added to any whole number, the sum is identical to that whole number.

\subsubsection{Sample Set C}

\section{Example 1.50}

Add the whole numbers.

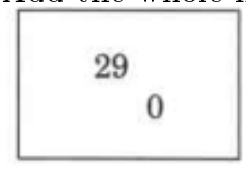

$29+0=29$

$0+29=29$

Zero added to 29 does not change the identity of 29 .

\subsubsection{Practice Set C}

Add the following whole numbers.

\section{Exercise 1.7.5}

(Solution on p. 87.)

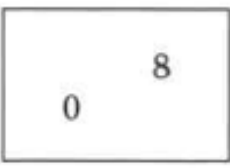

Exercise 1.7.6

(Solution on p. 87.)

0

5

Suppose we let the letter $\mathrm{x}$ represent a choice for some whole number. For the first two problems, find the sums. For the third problem, find the sum provided we now know that x represents the whole number 17. Exercise 1.7.7

(Solution on p. 87.)

$x$

0

Exercise 1.7.8

(Solution on p. 87.)

0

$x$ 
Exercise 1.7.9

(Solution on p. 87.)

0

$\boldsymbol{x}$

\subsubsection{Exercises}

For the following problems, add the numbers in two ways.

Exercise 1.7.10

(Solution on p. 87.)

8

29

Exercise 1.7.11

36

12

Exercise 1.7.12

(Solution on p. 87.)

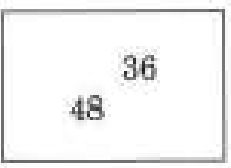

Exercise 1.7.13

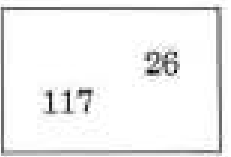

Exercise 1.7.14

(Solution on p. 87.)

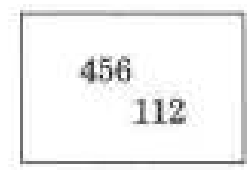

Exercise 1.7.15

$$
\begin{array}{r}
1,096 \\
4,251
\end{array}
$$

Exercise 1.7.16

(Solution on p. 87.)

73,205

49,118

Available for free at Connexions $<$ http://cnx.org/content/col10615/1.4 $>$ 
Exercise 1.7.17

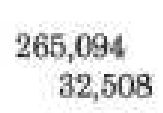

Exercise 1.7.18

(Solution on p. 87.)

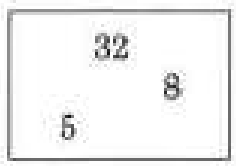

Exercise 1.7.19

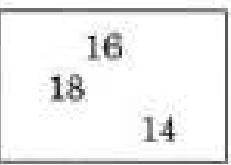

Exercise 1.7.20

(Solution on p. 87.)

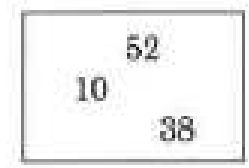

Exercise 1.7.21

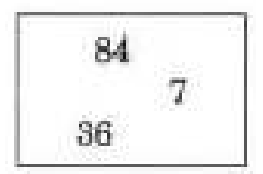

Exercise 1.7.22

(Solution on p. 87.)

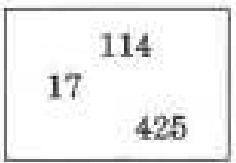

Exercise 1.7.23

\begin{tabular}{|cc|}
\hline & 1019 \\
11 & \\
& 586 \\
\hline
\end{tabular}

Exercise 1.7.24

(Solution on p. 87.)

\begin{tabular}{|c|}
\hline \multicolumn{1}{|c|}{1,261} \\
4,472
\end{tabular}

For the following problems, show that the pairs of quantities yield the same sum.

Exercise 1.7.25

$(11+27)+9$ and $11+(27+9)$

Exercise 1.7.26

$(80+52)+6$ and $80+(52+6)$

(Solution on p. 87.) 


\section{Exercise 1.7.27}

$(114+226)+108$ and $114+(226+108)$

Exercise 1.7.28

$(731+256)+171$ and $731+(256+171)$

(Solution on p. 87.)

Exercise 1.7.29

The fact that (a first number + a second number $)+$ third number $=$ a first number $+($ a second number + a third number) is an example of the property of addition.

Exercise 1.7.30

(Solution on p. 87.)

The fact that $0+$ any number $=$ that particular number is an example of the property of addition.

\section{Exercise 1.7.31}

The fact that a first number + a second number $=$ a second number + a first number is an example of the property of addition.

Exercise 1.7.32

Use the numbers 15 and 8 to illustrate the commutative property of addition.

(Solution on p. 87.)

Exercise 1.7.33

Use the numbers 6,5 , and 11 to illustrate the associative property of addition.

Exercise 1.7.34

(Solution on p. 87.)

The number zero is called the additive identity. Why is the term identity so appropriate?

\subsubsection{Exercises for Review}

Exercise 1.7.35

(Section 1.2) How many hundreds in 46,581 ?

Exercise 1.7.36

(Solution on p. 87.)

(Section 1.3) Write 2,218 as you would read it.

Exercise 1.7.37

(Section 1.4) Round 506,207 to the nearest thousand.

Exercise 1.7.38

(Solution on p. 87.)

(Section 1.5) Find the sum of

482

$$
+68
$$

Exercise 1.7.39

(Section 1.6) Find the difference: $\begin{array}{r}3,318 \\ -\quad 429 \\ \hline\end{array}$ 


\subsection{Summary of Key Concepts ${ }^{8}$}

\subsubsection{Summary of Key Concepts}

Number / Numeral (Section 1.2)

A number is a concept. It exists only in the mind. A numeral is a symbol that represents a number. It is customary not to distinguish between the two (but we should remain aware of the difference).

\section{Hindu-Arabic Numeration System (Section 1.2)}

In our society, we use the Hindu-Arabic numeration system. It was invented by the Hindus shortly before the third century and popularized by the Arabs about a thousand years later.

\section{Digits (Section 1.2)}

The numbers $0,1,2,3,4,5,6,7,8,9$ are called digits.

\section{Base Ten Positional System (Section 1.2)}

The Hindu-Arabic numeration system is a positional number system with base ten. Each position has value that is ten times the value of the position to its right.

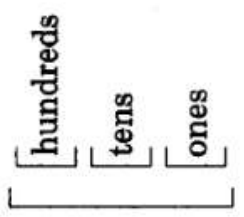

\section{Commas / Periods (Section 1.2)}

Commas are used to separate digits into groups of three. Each group of three is called a period. Each period has a name. From right to left, they are ones, thousands, millions, billions, etc.

\section{Whole Numbers (Section 1.2)}

A whole number is any number that is formed using only the digits $(0,1,2,3,4,5,6,7,8,9)$.

\section{Number Line (Section 1.2)}

The number line allows us to visually display the whole numbers.

\section{Graphing (Section 1.2)}

Graphing a whole number is a term used for visually displaying the whole number. The graph of 4 appears below.

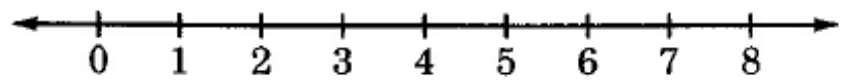

\section{Reading Whole Numbers (Section 1.3)}

To express a whole number as a verbal phrase:

1. Begin at the right and, working right to left, separate the number into distinct periods by inserting commas every three digits.

2. Begin at the left, and read each period individually.

\section{Writing Whole Numbers (Section 1.3)}

To rename a number that is expressed in words to a number expressed in digits:

\footnotetext{
${ }^{8}$ This content is available online at $<$ http://cnx.org/content/m34798/1.3/>.
} 
1. Notice that a number expressed as a verbal phrase will have its periods set off by commas.

2. Start at the beginning of the sentence, and write each period of numbers individually.

3. Use commas to separate periods, and combine the periods to form one number.

\section{Rounding (Section 1.4)}

Rounding is the process of approximating the number of a group of objects by mentally "seeing" the collection as occurring in groups of tens, hundreds, thousands, etc.

\section{Addition (Section 1.5)}

Addition is the process of combining two or more objects (real or intuitive) to form a new, third object, the total, or sum.

\section{Addends / Sum (Section 1.5)}

In addition, the numbers being added are called addends and the result, or total, the sum.

\section{Subtraction (Section 1.6)}

Subtraction is the process of determining the remainder when part of the total is removed.

Minuend / Subtrahend Difference (Section 1.6)

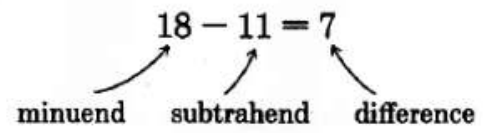

Commutative Property of Addition (Section 1.7)

If two whole numbers are added in either of two orders, the sum will not change.

$3+5=5+3$

\section{Associative Property of Addition (Section 1.7)}

If three whole numbers are to be added, the sum will be the same if the first two are added and that sum is then added to the third, or if the second two are added and the first is added to that sum.

$(3+5)+2=3+(5+2)$

\section{Parentheses in Addition (Section 1.7)}

Parentheses in addition indicate which numbers are to be added first.

\section{Additive Identity (Section 1.7)}

The whole number 0 is called the additive identity since, when it is added to any particular whole number, the sum is identical to that whole number.

$0+7=7$

$7+0=7$

\subsection{Exercise Supplement ${ }^{9}$}

\subsubsection{Exercise Supplement}

For problems 1-35, find the sums and differences.

Exercise 1.9.1

(Solution on p. 87.)

908

$+\quad 29$

\footnotetext{
${ }^{9}$ This content is available online at $<$ http://cnx.org/content $/ \mathrm{m} 34800 / 1.5 />$.
} 
Exercise 1.9.2

529

$+161$

Exercise 1.9.3

(Solution on p. 87.)

549

$+16$

Exercise 1.9.4

726

$+892$

Exercise 1.9.5

(Solution on p. 87.)

390

$+169$

Exercise 1.9.6

166

$+660$

Exercise 1.9.7

(Solution on p. 88.)

391

$+951$

Exercise 1.9.8

48

$+36$

Exercise 1.9.9

(Solution on p. 88.)

1,103

$\begin{array}{r}+\quad 898 \\ \hline\end{array}$

Exercise 1.9.10

1,642

$+899$

Exercise 1.9.11

(Solution on p. 88.)

807

$+1,156$

Exercise 1.9.12

80,349

$+2,679$

Exercise 1.9.13

(Solution on p. 88.)

70,070

$+9,386$

Exercise 1.9.14

90,874

$+2,945$ 
Exercise 1.9.15

(Solution on p. 88.)

45, 292

$+51,661$

Exercise 1.9.16

1,617

$+54,923$

Exercise 1.9.17

(Solution on p. 88.)

702, 607

$+89,217$

Exercise 1.9.18

$6,670,006$

$+\quad 2,495$

Exercise 1.9.19

(Solution on p. 88.)

267

$+8,034$

Exercise 1.9.20

7, 007

$+11,938$

Exercise 1.9.21

(Solution on p. 88.)

131,294

$+\quad 9,087$

Exercise 1.9.22

5,292

$+\quad 161$

Exercise 1.9.23

(Solution on p. 88.)

17,260

$+58,964$

Exercise 1.9.24

7, 006

$-5,382$

Exercise 1.9.25

(Solution on p. 88.)

7,973

$-3,018$

Exercise 1.9.26

16,608

$-1,660$

Exercise 1.9.27

(Solution on p. 88.)

209,527

- 23,916 
Exercise 1.9.28

584

$-226$

Exercise 1.9.29

(Solution on p. 88.)

3,313

$-1,075$

Exercise 1.9.30

458

$-122$

Exercise 1.9.31

(Solution on p. 88.)

1,007

$+331$

Exercise 1.9.32

16,082

$+2,013$

Exercise 1.9.33

(Solution on p. 88.)

926

$-48$

Exercise 1.9.34

736

$+5,869$

Exercise 1.9.35

(Solution on p. 88.)

676,504

- 58,277

For problems $36-39$, add the numbers.

Exercise 1.9.36

769

795

298

746

Exercise 1.9.37

(Solution on p. 88.)

554

184

883 
Exercise 1.9.38

30,188

79,731

16,600

66,085

39, 169

95,170

Exercise 1.9.39

(Solution on p. 88.)

$$
\begin{array}{r}
2,129 \\
6,190 \\
17,044 \\
30,447 \\
292 \\
41
\end{array}
$$

428,458

For problems 40-50, combine the numbers as indicated.

Exercise 1.9.40

$2,957+9,006$

Exercise 1.9.41

(Solution on p. 88.)

$19,040+813$

Exercise 1.9.42

$350,212+14,533$

Exercise 1.9.43

$970+702+22+8$

(Solution on p. 88.)

Exercise 1.9.44

$3,704+2,344+429+10,374+74$

Exercise 1.9.45

(Solution on p. 88.)

$874+845+295-900$

Exercise 1.9.46

$904+910-881$

Exercise 1.9.47

$521+453-334+600$

Exercise 1.9.48

$892-820-9$

Exercise 1.9.49

$159+4,085-918-608$

(Solution on p. 88.)

Exercise 1.9.50

$2,562+8,754-393-385-910$

For problems 51-63, add and subtract as indicated.

Exercise 1.9.51

(Solution on p. 88.)

Subtract 671 from 8,027. 
Exercise 1.9.52

Subtract 387 from 6,342 .

Exercise 1.9.53

(Solution on p. 88.)

Subtract 2,926 from 6,341.

Exercise 1.9.54

Subtract 4,355 from the sum of 74 and 7,319 .

Exercise 1.9.55

(Solution on p. 88.)

Subtract 325 from the sum of 7,188 and 4,964 .

Exercise 1.9.56

Subtract 496 from the difference of 60,321 and 99 .

Exercise 1.9.57

(Solution on p. 89.)

Subtract 20,663 from the difference of 523,150 and 95,225.

Exercise 1.9.58

Add the difference of 843 and 139 to the difference of 4,450 and 839 .

Exercise 1.9.59

(Solution on p. 89.)

Add the difference of 997,468 and 292,513 to the difference of 22,140 and 8,617 .

Exercise 1.9.60

Subtract the difference of 8,412 and 576 from the sum of 22,140 and 8,617 .

Exercise 1.9.61

(Solution on p. 89.)

Add the sum of $2,273,3,304,847$, and 16 to the difference of 4,365 and 864 .

Exercise 1.9.62

Add the sum of $19,161,201,166,127$, and 44 to the difference of the sums of $161,2,455$, and 85 , and $21,26,48$, and 187 .

Exercise 1.9.63

(Solution on p. 89.)

Is the sum of 626 and 1,242 the same as the sum of 1,242 and $626 ?$ Justify your claim.

\subsection{Proficiency Exam ${ }^{10}$}

\subsubsection{Proficiency Exam}

Exercise 1.10.1

(Solution on p. 89.)

(Section 1.2) What is the largest digit?

Exercise 1.10.2

(Solution on p. 89.)

(Section 1.2) In the Hindu-Arabic number system, each period has three values assigned to it. These values are the same for each period. From right to left, what are they?

Exercise 1.10.3

(Solution on p. 89.)

(Section 1.2) In the number 42,826 , how many hundreds are there?

Exercise 1.10.4

(Solution on p. 89.)

(Section 1.2) Is there a largest whole number? If so, what is it?

Exercise 1.10.5

(Solution on p. 89.)

(Section 1.2) Graph the following whole numbers on the number line: $2,3,5$.

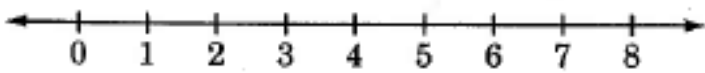

${ }^{10}$ This content is available online at $<\mathrm{http}: / / \mathrm{cnx} . o r g /$ content $/ \mathrm{m} 34805 / 1.4 />$. 
Exercise 1.10.6

(Solution on p. 89.)

(Section 1.3) Write the number 63,425 as you would read it aloud.

Exercise 1.10.7

(Solution on p. 89.)

(Section 1.3) Write the number eighteen million, three hundred fifty-nine thousand, seventy-two.

Exercise 1.10.8

(Solution on p. 89.)

(Section 1.4) Round 427 to the nearest hundred.

Exercise 1.10.9

(Solution on p. 89.)

(Section 1.4) Round 18,995 to the nearest ten.

Exercise 1.10.10

(Solution on p. 89.)

(Section 1.4) Round to the most reasonable digit: During a semester, a mathematics instructor uses 487 pieces of chalk.

For problems 11-17, find the sums and differences.

Exercise 1.10.11

(Solution on p. 89.)

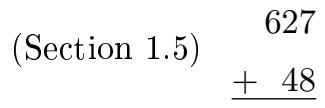

Exercise 1.10.12

(Section 1.5) $3106+921$

Exercise 1.10.13

(Solution on p. 89.)

152

(Section 1.5)

152

$+36$

Exercise 1.10.14

(Solution on p. 89.)

5,189

(Section 1.5)

6,189

4,122

$+8,001$

Exercise 1.10.15

(Solution on p. 89.)

(Section 1.5) $21+16+42+11$

(Solution on p. 89.)

Exercise 1.10.16

(Solution on p. 89.)

(Section 1.6) 520-216

Exercise 1.10.17

(Solution on p. 89.)

(Section 1.6)

80,001

$-9,878$

Exercise 1.10.18

(Solution on p. 89.)

(Section 1.6) Subtract 425 from 816.

Exercise 1.10.19

(Solution on p. 89.)

(Section 1.6) Subtract 712 from the sum of 507 and 387.

Exercise 1.10.20

(Solution on p. 89.)

(Section 1.7) Is the sum of 219 and 412 the same as the sum of 412 and 219? If so, what makes it so? 


\section{Solutions to Exercises in Chapter 1}

Solution to Exercise 1.2.1 (p. 10)

Yes. Letters are symbols. Taken as a collection (a written word), they represent a number.

Solution to Exercise 1.2.2 (p. 12)

five thousand

Solution to Exercise 1.2.3 (p. 12)

four hundred billion

Solution to Exercise 1.2.4 (p. 12)

zero tens, or zero

Solution to Exercise 1.2.5 (p. 13)

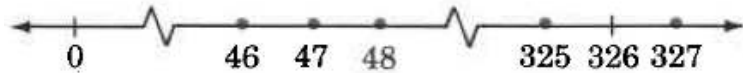

Solution to Exercise 1.2.6 (p. 13)

$4,5,6,113,978$

Solution to Exercise 1.2.7 (p. 14)

concept

Solution to Exercise 1.2.9 (p. 14)

Yes, since it is a symbol that represents a number.

Solution to Exercise 1.2.11 (p. 14)

positional; 10

Solution to Exercise 1.2.13 (p. 14)

ones, tens, hundreds

Solution to Exercise 1.2.15 (p. 14)

4

Solution to Exercise 1.2.17 (p. 14)

0

Solution to Exercise 1.2.19 (p. 14)

0

Solution to Exercise 1.2.21 (p. 14)

ten thousand

Solution to Exercise 1.2.23 (p. 14)

6 ten millions $=60$ million

Solution to Exercise 1.2.25 (p. 14)

1,340 (answers may vary)

Solution to Exercise 1.2.27 (p. 15)

900

Solution to Exercise 1.2.29 (p. 15)

yes; zero

Solution to Exercise 1.2.31 (p. 15)

graphing

Solution to Exercise 1.2.33 (p. 15)

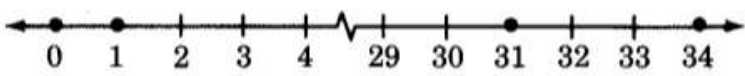

Solution to Exercise 1.2.35 (p. 15)

61, 99, 100, 102

Solution to Exercise 1.3.1 (p. 17)

Twelve thousand, five hundred forty-two

Solution to Exercise 1.3.2 (p. 17)

One hundred one million, seventy-four thousand, three 
Solution to Exercise 1.3.3 (p. 17)

One million, eight

Solution to Exercise 1.3.4 (p. 18)

103,025

Solution to Exercise 1.3.5 (p. 18)

$6,040,007$

Solution to Exercise 1.3.6 (p. 18)

20,003,080,109,402

Solution to Exercise 1.3.7 (p. 18)

$80,000,000,035$

Solution to Exercise 1.3.8 (p. 18)

nine hundred twelve

Solution to Exercise 1.3.10 (p. 19)

one thousand, four hundred ninety-one

Solution to Exercise 1.3.12 (p. 19)

thirty-five thousand, two hundred twenty-three

Solution to Exercise 1.3.14 (p. 19)

four hundred thirty-seven thousand, one hundred five

Solution to Exercise 1.3.16 (p. 19)

eight million, one thousand, one

Solution to Exercise 1.3.18 (p. 19)

seven hundred seventy million, three hundred eleven thousand, one hundred one

Solution to Exercise 1.3.20 (p. 19)

one hundred six billion, one hundred million, one thousand ten

Solution to Exercise 1.3.22 (p. 19)

eight hundred billion, eight hundred thousand

Solution to Exercise 1.3.24 (p. 19)

four; one thousand, four hundred sixty

Solution to Exercise 1.3.26 (p. 19)

twenty billion

Solution to Exercise 1.3.28 (p. 19)

four hundred twelve; fifty-two; twenty-one thousand, four hundred twenty-four

Solution to Exercise 1.3.30 (p. 19)

one thousand, nine hundred seventy-nine; eighty-five thousand; two million, nine hundred five thousand

Solution to Exercise 1.3.32 (p. 19)

one thousand, nine hundred eighty; two hundred seventeen

Solution to Exercise 1.3.34 (p. 20)

one thousand, nine hundred eighty one; one million, nine hundred fifty-six thousand

Solution to Exercise 1.3.36 (p. 20)

one thousand, nine hundred eighty; thirteen thousand, one hundred

Solution to Exercise 1.3.38 (p. 20)

twelve million, six hundred thirty thousand

Solution to Exercise 1.3.40 (p. 20)

681

Solution to Exercise 1.3.42 (p. 20)

7,201

Solution to Exercise 1.3.44 (p. 20)

512,003

Solution to Exercise 1.3.46 (p. 20)

$35,007,101$ 
Solution to Exercise 1.3.48 (p. 20)

$16,000,059,004$

Solution to Exercise 1.3.50 (p. 20) 23,000,000,000

Solution to Exercise 1.3.52 (p. 20)

$100,000,000,000,001$

Solution to Exercise 1.3.54 (p. 21) 4

Solution to Exercise 1.3.56 (p. 21) yes, zero

Solution to Exercise 1.4.1 (p. 24) 3400

Solution to Exercise 1.4.2 (p. 24) 27,000

Solution to Exercise 1.4.3 (p. 24)

$31,000,000$

Solution to Exercise 1.4.4 (p. 24)

0

Solution to Exercise 1.4.5 (p. 24) 60,000

Solution to Exercise 1.4.6 (p. 24)

\begin{tabular}{|l|l|l|l|}
\hline hundred & thousand & ten thousand & million \\
\hline 1,600 & 2000 & 0 & 0 \\
\hline
\end{tabular}

Table 1.27

Solution to Exercise 1.4.8 (p. 25)

\begin{tabular}{|l|l|l|l|}
\hline Hundred & thousand & ten thousand & million \\
\hline 91,800 & 92,000 & 90,000 & 0 \\
\hline
\end{tabular}

Table 1.28

Solution to Exercise 1.4.10 (p. 25)

\begin{tabular}{|l|l|l|l|}
\hline hundred & thousand & ten thousand & million \\
\hline 200 & 0 & 0 & 0 \\
\hline
\end{tabular}

Table 1.29

Solution to Exercise 1.4.12 (p. 25)

\begin{tabular}{|l|l|l|l|}
\hline hundred & thousand & ten thousand & million \\
\hline 900 & 1,000 & 0 & 0 \\
\hline
\end{tabular}

Available for free at Connexions $<$ http://cnx.org/content/col10615/1.4 $>$ 
Table 1.30

Solution to Exercise 1.4.14 (p. 26)

\begin{tabular}{|l|l|l|l|}
\hline hundred & thousand & ten thousand & million \\
\hline 900 & 1,000 & 0 & 0 \\
\hline
\end{tabular}

Table 1.31

Solution to Exercise 1.4.16 (p. 26)

\begin{tabular}{|l|l|l|l|}
\hline hundred & thousand & ten thousand & million \\
\hline 1,000 & 1,000 & 0 & 0 \\
\hline
\end{tabular}

Table 1.32

Solution to Exercise 1.4.18 (p. 26)

\begin{tabular}{|l|l|l|l|}
\hline hundred & thousand & ten thousand & million \\
\hline $551,061,300$ & $551,061,000$ & $551,060,000$ & $551,000,000$ \\
\hline
\end{tabular}

Table 1.33

Solution to Exercise 1.4.20 (p. 27)

\begin{tabular}{|l|l|l|l|}
\hline hundred & thousand & ten thousand & million \\
\hline $106,999,413,200$ & $106,999,413,000$ & $106,999,410,000$ & $106,999,000,000$ \\
\hline
\end{tabular}

Table 1.34

Solution to Exercise 1.4.22 (p. 27)

\begin{tabular}{|l|l|l|l|}
\hline Hundred & Thousand & ten thousand & Million \\
\hline $8,006,000$ & $8,006,000$ & $8,010,000$ & $8,000,000$ \\
\hline
\end{tabular}

Table 1.35

Solution to Exercise 1.4.24 (p. 27)

\begin{tabular}{|l|l|l|l|}
\hline hundred & thousand & ten thousand & million \\
\hline 33,500 & 33,000 & 30,000 & 0 \\
\hline
\end{tabular}

Available for free at Connexions $<$ http://cnx.org/content/col10615/1.4 $>$ 
Table 1.36

Solution to Exercise 1.4.26 (p. 28)

\begin{tabular}{|l|l|l|l|}
\hline hundred & thousand & ten thousand & million \\
\hline 388,600 & 389,000 & 390,000 & 0 \\
\hline
\end{tabular}

Table 1.37

Solution to Exercise 1.4.28 (p. 28)

\begin{tabular}{|l|l|l|l|}
\hline hundred & thousand & ten thousand & million \\
\hline 8,200 & 8,000 & 10,000 & 0 \\
\hline
\end{tabular}

Table 1.38

Solution to Exercise 1.4.30 (p. 28)

$19,310,000$

Solution to Exercise 1.4.32 (p. 29)

29,000,000

Solution to Exercise 1.4.34 (p. 29)

$70 \%$ or $75 \%$

Solution to Exercise 1.4.36 (p. 29)

$\$ 5,500,000,000$

Solution to Exercise 1.4.38 (p. 29)

230,000

Solution to Exercise 1.4.40 (p. 29)

$5,400,000$

Solution to Exercise 1.4.42 (p. 29)

graphing

Solution to Exercise 1.4.44 (p. 29)

Forty-two thousand, one hundred nine

Solution to Exercise 1.4.46 (p. 29)

4,000,000,008

Solution to Exercise 1.5.1 (p. 31)

88

6 tens +3 ones

+2 tens +5 ones

8 tens +8 ones

Solution to Exercise 1.5.2 (p. 31)

5,527

4 thousands +0 hundreds +2 tens +6 ones

+1 thousand +5 hundreds +0 tens +1 one

5 thousands +5 hundreds +2 tens +7 ones

Solution to Exercise 1.5.3 (p. 31)

267,166 
Solution to Exercise 1.5.4 (p. 34)

87

$$
\begin{gathered}
5 \text { tens }+8 \text { ones } \\
+\mathbf{2} \text { tens }+\mathbf{9} \text { ones } \\
\hline 7 \text { tens }+17 \text { ones } \\
=7 \text { tens }+1 \text { ten }+7 \text { ones } \\
=8 \text { tens }+7 \text { ones } \\
=87
\end{gathered}
$$

Solution to Exercise 1.5.5 (p. 34) 561

4 hundreds +7 tens +6 ones

$+\quad 8$ tens +5 ones

4 hundreds +15 tens +11 ones

$=4$ hundreds +15 tens +1 ten +1 one $=4$ hundreds +16 tens +1 one

$=4$ hundreds +1 hundred +6 tens +1 one

$$
=5 \text { hundreds }+6 \text { tens }+1 \text { one }
$$$$
=561
$$

Solution to Exercise 1.5.6 (p. 34)

115

$$
2 \text { tens }+7 \text { ones }
$$

+8 tens +8 ones

$=10$ tens +1 ten +5 ones

$$
=11 \text { tens }+5 \text { ones }
$$

$=1$ hundred +1 ten +5 ones

$$
=115
$$

Solution to Exercise 1.5.7 (p. 34) 153,525

Solution to Exercise 1.5.8 (p. 34) 167

Solution to Exercise 1.5.9 (p. 35) 2,468

Solution to Exercise 1.5.10 (p. 35) 434,826

Solution to Exercise 1.5.11 (p. 36) 155

Solution to Exercise 1.5.12 (p. 36) 19,750

Solution to Exercise 1.5.13 (p. 36) 30,682

Solution to Exercise 1.5.14 (p. 36) 19

Solution to Exercise 1.5.16 (p. 36) 48 
Solution to Exercise 1.5.18 (p. 36) 98

Solution to Exercise 1.5.20 (p. 36) 978

Solution to Exercise 1.5.22 (p. 37) 368

Solution to Exercise 1.5.24 (p. 37) 12,777

Solution to Exercise 1.5.26 (p. 37) 58,738

Solution to Exercise 1.5.28 (p. 37) $45,169,739$

Solution to Exercise 1.5.30 (p. 37) 33

Solution to Exercise 1.5.32 (p. 37) 81

Solution to Exercise 1.5.34 (p. 37) 91

Solution to Exercise 1.5.36 (p. 37) 809

Solution to Exercise 1.5.38 (p. 38) 862

Solution to Exercise 1.5.40 (p. 38) 2,321

Solution to Exercise 1.5.42 (p. 38) $1,141,204$

Solution to Exercise 1.5.44 (p. 38) $561,364,111$

Solution to Exercise 1.5.46 (p. 38) 942,302,364,207,060

Solution to Exercise 1.5.48 (p. 38) 234

Solution to Exercise 1.5.50 (p. 38) 95,365

Solution to Exercise 1.5.52 (p. 39) $1,972,128$

Solution to Exercise 1.5.54 (p. 39) 3,700

Solution to Exercise 1.5.56 (p. 39) $3,101,500$

Solution to Exercise 1.5.58 (p. 39) 100

Solution to Exercise 1.5.60 (p. 40) 0

Solution to Exercise 1.5.62 (p. 40) 1,000

Solution to Exercise 1.5.64 (p. 40) 5

Solution to Exercise 1.5.66 (p. 40) 19 
Solution to Exercise 1.5.68 (p. 40) 88

Solution to Exercise 1.5.70 (p. 41) $60,511,000$

Solution to Exercise 1.5.72 (p. 41)

$5,682,651$ square miles

Solution to Exercise 1.5.74 (p. 41) $1,190,000$

Solution to Exercise 1.5.76 (p. 42) $271,564,000$

Solution to Exercise 1.5.78 (p. 43)

20

Solution to Exercise 1.5.80 (p. 43) 25

Solution to Exercise 1.5.82 (p. 43)

40

Solution to Exercise 1.5.84 (p. 43)

50

Solution to Exercise 1.5.86 (p. 44)

50

Solution to Exercise 1.5.88 (p. 44)

50

Solution to Exercise 1.5.90 (p. 44)

0

Solution to Exercise 1.5.92 (p. 44)

6,800

Solution to Exercise 1.6.1 (p. 45)

$7-5=2$ since $2+5=7$

Solution to Exercise 1.6.2 (p. 45)

$9-1=8$ since $8+1=9$

Solution to Exercise 1.6.3 (p. 45)

$17-8=9$ since $9+8=17$

Solution to Exercise 1.6.4 (p. 47)

331

Solution to Exercise 1.6.5 (p. 47)

814

Solution to Exercise 1.6.6 (p. 47)

61,118

Solution to Exercise 1.6.7 (p. 47)

10,000

Solution to Exercise 1.6.8 (p. 47)

62,114 
Solution to Exercise 1.6.9 (p. 49)

$$
\begin{aligned}
& 18, 5 \text { tens }+3 \text { ones } \\
&-\quad \frac{3 \text { tens }+5 \text { ones }}{4 \text { tens }+1 \text { ten }+3 \text { ones }} \\
&-\quad \frac{3 \text { tens } \quad 5 \text { ones }}{4 \text { tens }+13 \text { ones }} \\
&-\quad \frac{3 \text { tens }+5 \text { ones }}{1 \text { ten }+8 \text { ones }} \\
&=18
\end{aligned}
$$

Solution to Exercise 1.6.10 (p. 49)

$$
\begin{aligned}
48, & 7 \text { tens }+6 \text { ones } \\
- & \frac{2 \text { tens }+8 \text { ones }}{6 \text { tens }+1 \text { ten }+6 \text { ones }} \\
-\quad & \frac{2 \text { tens }+8 \text { ones }}{6 \text { tens }+16 \text { ones }} \\
-\quad & \frac{2 \text { tens }+8 \text { ones }}{4 \text { tens }+8 \text { ones }} \\
=48 &
\end{aligned}
$$

Solution to Exercise 1.6.11 (p. 49)

307,8 hundreds +7 tens +2 ones

- $\quad 5$ hundreds +6 tens +5 ones

8 hundreds +6 tens +1 ten +2 ones

- 5 hundreds +6 tens +5 ones

8 hundreds +6 tens +12 ones

- $\quad 5$ hundreds +6 tens +5 ones

3 hundreds +0 tens +7 ones

$$
=307
$$

Solution to Exercise 1.6.12 (p. 49)

85

Solution to Exercise 1.6.13 (p. 49)

709

Solution to Exercise 1.6.14 (p. 49)

3,104

Solution to Exercise 1.6.15 (p. 50)

168

Solution to Exercise 1.6.16 (p. 50)

55,640

Solution to Exercise 1.6.17 (p. 50)

1,189

Solution to Exercise 1.6.18 (p. 52)

888 
Solution to Exercise 1.6.19 (p. 52) 4,543

Solution to Exercise 1.6.20 (p. 52) 8,669

Solution to Exercise 1.6.21 (p. 54) 16,134

Solution to Exercise 1.6.22 (p. 54) 4,839

Solution to Exercise 1.6.23 (p. 54) $15,789,940$

Solution to Exercise 1.6.24 (p. 55) 4,529

Solution to Exercise 1.6.25 (p. 55) $22,468,227$

Solution to Exercise 1.6.26 (p. 55) 7

Solution to Exercise 1.6.28 (p. 55) 6

Solution to Exercise 1.6.30 (p. 55) 3

Solution to Exercise 1.6.32 (p. 55) 41

Solution to Exercise 1.6.34 (p. 55) 209

Solution to Exercise 1.6.36 (p. 56) 21,001

Solution to Exercise 1.6.38 (p. 56) 279,253

Solution to Exercise 1.6.40 (p. 56) 77,472

Solution to Exercise 1.6.42 (p. 56) 24

Solution to Exercise 1.6.44 (p. 56) 188

Solution to Exercise 1.6.46 (p. 56) 2,377

Solution to Exercise 1.6.48 (p. 56) 26,686

Solution to Exercise 1.6.50 (p. 57) $63,143,259$

Solution to Exercise 1.6.52 (p. 57) 8,034

Solution to Exercise 1.6.54 (p. 57) 33

Solution to Exercise 1.6.56 (p. 57) $32,611,636,567$

Solution to Exercise 1.6.58 (p. 57) 3,938

Solution to Exercise 1.6.60 (p. 57) $8,273,955$ 
Solution to Exercise 1.6.62 (p. 58) 51

Solution to Exercise 1.6.64 (p. 58) 3,405

Solution to Exercise 1.6.66 (p. 58)

26

Solution to Exercise 1.6.68 (p. 58) 72,069

Solution to Exercise 1.6.70 (p. 58) 3,197

Solution to Exercise 1.6.72 (p. 58) 29

Solution to Exercise 1.6.74 (p. 58) 10,385

Solution to Exercise 1.6.76 (p. 58) $15 \%$

Solution to Exercise 1.6.78 (p. 58)

$11,247,000$

Solution to Exercise 1.6.80 (p. 59) 436

Solution to Exercise 1.6.82 (p. 59) $57,187,000$

Solution to Exercise 1.6.84 (p. 59) 165,000

Solution to Exercise 1.6.86 (p. 59) 74

Solution to Exercise 1.6.88 (p. 60) 4,547

Solution to Exercise 1.6.90 (p. 60) 11

Solution to Exercise 1.6.92 (p. 61) 10

Solution to Exercise 1.6.94 (p. 61) 12

Solution to Exercise 1.6.96 (p. 61) 76

Solution to Exercise 1.6.98 (p. 61) 51

Solution to Exercise 1.6.100 (p. 61)

60

Solution to Exercise 1.6.102 (p. 61)

165

Solution to Exercise 1.6.104 (p. 61)

214

Solution to Exercise 1.6.106 (p. 62) 330 (answers may vary)

Solution to Exercise 1.6.108 (p. 62) $27,000,000$

Solution to Exercise 1.7.1 (p. 63)

$12+41=53$ and $41+12=53$ 
Solution to Exercise 1.7.2 (p. 63)

$837+1,958=2,795$ and $1,958+837=2,795$

Solution to Exercise 1.7.3 (p. 63)

$(17+32)+25=49+25=74$ and $17+(32+25)=17+57=74$

Solution to Exercise 1.7.4 (p. 63)

$(1,629+806)+429=2,435+429=2,864$

$1,629+(806+429)=1,629+1,235=2,864$

Solution to Exercise 1.7.5 (p. 64)

8

Solution to Exercise 1.7.6 (p. 64)

5

Solution to Exercise 1.7.7 (p. 64)

$\mathrm{x}$

Solution to Exercise 1.7.8 (p. 64)

$\mathrm{x}$

Solution to Exercise 1.7.9 (p. 65)

17

Solution to Exercise 1.7.10 (p. 65)

37

Solution to Exercise 1.7.12 (p. 65)

45

Solution to Exercise 1.7.14 (p. 65)

568

Solution to Exercise 1.7.16 (p. 65) 122,323

Solution to Exercise 1.7.18 (p. 66)

45

Solution to Exercise 1.7.20 (p. 66)

100

Solution to Exercise 1.7.22 (p. 66)

556

Solution to Exercise 1.7.24 (p. 66)

43,461

Solution to Exercise 1.7.26 (p. 66)

$132+6=80+58=138$

Solution to Exercise 1.7.28 (p. 67)

$987+171=731+427=1,158$

Solution to Exercise 1.7.30 (p. 67)

Identity

Solution to Exercise 1.7.32 (p. 67)

$15+8=8+15=23$

Solution to Exercise 1.7.34 (p. 67)

...because its partner in addition remains identically the same after that addition

Solution to Exercise 1.7.36 (p. 67)

Two thousand, two hundred eighteen.

Solution to Exercise 1.7.38 (p. 67)

550

Solution to Exercise 1.9.1 (p. 69)

937

Solution to Exercise 1.9.3 (p. 70)

565 
Solution to Exercise 1.9.5 (p. 70)

559

Solution to Exercise 1.9.7 (p. 70)

1,342

Solution to Exercise 1.9.9 (p. 70)

2,001

Solution to Exercise 1.9.11 (p. 70)

1,963

Solution to Exercise 1.9.13 (p. 70)

79,456

Solution to Exercise 1.9.15 (p. 70)

96,953

Solution to Exercise 1.9.17 (p. 71)

791,824

Solution to Exercise 1.9.19 (p. 71)

8,301

Solution to Exercise 1.9.21 (p. 71)

140,381

Solution to Exercise 1.9.23 (p. 71)

76,224

Solution to Exercise 1.9.25 (p. 71)

4,955

Solution to Exercise 1.9.27 (p. 71)

185,611

Solution to Exercise 1.9.29 (p. 72)

2,238

Solution to Exercise 1.9.31 (p. 72)

1,338

Solution to Exercise 1.9.33 (p. 72)

878

Solution to Exercise 1.9.35 (p. 72)

618,227

Solution to Exercise 1.9.37 (p. 72)

1,621

Solution to Exercise 1.9.39 (p. 73)

484,601

Solution to Exercise 1.9.41 (p. 73)

19,853

Solution to Exercise 1.9.43 (p. 73)

1,702

Solution to Exercise 1.9.45 (p. 73)

1,114

Solution to Exercise 1.9.47 (p. 73)

1,300

Solution to Exercise 1.9.49 (p. 73)

2,718

Solution to Exercise 1.9.51 (p. 73)

7,356

Solution to Exercise 1.9.53 (p. 74)

3,415 
Solution to Exercise 1.9.55 (p. 74) 11,827

Solution to Exercise 1.9.57 (p. 74)

407,262

Solution to Exercise 1.9.59 (p. 74)

718,478

Solution to Exercise 1.9.61 (p. 74)

9,941

Solution to Exercise 1.9.63 (p. 74)

$626+1,242=1,242+626=1,868$

Solution to Exercise 1.10.1 (p. 74)

9

Solution to Exercise 1.10.2 (p. 74)

ones, tens, hundreds

Solution to Exercise 1.10.3 (p. 74)

8

Solution to Exercise 1.10.4 (p. 74)

no

Solution to Exercise 1.10.5 (p. 74)

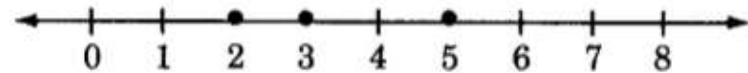

Solution to Exercise 1.10.6 (p. 75)

Sixty-three thousand, four hundred twenty-five

Solution to Exercise 1.10.7 (p. 75)

$18,359,072$

Solution to Exercise 1.10.8 (p. 75)

400

Solution to Exercise 1.10.9 (p. 75) 19,000

Solution to Exercise 1.10.10 (p. 75)

500

Solution to Exercise 1.10.11 (p. 75)

675

Solution to Exercise 1.10.12 (p. 75)

4,027

Solution to Exercise 1.10.13 (p. 75)

188

Solution to Exercise 1.10.14 (p. 75)

23,501

Solution to Exercise 1.10.15 (p. 75)

90

Solution to Exercise 1.10.16 (p. 75)

304

Solution to Exercise 1.10.17 (p. 75)

70,123

Solution to Exercise 1.10.18 (p. 75)

391

Solution to Exercise 1.10.19 (p. 75)

182

Solution to Exercise 1.10.20 (p. 75)

Yes, commutative property of addition 


\section{Chapter 2}

\section{Multiplication and Division of Whole Numbers}

\subsection{Objectives ${ }^{1}$}

After completing this chapter, you should

Multiplication of Whole Numbers (Section 2.2)

- understand the process of multiplication

- be able to multiply whole numbers

- be able to simplify multiplications with numbers ending in zero

- be able to use a calculator to multiply one whole number by another

\section{Concepts of Division of Whole Numbers (Section 2.3)}

- understand the process of division

- understand division of a nonzero number into zero

- understand why division by zero is undefined

- be able to use a calculator to divide one whole number by another

\section{Division of Whole Numbers (Section 2.4)}

- be able to divide a whole number by a single or multiple digit divisor

- be able to interpret a calculator statement that a division results in a remainder

\section{Some Interesting Facts about Division (Section 2.5)}

- be able to recognize a whole number that is divisible by $2,3,4,5,6,8,9$, or 10

Properties of Multiplication (Section 2.6)

- understand and appreciate the commutative and associative properties of multiplication

- understand why 1 is the multiplicative identity

\footnotetext{
${ }^{1}$ This content is available online at $<$ http://cnx.org/content $/ \mathrm{m} 18888 / 1.4 />$.
} 


\subsection{Multiplication of Whole Numbers ${ }^{2}$}

\subsubsection{Section Overview}

- Multiplication

- The Multiplication Process With a Single Digit Multiplier

- The Multiplication Process With a Multiple Digit Multiplier

- Multiplication With Numbers Ending in Zero

- Calculators

\subsubsection{Multiplication}

Multiplication is a description of repeated addition.

In the addition of

$5+5+5$

the number 5 is repeated 3 times. Therefore, we say we have three times five and describe it by writing

$3 \times 5$

Thus,

$3 \times 5=5+5+5$

\section{Multiplicand}

In a multiplication, the repeated addend (number being added) is called the multiplicand. In $3 \times 5$, the 5 is the multiplicand.

\section{Multiplier}

Also, in a multiplication, the number that records the number of times the multiplicand is used is called the multiplier. In $3 \times 5$, the 3 is the multiplier.

\subsubsection{Sample Set A}

Express each repeated addition as a multiplication. In each case, specify the multiplier and the multiplicand.

Example 2.1

$7+7+7+7+7+7$

$6 \times 7$. Multiplier is 6 . Multiplicand is 7 .

Example 2.2

$18+18+18$

$3 \times 18 . \quad$ Multiplier is $3 . \quad$ Multiplicand is 18 .

\footnotetext{
${ }^{2}$ This content is available online at $<$ http://cnx.org/content $/ \mathrm{m} 34863 / 1.2 />$.
} 


\subsubsection{Practice Set A}

Express each repeated addition as a multiplication. In each case, specify the multiplier and the multiplicand.

Exercise 2.2.1

$$
12+12+12+12
$$

(Solution on p. 142.)

. Multiplier is . Multiplicand is

Exercise 2.2.2

(Solution on p. 142.)

$36+36+36+36+36+36+36+36$ . Multiplier is . Multiplicand is

Exercise 2.2.3

$0+0+0+0+0$

(Solution on p. 142.) . Multiplier is Multiplicand is

Exercise 2.2.4

(Solution on p. 142.) $\underbrace{1847+1847+\ldots+1847}$

12, 000 times . Multiplier is . Multiplicand is

\section{Factors}

In a multiplication, the numbers being multiplied are also called factors.

\section{Products}

The result of a multiplication is called the product. In $3 \times 5=15$, the 3 and 5 are not only called the multiplier and multiplicand, but they are also called factors. The product is 15 .

\section{Indicators of Multiplication $\times, \cdot,()$}

The multiplication symbol $(x)$ is not the only symbol used to indicate multiplication. Other symbols include the $\operatorname{dot}(\cdot)$ and pairs of parentheses () . The expressions
$3 \times 5$,
$3 \cdot 5$,
$3(5)$,
(3) 5 ,
(3) (5)

all represent the same product.

\subsubsection{The Multiplication Process With a Single Digit Multiplier}

Since multiplication is repeated addition, we should not be surprised to notice that carrying can occur. Carrying occurs when we find the product of 38 and 7 :

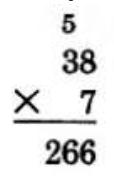

First, we compute $7 \times 8=56$. Write the 6 in the ones column. Carry the 5 . Then take $7 \times 3=21$. Add to 21 the 5 that was carried: $21+5=26$. The product is 266 . 


\subsubsection{Sample Set B}

Find the following products.

Example 2.3

1

64

$\begin{array}{r}\times \quad 3 \\ \hline 192\end{array}$

$3 \times 4=12$

Write the 2, carry the 1 .

$3 \times 6=18 \quad$ Add to 18 the 1 that was carried: $18+1=19$.

The product is 192 .

Example 2.4

$\begin{array}{r}13 \\ 526 \\ \times \quad 5 \\ \hline 2,630\end{array}$

$5 \times 6=30$

Write the 0 , carry the 3 .

$5 \times 2=10$

Add to 10 the 3 that was carried: $10+3=13$. Write the 3 , carry the 1 .

$5 \times 5=25$

Add to 25 the 1 that was carried: $25+1=6$.

The product is 2,630 .

\section{Example 2.5}

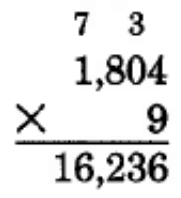

$$
\begin{gathered}
9 \times 4=36 \\
9 \times 0=0 \\
9 \times 8=72 \\
9 \times 1=9
\end{gathered}
$$

Write the 6 , carry the 3 .

Add to the 0 the 3 that was carried: $0+3=3$. Write the 3 .

Write the 2, carry the 7 .

Add to the 9 the 7 that was carried: $9+7=16$.

Since there are no more multiplications to perform, write both the 1 and 6 .

The product is 16,236 .

\subsubsection{Practice Set $B$}

Find the following products.

Exercise 2.2.5

(Solution on p. 142.)

37

$\underline{5}$ 
Exercise 2.2.6

(Solution on p. 142.)

78

$\times 8$

Exercise 2.2.7

(Solution on p. 142.)

536

$\begin{array}{r}\times \\ \hline\end{array}$

Exercise 2.2.8

(Solution on p. 142.)

40,019

$\times \quad 8$

Exercise 2.2.9

(Solution on p. 142.)

301, 599

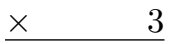

\subsubsection{The Multiplication Process With a Multiple Digit Multiplier}

In a multiplication in which the multiplier is composed of two or more digits, the multiplication must take place in parts. The process is as follows:

Part 1: First Partial Product Multiply the multiplicand by the ones digit of the multiplier. This product is called the first partial product.

Part 2: Second Partial Product Multiply the multiplicand by the tens digit of the multiplier. This product is called the second partial product. Since the tens digit is used as a factor, the second partial product is written below the first partial product so that its rightmost digit appears in the tens column.

Part 3: If necessary, continue this way finding partial products. Write each one below the previous one so that the rightmost digit appears in the column directly below the digit that was used as a factor.

Part 4: Total Product Add the partial products to obtain the total product.

NOTE: It may be necessary to carry when finding each partial product.

\subsubsection{Sample Set $\mathrm{C}$}

Example 2.6

Multiply 326 by 48 .

24

326

$\times 48$

$2608 \longleftarrow$ First partial product.

Part 1:

12

24

326

$\times 48$

$\overline{2608}$

Part 2:

$1304 \longleftarrow$ Second partial product. 
Part 3: This step is unnecessary since all of the digits in the multiplier have been used. Part 4: Add the partial products to obtain the total product.

$$
\begin{array}{r}
12 \\
24 \\
326 \\
\times \quad 48 \\
\frac{2608}{+1304} \\
\hline 15648
\end{array} \text { Total product. }
$$

The product is 15,648 .

Example 2.7

Multiply 5,369 by 842 .

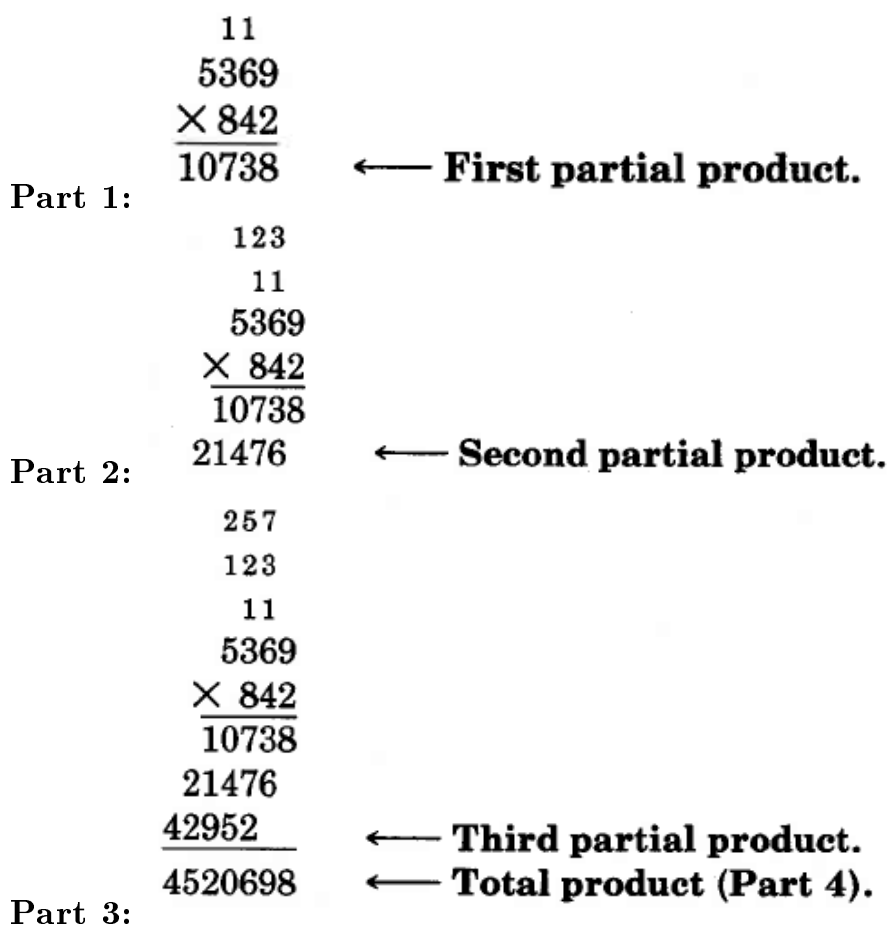

The product is $4,520,698$.

Example 2.8

Multiply 1,508 by 206 .

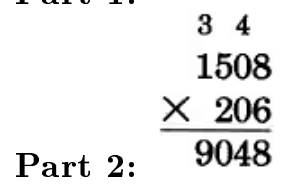


Since 0 times 1508 is 0 , the partial product will not change the identity of the total product (which is obtained by addition). Go to the next partial product.

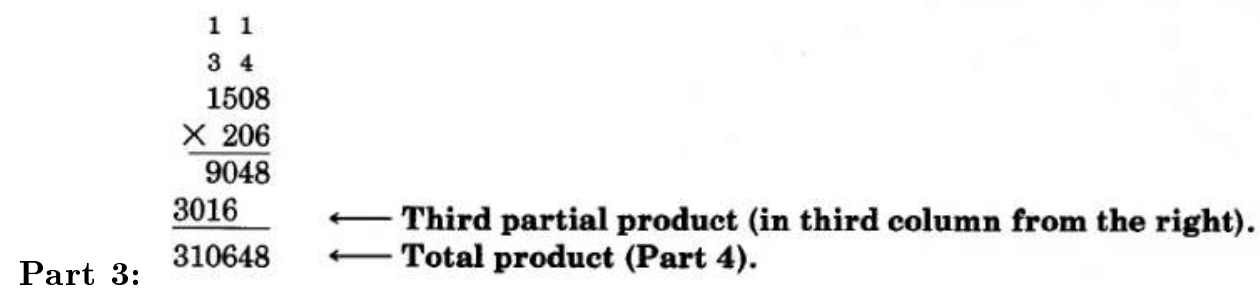

The product is 310,648

\subsubsection{Practice Set C}

Exercise 2.2.10

(Solution on p. 142.)

Multiply 73 by 14 .

Exercise 2.2.11

(Solution on p. 142.)

Multiply 86 by 52 .

Exercise 2.2.12

(Solution on p. 142.)

Multiply 419 by 85 .

Exercise 2.2.13

(Solution on p. 142.)

Multiply 2,376 by 613 .

Exercise 2.2.14

(Solution on p. 142.)

Multiply 8,107 by 304 .

Exercise 2.2.15

(Solution on p. 142.)

Multiply 66,260 by 1,008 .

Exercise 2.2.16

(Solution on p. 142.)

Multiply 209 by 501 .

Exercise 2.2.17

(Solution on p. 142.)

Multiply 24 by 10 .

Exercise 2.2.18

(Solution on p. 142.)

Multiply 3,809 by 1,000 .

Exercise 2.2.19

(Solution on p. 142.)

Multiply 813 by 10,000 .

\subsubsection{Multiplications With Numbers Ending in Zero}

Often, when performing a multiplication, one or both of the factors will end in zeros. Such multiplications can be done quickly by aligning the numbers so that the rightmost nonzero digits are in the same column. 


\subsubsection{Sample Set D}

Perform the multiplication $(49,000)(1,200)$.

$$
\begin{aligned}
&(49,000)(1,200)= 49000 \\
& \times \quad 1200 \\
& \hline
\end{aligned}
$$

Since 9 and 2 are the rightmost nonzero digits, put them in the same column. 49000

$\times 1200$

Draw (perhaps mentally) a vertical line to separate the zeros from the nonzeros.

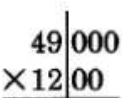

Multiply the numbers to the left of the vertical line as usual, then attach to the right end of this product the total number of zeros.

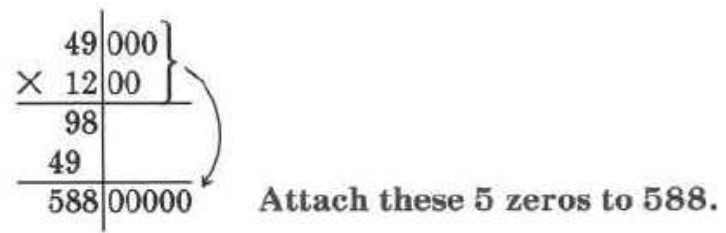

The product is $58,800,000$

\subsubsection{Practice Set $D$}

Exercise 2.2.20

(Solution on p. 142.)

Multiply 1,800 by 90 .

Exercise 2.2.21

(Solution on p. 142.)

Multiply 420,000 by 300 .

Exercise 2.2.22

(Solution on p. 142.)

Multiply $20,500,000$ by 140,000 .

\subsubsection{Calculators}

Most multiplications are performed using a calculator.

\subsubsection{Sample Set E}

Example 2.9

Multiply 75,891 by 263 .

\begin{tabular}{|c|l|r|}
\hline \multicolumn{3}{|r|}{ Display Reads } \\
\hline Type & 75891 & 75891 \\
\hline Press & $\times$ & 75891 \\
\hline Type & 263 & 263 \\
\hline Press & $=$ & 19959333 \\
\hline
\end{tabular}


Table 2.1

The product is $19,959,333$.

Example 2.10

Multiply $4,510,000,000,000$ by 1,700 .

\begin{tabular}{|l|l|r|}
\hline \multicolumn{3}{|c|}{ Display Reads } \\
\hline Type & 451 & 451 \\
\hline Press & $\times$ & 451 \\
\hline Type & 17 & 17 \\
\hline Press & $=$ & 7667 \\
\hline
\end{tabular}

Table 2.2

The display now reads 7667 . We'll have to add the zeros ourselves. There are a total of 12 zeros. Attaching 12 zeros to 7667, we get 7,667,000,000,000,000.

The product is $7,667,000,000,000,000$.

Example 2.11

Multiply $57,847,298$ by 38,976 .

\begin{tabular}{|l|l|r|}
\hline \multicolumn{2}{r}{} & Display Reads \\
\hline Type & 57847298 & 57847298 \\
\hline Press & $\times$ & 57847298 \\
\hline Type & 38976 & 38976 \\
\hline Press & $=$ & 2.254656312 \\
\hline
\end{tabular}

Table 2.3

The display now reads 2.254656312 . What kind of number is this? This is an example of a whole number written in scientific notation. We'll study this concept when we get to decimal numbers.

\subsubsection{Practice Set E}

Use a calculator to perform each multiplication.

Exercise 2.2.23

(Solution on p. 142.)

$52 \times 27$

Exercise 2.2.24

(Solution on p. 142.)

$1,448 \times 6,155$

Exercise 2.2.25

(Solution on p. 142.)

$8,940,000 \times 205,000$ 


\subsubsection{Exercises}

For the following problems, perform the multiplications. You may check each product with a calculator.

Exercise 2.2.26

(Solution on p. 142.)

$$
8
$$

$\times 3$

Exercise 2.2.27

3

$\times 5$

Exercise 2.2.28

(Solution on p. 143.)

8

$\underline{\times 6}$

Exercise 2.2.29

5

$\times 7$

Exercise 2.2.30

(Solution on p. 143.)

$6 \times 1$

Exercise 2.2.31

$4 \times 5$

Exercise 2.2.32

$75 \times 3$

(Solution on p. 143.)

Exercise 2.2.33

$35 \times 5$

Exercise 2.2.34

(Solution on p. 143.)

45

$\times 6$

Exercise 2.2.35

31

$\times 7$

Exercise 2.2.36

(Solution on p. 143.)

97

$\times 6$

Exercise 2.2.37

75

$\times 57$

Exercise 2.2.38

(Solution on p. 143.)

64

$\times 15$

Exercise 2.2.39

73

$\underline{\times 15}$ 
Exercise 2.2.40

(Solution on p. 143.)

81

$\times 95$

Exercise 2.2.41

31

$\times 33$

Exercise 2.2.42

(Solution on p. 143.)

$57 \times 64$

Exercise 2.2.43

$76 \times 42$

Exercise 2.2.44

(Solution on p. 143.)

$894 \times 52$

Exercise 2.2.45

$684 \times 38$

Exercise 2.2.46

(Solution on p. 143.)

115

$\times \quad 22$

Exercise 2.2.47 706

$\times 81$

Exercise 2.2.48

(Solution on p. 143.)

328

$\times \quad 21$

Exercise 2.2.49 550

$\times \quad 94$

Exercise 2.2.50

(Solution on p. 143.)

$930 \times 26$

Exercise 2.2.51

$318 \times 63$

Exercise 2.2.52

(Solution on p. 143.)

582

$\times 127$

Exercise 2.2.53

247

$\times 116$

Exercise 2.2.54

(Solution on p. 143.)

305

$\times 225$ 
Exercise 2.2.55

782

$\times 547$

Exercise 2.2.56

(Solution on p. 143.)

771

$\times 663$

Exercise 2.2.57

638

$\times 516$

Exercise 2.2.58

$1,905 \times 710$

(Solution on p. 143.)

Exercise 2.2.59

$5,757 \times 5,010$

Exercise 2.2.60

(Solution on p. 143.)

3,106

$\times 1,752$

Exercise 2.2.61

9,300

$\times 1,130$

Exercise 2.2.62

7,057

$\times 5,229$

Exercise 2.2.63

8,051

$\times 5,580$

Exercise 2.2.64

(Solution on p. 143.)

5,804

$\times 4,300$

Exercise 2.2.65

357

$\times 16$

Exercise 2.2.66

(Solution on p. 143.)

724

$\times \quad 0$

Exercise 2.2.67

2,649

$\times \quad 41$

Exercise 2.2.68

(Solution on p. 143.)

$$
5,173
$$

$\times \quad 8$

(Solution on p. 143.)

Available for free at Connexions $<\mathrm{http}: / / \operatorname{cnx} . o r g /$ content/col10615/1.4 $>$ 
Exercise 2.2.69

1,999

$\times \quad 0$

Exercise 2.2.70

(Solution on p. 143.)

1,666

$\times \quad 0$

Exercise 2.2.71

51,730

$\times \quad 142$

Exercise 2.2.72

(Solution on p. 143.)

387

$\times 190$

Exercise 2.2.73

3,400

$\times \quad 70$

Exercise 2.2.74

(Solution on p. 143.)

460,000

$\times \quad 14,000$

Exercise 2.2.75

$558,000,000$

$\times \quad 81,000$

Exercise 2.2.76

(Solution on p. 143.)

37,000

$\times \quad 120$

Exercise 2.2.77 498, 000

$\times \quad 0$

Exercise 2.2.78

(Solution on p. 144.)

$4,585,000$

$\times \quad 140$

Exercise 2.2.79

$30,700,000$

$\times \quad 180$

Exercise 2.2.80

(Solution on p. 144.)

8,000

$\times \quad 10$

Exercise 2.2.81

Suppose a theater holds 426 people. If the theater charges $\$ 4$ per ticket and sells every seat, how much money would they take in? 
Exercise 2.2.82

(Solution on p. 144.) In an English class, a student is expected to read 12 novels during the semester and prepare a report on each one of them. If there are 32 students in the class, how many reports will be prepared?

\section{Exercise 2.2.83}

In a mathematics class, a final exam consists of 65 problems. If this exam is given to 28 people, how many problems must the instructor grade?

Exercise 2.2.84

(Solution on p. 144.)

A business law instructor gives a 45 problem exam to two of her classes. If each class has 37 people in it, how many problems will the instructor have to grade?

Exercise 2.2.85

An algebra instructor gives an exam that consists of 43 problems to four of his classes. If the classes have $25,28,31$, and 35 students in them, how many problems will the instructor have to grade?

Exercise 2.2.86

(Solution on p. 144.)

In statistics, the term "standard deviation" refers to a number that is calculated from certain data. If the data indicate that one standard deviation is 38 units, how many units is three standard deviations?

Exercise 2.2.87

Soft drinks come in cases of 24 cans. If a supermarket sells 857 cases during one week, how many individual cans were sold?

Exercise 2.2.88

(Solution on p. 144.)

There are 60 seconds in 1 minute and 60 minutes in 1 hour. How many seconds are there in 1 hour?

Exercise 2.2.89

There are 60 seconds in 1 minute, 60 minutes in one hour, 24 hours in one day, and 365 days in one year. How many seconds are there in 1 year?

Exercise 2.2.90

(Solution on p. 144.)

Light travels 186,000 miles in one second. How many miles does light travel in one year? (Hint: Can you use the result of the previous problem?)

Exercise 2.2.91

An elementary school cafeteria sells 328 lunches every day. Each lunch costs $\$ 1$. How much money does the cafeteria bring in in 2 weeks?

Exercise 2.2.92

(Solution on p. 144.)

A computer company is selling stock for $\$ 23$ a share. If 87 people each buy 55 shares, how much money would be brought in?

\subsubsection{Exercises for Review}

Exercise 2.2.93

(Section 1.2) In the number 421,998 , how may ten thousands are there?

Exercise 2.2.94

(Solution on p. 144.)

(Section 1.4) Round 448,062,187 to the nearest hundred thousand.

Exercise 2.2.95

(Section 1.5) Find the sum. 22,451 + 18,976.

Exercise 2.2.96

(Solution on p. 144.)

(Section 1.6) Subtract 2,289 from 3,001.

Exercise 2.2.97

(Section 1.7) Specify which property of addition justifies the fact that (a first whole number $+\mathrm{a}$ second whole number $)=($ the second whole number + the first whole number $)$ 


\subsection{Concepts of Division of Whole Numbers ${ }^{3}$}

\subsubsection{Section Overview}

- Division

- Division into Zero (Zero As a Dividend: $\frac{0}{a}, a \neq 0$ )

- Division by Zero (Zero As a Divisor: $\frac{0}{a}, a \neq 0$ )

- Division by and into Zero (Zero As a Dividend and Divisor: $\frac{0}{0}$ )

- Calculators

\subsubsection{Division}

Division is a description of repeated subtraction.

In the process of division, the concern is how many times one number is contained in another number. For example, we might be interested in how many 5's are contained in 15 . The word times is significant because it implies a relationship between division and multiplication.

There are several notations used to indicate division. Suppose $Q$ records the number of times 5 is contained in 15 . We can indicate this by writing

$$
\begin{aligned}
& \underbrace{\frac{Q}{15}}_{5 \text { into } 15} \underbrace{\frac{15}{5}=Q}_{15} \\
& \underbrace{15 / 5=Q}_{15 \text { divided by } 5} \\
& \underbrace{15 \div 5=Q}_{15 \text { divided by } 5}
\end{aligned}
$$

Each of these division notations describes the same number, represented here by the symbol $Q$. Each notation also converts to the same multiplication form. It is $15=5 \times Q$

In division,

\section{Dividend}

the number being divided into is called the dividend.

\section{Divisor}

the number dividing into the dividend is the divisor.

\section{Quotient}

the result of the division is called the quotient.

$$
\begin{aligned}
& \text { quotient } \\
& \text { divisor } \overline{\text { dividend }} \\
& \frac{\text { dividend }}{\text { divisor }}=\text { quotient } \\
& \text { dividend } / \text { divisor }=\text { quotient dividend } \div \text { divisor }=\text { quotient }
\end{aligned}
$$

\footnotetext{
${ }^{3}$ This content is available online at $<\mathrm{http}: / / \mathrm{cnx}$. org/content $/ \mathrm{m} 34864 / 1.3 />$.
} 


\subsubsection{Sample Set A}

Find the following quotients using multiplication facts.

\section{Example 2.12}

$18 \div 6$

Since $6 \times 3=18$,

$18 \div 6=3$

Notice also that

18

$-6$

12

-6 \}Repeated subtraction

6

$-6$

0

Thus, 6 is contained in 18 three times.

\section{Example 2.13}

$\frac{24}{3}$

Since $3 \times 8=24$,

$\frac{24}{3}=8$

Notice also that 3 could be subtracted exactly 8 times from 24 . This implies that 3 is contained in 24 eight times.

\section{Example 2.14}

$\frac{36}{6}$

Since $6 \times 6=36$,

$\frac{36}{6}=6$

Thus, there are 6 sixes in 36 .

\section{Example 2.15}

$9 \overline{72}$

Since $9 \times 8=72$,
8
$9 \overline{72}$

Thus, there are 8 nines in 72 . 


\subsubsection{Practice Set A}

Use multiplication facts to determine the following quotients.

Exercise 2.3.1

(Solution on p. 144.)

$32 \div 8$

Exercise 2.3.2

(Solution on p. 144.)

$18 \div 9$

Exercise 2.3.3

(Solution on p. 144.)

$\frac{25}{5}$

Exercise 2.3.4

$\frac{48}{8}$

(Solution on p. 144.)

Exercise 2.3.5

$\frac{28}{7}$

Exercise 2.3.6

$4 \overline{36}$

(Solution on p. 144.)

(Solution on p. 144.)

\subsubsection{Division into Zero (Zero as a Dividend: $\frac{0}{a}, a \neq 0$ )}

Let's look at what happens when the dividend (the number being divided into) is zero, and the divisor (the number doing the dividing) is any whole number except zero. The question is

What number, if any, is $\frac{0}{\text { any nonzero whole number }}$ ?

Let's represent this unknown quotient by $Q$. Then,

$\frac{0}{\text { any nonzero whole number }}=Q$

Converting this division problem to its corresponding multiplication problem, we get

$0=Q \times($ any nonzero whole number $)$

From our knowledge of multiplication, we can understand that if the product of two whole numbers is zero, then one or both of the whole numbers must be zero. Since any nonzero whole number is certainly not zero, $Q$ must represent zero. Then,

$\frac{0}{\text { any nonzero whole number }}=0$

Zero Divided By Any Nonzero Whole Number Is Zero

Zero divided any nonzero whole number is zero.

\subsubsection{Division by Zero (Zero as a Divisor: $\frac{a}{0}, a \neq 0$ )}

Now we ask,

What number, if any, is $\frac{\text { any nonzero whole number }}{0}$ ?

Letting $Q$ represent a possible quotient, we get

$\frac{\text { any nonzero whole number }}{0}=Q$

Converting to the corresponding multiplication form, we have 
(any nonzero whole number $)=Q \times 0$

Since $Q \times 0=0$, (any nonzero whole number) $=0$. But this is absurd. This would mean that $6=0$, or $37=0$. A nonzero whole number cannot equal 0 ! Thus,

$\frac{\text { any nonzero whole number }}{0}$ does not name a number

Division by Zero is Undefined

Division by zero does not name a number. It is, therefore, undefined.

\subsubsection{Division by and Into Zero (Zero as a Dividend and Divisor: $\frac{0}{0}$ )}

We are now curious about zero divided by zero $\left(\frac{0}{0}\right)$. If we let $Q$ represent a potential quotient, we get

$\frac{0}{0}=Q$

Converting to the multiplication form,

$0=Q \times 0$

This results in

$0=0$

This is a statement that is true regardless of the number used in place of $Q$. For example,

$\frac{0}{0}=5$, since $0=5 \times 0$.

$\frac{0}{0}=31$, since $0=31 \times 0$.

$\frac{0}{0}=286$, since $0=286 \times 0$.

A unique quotient cannot be determined.

Indeterminant

Since the result of the division is inconclusive, we say that $\frac{0}{0}$ is indeterminant.

$\frac{0}{0}$ is Indeterminant

The division $\frac{0}{0}$ is indeterminant.

\subsubsection{Sample Set B}

Perform, if possible, each division.

Example 2.16

$\frac{19}{0}$. Since division by 0 does not name a whole number, no quotient exists, and we state $\frac{19}{0}$ is undefined

\section{Example 2.17}

$0 \overline{14}$. Since division by 0 does not name a defined number, no quotient exists, and we state

$0 \overline{14}$ is undefined

\section{Example 2.18}

$9 \overline{00}$. Since division into 0 by any nonzero whole number results in 0 , we have 


\section{Example 2.19}

$\frac{0}{7}$. Since division into 0 by any nonzero whole number results in 0 , we have $\frac{0}{7}=0$

\subsubsection{Practice Set B}

Perform, if possible, the following divisions.

Exercise 2.3.7

(Solution on p. 144.)

$\frac{5}{0}$

Exercise 2.3.8

$\frac{0}{4}$

Exercise 2.3.9

$0 \overline{0}$

(Solution on p. 144.)

(Solution on p. 144.)

Exercise 2.3.10

(Solution on p. 144.)

$0 \overline{8}$

Exercise 2.3.11

$\frac{9}{0}$

Exercise 2.3.12

$\frac{0}{1}$

(Solution on p. 144.)

(Solution on p. 144.)

\subsubsection{Calculators}

Divisions can also be performed using a calculator.

\subsubsection{Sample Set C}

Example 2.20

Divide 24 by 3 .

\begin{tabular}{|c|c|c|}
\hline \multicolumn{3}{|c|}{ Display Reads } \\
\hline Type & 24 & 24 \\
\hline Press & $\div$ & 24 \\
\hline Type & 3 & 3 \\
\hline Press & $=$ & 8 \\
\hline
\end{tabular}

Table 2.4

The display now reads 8 , and we conclude that $24 \div 3=8$.

Example 2.21

Divide 0 by 7 . 


\begin{tabular}{|c|c|c|}
\hline \multicolumn{3}{|c|}{ Display Reads } \\
\hline Type & 0 & 0 \\
\hline Press & $\div$ & 0 \\
\hline Type & 7 & 7 \\
\hline Press & $=$ & 0 \\
\hline
\end{tabular}

Table 2.5

The display now reads 0 , and we conclude that $0 \div 7=0$.

Example 2.22

Divide 7 by 0 .

Since division by zero is undefined, the calculator should register some kind of error message.

\begin{tabular}{|c|c|l|}
\hline \multicolumn{3}{|c|}{ Display Reads } \\
\hline Type & 7 & 7 \\
\hline Press & $\div$ & 7 \\
\hline Type & 0 & 0 \\
\hline Press & $=$ & Error \\
\hline
\end{tabular}

Table 2.6

The error message indicates an undefined operation was attempted, in this case, division by zero.

\subsubsection{Practice Set C}

Use a calculator to perform each division.

Exercise 2.3.13

(Solution on p. 144.)

$35 \div 7$

Exercise 2.3.14

(Solution on p. 144.)

$56 \div 8$

(Solution on p. 144.)

Exercise 2.3.15

$0 \div 6$

(Solution on p. 145.)

$3 \div 0$

Exercise 2.3.17

$0 \div 0$

(Solution on p. 145.) 


\subsubsection{Exercises}

For the following problems, determine the quotients (if possible). You may use a calculator to check the result.

Exercise 2.3.18

(Solution on p. 145.)

$4 \overline{32}$

Exercise 2.3.19

$7 \longdiv { 4 2 }$

Exercise 2.3.20

(Solution on p. 145.)

$6 \overline{18}$

Exercise 2.3.21

2) 14

Exercise 2.3.22 $3 \overline{27}$

(Solution on p. 145.)

Exercise 2.3.23 $1 \longdiv { 6 }$

Exercise 2.3.24

(Solution on p. 145.)

$4 \overline{28}$

Exercise 2.3.25 $\frac{30}{5}$

Exercise 2.3.26 $\frac{16}{4}$

(Solution on p. 145.)

Exercise 2.3.27 $24 \div 8$

Exercise 2.3.28

$10 \div 2$

(Solution on p. 145.)

Exercise 2.3.29

$21 \div 7$

Exercise 2.3.30

$21 \div 3$

(Solution on p. 145.)

Exercise 2.3.31 $0 \div 6$

Exercise 2.3.32

$8 \div 0$

(Solution on p. 145.)

Exercise 2.3.33 $12 \div 4$

Exercise 2.3.34

(Solution on p. 145.) $3 \overline{9}$

Exercise 2.3.35 $0 \overline{0}$

Exercise 2.3.36

(Solution on p. 145.) $7 \overline{0}$

Exercise 2.3.37 $6 \overline{48}$ 
Exercise 2.3.38

(Solution on p. 145.)

$\frac{15}{3}$

Exercise 2.3.39

$\frac{35}{0}$

Exercise 2.3.40

(Solution on p. 145.)

$56 \div 7$

Exercise 2.3.41

$\frac{0}{9}$

Exercise 2.3.42

$72 \div 8$

(Solution on p. 145.)

Exercise 2.3.43

Write $\frac{16}{2}=8$ using three different notations.

Exercise 2.3.44

(Solution on p. 145.)

Write $\frac{27}{9}=3$ using three different notations.

Exercise 2.3.45

In the statement $\frac{4}{6 \longdiv { 2 4 }}$

6 is called the

24 is called the

4 is called the

Exercise 2.3.46

(Solution on p. 145.)

In the statement $56 \div 8=7$,

7 is called the

8 is called the

56 is called the

\subsubsection{Exercises for Review}

Exercise 2.3.47

(Section 1.2) What is the largest digit?

Exercise 2.3.48

(Solution on p. 145.)

(Section 1.5) Find the sum.

$$
8,006
$$

$$
+4,118
$$

Exercise 2.3.49

(Section 1.6) Find the difference. $\begin{array}{r}631 \\ -589 \\ \hline\end{array}$

Exercise 2.3.50

(Solution on p. 145.)

(Section 1.7) Use the numbers 2, 3, and 7 to illustrate the associative property of addition.

Exercise 2.3.51

(Section 2.2) Find the product. 


\subsection{Division of Whole Numbers ${ }^{4}$}

\subsubsection{Section Overview}

- Division with a Single Digit Divisor

- Division with a Multiple Digit Divisor

- Division with a Remainder

- Calculators

\subsubsection{Division with a Single Digit Divisor}

Our experience with multiplication of whole numbers allows us to perform such divisions as $75 \div 5$. We perform the division by performing the corresponding multiplication, $5 \times Q=75$. Each division we considered in Section 2.3 had a one-digit quotient. Now we will consider divisions in which the quotient may consist of two or more digits. For example, $75 \div 5$.

Let's examine the division $75 \div 5$. We are asked to determine how many 5's are contained in 75 . We'll approach the problem in the following way.

1. Make an educated guess based on experience with multiplication.

2. Find how close the estimate is by multiplying the estimate by 5 .

3. If the product obtained in step 2 is less than 75 , find out how much less by subtracting it from 75 .

4. If the product obtained in step 2 is greater than 75 , decrease the estimate until the product is less than 75 . Decreasing the estimate makes sense because we do not wish to exceed 75 .

We can suggest from this discussion that the process of division consists of

\section{The Four Steps in Division}

1. an educated guess

2. a multiplication

3. a subtraction

4. bringing down the next digit (if necessary)

The educated guess can be made by determining how many times the divisor is contained in the dividend by using only one or two digits of the dividend.

\subsubsection{Sample Set A}

Example 2.23

Find $75 \div 5$.

$5 \overline{75}$ Rewrite the problem using a division bracket.

10

$5 \overline{75}$

Make an educated guess by noting that one 5 is contained in 75 at most 10 times.

Since 7 is the tens digit, we estimate that 5 goes into 75 at most 10 times.

\footnotetext{
${ }^{4}$ This content is available online at $<$ http://cnx.org/content/m34865/1.2/>.
} 


$$
\begin{array}{r}
10 \\
5 \longdiv { 7 5 } \\
-50 \\
25
\end{array}
$$

Now determine how close the estimate is.

10 fives is $10 \times 5=50$. Subtract 50 from 75 .

Estimate the number of 5 's in 25.

There are exactly 5 fives in 25 .

$$
\begin{aligned}
& \left.\begin{array}{l}
5 \\
10
\end{array}\right\} \quad \begin{array}{l}
10 \text { fives }+5 \text { fives }=15 \text { fives. } \\
5 \overline{75}
\end{array} \\
& \frac{-50}{25} \\
& \frac{-25}{0}
\end{aligned}
$$

Check: $75 \stackrel{75}{2} \underset{15}{ } \times 5$

Thus, $75 \div 5=15$.

The notation in this division can be shortened by writing.

$$
\begin{array}{r}
15 \\
5 \longdiv { 7 5 } \\
-5 \downarrow \\
\hline 25 \\
-25 \\
\hline 0
\end{array}
$$

Divide: 5 goes into 7 at most 1 time. $\quad$ Divide: 5 goes into 25 exactly 5 times.

\{ Multiply: $1 \times 5=5$. Write 5 below 7 . \{ Multiply: $5 \times 5=25$. Write 25 below 25 .

$$
\text { Subtract: } 7-5=2 \text {. Bring down the } 5 \text {. Subtract: } \quad 25-25=0 \text {. }
$$

\section{Example 2.24}

Find $4,944 \div 8$.

$$
8 \longdiv { 4 9 4 4 }
$$

Rewrite the problem using a division bracket.

600

$8 \longdiv { 4 9 4 4 }$

$-4800$

144 
8 goes into 49 at most 6 times, and 9 is in the hundreds column. We'll guess 600 .

Then, $8 \times 600=4800$.

$$
\begin{array}{r}
10 \\
600 \\
\hline 8 \lcm{4944} \\
-4800 \\
\hline 144 \\
-\quad 80 \\
\hline 64
\end{array}
$$

8 goes into 14 at most 1 time, and 4 is in the tens column. We'll guess 10 .

$$
\begin{array}{r}
8 \\
10 \\
600 \\
8 \longdiv { 4 9 4 4 } \\
-4800 \\
\hline 144 \\
-\quad 80 \\
\hline 64 \\
-64 \\
\hline 0
\end{array}
$$

8 goes into 64 exactly 8 times.

600 eights +10 eights +8 eights $=618$ eights.

\section{$4944 \stackrel{2}{\geq} \times 618$}

\section{Check: $4944 \cong 4944$}

Thus, $4,944 \div 8=618$.

As in the first problem, the notation in this division can be shortened by eliminating the subtraction signs and the zeros in each educated guess.

\section{8}

$8 \longdiv { 4 9 4 4 }$

48\}

14

$8 \downarrow$

64

$\frac{64}{0}$ 
Divide: 8 goes into 49 at most 6 times.

\{ Multiply: $6 \times 8=48$. Write 48 below 49 .

Subtract: $\quad 49-48=1$. Bring down the 4.

Divide: 8 goes into 64 exactly 8 times.

\{ Multiply: $8 \times 8=64$. Write 64 below 64 .

Subtract: $\quad 64-64=0$.
Divide: 8 goes into 14 at most 1 time.

\{ Multiply: $1 \times 8=8$. Write 8 below 14 .

Subtract: $14-8=6$. Bring down the 4 .

NOTE: Not all divisions end in zero. We will examine such divisions in a subsequent subsection.

\subsubsection{Practice Set A}

Perform the following divisions.

Exercise 2.4.1

(Solution on p. 145.)

$126 \div 7$

Exercise 2.4.2

(Solution on p. 145.)

$324 \div 4$

Exercise 2.4.3

(Solution on p. 145.)

$2,559 \div 3$

Exercise 2.4.4

(Solution on p. 145.)

$5,645 \div 5$

Exercise 2.4.5

$757,125 \div 9$

(Solution on p. 145.)

\subsubsection{Division with a Multiple Digit Divisor}

The process of division also works when the divisor consists of two or more digits. We now make educated guesses using the first digit of the divisor and one or two digits of the dividend.

\subsubsection{Sample Set B}

Example 2.25

Find $2,232 \div 36$.

\section{$3 6 \longdiv { 2 2 3 2 }$}

Use the first digit of the divisor and the first two digits of the dividend to make the educated guess.

3 goes into 22 at most 7 times.

Try $7: 7 \times 36=252$ which is greater than 223 . Reduce the estimate.

Try $6: 6 \times 36=216$ which is less than 223 . 


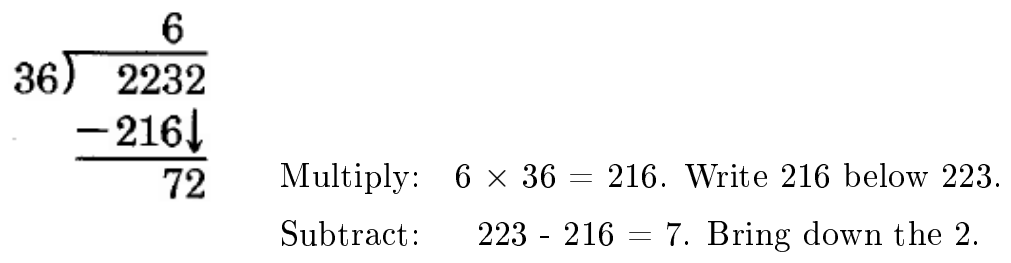

Divide 3 into 7 to estimate the number of times 36 goes into 72 . The 3 goes into 7 at most 2 times.

Try $2: 2 \times 36=72$.

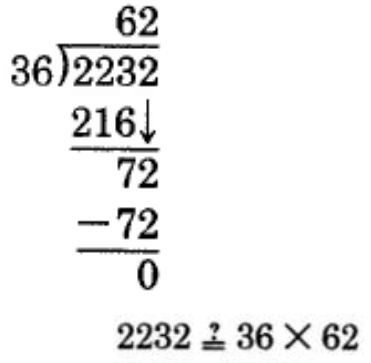

Check: $2232 \cong 2232$

Thus, $2,232 \div 36=62$.

Example 2.26

Find $2,417,228 \div 802$.

$8 0 2 \longdiv { 2 4 1 7 2 2 8 }$

First, the educated guess: $24 \div 8=3$. Then $3 \times 802=2406$, which is less than 2417 . Use 3 as the guess. Since $3 \times 802=2406$, and 2406 has four digits, place the 3 above the fourth digit of the dividend.

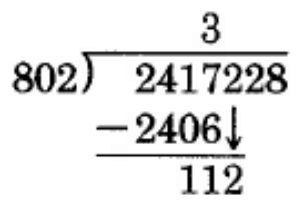

Subtract: $2417-2406=11$.

Bring down the 2 .

The divisor 802 goes into 112 at most 0 times. Use 0 .

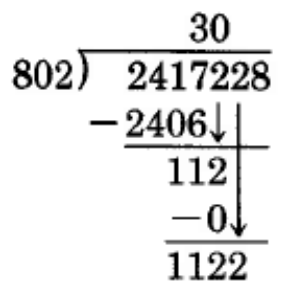




$$
\begin{array}{lc}
\text { Multiply: } & 0 \times 802=0 . \\
\text { Subtract: } & 112-0=112 .
\end{array}
$$

Bring down the 2 .

The 8 goes into 11 at most 1 time, and $1 \times 802=802$, which is less than 1122 . Try 1 .

$$
\begin{array}{r}
301 \\
\hline 2417228 \\
-2406 \downarrow \\
\hline 112 \\
\frac{-0 \downarrow}{1122} \\
-802 \downarrow \\
\hline 3208
\end{array}
$$

Subtract $1122-802=320$

Bring down the 8 .

8 goes into 32 at most 4 times.

$4 \times 802=3208$.

Use 4 .

$$
802 \begin{array}{r}
3014 \\
\\
-2406 \downarrow \\
\hline 112 \\
-0 \downarrow \\
\hline 1122 \\
-802 \downarrow \\
\hline 3208 \\
-\frac{3208}{0}
\end{array}
$$

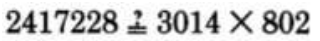

Check: $2417228 \cong 2417228$

Thus, $2,417,228 \div 802=3,014$.

\subsubsection{Practice Set B}

Perform the following divisions.

Exercise 2.4.6

(Solution on p. 146.)

$1,376 \div 32$

Exercise 2.4.7

$6,160 \div 55$

Exercise 2.4.8

(Solution on p. 146.)

$18,605 \div 61$

(Solution on p. 146.)

Exercise 2.4.9

(Solution on p. 146.)

$144,768 \div 48$ 


\subsubsection{Division with a Remainder}

We might wonder how many times 4 is contained in 10 . Repeated subtraction yields

$$
\begin{array}{r}
10 \\
-\quad 4 \\
\hline 6 \\
-4 \\
\hline 2
\end{array}
$$

Since the remainder is less than 4 , we stop the subtraction. Thus, 4 goes into 10 two times with 2 remaining. We can write this as a division as follows.

$$
\begin{array}{r}
2 \\
4 \longdiv { 1 0 } \\
-\quad 8 \\
\hline 2
\end{array}
$$

Divide: 4 goes into 10 at most 2 times.

Multiply: $2 \times 4=8$. Write 8 below 0 .

$$
\text { Subtract: } \quad 10-8=2 \text {. }
$$

Since 4 does not divide into 2 (the remainder is less than the divisor) and there are no digits to bring down to continue the process, we are done. We write

$$
\begin{array}{r}
2 \frac{2 R 2}{10} \\
\frac{-8}{2}
\end{array} \text { or } 10 \div 4=\underbrace{2 R 2}_{2 \text { with remainder } 2}
$$

\subsubsection{Sample Set C}

Example 2.27

Find $85 \div 3$.

$$
\begin{array}{r}
28 \\
3 \longdiv { 8 5 } \\
\frac{6 \downarrow}{25} \\
\frac{24}{1}
\end{array}
$$


Divide: 3 goes into 8 at most 2 times. $\quad$ Divide: 3 goes into 25 at most 8 times.

\{ Multiply: $2 \times 3=6$. Write 6 below 8 . \{ Multiply: $3 \times 8=24$. Write 24 below 25 .

Subtract: $\quad 8-6=2$. Bring down the 5. $\quad$ Subtract: $\quad 25-24=1$.

There are no more digits to bring down to continue the process. We are done. One is the remainder.

Check: Multiply 28 and 3, then add 1.

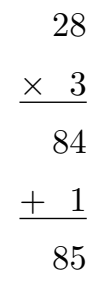

Thus, $85 \div 3=28$ R 1 .

Example 2.28

Find $726 \div 23$.

\begin{tabular}{r}
31 \\
$2 3 \longdiv { 7 2 6 }$ \\
$69 \downarrow$ \\
\hline 36 \\
$\frac{23}{13}$
\end{tabular}

Check: Multiply 31 by 23, then add 13 .
31
93
62
$\overline{713}$
$+13$
726

$\times 23$

Thus, $726 \div 23=31 R 13$.

\subsubsection{Practice Set C}

Perform the following divisions.

Exercise 2.4.10

(Solution on p. 146.)

$75 \div 4$

Exercise 2.4.11

(Solution on p. 146.)

$346 \div 8$

Exercise 2.4.12

(Solution on p. 146.)

$489 \div 21$

Exercise 2.4.13

$5,016 \div 82$

(Solution on p. 146.) 
Exercise 2.4.14

(Solution on p. 146.)

$41,196 \div 67$

\subsubsection{Calculators}

The calculator can be useful for finding quotients with single and multiple digit divisors. If, however, the division should result in a remainder, the calculator is unable to provide us with the particular value of the remainder. Also, some calculators (most nonscientific) are unable to perform divisions in which one of the numbers has more than eight digits.

\subsubsection{Sample Set D}

Use a calculator to perform each division.

Example 2.29

$328 \div 8$

\begin{tabular}{|l|l|}
\hline Type & 328 \\
\hline Press & $\div$ \\
\hline Type & 8 \\
\hline Press & $=$ \\
\hline
\end{tabular}

Table 2.7

The display now reads 41 .

Example 2.30

$53,136 \div 82$

\begin{tabular}{|l|l|}
\hline Type & 53136 \\
\hline Press & $\div$ \\
\hline Type & 82 \\
\hline Press & $=$ \\
\hline
\end{tabular}

Table 2.8

The display now reads 648 .

\section{Example 2.31}

$730,019,001 \div 326$

We first try to enter 730,019,001 but find that we can only enter 73001900 . If our calculator has only an eight-digit display (as most nonscientific calculators do), we will be unable to use the calculator to perform this division.

Example 2.32

$3727 \div 49$ 


\begin{tabular}{|c|l|}
\hline Type & 3727 \\
\hline Press & $\div$ \\
\hline Type & 49 \\
\hline Press & $=$ \\
\hline
\end{tabular}

Table 2.9

The display now reads 76.061224 .

This number is an example of a decimal number (see Section 6.1). When a decimal number results in a calculator division, we can conclude that the division produces a remainder.

\subsubsection{Practice Set D}

Use a calculator to perform each division.

Exercise 2.4.15

(Solution on p. 146.)

$3,330 \div 74$

Exercise 2.4.16

(Solution on p. 146.)

$63,365 \div 115$

Exercise 2.4.17

(Solution on p. 146.)

$21,996,385,287 \div 53$

Exercise 2.4.18

$4,558 \div 67$

(Solution on p. 146.)

\subsubsection{Exercises}

For the following problems, perform the divisions.

The first 38 problems can be checked with a calculator by multiplying the divisor and quotient then adding the remainder.

Exercise 2.4.19

(Solution on p. 146.)

$52 \div 4$

Exercise 2.4.20

$776 \div 8$

Exercise 2.4.21

$603 \div 9$

(Solution on p. 146.)

Exercise 2.4.22

$240 \div 8$

Exercise 2.4.23

$208 \div 4$

(Solution on p. 146.)

Exercise 2.4.24

$576 \div 6$

Exercise 2.4.25

$21 \div 7$

(Solution on p. 146.) 
Exercise 2.4.26

$0 \div 0$

Exercise 2.4.27

(Solution on p. 146.)

$140 \div 2$

Exercise 2.4.28

$528 \div 8$

Exercise 2.4.29

(Solution on p. 146.)

$244 \div 4$

Exercise 2.4.30

$0 \div 7$

Exercise 2.4.31

(Solution on p. 146.)

$177 \div 3$

Exercise 2.4.32

$96 \div 8$

Exercise 2.4.33

$67 \div 1$

(Solution on p. 146.)

Exercise 2.4.34

$896 \div 56$

Exercise 2.4.35

$1,044 \div 12$

(Solution on p. 146.)

Exercise 2.4.36

$988 \div 19$

Exercise 2.4.37

$5,238 \div 97$

Exercise 2.4.38

$2,530 \div 55$

Exercise 2.4.39

$4,264 \div 82$

(Solution on p. 146.)

Exercise 2.4.40

$637 \div 13$

Exercise 2.4.41

$3,420 \div 90$

(Solution on p. 147.)

Exercise 2.4.42

$5,655 \div 87$

Exercise 2.4.43

(Solution on p. 146.)

$2,115 \div 47$

Exercise 2.4.44

$9,328 \div 22$

Exercise 2.4.45

$55,167 \div 71$

(Solution on p. 147.)

Exercise 2.4.46

$68,356 \div 92$

Exercise 2.4.47

$27,702 \div 81$

(Solution on p. 147.)

Exercise 2.4.48

$6,510 \div 31$ 
Exercise 2.4.49

(Solution on p. 147.)

$60,536 \div 94$

Exercise 2.4.50

$31,844 \div 38$

Exercise 2.4.51

(Solution on p. 147.)

$23,985 \div 45$

Exercise 2.4.52

$60,606 \div 74$

Exercise 2.4.53

$2,975,400 \div 285$

(Solution on p. 147.)

Exercise 2.4.54 $1,389,660 \div 795$

Exercise 2.4.55

$7,162,060 \div 879$

Exercise 2.4.56

$7,561,060 \div 909$

Exercise 2.4.57 $38 \div 9$

Exercise 2.4.58

$97 \div 4$

Exercise 2.4.59

$199 \div 3$

Exercise 2.4.60 $573 \div 6$

Exercise 2.4.61

$10,701 \div 13$

(Solution on p. 147.)

Exercise 2.4.62

$13,521 \div 53$

Exercise 2.4.63

$3,628 \div 90$

(Solution on p. 147.)

Exercise 2.4.64

$10,592 \div 43$

Exercise 2.4.65

$19,965 \div 30$

(Solution on p. 147.)

(Solution on p. 147.)

Exercise 2.4.66

$8,320 \div 21$

Exercise 2.4.67

$61,282 \div 64$

(Solution on p. 147.)

Exercise 2.4.68

$1,030 \div 28$

Exercise 2.4.69

$7,319 \div 11$

(Solution on p. 147.)

Exercise 2.4.70

$3,628 \div 90$

Exercise 2.4.71

$35,279 \div 77$

(Solution on p. 147.)

(Solution on p. 147.)

Available for free at Connexions $<$ http://cnx.org/content/col10615/1.4 $>$ 
Exercise 2.4.72

$52,196 \div 55$

Exercise 2.4.73

(Solution on p. 147.)

$67,751 \div 68$

For the following 5 problems, use a calculator to find the quotients.

Exercise 2.4.74

$4,346 \div 53$

Exercise 2.4.75

(Solution on p. 147.)

$3,234 \div 77$

Exercise 2.4.76

$6,771 \div 37$

Exercise 2.4.77

$4,272,320 \div 520$

(Solution on p. 147.)

Exercise 2.4.78

$7,558,110 \div 651$

Exercise 2.4.79

(Solution on p. 147.)

A mathematics instructor at a high school is paid $\$ 17,775$ for 9 months. How much money does this instructor make each month?

Exercise 2.4.80

A couple pays $\$ 4,380$ a year for a one-bedroom apartment. How much does this couple pay each month for this apartment?

Exercise 2.4.81

(Solution on p. 147.)

Thirty-six people invest a total of $\$ 17,460$ in a particular stock. If they each invested the same amount, how much did each person invest?

\section{Exercise 2.4.82}

Each of the 28 students in a mathematics class buys a textbook. If the bookstore sells $\$ 644$ worth of books, what is the price of each book?

Exercise 2.4.83

(Solution on p. 147.)

A certain brand of refrigerator has an automatic ice cube maker that makes 336 ice cubes in one day. If the ice machine makes ice cubes at a constant rate, how many ice cubes does it make each hour?

\section{Exercise 2.4.84}

A beer manufacturer bottles 52,380 ounces of beer each hour. If each bottle contains the same number of ounces of beer, and the manufacturer fills 4,365 bottles per hour, how many ounces of beer does each bottle contain?

Exercise 2.4.85

(Solution on p. 147.)

A computer program consists of 68,112 bits. 68,112 bits equals 8,514 bytes. How many bits in one byte?

Exercise 2.4.86

A 26-story building in San Francisco has a total of 416 offices. If each floor has the same number of offices, how many floors does this building have?

Exercise 2.4.87

(Solution on p. 147.)

A college has 67 classrooms and a total of 2,546 desks. How many desks are in each classroom if each classroom has the same number of desks? 


\subsubsection{Exercises for Review}

Exercise 2.4.88

(Section 1.2) What is the value of 4 in the number 124,621 ?

Exercise 2.4.89

(Solution on p. 147.)

(Section 1.4) Round 604,092 to the nearest hundred thousand.

Exercise 2.4.90

(Section 1.7) What whole number is the additive identity?

Exercise 2.4.91

(Solution on p. 148.)

(Section 2.2) Find the product. 6, $256 \times 100$.

Exercise 2.4.92

(Section 2.3) Find the quotient. $0 \div 11$.

\subsection{Some Interesting Facts about Division ${ }^{5}$}

\subsubsection{Section Overview}

- Division by $2,3,4$, and 5

- Division by $6,8,9$, and 10

Quite often, we are able to determine if a whole number is divisible by another whole number just by observing some simple facts about the number. Some of these facts are listed in this section.

\subsubsection{Division by $2,3,4$, and 5}

Division by 2

A whole number is divisible by 2 if its last digit is $0,2,4,6$, or 8 .

The numbers $80,112,64,326$, and 1,008 are all divisible by 2 since the last digit of each is $0,2,4$, 6 , or 8 , respectively.

The numbers 85 and 731 are not divisible by 2 .

Division by 3

A whole number is divisible by 3 if the sum of its digits is divisible by 3 .

The number 432 is divisible by 3 since $4+3+2=9$ and 9 is divisible by 3 .

$432 \div 3=144$

The number 25 is not divisible by 3 since $2+5=7$, and 7 is not divisible by 3 .

Division by 4

A whole number is divisible by 4 if its last two digits form a number that is divisible by 4 .

The number 31,048 is divisible by 4 since the last two digits, 4 and 8 , form a number, 48 , that is divisible by 4 .

$31048 \div 4=7262$

\footnotetext{
${ }^{5}$ This content is available online at $<$ http://cnx.org/content $/ \mathrm{m} 34866 / 1.2 />$.
} 
The number 137 is not divisible by 4 since 37 is not divisible by 4 .

Division by 5

A whole number is divisible by 5 if its last digit is 0 or 5 .

\subsubsection{Sample Set A}

Example 2.33

The numbers $65,110,8,030$, and 16,955 are each divisible by 5 since the last digit of each is 0 or 5 .

\subsubsection{Practice Set A}

State which of the following whole numbers are divisible by $2,3,4$, or 5 . A number may be divisible by more than one number.

Exercise 2.5.1

26

Exercise 2.5.2

81

Exercise 2.5.3

51

Exercise 2.5.4

385

Exercise 2.5.5

6,112

Exercise 2.5.6

470

Exercise 2.5.7

113,154
(Solution on p. 148.)

(Solution on p. 148.)

(Solution on p. 148.)

(Solution on p. 148.)

(Solution on p. 148.)

(Solution on p. 148.)

(Solution on p. 148.)

\subsubsection{Division by $6,8,9,10$}

\section{Division by 6}

A number is divisible by 6 if it is divisible by both 2 and 3 .

The number 234 is divisible by 2 since its last digit is 4 . It is also divisible by 3 since $2+3+4=9$ and 9 is divisible by 3 . Therefore, 234 is divisible by 6 .

The number 6,532 is not divisible by 6 . Although its last digit is 2 , making it divisible by 2 , the sum of its digits, $6+5+3+2=16$, and 16 is not divisible by 3 .

\section{Division by 8}

A whole number is divisible by 8 if its last three digits form a number that is divisible by 8 .

The number 4,000 is divisible by 8 since 000 is divisible by 8 .

The number 13,128 is divisible by 8 since 128 is divisible by 8 .

The number 1,170 is not divisible by 8 since 170 is not divisible by 8 . 


\section{Division by 9}

A whole number is divisible by 9 if the sum of its digits is divisible by 9 .

The number 702 is divisible by 9 since $7+0+2$ is divisible by 9 .

The number 6588 is divisible by 9 since $6+5+8+8=27$ is divisible by 9 .

The number 14,123 is not divisible by 9 since $1+4+1+2+3=11$ is not divisible by 9 .

\section{Division by 10}

A Whole number is divisible by $\mathbf{1 0}$ if its last digit is 0 .

\subsubsection{Sample Set B}

\section{Example 2.34}

The numbers $30,170,16,240$, and 865,000 are all divisible by 10 .

\subsubsection{Practice Set B}

State which of the following whole numbers are divisible $6,8,9$, or 10 . Some numbers may be divisible by more than one number.

Exercise 2.5.8

(Solution on p. 148.)

900

Exercise 2.5.9

(Solution on p. 148.)

6,402

Exercise 2.5.10

(Solution on p. 148.)

6,660

Exercise 2.5.11

(Solution on p. 148.)

55,116

\subsubsection{Exercises}

For the following 30 problems, specify if the whole number is divisible by $2,3,4,5,6,8,9$, or 10 . Write "none" if the number is not divisible by any digit other than 1 . Some numbers may be divisible by more than one number.

Exercise 2.5.12

(Solution on p. 148.)

48

Exercise 2.5.13

85

Exercise 2.5.14

(Solution on p. 148.)

30

Exercise 2.5.15

83

Exercise 2.5.16

98

(Solution on p. 148.)

Exercise 2.5.17

972

Exercise 2.5.18

892

(Solution on p. 148.) 
Exercise 2.5.19

676

Exercise 2.5.20

(Solution on p. 148.)

903

Exercise 2.5.21

800

Exercise 2.5.22

223

(Solution on p. 148.)

Exercise 2.5.23

836

Exercise 2.5.24

(Solution on p. 148.)

665

Exercise 2.5.25

4,381

Exercise 2.5.26

2,195

(Solution on p. 148.)

Exercise 2.5.27

2,544

Exercise 2.5.28

5,172

(Solution on p. 148.)

Exercise 2.5.29

1,307

Exercise 2.5.30

1,050

(Solution on p. 148.)

Exercise 2.5.31

3,898

Exercise 2.5.32

1,621

(Solution on p. 148.)

Exercise 2.5.33 27,808

Exercise 2.5.34

45,764

(Solution on p. 148.)

Exercise 2.5.35

49,198

Exercise 2.5.36

296, 122

(Solution on p. 148.)

Exercise 2.5.37

178,656

Exercise 2.5.38

$5,102,417$

(Solution on p. 149.)

Exercise 2.5.39

16,990,792

Exercise 2.5.40

$620,157,659$

(Solution on p. 149.)

Exercise 2.5.41

$457,687,705$ 


\subsubsection{Exercises for Review}

Exercise 2.5.42

(Solution on p. 149.)

(Section 1.2) In the number 412, how many tens are there?

Exercise 2.5.43

(Section 1.6) Subtract 613 from 810.

Exercise 2.5.44

(Solution on p. 149.)

(Section 1.7) Add 35, 16, and 7 in two different ways.

Exercise 2.5.45

(Section 2.3) Find the quotient $35 \div 0$, if it exists.

Exercise 2.5.46

(Solution on p. 149.)

(Section 2.4) Find the quotient. $3654 \div 42$.

\subsection{Properties of Multiplication ${ }^{6}$}

\subsubsection{Section Overview}

- The Commutative Property of Multiplication

- The Associative Property of Multiplication

- The Multiplicative Identity

We will now examine three simple but very important properties of multiplication.

\subsubsection{The Commutative Property of Multiplication}

\section{Commutative Property of Multiplication}

The product of two whole numbers is the same regardless of the order of the factors.

\subsubsection{Sample Set A}

Example 2.35

Multiply the two whole numbers.

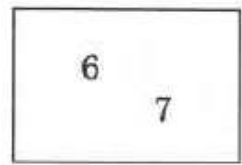

$6 \cdot 7=42$

$7 \cdot 6=42$

The numbers 6 and 7 can be multiplied in any order. Regardless of the order they are multiplied, the product is 42 .

\footnotetext{
${ }^{6}$ This content is available online at $<$ http://cnx.org/content $/ \mathrm{m} 34867 / 1.2 />$.
} 


\subsubsection{Practice Set A}

Use the commutative property of multiplication to find the products in two ways.

Exercise 2.6.1

(Solution on p. 149.)

15

6

Exercise 2.6.2

(Solution on p. 149.)

432

428

\subsubsection{The Associative Property of Multiplication}

\section{Associative Property of Multiplication}

If three whole numbers are multiplied, the product will be the same if the first two are multiplied first and then that product is multiplied by the third, or if the second two are multiplied first and that product is multiplied by the first. Note that the order of the factors is maintained.

It is a common mathematical practice to use parentheses to show which pair of numbers is to be combined first.

\subsubsection{Sample Set B}

Example 2.36

Multiply the whole numbers.

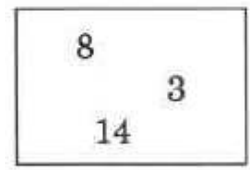

$(8 \cdot 3) \cdot 14=24 \cdot 14=336$

$8 \cdot(3 \cdot 14)=8 \cdot 42=336$

\subsubsection{Practice Set B}

Use the associative property of multiplication to find the products in two ways.

\section{Exercise 2.6.3}

7 8

(Solution on p. 149.) 
Exercise 2.6.4

(Solution on p. 149.)

73 18 126

\subsubsection{The Multiplicative Identity}

The Multiplicative Identity is 1

The whole number 1 is called the multiplicative identity, since any whole number multiplied by 1 is not changed.

\subsubsection{Sample Set C}

Example 2.37

Multiply the whole numbers.

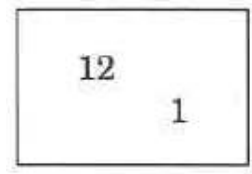

$12 \cdot 1=12$

$1 \cdot 12=12$

\subsubsection{Practice Set C}

Multiply the whole numbers.

Exercise 2.6.5

(Solution on p. 149.)

843

1

\subsubsection{Exercises}

For the following problems, multiply the numbers.

Exercise 2.6.6

(Solution on p. 149.)

\section{9}

26

Exercise 2.6.7

18

41 
Exercise 2.6.8

(Solution on p. 149.)

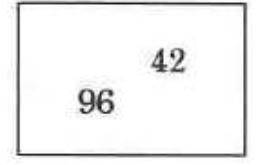

Exercise 2.6.9

$132^{6}$

Exercise 2.6.10

(Solution on p. 149.)

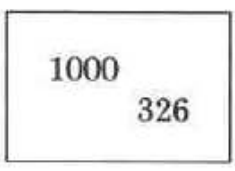

Exercise 2.6.11

\begin{tabular}{|ll|}
\hline & 1400 \\
70 & \\
\hline
\end{tabular}

Exercise 2.6.12

(Solution on p. 149.)

\begin{tabular}{|lll|}
\hline 3 & & \\
& & 7 \\
& 12 & \\
\hline
\end{tabular}

Exercise 2.6.13

\begin{tabular}{|ll|}
\hline \multicolumn{3}{|c|}{16} & 40 \\
& \\
& 5 \\
\hline
\end{tabular}

Exercise 2.6.14

(Solution on p. 149.)

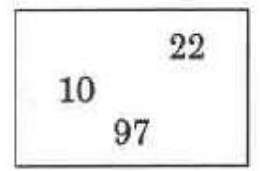

Exercise 2.6.15

\begin{tabular}{|rr|}
\hline 110 & \\
0 & 85 \\
\hline
\end{tabular}


Exercise 2.6.16

(Solution on p. 149.)

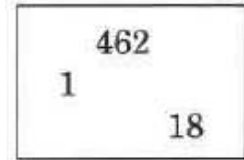

Exercise 2.6.17

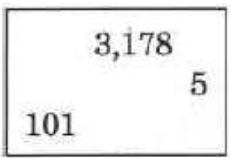

For the following 4 problems, show that the quantities yield the same products by performing the multiplications.

Exercise 2.6.18

(Solution on p. 149.)

$(4 \cdot 8) \cdot 2$ and $4 \cdot(8 \cdot 2)$

Exercise 2.6.19

$(100 \cdot 62) \cdot 4$ and $100 \cdot(62 \cdot 4)$

Exercise 2.6.20

(Solution on p. 149.)

$23 \cdot(11 \cdot 106)$ and $(23 \cdot 11) \cdot 106$

Exercise 2.6.21

$1 \cdot(5 \cdot 2)$ and $(1 \cdot 5) \cdot 2$

Exercise 2.6.22

(Solution on p. 149.)

The fact that (a first number . a second number) . a third number $=$ a first number . (a second number $\cdot$ a third number)is an example of the property of multiplication.

Exercise 2.6.23

The fact that 1 . any number = that particular numberis an example of the multiplication.

Exercise 2.6.24

(Solution on p. 149.)

Use the numbers 7 and 9 to illustrate the commutative property of multiplication.

Exercise 2.6.25

Use the numbers 6,4 , and 7 to illustrate the associative property of multiplication.

\subsubsection{Exercises for Review}

Exercise 2.6.26

(Solution on p. 149.)

(Section 1.2) In the number $84,526,098,441$, how many millions are there?

Exercise 2.6.27

(Section 1.5) Replace the letter $m$ with the whole number that makes the addition true. $+m$

Exercise 2.6.28

(Solution on p. 149.)

(Section 1.7) Use the numbers 4 and 15 to illustrate the commutative property of addition.

Exercise 2.6.29

(Section 2.3) Find the product. 8,000,000 $\times 1,000$. 
Exercise 2.6.30

(Solution on p. 149.)

(Section 2.5) Specify which of the digits $2,3,4,5,6,8,10$ are divisors of the number 2,244. 


\subsection{Summary of Key Concepts ${ }^{7}$}

\subsubsection{Summary of Key Concepts}

Multiplication (Section 2.2)

Multiplication is a description of repeated addition.

$\underbrace{7+7+7+7}_{7 \text { appears } 4 \text { times }}$

This expression is described by writing $4 \mathrm{X} 7$.

Multiplicand/Multiplier/Product (Section 2.2)

In a multiplication of whole numbers, the repeated addend is called the multiplicand, and the number that records the number of times the multiplicand is used is the multiplier. The result of the multiplication is the product.

Factors (Section 2.2)

In a multiplication, the numbers being multiplied are also called factors. Thus, the multiplicand and the multiplier can be called factors.

Division (Section 2.3)

Division is a description of repeated subtraction.

Dividend/Divisor/Quotient (Section 2.3)

In a division, the number divided into is called the dividend, and the number dividing into the dividend is called the divisor. The result of the division is called the quotient.

$$
\text { divisor) } \frac{\text { quotient }}{\text { dividend }}
$$

Division into Zero (Section 2.3)

Zero divided by any nonzero whole number is zero.

Division by Zero (Section 2.3)

Division by zero does not name a whole number. It is, therefore, undefined. The quotient $\frac{0}{0}$ is indeterminant.

Division by 2, 3, 4, 5, 6, 8, 9, 10 (Section 2.5)

Division by the whole numbers $2,3,4,5,6,8,9$, and 10 can be determined by noting some certain properties of the particular whole number.

Commutative Property of Multiplication (Section 2.6)

The product of two whole numbers is the same regardless of the order of the factors. $3 \times 5=5 \times 3$

\section{Associative Property of Multiplication (Section 2.6)}

If three whole numbers are to be multiplied, the product will be the same if the first two are multiplied first and then that product is multiplied by the third, or if the second two are multiplied first and then that product is multiplied by the first.

$(3 \times 5) \times 2=3 \times(5 \times 2)$

Note that the order of the factors is maintained.

Multiplicative Identity (Section 2.6)

The whole number 1 is called the multiplicative identity since any whole number multiplied by 1 is not changed.

\footnotetext{
${ }^{7}$ This content is available online at $<$ http://cnx.org/content $/ \mathrm{m} 34868 / 1.2 />$.
} 
$4 \times 1=4$

$1 \times 4=4$

\subsection{Exercise Supplement ${ }^{8}$}

\subsubsection{Exercise Supplement}

\subsubsection{Multiplication of Whole Numbers (Section 2.2)}

Exercise 2.8.1

In the multiplication $5 \times 9=45,5$ and 9 are called

(Solution on p. 149.)

Exercise 2.8.2

In the multiplication $4 \times 8=32,4$ and 8 are called and 45 is called the and 32 is called the

\subsubsection{Concepts of Division of Whole Numbers (Section 2.3)}

Exercise 2.8.3

In the division $24 \div 6=4,6$ is called the , and 4 is called the

(Solution on p. 150.)

Exercise 2.8.4

In the division $36 \div 2=18,2$ is called the , and 18 is called the

\subsubsection{Some Interesting Facts about Division (Section 2.5)}

Exercise 2.8.5

(Solution on p. 150.)

A number is divisible by 2 only if its last digit is

Exercise 2.8.6

A number is divisible by 3 only if of its digits is divisible by 3 .

Exercise 2.8.7

(Solution on p. 150.)

A number is divisible by 4 only if the rightmost two digits form a number that is

\subsubsection{Multiplication and Division of Whole Numbers (Section 2.2,Section 2.4)}

Find each product or quotient.

Exercise 2.8.8

24

$\times 3$

Exercise 2.8.9

(Solution on p. 150.)

14

$\underline{\times 8}$

Exercise 2.8.10

$21 \div 7$

Exercise 2.8.11

(Solution on p. 150.)

$35 \div 5$

\footnotetext{
${ }^{8}$ This content is available online at $<\mathrm{http}: / / \mathrm{cnx} .0 \mathrm{rg} /$ content $/ \mathrm{m} 34869 / 1.2 />$.
} 
Exercise 2.8.12

36

$\times 22$

Exercise 2.8.13

(Solution on p. 150.)

87

$\times 35$

Exercise 2.8.14

117

$\times 42$

Exercise 2.8.15

(Solution on p. 150.)

$208 \div 52$

Exercise 2.8.16

521

$\begin{array}{r}87 \\ \hline\end{array}$

Exercise 2.8.17

(Solution on p. 150.)

1005

$\times \quad 15$

Exercise 2.8.18 $1338 \div 446$

Exercise 2.8.19

(Solution on p. 150.)

$2814 \div 201$

Exercise 2.8.20

5521

$\times 8$

Exercise 2.8.21

(Solution on p. 150.)

6016

$\times \quad 7$

Exercise 2.8.22

$576 \div 24$

Exercise 2.8.23

$3969 \div 63$

(Solution on p. 150.)

Exercise 2.8.24

5482

$\times \quad 322$

Exercise 2.8.25

(Solution on p. 150.)

9104

$\times 115$

Exercise 2.8.26

6102

$\times 1000$ 
Exercise 2.8.27

(Solution on p. 150.)

10101

$\times 10000$

Exercise 2.8.28

$162,006 \div 31$

Exercise 2.8.29

(Solution on p. 150.)

$0 \div 25$

Exercise 2.8.30

$25 \div 0$

Exercise 2.8.31

(Solution on p. 150.)

$4280 \div 10$

Exercise 2.8.32

$2126000 \div 100$

Exercise 2.8.33

$84 \div 15$

(Solution on p. 150.)

Exercise 2.8.34

$126 \div 4$

Exercise 2.8.35

$424 \div 0$

(Solution on p. 150.)

Exercise 2.8.36

$1198 \div 46$

Exercise 2.8.37

$995 \div 31$

(Solution on p. 150.)

Exercise 2.8.38

$0 \div 18$

Exercise 2.8.39

(Solution on p. 150.) 2162

$\times 1421$

Exercise 2.8.40

$0 \times 0$

Exercise 2.8.41

$5 \times 0$

(Solution on p. 150.)

Exercise 2.8.42 $64 \times 1$

Exercise 2.8.43

(Solution on p. 150.)

$1 \times 0$

Exercise 2.8.44

$0 \div 3$

Exercise 2.8.45

$14 \div 0$

(Solution on p. 150.)

Exercise 2.8.46

$35 \div 1$

Exercise 2.8.47

$1 \div 1$

(Solution on p. 150.) 


\subsubsection{Properties of Multiplication (Section 2.6)}

\section{Exercise 2.8.48}

Use the commutative property of multiplication to rewrite $36 \times 128$.

Exercise 2.8.49

(Solution on p. 150.)

Use the commutative property of multiplication to rewrite $114 \times 226$.

Exercise 2.8.50

Use the associative property of multiplication to rewrite $(5 \cdot 4) \cdot 8$.

Exercise 2.8.51

(Solution on p. 150.)

Use the associative property of multiplication to rewrite $16 \cdot(14 \cdot 0)$.

\subsubsection{Multiplication and Division of Whole Numbers (Section 2.2,Section 2.4)}

\section{Exercise 2.8.52}

A computer store is selling diskettes for $\$ 4$ each. At this price, how much would 15 diskettes cost?

Exercise 2.8.53

(Solution on p. 151.)

Light travels 186,000 miles in one second. How far does light travel in 23 seconds?

Exercise 2.8.54

A dinner bill for eight people comes to exactly $\$ 112$. How much should each person pay if they all agree to split the bill equally?

Exercise 2.8.55

(Solution on p. 151.)

Each of the 33 students in a math class buys a textbook. If the bookstore sells $\$ 1089$ worth of books, what is the price of each book?

\subsection{Proficiency Exam ${ }^{9}$}

\subsubsection{Proficiency Exam}

Exercise 2.9.1

(Solution on p. 151.)

(Section 2.2) In the multiplication of $8 \times 7=56$, what are the names given to the 8 and 7 and the 56 ?

Exercise 2.9.2

(Section 2.2) Multiplication is a description of what repeated process?

Exercise 2.9.3

(Solution on p. 151.)

(Section 2.3) In the division $12 \div 3=4$, what are the names given to the 3 and the 4 ?

Exercise 2.9.4

(Section 2.5) Name the digits that a number must end in to be divisible by 2 .

(Solution on p. 151.)

Exercise 2.9.5

(Solution on p. 151.)

(Section 2.6) Name the property of multiplication that states that the order of the factors in a multiplication can be changed without changing the product.

Exercise 2.9.6

(Solution on p. 151.)

(Section 2.6) Which number is called the multiplicative identity?

For problems 7-17, find the product or quotient.

Exercise 2.9.7

(Solution on p. 151.)

(Section 2.2) $14 \times 6$

${ }^{9}$ This content is available online at $<$ http://cnx.org/content $/ \mathrm{m} 34870 / 1.2 />$. 
Exercise 2.9.8

(Section 2.2) $37 \times 0$

Exercise 2.9.9

(Section 2.2) $352 \times 1000$

Exercise 2.9.10

(Section 2.2) $5986 \times 70$

Exercise 2.9.11

(Section 2.2) $12 \times 12$

Exercise 2.9.12

(Section 2.3) $856 \div 0$

Exercise 2.9.13

(Section 2.3) $0 \div 8$

Exercise 2.9.14

(Section 2.4) $136 \div 8$

Exercise 2.9.15

(Section 2.4) $432 \div 24$

Exercise 2.9.16

(Section 2.4) $5286 \div 37$

Exercise 2.9.17

(Section 2.6) $211 \times 1$
(Solution on p. 151.)

(Solution on p. 151.)

(Solution on p. 151.)

(Solution on p. 151.)

(Solution on p. 151.)

(Solution on p. 151.)

(Solution on p. 151.)

(Solution on p. 151.)

(Solution on p. 151.)

(Solution on p. 151.)

(Solution on p. 151.)

(Solution on p. 151.)

(Solution on p. 151.)

Exercise 2.9.20

(Section 2.5) Which number(s) is divisible by 5 ?

Exercise 2.9.18

(Section 2.5) Which numbers are divisible by 3 ?

Exercise 2.9.19

(Section 2.5) Which number is divisible by 4 ? 


\section{Solutions to Exercises in Chapter 2}

Solution to Exercise 2.2.1 (p. 93)

$4 \times 12$. Multiplier is 4. Multiplicand is 12 .

Solution to Exercise 2.2.2 (p. 93)

$8 \times 36$. Multiplier is 8 . Multiplicand is 36 .

Solution to Exercise 2.2.3 (p. 93)

$5 \times 0$. Multiplier is 5 . Multiplicand is 0 .

Solution to Exercise 2.2.4 (p. 93)

$12,000 \times 1,847$. Multiplier is 12,000 . Multiplicand is 1,847 .

Solution to Exercise 2.2.5 (p. 94)

185

Solution to Exercise 2.2.6 (p. 94)

624

Solution to Exercise 2.2.7 (p. 95)

3,752

Solution to Exercise 2.2.8 (p. 95)

320,152

Solution to Exercise 2.2.9 (p. 95)

904,797

Solution to Exercise 2.2.10 (p. 97)

1,022

Solution to Exercise 2.2.11 (p. 97)

4,472

Solution to Exercise 2.2.12 (p. 97)

35,615

Solution to Exercise 2.2.13 (p. 97)

$1,456,488$

Solution to Exercise 2.2.14 (p. 97)

$2,464,528$

Solution to Exercise 2.2.15 (p. 97)

$66,790,080$

Solution to Exercise 2.2.16 (p. 97)

104,709

Solution to Exercise 2.2.17 (p. 97)

240

Solution to Exercise 2.2.18 (p. 97)

$3,809,000$

Solution to Exercise 2.2.19 (p. 97)

$8,130,000$

Solution to Exercise 2.2.20 (p. 98)

162,000

Solution to Exercise 2.2.21 (p. 98)

$126,000,000$

Solution to Exercise 2.2.22 (p. 98)

2,870,000,000,000

Solution to Exercise 2.2.23 (p. 99)

1,404

Solution to Exercise 2.2.24 (p. 99)

$8,912,440$

Solution to Exercise 2.2.25 (p. 99)

$1,832,700,000,000$ 
Solution to Exercise 2.2.26 (p. 100) 24

Solution to Exercise 2.2.28 (p. 100) 48

Solution to Exercise 2.2.30 (p. 100) 6

Solution to Exercise 2.2.32 (p. 100) 225

Solution to Exercise 2.2.34 (p. 100) 270

Solution to Exercise 2.2.36 (p. 100) 582

Solution to Exercise 2.2.38 (p. 100) 960

Solution to Exercise 2.2.40 (p. 100) 7,695

Solution to Exercise 2.2.42 (p. 101) 3,648

Solution to Exercise 2.2.44 (p. 101) 46,488

Solution to Exercise 2.2.46 (p. 101) 2,530

Solution to Exercise 2.2.48 (p. 101) 6,888

Solution to Exercise 2.2.50 (p. 101) 24,180

Solution to Exercise 2.2.52 (p. 101) 73,914

Solution to Exercise 2.2.54 (p. 101) 68,625

Solution to Exercise 2.2.56 (p. 102) 511,173

Solution to Exercise 2.2.58 (p. 102) $1,352,550$

Solution to Exercise 2.2.60 (p. 102) $5,441,712$

Solution to Exercise 2.2.62 (p. 102) $36,901,053$

Solution to Exercise 2.2.64 (p. 102) $24,957,200$

Solution to Exercise 2.2.66 (p. 102) 0

Solution to Exercise 2.2.68 (p. 102) 41,384

Solution to Exercise 2.2.70 (p. 103) 0

Solution to Exercise 2.2.72 (p. 103) 73,530

Solution to Exercise 2.2.74 (p. 103) $6,440,000,000$ 
Solution to Exercise 2.2.76 (p. 103) $4,440,000$

Solution to Exercise 2.2.78 (p. 103) $641,900,000$

Solution to Exercise 2.2.80 (p. 103)

80,000

Solution to Exercise 2.2.82 (p. 104)

384 reports

Solution to Exercise 2.2.84 (p. 104)

3,330 problems

Solution to Exercise 2.2.86 (p. 104)

114 units

Solution to Exercise 2.2.88 (p. 104)

3,600 seconds

Solution to Exercise 2.2.90 (p. 104)

$5,865,696,000,000$ miles per year

Solution to Exercise 2.2.92 (p. 104)

$\$ 110,055$

Solution to Exercise 2.2.94 (p. 104)

$448,100,000$

Solution to Exercise 2.2.96 (p. 104)

712

Solution to Exercise 2.3.1 (p. 107) 4

Solution to Exercise 2.3.2 (p. 107)

2

Solution to Exercise 2.3.3 (p. 107)

5

Solution to Exercise 2.3.4 (p. 107)

6

Solution to Exercise 2.3.5 (p. 107)

4

Solution to Exercise 2.3.6 (p. 107)

9

Solution to Exercise 2.3.7 (p. 109) undefined

Solution to Exercise 2.3.8 (p. 109)

0

Solution to Exercise 2.3.9 (p. 109)

indeterminant

Solution to Exercise 2.3.10 (p. 109)

undefined

Solution to Exercise 2.3.11 (p. 109)

undefined

Solution to Exercise 2.3.12 (p. 109)

0

Solution to Exercise 2.3.13 (p. 110)

5

Solution to Exercise 2.3.14 (p. 110)

7 
Solution to Exercise 2.3.15 (p. 110)

0

Solution to Exercise 2.3.16 (p. 110)

An error message tells us that this operation is undefined. The particular message depends on the calculator. Solution to Exercise 2.3.17 (p. 110)

An error message tells us that this operation cannot be performed. Some calculators actually set $0 \div 0$ equal to 1 . We know better! $0 \div 0$ is indeterminant.

Solution to Exercise 2.3.18 (p. 111) 8

Solution to Exercise 2.3.20 (p. 111)

3

Solution to Exercise 2.3.22 (p. 111)

9

Solution to Exercise 2.3.24 (p. 111)

7

Solution to Exercise 2.3.26 (p. 111)

4

Solution to Exercise 2.3.28 (p. 111)

5

Solution to Exercise 2.3.30 (p. 111)

7

Solution to Exercise 2.3.32 (p. 111) not defined

Solution to Exercise 2.3.34 (p. 111) 3

Solution to Exercise 2.3.36 (p. 111)

0

Solution to Exercise 2.3.38 (p. 111)

5

Solution to Exercise 2.3.40 (p. 112)

8

Solution to Exercise 2.3.42 (p. 112)

9

Solution to Exercise 2.3.44 (p. 112)

$27 \div 9=3 ; 9) \overline{27}=3 ; \frac{27}{9}=3$

Solution to Exercise 2.3.46 (p. 112)

7 is quotient; 8 is divisor; 56 is dividend

Solution to Exercise 2.3.48 (p. 112)

12,124

Solution to Exercise 2.3.50 (p. 112)

$(2+3)+7=2+(3+7)=12$

$5+7=2+10=12$

Solution to Exercise 2.4.1 (p. 116)

18

Solution to Exercise 2.4.2 (p. 116)

81

Solution to Exercise 2.4.3 (p. 116)

853

Solution to Exercise 2.4.4 (p. 116)

1,129 
Solution to Exercise 2.4.5 (p. 116) 84,125

Solution to Exercise 2.4.6 (p. 118)

43

Solution to Exercise 2.4.7 (p. 118)

112

Solution to Exercise 2.4.8 (p. 118)

305

Solution to Exercise 2.4.9 (p. 118)

3,016

Solution to Exercise 2.4.10 (p. 120)

18 R3

Solution to Exercise 2.4.11 (p. 120)

$43 \mathrm{R} 2$

Solution to Exercise 2.4.12 (p. 120)

23 R6

Solution to Exercise 2.4.13 (p. 120)

61 R14

Solution to Exercise 2.4.14 (p. 121)

614 R58

Solution to Exercise 2.4.15 (p. 122)

45

Solution to Exercise 2.4.16 (p. 122)

551

Solution to Exercise 2.4.17 (p. 122)

Since the dividend has more than eight digits, this division cannot be performed on most nonscientific calculators. On others, the answer is 415,026,137.4

Solution to Exercise 2.4.18 (p. 122)

This division results in 68.02985075 , a decimal number, and therefore, we cannot, at this time, find the value of the remainder. Later, we will discuss decimal numbers.

Solution to Exercise 2.4.19 (p. 122)

13

Solution to Exercise 2.4.21 (p. 122)

67

Solution to Exercise 2.4.23 (p. 122)

52

Solution to Exercise 2.4.25 (p. 122) 3

Solution to Exercise 2.4.27 (p. 123)

70

Solution to Exercise 2.4.29 (p. 123)

61

Solution to Exercise 2.4.31 (p. 123)

59

Solution to Exercise 2.4.33 (p. 123)

67

Solution to Exercise 2.4.35 (p. 123)

87

Solution to Exercise 2.4.37 (p. 123)

54 
Solution to Exercise 2.4.39 (p. 123)

52

Solution to Exercise 2.4.41 (p. 123)

38

Solution to Exercise 2.4.43 (p. 123)

45

Solution to Exercise 2.4.45 (p. 123)

777

Solution to Exercise 2.4.47 (p. 123)

342

Solution to Exercise 2.4.49 (p. 124)

644

Solution to Exercise 2.4.51 (p. 124)

533

Solution to Exercise 2.4.53 (p. 124)

10,440

Solution to Exercise 2.4.55 (p. 124)

8,147 remainder 847

Solution to Exercise 2.4.57 (p. 124)

4 remainder 2

Solution to Exercise 2.4.59 (p. 124)

66 remainder 1

Solution to Exercise 2.4.61 (p. 124)

823 remainder 2

Solution to Exercise 2.4.63 (p. 124)

40 remainder 28

Solution to Exercise 2.4.65 (p. 124)

665 remainder 15

Solution to Exercise 2.4.67 (p. 124)

957 remainder 34

Solution to Exercise 2.4.69 (p. 124)

665 remainder 4

Solution to Exercise 2.4.71 (p. 124)

458 remainder 13

Solution to Exercise 2.4.73 (p. 125)

996 remainder 23

Solution to Exercise 2.4.75 (p. 125) 42

Solution to Exercise 2.4.77 (p. 125)

8,216

Solution to Exercise 2.4.79 (p. 125)

$\$ 1,975$ per month

Solution to Exercise 2.4.81 (p. 125)

$\$ 485$ each person invested

Solution to Exercise 2.4.83 (p. 125)

14 cubes per hour

Solution to Exercise 2.4.85 (p. 125)

8 bits in each byte

Solution to Exercise 2.4.87 (p. 125)

38 
Solution to Exercise 2.4.89 (p. 126) 600,000

Solution to Exercise 2.4.91 (p. 126) 625,600

Solution to Exercise 2.5.1 (p. 127) 2

Solution to Exercise 2.5.2 (p. 127)

3

Solution to Exercise 2.5.3 (p. 127)

3

Solution to Exercise 2.5.4 (p. 127)

5

Solution to Exercise 2.5.5 (p. 127)

2,4

Solution to Exercise 2.5.6 (p. 127)

2,5

Solution to Exercise 2.5.7 (p. 127)

2,3

Solution to Exercise 2.5.8 (p. 128)

$6,9,10$

Solution to Exercise 2.5.9 (p. 128)

6

Solution to Exercise 2.5.10 (p. 128)

$6,9,10$

Solution to Exercise 2.5.11 (p. 128)

6,9

Solution to Exercise 2.5.12 (p. 128)

$2,3,4,6,8$

Solution to Exercise 2.5.14 (p. 128)

$2,3,5,6,10$

Solution to Exercise 2.5.16 (p. 128)

2

Solution to Exercise 2.5.18 (p. 128)

2,4

Solution to Exercise 2.5.20 (p. 129) 3

Solution to Exercise 2.5.22 (p. 129) none

Solution to Exercise 2.5.24 (p. 129) 5

Solution to Exercise 2.5.26 (p. 129) 5

Solution to Exercise 2.5.28 (p. 129)

$2,3,4,6$

Solution to Exercise 2.5.30 (p. 129)

$2,3,5,6,10$

Solution to Exercise 2.5.32 (p. 129)

none

Solution to Exercise 2.5.34 (p. 129)

2,4 
Solution to Exercise 2.5.36 (p. 129) 2

Solution to Exercise 2.5.38 (p. 129) none

Solution to Exercise 2.5.40 (p. 129)

none

Solution to Exercise 2.5.42 (p. 130) 1

Solution to Exercise 2.5.44 (p. 130)

$(35+16)+7=51+7=58$

$35+(16+7)=35+23=58$

Solution to Exercise 2.5.46 (p. 130) 87

Solution to Exercise 2.6.1 (p. 131)

$15 \cdot 6=90$ and $6 \cdot 15=90$

Solution to Exercise 2.6.2 (p. 131)

$432 \cdot 428=184,896$ and $428 \cdot 432=184,896$

Solution to Exercise 2.6.3 (p. 131)

168

Solution to Exercise 2.6.4 (p. 132)

165,564

Solution to Exercise 2.6.5 (p. 132)

843

Solution to Exercise 2.6.6 (p. 132)

234

Solution to Exercise 2.6.8 (p. 133)

4,032

Solution to Exercise 2.6.10 (p. 133)

326,000

Solution to Exercise 2.6.12 (p. 133)

252

Solution to Exercise 2.6.14 (p. 133)

21,340

Solution to Exercise 2.6.16 (p. 134)

8,316

Solution to Exercise 2.6.18 (p. 134)

$32 \cdot 2=64=4 \cdot 16$

Solution to Exercise 2.6.20 (p. 134)

$23 \cdot 1,166=26,818=253 \cdot 106$

Solution to Exercise 2.6.22 (p. 134)

associative

Solution to Exercise 2.6.24 (p. 134)

$7 \cdot 9=63=9 \cdot 7$

Solution to Exercise 2.6.26 (p. 134)

6

Solution to Exercise 2.6.28 (p. 134)

$4+15=19$

$15+4=19$

Solution to Exercise 2.6.30 (p. 135)

$2,3,4,6$ 
Solution to Exercise 2.8.1 (p. 137)

factors; product

Solution to Exercise 2.8.3 (p. 137)

divisor; quotient

Solution to Exercise 2.8.5 (p. 137)

an even digit $(0,2,4,6$, or 8$)$

Solution to Exercise 2.8.7 (p. 137)

divisible by 4

Solution to Exercise 2.8.9 (p. 137)

112

Solution to Exercise 2.8.11 (p. 137)

7

Solution to Exercise 2.8.13 (p. 138)

3,045

Solution to Exercise 2.8.15 (p. 138)

4

Solution to Exercise 2.8.17 (p. 138)

15,075

Solution to Exercise 2.8.19 (p. 138)

14

Solution to Exercise 2.8.21 (p. 138)

42,112

Solution to Exercise 2.8.23 (p. 138)

63

Solution to Exercise 2.8.25 (p. 138)

$1,046,960$

Solution to Exercise 2.8.27 (p. 138)

$101,010,000$

Solution to Exercise 2.8.29 (p. 139)

0

Solution to Exercise 2.8.31 (p. 139)

428

Solution to Exercise 2.8.33 (p. 139)

5 remainder 9

Solution to Exercise 2.8.35 (p. 139)

not defined

Solution to Exercise 2.8.37 (p. 139)

32 remainder 3

Solution to Exercise 2.8.39 (p. 139)

$3,072,202$

Solution to Exercise 2.8.41 (p. 139)

0

Solution to Exercise 2.8.43 (p. 139)

0

Solution to Exercise 2.8.45 (p. 139)

not defined

Solution to Exercise 2.8.47 (p. 139)

1

Solution to Exercise 2.8.49 (p. 140)

$226 \cdot 114$ 
Solution to Exercise 2.8.51 (p. 140) $(16 \cdot 14) \cdot 0$

Solution to Exercise 2.8.53 (p. 140) $4,278,000$

Solution to Exercise 2.8.55 (p. 140)

$\$ 33$

Solution to Exercise 2.9.1 (p. 140)

8 and 7 are factors; 56 is the product

Solution to Exercise 2.9.2 (p. 140)

Addition

Solution to Exercise 2.9.3 (p. 140)

3 is the divisor; 4 is the quotient

Solution to Exercise 2.9.4 (p. 140)

$0,2,4,6$, or 8

Solution to Exercise 2.9.5 (p. 140)

commutative

Solution to Exercise 2.9.6 (p. 140)

1

Solution to Exercise 2.9.7 (p. 140)

84

Solution to Exercise 2.9.8 (p. 141)

0

Solution to Exercise 2.9.9 (p. 141)

352,000

Solution to Exercise 2.9.10 (p. 141)

419,020

Solution to Exercise 2.9.11 (p. 141)

252

Solution to Exercise 2.9.12 (p. 141)

not defined

Solution to Exercise 2.9.13 (p. 141)

0

Solution to Exercise 2.9.14 (p. 141)

17

Solution to Exercise 2.9.15 (p. 141) 18

Solution to Exercise 2.9.16 (p. 141)

142 remainder 32

Solution to Exercise 2.9.17 (p. 141)

211

Solution to Exercise 2.9.18 (p. 141)

216; 1,005

Solution to Exercise 2.9.19 (p. 141)

$216 ; 640$

Solution to Exercise 2.9.20 (p. 141)

1,$005 ; 640$ 


\section{Chapter 3}

\section{Exponents, Roots, and Factorization of Whole Numbers}

\subsection{Objectives ${ }^{1}$}

After completing this chapter, you should

Exponents and Roots (Section 3.2)

- understand and be able to read exponential notation

- understand the concept of root and be able to read root notation

- be able to use a calculator having the $y^{x}$ key to determine a root

Grouping Symbols and the Order of Operations (Section 3.3)

- understand the use of grouping symbols

- understand and be able to use the order of operations

- use the calculator to determine the value of a numerical expression

\section{Prime Factorization of Natural Numbers (Section 3.4)}

- be able to determine the factors of a whole number

- be able to distinguish between prime and composite numbers

- be familiar with the fundamental principle of arithmetic

- be able to find the prime factorization of a whole number

\section{The Greatest Common Factor (Section 3.5)}

- be able to find the greatest common factor of two or more whole numbers

The Least Common Multiple (Section 3.6)

- be able to find the least common multiple of two or more whole numbers

\footnotetext{
${ }^{1}$ This content is available online at $<\mathrm{http}: / / \mathrm{cnx} . o r g /$ content $/ \mathrm{m} 18890 / 1.4 />$.
} 


\subsection{Exponents and Roots ${ }^{2}$}

\subsubsection{Section Overview}

- Exponential Notation

- Reading Exponential Notation

- Roots

- Reading Root Notation

- Calculators

\subsubsection{Exponential Notation}

\section{Exponential Notation}

We have noted that multiplication is a description of repeated addition. Exponential notation is a description of repeated multiplication.

Suppose we have the repeated multiplication

$8 \cdot 8 \cdot 8 \cdot 8 \cdot 8$

\section{Exponent}

The factor 8 is repeated 5 times. Exponential notation uses a superscript for the number of times the factor is repeated. The superscript is placed on the repeated factor, $8^{5}$, in this case. The superscript is called an exponent.

\section{The Function of an Exponent}

An exponent records the number of identical factors that are repeated in a multiplication.

\subsubsection{Sample Set A}

Write the following multiplication using exponents.

\section{Example 3.1}

$3 \cdot 3$. Since the factor 3 appears 2 times, we record this as

$3^{2}$

Example 3.2

$62 \cdot 62 \cdot 62 \cdot 62 \cdot 62 \cdot 62 \cdot 62 \cdot 62 \cdot 62$. Since the factor 62 appears 9 times, we record this as

$62^{9}$

Expand (write without exponents) each number.

Example 3.3

$12^{4}$. The exponent 4 is recording 4 factors of 12 in a multiplication. Thus,

$12^{4}=12 \cdot 12 \cdot 12 \cdot 12$

Example 3.4

$706^{3}$. The exponent 3 is recording 3 factors of 706 in a multiplication. Thus,

$706^{3}=706 \cdot 706 \cdot 706$

\footnotetext{
${ }^{2}$ This content is available online at $<$ http://cnx.org/content $/ \mathrm{m} 34871 / 1.2 />$.
} 


\subsubsection{Practice Set A}

Write the following using exponents.

Exercise 3.2.1

(Solution on p. 200.)

$37 \cdot 37$

Exercise 3.2.2

(Solution on p. 200.)

$16 \cdot 16 \cdot 16 \cdot 16 \cdot 16$

Exercise 3.2.3

$9 \cdot 9 \cdot 9 \cdot 9 \cdot 9 \cdot 9 \cdot 9 \cdot 9 \cdot 9 \cdot 9$

(Solution on p. 200.)

Write each number without exponents.

Exercise 3.2.4

$85^{3}$

Exercise 3.2.5

$4^{7}$

Exercise 3.2.6

$1,739^{2}$

(Solution on p. 200.)

(Solution on p. 200.)

(Solution on p. 200.)

\subsubsection{Reading Exponential Notation}

In a number such as $8^{5}$,

Base

8 is called the base.

Exponent, Power

5 is called the exponent, or power. $8^{5}$ is read as "eight to the fifth power," or more simply as "eight to the fifth," or "the fifth power of eight."

\section{Squared}

When a whole number is raised to the second power, it is said to be squared. The number $5^{2}$ can be read as

5 to the second power, or

5 to the second, or

5 squared.

\section{Cubed}

When a whole number is raised to the third power, it is said to be cubed. The number $5^{3}$ can be read as

5 to the third power, or

5 to the third, or

5 cubed.

When a whole number is raised to the power of 4 or higher, we simply say that that number is raised to that particular power. The number $5^{8}$ can be read as

5 to the eighth power, or just

5 to the eighth. 


\subsubsection{Roots}

In the English language, the word "root" can mean a source of something. In mathematical terms, the word "root" is used to indicate that one number is the source of another number through repeated multiplication.

\section{Square Root}

We know that $49=7^{2}$, that is, $49=7 \cdot 7$. Through repeated multiplication, 7 is the source of 49 . Thus, 7 is a root of 49. Since two 7's must be multiplied together to produce 49, the 7 is called the second or square root of 49 .

\section{Cube Root}

We know that $8=2^{3}$, that is, $8=2 \cdot 2 \cdot 2$. Through repeated multiplication, 2 is the source of 8 . Thus, 2 is a root of 8 . Since three 2 's must be multiplied together to produce 8,2 is called the third or cube root of 8 .

We can continue this way to see such roots as fourth roots, fifth roots, sixth roots, and so on.

\subsubsection{Reading Root Notation}

There is a symbol used to indicate roots of a number. It is called the radical sign $\sqrt[n]{ }$

The Radical Sign

The symbol $\sqrt[n]{ }$ is called a radical sign and indicates the nth root of a number.

We discuss particular roots using the radical sign as follows:

\section{Square Root}

$\sqrt[2]{\text { number }}$ indicates the square root of the number under the radical sign. It is customary to drop the 2 in the radical sign when discussing square roots. The symbol $\sqrt{ }$ is understood to be the square root radical sign.

$\sqrt{49}=7$ since $7 \cdot 7=7^{2}=49$

\section{Cube Root}

$\sqrt[3]{\text { number }}$ indicates the cube root of the number under the radical sign.

$\sqrt[3]{8}=2$ since $2 \cdot 2 \cdot 2=2^{3}=8$

\section{Fourth Root}

$\sqrt[4]{\text { number }}$ indicates the fourth root of the number under the radical sign.

$\sqrt[4]{81}=3$ since $3 \cdot 3 \cdot 3 \cdot 3=3^{4}=81$

In an expression such as $\sqrt[5]{32}$

\section{Radical Sign}

$\sqrt{ }$ is called the radical sign.

\section{Index}

5 is called the index. (The index describes the indicated root.)

\section{Radicand}

32 is called the radicand.

\section{Radical}

$\sqrt[5]{32}$ is called a radical (or radical expression). 


\subsubsection{Sample Set B}

Find each root.

\section{Example 3.5}

$\sqrt{25}$ To determine the square root of 25 , we ask, "What whole number squared equals 25 ?" From our experience with multiplication, we know this number to be 5 . Thus,

$\sqrt{25}=5$

Check: $5 \cdot 5=5^{2}=25$

Example 3.6

$\sqrt[5]{32}$ To determine the fifth root of 32 , we ask, "What whole number raised to the fifth power equals 32?" This number is 2 .

$\sqrt[5]{32}=2$

Check: $2 \cdot 2 \cdot 2 \cdot 2 \cdot 2=2^{5}=32$

\subsubsection{Practice Set B}

Find the following roots using only a knowledge of multiplication.

Exercise 3.2.7 $\sqrt{64}$

Exercise 3.2.8

(Solution on p. 200.) $\sqrt{100}$

Exercise 3.2.9 $\sqrt[3]{64}$

Exercise 3.2.10 $\sqrt[6]{64}$

(Solution on p. 200.)

(Solution on p. 200.)

(Solution on p. 200.)

\subsubsection{Calculators}

Calculators with the $\sqrt{x}, y^{x}$, and $1 / x$ keys can be used to find or approximate roots.

\subsubsection{Sample Set C}

\section{Example 3.7}

Use the calculator to find $\sqrt{121}$

\begin{tabular}{|l|l|l|}
\hline & & Display Reads \\
\hline Type & 121 & 121 \\
\hline Press & $\sqrt{x}$ & 11 \\
\hline
\end{tabular}

Table 3.1

\section{Example 3.8}

Find $\sqrt[7]{2187}$. 


\begin{tabular}{|c|l|l|}
\hline & & Display Reads \\
\hline Type & 2187 & 2187 \\
\hline Press & $y^{x}$ & 2187 \\
\hline Type & 7 & 7 \\
\hline Press & $1 / x$ & .14285714 \\
\hline Press & $=$ & 3 \\
\hline
\end{tabular}

Table 3.2

$\sqrt[7]{2187}=3\left(\right.$ Which means that $3^{7}=2187$.)

\subsubsection{Practice Set C}

Use a calculator to find the following roots.

Exercise 3.2.11

(Solution on p. 200.) $\sqrt[3]{729}$

Exercise 3.2.12

(Solution on p. 200.) $\sqrt[4]{8503056}$

Exercise 3.2.13

(Solution on p. 200.) $\sqrt{53361}$

(Solution on p. 200.)

Exercise 3.2.14 $\sqrt[12]{16777216}$

\subsubsection{Exercises}

For the following problems, write the expressions using exponential notation.

Exercise 3.2.15

(Solution on p. 200.)

$4 \cdot 4$

Exercise 3.2.16

$12 \cdot 12$

Exercise 3.2.17

$9 \cdot 9 \cdot 9 \cdot 9$

(Solution on p. 200.)

Exercise 3.2.18

$10 \cdot 10 \cdot 10 \cdot 10 \cdot 10 \cdot 10$

Exercise 3.2.19

$826 \cdot 826 \cdot 826$

(Solution on p. 200.)

Exercise 3.2.20

$3,021 \cdot 3,021 \cdot 3,021 \cdot 3,021 \cdot 3,021$

Exercise 3.2.21

$\underbrace{6 \cdot 6 \cdots \cdots 6}_{85 \text { factors of } 6}$

(Solution on p. 200.)

Exercise 3.2.22

$\underbrace{2 \cdot 2 \cdots \cdots 2}_{112 \text { factors of } 2}$ 
Exercise 3.2.23

(Solution on p. 200.)

$$
\underbrace{1 \cdot 1 \cdots \cdots 1}_{3,008 \text { factors of } 1}
$$

For the following problems, expand the terms. (Do not find the actual value.)

Exercise 3.2.24

$5^{3}$

Exercise 3.2.25

$7^{4}$

(Solution on p. 200.)

Exercise 3.2.26

$15^{2}$

Exercise 3.2.27

$117^{5}$

(Solution on p. 200.)

Exercise 3.2.28

$61^{6}$

Exercise 3.2.29

$30^{2}$

(Solution on p. 200.)

For the following problems, determine the value of each of the powers. Use a calculator to check each result.

Exercise 3.2.30

$3^{2}$

Exercise 3.2.31

(Solution on p. 200.)

$4^{2}$

Exercise 3.2.32

$1^{2}$

Exercise 3.2.33

$10^{2}$

(Solution on p. 200.)

Exercise 3.2.34

$11^{2}$

Exercise 3.2.35

$12^{2}$

(Solution on p. 200.)

Exercise 3.2.36

$13^{2}$

Exercise 3.2.37

$15^{2}$

(Solution on p. 200.)

Exercise 3.2.38

$1^{4}$

Exercise 3.2.39

$3^{4}$

(Solution on p. 201.)

Exercise 3.2.40

$7^{3}$

Exercise 3.2.41

$10^{3}$

(Solution on p. 201.)

Exercise 3.2.42

$100^{2}$

Exercise 3.2.43

$8^{3}$

(Solution on p. 201.) 
Exercise 3.2.44

$5^{5}$

Exercise 3.2.45

$9^{3}$

(Solution on p. 201.)

Exercise 3.2.46

$6^{2}$

Exercise 3.2.47

$7^{1}$

(Solution on p. 201.)

Exercise 3.2.48

$1^{28}$

Exercise 3.2.49

$2^{7}$

(Solution on p. 201.)

Exercise 3.2.50

$0^{5}$

Exercise 3.2.51

$8^{4}$

(Solution on p. 201.)

Exercise 3.2.52

$5^{8}$

Exercise 3.2.53

$6^{9}$

Exercise 3.2.54

$25^{3}$

Exercise 3.2.55

$42^{2}$

Exercise 3.2.56

$31^{3}$

Exercise 3.2.57

$15^{5}$

(Solution on p. 201.)

Exercise 3.2.58

$2^{20}$

Exercise 3.2.59

(Solution on p. 201.)

$816^{2}$

(Solution on p. 201.)

For the following problems, find the roots (using your knowledge of multiplication). Use a calculator to check each result.

Exercise 3.2.60

$\sqrt{9}$

Exercise 3.2.61

$\sqrt{16}$

(Solution on p. 201.)

Exercise 3.2.62

$\sqrt{36}$

Exercise 3.2.63

$\sqrt{64}$

(Solution on p. 201.)

Exercise 3.2.64

$\sqrt{121}$

Exercise 3.2.65

$\sqrt{144}$

(Solution on p. 201.) 
Exercise 3.2.66

$\sqrt{169}$

Exercise 3.2.67

(Solution on p. 201.)

$\sqrt{225}$

Exercise 3.2.68

$\sqrt[3]{27}$

Exercise 3.2.69

(Solution on p. 201.)

$\sqrt[5]{32}$

Exercise 3.2.70

$\sqrt[4]{256}$

Exercise 3.2.71

(Solution on p. 201.)

$\sqrt[3]{216}$

Exercise 3.2.72

$\sqrt[7]{1}$

Exercise 3.2.73

$\sqrt{400}$

(Solution on p. 201.)

Exercise 3.2.74

$\sqrt{900}$

Exercise 3.2.75

(Solution on p. 201.)

$\sqrt{10,000}$

Exercise 3.2.76

$\sqrt{324}$

Exercise 3.2.77

$\sqrt{3,600}$

(Solution on p. 201.)

For the following problems, use a calculator with the keys $\sqrt{x}, y^{x}$, and $1 / x$ to find each of the values.

Exercise 3.2.78

$\sqrt{676}$

Exercise 3.2.79

(Solution on p. 201.)

$\sqrt{1,156}$

Exercise 3.2.80

$\sqrt{46,225}$

Exercise 3.2.81

$\sqrt{17,288,964}$

(Solution on p. 201.)

Exercise 3.2.82

$\sqrt[3]{3,375}$

Exercise 3.2.83

$\sqrt[4]{331,776}$

(Solution on p. 201.)

Exercise 3.2.84

$\sqrt[8]{5,764,801}$

Exercise 3.2.85

$\sqrt[12]{16,777,216}$

Exercise 3.2.86

$\sqrt[8]{16,777,216}$

Exercise 3.2.87

$\sqrt[10]{9,765,625}$

(Solution on p. 201.)

(Solution on p. 201.) 
Exercise 3.2.88

$\sqrt[4]{160,000}$

Exercise 3.2.89

$\sqrt[3]{531,441}$

(Solution on p. 202.)

\subsubsection{Exercises for Review}

Exercise 3.2.90

(Section 1.7) Use the numbers 3,8 , and 9 to illustrate the associative property of addition.

Exercise 3.2.91

(Solution on p. 202.)

(Section 2.2) In the multiplication $8 \cdot 4=32$, specify the name given to the numbers 8 and 4 .

Exercise 3.2.92

(Section 2.3) Does the quotient $15 \div 0$ exist? If so, what is it?

Exercise 3.2.93

(Solution on p. 202.)

(Section 2.3) Does the quotient $0 \div 15$ exist? If so, what is it?

Exercise 3.2.94

(Section 2.6) Use the numbers 4 and 7 to illustrate the commutative property of multiplication.

\subsection{Grouping Symbols and the Order of Operations ${ }^{3}$}

\subsubsection{Section Overview}

- Grouping Symbols

- Multiple Grouping Symbols

- The Order of Operations

- Calculators

\subsubsection{Grouping Symbols}

Grouping symbols are used to indicate that a particular collection of numbers and meaningful operations are to be grouped together and considered as one number. The grouping symbols commonly used in mathematics are the following:

()$,[],\{\}$,

Parentheses: ( )

Brackets: []

Braces: \{\}

Bar:

In a computation in which more than one operation is involved, grouping symbols indicate which operation to perform first. If possible, we perform operations inside grouping symbols first.

\footnotetext{
${ }^{3}$ This content is available online at $<$ http://cnx.org/content $/ \mathrm{m} 34872 / 1.2 />$.
} 


\subsubsection{Sample Set A}

If possible, determine the value of each of the following.

Example 3.9

$9+(3 \cdot 8)$

Since 3 and 8 are within parentheses, they are to be combined first.

$$
\begin{aligned}
9+(3 \cdot 8)= & 9+24 \\
& =33
\end{aligned}
$$

Thus,

$9+(3 \cdot 8)=33$

Example 3.10

$(10 \div 0) \cdot 6$

Since $10 \div 0$ is undefined, this operation is meaningless, and we attach no value to it. We write, "undefined."

\subsubsection{Practice Set A}

If possible, determine the value of each of the following.

Exercise 3.3.1

(Solution on p. 202.)

$16-(3 \cdot 2)$

Exercise 3.3.2

$5+(7 \cdot 9)$

Exercise 3.3.3

(Solution on p. 202.)

$(4+8) \cdot 2$

Exercise 3.3.4

$28 \div(18-11)$

Exercise 3.3.5

$(33 \div 3)-11$

Exercise 3.3.6

$4+(0 \div 0)$

(Solution on p. 202.)

(Solution on p. 202.)

(Solution on p. 202.)

(Solution on p. 202.)

\subsubsection{Multiple Grouping Symbols}

When a set of grouping symbols occurs inside another set of grouping symbols, we perform the operations within the innermost set first. 


\subsubsection{Sample Set B}

Determine the value of each of the following.

Example 3.11

$2+(8 \cdot 3)-(5+6)$

Combine 8 and 3 first, then combine 5 and 6.

$2+24-11$ Now combine left to right.

$26-11$

15

Example 3.12

$10+[30-(2 \cdot 9)]$

Combine 2 and 9 since they occur in the innermost set of parentheses.

$10+[30-18] \quad$ Now combine 30 and 18 .

$10+12$

22

\subsubsection{Practice Set B}

Determine the value of each of the following.

Exercise 3.3.7

(Solution on p. 202.)

$(17+8)+(9+20)$

Exercise 3.3.8

(Solution on p. 202.)

$(55-6)-(13 \cdot 2)$

Exercise 3.3.9

(Solution on p. 202.)

$23+(12 \div 4)-(11 \cdot 2)$

Exercise 3.3.10

(Solution on p. 202.)

$86+[14 \div(10-8)]$

Exercise 3.3.11

$31+\{9+[1+(35-2)]\}$

(Solution on p. 202.)

Exercise 3.3.12

$\{6-[24 \div(4 \cdot 2)]\}^{3}$

(Solution on p. 202.)

\subsubsection{The Order of Operations}

Sometimes there are no grouping symbols indicating which operations to perform first. For example, suppose we wish to find the value of $3+5 \cdot 2$. We could do either of two things:

Add 3 and 5 , then multiply this sum by 2 .

$$
\begin{aligned}
3+5 \cdot 2 & =8 \cdot 2 \\
& =16
\end{aligned}
$$

Multiply 5 and 2, then add 3 to this product. 


$$
\begin{aligned}
3+5 \cdot 2= & 3+10 \\
& =13
\end{aligned}
$$

We now have two values for one number. To determine the correct value, we must use the accepted order of operations.

\section{Order of Operations}

1. Perform all operations inside grouping symbols, beginning with the innermost set, in the order $2,3,4$ described below,

2. Perform all exponential and root operations.

3. Perform all multiplications and divisions, moving left to right.

4. Perform all additions and subtractions, moving left to right.

\subsubsection{Sample Set C}

Determine the value of each of the following.

\section{Example 3.13}

$21+3 \cdot 12$ Multiply first.

$21+36 \quad$ Add.

57

Example 3.14

$(15-8)+5 \cdot(6+4)$. Simplify inside parentheses first.

$$
\begin{array}{cc}
7+5 \cdot 10 & \text { Multiply. } \\
7+50 & \text { Add. }
\end{array}
$$

57

\section{Example 3.15}

$63-(4+6 \cdot 3)+76-4$ Simplify first within the parenthesis by multiplying, then adding.

$63-(4+18)+76-4$

$63-22+76-4 \quad$ Now perform the additions and subtractions, moving left to right.

$$
\begin{aligned}
& 41+76-4 \quad \text { Add } 41 \text { and } 76: \quad 41+76=117 . \\
& 117-4 \quad \text { Subtract } 4 \text { from 117: } \quad 117-4=113 .
\end{aligned}
$$

113

\section{Example 3.16}

$7 \cdot 6-4^{2}+1^{5}$ Evaluate the exponential forms, moving left to right.

$$
\begin{array}{lrl}
7 \cdot 6-16+1 & \text { Multiply } 7 \text { and } 6: & 7 \cdot 6=42 \\
42-16+1 & \text { Subtract } 16 \text { from } 42: & 42-16=26
\end{array}
$$

$26+1$

Add 26 and 1: $26+1=27$ 


\section{Example 3.17}

$6 \cdot\left(3^{2}+2^{2}\right)+4^{2} \quad$ Evaluate the exponential forms in the parentheses: $3^{2}=9$ and $2^{2}=4$

$6 \cdot(9+4)+4^{2}$

$6 \cdot(13)+4^{2}$

$6 \cdot(13)+16$

$78+16$

94
Add the 9 and 4 in the parentheses: $9+4=13$

Evaluate the exponential form: $4^{2}=16$

Multiply 6 and 13: $6 \cdot 13=78$

Add 78 and 16: $78+16=94$

\section{Example 3.18}

$$
\begin{gathered}
\frac{6^{2}+2^{2}}{4^{2}+6 \cdot 2^{2}}+\frac{1^{3}+8^{2}}{10^{2}-19 \cdot 5} \\
\frac{36+4}{16+6 \cdot 4}+\frac{1+64}{100-19 \cdot 5} \\
\frac{36+4}{16+24}+\frac{1+64}{100-95} \\
\frac{40}{40}+\frac{65}{5} \\
1+13
\end{gathered}
$$

Recall that the bar is a grouping symbol.

The fraction $\frac{6^{2}+2^{2}}{4^{2}+6 \cdot 2^{2}}$ is equivalent to $\left(6^{2}+2^{2}\right) \div\left(4^{2}+6 \cdot 2^{2}\right)$

14

\subsubsection{Practice Set C}

Determine the value of each of the following.

Exercise 3.3.13

(Solution on p. 202.)

$8+(32-7)$

Exercise 3.3.14

(Solution on p. 202.)

$(34+18-2 \cdot 3)+11$

Exercise 3.3.15

$8(10)+4(2+3)-(20+3 \cdot 15+40-5)$

Exercise 3.3.16

$5 \cdot 8+4^{2}-2^{2}$

Exercise 3.3.17

$4\left(6^{2}-3^{3}\right) \div\left(4^{2}-4\right)$

Exercise 3.3.18

$$
(8+9 \cdot 3) \div 7+5 \cdot(8 \div 4+7+3 \cdot 5)
$$

Exercise 3.3.19

$$
\frac{3^{3}+2^{3}}{6^{2}-29}+5\left(\frac{8^{2}+2^{4}}{7^{2}-3^{2}}\right) \div \frac{8 \cdot 3+1^{8}}{2^{3}-3}
$$

(Solution on p. 202.)

(Solution on p. 202.)

(Solution on p. 202.)

(Solution on p. 202.)

(Solution on p. 202.)

\subsubsection{Calculators}

Using a calculator is helpful for simplifying computations that involve large numbers. 


\subsubsection{Sample Set D}

Use a calculator to determine each value.

Example 3.19

$9,842+56 \cdot 85$

\begin{tabular}{|l|l|l|l|}
\hline & Key & & Display Reads \\
\hline Perform the multiplication first. & Type & 56 & 56 \\
\hline & Press & $\times$ & 56 \\
\hline & Type & 85 & 85 \\
\hline Now perform the addition. & Press & + & 4760 \\
\hline & Type & 9842 & 9842 \\
\hline & Press & $=$ & 14602 \\
\hline
\end{tabular}

Table 3.3

The display now reads 14,602 .

Example 3.20

$42(27+18)+105(810 \div 18)$

\begin{tabular}{|l|l|l|l|}
\hline & Key & & Display Reads \\
\hline Operate inside the parentheses & Type & 27 & 27 \\
\hline & Press & + & 27 \\
\hline & Type & 18 & 18 \\
\hline & Press & $=$ & 45 \\
\hline Multiply by 42. & Press & $\times$ & 45 \\
\hline & Type & 42 & 42 \\
\hline & Press & $=$ & 1890 \\
\hline
\end{tabular}

Table 3.4

Place this result into memory by pressing the memory key. 


\begin{tabular}{|l|l|l|l|}
\hline & Key & & Display Reads \\
\hline Now operate in the other parentheses. & Type & 810 & 810 \\
\hline & Press & $\div$ & 810 \\
\hline & Type & 18 & 18 \\
\hline Now multiply by 105. & Press & $=$ & 45 \\
\hline & Press & $\times$ & 45 \\
\hline & Type & 105 & 105 \\
\hline We are now ready to add these two quantities together. & Press & + & 4725 \\
\hline Press the memory recall key. & & & 1890 \\
\hline & Press & $=$ & 6615 \\
\hline
\end{tabular}

Table 3.5

Thus, $42(27+18)+105(810 \div 18)=6,615$

Example 3.21

$16^{4}+37^{3}$

\begin{tabular}{|l|l|l|}
\hline \multicolumn{3}{|c|}{ Nonscientific Calculators } \\
\hline Key & & Display Reads \\
\hline Type & 16 & 16 \\
\hline Press & $\times$ & 16 \\
\hline Type & 16 & 16 \\
\hline Press & $\times$ & 256 \\
\hline Type & 16 & 16 \\
\hline Press & $\times$ & 4096 \\
\hline Type & 16 & 16 \\
\hline Press & $=$ & 65536 \\
\hline Press the memory key & & \\
\hline Type & 37 & 37 \\
\hline Press & $\times$ & 37 \\
\hline Type & 37 & 37 \\
\hline Press & $\times$ & 1396 \\
\hline Type & 37 & 37 \\
\hline Press & $\times$ & 50653 \\
\hline Press & + & 50653 \\
\hline Press memory recall key & & 65536 \\
\hline Press & $=$ & 116189 \\
\hline
\end{tabular}


Table 3.6

\begin{tabular}{|l|l|l|}
\hline \multicolumn{3}{|c|}{ Calculators with $y^{x}$ Key } \\
\hline Key & & Display Reads \\
\hline Type & 16 & 16 \\
\hline Press & $y^{x}$ & 16 \\
\hline Type & 4 & 4 \\
\hline Press & $=$ & 4096 \\
\hline Press & + & 4096 \\
\hline Type & 37 & 37 \\
\hline Press & $y^{x}$ & 37 \\
\hline Type & 3 & 3 \\
\hline Press & $=$ & 116189 \\
\hline
\end{tabular}

Table 3.7

Thus, $16^{4}+37^{3}=116,189$

We can certainly see that the more powerful calculator simplifies computations.

Example 3.22

Nonscientific calculators are unable to handle calculations involving very large numbers.

$85612 \cdot 21065$

\begin{tabular}{|l|l|l|}
\hline Key & & Display Reads \\
\hline Type & 85612 & 85612 \\
\hline Press & $\times$ & 85612 \\
\hline Type & 21065 & 21065 \\
\hline Press & $=$ & \\
\hline
\end{tabular}

Table 3.8

This number is too big for the display of some calculators and we'll probably get some kind of error message. On some scientific calculators such large numbers are coped with by placing them in a form called "scientific notation." Others can do the multiplication directly. (1803416780)

\subsubsection{Practice Set D}

Use a calculator to find each value.

Exercise 3.3.20

(Solution on p. 202.)

$9,285+86(49)$

Exercise 3.3.21

(Solution on p. 202.)

$55(84-26)+120(512-488)$ 
Exercise 3.3.22

$106^{3}-17^{4}$

Exercise 3.3.23 $6,053^{3}$
(Solution on p. 202.)

(Solution on p. 203.)

\subsubsection{Exercises}

For the following problems, find each value. Check each result with a calculator.

Exercise 3.3.24

(Solution on p. 203.)

$2+3 \cdot(8)$

Exercise 3.3.25

$18+7 \cdot(4-1)$

Exercise 3.3.26

(Solution on p. 203.)

$3+8 \cdot(6-2)+11$

Exercise 3.3.27

$1-5 \cdot(8-8)$

Exercise 3.3.28

$37-1 \cdot 6^{2}$

(Solution on p. 203.)

Exercise 3.3.29

$98 \div 2 \div 7^{2}$

Exercise 3.3.30 $\left(4^{2}-2 \cdot 4\right)-2^{3}$

(Solution on p. 203.)

Exercise 3.3.31 $\sqrt{9}+14$

Exercise 3.3.32 $\sqrt{100}+\sqrt{81}-4^{2}$

(Solution on p. 203.)

Exercise 3.3.33 $\sqrt[3]{8}+8-2 \cdot 5$

Exercise 3.3.34 $\sqrt[4]{16}-1+5^{2}$

(Solution on p. 203.)

Exercise 3.3.35 $61-22+4[3 \cdot(10)+11]$

Exercise 3.3.36 $121-4 \cdot[(4) \cdot(5)-12]+\frac{16}{2}$

Exercise 3.3.37 $\frac{(1+16)-3}{7}+5 \cdot(12)$

Exercise 3.3.38 $\frac{8 \cdot(6+20)}{8}+\frac{3 \cdot(6+16)}{22}$

(Solution on p. 203.)

Exercise 3.3.39 $10 \cdot[8+2 \cdot(6+7)]$

Exercise 3.3.40 $21 \div 7 \div 3$

(Solution on p. 203.)

Exercise 3.3.41 $10^{2} \cdot 3 \div 5^{2} \cdot 3-2 \cdot 3$ 
Exercise 3.3.42

(Solution on p. 203.)

$85 \div 5 \cdot 5-85$

Exercise 3.3.43

$\frac{51}{17}+7-2 \cdot 5 \cdot\left(\frac{12}{3}\right)$

Exercise 3.3.44

$2^{2} \cdot 3+2^{3} \cdot(6-2)-(3+17)+11(6)$

(Solution on p. 203.)

Exercise 3.3.45

$26-2 \cdot\left\{\frac{6+20}{13}\right\}$

Exercise 3.3.46

$$
2 \cdot\{(7+7)+6 \cdot[4 \cdot(8+2)]\}
$$

(Solution on p. 203.)

Exercise 3.3.47

$0+10(0)+15 \cdot\{4 \cdot 3+1\}$

Exercise 3.3.48

$18+\frac{7+2}{9}$

(Solution on p. 203.)

Exercise 3.3.49

$(4+7) \cdot(8-3)$

Exercise 3.3.50

$(6+8) \cdot(5+2-4)$

(Solution on p. 203.)

Exercise 3.3.51

$(21-3) \cdot(6-1) \cdot(7)+4(6+3)$

Exercise 3.3.52

$(10+5) \cdot(10+5)-4 \cdot(60-4)$

(Solution on p. 203.)

Exercise 3.3.53

$6 \cdot\{2 \cdot 8+3\}-(5) \cdot(2)+\frac{8}{4}+(1+8) \cdot(1+11)$

Exercise 3.3.54

$2^{5}+3 \cdot(8+1)$

(Solution on p. 203.)

Exercise 3.3.55

$3^{4}+2^{4} \cdot(1+5)$

Exercise 3.3.56

$1^{6}+0^{8}+5^{2} \cdot(2+8)^{3}$

(Solution on p. 203.)

Exercise 3.3.57

$(7) \cdot(16)-3^{4}+2^{2} \cdot\left(1^{7}+3^{2}\right)$

Exercise 3.3.58 $\frac{2^{3}-7}{5^{2}}$

(Solution on p. 203.)

Exercise 3.3.59 $\frac{(1+6)^{2}+2}{3 \cdot 6+1}$

Exercise 3.3.60

$\frac{6^{2}-1}{2^{3}-3}+\frac{4^{3}+2 \cdot 3}{2 \cdot 5}$

Exercise 3.3.61

$\frac{5\left(8^{2}-9 \cdot 6\right)}{2^{5}-7}+\frac{7^{2}-4^{2}}{2^{4}-5}$

Exercise 3.3.62

$\frac{(2+1)^{3}+2^{3}+1^{10}}{6^{2}}-\frac{15^{2}-[2 \cdot 5]^{2}}{5 \cdot 5^{2}}$

(Solution on p. 203.)

Exercise 3.3.63

$\frac{6^{3}-2 \cdot 10^{2}}{2^{2}}+\frac{18\left(2^{3}+7^{2}\right)}{2(19)-3^{3}}$

(Solution on p. 203.) 
Exercise 3.3.64

(Solution on p. 203.)

$2 \cdot\left\{6+\left[10^{2}-6 \sqrt{25}\right]\right\}$

Exercise 3.3.65

$181-3 \cdot(2 \sqrt{36}+3 \sqrt[3]{64})$

Exercise 3.3.66

(Solution on p. 203.)

$\frac{2 \cdot(\sqrt{81}-\sqrt[3]{125})}{4^{2}-10+2^{2}}$

\subsubsection{Exercises for Review}

Exercise 3.3.67

(Section 1.7) The fact that $0+$ any whole number $=$ that particular whole number is an example of which property of addition?

Exercise 3.3.68

(Solution on p. 203.)

(Section 2.2) Find the product. 4,271 $\times 630$.

Exercise 3.3.69

(Section 2.3) In the statement $27 \div 3=9$, what name is given to the result 9 ?

Exercise 3.3.70

(Solution on p. 203.)

(Section 2.7) What number is the multiplicative identity?

Exercise 3.3.71

(Section 2.7) Find the value of $2^{4}$.

\subsection{Prime Factorization of Natural Numbers ${ }^{4}$}

\subsubsection{Section Overview}

- Factors

- Determining the Factors of a Whole Number

- Prime and Composite Numbers

- The Fundamental Principle of Arithmetic

- The Prime Factorization of a Natural Number

\subsubsection{Factors}

From observations made in the process of multiplication, we have seen that

$($ factor $) \cdot($ factor $)=$ product

\section{Factors, Product}

The two numbers being multiplied are the factors and the result of the multiplication is the product. Now, using our knowledge of division, we can see that a first number is a factor of a second number if the first number divides into the second number a whole number of times (without a remainder).

One Number as a Factor of Another

A first number is a factor of a second number if the first number divides into the second number a whole number of times (without a remainder).

\footnotetext{
${ }^{4}$ This content is available online at $<$ http://cnx.org/content $/ \mathrm{m} 34873 / 1.2 />$.
} 
We show this in the following examples:

Example 3.23

3 is a factor of 27 , since $27 \div 3=9$, or $3 \cdot 9=27$.

Example 3.24

7 is a factor of 56 , since $56 \div 7=8$, or $7 \cdot 8=56$.

Example 3.25

4 is not a factor of 10 , since $10 \div 4=2 R 2$. (There is a remainder.)

\subsubsection{Determining the Factors of a Whole Number}

We can use the tests for divisibility from Section 2.5 to determine all the factors of a whole number.

\subsubsection{Sample Set A}

Example 3.26

Find all the factors of 24 .
Try 1:
$24 \div 1=24$
1 and 24 are factors

Try 2:

24 is even, so 24 is divisible by 2 .

$$
24 \div 2=12 \quad 2 \text { and } 12 \text { are factors }
$$

Try 3: $2+4=6$ and 6 is divisible by 3 , so 24 is divisible by 3 .

$\begin{array}{lcc} & 24 \div 3=8 & 3 \text { and } 8 \text { are factors } \\ \text { Try 4: } & 24 \div 4=6 & 4 \text { and } 6 \text { are factors } \\ \text { Try 5: } & 24 \div 5=4 R 4 & 5 \text { is not a factor. }\end{array}$

The next number to try is 6 , but we already have that 6 is a factor. Once we come upon a factor that we already have discovered, we can stop.

All the whole number factors of 24 are 1, 2, 3, 4, 6, 8, 12, and 24 .

\subsubsection{Practice Set A}

Find all the factors of each of the following numbers.

Exercise 3.4.1

(Solution on p. 204.)

6

Exercise 3.4.2

(Solution on p. 204.)

12

Exercise 3.4.3

(Solution on p. 204.)

18

Exercise 3.4.4

(Solution on p. 204.)

5

Exercise 3.4.5

10

(Solution on p. 204.)

Exercise 3.4.6

33

(Solution on p. 204.) 


\subsubsection{Prime and Composite Numbers}

Notice that the only factors of 7 are 1 and 7 itself, and that the only factors of 3 are 1 and 3 itself. However, the number 8 has the factors $1,2,4$, and 8 , and the number 10 has the factors $1,2,5$, and 10 . Thus, we can see that a whole number can have only two factors (itself and 1) and another whole number can have several factors.

We can use this observation to make a useful classification for whole numbers: prime numbers and composite numbers.

\section{Prime Number}

A whole number (greater than one) whose only factors are itself and 1 is called a prime number.

\section{The Number 1 is Not a Prime Number}

The first seven prime numbers are $2,3,5,7,11,13$, and 17 . Notice that the whole number 1 is not considered to be a prime number, and the whole number 2 is the first prime and the only even prime number.

\section{Composite Number}

A whole number composed of factors other than itself and 1 is called a composite number. Composite numbers are not prime numbers.

Some composite numbers are $4,6,8,9,10,12$, and 15 .

\subsubsection{Sample Set B}

Determine which whole numbers are prime and which are composite.

\section{Example 3.27}

39. Since 3 divides into 39 , the number 39 is composite: $39 \div 3=13$

Example 3.28

47. A few division trials will assure us that 47 is only divisible by 1 and 47 . Therefore, 47 is prime.

\subsubsection{Practice Set B}

Determine which of the following whole numbers are prime and which are composite.

Exercise 3.4.8

3

Exercise 3.4.9

16

Exercise 3.4.10

21

Exercise 3.4.11

35

Exercise 3.4.12

47

Exercise 3.4.13

29
(Solution on p. 204.)

(Solution on p. 204.)

(Solution on p. 204.)

(Solution on p. 204.)

(Solution on p. 204.)

(Solution on p. 204.) 


\subsubsection{The Fundamental Principle of Arithmetic}

Prime numbers are very useful in the study of mathematics. We will see how they are used in subsequent sections. We now state the Fundamental Principle of Arithmetic.

Fundamental Principle of Arithmetic

Except for the order of the factors, every natural number other than 1 can be factored in one and only one way as a product of prime numbers.

\section{Prime Factorization}

When a number is factored so that all its factors are prime numbers. the factorization is called the prime factorization of the number.

The technique of prime factorization is illustrated in the following three examples.

1. $10=5 \cdot 2$. Both 2 and 5 are primes. Therefore, $2 \cdot 5$ is the prime factorization of 10 .

2. 11. The number 11 is a prime number. Prime factorization applies only to composite numbers. Thus, 11 has no prime factorization.

3. $60=2 \cdot 30$. The number 30 is not prime: $30=2 \cdot 15$.

$60=2 \cdot 2 \cdot 15$

The number 15 is not prime: $15=3 \cdot 5$

$60=2 \cdot 2 \cdot 3 \cdot 5$

We'll use exponents.

$60=2^{2} \cdot 3 \cdot 5$

The numbers 2,3 , and 5 are each prime. Therefore, $2^{2} \cdot 3 \cdot 5$ is the prime factorization of 60 .

\subsubsection{The Prime Factorization of a Natural Number}

The following method provides a way of finding the prime factorization of a natural number.

\section{The Method of Finding the Prime Factorization of a Natural Number}

1. Divide the number repeatedly by the smallest prime number that will divide into it a whole number of times (without a remainder).

2. When the prime number used in step 1 no longer divides into the given number without a remainder, repeat the division process with the next largest prime that divides the given number.

3. Continue this process until the quotient is smaller than the divisor.

4. The prime factorization of the given number is the product of all these prime divisors. If the number has no prime divisors, it is a prime number.

We may be able to use some of the tests for divisibility we studied in Section 2.5 to help find the primes that divide the given number. 


\subsubsection{Sample Set C}

\section{Example 3.29}

Find the prime factorization of 60 .

Since the last digit of 60 is 0 , which is even, 60 is divisible by 2 . We will repeatedly divide by 2 until we no longer can. We shall divide as follows:

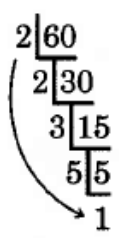

30 is divisible by 2 again.

15 is not divisible by 2 , but it is divisible by 3 , the next prime.

5 is not divisble by 3 , but it is divisible by 5 , the next prime.

The quotient 1 is finally smaller than the divisor 5 , and the prime factorization of 60 is the product of these prime divisors.

$60=2 \cdot 2 \cdot 3 \cdot 5$

We use exponents when possible.

$60=2^{2} \cdot 3 \cdot 5$

\section{Example 3.30}

Find the prime factorization of 441 .

441 is not divisible by 2 since its last digit is not divisible by 2 .

441 is divisible by 3 since $4+4+1=9$ and 9 is divisible by 3 .

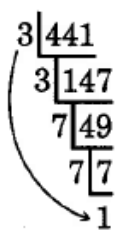

147 is divisible by $3(1+4+7=12)$.

49 is not divisible by 3 , nor is it divisible by 5 . It is divisible by 7 .

The quotient 1 is finally smaller than the divisor 7 , and the prime factorization of 441 is the product of these prime divisors.

$441=3 \cdot 3 \cdot 7 \cdot 7$

Use exponents.

$441=3^{2} \cdot 7^{2}$

\section{Example 3.31}

Find the prime factorization of 31 . 
31 is not divisible by 2
Its last digit is not even

$$
31 \div 2=15 \mathrm{R} 1
$$

The quotient, 15, is larger than the divisor, 3 . Continue.

The digits $3+1=4$, and 4 is not divisible by 3 .

$$
31 \div 3=10 \mathrm{R} 1
$$

The quotient, 10, is larger than the divisor, 3 . Continue.

31 is not divisible by 5

The last digit of 31 is not 0 or 5 .

$$
31 \div 5=6 \mathrm{R} 1
$$

The quotient, 6 , is larger than the divisor, 5 . Continue.

31 is not divisible by 7 .

Divide by 7 .

$$
31 \div 7=4 \mathrm{R} 1
$$

The quotient, 4 , is smaller than the divisor, 7 .

We can stop the process and conclude that 31 is a prime number.

The number 31 is a prime number

\subsubsection{Practice Set C}

Find the prime factorization of each whole number.

Exercise 3.4.16

22

Exercise 3.4.17

40

Exercise 3.4.18

48

Exercise 3.4.19

63

Exercise 3.4.20

945

Exercise 3.4.21

1,617

Exercise 3.4.22

17

Exercise 3.4.23

61

\subsubsection{Exercises}

For the following problems, determine the missing factor(s).

Exercise 3.4.24

$14=7$.
(Solution on p. 204.)

(Solution on p. 204.)

(Solution on p. 204.)

(Solution on p. 204.)

(Solution on p. 204.)

(Solution on p. 204.)

(Solution on p. 204.)

(Solution on p. 204.) 
Exercise 3.4.25

$20=4$.

Exercise 3.4.26

(Solution on p. 204.)

$36=9$.

Exercise 3.4.27 $42=21$.

Exercise 3.4.28

(Solution on p. 205.)

$44=4$.

Exercise 3.4.29

$38=2$.

Exercise 3.4.30

(Solution on p. 205.)

$18=3$.

Exercise 3.4.31

$28=2$.

Exercise 3.4.32

(Solution on p. 205.)

$300=2 \cdot 5$

Exercise 3.4.33

$840=2$.

For the following problems, find all the factors of each of the numbers.

Exercise 3.4.34

(Solution on p. 205.)

16

Exercise 3.4.35

22

Exercise 3.4.36

56

(Solution on p. 205.)

Exercise 3.4.37

105

Exercise 3.4.38

220

(Solution on p. 205.)

Exercise 3.4.39

15

Exercise 3.4.40

32

(Solution on p. 205.)

Exercise 3.4.41

80

Exercise 3.4.42

142

(Solution on p. 205.)

Exercise 3.4.43

218

For the following problems, determine which of the whole numbers are prime and which are composite.

Exercise 3.4.44

(Solution on p. 205.)

23

Exercise 3.4.45

25 
Exercise 3.4.46

(Solution on p. 205.)

27

Exercise 3.4.47

2

Exercise 3.4.48

(Solution on p. 205.)

3

Exercise 3.4.49

5

Exercise 3.4.50

7

(Solution on p. 205.)

Exercise 3.4.51

9

Exercise 3.4.52

11

(Solution on p. 205.)

Exercise 3.4.53

34

Exercise 3.4.54

(Solution on p. 205.)

55

Exercise 3.4.55

63

Exercise 3.4.56

1,044

(Solution on p. 205.)

Exercise 3.4.57

924

Exercise 3.4.58

339

(Solution on p. 205.)

Exercise 3.4.59

103

Exercise 3.4.60

209

(Solution on p. 205.)

Exercise 3.4.61

667

Exercise 3.4.62

4,575

(Solution on p. 205.)

Exercise 3.4.63

119

For the following problems, find the prime factorization of each of the whole numbers.

Exercise 3.4.64

(Solution on p. 205.)

26

Exercise 3.4.65

38

Exercise 3.4.66

54

(Solution on p. 205.)

Exercise 3.4.67

62 
Exercise 3.4.68

(Solution on p. 205.)

56

Exercise 3.4.69

176

Exercise 3.4.70

(Solution on p. 205.)

480

Exercise 3.4.71

819

Exercise 3.4.72

(Solution on p. 205.)

2,025

Exercise 3.4.73

148,225

\subsubsection{Exercises For Review}

Exercise 3.4.74

(Solution on p. 205.)

(Section 1.4) Round 26,584 to the nearest ten.

Exercise 3.4.75

(Section 1.6) How much bigger is 106 than 79 ?

Exercise 3.4.76

(Solution on p. 205.)

(Section 2.3) True or false? Zero divided by any nonzero whole number is zero.

Exercise 3.4.77

(Section 2.4) Find the quotient. 10,584 $\div 126$.

Exercise 3.4.78

(Section 3.3) Find the value of $\sqrt{121}-\sqrt{81}+6^{2} \div 3$.

(Solution on p. 206.)

\subsection{The Greatest Common Factor ${ }^{5}$}

\subsubsection{Section Overview}

- The Greatest Common Factor (GCF)

- A Method for Determining the Greatest Common Factor

\subsubsection{The Greatest Common Factor (GCF)}

Using the method we studied in Section 3.4, we could obtain the prime factorizations of 30 and 42 .

$30=2 \cdot 3 \cdot 5$

$42=2 \cdot 3 \cdot 7$

\section{Common Factor}

We notice that 2 appears as a factor in both numbers, that is, 2 is a common factor of 30 and 42 . We also notice that 3 appears as a factor in both numbers. Three is also a common factor of 30 and 42 .

\footnotetext{
${ }^{5}$ This content is available online at $<$ http://cnx.org/content $/ \mathrm{m} 34874 / 1.2 />$.
} 


\section{Greatest Common Factor (GCF)}

When considering two or more numbers, it is often useful to know if there is a largest common factor of the numbers, and if so, what that number is. The largest common factor of two or more whole numbers is called the greatest common factor, and is abbreviated by GCF. The greatest common factor of a collection of whole numbers is useful in working with fractions (which we will do in Section 4.1).

\subsubsection{A Method for Determining the Greatest Common Factor}

A straightforward method for determining the GCF of two or more whole numbers makes use of both the prime factorization of the numbers and exponents.

\section{Finding the GCF}

To find the greatest common factor (GCF) of two or more whole numbers:

1. Write the prime factorization of each number, using exponents on repeated factors.

2. Write each base that is common to each of the numbers.

3. To each base listed in step 2, attach the smallest exponent that appears on it in either of the prime factorizations.

4. The GCF is the product of the numbers found in step 3.

\subsubsection{Sample Set A}

Find the GCF of the following numbers.

\section{Example 3.32}

12 and 18

$12=2 \cdot 6=2 \cdot 2 \cdot 3=2^{2} \cdot 3$

$$
18=2 \cdot 9=2 \cdot 3 \cdot 3=2 \cdot 3^{2}
$$

2. The common bases are 2 and 3 .

3. The smallest exponents appearing on 2 and 3 in the prime factorizations are, respectively, 1 and $1\left(2^{1}\right.$ and $\left.3^{1}\right)$, or 2 and 3 .

4. The GCF is the product of these numbers. $2 \cdot 3=6$

The GCF of 30 and 42 is 6 because 6 is the largest number that divides both 30 and 42 without a remainder.

\section{Example 3.33}

18,60 , and 72

$$
\begin{gathered}
18=2 \cdot 9=2 \cdot 3 \cdot 3=2 \cdot 3^{2} \\
\text { 1. } \quad 60=2 \cdot 30=2 \cdot 2 \cdot 15=2 \cdot 2 \cdot 3 \cdot 5=2^{2} \cdot 3 \cdot 5 \\
72=2 \cdot 36=2 \cdot 2 \cdot 18=2 \cdot 2 \cdot 2 \cdot 9=2 \cdot 2 \cdot 2 \cdot 3 \cdot 3=2^{3} \cdot 3^{2}
\end{gathered}
$$

2. The common bases are 2 and 3 .

3. The smallest exponents appearing on 2 and 3 in the prime factorizations are, respectively, 1 and 1:

$2^{1}$ from 18

$3^{1}$ from 60

4. The GCF is the product of these numbers.

$\mathrm{GCF}$ is $2 \cdot 3=6$ 
Thus, 6 is the largest number that divides 18,60 , and 72 without a remainder.

\section{Example 3.34}

$700,1,880$, and 6,160

$$
\begin{aligned}
700=2 \cdot 350=2 \cdot 2 \cdot 175 & = & 2 \cdot 2 \cdot 5 \cdot 35 \\
& = & 2 \cdot 2 \cdot 5 \cdot 5 \cdot 7 \\
& = & 2^{2} \cdot 5^{2} \cdot 7 \\
1,880=2 \cdot 940=2 \cdot 2 \cdot 470 & = & 2 \cdot 2 \cdot 2 \cdot 235 \\
& = & 2 \cdot 2 \cdot 2 \cdot 5 \cdot 47 \\
& = & 2^{3} \cdot 5 \cdot 47 \\
6,160=2 \cdot 3,080=2 \cdot 2 \cdot 1,540 & = & 2 \cdot 2 \cdot 2 \cdot 770 \\
& = & 2 \cdot 2 \cdot 2 \cdot 2 \cdot 385 \\
& = & 2 \cdot 2 \cdot 2 \cdot 2 \cdot 5 \cdot 77 \\
& = & 2 \cdot 2 \cdot 2 \cdot 2 \cdot 5 \cdot 7 \cdot 11 \\
& = & 2^{4} \cdot 5 \cdot 7 \cdot 11
\end{aligned}
$$

1.

2. The common bases are 2 and 5

3. The smallest exponents appearing on 2 and 5 in the prime factorizations are, respectively, 2 and 1.

$2^{2}$ from 700 .

$5^{1}$ from either 1,880 or 6,160 .

4. The GCF is the product of these numbers.

$\mathrm{GCF}$ is $2^{2} \cdot 5=4 \cdot 5=20$

Thus, 20 is the largest number that divides $700,1,880$, and 6,160 without a remainder.

\subsubsection{Practice Set A}

Find the GCF of the following numbers.

Exercise 3.5.1

(Solution on p. 206.)

24 and 36

Exercise 3.5.2

(Solution on p. 206.)

48 and 72

Exercise 3.5.3

(Solution on p. 206.)

50 and 140

Exercise 3.5.4

(Solution on p. 206.)

21 and 225

Exercise 3.5.5

(Solution on p. 206.)

450,600 , and 540 


\subsubsection{Exercises}

For the following problems, find the greatest common factor (GCF) of the numbers.

Exercise 3.5.6

(Solution on p. 206.)

6 and 8

Exercise 3.5.7

5 and 10

Exercise 3.5.8

8 and 12

(Solution on p. 206.)

Exercise 3.5.9

9 and 12

Exercise 3.5.10

20 and 24

(Solution on p. 206.)

Exercise 3.5.11

35 and 175

Exercise 3.5.12

25 and 45

(Solution on p. 206.)

Exercise 3.5.13

45 and 189

Exercise 3.5.14

66 and 165

(Solution on p. 206.)

Exercise 3.5.15

264 and 132

Exercise 3.5.16

99 and 135

Exercise 3.5.17

65 and 15

Exercise 3.5.18

33 and 77

(Solution on p. 206.)

Exercise 3.5.19

245 and 80

Exercise 3.5.20

351 and 165

Exercise 3.5.21

60, 140, and 100

Exercise 3.5.22

147, 343, and 231

(Solution on p. 206.)

Exercise 3.5.23

24, 30, and 45

Exercise 3.5.24

175,225 , and 400

(Solution on p. 206.)

Exercise 3.5.25

210, 630, and 182

Exercise 3.5.26

(Solution on p. 206.)

14,44 , and 616

(Solution on p. 206.) 
Exercise 3.5.27

$1,617,735$, and 429

Exercise 3.5.28

(Solution on p. 206.)

$1,573,4,862$, and 3,553

Exercise 3.5.29

$3,672,68$, and 920

Exercise 3.5.30

(Solution on p. 206.)

$7,2,401,343,16$, and 807

Exercise 3.5.31

500,77 , and 39

Exercise 3.5.32

(Solution on p. 206.)

441,275 , and 221

\subsubsection{Exercises for Review}

Exercise 3.5.33

(Section 2.2) Find the product. 2,753 $\times 4,006$.

Exercise 3.5.34

(Solution on p. 206.)

(Section 2.4) Find the quotient. $954 \div 18$.

Exercise 3.5.35

(Section 2.5) Specify which of the digits 2, 3, or 4 divide into 9,462.

Exercise 3.5.36

(Solution on p. 206.)

(Section 3.2) Write $8 \times 8 \times 8 \times 8 \times 8 \times 8$ using exponents.

Exercise 3.5.37

(Section 3.4) Find the prime factorization of 378.

\subsection{The Least Common Multiple}

\subsubsection{Section Overview}

- Multiples

- Common Multiples

- The Least Common Multiple (LCM)

- Finding the Least Common Multiple

\subsubsection{Multiples}

When a whole number is multiplied by other whole numbers, with the exception of zero, the resulting products are called multiples of the given whole number. Note that any whole number is a multiple of itself.

\footnotetext{
${ }^{6}$ This content is available online at $<$ http://cnx.org/content $/ \mathrm{m} 34876 / 1.2 />$.
} 


\subsubsection{Sample Set A}

\begin{tabular}{|l|l|l|l|}
\hline Multiples of 2 & Multiples of 3 & Multiples of 8 & Multiples of 10 \\
\hline $2 \times 1=2$ & $3 \times 1=3$ & $8 \times 1=8$ & $10 \times 1=10$ \\
\hline $2 \times 2=4$ & $3 \times 2=6$ & $8 \times 2=16$ & $10 \times 2=20$ \\
\hline $2 \times 3=6$ & $3 \times 3=9$ & $8 \times 3=24$ & $10 \times 3=30$ \\
\hline $2 \times 4=8$ & $3 \times 4=12$ & $8 \times 4=32$ & $10 \times 4=40$ \\
\hline $2 \times 5=10$ & $3 \times 5=15$ & $8 \times 5=40$ & $10 \times 5=50$ \\
\hline$\vdots$ & $\vdots$ & $\vdots$ & $\vdots$ \\
\hline
\end{tabular}

Table 3.9

\subsubsection{Practice Set A}

Find the first five multiples of the following numbers.

Exercise 3.6.1

(Solution on p. 206.)

4

Exercise 3.6.2

(Solution on p. 206.)

5

Exercise 3.6.3

(Solution on p. 206.)

6

(Solution on p. 207.)

Exercise 3.6.4

7

(Solution on p. 207.)

Exercise 3.6.5

9

\subsubsection{Common Multiples}

There will be times when we are given two or more whole numbers and we will need to know if there are any multiples that are common to each of them. If there are, we will need to know what they are. For example, some of the multiples that are common to 2 and 3 are 6,12 , and 18 .

\subsubsection{Sample Set B}

\section{Example 3.35}

We can visualize common multiples using the number line.

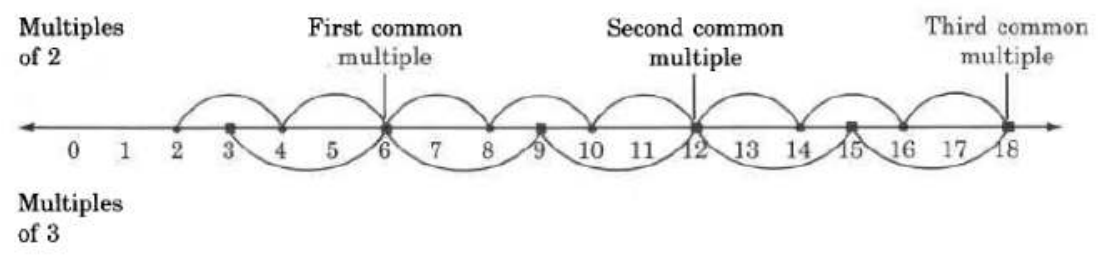

Notice that the common multiples can be divided by both whole numbers. 


\subsubsection{Practice Set B}

Find the first five common multiples of the following numbers.

Exercise 3.6.6

2 and 4

Exercise 3.6.7

3 and 4

Exercise 3.6.8

2 and 5

Exercise 3.6.9

3 and 6

Exercise 3.6.10

4 and 5
(Solution on p. 207.)

(Solution on p. 207.)

(Solution on p. 207.)

(Solution on p. 207.)

(Solution on p. 207.)

\subsubsection{The Least Common Multiple (LCM)}

Notice that in our number line visualization of common multiples (above), the first common multiple is also the smallest, or least common multiple, abbreviated by LCM.

\section{Least Common Multiple}

The least common multiple, LCM, of two or more whole numbers is the smallest whole number that each of the given numbers will divide into without a remainder.

The least common multiple will be extremely useful in working with fractions (Section 4.1).

\subsubsection{Finding the Least Common Multiple}

\section{Finding the LCM}

To find the LCM of two or more numbers:

1. Write the prime factorization of each number, using exponents on repeated factors.

2. Write each base that appears in each of the prime factorizations.

3. To each base, attach the largest exponent that appears on it in the prime factorizations.

4. The LCM is the product of the numbers found in step 3.

There are some major differences between using the processes for obtaining the GCF and the LCM that we must note carefully:

\section{The Difference Between the Processes for Obtaining the GCF and the LCM}

1. Notice the difference between step 2 for the LCM and step 2 for the GCF. For the GCF, we use only the bases that are common in the prime factorizations, whereas for the LCM, we use each base that appears in the prime factorizations.

2. Notice the difference between step 3 for the LCM and step 3 for the GCF. For the GCF, we attach the smallest exponents to the common bases, whereas for the LCM, we attach the largest exponents to the bases. 


\subsubsection{Sample Set C}

Find the LCM of the following numbers.

\section{Example 3.36}

9 and 12

1.

$$
9=3 \cdot 3=3^{2}
$$

$12=2 \cdot 6=2 \cdot 2 \cdot 3=2^{2} \cdot 3$

2. The bases that appear in the prime factorizations are 2 and 3 .

3. The largest exponents appearing on 2 and 3 in the prime factorizations are, respectively, 2 and 2: $2^{2}$ from 12 .

\section{$3^{2}$ from 9.}

4. The LCM is the product of these numbers. $\mathrm{LCM}=2^{2} \cdot 3^{2}=4 \cdot 9=36$

Thus, 36 is the smallest number that both 9 and 12 divide into without remainders.

\section{Example 3.37}

90 and 630

$$
90=2 \cdot 45=2 \cdot 3 \cdot 15=2 \cdot 3 \cdot 3 \cdot 5=2 \cdot 3^{2} \cdot 5
$$

1. $630=2 \cdot 315=2 \cdot 3 \cdot 105=2 \cdot 3 \cdot 3 \cdot 35=2 \cdot 3 \cdot 3 \cdot 5 \cdot 7$

$$
=2 \cdot 3^{2} \cdot 5 \cdot 7
$$

2. The bases that appear in the prime factorizations are $2,3,5$, and 7 .

3. The largest exponents that appear on $2,3,5$, and 7 are, respectively, $1,2,1$, and 1 :

$$
\begin{aligned}
& 2^{1} \text { from either } 90 \text { or } 630 . \\
& 3^{2} \text { from either } 90 \text { or } 630 . \\
& 5^{1} \text { from either } 90 \text { or } 630 . \\
& 7^{1} \text { from } 630 .
\end{aligned}
$$

4. The LCM is the product of these numbers. $\mathrm{LCM}=2 \cdot 3^{2} \cdot 5 \cdot 7=2 \cdot 9 \cdot 5 \cdot 7=630$

Thus, 630 is the smallest number that both 90 and 630 divide into with no remainders.

\section{Example 3.38}

33,110 , and 484

$$
33=3 \cdot 11
$$

1. $110=2 \cdot 55=2 \cdot 5 \cdot 11$

$$
484=2 \cdot 242=2 \cdot 2 \cdot 121=2 \cdot 2 \cdot 11 \cdot 11=2^{2} \cdot 11^{2} .
$$

2. The bases that appear in the prime factorizations are $2,3,5$, and 11 .

3. The largest exponents that appear on $2,3,5$, and 11 are, respectively, 2, 1, 1, and 2:

$$
\begin{aligned}
& 2^{2} \text { from } 484 . \\
& 3^{1} \text { from } 33 . \\
& 5^{1} \text { from } 110 \\
& 11^{2} \text { from } 484 .
\end{aligned}
$$

$$
\begin{aligned}
\text { 4CM } & =2^{2} \cdot 3 \cdot 5 \cdot 11^{2} \\
& =4 \cdot 3 \cdot 5 \cdot 121 \\
& =
\end{aligned}
$$

Thus, 7260 is the smallest number that 33,110 , and 484 divide into without remainders. 


\subsubsection{Practice Set C}

Find the LCM of the following numbers.

Exercise 3.6.11

(Solution on p. 207.)

20 and 54

Exercise 3.6.12

(Solution on p. 207.)

14 and 28

Exercise 3.6.13

6 and 63

Exercise 3.6.14

28,40 , and 98

Exercise 3.6.15

(Solution on p. 207.)

(Solution on p. 207.)

$16,27,125$, and 363

(Solution on p. 207.)

\subsubsection{Exercises}

For the following problems, find the least common multiple of the numbers.

Exercise 3.6.16

(Solution on p. 207.)

8 and 12

Exercise 3.6.17

6 and 15

Exercise 3.6.18

8 and 10

(Solution on p. 207.)

Exercise 3.6.19

10 and 14

Exercise 3.6.20

4 and 6

(Solution on p. 207.)

Exercise 3.6.21

6 and 12

Exercise 3.6.22

(Solution on p. 207.)

9 and 18

Exercise 3.6.23

6 and 8

Exercise 3.6.24

5 and 6

(Solution on p. 207.)

Exercise 3.6.25

7 and 8

Exercise 3.6.26

3 and 4

(Solution on p. 207.)

Exercise 3.6.27

2 and 9

Exercise 3.6.28

7 and 9

(Solution on p. 207.)

Exercise 3.6.29

28 and 36 
Exercise 3.6.30

(Solution on p. 207.)

24 and 36

Exercise 3.6.31

28 and 42

Exercise 3.6.32

(Solution on p. 207.)

240 and 360

Exercise 3.6.33

162 and 270

Exercise 3.6.34

20 and 24

(Solution on p. 207.)

Exercise 3.6.35

25 and 30

Exercise 3.6.36

24 and 54

(Solution on p. 207.)

Exercise 3.6.37

16 and 24

Exercise 3.6.38

(Solution on p. 207.)

36 and 48

Exercise 3.6.39

24 and 40

Exercise 3.6.40

15 and 21

(Solution on p. 207.)

Exercise 3.6.41

50 and 140

Exercise 3.6.42

7, 11, and 33

Exercise 3.6.43

8, 10, and 15

Exercise 3.6.44

18, 21, and 42

(Solution on p. 208.)

Exercise 3.6.45

4,5 , and 21

Exercise 3.6.46

45, 63, and 98

(Solution on p. 208.)

Exercise 3.6.47

15, 25, and 40

Exercise 3.6.48

(Solution on p. 208.)

12,16 , and 20

Exercise 3.6.49

84 and 96

Exercise 3.6.50

48 and 54

(Solution on p. 208.)

Exercise 3.6.51

12,16 , and 24

Exercise 3.6.52

(Solution on p. 208.)

$12,16,24$, and 36

(Solution on p. 208.) 
Exercise 3.6.53

$6,9,12$, and 18

Exercise 3.6.54

(Solution on p. 208.)

8, 14, 28, and 32

Exercise 3.6.55

$18,80,108$, and 490

Exercise 3.6.56

(Solution on p. 208.)

$22,27,130$, and 225

Exercise 3.6.57

$38,92,115$, and 189

Exercise 3.6.58

(Solution on p. 208.)

8 and 8

Exercise 3.6.59

12,12 , and 12

Exercise 3.6.60

$3,9,12$, and 3

(Solution on p. 208.)

\subsubsection{Exercises for Review}

Exercise 3.6.61

(Section 1.4) Round 434,892 to the nearest ten thousand.

Exercise 3.6.62

(Solution on p. 208.)

(Section 1.6) How much bigger is 14,061 than 7,509 ?

Exercise 3.6.63

(Section 2.4) Find the quotient. 22, $428 \div 14$.

Exercise 3.6.64

(Section 3.2) Expand $84^{3}$. Do not find the value.

(Solution on p. 208.)

Exercise 3.6.65

(Section 3.5) Find the greatest common factor of 48 and 72. 


\subsection{Summary of Key Concepts ${ }^{7}$}

\subsubsection{Summary of Key Concepts}

Exponential Notation (Section 3.2)

Exponential notation is a description of repeated multiplication.

Exponent (Section 3.2)

An exponent records the number of identical factors repeated in a multiplication.

In a number such as $7^{3}$,

Base (Section 3.2)

7 is called the base.

Exponent (Section 3.2)

3 is called the exponent, or power.

Power (Section 3.2)

$7^{3}$ is read "seven to the third power," or "seven cubed."

\section{Squared, Cubed (Section 3.2)}

A number raised to the second power is often called squared. A number raised to the third power is often called cubed.

\section{Root (Section 3.2)}

In mathematics, the word root is used to indicate that, through repeated multiplication, one number is the source of another number.

The Radical Sign $\sqrt{ }$ (Section 3.2)

The symbol $\sqrt{ }$ is called a radical sign and indicates the square root of a number. The symbol $\sqrt[n]{ }$ represents the $n$th root.

\section{Radical, Index, Radicand (Section 3.2)}

An expression such as $\sqrt[4]{16}$ is called a radical and 4 is called the index. The number 16 is called the radicand.

\section{Grouping Symbols (Section 3.3)}

Grouping symbols are used to indicate that a particular collection of numbers and meaningful operations are to be grouped together and considered as one number. The grouping symbols commonly used in mathematics are

Parentheses: ( )

Brackets: [ ]

Braces: \{\}

Bar:

\section{Order of Operations (Section 3.3)}

1. Perform all operations inside grouping symbols, beginning with the innermost set, in the order of 2,3 , and 4 below.

2. Perform all exponential and root operations, moving left to right.

3. Perform all multiplications and division, moving left to right.

\footnotetext{
${ }^{7}$ This content is available online at $<$ http://cnx.org/content $/ \mathrm{m} 34877 / 1.2 />$.
} 
4. Perform all additions and subtractions, moving left to right.

One Number as the Factor of Another (Section 3.4)

A first number is a factor of a second number if the first number divides into the second number a whole number of times.

Prime Number (Section 3.4)

A whole number greater than one whose only factors are itself and 1 is called a prime number. The whole number 1 is not a prime number. The whole number 2 is the first prime number and the only even prime number.

\section{Composite Number (Section 3.4)}

A whole number greater than one that is composed of factors other than itself and 1 is called a composite number.

Fundamental Principle of Arithmetic (Section 3.4)

Except for the order of factors, every whole number other than 1 can be written in one and only one way as a product of prime numbers.

\section{Prime Factorization (Section 3.4)}

The prime factorization of 45 is $3 \cdot 3 \cdot 5$. The numbers that occur in this factorization of 45 are each prime.

\section{Determining the Prime Factorization of a Whole Number (Section 3.4)}

There is a simple method, based on division by prime numbers, that produces the prime factorization of a whole number. For example, we determine the prime factorization of 132 as follows.

$$
\begin{array}{r|r}
2 \lcm{132} \\
2 \lcm{66} \\
3 \lcm{33} \\
\hline 11
\end{array}
$$

The prime factorization of 132 is $2 \cdot 2 \cdot 3 \cdot 11=2^{2} \cdot 3 \cdot 11$.

\section{Common Factor (Section 3.5)}

A factor that occurs in each number of a group of numbers is called a common factor. 3 is a common factor to the group 18,6 , and 45

\section{Greatest Common Factor (GCF) (Section 3.5)}

The largest common factor of a group of whole numbers is called the greatest common factor. For example, to find the greatest common factor of 12 and 20 ,

$$
\text { 1. Write the prime factorization of each number. } \begin{aligned}
& 12=2 \cdot 2 \cdot 3=2^{2} \cdot 3 \\
& 60=2 \cdot 2 \cdot 3 \cdot 5=2^{2} \cdot 3 \cdot
\end{aligned}
$$

2. Write each base that is common to each of the numbers: 2 and 3

3 . The smallest exponent appearing on 2 is 2 .

The smallest exponent appearing on 3 is 1 .

4. The GCF of 12 and 60 is the product of the numbers $2^{2}$ and $3 \cdot 2^{2} \cdot 3=4 \cdot 3=12$

Thus, 12 is the largest number that divides both 12 and 60 without a remainder.

\section{Finding the GCF (Section 3.5)}

There is a simple method, based on prime factorization, that determines the GCF of a group of whole numbers. 
Multiple (Section 3.6)

When a whole number is multiplied by all other whole numbers, with the exception of zero, the resulting individual products are called multiples of that whole number. Some multiples of 7 are 7, 14, 21, and 28 .

\section{Common Multiples (Section 3.6)}

Multiples that are common to a group of whole numbers are called common multiples. Some common multiples of 6 and 9 are 18, 36, and 54 .

\section{The LCM (Section 3.6)}

The least common multiple (LCM) of a group of whole numbers is the smallest whole number that each of the given whole numbers divides into without a remainder. The least common multiple of 9 and 6 is 18 .

\section{Finding the LCM (Section 3.6)}

There is a simple method, based on prime factorization, that determines the LCM of a group of whole numbers. For example, the least common multiple of 28 and 72 is found in the following way.

1. Write the prime factorization of each number $\begin{aligned} & 28=2 \cdot 2 \cdot 7=2^{2} \cdot 7 \\ & 72=2 \cdot 2 \cdot 2 \cdot 3 \cdot 3=2^{3} \cdot 3^{2}\end{aligned}$

2. Write each base that appears in each of the prime factorizations, 2,3 , and 7 .

3. To each of the bases listed in step 2, attach the largest exponent that appears on it in the prime factorization. $2^{3}, 3^{2}$, and 7

4. The LCM is the product of the numbers found in step $3.2^{3} \cdot 3^{2} \cdot 7=8 \cdot 9 \cdot 7=504$

Thus, 504 is the smallest number that both 28 and 72 will divide into without a remainder.

The Difference Between the GCF and the LCM (Section 3.6)

The GCF of two or more whole numbers is the largest number that divides into each of the given whole numbers. The LCM of two or more whole numbers is the smallest whole number that each of the given numbers divides into without a remainder.

\subsection{Exercise Supplement ${ }^{8}$}

\subsubsection{Exercise Supplement}

\subsubsection{Exponents and Roots (Section 3.2)}

For problems $1-25$, determine the value of each power and root.

Exercise 3.8.1 $3^{3}$

Exercise 3.8.2 $4^{3}$

Exercise 3.8.3 $0^{5}$

Exercise 3.8.4 $1^{4}$

Exercise 3.8.5 $12^{2}$

Exercise 3.8.6 $7^{2}$
(Solution on p. 208.)

(Solution on p. 208.)

(Solution on p. 208.)

\footnotetext{
${ }^{8}$ This content is available online at $<$ http://cnx.org/content $/ \mathrm{m} 34878 / 1.2 />$.
} 
Exercise 3.8.7 $8^{2}$

Exercise 3.8.8 $11^{2}$

Exercise 3.8.9 $2^{5}$

Exercise 3.8.10 $3^{4}$

Exercise 3.8.11 $15^{2}$

Exercise 3.8.12 $20^{2}$

Exercise 3.8.13 $25^{2}$

Exercise 3.8.14 $\sqrt{36}$

Exercise 3.8.15 $\sqrt{225}$

Exercise 3.8.16 $\sqrt[3]{64}$

Exercise 3.8.17 $\sqrt[4]{16}$

Exercise 3.8.18 $\sqrt{0}$

Exercise 3.8.19 $\sqrt[3]{1}$

Exercise 3.8.20 $\sqrt[3]{216}$

Exercise 3.8.21 $\sqrt{144}$

Exercise 3.8.22 $\sqrt{196}$

Exercise 3.8.23 $\sqrt{1}$

Exercise 3.8.24 $\sqrt[4]{0}$

Exercise 3.8.25 $\sqrt[6]{64}$
(Solution on p. 208.)

(Solution on p. 208.)

(Solution on p. 208.)

(Solution on p. 208.)

(Solution on p. 208.)

(Solution on p. 208.)

(Solution on p. 208.)

(Solution on p. 208.)

(Solution on p. 208.)

(Solution on p. 208.) 


\subsubsection{Section 3.2}

For problems 26-45, use the order of operations to determine each value.

Exercise 3.8.26

$2^{3}-2 \cdot 4$

Exercise 3.8.27

$5^{2}-10 \cdot 2-5$

(Solution on p. 209.)

Exercise 3.8.28

$\sqrt{81}-3^{2}+6 \cdot 2$

Exercise 3.8.29

$15^{2}+5^{2} \cdot 2^{2}$

(Solution on p. 209.)

Exercise 3.8.30

$3 \cdot\left(2^{2}+3^{2}\right)$

Exercise 3.8.31

$64 \cdot\left(3^{2}-2^{3}\right)$

(Solution on p. 209.)

Exercise 3.8.32 $\frac{5^{2}+1}{13}+\frac{3^{3}+1}{14}$

Exercise 3.8.33 $\frac{6^{2}-1}{5 \cdot 7}-\frac{49+7}{2 \cdot 7}$

(Solution on p. 209.)

Exercise 3.8.34 $\frac{2 \cdot\left[3+5\left(2^{2}+1\right)\right]}{5 \cdot 2^{3}-3^{2}}$

Exercise 3.8.35

$\frac{3^{2} \cdot\left[2^{5}-1^{4}\left(2^{3}+25\right)\right]}{2 \cdot 5^{2}+5+2}$

Exercise 3.8.36

$\frac{\left(5^{2}-2^{3}\right)-2 \cdot 7}{2^{2}-1}+5 \cdot\left[\frac{3^{2}-3}{2}+1\right]$

Exercise 3.8.37

$(8-3)^{2}+\left(2+3^{2}\right)^{2}$

(Solution on p. 209.)

Exercise 3.8.38

$3^{2} \cdot\left(4^{2}+\sqrt{25}\right)+2^{3} \cdot\left(\sqrt{81}-3^{2}\right)$

Exercise 3.8.39

$\sqrt{16+9}$

(Solution on p. 209.)

Exercise 3.8.40

$\sqrt{16}+\sqrt{9}$

Exercise 3.8.41

Compare the results of problems 39 and 40 . What might we conclude?

(Solution on p. 209.)

Exercise 3.8.42 $\sqrt{18 \cdot 2}$

Exercise 3.8.43 $\sqrt{6 \cdot 6}$

(Solution on p. 209.)

Exercise 3.8.44 $\sqrt{7 \cdot 7}$

Exercise 3.8.45 $\sqrt{8 \cdot 8}$

(Solution on p. 209.)

Exercise 3.8.46

An records the number of identical factors that are repeated in a multiplication. 


\subsubsection{Prime Factorization of Natural Numbers (Section 3.4)}

For problems 47-53, find all the factors of each number.

Exercise 3.8.47

18

Exercise 3.8.48

24

Exercise 3.8.49

11

(Solution on p. 209.)

Exercise 3.8.50

12

Exercise 3.8.51

51

(Solution on p. 209.)

Exercise 3.8.52

25

Exercise 3.8.53

2

(Solution on p. 209.)

Exercise 3.8.54

What number is the smallest prime number?

\subsubsection{Grouping Symbol and the Order of Operations (Section 3.3)}

For problems $55-64$, write each number as a product of prime factors.

Exercise 3.8.55

55

Exercise 3.8.56

20

Exercise 3.8.57

80

(Solution on p. 209.)

Exercise 3.8.58

284

Exercise 3.8.59

700

(Solution on p. 209.)

Exercise 3.8.60

845

Exercise 3.8.61 1,614

(Solution on p. 209.)

Exercise 3.8.62

921

Exercise 3.8.63 29

(Solution on p. 209.)

Exercise 3.8.64

37 


\subsubsection{The Greatest Common Factor (Section 3.5)}

For problems $65-75$, find the greatest common factor of each collection of numbers.

Exercise 3.8.65

(Solution on p. 209.)

5 and 15

Exercise 3.8.66

6 and 14

Exercise 3.8.67

10 and 15

(Solution on p. 209.)

Exercise 3.8.68

6,8 , and 12

Exercise 3.8.69

18 and 24

Exercise 3.8.70

42 and 54

Exercise 3.8.71

40 and 60

Exercise 3.8.72

18,48 , and 72

Exercise 3.8.73

147,189 , and 315

Exercise 3.8.74

64, 72, and 108

Exercise 3.8.75

275, 297, and 539

(Solution on p. 209.)

(Solution on p. 209.)

(Solution on p. 209.)

(Solution on p. 209.)

\subsubsection{The Least Common Multiple (Section 3.6)}

For problems $76-86$, find the least common multiple of each collection of numbers.

Exercise 3.8.76

5 and 15

Exercise 3.8.77

6 and 14

Exercise 3.8.78

10 and 15

Exercise 3.8.79

36 and 90

(Solution on p. 210.)

Exercise 3.8.80

42 and 54

Exercise 3.8.81

8, 12 , and 20

Exercise 3.8.82

40, 50, and 180

Exercise 3.8.83

135,147 , and 324

(Solution on p. 210.)

(Solution on p. 210.)

Available for free at Connexions $<$ http://cnx.org/content/col10615/1.4 $>$ 
Exercise 3.8.84

108,144 , and 324

Exercise 3.8.85

(Solution on p. 210.)

$5,18,25$, and 30

Exercise 3.8.86

$12,15,18$, and 20

Exercise 3.8.87

(Solution on p. 210.)

Find all divisors of 24 .

Exercise 3.8.88

Find all factors of 24 .

Exercise 3.8.89

Write all divisors of $2^{3} \cdot 5^{2} \cdot 7$.

(Solution on p. 210.)

Exercise 3.8.90

Write all divisors of $6 \cdot 8^{2} \cdot 10^{3}$.

Exercise 3.8.91

Does 7 divide $5^{3} \cdot 6^{4} \cdot 7^{2} \cdot 8^{5}$ ?

(Solution on p. 210.)

Exercise 3.8.92

Does 13 divide $8^{3} \cdot 10^{2} \cdot 11^{4} \cdot 13^{2} \cdot 15$ ?

\subsection{Proficiency Exam ${ }^{9}$}

\subsubsection{Proficiency Exam}

Exercise 3.9.1

(Solution on p. 210.)

(Section 3.2) In the number $8^{5}$, write the names used for the number 8 and the number 5 .

Exercise 3.9.2

(Solution on p. 210.)

(Section 3.2) Write using exponents. $12 \times 12 \times 12 \times 12 \times 12 \times 12 \times 12$

(Solution on p. 210.)

Exercise 3.9.3

(Section 3.2) Expand $9^{4}$.

For problems 4-15, determine the value of each expression.

Exercise 3.9.4

(Section 3.3) $4^{3}$

Exercise 3.9.5

(Section 3.3) $1^{5}$

Exercise 3.9.6

(Section 3.3) $0^{3}$

Exercise 3.9.7

(Section 3.3) $2^{6}$

Exercise 3.9.8

(Section 3.3) $\sqrt{49}$

(Solution on p. 210.)

(Solution on p. 210.)

(Solution on p. 210.)

(Solution on p. 210.)

(Solution on p. 210.)

Exercise 3.9.9

(Solution on p. 210.)

(Section 3.3) $\sqrt[3]{27}$

Exercise 3.9.10

(Solution on p. 210.) (Section 3.3) $\sqrt[8]{1}$

\footnotetext{
${ }^{9}$ This content is available online at $<$ http://cnx.org/content $/ \mathrm{m} 34879 / 1.2 />$.
} 
Exercise 3.9.11

(Solution on p. 210.)

(Section 3.3) $16+2 \cdot(8-6)$

(Solution on p. 210.)

Exercise 3.9.12

(Section 3.3) $5^{3}-\sqrt{100}+8 \cdot 2-20 \div 5$

(Solution on p. 210.)

Exercise 3.9.13

(Section 3.3) $3 \cdot \frac{8^{2}-2 \cdot 3^{2}}{5^{2}-2} \cdot \frac{6^{3}-4 \cdot 5^{2}}{29}$

Exercise 3.9.14

(Section 3.3$) \frac{20+2^{4}}{2^{3} \cdot 2-5 \cdot 2} \cdot \frac{5 \cdot 7-\sqrt{81}}{7+3 \cdot 2}$

Exercise 3.9.15

(Section 3.3) $\left[(8-3)^{2}+(33-4 \sqrt{49})\right]-2\left[\left(10-3^{2}\right)+9\right]-5$

(Solution on p. 210.)

(Solution on p. 210.)

For problems 16-20, find the prime factorization of each whole number. If the number is prime, write "prime."

Exercise 3.9.16

(Section 3.4) 18

Exercise 3.9.17

(Section 3.4) 68

Exercise 3.9.18

(Solution on p. 210.)

(Section 3.4) 142

Exercise 3.9.19

(Section 3.4) 151

Exercise 3.9.20

(Solution on p. 210.)

(Solution on p. 211.)

(Section 3.4) 468

(Solution on p. 211.)

(Solution on p. 211.)

For problems 21 and 22, find the greatest common factor.

Exercise 3.9.21

(Solution on p. 211.)

(Section 3.5) 200 and 36

Exercise 3.9.22

(Section 3.5) 900 and 135

Exercise 3.9.23

(Solution on p. 211.)

(Section 3.5) Write all the factors of 36.

Exercise 3.9.24

(Solution on p. 211.)

(Section 3.5) Write all the divisors of 18.

Exercise 3.9.25

(Section 3.5) Does 7 divide into $5^{2} \cdot 6^{3} \cdot 7^{4} \cdot 8$ ? Explain.

Exercise 3.9.26

(Section 3.5) Is 3 a factor of $2^{6} \cdot 3^{2} \cdot 5^{3} \cdot 4^{6}$ ? Explain.

Exercise 3.9.27

(Section 3.5) Does 13 divide into $11^{3} \cdot 12^{4} \cdot 15^{2}$ ? Explain.

(Solution on p. 211.)

(Solution on p. 211.)

(Solution on p. 211.)

(Solution on p. 211.)

For problems 28 and 29, find the least common multiple.

Exercise 3.9.28

(Solution on p. 211.)

(Section 3.6) 432 and 180

Exercise 3.9.29

(Section 3.6) 28, 40, and 95

(Solution on p. 211.) 


\section{Solutions to Exercises in Chapter 3}

Solution to Exercise 3.2.1 (p. 155)

$37^{2}$

Solution to Exercise 3.2.2 (p. 155)

$16^{5}$

Solution to Exercise 3.2.3 (p. 155) $9^{10}$

Solution to Exercise 3.2.4 (p. 155) $85 \cdot 85 \cdot 85$

Solution to Exercise 3.2.5 (p. 155)

$4 \cdot 4 \cdot 4 \cdot 4 \cdot 4 \cdot 4 \cdot 4$

Solution to Exercise 3.2.6 (p. 155)

$1,739 \cdot 1,739$

Solution to Exercise 3.2.7 (p. 157)

8

Solution to Exercise 3.2.8 (p. 157)

10

Solution to Exercise 3.2.9 (p. 157)

4

Solution to Exercise 3.2.10 (p. 157)

2

Solution to Exercise 3.2.11 (p. 158)

9

Solution to Exercise 3.2.12 (p. 158)

54

Solution to Exercise 3.2.13 (p. 158)

231

Solution to Exercise 3.2.14 (p. 158)

4

Solution to Exercise 3.2.15 (p. 158) $4^{2}$

Solution to Exercise 3.2.17 (p. 158)

$9^{4}$

Solution to Exercise 3.2.19 (p. 158) $826^{3}$

Solution to Exercise 3.2.21 (p. 158) $6^{85}$

Solution to Exercise 3.2.23 (p. 159) $1^{3008}$

Solution to Exercise 3.2.25 (p. 159)

$7 \cdot 7 \cdot 7 \cdot 7$

Solution to Exercise 3.2.27 (p. 159)

$117 \cdot 117 \cdot 117 \cdot 117 \cdot 117$

Solution to Exercise 3.2.29 (p. 159) $30 \cdot 30$

Solution to Exercise 3.2.31 (p. 159)

$4 \cdot 4=16$

Solution to Exercise 3.2.33 (p. 159)

$10 \cdot 10=100$

Solution to Exercise 3.2.35 (p. 159) $12 \cdot 12=144$ 
Solution to Exercise 3.2.37 (p. 159) $15 \cdot 15=225$

Solution to Exercise 3.2.39 (p. 159) $3 \cdot 3 \cdot 3 \cdot 3=81$

Solution to Exercise 3.2.41 (p. 159) $10 \cdot 10 \cdot 10=1,000$

Solution to Exercise 3.2.43 (p. 159) $8 \cdot 8 \cdot 8=512$

Solution to Exercise 3.2.45 (p. 160) $9 \cdot 9 \cdot 9=729$

Solution to Exercise 3.2.47 (p. 160) $7^{1}=7$

Solution to Exercise 3.2.49 (p. 160) $2 \cdot 2 \cdot 2 \cdot 2 \cdot 2 \cdot 2 \cdot 2=128$

Solution to Exercise 3.2.51 (p. 160) $8 \cdot 8 \cdot 8 \cdot 8=4,096$

Solution to Exercise 3.2.53 (p. 160) $6 \cdot 6 \cdot 6 \cdot 6 \cdot 6 \cdot 6 \cdot 6 \cdot 6 \cdot 6=10,077,696$

Solution to Exercise 3.2.55 (p. 160) $42 \cdot 42=1,764$

Solution to Exercise 3.2.57 (p. 160) $15 \cdot 15 \cdot 15 \cdot 15 \cdot 15=759,375$

Solution to Exercise 3.2.59 (p. 160) $816 \cdot 816=665,856$

Solution to Exercise 3.2.61 (p. 160) 4

Solution to Exercise 3.2.63 (p. 160) 8

Solution to Exercise 3.2.65 (p. 160) 12

Solution to Exercise 3.2.67 (p. 161) 15

Solution to Exercise 3.2.69 (p. 161) 2

Solution to Exercise 3.2.71 (p. 161) 6

Solution to Exercise 3.2.73 (p. 161) 20

Solution to Exercise 3.2.75 (p. 161) 100

Solution to Exercise 3.2.77 (p. 161) 60

Solution to Exercise 3.2.79 (p. 161) 34

Solution to Exercise 3.2.81 (p. 161) 4,158

Solution to Exercise 3.2 .83 (p. 161) 24

Solution to Exercise 3.2.85 (p. 161) 4 
Solution to Exercise 3.2.87 (p. 161) 5

Solution to Exercise 3.2.89 (p. 162)

81

Solution to Exercise 3.2.91 (p. 162)

8 is the multiplier; 4 is the multiplicand

Solution to Exercise 3.2.93 (p. 162)

Yes; 0

Solution to Exercise 3.3.1 (p. 163)

10

Solution to Exercise 3.3.2 (p. 163)

68

Solution to Exercise 3.3.3 (p. 163)

24

Solution to Exercise 3.3.4 (p. 163)

4

Solution to Exercise 3.3.5 (p. 163)

0

Solution to Exercise 3.3.6 (p. 163)

not possible (indeterminant)

Solution to Exercise 3.3.7 (p. 164)

54

Solution to Exercise 3.3.8 (p. 164)

23

Solution to Exercise 3.3.9 (p. 164)

4

Solution to Exercise 3.3.10 (p. 164)

93

Solution to Exercise 3.3.11 (p. 164)

74

Solution to Exercise 3.3.12 (p. 164)

27

Solution to Exercise 3.3.13 (p. 166)

33

Solution to Exercise 3.3.14 (p. 166) 57

Solution to Exercise 3.3.15 (p. 166) 0

Solution to Exercise 3.3.16 (p. 166)

52

Solution to Exercise 3.3.17 (p. 166)

3

Solution to Exercise 3.3.18 (p. 166)

125

Solution to Exercise 3.3.19 (p. 166)

7

Solution to Exercise 3.3.20 (p. 169) 13,499

Solution to Exercise 3.3.21 (p. 169) 6,070 
Solution to Exercise 3.3.22 (p. 170)

$1,107,495$

Solution to Exercise 3.3.23 (p. 170)

This number is too big for a nonscientific calculator. A scientific calculator will probably give you $2.217747109 \times 10^{11}$

Solution to Exercise 3.3.24 (p. 170)

26

Solution to Exercise 3.3.26 (p. 170)

46

Solution to Exercise 3.3.28 (p. 170)

1

Solution to Exercise 3.3.30 (p. 170)

0

Solution to Exercise 3.3.32 (p. 170) 3

Solution to Exercise 3.3.34 (p. 170)

26

Solution to Exercise 3.3.36 (p. 170)

97

Solution to Exercise 3.3.38 (p. 170)

29

Solution to Exercise 3.3.40 (p. 170)

1

Solution to Exercise 3.3.42 (p. 171)

0

Solution to Exercise 3.3.44 (p. 171)

90

Solution to Exercise 3.3.46 (p. 171)

508

Solution to Exercise 3.3.48 (p. 171)

19

Solution to Exercise 3.3.50 (p. 171)

144

Solution to Exercise 3.3.52 (p. 171)

1

Solution to Exercise 3.3.54 (p. 171)

52

Solution to Exercise 3.3.56 (p. 171)

25,001

Solution to Exercise 3.3.58 (p. 171)

$\frac{1}{25}$

Solution to Exercise 3.3.60 (p. 171)

14

Solution to Exercise 3.3.62 (p. 171)

0

Solution to Exercise 3.3.64 (p. 172) 152

Solution to Exercise 3.3.66 (p. 172)

$\frac{4}{5}$

Solution to Exercise 3.3.68 (p. 172)

$2,690,730$ 
Solution to Exercise 3.3.70 (p. 172)

1

Solution to Exercise 3.4.1 (p. 173)

$1,2,3,6$

Solution to Exercise 3.4.2 (p. 173)

$1,2,3,4,6,12$

Solution to Exercise 3.4.3 (p. 173)

$1,2,3,6,9,18$

Solution to Exercise 3.4.4 (p. 173)

1,5

Solution to Exercise 3.4.5 (p. 173)

$1,2,5,10$

Solution to Exercise 3.4.6 (p. 173)

$1,3,11,33$

Solution to Exercise 3.4.7 (p. 174)

1,19

Solution to Exercise 3.4.8 (p. 174)

prime

Solution to Exercise 3.4.9 (p. 174)

composite

Solution to Exercise 3.4.10 (p. 174)

composite

Solution to Exercise 3.4.11 (p. 174)

composite

Solution to Exercise 3.4.12 (p. 174)

prime

Solution to Exercise 3.4.13 (p. 174)

prime

Solution to Exercise 3.4.14 (p. 175)

prime

Solution to Exercise 3.4.15 (p. 175)

composite

Solution to Exercise 3.4.16 (p. 177)

$22=2 \cdot 11$

Solution to Exercise 3.4.17 (p. 177)

$40=2^{3} \cdot 5$

Solution to Exercise 3.4.18 (p. 177)

$48=2^{4} \cdot 3$

Solution to Exercise 3.4.19 (p. 177)

$63=3^{2} \cdot 7$

Solution to Exercise 3.4.20 (p. 177)

$945=3^{3} \cdot 5 \cdot 7$

Solution to Exercise 3.4.21 (p. 177)

$1617=3 \cdot 7^{2} \cdot 11$

Solution to Exercise 3.4.22 (p. 177)

17 is prime

Solution to Exercise 3.4.23 (p. 177)

61 is prime

Solution to Exercise 3.4.24 (p. 177)

2 
Solution to Exercise 3.4.26 (p. 178) 4

Solution to Exercise 3.4.28 (p. 178) 11

Solution to Exercise 3.4.30 (p. 178)

$3 \cdot 2$

Solution to Exercise 3.4.32 (p. 178)

$2 \cdot 3 \cdot 5$

Solution to Exercise 3.4.34 (p. 178) $1,2,4,8,16$

Solution to Exercise 3.4.36 (p. 178) $1,2,4,7,8,14,28,56$

Solution to Exercise 3.4.38 (p. 178) $1,2,4,5,10,11,20,22,44,55,110,220$ Solution to Exercise 3.4.40 (p. 178) $1,2,4,8,16,32$

Solution to Exercise 3.4.42 (p. 178) 1, 2, 71, 142

Solution to Exercise 3.4.44 (p. 178) prime

Solution to Exercise 3.4.46 (p. 179) composite

Solution to Exercise 3.4.48 (p. 179) prime

Solution to Exercise 3.4.50 (p. 179) prime

Solution to Exercise 3.4.52 (p. 179) prime

Solution to Exercise 3.4.54 (p. 179) composite $(5 \cdot 11)$

Solution to Exercise 3.4.56 (p. 179) composite

Solution to Exercise 3.4.58 (p. 179) composite

Solution to Exercise 3.4.60 (p. 179) composite $(11 \cdot 19)$

Solution to Exercise 3.4.62 (p. 179) composite

Solution to Exercise 3.4.64 (p. 179) $2 \cdot 13$

Solution to Exercise 3.4.66 (p. 179) $2 \cdot 3^{3}$

Solution to Exercise 3.4.68 (p. 180) $2^{3} \cdot 7$

Solution to Exercise 3.4.70 (p. 180) $2^{5} \cdot 3 \cdot 5$

Solution to Exercise 3.4.72 (p. 180) $3^{4} \cdot 5^{2}$

Solution to Exercise 3.4.74 (p. 180) 26,580 
Solution to Exercise 3.4.76 (p. 180)

true

Solution to Exercise 3.4.78 (p. 180)

14

Solution to Exercise 3.5.1 (p. 182)

12

Solution to Exercise 3.5.2 (p. 182)

24

Solution to Exercise 3.5.3 (p. 182)

10

Solution to Exercise 3.5.4 (p. 182)

3

Solution to Exercise 3.5.5 (p. 182)

30

Solution to Exercise 3.5.6 (p. 183)

2

Solution to Exercise 3.5.8 (p. 183)

4

Solution to Exercise 3.5.10 (p. 183)

4

Solution to Exercise 3.5.12 (p. 183)

5

Solution to Exercise 3.5.14 (p. 183) 33

Solution to Exercise 3.5.16 (p. 183) 9

Solution to Exercise 3.5.18 (p. 183)

11

Solution to Exercise 3.5.20 (p. 183)

3

Solution to Exercise 3.5.22 (p. 183)

7

Solution to Exercise 3.5.24 (p. 183)

25

Solution to Exercise 3.5.26 (p. 183)

2

Solution to Exercise 3.5.28 (p. 184)

11

Solution to Exercise 3.5.30 (p. 184)

1

Solution to Exercise 3.5.32 (p. 184)

1

Solution to Exercise 3.5.34 (p. 184)

53

Solution to Exercise 3.5.36 (p. 184)

$8^{6}=262,144$

Solution to Exercise 3.6.1 (p. 185)

$4,8,12,16,20$

Solution to Exercise 3.6.2 (p. 185)

$5,10,15,20,25$ 
Solution to Exercise 3.6.3 (p. 185)

$6,12,18,24,30$

Solution to Exercise 3.6.4 (p. 185)

$7,14,21,28,35$

Solution to Exercise 3.6.5 (p. 185)

$9,18,27,36,45$

Solution to Exercise 3.6.6 (p. 186)

$4,8,12,16,20$

Solution to Exercise 3.6.7 (p. 186)

12, 24, 36, 48, 60

Solution to Exercise 3.6.8 (p. 186)

10, 20, 30, 40, 50

Solution to Exercise 3.6.9 (p. 186)

$6,12,18,24,30$

Solution to Exercise 3.6.10 (p. 186)

$20,40,60,80,100$

Solution to Exercise 3.6.11 (p. 188)

540

Solution to Exercise 3.6.12 (p. 188)

28

Solution to Exercise 3.6.13 (p. 188)

126

Solution to Exercise 3.6.14 (p. 188)

1,960

Solution to Exercise 3.6.15 (p. 188)

$6,534,000$

Solution to Exercise 3.6.16 (p. 188)

24

Solution to Exercise 3.6.18 (p. 188)

40

Solution to Exercise 3.6.20 (p. 188)

12

Solution to Exercise 3.6.22 (p. 188)

18

Solution to Exercise 3.6.24 (p. 188)

30

Solution to Exercise 3.6.26 (p. 188)

12

Solution to Exercise 3.6.28 (p. 188)

63

Solution to Exercise 3.6.30 (p. 189)

72

Solution to Exercise 3.6.32 (p. 189)

720

Solution to Exercise 3.6.34 (p. 189)

120

Solution to Exercise 3.6.36 (p. 189)

216

Solution to Exercise 3.6.38 (p. 189)

144 
Solution to Exercise 3.6.40 (p. 189)

105

Solution to Exercise 3.6.42 (p. 189)

231

Solution to Exercise 3.6.44 (p. 189)

126

Solution to Exercise 3.6.46 (p. 189)

4,410

Solution to Exercise 3.6.48 (p. 189)

240

Solution to Exercise 3.6.50 (p. 189)

432

Solution to Exercise 3.6.52 (p. 189)

144

Solution to Exercise 3.6.54 (p. 190)

224

Solution to Exercise 3.6.56 (p. 190)

193,050

Solution to Exercise 3.6.58 (p. 190)

8

Solution to Exercise 3.6.60 (p. 190)

36

Solution to Exercise 3.6.62 (p. 190)

6,552

Solution to Exercise 3.6.64 (p. 190)

$84 \cdot 84 \cdot 84$

Solution to Exercise 3.8.1 (p. 193)

27

Solution to Exercise 3.8.3 (p. 193)

0

Solution to Exercise 3.8.5 (p. 193)

144

Solution to Exercise 3.8.7 (p. 194)

64

Solution to Exercise 3.8.9 (p. 194)

32

Solution to Exercise 3.8.11 (p. 194)

225

Solution to Exercise 3.8.13 (p. 194)

625

Solution to Exercise 3.8.15 (p. 194)

15

Solution to Exercise 3.8.17 (p. 194)

2

Solution to Exercise 3.8.19 (p. 194)

1

Solution to Exercise 3.8.21 (p. 194)

12

Solution to Exercise 3.8.23 (p. 194)

1 
Solution to Exercise 3.8.25 (p. 194)

2

Solution to Exercise 3.8.27 (p. 195)

0

Solution to Exercise 3.8.29 (p. 195)

325

Solution to Exercise 3.8.31 (p. 195)

64

Solution to Exercise 3.8.33 (p. 195)

$-3$

Solution to Exercise 3.8.35 (p. 195)

$-\frac{9}{57}$

Solution to Exercise 3.8.37 (p. 195)

146

Solution to Exercise 3.8.39 (p. 195) 5

Solution to Exercise 3.8.41 (p. 195)

The sum of square roots is not necessarily equal to the square root of the sum.

Solution to Exercise 3.8.43 (p. 195)

6

Solution to Exercise 3.8.45 (p. 195)

8

Solution to Exercise 3.8.47 (p. 196)

$1,2,3,6,9,18$

Solution to Exercise 3.8.49 (p. 196)

1,11

Solution to Exercise 3.8.51 (p. 196)

$1,3,17,51$,

Solution to Exercise 3.8.53 (p. 196)

1,2

Solution to Exercise 3.8.55 (p. 196)

$5 \cdot 11$

Solution to Exercise 3.8.57 (p. 196)

$2^{4} \cdot 5$

Solution to Exercise 3.8.59 (p. 196)

$2^{2} \cdot 5^{2} \cdot 7$

Solution to Exercise 3.8.61 (p. 196)

$2 \cdot 3 \cdot 269$

Solution to Exercise 3.8.63 (p. 196)

29 is a prime number

Solution to Exercise 3.8.65 (p. 197)

5

Solution to Exercise 3.8.67 (p. 197)

5

Solution to Exercise 3.8.69 (p. 197)

6

Solution to Exercise 3.8.71 (p. 197)

20

Solution to Exercise 3.8.73 (p. 197)

21 
Solution to Exercise 3.8.75 (p. 197)

11

Solution to Exercise 3.8.77 (p. 197)

42

Solution to Exercise 3.8.79 (p. 197)

180

Solution to Exercise 3.8.81 (p. 197)

120

Solution to Exercise 3.8.83 (p. 197)

79,380

Solution to Exercise 3.8.85 (p. 198)

450

Solution to Exercise 3.8.87 (p. 198)

$1,2,3,4,6,8,12,24$

Solution to Exercise 3.8.89 (p. 198)

$1,2,4,5,7,8,10,14,20,25,35,40,50,56,70,100,140,175,200,280,700,1,400$

Solution to Exercise 3.8.91 (p. 198)

yes

Solution to Exercise 3.9.1 (p. 198)

base; exponent

Solution to Exercise 3.9.2 (p. 198)

$12^{7}$

Solution to Exercise 3.9.3 (p. 198)

$9^{4}=9 \cdot 9 \cdot 9 \cdot 9=6,561$

Solution to Exercise 3.9.4 (p. 198)

64

Solution to Exercise 3.9.5 (p. 198)

1

Solution to Exercise 3.9.6 (p. 198)

0

Solution to Exercise 3.9.7 (p. 198)

64

Solution to Exercise 3.9.8 (p. 198)

7

Solution to Exercise 3.9.9 (p. 198)

3

Solution to Exercise 3.9.10 (p. 198)

1

Solution to Exercise 3.9.11 (p. 199)

20

Solution to Exercise 3.9.12 (p. 199)

127

Solution to Exercise 3.9.13 (p. 199)

24

Solution to Exercise 3.9.14 (p. 199)

8

Solution to Exercise 3.9.15 (p. 199)

5

Solution to Exercise 3.9.16 (p. 199)

$3^{2} \cdot 2$ 
Solution to Exercise 3.9.17 (p. 199) $2^{2} \cdot 17$

Solution to Exercise 3.9.18 (p. 199)

$2 \cdot 71$

Solution to Exercise 3.9.19 (p. 199)

prime

Solution to Exercise 3.9.20 (p. 199)

$2^{2} \cdot 3^{2} \cdot 13$

Solution to Exercise 3.9.21 (p. 199)

4

Solution to Exercise 3.9.22 (p. 199)

45

Solution to Exercise 3.9.23 (p. 199)

$1,2,3,4,6,9,12,18,36$

Solution to Exercise 3.9.24 (p. 199)

$1,2,3,6,9,18$

Solution to Exercise 3.9.25 (p. 199)

Yes, because one of the (prime) factors of the number is 7 .

Solution to Exercise 3.9.26 (p. 199)

Yes, because it is one of the factors of the number.

Solution to Exercise 3.9.27 (p. 199)

No, because the prime 13 is not a factor any of the listed factors of the number.

Solution to Exercise 3.9.28 (p. 199)

2,160

Solution to Exercise 3.9 .29 (p. 199)

5,320 


\section{Chapter 4}

\section{Introduction to Fractions and Multiplication and Division of Fractions}

\subsection{Objectives ${ }^{1}$}

After completing this chapter, you should

Fractions of Whole Numbers (Section 4.2)

- understand the concept of fractions of whole numbers

- be able to recognize the parts of a fraction

Proper Fractions, improper Fractions, and Mixed Numbers (Section 4.3)

- be able to distinguish between proper fractions, improper fractions, and mixed numbers

- be able to convert an improper fraction to a mixed number

- be able to convert a mixed number to an improper fraction

Equivalent Fractions, Reducing Fractions to Lowest Terms, and Raising Fractions to Higher Terms (Section 4.4)

- be able to recognize equivalent fractions

- be able to reduce a fraction to lowest terms

- be able to raise a fraction to higher terms

\section{Multiplication of Fractions (Section 4.5)}

- understand the concept of multiplication of fractions

- be able to multiply one fraction by another

- be able to multiply mixed numbers

- be able to find powers and roots of various fractions

\section{Division of Fractions (Section 4.6)}

- be able to determine the reciprocal of a number

- be able to divide one fraction by another

\section{Applications Involving Fractions (Section 4.7)}

- be able to solve missing product statements

- be able to solve missing factor statements

\footnotetext{
${ }^{1}$ This content is available online at $<$ http://cnx.org/content $/ \mathrm{m} 18892 / 1.3 />$.
} 


\subsection{Fractions of Whole Numbers ${ }^{2}$}

\subsubsection{Section Overview}

- More Numbers on the Number Line

- Fractions of Whole Numbers

- The Parts of a Fraction

- Reading and Writing Fractions

\subsubsection{More Numbers on the Number Line}

In Chapters Section 1.1, Section 2.1, and Section 3.1, we studied the whole numbers and methods of combining them. We noted that we could visually display the whole numbers by drawing a number line and placing closed circles at whole number locations.

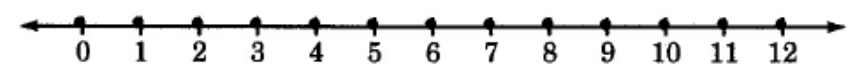

By observing this number line, we can see that the whole numbers do not account for every point on the line. What numbers, if any, can be associated with these points? In this section we will see that many of the points on the number line, including the points already associated with whole numbers, can be associated with numbers called fractions.

\subsubsection{Fractions of Whole Numbers}

\section{The Nature of the Positive Fractions}

We can extend our collection of numbers, which now contains only the whole numbers, by including fractions of whole numbers. We can determine the nature of these fractions using the number line.

If we place a pencil at some whole number and proceed to travel to the right to the next whole number, we see that our journey can be broken into different types of equal parts as shown in the following examples.

a. 1 part.

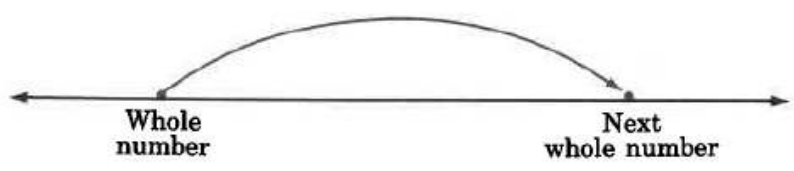

b. 2 equal parts.

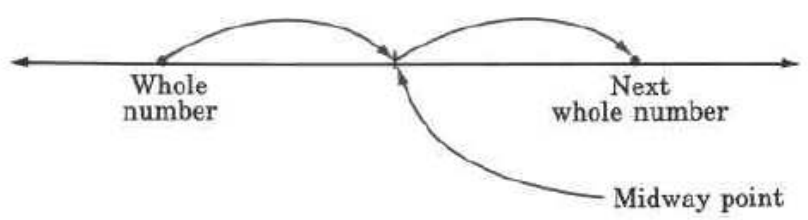

c. 3 equal parts.

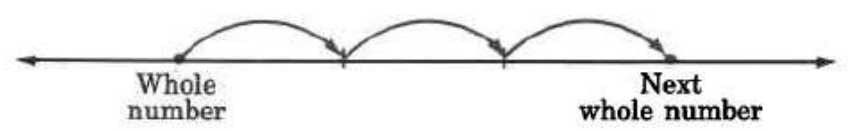

\footnotetext{
${ }^{2}$ This content is available online at $<$ http://cnx.org/content $/ \mathrm{m} 34908 / 1.2 />$.
} 
d. 4 equal parts.

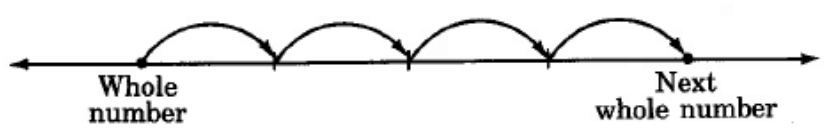

\section{The Latin Word Fractio}

Notice that the number of parts, 2, 3, and 4, that we are breaking the original quantity into is always a nonzero whole number. The idea of breaking up a whole quantity gives us the word fraction. The word fraction comes from the Latin word "fractio" which means a breaking, or fracture.

Suppose we break up the interval from some whole number to the next whole number into five equal parts.

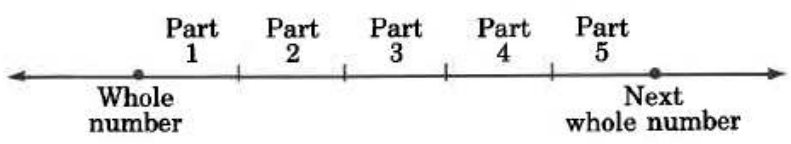

After starting to move from one whole number to the next, we decide to stop after covering only two parts. We have covered 2 parts of 5 equal parts. This situation is described by writing $\frac{2}{5}$.

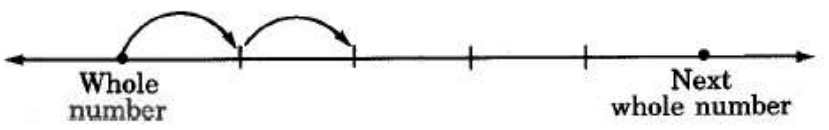

\section{Positive Fraction}

A number such as $\frac{2}{5}$ is called a positive fraction, or more simply, a fraction.

\subsubsection{The Parts of a Fraction}

A fraction has three parts.

\section{The fraction bar - .Fraction Bar}

The fraction bar serves as a grouping symbol. It separates a quantity into individual groups. These groups have names, as noted in 2 and 3 below.

2. The nonzero number below the fraction bar.Denominator

This number is called the denominator of the fraction, and it indicates the number of parts the whole quantity has been divided into. Notice that the denominator must be a nonzero whole number since the least number of parts any quantity can have is one.

3. The number above the fraction bar. Numerator

This number is called the numerator of the fraction, and it indicates how many of the specified parts are being considered. Notice that the numerator can be any whole number (including zero) since any number of the specified parts can be considered.

$$
\frac{\text { whole number }}{\text { nonzero whole number }} \leftrightarrow \frac{\text { numerator }}{\text { denominator }}
$$

\subsubsection{Sample Set A}

The diagrams in the following problems are illustrations of fractions.

\section{Example 4.1}




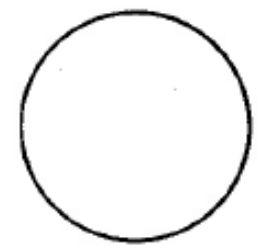

(a)

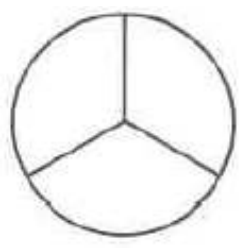

(b)

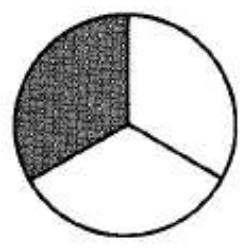

(c)

Diagrams 4.1: (a) A whole circle (b) The whole circle divided into 3 equal parts (c) 1 of the 3 equal parts

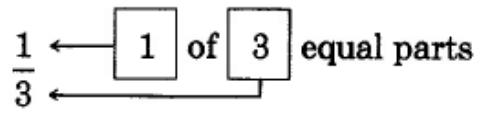

The fraction $\frac{1}{3}$ is read as "one third."

Example 4.2

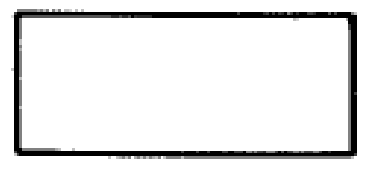

(a)

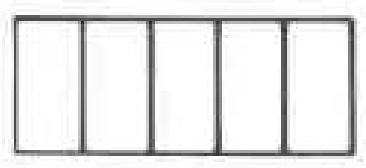

(b)

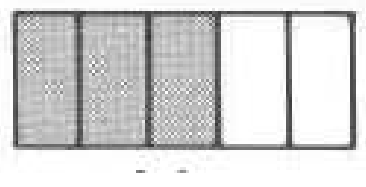

(c)

Figure 4.2: (a) A whole rectangle (b) The whole rectangle divided into 5 equal parts (c) 3 of the 5 equal parts

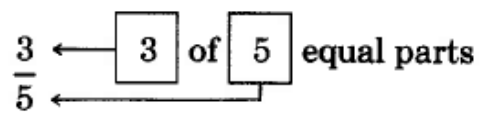

The fraction $\frac{3}{5}$ "is read as "three fifths."

Example 4.3 


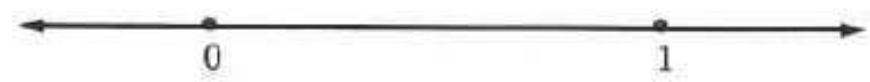

(a)

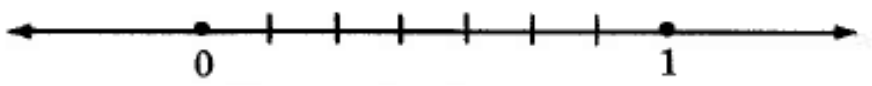

(b)

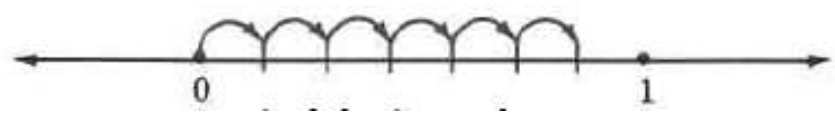

(c)

Figure 4.3: (a) The number line between 0 and 1 (b) The number line between 0 and 1 divided into 7 equal parts (c) 6 of the 7 equal parts

$\frac{6}{7} 6$ of the 7 equal parts

The fraction $\frac{6}{7}$ is read as "six sevenths."

Example 4.4

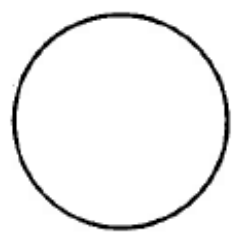

(a)

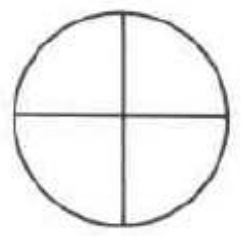

(b)

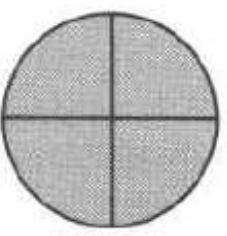

(c)

Figure 4.4: (a) A whole circle (b) The whole circle divided into 4 equal parts (c) 4 of the 4 equal parts

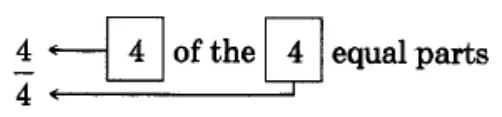

When the numerator and denominator are equal, the fraction represents the entire quantity, and its value is 1 .

$\frac{\text { nonzero whole number }}{\text { same nonzero whole number }}=1$

\subsubsection{Practice Set A}

Specify the numerator and denominator of the following fractions. 


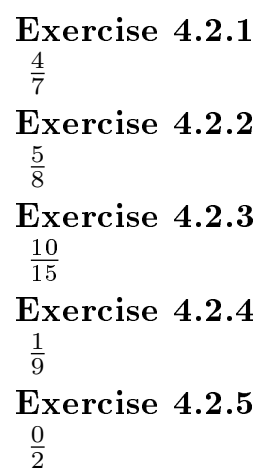

(Solution on p. 278.)

(Solution on p. 278.)

(Solution on p. 278.)

(Solution on p. 278.)

(Solution on p. 278.)

\subsubsection{Reading and Writing Fractions}

In order to properly translate fractions from word form to number form, or from number form to word form, it is necessary to understand the use of the hyphen.

\section{Use of the Hyphen}

One of the main uses of the hyphen is to tell the reader that two words not ordinarily joined are to be taken in combination as a unit. Hyphens are always used for numbers between and including 21 and 99 (except those ending in zero).

\subsubsection{Sample Set B}

Write each fraction using whole numbers.

\section{Example 4.5}

Fifty three-hundredths. The hyphen joins the words three and hundredths and tells us to consider them as a single unit. Therefore,

fifty three-hundredths translates as $\frac{50}{300}$

\section{Example 4.6}

Fifty-three hundredths. The hyphen joins the numbers fifty and three and tells us to consider them as a single unit. Therefore, fifty-three hundredths translates as $\frac{53}{100}$

\section{Example 4.7}

Four hundred seven-thousandths. The hyphen joins the words seven and thousandths and tells us to consider them as a single unit. Therefore, four hundred seven-thousandths translates as $\frac{400}{7,000}$

\section{Example 4.8}

Four hundred seven thousandths. The absence of hyphens indicates that the words seven and thousandths are to be considered individually.

four hundred seven thousandths translates as $\frac{407}{1000}$

Write each fraction using words.

\section{Example 4.9}

$\frac{21}{85}$ translates as twenty-one eighty-fifths.

\section{Example 4.10}

$\frac{200}{3,000}$ translates as two hundred three-thousandths. A hyphen is needed between the words three and thousandths to tell the reader that these words are to be considered as a single unit. 


\section{Example 4.11}

$\frac{203}{1,000}$ translates as two hundred three thousandths.

\subsubsection{Practice Set $B$}

Write the following fractions using whole numbers.

Exercise 4.2.6

(Solution on p. 278.)

one tenth

Exercise 4.2.7

(Solution on p. 278.)

eleven fourteenths

Exercise 4.2.8

(Solution on p. 278.)

sixteen thirty-fifths

Exercise 4.2.9

(Solution on p. 278.)

eight hundred seven-thousandths

Write the following using words.

Exercise 4.2.10

$\frac{3}{8}$

Exercise 4.2.11

$\frac{1}{10}$

Exercise 4.2.12

$\frac{3}{250}$

Exercise 4.2.13

$\frac{114}{3,190}$

Name the fraction that describes each shaded portion.

Exercise 4.2.14

(Solution on p. 278.)

(Solution on p. 278.)

(Solution on p. 278.)

(Solution on p. 278.)

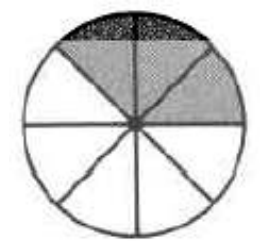

(Solution on p. 278.)

Exercise 4.2.15

(Solution on p. 278.)

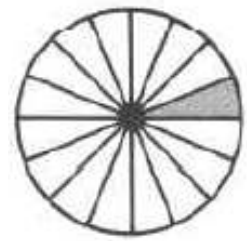

In the following 2 problems, state the numerator and denominator, and write each fraction in words.

Exercise 4.2.16

(Solution on p. 278.)

The number $\frac{5}{9}$ is used in converting from Fahrenheit to Celsius.

Exercise 4.2.17

A dime is $\frac{1}{10}$ of a dollar.

(Solution on p. 278.) 


\subsubsection{Exercises}

For the following 10 problems, specify the numerator and denominator in each fraction.

Exercise 4.2.18

(Solution on p. 278.)

$\frac{3}{4}$

Exercise 4.2.19

$\frac{9}{10}$

Exercise 4.2.20

$\frac{1}{5}$

(Solution on p. 278.)

Exercise 4.2.21

$\frac{5}{6}$

Exercise 4.2.22

$\frac{7}{7}$

Exercise 4.2.23

$\frac{4}{6}$

Exercise 4.2.24

$\frac{0}{12}$

(Solution on p. 278.)

Exercise 4.2.25

$\frac{25}{25}$

Exercise 4.2.26

$\frac{18}{1}$

(Solution on p. 278.)

Exercise 4.2.27

$\frac{0}{16}$

For the following 10 problems, write the fractions using whole numbers.

Exercise 4.2.28

four fifths

(Solution on p. 278.)

Exercise 4.2.29

two ninths

Exercise 4.2.30

fifteen twentieths

(Solution on p. 278.)

Exercise 4.2.31

forty-seven eighty-thirds

Exercise 4.2.32

(Solution on p. 278.)

ninety-one one hundred sevenths

(Solution on p. 278.)

Exercise 4.2.33

twenty-two four hundred elevenths

Exercise 4.2.34

(Solution on p. 279.)

six hundred five eight hundred thirty-fourths

Exercise 4.2.35

three thousand three forty-four ten-thousandths

Exercise 4.2.36

(Solution on p. 279.)

ninety-two one-millionths

Exercise 4.2.37

one three-billionths

For the following 10 problems, write the fractions using words. 
Exercise 4.2.38

(Solution on p. 279.)

$\frac{5}{9}$

Exercise 4.2.39 $\frac{6}{10}$

Exercise 4.2.40

(Solution on p. 279.)

$\frac{8}{15}$

Exercise 4.2.41

$\frac{10}{13}$

Exercise 4.2.42

$\frac{75}{100}$

(Solution on p. 279.)

Exercise 4.2.43

$\frac{86}{135}$

Exercise 4.2.44

$\frac{916}{1,014}$

Exercise 4.2.45

$\frac{501}{10,001}$

Exercise 4.2.46

$\frac{18}{31,608}$

Exercise 4.2.47

$\frac{1}{500,000}$

For the following 4 problems, name the fraction corresponding to the shaded portion.

Exercise 4.2.48

(Solution on p. 279.)

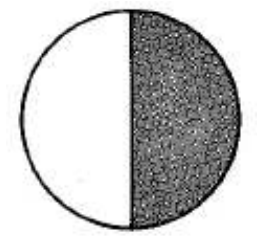

Exercise 4.2.49

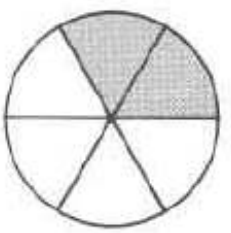

Exercise 4.2.50

(Solution on p. 279.)

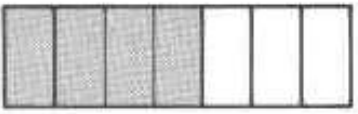

Exercise 4.2.51

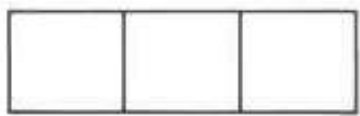

For the following 4 problems, shade the portion corresponding to the given fraction on the given figure. 
Exercise 4.2.52

(Solution on p. 279.)

$\frac{3}{5}$

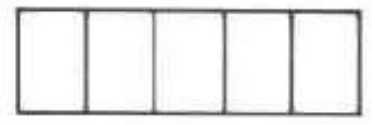

Exercise 4.2.53

$\frac{1}{8}$

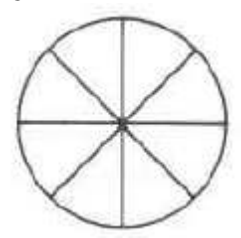

Exercise 4.2.54

$\frac{6}{6}$

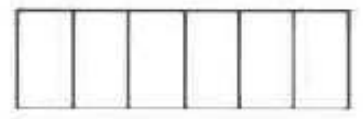

Exercise 4.2.55

$\frac{0}{3}$

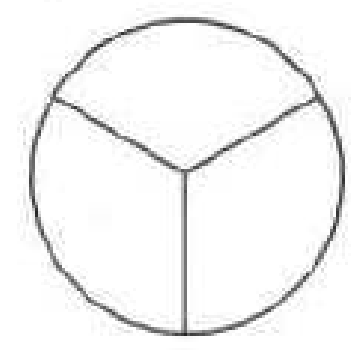

State the numerator and denominator and write in words each of the fractions appearing in the statements for the following 10 problems.

Exercise 4.2.56

A contractor is selling houses on $\frac{1}{4}$ acre lots.

(Solution on p. 279.)

\section{Exercise 4.2.57}

The fraction $\frac{22}{7}$ is sometimes used as an approximation to the number $\pi$. (The symbol is read "pi.")

Exercise 4.2.58

(Solution on p. 279.)

The fraction $\frac{4}{3}$ is used in finding the volume of a sphere.

(Solution on p. 279.)

Exercise 4.2.59

One inch is $\frac{1}{12}$ of a foot.

Exercise 4.2.60

(Solution on p. 279.)

About $\frac{2}{7}$ of the students in a college statistics class received a "B" in the course.

Exercise 4.2.61

The probability of randomly selecting a club when drawing one card from a standard deck of 52 cards is $\frac{13}{52}$. 
Exercise 4.2.62

(Solution on p. 279.)

In a box that contains eight computer chips, five are known to be good and three are known to be defective. If three chips are selected at random, the probability that all three are defective is $\frac{1}{56}$.

Exercise 4.2.63

In a room of 25 people, the probability that at least two people have the same birthdate (date and month, not year) is $\frac{569}{1000}$.

Exercise 4.2.64

The mean (average) of the numbers $21,25,43$, and 36 is $\frac{125}{4}$.

(Solution on p. 279.)

Exercise 4.2.65

If a rock falls from a height of 20 meters on Jupiter, the rock will be $\frac{32}{25}$ meters high after $\frac{6}{5}$ seconds.

\subsubsection{Exercises For Review}

Exercise 4.2.66

(Solution on p. 279.)

(Section 1.7) Use the numbers 3 and 11 to illustrate the commutative property of addition.

Exercise 4.2.67

(Section 2.4) Find the quotient. $676 \div 26$

Exercise 4.2.68

(Solution on p. 279.)

(Section 3.2 ) Write $7 \cdot 7 \cdot 7 \cdot 7 \cdot 7$ using exponents.

Exercise 4.2.69

(Section 3.3) Find the value of $\frac{8 \cdot(6+20)}{8}+\frac{3 \cdot(6+16)}{22}$.

Exercise 4.2.70

(Solution on p. 279.)

(Section 3.6) Find the least common multiple of 12, 16, and 18.

\subsection{Proper Fractions, Improper Fractions, and Mixed Numbers ${ }^{3}$}

\subsubsection{Section Overview}

- Positive Proper Fractions

- Positive Improper Fractions

- Positive Mixed Numbers

- Relating Positive Improper Fractions and Positive Mixed Numbers

- Converting an Improper Fraction to a Mixed Number

- Converting a Mixed Number to an Improper Fraction

Now that we know what positive fractions are, we consider three types of positive fractions: proper fractions, improper fractions, and mixed numbers.

\subsubsection{Positive Proper Fractions}

\section{Positive Proper Fraction}

Fractions in which the whole number in the numerator is strictly less than the whole number in the denominator are called positive proper fractions. On the number line, proper fractions are located in the interval from 0 to 1 . Positive proper fractions are always less than one.

\footnotetext{
${ }^{3}$ This content is available online at $<\mathrm{http}: / / \mathrm{cnx} . o r g /$ content $/ \mathrm{m} 34912 / 1.2 />$.
} 


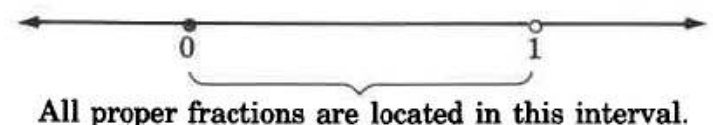

The closed circle at 0 indicates that 0 is included, while the open circle at 1 indicates that 1 is not included.

Some examples of positive proper fractions are

$\frac{1}{2}, \frac{3}{5}, \frac{20}{27}$, and $\frac{106}{255}$

Note that $1<2,3<5,20<27$, and $106<225$.

\subsubsection{Positive Improper Fractions}

Positive Improper Fractions

Fractions in which the whole number in the numerator is greater than or equal to the whole number in the denominator are called positive improper fractions. On the number line, improper fractions lie to the right of (and including) 1. Positive improper fractions are always greater than or equal to 1.

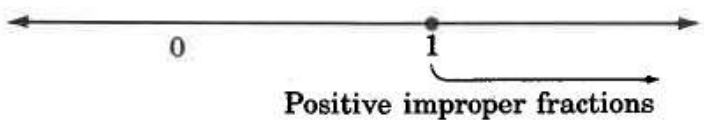

Some examples of positive improper fractions are

$\frac{3}{2}, \frac{8}{5}, \frac{4}{4}$, and $\frac{105}{16}$

Note that $3 \geq 2,8 \geq 5,4 \geq 4$, and $105 \geq 16$.

\subsubsection{Positive Mixed Numbers}

\section{Positive Mixed Numbers}

A number of the form

nonzero whole number + proper fraction

is called a positive mixed number. For example, $2 \frac{3}{5}$ is a mixed number. On the number line, mixed numbers are located in the interval to the right of (and including) 1. Mixed numbers are always greater than or equal to 1.

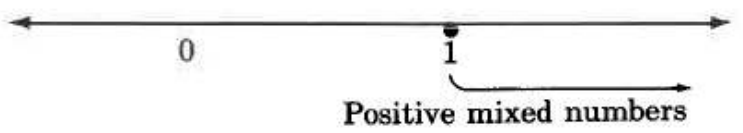

\subsubsection{Relating Positive Improper Fractions and Positive Mixed Numbers}

A relationship between improper fractions and mixed numbers is suggested by two facts. The first is that improper fractions and mixed numbers are located in the same interval on the number line. The second fact, that mixed numbers are the sum of a natural number and a fraction, can be seen by making the following observations.

Divide a whole quantity into 3 equal parts. 


\begin{tabular}{|l|l|l|}
\hline$\frac{1}{3}$ & $\frac{1}{3}$ & $\frac{1}{3}$ \\
\hline
\end{tabular}

Now, consider the following examples by observing the respective shaded areas.

\begin{tabular}{|l|l|l|}
\hline$\frac{1}{3}$ & $\frac{1}{3}$ & $\frac{1}{3}$ \\
\hline
\end{tabular}

In the shaded region, there are 2 one thirds, or $\frac{2}{3}$.

$2\left(\frac{1}{3}\right)=\frac{2}{3}$

\begin{tabular}{|l|l|l|}
\hline$\frac{1}{3}$ & $\frac{1}{3}$ & $\frac{1}{3}$ \\
\hline
\end{tabular}

There are 3 one thirds, or $\frac{3}{3}$, or 1 .

$3\left(\frac{1}{3}\right)=\frac{3}{3}$ or 1

Thus,

$\frac{3}{3}=1$

Improper fraction $=$ whole number.

\begin{tabular}{|l|l|l|}
\hline$\frac{1}{3}$ & $\frac{1}{3}$ & $\frac{1}{3}$ \\
\hline
\end{tabular}

\begin{tabular}{|l|l|l|}
\hline$\frac{1}{3}$ & $\frac{1}{3}$ & $\frac{1}{3}$ \\
\hline
\end{tabular}

There are 4 one thirds, or $\frac{4}{3}$, or 1 and $\frac{1}{3}$.

$4\left(\frac{1}{3}\right)=\frac{4}{3}$ or 1 and $\frac{1}{3}$

The terms 1 and $\frac{1}{3}$ can be represented as $1+\frac{1}{3}$ or $1 \frac{1}{3}$

Thus,

$\frac{4}{3}=1 \frac{1}{3}$.

Improper fraction $=$ mixed number.

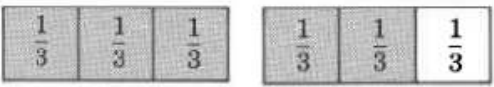

There are 5 one thirds, or $\frac{5}{3}$, or 1 and $\frac{2}{3}$.

$5\left(\frac{1}{3}\right)=\frac{5}{3}$ or 1 and $\frac{2}{3}$

The terms 1 and $\frac{2}{3}$ can be represented as $1+\frac{2}{3}$ or $1 \frac{2}{3}$.

Thus,

$\frac{5}{3}=1 \frac{2}{3}$.

Improper fraction $=$ mixed number.

\begin{tabular}{|l|l|l|}
\hline$\frac{1}{3}$ & $\frac{1}{3}$ & $\frac{1}{3}$ \\
\hline
\end{tabular}

\begin{tabular}{|l|l|l|}
\hline$\frac{1}{3}$ & $\frac{1}{3}$ & $\frac{1}{3}$ \\
\hline
\end{tabular}

There are 6 one thirds, or $\frac{6}{3}$, or 2 .

$6\left(\frac{1}{3}\right)=\frac{6}{3}=2$ 
Thus,

$\frac{6}{3}=2$

Improper fraction $=$ whole number.

The following important fact is illustrated in the preceding examples.

Mixed Number $=$ Natural Number + Proper Fraction

Mixed numbers are the sum of a natural number and a proper fraction. Mixed number $=$ (natural number)

+ (proper fraction)

For example $1 \frac{1}{3}$ can be expressed as $1+\frac{1}{3}$ The fraction $5 \frac{7}{8}$ can be expressed as $5+\frac{7}{8}$.

It is important to note that a number such as $5+\frac{7}{8}$ does not indicate multiplication. To indicate multiplication, we would need to use a multiplication symbol (such as $\cdot$ )

NOTE: $5 \frac{7}{8}$ means $5+\frac{7}{8}$ and not $5 \cdot \frac{7}{8}$, which means 5 times $\frac{7}{8}$ or 5 multiplied by $\frac{7}{8}$.

Thus, mixed numbers may be represented by improper fractions, and improper fractions may be represented by mixed numbers.

\subsubsection{Converting Improper Fractions to Mixed Numbers}

To understand how we might convert an improper fraction to a mixed number, let's consider the fraction, $\frac{4}{3}$.

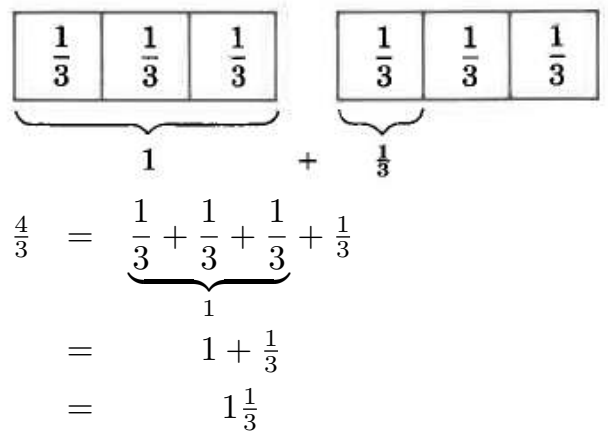

Thus, $\frac{4}{3}=1 \frac{1}{3}$.

We can illustrate a procedure for converting an improper fraction to a mixed number using this example. However, the conversion is more easily accomplished by dividing the numerator by the denominator and using the result to write the mixed number.

Converting an Improper Fraction to a Mixed Number

To convert an improper fraction to a mixed number, divide the numerator by the denominator.

1. The whole number part of the mixed number is the quotient.

2. The fractional part of the mixed number is the remainder written over the divisor (the denominator of the improper fraction). 


\subsubsection{Sample Set A}

Convert each improper fraction to its corresponding mixed number.

Example 4.12

$\frac{5}{3}$ Divide 5 by 3 .

$$
\begin{aligned}
& 1 \longleftarrow \text { whole number part } \\
& 3 \longdiv { 5 } \\
& \frac{3}{2} \longleftarrow \text { numerator of the fractional part } \\
& \text { denominator of the fractional part }
\end{aligned}
$$

The improper fraction $\frac{5}{3}=1 \frac{2}{3}$.

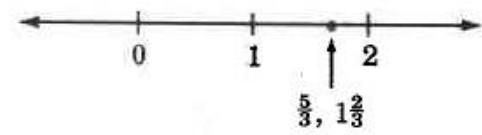

Example 4.13

$\frac{46}{9}$. Divide 46 by 9 .

$$
\begin{aligned}
& 9 \longdiv { 5 6 } \longleftarrow \text { whole number part } \\
& \uparrow \frac{45}{1} \longleftarrow \text { numerator of the fractional part } \\
& \text { denominator of the fractional part }
\end{aligned}
$$

The improper fraction $\frac{46}{9}=5 \frac{1}{9}$.

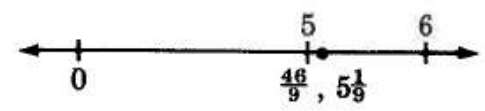

Example 4.14

$\frac{83}{11}$. Divide 83 by 11 .

$$
\begin{aligned}
& 1 1 \longdiv { 8 3 } \longleftarrow \text { whole number part } \\
& \frac{77}{6} \longleftarrow \text { numerator of the fractional part } \\
& \text { denominator of the fractional part }
\end{aligned}
$$

The improper fraction $\frac{83}{11}=7 \frac{6}{11}$.

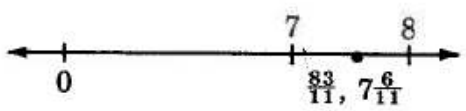

Example 4.15

$\frac{104}{4}$ Divide 104 by 4 . 


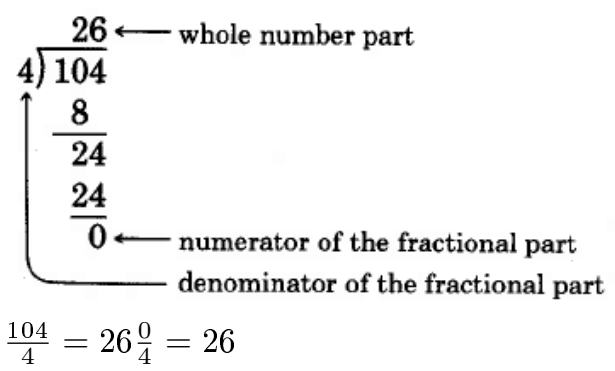

The improper fraction $\frac{104}{4}=26$.

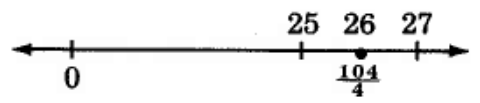

\subsubsection{Practice Set A}

Convert each improper fraction to its corresponding mixed number.

Exercise 4.3.1

$\frac{9}{2}$

Exercise 4.3.2

$\frac{11}{3}$

Exercise 4.3.3

$\frac{14}{11}$

Exercise 4.3.4

$\frac{31}{13}$

Exercise 4.3.5

$\frac{79}{4}$

Exercise 4.3.6

$\frac{496}{8}$
(Solution on p. 279.)

(Solution on p. 279.)

(Solution on p. 279.)

(Solution on p. 279.)

(Solution on p. 280.)

(Solution on p. 280.)

\subsubsection{Converting Mixed Numbers to Improper Fractions}

To understand how to convert a mixed number to an improper fraction, we'll recall mixed number $=($ natural number $)+($ proper fraction $)$

and consider the following diagram.

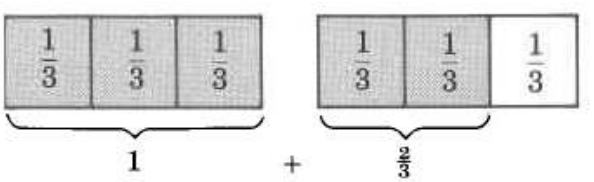




$$
1 \frac{2}{3}=\underbrace{\overbrace{\frac{1}{3}+\frac{1}{3}+\frac{1}{3}+\frac{1}{3}+\frac{1}{3}}^{\frac{2}{3}}}_{5 \cdot \frac{1}{3}=\frac{5}{3}}
$$

Recall that multiplication describes repeated addition.

Notice that $\frac{5}{3}$ can be obtained from $1 \frac{2}{3}$ using multiplication in the following way.

Multiply: $3 \cdot 1=3$

$$
1 \frac{2}{3}
$$

Add: $3+2=5$. Place the 5 over the $3: \frac{5}{3}$

The procedure for converting a mixed number to an improper fraction is illustrated in this example.

\section{Converting a Mixed Number to an Improper Fraction}

To convert a mixed number to an improper fraction,

1. Multiply the denominator of the fractional part of the mixed number by the whole number part.

2. To this product, add the numerator of the fractional part.

3. Place this result over the denominator of the fractional part.

\subsubsection{Sample Set B}

Convert each mixed number to an improper fraction.

\section{Example 4.16}

$5 \frac{7}{8}$

1. Multiply: $8 \cdot 5=40$.

2. Add: $40+7=47$.

3. Place 47 over $8: \frac{47}{8}$.

Thus, $5 \frac{7}{8}=\frac{47}{8}$.

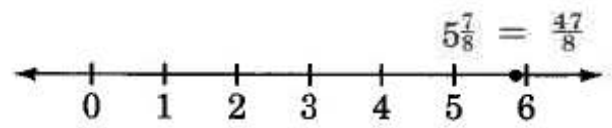

\section{Example 4.17}

$16 \frac{2}{3}$

1. Multiply: $3 \cdot 16=48$.

2. Add: $48+2=50$.

3. Place 50 over 3 : $\frac{50}{3}$

Thus, $16 \frac{2}{3}=\frac{50}{3}$ 


\subsubsection{Practice Set B}

Convert each mixed number to its corresponding improper fraction.

Exercise 4.3.7

$8 \frac{1}{4}$

Exercise 4.3.8

$5 \frac{3}{5}$

Exercise 4.3.9 $1 \frac{4}{15}$

Exercise 4.3.10 $12 \frac{2}{7}$
(Solution on p. 280.)

(Solution on p. 280.)

(Solution on p. 280.)

(Solution on p. 280.)

\subsubsection{Exercises}

For the following 15 problems, identify each expression as a proper fraction, an improper fraction, or a mixed number.

Exercise 4.3.11

(Solution on p. 280.)

$\frac{3}{2}$

Exercise 4.3.12

$\frac{4}{9}$

Exercise 4.3.13

$\frac{5}{7}$

(Solution on p. 280.)

Exercise 4.3.14

$\frac{1}{8}$

Exercise 4.3.15

$6 \frac{1}{4}$

Exercise 4.3.16

$\frac{11}{8}$

Exercise 4.3.17 $\frac{1,001}{12}$

Exercise 4.3.18 $191 \frac{4}{5}$

Exercise 4.3.19 $1 \frac{9}{13}$

(Solution on p. 280.)

Exercise 4.3.20 $31 \frac{6}{7}$

Exercise 4.3.21 $3 \frac{1}{40}$

(Solution on p. 280.)

(Solution on p. 280.)

Exercise 4.3.22

$\frac{55}{12}$

Exercise 4.3.23 $\frac{0}{9}$

Exercise 4.3.24 $\frac{8}{9}$

Exercise 4.3.25 $101 \frac{1}{11}$

(Solution on p. 280.)

(Solution on p. 280.)

(Solution on p. 280.) 
For the following 15 problems, convert each of the improper fractions to its corresponding mixed number.

Exercise 4.3.26

$\frac{11}{6}$

Exercise 4.3.27

$\frac{14}{3}$

(Solution on p. 280.)

Exercise 4.3.28 $\frac{25}{4}$

Exercise 4.3.29 $\frac{35}{4}$

Exercise 4.3.30 $\frac{71}{8}$

Exercise 4.3.31 $\frac{63}{7}$

(Solution on p. 280.)

Exercise 4.3.32 $\frac{121}{11}$

Exercise 4.3.33

(Solution on p. 280.) $\frac{165}{12}$

Exercise 4.3.34 $\frac{346}{15}$

Exercise 4.3.35 $\frac{5,000}{9}$

Exercise 4.3.36 $\frac{23}{5}$

Exercise 4.3.37 $\frac{73}{2}$

Exercise 4.3.38 $\frac{19}{2}$

Exercise 4.3.39 $\frac{316}{41}$

Exercise 4.3.40 $\frac{800}{3}$

(Solution on p. 280.)

(Solution on p. 280.)

(Solution on p. 280.)

(Solution on p. 280.)

For the following 15 problems, convert each of the mixed numbers to its corresponding improper fraction.

Exercise 4.3.41 $4 \frac{1}{8}$

(Solution on p. 280.)

Exercise 4.3.42 $1 \frac{5}{12}$

Exercise 4.3.43 $6 \frac{7}{9}$

(Solution on p. 280.)

Exercise 4.3.44 $15 \frac{1}{4}$

Exercise 4.3.45 $10 \frac{5}{11}$

(Solution on p. 280.)

Exercise 4.3.46 $15 \frac{3}{10}$

Exercise 4.3.47 $8 \frac{2}{3}$

(Solution on p. 280.) 
Exercise 4.3.48

$4 \frac{3}{4}$

Exercise 4.3.49

$21 \frac{2}{5}$

(Solution on p. 281.)

Exercise 4.3.50

$17 \frac{9}{10}$

Exercise 4.3.51 $9 \frac{20}{21}$

(Solution on p. 281.)

Exercise 4.3.52 $5 \frac{1}{16}$

Exercise 4.3.53 $90 \frac{1}{100}$

Exercise 4.3.54 $300 \frac{43}{1,000}$

Exercise 4.3.55 $19 \frac{7}{8}$

(Solution on p. 281.)

Exercise 4.3.56

Why does $0 \frac{4}{7}$ not qualify as a mixed number?

HinT: See the definition of a mixed number.

Exercise 4.3.57

Why does 5 qualify as a mixed number?

(Solution on p. 281.)

NOTE: See the definition of a mixed number.

\section{Calculator Problems}

For the following 8 problems, use a calculator to convert each mixed number to its corresponding improper fraction.

Exercise 4.3.58 $35 \frac{11}{12}$

Exercise 4.3.59 $27 \frac{5}{61}$

(Solution on p. 281.)

Exercise 4.3.60 $83 \frac{40}{41}$

Exercise 4.3.61 $105 \frac{21}{23}$

(Solution on p. 281.)

Exercise 4.3.62 $72 \frac{605}{606}$

Exercise 4.3.63 $816 \frac{19}{25}$

Exercise 4.3.64 $708 \frac{42}{51}$

Exercise 4.3.65 $6,012 \frac{4,216}{8,117}$

(Solution on p. 281.) 


\subsubsection{Exercises For Review}

Exercise 4.3.66

(Section 1.4) Round 2,614,000 to the nearest thousand.

Exercise 4.3.67

(Solution on p. 281.)

(Section 2.2) Find the product. 1,004 $\cdot 1,005$.

Exercise 4.3.68

(Section 2.5) Determine if 41,826 is divisible by 2 and 3 .

Exercise 4.3.69

(Solution on p. 281.)

(Section 3.6) Find the least common multiple of 28 and 36.

Exercise 4.3.70

(Section 4.2) Specify the numerator and denominator of the fraction $\frac{12}{19}$.

\subsection{Equivalent Fractions, Reducing Fractions to Lowest Terms, and Raising Fractions to Higher Terms ${ }^{4}$}

\subsubsection{Section Overview}

- Equivalent Fractions

- Reducing Fractions to Lowest Terms

- Raising Fractions to Higher Terms

\subsubsection{Equivalent Fractions}

Let's examine the following two diagrams.

\begin{tabular}{|c|c|c|c|c|c|}
\hline \multicolumn{1}{|c|}{$\frac{1}{3}$} & \multicolumn{2}{|c|}{$\frac{1}{3}$} & \multicolumn{2}{|c|}{$\frac{1}{3}$} \\
\hline$\frac{1}{6}$ & $\frac{1}{6}$ & $\frac{1}{6}$ & $\frac{1}{6}$ & $\frac{1}{6}$ & $\frac{1}{6}$ \\
\hline
\end{tabular}

$\frac{2}{3}$ of the whole is shaded.

$\frac{4}{6}$ of the whole is shaded.

Notice that both $\frac{2}{3}$ and $\frac{4}{6}$ represent the same part of the whole, that is, they represent the same number.

Equivalent Fractions

Fractions that have the same value are called equivalent fractions. Equivalent fractions may look different, but they are still the same point on the number line.

There is an interesting property that equivalent fractions satisfy.

$\frac{2}{3} \times \frac{4}{6}$

A Test for Equivalent Fractions Using the Cross Product These pairs of products are called cross products.

\section{$2 \cdot 6 \stackrel{?}{=} 3 \cdot 4$}

$12 \unrhd 12$

\footnotetext{
${ }^{4}$ This content is available online at $<$ http://cnx.org/content $/ \mathrm{m} 34927 / 1.3 />$.
} 
If the cross products are equal, the fractions are equivalent. If the cross products are not equal, the fractions are not equivalent.

Thus, $\frac{2}{3}$ and $\frac{4}{6}$ are equivalent, that is, $\frac{2}{3}=\frac{4}{6}$.

\subsubsection{Sample Set A}

Determine if the following pairs of fractions are equivalent.

\section{Example 4.18}

$\frac{3}{4}$ and $\frac{6}{8}$. Test for equality of the cross products.

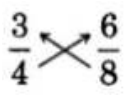

\section{$3 \cdot 8 \stackrel{?}{=} 6 \cdot 4$}

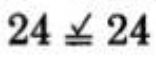

The cross products are equals.

The fractions $\frac{3}{4}$ and $\frac{6}{8}$ are equivalent, so $\frac{3}{4}=\frac{6}{8}$.

Example 4.19

$\frac{3}{8}$ and $\frac{9}{16}$. Test for equality of the cross products.

$\frac{3}{8} \times \frac{9}{16}$

$3 \cdot 16 \stackrel{?}{=} 9 \cdot 8$

$48 \neq 72$

The cross products are not equal.

The fractions $\frac{3}{8}$ and $\frac{9}{16}$ are not equivalent.

\subsubsection{Practice Set A}

Determine if the pairs of fractions are equivalent.

Exercise 4.4.1

$\frac{1}{2}, \frac{3}{6}$

Exercise 4.4.2

$\frac{4}{5}, \frac{12}{15}$

Exercise 4.4.3

$\frac{2}{3}, \frac{8}{15}$

Exercise 4.4.4

$\frac{1}{8}, \frac{5}{40}$

Exercise 4.4.5

$\frac{3}{12}, \frac{1}{4}$

(Solution on p. 281.)

(Solution on p. 281.)

(Solution on p. 281.)

(Solution on p. 281.)

(Solution on p. 281.)

\subsubsection{Reducing Fractions to Lowest Terms}

It is often very useful to convert one fraction to an equivalent fraction that has reduced values in the numerator and denominator. We can suggest a method for doing so by considering the equivalent fractions $\frac{9}{15}$ and $\frac{3}{5}$. First, divide both the numerator and denominator of $\frac{9}{15}$ by 3 . The fractions $\frac{9}{15}$ and $\frac{3}{5}$ are equivalent. 
(Can you prove this?) So, $\frac{9}{15}=\frac{3}{5}$. We wish to convert $\frac{9}{15}$ to $\frac{3}{5}$. Now divide the numerator and denominator of $\frac{9}{15}$ by 3 , and see what happens.

$\frac{9 \div 3}{15 \div 3}=\frac{3}{5}$

The fraction $\frac{9}{15}$ is converted to $\frac{3}{5}$.

A natural question is "Why did we choose to divide by 3 ?" Notice that

$\frac{9}{15}=\frac{3 \cdot 3}{5 \cdot 3}$

We can see that the factor 3 is common to both the numerator and denominator.

\section{Reducing a Fraction}

From these observations we can suggest the following method for converting one fraction to an equivalent fraction that has reduced values in the numerator and denominator. The method is called reducing a fraction.

A fraction can be reduced by dividing both the numerator and denominator by the same nonzero whole number.

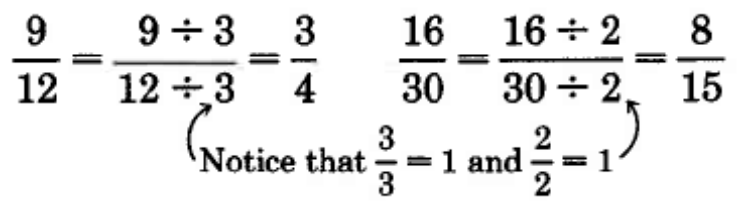

Consider the collection of equivalent fractions

$\frac{5}{20}, \frac{4}{16}, \frac{3}{12}, \frac{2}{8}, \frac{1}{4}$

\section{Reduced to Lowest Terms}

Notice that each of the first four fractions can be reduced to the last fraction, $\frac{1}{4}$, by dividing both the numerator and denominator by, respectively, 5, 4, 3, and 2. When a fraction is converted to the fraction that has the smallest numerator and denominator in its collection of equivalent fractions, it is said to be reduced to lowest terms. The fractions $\frac{1}{4}, \frac{3}{8}, \frac{2}{5}$, and $\frac{7}{10}$ are all reduced to lowest terms.

Observe a very important property of a fraction that has been reduced to lowest terms. The only whole number that divides both the numerator and denominator without a remainder is the number 1 . When 1 is the only whole number that divides two whole numbers, the two whole numbers are said to be relatively prime.

\section{Relatively Prime}

A fraction is reduced to lowest terms if its numerator and denominator are relatively prime.

\subsubsection{Methods of Reducing Fractions to Lowest Terms}

\section{Method 1: Dividing Out Common Primes}

1. Write the numerator and denominator as a product of primes.

2. Divide the numerator and denominator by each of the common prime factors. We often indicate this division by drawing a slanted line through each divided out factor. This process is also called cancelling common factors.

3. The product of the remaining factors in the numerator and the product of remaining factors of the denominator are relatively prime, and this fraction is reduced to lowest terms. 


\subsection{Sample Set B}

Reduce each fraction to lowest terms.

\section{Example 4.20}

$\frac{6}{18}=\frac{\frac{1}{22} \cdot \frac{1}{3}}{\frac{2}{2} \cdot \overline{3} \cdot 3}=\frac{1}{3} 1$ and 3 are relatively prime.

\section{Example 4.21}

$\frac{16}{20}=\frac{\frac{1}{2 \cdot} \cdot \frac{1}{2} \cdot 2 \cdot 2}{\overline{\frac{2}{2} \cdot() 2} \cdot 5}=\frac{4}{5} 4$ and 5 are relatively prime.

\section{Example 4.22}

$\frac{56}{104}=\frac{\frac{1}{22} \cdot \frac{1}{22} \cdot \frac{1}{22} \cdot 7}{\frac{2}{2} \cdot \overline{2} \cdot \overline{2} \cdot \frac{1}{1} \cdot 13}=\frac{7}{13} 7$ and 13 are relatively prime (and also truly prime)

Example 4.23

$$
\frac{315}{336}=\frac{\frac{1}{3 \cdot} \cdot 3 \cdot 5 \cdot \frac{1}{7}}{2 \cdot 2 \cdot 2 \cdot 2 \cdot \overline{3} \cdot \overline{1} \cdot \overline{1} \cdot}=\frac{15}{16} \quad 15 \text { and } 16 \text { are relatively prime. }
$$

Example 4.24

$\frac{8}{15}=\frac{2 \cdot 2 \cdot 2}{3 \cdot 5}$ No common prime factors, so 8 and 15 are relatively prime.

The fraction $\frac{8}{15}$ is reduced to lowest terms.

\subsection{Practice Set B}

Reduce each fraction to lowest terms.

Exercise 4.4.6

(Solution on p. 281.)

$\frac{4}{8}$

Exercise 4.4.7

$\frac{6}{15}$

Exercise 4.4.8

$\frac{6}{48}$

Exercise 4.4.9

$\frac{21}{48}$

Exercise 4.4.10

$\frac{72}{42}$

Exercise 4.4.11

$\frac{135}{243}$

(Solution on p. 281.)

(Solution on p. 281.)

(Solution on p. 281.)

(Solution on p. 281.)

(Solution on p. 281.)

\section{Method 2: Dividing Out Common Factors}

1. Mentally divide the numerator and the denominator by a factor that is common to each. Write the quotient above the original number.

2. Continue this process until the numerator and denominator are relatively prime. 


\subsection{Sample Set C}

Reduce each fraction to lowest terms.

\section{Example 4.25}

$\frac{25}{30} .5$ divides into both 25 and 30 .

$\frac{\frac{5}{25}}{\frac{25}{30}}=\frac{5}{6} 5$ and 6 are relatively prime.

\section{Example 4.26}

$\frac{18}{24}$. Both numbers are even so we can divide by 2 .

$\frac{\frac{9}{\frac{18}{224}}}{\frac{12}{12}}$ Now, both 9 and 12 are divisible by 3 .

$\frac{\frac{\frac{3}{99}}{\frac{18}{\partial 24}}}{\frac{\sqrt{12}}{4}}=\frac{3}{4} 3$ and 4 are relatively prime.

\section{Example 4.27}

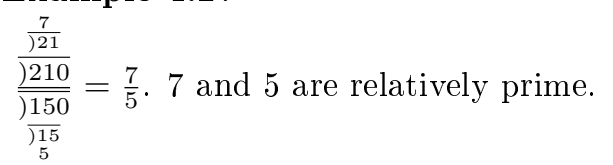

\section{Example 4.28}

$\frac{36}{96}=\frac{18}{48}=\frac{9}{24}=\frac{3}{8} .3$ and 8 are relatively prime.

\subsection{Practice Set C}

Reduce each fraction to lowest terms.

Exercise 4.4.12

$\frac{12}{16}$

Exercise 4.4.13 $\frac{9}{24}$

Exercise 4.4.14

$\frac{21}{84}$

Exercise 4.4.15

$\frac{48}{64}$

Exercise 4.4.16

$\frac{63}{81}$

Exercise 4.4.17

$\frac{150}{240}$

(Solution on p. 281.)

(Solution on p. 281.)

(Solution on p. 281.)

(Solution on p. 282.)

(Solution on p. 282.)

(Solution on p. 282.) 


\subsubsection{Raising Fractions to Higher Terms}

Equally as important as reducing fractions is raising fractions to higher terms. Raising a fraction to higher terms is the process of constructing an equivalent fraction that has higher values in the numerator and denominator than the original fraction.

The fractions $\frac{3}{5}$ and $\frac{9}{15}$ are equivalent, that is, $\frac{3}{5}=\frac{9}{15}$. Notice also,

$\frac{3 \cdot 3}{5 \cdot 3}=\frac{9}{15}$

Notice that $\frac{3}{3}=1$ and that $\frac{3}{5} \cdot 1=\frac{3}{5}$. We are not changing the value of $\frac{3}{5}$.

From these observations we can suggest the following method for converting one fraction to an equivalent fraction that has higher values in the numerator and denominator. This method is called raising a fraction to higher terms.

Raising a Fraction to Higher Terms

A fraction can be raised to an equivalent fraction that has higher terms in the numerator and denominator by multiplying both the numerator and denominator by the same nonzero whole number.

The fraction $\frac{3}{4}$ can be raised to $\frac{24}{32}$ by multiplying both the numerator and denominator by 8 .

$$
\frac{3}{4}=\frac{3 \cdot 8}{4 \cdot 8}=\frac{24}{32} \text { Notice that } \frac{8}{8}=1 .
$$

Most often, we will want to convert a given fraction to an equivalent fraction with a higher specified denominator. For example, we may wish to convert $\frac{5}{8}$ to an equivalent fraction that has denominator 32 , that is,

$\frac{5}{8}=\frac{?}{32}$

This is possible to do because we know the process. We must multiply both the numerator and denominator of $\frac{5}{8}$ by the same nonzero whole number in order to 8 obtain an equivalent fraction.

We have some information. The denominator 8 was raised to 32 by multiplying it by some nonzero whole number. Division will give us the proper factor. Divide the original denominator into the new denominator.

$32 \div 8=4$

Now, multiply the numerator 5 by 4 .

$5 \cdot 4=20$

Thus,

$\frac{5}{8}=\frac{5 \cdot 4}{8 \cdot 4}=\frac{20}{32}$

So,

$\frac{5}{8}=\frac{20}{32}$

\subsubsection{Sample Set D}

Determine the missing numerator or denominator. 
Example 4.29

$\frac{3}{7}=\frac{?}{35}$. Divide the original denominator into the new denominator.

$35 \div 7=5$ The quotient is 5 . Multiply the original numerator by 5 .

$\frac{3}{7}=\frac{3 \cdot 5}{7 \cdot 5}=\frac{15}{35}$ The missing numerator is 15 .

Example 4.30

$\frac{5}{6}=\frac{45}{?}$. Divide the original numerator into the new numerator.

$45 \div 5=9$ The quotient is 9 . Multiply the original denominator by 9 .

$\frac{5}{6}=\frac{5 \cdot 9}{6 \cdot 9}=\frac{45}{54}$ The missing denominator is 45 .

\subsubsection{Practice Set D}

Determine the missing numerator or denominator.

Exercise 4.4.18

$\frac{4}{5}=\frac{?}{40}$

Exercise 4.4.19

$\frac{3}{7}=\frac{?}{28}$

Exercise 4.4.20

$\frac{1}{6}=\frac{?}{24}$

Exercise 4.4.21

$\frac{3}{10}=\frac{45}{?}$

Exercise 4.4.22

$\frac{8}{15}=\frac{?}{165}$

(Solution on p. 282.)

(Solution on p. 282.)

(Solution on p. 282.)

(Solution on p. 282.)

(Solution on p. 282.)

\subsubsection{Exercises}

For the following problems, determine if the pairs of fractions are equivalent.

Exercise 4.4.23

(Solution on p. 282.)

$\frac{1}{2}, \frac{5}{10}$

Exercise 4.4.24

$\frac{2}{3}, \frac{8}{12}$

Exercise 4.4.25

$\frac{5}{12}, \frac{10}{24}$

(Solution on p. 282.)

Exercise 4.4.26 $\frac{1}{2}, \frac{3}{6}$

Exercise 4.4.27

$\frac{3}{5}, \frac{12}{15}$

Exercise 4.4.28

$\frac{1}{6}, \frac{7}{42}$

Exercise 4.4.29

$\frac{16}{25}, \frac{49}{75}$

Exercise 4.4.30

$\frac{5}{28}, \frac{20}{112}$

(Solution on p. 282.)

(Solution on p. 282.) 
Exercise 4.4.31 $\frac{3}{10}, \frac{36}{110}$

Exercise 4.4.32 $\frac{6}{10}, \frac{18}{32}$

Exercise 4.4.33 $\frac{5}{8}, \frac{15}{24}$

Exercise 4.4.34 $\frac{10}{16}, \frac{15}{24}$

Exercise 4.4.35 $\frac{4}{5}, \frac{3}{4}$

Exercise 4.4.36 $\frac{5}{7}, \frac{15}{21}$

Exercise 4.4.37 $\frac{9}{11}, \frac{11}{9}$
(Solution on p. 282.)

(Solution on p. 282.)

(Solution on p. 282.)

(Solution on p. 282.)

For the following problems, determine the missing numerator or denominator.

Exercise 4.4.38 $\frac{1}{3}=\frac{?}{12}$

Exercise 4.4.39 $\frac{1}{5}=\frac{?}{30}$

(Solution on p. 282.)

Exercise 4.4.40 $\frac{2}{3}=\frac{?}{9}$

Exercise 4.4.41 $\frac{1}{5}=\frac{?}{30}$

(Solution on p. 282.)

Exercise 4.4.42 $\frac{2}{3}=\frac{?}{9}$

Exercise 4.4.43 $\frac{3}{4}=\frac{?}{16}$

Exercise 4.4.44 $\frac{5}{6}=\frac{?}{18}$

Exercise 4.4.45 $\frac{4}{5}=\frac{?}{25}$

(Solution on p. 282.)

Exercise 4.4.46 $\frac{1}{2}=\frac{4}{?}$

Exercise 4.4.47 $\frac{9}{25}=\frac{27}{?}$

(Solution on p. 282.)

Exercise 4.4.48 $\frac{3}{2}=\frac{18}{?}$

Exercise 4.4.49 $\frac{5}{3}=\frac{80}{?}$

Exercise 4.4.50 $\frac{1}{8}=\frac{3}{?}$

Exercise 4.4.51 $\frac{4}{5}=\frac{?}{100}$

(Solution on p. 282.)

Exercise 4.4.52 $\frac{1}{2}=\frac{25}{?}$ 
Exercise 4.4.53

(Solution on p. 282.)

$\frac{3}{16}=\frac{?}{96}$

Exercise 4.4.54

$\frac{15}{16}=\frac{225}{?}$

Exercise 4.4.55

$\frac{11}{12}=\frac{?}{168}$

(Solution on p. 282.)

Exercise 4.4.56

$\frac{9}{13}=\frac{?}{286}$

Exercise 4.4.57

$\frac{32}{33}=\frac{?}{1518}$

Exercise 4.4.58

$\frac{19}{20}=\frac{1045}{?}$

Exercise 4.4.59 $\frac{37}{50}=\frac{1369}{?}$

(Solution on p. 283.)

(Solution on p. 283.)

For the following problems, reduce, if possible, each of the fractions to lowest terms.

Exercise 4.4.60 $\frac{6}{8}$

Exercise 4.4.61

(Solution on p. 283.) $\frac{8}{10}$

Exercise 4.4.62 $\frac{5}{10}$

Exercise 4.4.63

(Solution on p. 283.) $\frac{6}{14}$

Exercise 4.4.64 $\frac{3}{12}$

Exercise 4.4.65 $\frac{4}{14}$

(Solution on p. 283.)

Exercise 4.4.66

$\frac{1}{6}$

Exercise 4.4.67

$\frac{4}{6}$

(Solution on p. 283.)

Exercise 4.4.68

$\frac{18}{14}$

Exercise 4.4.69 $\frac{20}{8}$

(Solution on p. 283.)

Exercise 4.4.70

$\frac{4}{6}$

Exercise 4.4.71 $\frac{10}{6}$

(Solution on p. 283.)

Exercise 4.4.72 $\frac{6}{14}$

Exercise 4.4.73 $\frac{14}{6}$

Exercise 4.4.74 $\frac{10}{12}$ 
Exercise 4.4.75 $\frac{16}{70}$

Exercise 4.4.76 $\frac{40}{60}$

Exercise 4.4.77 $\frac{20}{12}$

Exercise 4.4.78 $\frac{32}{28}$

Exercise 4.4.79 $\frac{36}{10}$

Exercise 4.4.80 $\frac{36}{60}$

Exercise 4.4.81 $\frac{12}{18}$

Exercise 4.4.82 $\frac{18}{27}$

Exercise 4.4.83 $\frac{18}{24}$

Exercise 4.4.84 $\frac{32}{40}$

Exercise 4.4.85 $\frac{11}{22}$

Exercise 4.4.86 $\frac{27}{81}$

Exercise 4.4.87 $\frac{17}{51}$

Exercise 4.4.88 $\frac{16}{42}$

Exercise 4.4.89 $\frac{39}{13}$

Exercise 4.4.90 $\frac{44}{11}$

Exercise 4.4.91 $\frac{66}{33}$

Exercise 4.4.92 $\frac{15}{1}$

Exercise 4.4.93 $\frac{15}{16}$

Exercise 4.4.94 $\frac{15}{40}$

Exercise 4.4.95 $\frac{36}{100}$

Exercise 4.4.96 $\frac{45}{32}$

Exercise 4.4.97 $\frac{30}{75}$
(Solution on p. 283.)

(Solution on p. 283.)

(Solution on p. 283.)

(Solution on p. 283.)

(Solution on p. 283.)

(Solution on p. 283.)

(Solution on p. 283.)

(Solution on p. 283.)

(Solution on p. 283.)

(Solution on p. 283.)

(Solution on p. 283.)

(Solution on p. 283.) 
Exercise 4.4.98

$\frac{121}{132}$

Exercise 4.4.99

$\frac{72}{64}$

(Solution on p. 283.)

Exercise 4.4.100

$\frac{30}{105}$

Exercise 4.4.101

$\frac{46}{60}$

(Solution on p. 283.)

Exercise 4.4.102

$\frac{75}{45}$

Exercise 4.4.103

$\frac{40}{18}$

Exercise 4.4.104 $\frac{108}{76}$

Exercise 4.4.105 $\frac{7}{21}$

Exercise 4.4.106 $\frac{6}{51}$

Exercise 4.4.107 $\frac{51}{12}$

Exercise 4.4.108 $\frac{8}{100}$

Exercise 4.4.109

$\frac{51}{54}$

Exercise 4.4.110

A ream of paper contains 500 sheets. What fraction of a ream of paper is 200 sheets? Be sure to reduce.

Exercise 4.4.111

(Solution on p. 284.)

There are 24 hours in a day. What fraction of a day is 14 hours?

(Solution on p. 283.)

(Solution on p. 283.)

(Solution on p. 284.)

Exercise 4.4.112

A full box contains 80 calculators. How many calculators are in $\frac{1}{4}$ of a box?

Exercise 4.4.113

There are 48 plants per flat. How many plants are there in $\frac{1}{3}$ of a flat?

(Solution on p. 284.)

Exercise 4.4.114

A person making $\$ 18,000$ per year must pay $\$ 3,960$ in income tax. What fraction of this person's yearly salary goes to the IRS?

For the following problems, find the mistake.

Exercise 4.4.115

(Solution on p. 284.)

$\frac{3}{24}=\frac{\overline{3}}{\overline{3} \cdot 8}=\frac{0}{8}=0$

Exercise 4.4.116

$\frac{8}{10}=\frac{\overline{2}+6}{\overline{2}+8}=\frac{6}{8}=\frac{3}{4}$

Exercise 4.4.117

(Solution on p. 284.)

$\frac{7}{15}=\frac{\overline{7}}{\overline{7}+8}=\frac{1}{8}$

Exercise 4.4.118

$\frac{6}{7}=\frac{\overline{5}+1}{\overline{5}+2}=\frac{1}{2}$ 
Exercise 4.4.119

(Solution on p. 284.)

$\frac{\overline{9}}{\overline{) 9}}=\frac{0}{0}=0$

\subsubsection{Exercises for Review}

Exercise 4.4.120

(Section 1.4) Round 816 to the nearest thousand.

Exercise 4.4.121

(Solution on p. 284.)

(Section 2.3) Perform the division: $0 \div 6$.

Exercise 4.4.122

(Section 3.4) Find all the factors of 24.

Exercise 4.4.123

(Solution on p. 284.)

(Section 3.5) Find the greatest common factor of 12 and 18.

Exercise 4.4.124

(Section 4.3) Convert $\frac{15}{8}$ to a mixed number.

\subsection{Multiplication of Fractions ${ }^{5}$}

\subsubsection{Section Overview}

- Fractions of Fractions

- Multiplication of Fractions

- Multiplication of Fractions by Dividing Out Common Factors

- Multiplication of Mixed Numbers

- Powers and Roots of Fractions

\subsubsection{Fractions of Fractions}

We know that a fraction represents a part of a whole quantity. For example, two fifths of one unit can be represented by

\begin{tabular}{|l|l|l|l|l|}
\hline$\frac{1}{5}$ & $\frac{1}{5}$ & $\frac{1}{5}$ & $\frac{1}{5}$ & $\frac{1}{5}$ \\
\hline
\end{tabular}

$\frac{2}{5}$ of the whole is shaded.

A natural question is, what is a fractional part of a fractional quantity, or, what is a fraction of a fraction? For example, what $\frac{2}{3}$ of $\frac{1}{2}$ ?

We can suggest an answer to this question by using a picture to examine $\frac{2}{3}$ of $\frac{1}{2}$.

First, let's represent $\frac{1}{2}$.

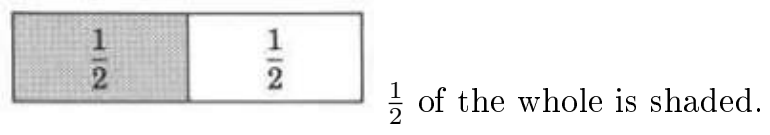

Then divide each of the $\frac{1}{2}$ parts into 3 equal parts.

\footnotetext{
${ }^{5}$ This content is available online at $<$ http://cnx.org/content $/ \mathrm{m} 34928 / 1.2 />$.
} 


\begin{tabular}{|c|c|}
\hline$\frac{1}{6}$ & $\frac{1}{6}$ \\
\hline$\frac{1}{6}$ & $\frac{1}{6}$ \\
\hline$\frac{1}{6}$ & $\frac{1}{6}$ \\
\hline
\end{tabular}$\underbrace{\frac{1}{2}}_{\frac{1}{2}}$

Each part is $\frac{1}{6}$ of the whole.

Now we'll take $\frac{2}{3}$ of the $\frac{1}{2}$ unit.

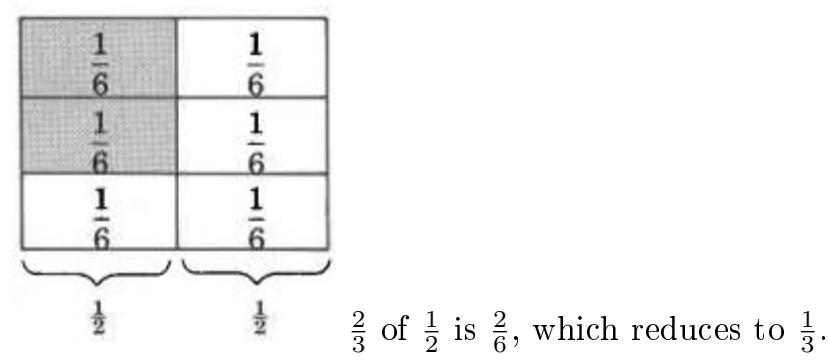

\subsubsection{Multiplication of Fractions}

Now we ask, what arithmetic operation $(+,-, \times, \div)$ will produce $\frac{2}{6}$ from $\frac{2}{3}$ of $\frac{1}{2}$ ?

Notice that, if in the fractions $\frac{2}{3}$ and $\frac{1}{2}$, we multiply the numerators together and the denominators together, we get precisely $\frac{2}{6}$.

$\frac{2 \cdot 1}{3 \cdot 2}=\frac{2}{6}$

This reduces to $\frac{1}{3}$ as before.

Using this observation, we can suggest the following:

1. The word "of" translates to the arithmetic operation "times."

2. To multiply two or more fractions, multiply the numerators together and then multiply the denominators together. Reduce if necessary.

$\frac{\text { numerator } 1}{\text { denominator } 1} \cdot \frac{\text { numerator } 2}{\text { denominator } 2}=\frac{\text { numerator } 1}{\text { denominator } 1} \cdot \frac{\text { numerator } 2}{\text { denominator } 2}$

\subsubsection{Sample Set A}

Perform the following multiplications.

\section{Example 4.31}

$$
\begin{aligned}
& \frac{3}{4} \cdot \frac{1}{6}=\frac{3 \cdot 1}{4 \cdot 6}=\frac{3}{24} \quad \text { Now, reduce. } \\
= & \frac{\frac{1}{3}}{\frac{3}{24}}=\frac{1}{8}
\end{aligned}
$$

Thus

$$
\frac{3}{4} \cdot \frac{1}{6}=\frac{1}{8}
$$

This means that $\frac{3}{4}$ of $\frac{1}{6}$ is $\frac{1}{8}$, that is, $\frac{3}{4}$ of $\frac{1}{6}$ of a unit is $\frac{1}{8}$ of the original unit. 


\section{Example 4.32}

$\frac{3}{8} \cdot 4$. Write 4 as a fraction by writing $\frac{4}{1}$

$\frac{3}{8} \cdot \frac{4}{1}=\frac{3 \cdot 4}{8 \cdot 1}=\frac{12}{8}=\frac{\frac{3}{12}}{\overline{18}}=\frac{3}{2}$

$\frac{3}{8} \cdot 4=\frac{3}{2}$

This means that $\frac{3}{8}$ of 4 whole units is $\frac{3}{2}$ of one whole unit.

Example 4.33

$\frac{2}{5} \cdot \frac{5}{8} \cdot \frac{1}{4}=\frac{2 \cdot 5 \cdot 1}{5 \cdot 8 \cdot 4}=\frac{\frac{1}{10}}{\frac{\frac{10}{160}}{16}}=\frac{1}{16}$

This means that $\frac{2}{5}$ of $\frac{5}{8}$ of $\frac{1}{4}$ of a whole unit is $\frac{1}{16}$ of the original unit.

\subsubsection{Practice Set A}

Perform the following multiplications.

Exercise 4.5.1

(Solution on p. 284.)

$$
\frac{2}{5} \cdot \frac{1}{6}
$$

Exercise 4.5.2

$$
\frac{1}{4} \cdot \frac{8}{9}
$$

Exercise 4.5.3

$\frac{4}{9} \cdot \frac{15}{16}$

Exercise 4.5.4

$$
\left(\frac{2}{3}\right)\left(\frac{2}{3}\right)
$$

Exercise 4.5.5

$$
\left(\frac{7}{4}\right)\left(\frac{8}{5}\right)
$$

Exercise 4.5.6

$$
\frac{5}{6} \cdot \frac{7}{8}
$$

Exercise 4.5.7

$$
\frac{2}{3} \cdot 5
$$

Exercise 4.5.8

$\left(\frac{3}{4}\right)(10)$

Exercise 4.5.9

$$
\frac{3}{4} \cdot \frac{8}{9} \cdot \frac{5}{12}
$$

(Solution on p. 284.)

(Solution on p. 284.)

(Solution on p. 284.)

(Solution on p. 284.)

(Solution on p. 284.)

(Solution on p. 284.)

(Solution on p. 284.)

(Solution on p. 284.)

\subsubsection{Multiplying Fractions by Dividing Out Common Factors}

We have seen that to multiply two fractions together, we multiply numerators together, then denominators together, then reduce to lowest terms, if necessary. The reduction can be tedious if the numbers in the fractions are large. For example,

$\frac{9}{16} \cdot \frac{10}{21}=\frac{9 \cdot 10}{16 \cdot 21}=\frac{90}{336}=\frac{45}{168}=\frac{15}{28}$

We avoid the process of reducing if we divide out common factors before we multiply. 
$\frac{9}{16} \cdot \frac{10}{21}=\frac{\frac{3}{29}}{\frac{16}{16}} \cdot \frac{\frac{5}{10}}{\frac{10}{21}}=\frac{3 \cdot 5}{8 \cdot 7}=\frac{15}{56}$

Divide 3 into 9 and 21, and divide 2 into 10 and 16. The product is a fraction that is reduced to lowest terms.

\section{The Process of Multiplication by Dividing Out Common Factors}

To multiply fractions by dividing out common factors, divide out factors that are common to both a numerator and a denominator. The factor being divided out can appear in any numerator and any denominator.

\subsubsection{Sample Set B}

Perform the following multiplications.

$$
\begin{aligned}
& \text { Example } 4.34 \\
& \frac{4}{5} \cdot \frac{5}{6} \\
& \frac{2}{\frac{24}{55}} \cdot \frac{\frac{1}{5}}{\frac{j}{3}}=\frac{2 \cdot 1}{1 \cdot 3}=\frac{2}{3}
\end{aligned}
$$

Divide 4 and 6 by 2

Divide 5 and 5 by 5

$$
\begin{aligned}
& \text { Example } 4.35 \\
& \frac{8}{12} \cdot \frac{8}{10} \\
& \frac{\frac{4}{8}}{\frac{8}{12}} \cdot \frac{\frac{2}{8}}{\frac{78}{10}}=\frac{4 \cdot 2}{3 \cdot 5}=\frac{8}{15}
\end{aligned}
$$

Divide 8 and 10 by 2 .

Divide 8 and 12 by 4 .

\section{Example 4.36}

$$
8 \cdot \frac{5}{12}=\frac{\frac{2}{8}}{1} \cdot \frac{5}{\frac{5}{32}}=\frac{2 \cdot 5}{1 \cdot 3}=\frac{10}{3}
$$

\section{Example 4.37}

$$
\begin{aligned}
& \frac{35}{18} \cdot \frac{63}{105} \\
& \frac{\frac{1}{77}}{\frac{\frac{75}{38}}{\frac{36}{2}} \frac{\frac{7}{63}}{\frac{705}{21}}}=\frac{1 \cdot 7}{2 \cdot 3}=\frac{7}{6}
\end{aligned}
$$

\section{Example 4.38}

$$
\begin{aligned}
& \frac{13}{9} \cdot \frac{6}{39} \cdot \frac{1}{12} \\
& \frac{1}{9} \cdot \frac{\frac{1}{\frac{12}{13}}}{\frac{\frac{\partial 6}{39}}{\frac{39}{3}}} \cdot \frac{1}{\frac{12}{12}}=\frac{1 \cdot 1 \cdot 1}{9 \cdot 1 \cdot 6}=\frac{1}{54}
\end{aligned}
$$




\subsubsection{Practice Set B}

Perform the following multiplications.

Exercise 4.5.10

$\frac{2}{3} \cdot \frac{7}{8}$

Exercise 4.5.11

$\frac{25}{12} \cdot \frac{10}{45}$

Exercise 4.5.12

$\frac{40}{48} \cdot \frac{72}{90}$

Exercise 4.5.13

$7 \cdot \frac{2}{49}$

Exercise 4.5.14

(Solution on p. 284.)

(Solution on p. 284.)

(Solution on p. 284.)

(Solution on p. 284.)

$12 \cdot \frac{3}{8}$

Exercise 4.5.15

$\left(\frac{13}{7}\right)\left(\frac{14}{26}\right)$

Exercise 4.5.16

$\frac{16}{10} \cdot \frac{22}{6} \cdot \frac{21}{44}$

(Solution on p. 284.)

(Solution on p. 284.)

(Solution on p. 284.)

\subsubsection{Multiplication of Mixed Numbers}

\section{Multiplying Mixed Numbers}

To perform a multiplication in which there are mixed numbers, it is convenient to first convert each mixed number to an improper fraction, then multiply.

\subsubsection{Sample Set C}

Perform the following multiplications. Convert improper fractions to mixed numbers.

Example 4.39

$1 \frac{1}{8} \cdot 4 \frac{2}{3}$

Convert each mixed number to an improper fraction.

$1 \frac{1}{8}=\frac{8 \cdot 1+1}{8}=\frac{9}{8}$

$4 \frac{2}{3}=\frac{4 \cdot 3+2}{3}=\frac{14}{3}$

$\frac{\frac{3}{9}}{\frac{9}{88}} \cdot \frac{\frac{7}{14}}{\frac{14}{13}}=\frac{3 \cdot 7}{4 \cdot 1}=\frac{21}{4}=5 \frac{1}{4}$

Example 4.40

$16 \cdot 8 \frac{1}{5}$

Convert $8 \frac{1}{5}$ to an improper fraction.

$8 \frac{1}{5}=\frac{5 \cdot 8+1}{5}=\frac{41}{5}$

$\frac{16}{1} \cdot \frac{41}{5}$.

There are no common factors to divide out.

$\frac{16}{1} \cdot \frac{41}{5}=\frac{16 \cdot 41}{1 \cdot 5}=\frac{656}{5}=131 \frac{1}{5}$ 


\section{Example 4.41}

$9 \frac{1}{6} \cdot 12 \frac{3}{5}$

Convert to improper fractions.

$9 \frac{1}{6}=\frac{6 \cdot 9+1}{6}=\frac{55}{6}$
$12 \frac{3}{5}=\frac{5 \cdot 12+3}{5}=\frac{63}{5}$
$\frac{11}{\frac{55}{56}} \cdot \frac{\frac{21}{663}}{\overline{5}}=\frac{11 \cdot 21}{2 \cdot 1}=\frac{231}{2}=115 \frac{1}{2}$

Example 4.42

$$
\begin{aligned}
\frac{11}{8} \cdot 4 \frac{1}{2} \cdot 3 \frac{1}{8} & =\quad \frac{11}{8} \cdot \frac{\frac{3}{9}}{\frac{79}{2}} \cdot \frac{\frac{5}{10}}{\frac{10}{3}} \\
& =\frac{11 \cdot 3 \cdot 5}{8 \cdot 1 \cdot 1}=\frac{165}{8}=20 \frac{5}{8}
\end{aligned}
$$

\subsubsection{Practice Set C}

Perform the following multiplications. Convert improper fractions to mixed numbers.

Exercise 4.5.17 $2 \frac{2}{3} \cdot 2 \frac{1}{4}$

Exercise 4.5.18 $6 \frac{2}{3} \cdot 3 \frac{3}{10}$

Exercise 4.5.19 $7 \frac{1}{8} \cdot 12$

Exercise 4.5.20

(Solution on p. 285.)

(Solution on p. 285.)

(Solution on p. 285.)

(Solution on p. 285.) $2 \frac{2}{5} \cdot 3 \frac{3}{4} \cdot 3 \frac{1}{3}$

\subsubsection{Powers and Roots of Fractions}

\subsubsection{Sample Set D}

Find the value of each of the following.

Example 4.43

$\left(\frac{1}{6}\right)^{2}=\frac{1}{6} \cdot \frac{1}{6}=\frac{1 \cdot 1}{6 \cdot 6}=\frac{1}{36}$

Example 4.44

$\sqrt{\frac{9}{100}}$. We're looking for a number, call it ?, such that when it is squared, $\frac{9}{100}$ is produced.

$(?)^{2}=\frac{9}{100}$

We know that

$3^{2}=9$ and $10^{2}=100$

We'll try $\frac{3}{10}$. Since

$\left(\frac{3}{10}\right)^{2}=\frac{3}{10} \cdot \frac{3}{10}=\frac{3 \cdot 3}{10 \cdot 10}=\frac{9}{100}$ 
$\sqrt{\frac{9}{100}}=\frac{3}{10}$

Example 4.45

$4 \frac{2}{5} \cdot \sqrt{\frac{100}{121}}$

$\frac{\frac{2}{22}}{\overline{15}} \cdot \frac{\frac{2}{\frac{10}{10}}}{\underset{1}{\overline{11}}}=\frac{2 \cdot 2}{1 \cdot 1}=\frac{4}{1}=4$

$4 \frac{2}{5} \cdot \sqrt{\frac{100}{121}}=4$

\subsubsection{Practice Set D}

Find the value of each of the following.

Exercise 4.5.21

$\left(\frac{1}{8}\right)^{2}$

Exercise 4.5.22 $\left(\frac{3}{10}\right)^{2}$

Exercise 4.5.23

(Solution on p. 285.)

$\sqrt{\frac{4}{9}}$

Exercise 4.5.24

$\sqrt{\frac{1}{4}}$

Exercise 4.5.25

$\frac{3}{8} \cdot \sqrt{\frac{1}{9}}$

Exercise 4.5.26

$9 \frac{1}{3} \cdot \sqrt{\frac{81}{100}}$

Exercise 4.5.27

$2 \frac{8}{13} \cdot \sqrt{\frac{169}{16}}$

(Solution on p. 285.)

(Solution on p. 285.)

(Solution on p. 285.)

(Solution on p. 285.)

(Solution on p. 285.)

(Solution on p. 285.)

\subsubsection{Exercises}

For the following six problems, use the diagrams to find each of the following parts. Use multiplication to verify your result.

Exercise 4.5.28

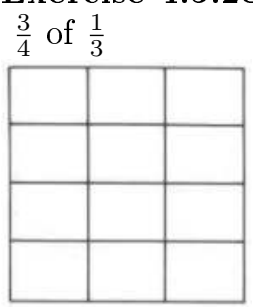

(Solution on p. 285.)

Exercise 4.5.29

$\frac{2}{3}$ of $\frac{3}{5}$ 


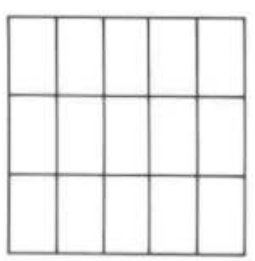

Exercise 4.5.30

(Solution on p. 285.) $\frac{2}{7}$ of $\frac{7}{8}$

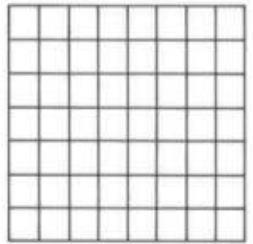

Exercise 4.5.31

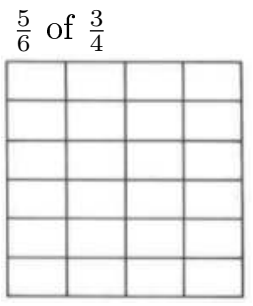

Exercise 4.5.32

$\frac{1}{8}$ of $\frac{1}{8}$

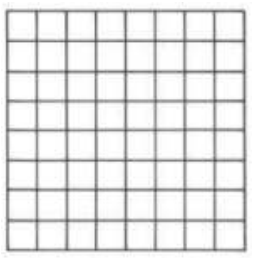

(Solution on p. 285.)

Exercise 4.5.33

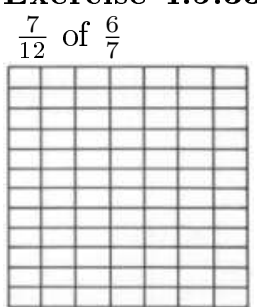

For the following problems, find each part without using a diagram.

Exercise 4.5.34

(Solution on p. 285.)

$\frac{1}{2}$ of $\frac{4}{5}$

Exercise 4.5.35

$\frac{3}{5}$ of $\frac{5}{12}$

Exercise 4.5.36

$\frac{1}{4}$ of $\frac{8}{9}$

Exercise 4.5.37

$\frac{3}{16}$ of $\frac{12}{15}$

(Solution on p. 285.) 
Exercise 4.5.38

(Solution on p. 286.)

$\frac{2}{9}$ of $\frac{6}{5}$

Exercise 4.5.39

$\frac{1}{8}$ of $\frac{3}{8}$

Exercise 4.5.40

(Solution on p. 286.)

$\frac{2}{3}$ of $\frac{9}{10}$

Exercise 4.5.41

$\frac{18}{19}$ of $\frac{38}{54}$

Exercise 4.5.42

$\frac{5}{6}$ of $2 \frac{2}{5}$

(Solution on p. 286.)

Exercise 4.5.43

$\frac{3}{4}$ of $3 \frac{3}{5}$

Exercise 4.5.44

$\frac{3}{2}$ of $2 \frac{2}{9}$

(Solution on p. 286.)

Exercise 4.5.45

$\frac{15}{4}$ of $4 \frac{4}{5}$

Exercise 4.5.46

$5 \frac{1}{3}$ of $9 \frac{3}{4}$

(Solution on p. 286.)

Exercise 4.5.47

$1 \frac{13}{15}$ of $8 \frac{3}{4}$

Exercise 4.5.48

$\frac{8}{9}$ of $\frac{3}{4}$ of $\frac{2}{3}$

Exercise 4.5.49

$\frac{1}{6}$ of $\frac{12}{13}$ of $\frac{26}{36}$

Exercise 4.5.50

$\frac{1}{2}$ of $\frac{1}{3}$ of $\frac{1}{4}$

Exercise 4.5.51

$1 \frac{3}{7}$ of $5 \frac{1}{5}$ of $8 \frac{1}{3}$

Exercise 4.5.52

$2 \frac{4}{5}$ of $5 \frac{5}{6}$ of $7 \frac{5}{7}$

(Solution on p. 286.)

(Solution on p. 286.)

For the following problems, find the products. Be sure to reduce.

Exercise 4.5.53

$\frac{1}{3} \cdot \frac{2}{3}$

Exercise 4.5.54

$\frac{1}{2} \cdot \frac{1}{2}$

Exercise 4.5.55

$\frac{3}{4} \cdot \frac{3}{8}$

Exercise 4.5.56

$\frac{2}{5} \cdot \frac{5}{6}$

Exercise 4.5.57

$\frac{3}{8} \cdot \frac{8}{9}$

Exercise 4.5.58

(Solution on p. 286.)

$\frac{5}{6} \cdot \frac{14}{15}$

(Solution on p. 286.)

Exercise 4.5.59

$\frac{4}{7} \cdot \frac{7}{4}$ 
Exercise 4.5.60

(Solution on p. 286.)

$\frac{3}{11} \cdot \frac{11}{3}$

Exercise 4.5.61

$\frac{9}{16} \cdot \frac{20}{27}$

Exercise 4.5.62

(Solution on p. 286.)

$\frac{35}{36} \cdot \frac{48}{55}$

Exercise 4.5.63

$\frac{21}{25} \cdot \frac{15}{14}$

Exercise 4.5.64

$\frac{76}{99} \cdot \frac{66}{38}$

Exercise 4.5.65

$\frac{3}{7} \cdot \frac{14}{18} \cdot \frac{6}{2}$

Exercise 4.5.66

$\frac{4}{15} \cdot \frac{10}{3} \cdot \frac{27}{2}$

Exercise 4.5.67

$\frac{14}{15} \cdot \frac{21}{28} \cdot \frac{45}{7}$

Exercise 4.5.68

$\frac{8}{3} \cdot \frac{15}{4} \cdot \frac{16}{21}$

Exercise 4.5.69

$\frac{18}{14} \cdot \frac{21}{35} \cdot \frac{36}{7}$

Exercise 4.5.70

$\frac{3}{5} \cdot 20$

Exercise 4.5.71

$\frac{8}{9} \cdot 18$

Exercise 4.5.72

$\frac{6}{11} \cdot 33$

Exercise 4.5.73

$\frac{18}{19} \cdot 38$

Exercise 4.5.74

$\frac{5}{6} \cdot 10$

Exercise 4.5.75

$\frac{1}{9} \cdot 3$

Exercise 4.5.76 $5 \cdot \frac{3}{8}$

Exercise 4.5.77 $16 \cdot \frac{1}{4}$

Exercise 4.5.78 $\frac{2}{3} \cdot 12 \cdot \frac{3}{4}$

Exercise 4.5.79

$\frac{3}{8} \cdot 24 \cdot \frac{2}{3}$

Exercise 4.5.80

$\frac{5}{18} \cdot 10 \cdot \frac{2}{5}$

Exercise 4.5.81

$\frac{16}{15} \cdot 50 \cdot \frac{3}{10}$

Exercise 4.5.82

$5 \frac{1}{3} \cdot \frac{27}{32}$

(Solution on p. 286.)

(Solution on p. 286.)

(Solution on p. 286.)

(Solution on p. 286.)

(Solution on p. 286.)

(Solution on p. 286.)

(Solution on p. 286.)

(Solution on p. 286.)

(Solution on p. 286.)

Available for free at Connexions $<$ http://cnx.org/content/col10615/1.4 $>$ 
Exercise 4.5.83

$2 \frac{6}{7} \cdot 5 \frac{3}{5}$

Exercise 4.5.84

$6 \frac{1}{4} \cdot 2 \frac{4}{15}$

(Solution on p. 286.)

Exercise 4.5.85

$9 \frac{1}{3} \cdot \frac{9}{16} \cdot 1 \frac{1}{3}$

Exercise 4.5.86

$3 \frac{5}{9} \cdot 1 \frac{13}{14} \cdot 10 \frac{1}{2}$

Exercise 4.5.87

$20 \frac{1}{4} \cdot 8 \frac{2}{3} \cdot 16 \frac{4}{5}$

Exercise 4.5.88

$\left(\frac{2}{3}\right)^{2}$

(Solution on p. 287.)

Exercise 4.5.89

$\left(\frac{3}{8}\right)^{2}$

Exercise 4.5.90

$\left(\frac{2}{11}\right)^{2}$

(Solution on p. 287.)

Exercise 4.5.91

$\left(\frac{8}{9}\right)^{2}$

Exercise 4.5.92

$\left(\frac{1}{2}\right)^{2}$

(Solution on p. 286.)

Exercise 4.5.93

$\left(\frac{3}{5}\right)^{2} \cdot \frac{20}{3}$

Exercise 4.5.94

$\left(\frac{1}{4}\right)^{2} \cdot \frac{16}{15}$

Exercise 4.5.95

$\left(\frac{1}{2}\right)^{2} \cdot \frac{8}{9}$

Exercise 4.5.96

$\left(\frac{1}{2}\right)^{2} \cdot\left(\frac{2}{5}\right)^{2}$

(Solution on p. 287.)

Exercise 4.5.97

$\left(\frac{3}{7}\right)^{2} \cdot\left(\frac{1}{9}\right)^{2}$

For the following problems, find each value. Reduce answers to lowest terms or convert to mixed numbers.

Exercise 4.5.98

(Solution on p. 287.)

$\sqrt{\frac{4}{9}}$

Exercise 4.5.99

$\sqrt{\frac{16}{25}}$

Exercise 4.5.100

$\sqrt{\frac{81}{121}}$

(Solution on p. 287.)

Exercise 4.5.101

$\sqrt{\frac{36}{49}}$

Exercise 4.5.102

(Solution on p. 287.)

$\sqrt{\frac{144}{25}}$

Exercise 4.5.103

$\frac{2}{3} \cdot \sqrt{\frac{9}{16}}$ 
Exercise 4.5.104

(Solution on p. 287.)

$\frac{3}{5} \cdot \sqrt{\frac{25}{81}}$

Exercise 4.5.105

$\left(\frac{8}{5}\right)^{2} \cdot \sqrt{\frac{25}{64}}$

Exercise 4.5.106

(Solution on p. 287.)

$\left(1 \frac{3}{4}\right)^{2} \cdot \sqrt{\frac{4}{49}}$

Exercise 4.5.107

$\left(2 \frac{2}{3}\right)^{2} \cdot \sqrt{\frac{36}{49}} \cdot \sqrt{\frac{64}{81}}$

\subsubsection{Exercises for Review}

Exercise 4.5.108

(Solution on p. 287.)

(Section 1.2) How many thousands in 342,810 ?

Exercise 4.5.109

(Section 1.5) Find the sum of 22, 42, and 101.

Exercise 4.5.110

(Solution on p. 287.)

(Section 2.5) Is 634,281 divisible by 3 ?

Exercise 4.5.111

(Section 3.4) Is the whole number 51 prime or composite?

Exercise 4.5.112

(Section 4.4) Reduce $\frac{36}{150}$ to lowest terms.

(Solution on p. 287.)

\subsection{Division of Fractions ${ }^{6}$}

\subsubsection{Section Overview}

- Reciprocals

- Dividing Fractions

\subsubsection{Reciprocals}

\section{Reciprocals}

Two numbers whose product is 1 are called reciprocals of each other.

\subsubsection{Sample Set A}

The following pairs of numbers are reciprocals.

\section{Example 4.46}

$$
\underbrace{\frac{3}{4} \text { and } \frac{4}{3}}_{\frac{3}{4} \cdot \frac{4}{3}=1}
$$

\footnotetext{
${ }^{6}$ This content is available online at $<$ http://cnx.org/content $/ \mathrm{m} 34929 / 1.2 />$.
} 


\section{Example 4.47}

$$
\underbrace{\frac{7}{16} \text { and } \frac{16}{7}}_{\frac{7}{16} \cdot \frac{16}{7}=1}
$$

Example 4.48

$$
\underbrace{\frac{1}{6} \text { and } \frac{6}{1}}_{\frac{1}{6} \cdot \frac{6}{1}=1}
$$

Notice that we can find the reciprocal of a nonzero number in fractional form by inverting it (exchanging positions of the numerator and denominator).

\subsubsection{Practice Set A}

Find the reciprocal of each number.

Exercise 4.6.1

Exercise 4.6.2

Exercise 4.6.3 $\frac{7}{8}$

Exercise 4.6.4 $\frac{1}{5}$

Exercise 4.6.5 $2 \frac{2}{7}$

(Solution on p. 287.)

(Solution on p. 287.)

(Solution on p. 287.)

(Solution on p. 287.)

(Solution on p. 287.)

Hint: Write this number as an improper fraction first.

Exercise 4.6.6

$5 \frac{1}{4}$

Exercise 4.6.7 $10 \frac{3}{16}$

(Solution on p. 287.)

(Solution on p. 287.)

\subsubsection{Dividing Fractions}

Our concept of division is that it indicates how many times one quantity is contained in another quantity. For example, using the diagram we can see that there are 6 one-thirds in 2.

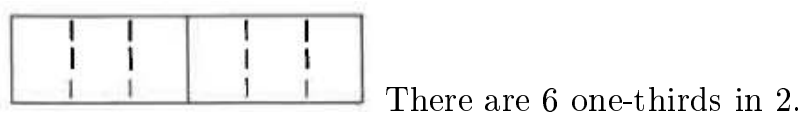

Since 2 contains six $\frac{1}{3}$ 's we express this as 
$\begin{aligned} 2 \div \frac{1}{3} & =6 \\ \text { Note also that } 2 \cdot \frac{1}{3} & =6 \\ \downarrow & \frac{1}{3} \text { and } 3 \text { are reciprocals }\end{aligned}$

Using these observations, we can suggest the following method for dividing a number by a fraction.

Dividing One Fraction by Another Fraction

To divide a first fraction by a second, nonzero fraction, multiply the first traction by the reciprocal of the second fraction.

Invert and Multiply

This method is commonly referred to as "invert the divisor and multiply."

\subsubsection{Sample Set B}

Perform the following divisions.

Example 4.49

$\frac{1}{3} \div \frac{3}{4}$. The divisor is $\frac{3}{4}$. Its reciprocal is $\frac{4}{3}$. Multiply $\frac{1}{3}$ by $\frac{4}{3}$.

$\frac{1}{3} \cdot \frac{4}{3}=\frac{1 \cdot 4}{3 \cdot 3}=\frac{4}{9}$

$\frac{1}{3} \div \frac{3}{4}=\frac{4}{9}$

Example 4.50

$\frac{3}{8} \div \frac{5}{4}$ The divisor is $\frac{5}{4}$. Its reciprocal is $\frac{4}{5}$. Multiply $\frac{3}{8}$ by $\frac{4}{5}$.

$\frac{3}{\frac{3}{3}} \cdot \frac{\frac{1}{4}}{5}=\frac{3 \cdot 1}{2 \cdot 5}=\frac{3}{10}$

$\frac{3}{8} \div \frac{5}{4}=\frac{3}{10}$

Example 4.51

$\frac{5}{6} \div \frac{5}{12}$. The divisor is $\frac{5}{12}$. Its reciprocal is $\frac{12}{5}$. Multiply $\frac{5}{6}$ by $\frac{12}{5}$.

$\frac{\frac{1}{55}}{\frac{5}{\sqrt{6}}} \cdot \frac{\frac{2}{12}}{\overline{1}}=\frac{1 \cdot 2}{1}=\frac{2}{1}=2$

$\frac{5}{6} \div \frac{5}{12}=2$

Example 4.52

$2 \frac{2}{9} \div 3 \frac{1}{3}$. Convert each mixed number to an improper fraction.

$2 \frac{2}{9}=\frac{9 \cdot 2+2}{9}=\frac{20}{9}$.

$3 \frac{1}{3}=\frac{3 \cdot 3+1}{3}=\frac{10}{3}$.

$\frac{20}{9} \div \frac{10}{3}$ The divisor is $\frac{10}{3}$. Its reciprocal is $\frac{3}{10}$. Multiply $\frac{20}{9}$ by $\frac{3}{10}$.

$\frac{\frac{2}{20}}{\frac{29}{3}} \cdot \frac{\frac{1}{33}}{\frac{\partial 3}{10}}=\frac{2 \cdot 1}{3 \cdot 1}=\frac{2}{3}$ 
$2 \frac{2}{9} \div 3 \frac{1}{3}=\frac{2}{3}$

Example 4.53

$\frac{12}{11} \div 8$. First conveniently write 8 as $\frac{8}{1}$.

$\frac{12}{11} \div \frac{8}{1}$ The divisor is $\frac{8}{1}$. Its reciprocal is $\frac{1}{8}$. Multiply $\frac{12}{11}$ by $\frac{1}{8}$.

$\frac{\frac{3}{212}}{11} \cdot \frac{1}{\frac{1}{2}}=\frac{3 \cdot 1}{11 \cdot 2}=\frac{3}{22}$

$\frac{12}{11} \div 8=\frac{3}{22}$

Example 4.54

$\frac{7}{8} \div \frac{21}{20} \cdot \frac{3}{35}$. The divisor is $\frac{21}{20}$. Its reciprocal is $\frac{20}{21}$.

$\frac{\frac{1}{77}}{\frac{27}{\sqrt{8}}} \cdot \frac{\frac{1}{\sqrt{5}}}{\frac{220}{\sqrt[20]{33}}} \frac{\frac{1}{33}}{\frac{33}{35}}=\frac{1 \cdot 1 \cdot 1}{2 \cdot 1 \cdot 7}=\frac{1}{14}$

$\frac{7}{8} \div \frac{21}{20} \cdot \frac{3}{25}=\frac{1}{14}$

Example 4.55

How many $2 \frac{3}{8}$-inch-wide packages can be placed in a box 19 inches wide?

The problem is to determine how many two and three eighths are contained in 19, that is, what is $19 \div 2 \frac{3}{8} ?$

$2 \frac{3}{8}=\frac{19}{8}$ Convert the divisor $2 \frac{3}{8}$ to an improper fraction.

$19=\frac{19}{1}$ Write the dividend 19 as $\frac{19}{1}$.

$\frac{19}{1} \div \frac{19}{8}$ The divisor is $\frac{19}{8}$. Its reciprocal is $\frac{8}{19}$.

$\frac{\frac{1}{219}}{1} \cdot \frac{8}{\underset{1}{19}}=\frac{1 \cdot 8}{1 \cdot 1}=\frac{8}{1}=8$

Thus, 8 packages will fit into the box.

\subsubsection{Practice Set B}

Perform the following divisions.

Exercise 4.6.8

(Solution on p. 287.)

$\frac{1}{2} \div \frac{9}{8}$

Exercise 4.6.9

(Solution on p. 287.)

$\frac{3}{8} \div \frac{9}{24}$

Exercise 4.6.10

$\frac{7}{15} \div \frac{14}{15}$

Exercise 4.6.11

$8 \div \frac{8}{15}$

Exercise 4.6.12

$6 \frac{1}{4} \div \frac{5}{12}$

(Solution on p. 287.)

(Solution on p. 287.)

(Solution on p. 287.) 
Exercise 4.6.13

(Solution on p. 288.)

$3 \frac{1}{3} \div 1 \frac{2}{3}$

Exercise 4.6.14

(Solution on p. 288.)

$\frac{5}{6} \div \frac{2}{3} \cdot \frac{8}{25}$

Exercise 4.6.15

(Solution on p. 288.)

A container will hold 106 ounces of grape juice. How many $6 \frac{5}{8}$-ounce glasses of grape juice can be served from this container?

Determine each of the following quotients and then write a rule for this type of division.

Exercise 4.6.16

(Solution on p. 288.)

$$
1 \div \frac{2}{3}
$$

Exercise 4.6.17

(Solution on p. 288.)

$1 \div \frac{3}{8}$

Exercise 4.6.18

(Solution on p. 288.)

$1 \div \frac{3}{4}$

Exercise 4.6.19

$1 \div \frac{5}{2}$

Exercise 4.6.20

(Solution on p. 288.)

When dividing 1 by a fraction, the quotient is the

(Solution on p. 288.)

\subsubsection{Exercises}

For the following problems, find the reciprocal of each number.

Exercise 4.6.21

$\frac{4}{5}$

(Solution on p. 288.)

Exercise 4.6.22

$\frac{8}{11}$

Exercise 4.6.23

$\frac{2}{9}$

(Solution on p. 288.)

Exercise 4.6.24

$\frac{1}{5}$

Exercise 4.6.25

$3 \frac{1}{4}$

(Solution on p. 288.)

Exercise 4.6.26

$8 \frac{1}{4}$

Exercise 4.6.27

$3 \frac{2}{7}$

Exercise 4.6.28

$5 \frac{3}{4}$

Exercise 4.6.29

1

(Solution on p. 288.)

Exercise 4.6.30

4

For the following problems, find each value.

Exercise 4.6.31 $\frac{3}{8} \div \frac{3}{5}$

(Solution on p. 288.) 
Exercise 4.6.32 $\frac{5}{9} \div \frac{5}{6}$

Exercise 4.6.33 $\frac{9}{16} \div \frac{15}{8}$

(Solution on p. 288.)

Exercise 4.6.34 $\frac{4}{9} \div \frac{6}{15}$

Exercise 4.6.35 $\frac{25}{49} \div \frac{4}{9}$

Exercise 4.6.36 $\frac{15}{4} \div \frac{27}{8}$

Exercise 4.6.37 $\frac{24}{75} \div \frac{8}{15}$

(Solution on p. 288.)

Exercise 4.6.38 $\frac{5}{7} \div 0$

Exercise 4.6.39 $\frac{7}{8} \div \frac{7}{8}$

Exercise 4.6.40 $0 \div \frac{3}{5}$

Exercise 4.6.41 $\frac{4}{11} \div \frac{4}{11}$

(Solution on p. 288.)

Exercise 4.6.42 $\frac{2}{3} \div \frac{2}{3}$

Exercise 4.6.43 $\frac{7}{10} \div \frac{10}{7}$

(Solution on p. 288.)

Exercise 4.6.44 $\frac{3}{4} \div 6$

Exercise 4.6.45 $\frac{9}{5} \div 3$

Exercise 4.6.46 $4 \frac{1}{6} \div 3 \frac{1}{3}$

Exercise 4.6.47 $7 \frac{1}{7} \div 8 \frac{1}{3}$

(Solution on p. 288.)

(Solution on p. 288.)

Exercise 4.6.48 $1 \frac{1}{2} \div 1 \frac{1}{5}$

Exercise 4.6.49 $3 \frac{2}{5} \div \frac{6}{25}$

(Solution on p. 288.)

Exercise 4.6.50 $5 \frac{1}{6} \div \frac{31}{6}$

Exercise 4.6.51 $\frac{35}{6} \div 3 \frac{3}{4}$

Exercise 4.6.52 $5 \frac{1}{9} \div \frac{1}{18}$

Exercise 4.6.53 $8 \frac{3}{4} \div \frac{7}{8}$

(Solution on p. 288.)

(Solution on p. 288.)

Exercise 4.6.54 $\frac{12}{8} \div 1 \frac{1}{2}$ 
Exercise 4.6.55

(Solution on p. 289.)

$3 \frac{1}{8} \div \frac{15}{16}$

Exercise 4.6.56

$11 \frac{11}{12} \div 9 \frac{5}{8}$

Exercise 4.6.57

(Solution on p. 289.)

$2 \frac{2}{9} \div 11 \frac{2}{3}$

Exercise 4.6.58

$\frac{16}{3} \div 6 \frac{2}{5}$

Exercise 4.6.59

$4 \frac{3}{25} \div 2 \frac{56}{75}$

(Solution on p. 289.)

Exercise 4.6.60

$\frac{1}{1000} \div \frac{1}{100}$

Exercise 4.6.61

$\frac{3}{8} \div \frac{9}{16} \cdot \frac{6}{5}$

Exercise 4.6.62

$\frac{3}{16} \cdot \frac{9}{8} \cdot \frac{6}{5}$

Exercise 4.6.63

$\frac{4}{15} \div \frac{2}{25} \cdot \frac{9}{10}$

Exercise 4.6.64

$\frac{21}{30} \cdot 1 \frac{1}{4} \div \frac{9}{10}$

Exercise 4.6.65

$8 \frac{1}{3} \cdot \frac{36}{75} \div 4$

(Solution on p. 289.)

(Solution on p. 289.)

(Solution on p. 289.)

\subsubsection{Exercises for Review}

Exercise 4.6.66

(Section 1.2) What is the value of 5 in the number 504,216 ?

Exercise 4.6.67

(Solution on p. 289.)

(Section 2.2) Find the product of 2,010 and 160.

Exercise 4.6.68

(Section 2.6) Use the numbers 8 and 5 to illustrate the commutative property of multiplication.

Exercise 4.6.69

(Solution on p. 289.)

(Section 3.6) Find the least common multiple of 6, 16, and 72.

Exercise 4.6.70

(Section 4.5) Find $\frac{8}{9}$ of $6 \frac{3}{4}$.

\subsection{Applications Involving Fractions ${ }^{7}$}

\subsubsection{Section Overview}

- Multiplication Statements

- Missing Product Statements

- Missing Factor Statements

\footnotetext{
${ }^{7}$ This content is available online at $<$ http://cnx.org/content $/ \mathrm{m} 34930 / 1.2 />$.
} 


\subsubsection{Multiplication Statements}

\section{Statement, Multiplication Statement}

A statement is a sentence that is either true or false. A mathematical statement of the form

product $=($ factor 1$) \cdot($ factor 2$)$

is a multiplication statement. Depending on the numbers that are used, it can be either true or false.

Omitting exactly one of the three numbers in the statement will produce exactly one of the following three problems. For convenience, we'll represent the omitted (or missing) number with the letter $M$ ( $M$ for Missing).

1. $M=$ (factor 1$) \cdot($ factor 2) Missing product statement.

2. $M \cdot($ factor 2$)=$ product Missing factor statement.

3. (factor 1$) \cdot M=$ product Missing factor statement.

We are interested in developing and working with methods to determine the missing number that makes the statement true. Fundamental to these methods is the ability to translate two words to mathematical symbols. The word

of translates to times

is translates to equals

\subsubsection{Missing Products Statements}

The equation $\mathrm{M}=8 \cdot 4$ is a missing product statement. We can find the value of $M$ that makes this statement true by multiplying the known factors.

Missing product statements can be used to determine the answer to a question such as, "What number is fraction 1 of fraction 2 ?

\subsubsection{Sample Set A}

Find $\frac{3}{4}$ of $\frac{8}{9}$. We are being asked the question, "What number is $\frac{3}{4}$ of $\frac{8}{9}$ ?" We must translate from words to mathematical symbols.

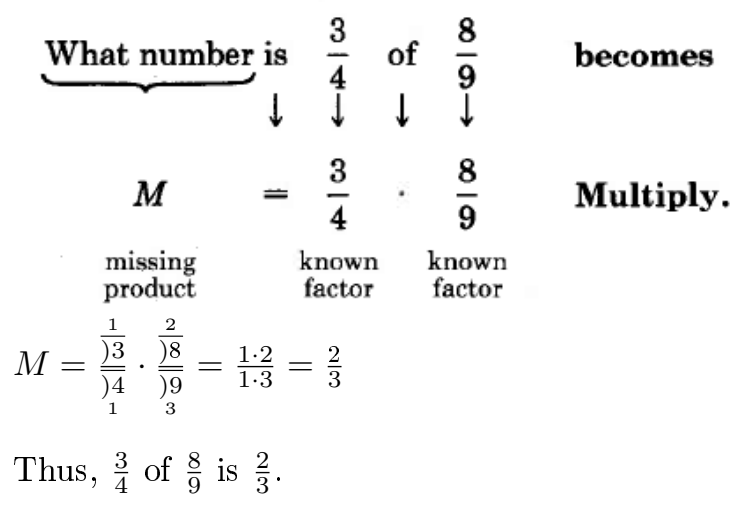




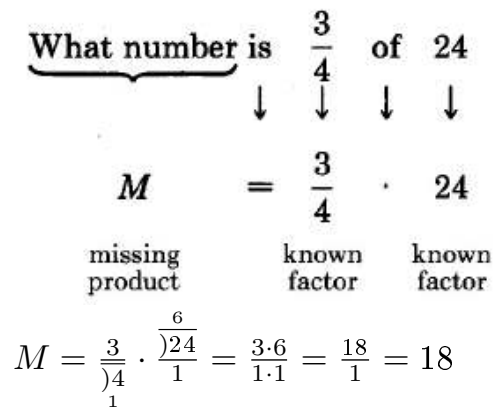

Thus, 18 is $\frac{3}{4}$ of 24 .

\subsubsection{Practice Set A}

Exercise 4.7.1

(Solution on p. 289.)

Find $\frac{3}{8}$ of $\frac{16}{15}$.

Exercise 4.7.2

What number is $\frac{9}{10}$ of $\frac{5}{6}$ ?

(Solution on p. 289.)

Exercise 4.7.3

$\frac{11}{16}$ of $\frac{8}{33}$ is what number?

(Solution on p. 289.)

\subsubsection{Missing Factor Statements}

The equation $8 \cdot M=32$ is a missing factor statement. We can find the value of $M$ that makes this statement true by dividing (since we know that $32 \div 8=4$ ).

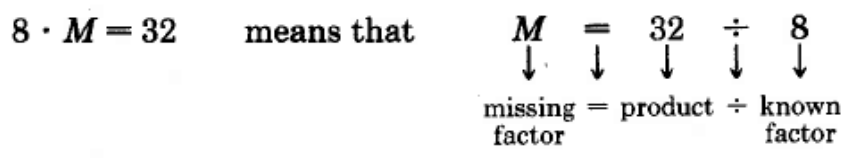

Finding the Missing Factor

To find the missing factor in a missing factor statement, divide the product by the known factor. missing factor $=$ (product $) \div($ known factor $)$

Missing factor statements can be used to answer such questions as

1. $\frac{3}{8}$ of what number is $\frac{9}{4}$ ?

2. What part of $1 \frac{2}{7}$ is $1 \frac{13}{14}$ ?

\subsubsection{Sample Set B}

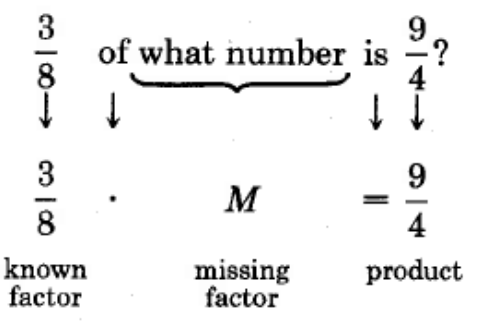

Now, using 
missing factor $=($ product $) \div($ known factor $)$

We get

$$
\begin{aligned}
M=\frac{9}{4} \div \frac{3}{8}=\frac{9}{4} \cdot \frac{8}{3} & =\frac{\frac{3}{\frac{9}{9}} \cdot \frac{2}{\frac{8}{13}}}{1}{ }_{1} \\
& =\quad \frac{3 \cdot 2}{1 \cdot 1} \\
& =6
\end{aligned}
$$

Check: $\quad \frac{3}{8} \cdot 6 \stackrel{9}{=} \frac{9}{4}$

$$
\begin{array}{r}
\frac{3}{\phi} \cdot \frac{3}{1} \stackrel{?}{=} \frac{9}{4} \\
\frac{3 \cdot 3}{4 \cdot 1} \stackrel{?}{=} \frac{9}{4} \\
\frac{9}{4} \stackrel{ }{=} \frac{9}{4}
\end{array}
$$

Thus, $\frac{3}{8}$ of 6 is $\frac{9}{4}$.

$$
\begin{aligned}
& \underbrace{\text { What part of }}_{\downarrow} 1 \frac{2}{7} \text { is } 1 \frac{13}{14} \text { ? } \\
& M \quad \cdot 1 \frac{2}{7}=1 \frac{13}{14} \\
& \begin{array}{ll}
\text { missing } & \text { known product } \\
\text { factor } & \text { factor }
\end{array}
\end{aligned}
$$

For convenience, let's convert the mixed numbers to improper fractions.

$M \cdot \frac{9}{7}=\frac{27}{14}$

Now, using

missing factor $=($ product $) \div($ known factor $)$

we get

$$
\begin{aligned}
M=\frac{27}{14} \div \frac{9}{7}=\frac{27}{14} \cdot \frac{7}{9}= & \frac{\frac{3}{27}}{\frac{27}{14}} \cdot \frac{\frac{1}{7}}{\frac{7}{99}} \\
& =\frac{3 \cdot 1}{2 \cdot 1} \\
& =\frac{3}{2}
\end{aligned}
$$

Check: $\quad \frac{3}{2} \cdot \frac{9}{7} \geq \frac{27}{14}$

$$
\begin{aligned}
& \frac{3 \cdot 9}{2 \cdot 7} \stackrel{27}{14} \\
& \frac{27}{14} \stackrel{27}{14}
\end{aligned}
$$


Thus, $\frac{3}{2}$ of $1 \frac{2}{7}$ is $1 \frac{13}{14}$.

\subsubsection{Practice Set B}

Exercise 4.7.4

(Solution on p. 289.)

$\frac{3}{5}$ of what number is $\frac{9}{20}$ ?

Exercise 4.7.5

$3 \frac{3}{4}$ of what number is $2 \frac{2}{9}$ ?

(Solution on p. 289.)

Exercise 4.7.6

What part of $\frac{3}{5}$ is $\frac{9}{10}$ ?

Exercise 4.7.7

What part of $1 \frac{1}{4}$ is $1 \frac{7}{8}$ ?

(Solution on p. 289.)

(Solution on p. 289.)

\subsubsection{Exercises}

Exercise 4.7.8

(Solution on p. 289.)

Find $\frac{2}{3}$ of $\frac{3}{4}$.

Exercise 4.7.9

Find $\frac{5}{8}$ of $\frac{1}{10}$.

Exercise 4.7.10

Find $\frac{12}{13}$ of $\frac{13}{36}$.

Exercise 4.7.11

Find $\frac{1}{4}$ of $\frac{4}{7}$.

Exercise 4.7.12

(Solution on p. 289.)

$\frac{3}{10}$ of $\frac{15}{4}$ is what number?

Exercise 4.7.13

$\frac{14}{15}$ of $\frac{20}{21}$ is what number?

Exercise 4.7.14

$\frac{3}{44}$ of $\frac{11}{12}$ is what number?

(Solution on p. 289.)

Exercise 4.7.15

$\frac{1}{3}$ of 2 is what number?

Exercise 4.7.16

$\frac{1}{4}$ of 3 is what number?

(Solution on p. 289.)

Exercise 4.7.17

$\frac{1}{10}$ of $\frac{1}{100}$ is what number?

Exercise 4.7.18

$\frac{1}{100}$ of $\frac{1}{10}$ is what number?

(Solution on p. 289.)

Exercise 4.7.19

$1 \frac{5}{9}$ of $2 \frac{4}{7}$ is what number?

Exercise 4.7.20

$1 \frac{7}{18}$ of $\frac{4}{15}$ is what number?

(Solution on p. 289.)

Exercise 4.7.21

$1 \frac{1}{8}$ of $1 \frac{11}{16}$ is what number?

Exercise 4.7.22

Find $\frac{2}{3}$ of $\frac{1}{6}$ of $\frac{9}{2}$.

(Solution on p. 289.) 
Exercise 4.7.23

Find $\frac{5}{8}$ of $\frac{9}{20}$ of $\frac{4}{9}$.

Exercise 4.7.24

$\frac{5}{12}$ of what number is $\frac{5}{6}$ ?

(Solution on p. 289.)

Exercise 4.7.25

$\frac{3}{14}$ of what number is $\frac{6}{7}$ ?

Exercise 4.7.26

$\frac{10}{3}$ of what number is $\frac{5}{9}$ ?

(Solution on p. 289.)

Exercise 4.7.27

$\frac{15}{7}$ of what number is $\frac{20}{21}$ ?

Exercise 4.7.28

$\frac{8}{3}$ of what number is $1 \frac{7}{9}$ ?

(Solution on p. 290.)

Exercise 4.7.29

$\frac{1}{3}$ of what number is $\frac{1}{3}$ ?

Exercise 4.7.30

$\frac{1}{6}$ of what number is $\frac{1}{6}$ ?

Exercise 4.7.31

$\frac{3}{4}$ of what number is $\frac{3}{4}$ ?

Exercise 4.7.32

$\frac{8}{11}$ of what number is $\frac{8}{11}$ ?

(Solution on p. 290.)

Exercise 4.7.33

$\frac{3}{8}$ of what number is 0 ?

Exercise 4.7.34

$\frac{2}{3}$ of what number is 1 ?

(Solution on p. 290.)

Exercise 4.7.35

$3 \frac{1}{5}$ of what number is 1 ?

Exercise 4.7.36

$1 \frac{9}{12}$ of what number is $5 \frac{1}{4}$ ?

(Solution on p. 290.)

Exercise 4.7.37

$3 \frac{1}{25}$ of what number is $2 \frac{8}{15}$ ?

Exercise 4.7.38

What part of $\frac{2}{3}$ is $1 \frac{1}{9}$ ?

Exercise 4.7.39

What part of $\frac{9}{10}$ is $3 \frac{3}{5}$ ?

Exercise 4.7.40

What part of $\frac{8}{9}$ is $\frac{3}{5}$ ?

(Solution on p. 290.)

Exercise 4.7.41

What part of $\frac{14}{15}$ is $\frac{7}{30}$ ?

Exercise 4.7.42

What part of 3 is $\frac{1}{5}$ ?

Exercise 4.7.43

What part of 8 is $\frac{2}{3}$ ?

Exercise 4.7.44

What part of 24 is 9 ?

(Solution on p. 290.)

Exercise 4.7.45

What part of 42 is 26 ? 
Exercise 4.7.46

(Solution on p. 290.)

Find $\frac{12}{13}$ of $\frac{39}{40}$.

Exercise 4.7.47

$\frac{14}{15}$ of $\frac{12}{21}$ is what number?

Exercise 4.7.48

$\frac{8}{15}$ of what number is $2 \frac{2}{5}$ ?

(Solution on p. 290.)

Exercise 4.7.49

$\frac{11}{15}$ of what number is $\frac{22}{35}$ ?

Exercise 4.7.50

$\frac{11}{16}$ of what number is 1 ?

(Solution on p. 290.)

Exercise 4.7.51

What part of $\frac{23}{40}$ is $3 \frac{9}{20}$ ?

Exercise 4.7.52

$\frac{4}{35}$ of $3 \frac{9}{22}$ is what number?

(Solution on p. 290.)

\subsubsection{Exercises for Review}

Exercise 4.7.53

(Section 1.7) Use the numbers 2 and 7 to illustrate the commutative property of addition.

Exercise 4.7.54

(Solution on p. 290.)

(Section 2.3) Is 4 divisible by 0 ?

Exercise 4.7.55

(Section 3.5) Expand $3^{7}$. Do not find the actual value.

Exercise 4.7.56

(Section 4.3) Convert $3 \frac{5}{12}$ to an improper fraction.

(Solution on p. 290.)

Exercise 4.7.57

(Section 4.4) Find the value of $\frac{3}{8} \div \frac{9}{16} \cdot \frac{6}{5}$. 


\subsection{Summary of Key Concepts ${ }^{8}$}

\subsubsection{Summary of Key Concepts}

\section{Fraction (Section 4.2)}

The idea of breaking up a whole quantity into equal parts gives us the word fraction.

Fraction Bar, Denominator, Numerator (Section 4.2)

A fraction has three parts:

1. The fraction bar -

2. The nonzero whole number below the fraction bar is the denominator.

3. The whole number above the fraction bar is the numerator.

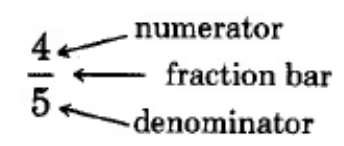

Proper Fraction (Section 4.3)

Proper fractions are fractions in which the numerator is strictly less than the denominator.

$\frac{4}{5}$ is a proper fraction

Improper Fraction (Section 4.3)

Improper fractions are fractions in which the numerator is greater than or equal to the denominator. Also, any nonzero number placed over 1 is an improper fraction.

$\frac{5}{4}, \frac{5}{5}$, and $\frac{5}{1}$ are improper fractions

Mixed Number (Section 4.3)

A mixed number is a number that is the sum of a whole number and a proper fraction.

$1 \frac{1}{5}$ is a mixed number $\left(1 \frac{1}{5}=1+\frac{1}{5}\right)$

Correspondence Between Improper Fractions and Mixed Numbers (Section 4.3)

Each improper fraction corresponds to a particular mixed number, and each mixed number corresponds to a particular improper fraction.

Converting an Improper Fraction to a Mixed Number (Section 4.3)

A method, based on division, converts an improper fraction to an equivalent mixed number.

$\frac{5}{4}$ can be converted to $1 \frac{1}{4}$

Converting a Mixed Number to an Improper Fraction (Section 4.3)

A method, based on multiplication, converts a mixed number to an equivalent improper fraction.

$5 \frac{7}{8}$ can be converted to $\frac{47}{8}$

Equivalent Fractions (Section 4.4)

Fractions that represent the same quantity are equivalent fractions.

$\frac{3}{4}$ and $\frac{6}{8}$ are equivalent fractions

\footnotetext{
${ }^{8}$ This content is available online at $<$ http://cnx.org/content $/ \mathrm{m} 34931 / 1.2 />$.
} 
Test for Equivalent Fractions (Section 4.4)

If the cross products of two fractions are equal, then the two fractions are equivalent.

$\frac{3}{4} \times \frac{6}{8}$

$3 \cdot 8 \stackrel{2}{ \pm} \cdot 6$

$24=24$

Thus, $\frac{3}{4}$ and $\frac{6}{8}$ are equivalent.

Relatively Prime (Section 4.4)

Two whole numbers are relatively prime when 1 is the only number that divides both of them.

3 and 4 are relatively prime

Reduced to Lowest Terms (Section 4.4)

A fraction is reduced to lowest terms if its numerator and denominator are relatively prime.

The number $\frac{3}{4}$ is reduced to lowest terms, since 3 and 4 are relatively prime.

The number $\frac{6}{8}$ is not reduced to lowest terms since 6 and 8 are not relatively prime.

Reducing Fractions to Lowest Terms (Section 4.4)

Two methods, one based on dividing out common primes and one based on dividing out any common factors, are available for reducing a fraction to lowest terms.

Raising Fractions to Higher Terms (Section 4.4)

A fraction can be raised to higher terms by multiplying both the numerator and denominator by the same nonzero number.

$\frac{3}{4}=\frac{3 \cdot 2}{4 \cdot 2}=\frac{6}{8}$

\section{The Word "OF" Means Multiplication (Section 4.5)}

In many mathematical applications, the word "of" means multiplication.

Multiplication of Fractions (Section 4.5)

To multiply two or more fractions, multiply the numerators together and multiply the denominators together. Reduce if possible.

$\frac{5}{8} \cdot \frac{4}{15}=\frac{5 \cdot 4}{8 \cdot 15}=\frac{20}{120}=\frac{1}{6}$

Multiplying Fractions by Dividing Out Common Factors (Section 4.5)

Two or more fractions can be multiplied by first dividing out common factors and then using the rule for multiplying fractions.

$\frac{\frac{1}{\frac{5}{15}}}{\frac{\frac{1}{2}}{2}} \cdot \frac{\frac{1}{315}}{\frac{3}{3}}=\frac{1 \cdot 1}{2 \cdot 3}=\frac{1}{6}$

\section{Multiplication of Mixed Numbers (Section 4.5)}

To perform a multiplication in which there are mixed numbers, first convert each mixed number to an improper fraction, then multiply. This idea also applies to division of mixed numbers.

\section{Reciprocals (Section 4.6)}

Two numbers whose product is 1 are reciprocals.

7 and $\frac{1}{7}$ are reciprocals 


\section{Division of Fractions (Section 4.6)}

To divide one fraction by another fraction, multiply the dividend by the reciprocal of the divisor.

$\frac{4}{5} \div \frac{2}{15}=\frac{4}{5} \cdot \frac{15}{2}$

\section{Dividing 1 by a Fraction (Section 4.6)}

When dividing 1 by a fraction, the quotient is the reciprocal of the fraction.

$\frac{1}{\frac{3}{7}}=\frac{7}{3}$

\section{Multiplication Statements (Section 4.7)}

A mathematical statement of the form

product $=($ factor 1$)($ factor 2$)$

is a multiplication statement.

By omitting one of the three numbers, one of three following problems result:

1. $M=($ factor 1$) \cdot($ factor 2$)$ Missing product statement.

2. product $=($ factor 1$) \cdot M$ Missing factor statement.

3. product $=M \cdot($ factor 2$)$ Missing factor statement.

Missing products are determined by simply multiplying the known factors. Missing factors are determined by

missing factor $=($ product $) \div($ known factor $)$

\subsection{Exercise Supplement ${ }^{9}$}

\subsubsection{Exercise Supplement}

\subsubsection{Fractions of Whole Numbers (Section 4.2)}

For Problems 1 and 2, name the suggested fraction.

Exercise 4.9.1

(Solution on p. 290.)

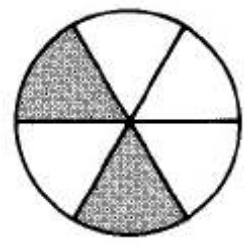

\section{Exercise 4.9.2}

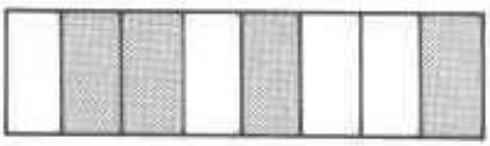

For problems 3-5, specify the numerator and denominator.

Exercise 4.9.3 $\frac{4}{5}$

\footnotetext{
${ }^{9}$ This content is available online at $<\mathrm{http}: / / \mathrm{cnx} .0 r g /$ content $/ \mathrm{m} 34932 / 1.2 />$.
} 
Exercise 4.9.4

$\frac{5}{12}$

Exercise 4.9.5

$\frac{1}{3}$

(Solution on p. 290.)

For problems 6-10, write each fraction using digits.

\section{Exercise 4.9.6}

Three fifths

Exercise 4.9.7

(Solution on p. 290.)

Eight elevenths

Exercise 4.9.8

Sixty-one forty firsts

Exercise 4.9.9

(Solution on p. 290.)

Two hundred six-thousandths

Exercise 4.9.10

zero tenths

For problems 11-15, write each fraction using words.

Exercise 4.9.11

$\frac{10}{17}$

(Solution on p. 290.)

Exercise 4.9.12

$\frac{21}{38}$

Exercise 4.9.13

$\frac{606}{1431}$

Exercise 4.9.14

$\frac{0}{8}$

Exercise 4.9.15

$\frac{1}{16}$

(Solution on p. 290.)

For problems 16-18, state each numerator and denominator and write each fraction using digits.

Exercise 4.9.16

One minute is one sixtieth of an hour.

Exercise 4.9.17

(Solution on p. 290.)

In a box that contains forty-five electronic components, eight are known to be defective. If three components are chosen at random from the box, the probability that all three are defective is fifty-six fourteen thousand one hundred ninetieths.

Exercise 4.9.18

About three fifths of the students in a college algebra class received a "B" in the course.

For problems 19 and 20, shade the region corresponding to the given fraction.

Exercise 4.9.19

$\frac{1}{4}$

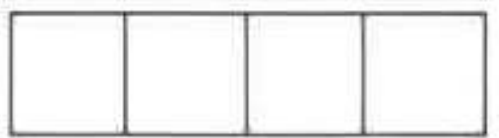

(Solution on p. 290.)

\section{Exercise 4.9.20}

$\frac{3}{7}$ 


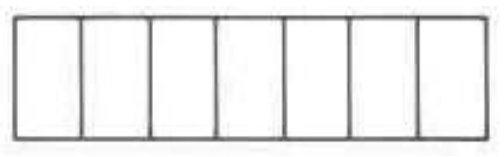

4.9.1.2 Proper Fraction, Improper Fraction, and Mixed Numbers (Section 4.3)

For problems 21-29, convert each improper fraction to a mixed number.

Exercise 4.9.21

(Solution on p. 291.)

$\frac{11}{4}$

Exercise 4.9.22

$\frac{15}{2}$

Exercise 4.9.23

$\frac{51}{8}$

(Solution on p. 291.)

Exercise 4.9.24 $\frac{121}{15}$

Exercise 4.9.25 $\frac{356}{3}$

Exercise 4.9.26

$\frac{3}{2}$

Exercise 4.9.27

$\frac{5}{4}$

(Solution on p. 291.)

Exercise 4.9.28

$\frac{20}{5}$

Exercise 4.9.29

$\frac{9}{3}$

(Solution on p. 291.)

For problems 30-40, convert each mixed number to an improper fraction.

Exercise 4.9.30 $5 \frac{2}{3}$

Exercise 4.9.31 $16 \frac{1}{8}$

(Solution on p. 291.)

Exercise 4.9.32 $18 \frac{1}{3}$

Exercise 4.9.33 $3 \frac{1}{5}$

(Solution on p. 291.)

Exercise 4.9.34 $2 \frac{9}{16}$

Exercise 4.9.35 $17 \frac{20}{21}$

(Solution on p. 291.)

Exercise 4.9.36 $1 \frac{7}{8}$

Exercise 4.9.37 $1 \frac{1}{2}$

Exercise 4.9.38 $2 \frac{1}{2}$ 
Exercise 4.9.39

(Solution on p. 291.)

$8 \frac{6}{7}$

Exercise 4.9.40

$2 \frac{9}{2}$

Exercise 4.9.41

Why does $0 \frac{1}{12}$ not qualify as a mixed number?

(Solution on p. 291.)

Exercise 4.9.42

Why does 8 qualify as a mixed number?

4.9.1.3 Equivalent Fractions, Reducing Fractions to Lowest Terms, and Raising Fractions to Higher Term (Section 4.4)

For problems $43-47$, determine if the pairs of fractions are equivalent.

Exercise 4.9.43

$\frac{1}{2}, \frac{15}{30}$

(Solution on p. 291.)

Exercise 4.9.44

$\frac{8}{9}, \frac{32}{36}$

Exercise 4.9.45

$\frac{3}{14}, \frac{24}{110}$

(Solution on p. 291.)

Exercise 4.9.46

$2 \frac{3}{8}, \frac{38}{16}$

Exercise 4.9.47

$\frac{108}{77}, 1 \frac{5}{13}$

For problems 48-60, reduce, if possible, each fraction.

Exercise 4.9.48

$\frac{10}{25}$

Exercise 4.9.49

$\frac{32}{44}$

(Solution on p. 291.)

Exercise 4.9.50

$\frac{102}{266}$

Exercise 4.9.51

$\frac{15}{33}$

Exercise 4.9.52

$\frac{18}{25}$

Exercise 4.9.53

$\frac{21}{35}$

Exercise 4.9.54

$\frac{9}{16}$

Exercise 4.9.55

$\frac{45}{85}$

Exercise 4.9.56

$\frac{24}{42}$

Exercise 4.9.57

$\frac{70}{136}$

(Solution on p. 291.)

(Solution on p. 291.)

(Solution on p. 291.)

(Solution on p. 291.) 
Exercise 4.9.58

$$
\frac{182}{580}
$$

Exercise 4.9.59

$\frac{325}{810}$

(Solution on p. 291.)

Exercise 4.9.60 $\frac{250}{1000}$

For problems 61-72, determine the missing numerator or denominator.

Exercise 4.9.61 $\frac{3}{7}=\frac{?}{35}$

(Solution on p. 291.)

Exercise 4.9.62 $\frac{4}{11}=\frac{?}{99}$

Exercise 4.9.63 $\frac{1}{12}=\frac{?}{72}$

Exercise 4.9.64 $\frac{5}{8}=\frac{25}{?}$

Exercise 4.9.65 $\frac{11}{9}=\frac{33}{?}$

Exercise 4.9.66 $\frac{4}{15}=\frac{24}{?}$

Exercise 4.9.67 $\frac{14}{15}=\frac{?}{45}$

Exercise 4.9.68

$\frac{0}{5}=\frac{?}{20}$

Exercise 4.9.69 $\frac{12}{21}=\frac{96}{?}$

(Solution on p. 291.)

Exercise 4.9.70 $\frac{14}{23}=\frac{?}{253}$

Exercise 4.9.71 $\frac{15}{16}=\frac{180}{?}$

Exercise 4.9.72 $\frac{21}{22}=\frac{336}{?}$

(Solution on p. 291.)

(Solution on p. 291.)

(Solution on p. 292.)

(Solution on p. 292.)

\subsubsection{Multiplication and Division of Fractions (Section 4.5, Section 4.6)}

For problems 73-95, perform each multiplication and division.

Exercise 4.9.73

$\frac{4}{5} \cdot \frac{15}{16}$

(Solution on p. 292.)

Exercise 4.9.74 $\frac{8}{9} \cdot \frac{3}{24}$

Exercise 4.9.75 $\frac{1}{10} \cdot \frac{5}{12}$

Exercise 4.9.76 $\frac{14}{15} \cdot \frac{7}{5}$

Exercise 4.9.77 $\frac{5}{6} \cdot \frac{13}{22} \cdot \frac{11}{39}$ 
Exercise 4.9.78 $\frac{2}{3} \div \frac{15}{7} \cdot \frac{5}{6}$

Exercise 4.9.79 $3 \frac{1}{2} \div \frac{7}{2}$

(Solution on p. 292.)

Exercise 4.9.80 $2 \frac{4}{9} \div \frac{11}{45}$

Exercise 4.9.81 $\frac{8}{15} \cdot \frac{3}{16} \cdot \frac{5}{24}$

Exercise 4.9.82 $\frac{8}{15} \div 3 \frac{3}{5} \cdot \frac{9}{16}$

Exercise 4.9.83 $\frac{14}{15} \div 3 \frac{8}{9} \cdot \frac{10}{21}$

(Solution on p. 292.)

Exercise 4.9.84 $18 \cdot 5 \frac{3}{4}$

Exercise 4.9.85 $3 \frac{3}{7} \cdot 2 \frac{1}{12}$

(Solution on p. 292.)

Exercise 4.9.86 $4 \frac{1}{2} \div 2 \frac{4}{7}$

Exercise 4.9.87

$6 \frac{1}{2} \div 3 \frac{1}{4}$

Exercise 4.9.88 $3 \frac{5}{16} \div 2 \frac{7}{18}$

Exercise 4.9.89 $7 \div 2 \frac{1}{3}$

(Solution on p. 292.)

Exercise 4.9.90 $17 \div 4 \frac{1}{4}$

Exercise 4.9.91 $\frac{5}{8} \div 1 \frac{1}{4}$

Exercise 4.9.92 $2 \frac{2}{3} \cdot 3 \frac{3}{4}$

Exercise 4.9.93 $20 \cdot \frac{18}{4}$

Exercise 4.9.94 $0 \div 4 \frac{1}{8}$

Exercise 4.9.95 $1 \div 6 \frac{1}{4} \cdot \frac{25}{4}$

(Solution on p. 292.)

(Solution on p. 292.)

(Solution on p. 292.)

(Solution on p. 292.) 


\subsubsection{Applications Involving Fractions (Section 4.7)}

Exercise 4.9.96

Find $\frac{8}{9}$ of $\frac{27}{2}$.

Exercise 4.9.97

What part of $\frac{3}{8}$ is $\frac{21}{16}$ ?

(Solution on p. 292.)

Exercise 4.9.98

What part of $3 \frac{1}{5}$ is $1 \frac{7}{25}$ ?

Exercise 4.9.99

Find $6 \frac{2}{3}$ of $\frac{9}{15}$.

(Solution on p. 292.)

Exercise 4.9.100

$\frac{7}{20}$ of what number is $\frac{14}{35}$ ?

Exercise 4.9.101

What part of $4 \frac{1}{16}$ is $3 \frac{3}{4}$ ?

Exercise 4.9.102

Find $8 \frac{3}{10}$ of $16 \frac{2}{3}$.

Exercise 4.9.103

$\frac{3}{20}$ of what number is $\frac{18}{30}$ ?

(Solution on p. 292.)

Exercise 4.9.104

Find $\frac{1}{3}$ of 0 .

Exercise 4.9.105

Find $\frac{11}{12}$ of 1 .

(Solution on p. 292.)

(Solution on p. 292.)

\subsection{Proficiency Exam ${ }^{10}$}

\subsubsection{Proficiency Exam}

Exercise 4.10.1

(Section 4.2) Shade a portion that corresponds to the fraction $\frac{5}{8}$.

(Solution on p. 292.)

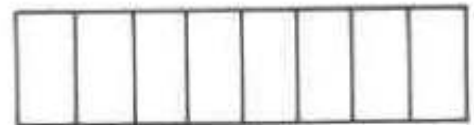

Exercise 4.10.2

(Section 4.2) Specify the numerator and denominator of the fraction $\frac{5}{9}$.

(Solution on p. 292.)

Exercise 4.10.3

(Solution on p. 292.)

(Section 4.2) Write the fraction five elevenths.

Exercise 4.10.4

(Section 4.2) Write, in words, $\frac{4}{5}$.

Exercise 4.10.5

(Section 4.3) Which of the fractions is a proper fraction? $4 \frac{1}{12}, \frac{5}{12}, \frac{12}{5}$

Exercise 4.10.6

(Solution on p. 292.)

(Section 4.3) Convert $3 \frac{4}{7}$ to an improper fraction.

Exercise 4.10.7

(Section 4.3) Convert $\frac{16}{5}$ to a mixed number.

(Solution on p. 292.)

(Solution on p. 293.)

(Solution on p. 293.)

\footnotetext{
${ }^{10}$ This content is available online at $<\mathrm{http}: / / \mathrm{cnx}$. org/content $/ \mathrm{m} 34933 / 1.2 />$.
} 
Exercise 4.10.8

(Solution on p. 293.)

(Section 4.4) Determine if $\frac{5}{12}$ and $\frac{20}{48}$ are equivalent fractions.

For problems 9-11, reduce, if possible, each fraction to lowest terms.

Exercise 4.10.9

(Section 4.4) $\frac{21}{35}$

Exercise 4.10.10

(Section 4.4) $\frac{15}{51}$

Exercise 4.10.11

(Section 4.4) $\frac{104}{480}$

(Solution on p. 293.)

(Solution on p. 293.)

(Solution on p. 293.)

For problems 12 and 13, determine the missing numerator or denominator.

Exercise 4.10.12

(Section 4.4) $\frac{5}{9}=\frac{?}{36}$

Exercise 4.10.13

(Section 4.4$) \frac{4}{3}=\frac{32}{?}$

(Solution on p. 293.)

(Solution on p. 293.)

For problems 14-25, find each value.

Exercise 4.10.14

(Section 4.5$) \frac{15}{16} \cdot \frac{4}{25}$

Exercise 4.10.15

(Section 4.5 ) $3 \frac{3}{4} \cdot 2 \frac{2}{9} \cdot 6 \frac{3}{5}$

Exercise 4.10.16

(Section 4.5) $\sqrt{\frac{25}{36}}$

Exercise 4.10.17

(Section 4.5$) \sqrt{\frac{4}{9}} \cdot \sqrt{\frac{81}{64}}$

Exercise 4.10.18

(Section 4.5$) \frac{11}{30} \cdot \sqrt{\frac{225}{121}}$

Exercise 4.10.19

(Section 4.6$) \frac{4}{15} \div 8$

Exercise 4.10.20

(Section 4.6$) \frac{8}{15} \cdot \frac{5}{12} \div 2 \frac{4}{9}$

Exercise 4.10.21

(Section 4.6) $\left(\frac{6}{5}\right)^{3} \div \sqrt{1 \frac{11}{25}}$

(Solution on p. 293.)

(Solution on p. 293.)

(Solution on p. 293.)

(Solution on p. 293.)

(Solution on p. 293.)

Exercise 4.10.22

(Section 4.7) Find $\frac{5}{12}$ of $\frac{24}{25}$.

Exercise 4.10.23

(Section 4.7) $\frac{2}{9}$ of what number is $\frac{1}{18}$ ?

Exercise 4.10.24

(Section 4.7) $1 \frac{5}{7}$ of $\frac{21}{20}$ is what number?

(Solution on p. 293.)

(Solution on p. 293.)

(Solution on p. 293.)

Exercise 4.10.25

(Section 4.7) What part of $\frac{9}{14}$ is $\frac{6}{7}$ ?

(Solution on p. 293.)

(Solution on p. 293.)

(Solution on p. 293.)

(Solution on p. 293.) 


\section{Solutions to Exercises in Chapter 4}

Solution to Exercise 4.2.1 (p. 217)

4,7

Solution to Exercise 4.2.2 (p. 218)

5,8

Solution to Exercise 4.2.3 (p. 218)

10,15

Solution to Exercise 4.2.4 (p. 218)

1,9

Solution to Exercise 4.2.5 (p. 218)

0,2

Solution to Exercise 4.2.6 (p. 219)

$\frac{1}{10}$

Solution to Exercise 4.2.7 (p. 219)

$\frac{11}{14}$

Solution to Exercise 4.2.8 (p. 219)

$\frac{16}{35}$

Solution to Exercise 4.2.9 (p. 219)

$\frac{800}{7,000}$

Solution to Exercise 4.2.10 (p. 219)

three eighths

Solution to Exercise 4.2.11 (p. 219) one tenth

Solution to Exercise 4.2.12 (p. 219) three two hundred fiftieths

Solution to Exercise 4.2.13 (p. 219)

one hundred fourteen three thousand one hundred ninetieths

Solution to Exercise 4.2.14 (p. 219)

$\frac{3}{8}$

Solution to Exercise 4.2.15 (p. 219)

$\frac{1}{16}$

Solution to Exercise 4.2.16 (p. 219)

5,9 , five ninths

Solution to Exercise 4.2.17 (p. 219)

1,10 , one tenth

Solution to Exercise 4.2.18 (p. 220)

numerator, 3 ; denominator, 4

Solution to Exercise 4.2.20 (p. 220)

numerator, 1 ; denominator, 5

Solution to Exercise 4.2.22 (p. 220)

numerator, 7 ; denominator, 7

Solution to Exercise 4.2.24 (p. 220)

numerator, 0; denominator, 12

Solution to Exercise 4.2.26 (p. 220)

numerator, 18; denominator, 1

Solution to Exercise 4.2.28 (p. 220)

$\frac{4}{5}$

Solution to Exercise 4.2.30 (p. 220)

$\frac{15}{20}$ 
Solution to Exercise 4.2.32 (p. 220)

$\frac{91}{107}$

Solution to Exercise 4.2.34 (p. 220)

$\frac{605}{834}$

Solution to Exercise 4.2.36 (p. 220)

$\frac{92}{1,000,000}$

Solution to Exercise 4.2.38 (p. 220)

five ninths

Solution to Exercise 4.2.40 (p. 221)

eight fifteenths

Solution to Exercise 4.2.42 (p. 221)

seventy-five one hundredths

Solution to Exercise 4.2.44 (p. 221)

nine hundred sixteen one thousand fourteenths

Solution to Exercise 4.2.46 (p. 221)

eighteen thirty-one thousand six hundred eighths

Solution to Exercise 4.2.48 (p. 221)

$\frac{1}{2}$

Solution to Exercise 4.2.50 (p. 221)

$\frac{4}{7}$

Solution to Exercise 4.2.52 (p. 221)

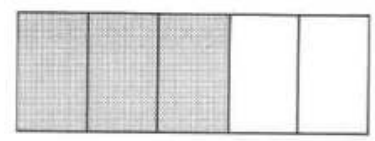

Solution to Exercise 4.2.54 (p. 222)

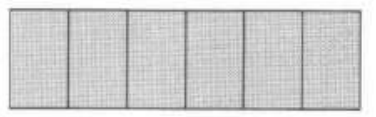

Solution to Exercise 4.2.56 (p. 222)

Numerator, 1; denominator, 4; one fourth

Solution to Exercise 4.2.58 (p. 222)

Numerator, 4; denominator, 3 ; four thirds

Solution to Exercise 4.2.60 (p. 222)

Numerator, 2; denominator, 7; two sevenths

Solution to Exercise 4.2.62 (p. 223)

Numerator, 1; denominator, 56; one fifty-sixth

Solution to Exercise 4.2.64 (p. 223)

Numerator, 125; denominator, 4; one hundred twenty-five fourths

Solution to Exercise 4.2.66 (p. 223)

$3+11=11+3=14$

Solution to Exercise 4.2.68 (p. 223)

$7^{5}$

Solution to Exercise 4.2.70 (p. 223)

144

Solution to Exercise 4.3.1 (p. 228)

$4 \frac{1}{2}$

Solution to Exercise 4.3.2 (p. 228)

$3 \frac{2}{3}$

Solution to Exercise 4.3.3 (p. 228)

$1 \frac{3}{11}$ 
Solution to Exercise 4.3.4 (p. 228)

$2 \frac{5}{13}$

Solution to Exercise 4.3.5 (p. 228)

$19 \frac{3}{4}$

Solution to Exercise 4.3.6 (p. 228)

62

Solution to Exercise 4.3.7 (p. 230)

$\frac{33}{4}$

Solution to Exercise 4.3.8 (p. 230)

$\frac{28}{5}$

Solution to Exercise 4.3.9 (p. 230)

$\frac{19}{15}$

Solution to Exercise 4.3.10 (p. 230)

$\frac{86}{7}$

Solution to Exercise 4.3.11 (p. 230)

improper fraction

Solution to Exercise 4.3.13 (p. 230)

proper fraction

Solution to Exercise 4.3.15 (p. 230)

mixed number

Solution to Exercise 4.3.17 (p. 230)

improper fraction

Solution to Exercise 4.3.19 (p. 230)

mixed number

Solution to Exercise 4.3.21 (p. 230) mixed number

Solution to Exercise 4.3.23 (p. 230) proper fraction

Solution to Exercise 4.3.25 (p. 230)

mixed number

Solution to Exercise 4.3.27 (p. 231)

$4 \frac{2}{3}$

Solution to Exercise 4.3.29 (p. 231)

$8 \frac{3}{4}$

Solution to Exercise 4.3.31 (p. 231) 9

Solution to Exercise 4.3.33 (p. 231) $13 \frac{9}{12}$ or $13 \frac{3}{4}$

Solution to Exercise 4.3.35 (p. 231) $555 \frac{5}{9}$

Solution to Exercise 4.3.37 (p. 231)

$36 \frac{1}{2}$

Solution to Exercise 4.3.39 (p. 231)

$7 \frac{29}{41}$

Solution to Exercise 4.3.41 (p. 231)

$\frac{33}{8}$

Solution to Exercise 4.3.43 (p. 231)

$\frac{61}{9}$

Solution to Exercise 4.3.45 (p. 231)

$\frac{115}{11}$ 
Solution to Exercise 4.3.47 (p. 231)

$\frac{26}{3}$

Solution to Exercise 4.3.49 (p. 232)

$\frac{107}{5}$

Solution to Exercise 4.3.51 (p. 232)

$\frac{209}{21}$

Solution to Exercise 4.3.53 (p. 232)

$\frac{9001}{100}$

Solution to Exercise 4.3.55 (p. 232)

$\frac{159}{8}$

Solution to Exercise 4.3.57 (p. 232)

... because it may be written as $5 \frac{0}{n}$, where $n$ is any positive whole number.

Solution to Exercise 4.3.59 (p. 232)

$\frac{1,652}{61}$

Solution to Exercise 4.3.61 (p. 232)

$\frac{2,436}{23}$

Solution to Exercise 4.3.63 (p. 232)

$\frac{20,419}{25}$

Solution to Exercise 4.3.65 (p. 232)

$\frac{48,803,620}{8,117}$

Solution to Exercise 4.3.67 (p. 233)

$1,009,020$

Solution to Exercise 4.3.69 (p. 233)

252

Solution to Exercise 4.4.1 (p. 234)

$6 \unrhd 6$, yes

Solution to Exercise 4.4.2 (p. 234)

$60 \cong 60$, yes

Solution to Exercise 4.4.3 (p. 234)

$30 \neq 24$, no

Solution to Exercise 4.4.4 (p. 234)

$40 \unrhd 40$, yes

Solution to Exercise 4.4.5 (p. 234)

$12 \triangleq 12$, yes

Solution to Exercise 4.4.6 (p. 236)

$\frac{1}{2}$

Solution to Exercise 4.4.7 (p. 236)

$\frac{2}{5}$

Solution to Exercise 4.4.8 (p. 236)

$\frac{1}{8}$

Solution to Exercise 4.4.9 (p. 236)

$\frac{7}{16}$

Solution to Exercise 4.4.10 (p. 236)

$\frac{12}{7}$

Solution to Exercise 4.4.11 (p. 236)

$\frac{5}{9}$

Solution to Exercise 4.4.12 (p. 237)

$\frac{3}{4}$

Solution to Exercise 4.4.13 (p. 237)

$\frac{3}{8}$ 
Solution to Exercise 4.4.14 (p. 237)

$\frac{1}{4}$

Solution to Exercise 4.4.15 (p. 237)

$\frac{3}{4}$

Solution to Exercise 4.4.16 (p. 237)

$\frac{7}{9}$

Solution to Exercise 4.4.17 (p. 237)

$\frac{5}{8}$

Solution to Exercise 4.4.18 (p. 239)

32

Solution to Exercise 4.4.19 (p. 239) 12

Solution to Exercise 4.4.20 (p. 239) 4

Solution to Exercise 4.4.21 (p. 239)

150

Solution to Exercise 4.4.22 (p. 239)

88

Solution to Exercise 4.4.23 (p. 239) equivalent

Solution to Exercise 4.4.25 (p. 239) equivalent

Solution to Exercise 4.4.27 (p. 239) not equivalent

Solution to Exercise 4.4.29 (p. 239) not equivalent

Solution to Exercise 4.4.31 (p. 240) not equivalent

Solution to Exercise 4.4.33 (p. 240) equivalent

Solution to Exercise 4.4.35 (p. 240) not equivalent

Solution to Exercise 4.4.37 (p. 240) not equivalent

Solution to Exercise 4.4.39 (p. 240) 6

Solution to Exercise 4.4.41 (p. 240) 12

Solution to Exercise 4.4.43 (p. 240)

12

Solution to Exercise 4.4.45 (p. 240)

20

Solution to Exercise 4.4.47 (p. 240) 75

Solution to Exercise 4.4.49 (p. 240) 48

Solution to Exercise 4.4.51 (p. 240) 80

Solution to Exercise 4.4.53 (p. 241) 18 
Solution to Exercise 4.4.55 (p. 241) 154

Solution to Exercise 4.4.57 (p. 241) 1,472

Solution to Exercise 4.4.59 (p. 241) 1,850

Solution to Exercise 4.4.61 (p. 241) $\frac{4}{5}$

Solution to Exercise 4.4.63 (p. 241) $\frac{3}{7}$

Solution to Exercise 4.4.65 (p. 241) $\frac{2}{7}$

Solution to Exercise 4.4.67 (p. 241) $\frac{2}{3}$

Solution to Exercise 4.4.69 (p. 241)

$\frac{5}{2}$

Solution to Exercise 4.4.71 (p. 241)

$\frac{5}{3}$

Solution to Exercise 4.4.73 (p. 241)

$\frac{7}{3}$

Solution to Exercise 4.4.75 (p. 242)

$\frac{8}{35}$

Solution to Exercise 4.4.77 (p. 242)

$\frac{5}{3}$

Solution to Exercise 4.4.79 (p. 242)

$\frac{18}{5}$

Solution to Exercise 4.4.81 (p. 242)

$\frac{2}{3}$

Solution to Exercise 4.4.83 (p. 242)

3

Solution to Exercise 4.4.85 (p. 242)

$\frac{1}{2}$

Solution to Exercise 4.4.87 (p. 242)

$\frac{1}{3}$

Solution to Exercise 4.4.89 (p. 242)

3

Solution to Exercise 4.4.91 (p. 242) 2

Solution to Exercise 4.4.93 (p. 242)

already reduced

Solution to Exercise 4.4.95 (p. 242)

$\frac{9}{25}$

Solution to Exercise 4.4.97 (p. 242)

$\frac{2}{5}$

Solution to Exercise 4.4.99 (p. 243)

$\frac{9}{8}$

Solution to Exercise 4.4.101 (p. 243)

$\frac{23}{30}$

Solution to Exercise 4.4.103 (p. 243)

$\frac{20}{9}$ 
Solution to Exercise 4.4.105 (p. 243)

$\frac{1}{3}$

Solution to Exercise 4.4.107 (p. 243)

$\frac{17}{4}$

Solution to Exercise 4.4.109 (p. 243)

$\frac{17}{18}$

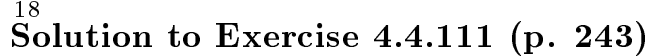

$\frac{7}{12}$

Solution to Exercise 4.4.113 (p. 243)

16

Solution to Exercise 4.4.115 (p. 243)

Should be $\frac{1}{8}$; the cancellation is division, so the numerator should be 1 .

Solution to Exercise 4.4.117 (p. 243)

Cancel factors only, not addends; $\frac{7}{15}$ is already reduced.

Solution to Exercise 4.4.119 (p. 244)

Same as Exercise 4.4.115; answer is $\frac{1}{1}$ or 1 .

Solution to Exercise 4.4.121 (p. 244)

0

Solution to Exercise 4.4.123 (p. 244)

6

Solution to Exercise 4.5.1 (p. 246)

$\frac{1}{15}$

Solution to Exercise 4.5.2 (p. 246)

$\frac{2}{9}$

Solution to Exercise 4.5.3 (p. 246)

$\frac{5}{12}$

Solution to Exercise 4.5.4 (p. 246)

$\frac{4}{9}$

Solution to Exercise 4.5.5 (p. 246)

$\frac{14}{5}$

Solution to Exercise 4.5.6 (p. 246)

$\frac{35}{48}$

Solution to Exercise 4.5.7 (p. 246)

$\frac{10}{3}$

Solution to Exercise 4.5.8 (p. 246)

$\frac{15}{2}$

Solution to Exercise 4.5.9 (p. 246)

$\frac{5}{18}$

Solution to Exercise 4.5.10 (p. 248)

$\frac{7}{12}$

Solution to Exercise 4.5.11 (p. 248)

$\frac{25}{54}$

Solution to Exercise 4.5.12 (p. 248)

$\frac{2}{3}$

Solution to Exercise 4.5.13 (p. 248)

$\frac{2}{7}$

Solution to Exercise 4.5.14 (p. 248)

$\frac{9}{2}$

Solution to Exercise 4.5.15 (p. 248)

1 
Solution to Exercise 4.5.16 (p. 248)

$\frac{14}{5}$

Solution to Exercise 4.5.17 (p. 249)

6

Solution to Exercise 4.5.18 (p. 249)

22

Solution to Exercise 4.5.19 (p. 249)

$85 \frac{1}{2}$

Solution to Exercise 4.5.20 (p. 249)

30

Solution to Exercise 4.5.21 (p. 250)

$\frac{1}{64}$

Solution to Exercise 4.5.22 (p. 250)

$\frac{9}{100}$

Solution to Exercise 4.5.23 (p. 250)

$\frac{2}{3}$

Solution to Exercise 4.5.24 (p. 250)

$\frac{1}{2}$

Solution to Exercise 4.5.25 (p. 250)

$\frac{1}{8}$

Solution to Exercise 4.5.26 (p. 250)

$8 \frac{2}{5}$

Solution to Exercise 4.5.27 (p. 250)

$8 \frac{1}{2}$

Solution to Exercise 4.5.28 (p. 250)

$\frac{1}{4}$

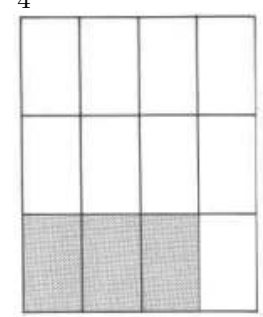

Solution to Exercise 4.5.30 (p. 251)

$\frac{1}{4}$

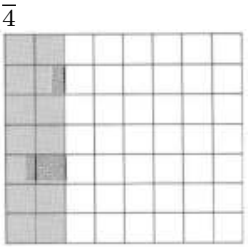

Solution to Exercise 4.5.32 (p. 251)

$\frac{1}{64}$

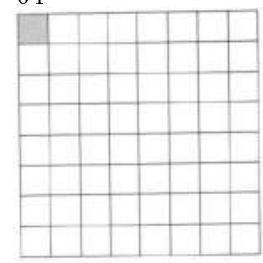

Solution to Exercise 4.5.34 (p. 251) $\frac{2}{5}$ 
Solution to Exercise 4.5.36 (p. 251)

$\frac{2}{9}$

Solution to Exercise 4.5.38 (p. 252)

$\frac{4}{15}$

Solution to Exercise 4.5.40 (p. 252)

$\frac{3}{5}$

Solution to Exercise 4.5.42 (p. 252)

2

Solution to Exercise 4.5.44 (p. 252)

$\frac{10}{3}$ or $3 \frac{1}{3}$

Solution to Exercise 4.5.46 (p. 252)

52

Solution to Exercise 4.5.48 (p. 252)

$\frac{4}{9}$

Solution to Exercise 4.5.50 (p. 252)

$\frac{1}{24}$

Solution to Exercise 4.5.52 (p. 252)

126

Solution to Exercise 4.5.54 (p. 252)

$\frac{1}{4}$

Solution to Exercise 4.5.56 (p. 252)

$\frac{1}{3}$

Solution to Exercise 4.5.58 (p. 252)

$\frac{7}{9}$

Solution to Exercise 4.5.60 (p. 253)

1

Solution to Exercise 4.5.62 (p. 253)

$\frac{28}{33}$

Solution to Exercise 4.5.64 (p. 253)

$\frac{4}{3}$

Solution to Exercise 4.5.66 (p. 253)

12

Solution to Exercise 4.5.68 (p. 253)

$7 \frac{13}{21}$ or $\frac{160}{21}$

Solution to Exercise 4.5.70 (p. 253)

12

Solution to Exercise 4.5.72 (p. 253) 18

Solution to Exercise 4.5.74 (p. 253)

$\frac{25}{3}$ or $8 \frac{1}{3}$

Solution to Exercise 4.5.76 (p. 253)

$\frac{15}{8}=1 \frac{7}{8}$

Solution to Exercise 4.5.78 (p. 253)

6

Solution to Exercise 4.5.80 (p. 253)

$\frac{10}{9}=1 \frac{1}{9}$

Solution to Exercise 4.5.82 (p. 253)

$\frac{9}{2}=4 \frac{1}{2}$

Solution to Exercise 4.5.84 (p. 254)

$\frac{85}{6}=14 \frac{1}{6}$ 
Solution to Exercise 4.5.86 (p. 254) 72

Solution to Exercise 4.5.88 (p. 254)

$\frac{4}{9}$

Solution to Exercise 4.5.90 (p. 254)

$\frac{4}{121}$

Solution to Exercise 4.5.92 (p. 254)

$\frac{1}{4}$

Solution to Exercise 4.5.94 (p. 254)

$\frac{1}{15}$

Solution to Exercise 4.5.96 (p. 254)

$\frac{1}{25}$

Solution to Exercise 4.5.98 (p. 254)

$\frac{2}{3}$

Solution to Exercise 4.5.100 (p. 254)

$\frac{9}{11}$

Solution to Exercise 4.5.102 (p. 254)

$\frac{12}{5}=2 \frac{2}{5}$

Solution to Exercise 4.5.104 (p. 255)

$\frac{1}{3}$

Solution to Exercise 4.5.106 (p. 255)

$\frac{7}{8}$

Solution to Exercise 4.5.108 (p. 255)

2

Solution to Exercise 4.5.110 (p. 255)

yes

Solution to Exercise 4.5.112 (p. 255)

$\frac{6}{25}$

Solution to Exercise 4.6.1 (p. 256)

$\frac{10}{3}$

Solution to Exercise 4.6.2 (p. 256)

$\frac{3}{2}$

Solution to Exercise 4.6.3 (p. 256)

$\frac{8}{7}$

Solution to Exercise 4.6.4 (p. 256) 5

Solution to Exercise 4.6.5 (p. 256)

$\frac{7}{16}$

Solution to Exercise 4.6.6 (p. 256)

$\frac{4}{21}$

Solution to Exercise 4.6.7 (p. 256)

$\frac{16}{163}$

Solution to Exercise 4.6.8 (p. 258)

$\frac{4}{9}$

Solution to Exercise 4.6.9 (p. 258)

1

Solution to Exercise 4.6.10 (p. 258)

$\frac{1}{2}$

Solution to Exercise 4.6.11 (p. 258)

15 
Solution to Exercise 4.6.12 (p. 258) 15

Solution to Exercise 4.6.13 (p. 259)

2

Solution to Exercise 4.6.14 (p. 259)

$\frac{2}{5}$

Solution to Exercise 4.6.15 (p. 259)

16 glasses

Solution to Exercise 4.6.16 (p. 259)

$\frac{3}{2}$

Solution to Exercise 4.6.17 (p. 259)

$\frac{8}{3}$

Solution to Exercise 4.6.18 (p. 259)

$\frac{4}{3}$

Solution to Exercise 4.6.19 (p. 259)

$\frac{2}{5}$

Solution to Exercise 4.6.20 (p. 259)

is the reciprocal of the fraction.

Solution to Exercise 4.6.21 (p. 259) $\frac{5}{4}$ or $1 \frac{1}{4}$

Solution to Exercise 4.6.23 (p. 259)

$\frac{9}{2}$ or $4 \frac{1}{2}$

Solution to Exercise 4.6.25 (p. 259)

$\frac{4}{13}$

Solution to Exercise 4.6.27 (p. 259)

$\frac{7}{23}$

Solution to Exercise 4.6.29 (p. 259)

1

Solution to Exercise 4.6.31 (p. 259)

$\frac{5}{8}$

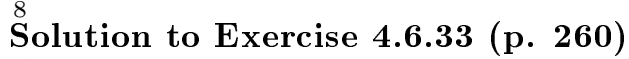

$\frac{3}{10}$

Solution to Exercise 4.6.35 (p. 260)

$\frac{225}{196}$ or $1 \frac{29}{196}$

Solution to Exercise 4.6.37 (p. 260)

$\frac{3}{5}$

Solution to Exercise 4.6.39 (p. 260)

1

Solution to Exercise 4.6.41 (p. 260)

1

Solution to Exercise 4.6.43 (p. 260)

$\frac{49}{100}$

Solution to Exercise 4.6.45 (p. 260)

$\frac{3}{5}$

Solution to Exercise 4.6.47 (p. 260)

$\frac{6}{7}$

Solution to Exercise 4.6.49 (p. 260)

$\frac{85}{6}$ or $14 \frac{1}{6}$

Solution to Exercise 4.6.51 (p. 260)

$\frac{28}{18}=\frac{14}{9}$ or $1 \frac{5}{9}$ 
Solution to Exercise 4.6.53 (p. 260) 10

Solution to Exercise 4.6.55 (p. 261) $\frac{10}{3}$ or $3 \frac{1}{3}$

Solution to Exercise 4.6.57 (p. 261)

$\frac{4}{21}$

Solution to Exercise 4.6.59 (p. 261) $\frac{3}{2}$ or $1 \frac{1}{2}$

Solution to Exercise 4.6.61 (p. 261) $\frac{4}{5}$

Solution to Exercise 4.6.63 (p. 261) 3

Solution to Exercise 4.6.65 (p. 261) 1

Solution to Exercise 4.6.67 (p. 261) 321,600

Solution to Exercise 4.6.69 (p. 261) 144

Solution to Exercise 4.7.1 (p. 263)

$\frac{2}{5}$

Solution to Exercise 4.7.2 (p. 263) $\frac{3}{4}$

Solution to Exercise 4.7.3 (p. 263)

$\frac{1}{6}$

Solution to Exercise 4.7.4 (p. 265)

$\frac{3}{4}$

Solution to Exercise 4.7.5 (p. 265)

$\frac{16}{27}$

Solution to Exercise 4.7.6 (p. 265)

$1 \frac{1}{2}$

Solution to Exercise 4.7.7 (p. 265)

$1 \frac{1}{2}$

Solution to Exercise 4.7.8 (p. 265)

$\frac{1}{2}$

Solution to Exercise 4.7.10 (p. 265)

$\frac{1}{3}$

Solution to Exercise 4.7.12 (p. 265)

$\frac{9}{8}$ or $1 \frac{1}{8}$

Solution to Exercise 4.7.14 (p. 265)

$\frac{1}{16}$

Solution to Exercise 4.7.16 (p. 265)

$\frac{3}{4}$

Solution to Exercise 4.7.18 (p. 265)

$\frac{1}{1,000}$

Solution to Exercise 4.7.20 (p. 265)

$\frac{10}{27}$

Solution to Exercise 4.7.22 (p. 265)

$\frac{1}{2}$

Solution to Exercise 4.7.24 (p. 266)

2 
Solution to Exercise 4.7.26 (p. 266)

$\frac{1}{6}$

Solution to Exercise 4.7.28 (p. 266)

$\frac{2}{3}$

Solution to Exercise 4.7.30 (p. 266)

1

Solution to Exercise 4.7.32 (p. 266)

1

Solution to Exercise 4.7.34 (p. 266)

$\frac{3}{2}$ or $1 \frac{2}{3}$

Solution to Exercise 4.7.36 (p. 266)

3

Solution to Exercise 4.7.38 (p. 266)

$\frac{5}{3}$ or $1 \frac{2}{3}$

Solution to Exercise 4.7.40 (p. 266)

$\frac{27}{40}$

Solution to Exercise 4.7.42 (p. 266)

$\frac{1}{15}$

Solution to Exercise 4.7.44 (p. 266)

$\frac{3}{8}$

Solution to Exercise 4.7.46 (p. 267)

$\frac{9}{10}$

Solution to Exercise 4.7.48 (p. 267)

$\frac{9}{2}=4 \frac{1}{2}$

Solution to Exercise 4.7.50 (p. 267)

$\frac{16}{11}$ or $1 \frac{5}{11}$

Solution to Exercise 4.7.52 (p. 267)

$\frac{30}{77}$

Solution to Exercise 4.7.54 (p. 267)

no

Solution to Exercise 4.7.56 (p. 267)

$\frac{41}{12}$

Solution to Exercise 4.9.1 (p. 270)

$\frac{2}{6}$ or $\frac{1}{3}$

Solution to Exercise 4.9.3 (p. 270)

numerator, 4; denominator, 5

Solution to Exercise 4.9.5 (p. 271)

numerator, 1 ; denominator, 3

Solution to Exercise 4.9.7 (p. 271)

$\frac{8}{11}$

Solution to Exercise 4.9.9 (p. 271)

$\frac{200}{6,000}$

Solution to Exercise 4.9.11 (p. 271)

ten seventeenths

Solution to Exercise 4.9.13 (p. 271)

six hundred six, one thousand four hundred thirty-firsts

Solution to Exercise 4.9.15 (p. 271)

one sixteenth

Solution to Exercise 4.9.17 (p. 271)

numerator, 56; denominator, 14,190 
Solution to Exercise 4.9.19 (p. 271)

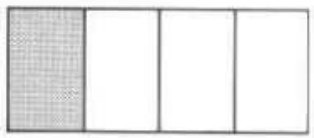

Solution to Exercise 4.9.21 (p. 272) $2 \frac{3}{4}$

Solution to Exercise 4.9.23 (p. 272) $6 \frac{3}{8}$

Solution to Exercise 4.9.25 (p. 272) $118 \frac{2}{3}$

Solution to Exercise 4.9.27 (p. 272) $1 \frac{1}{4}$

Solution to Exercise 4.9.29 (p. 272) 3

Solution to Exercise 4.9.31 (p. 272) $\frac{129}{8}$

Solution to Exercise 4.9.33 (p. 272)

$\frac{16}{5}$

Solution to Exercise 4.9.35 (p. 272)

$\frac{377}{21}$

Solution to Exercise 4.9.37 (p. 272) $\frac{3}{2}$

Solution to Exercise 4.9.39 (p. 273) $\frac{62}{7}$

Solution to Exercise 4.9.41 (p. 273) because the whole number part is zero

Solution to Exercise 4.9.43 (p. 273) equivalent

Solution to Exercise 4.9.45 (p. 273) not equivalent

Solution to Exercise 4.9.47 (p. 273) not equivalent

Solution to Exercise 4.9.49 (p. 273) $\frac{8}{11}$

Solution to Exercise 4.9.51 (p. 273) $\frac{5}{11}$

Solution to Exercise 4.9.53 (p. 273) $\frac{3}{5}$

Solution to Exercise 4.9.55 (p. 273)

$\frac{9}{17}$

Solution to Exercise 4.9.57 (p. 273) $\frac{35}{68}$

Solution to Exercise 4.9.59 (p. 274)

$\frac{65}{162}$

Solution to Exercise 4.9.61 (p. 274)

15

Solution to Exercise 4.9.63 (p. 274) 6

Solution to Exercise 4.9.65 (p. 274) 27 
Solution to Exercise 4.9.67 (p. 274)

42

Solution to Exercise 4.9.69 (p. 274)

168

Solution to Exercise 4.9.71 (p. 274)

192

Solution to Exercise 4.9.73 (p. 274)

$\frac{3}{4}$

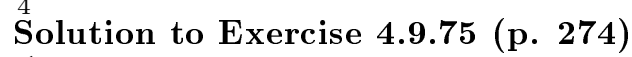

$\frac{1}{24}$

Solution to Exercise 4.9.77 (p. 274)

$\frac{5}{36}$

Solution to Exercise 4.9.79 (p. 275) 1

Solution to Exercise 4.9.81 (p. 275) $\frac{1}{48}$

Solution to Exercise 4.9.83 (p. 275)

$\frac{4}{35}$

Solution to Exercise 4.9.85 (p. 275) $\frac{50}{7}=7 \frac{1}{7}$

Solution to Exercise 4.9.87 (p. 275) 2

Solution to Exercise 4.9.89 (p. 275) 3

Solution to Exercise 4.9.91 (p. 275) $\frac{1}{2}$

Solution to Exercise 4.9.93 (p. 275) 90

Solution to Exercise 4.9.95 (p. 275) 1

Solution to Exercise 4.9.97 (p. 276) $\frac{7}{2}$ or $3 \frac{1}{2}$

Solution to Exercise 4.9.99 (p. 276) 4

Solution to Exercise 4.9.101 (p. 276) $\frac{12}{13}$

Solution to Exercise 4.9.103 (p. 276) 4

Solution to Exercise 4.9.105 (p. 276)

$\frac{11}{12}$

Solution to Exercise 4.10.1 (p. 276)

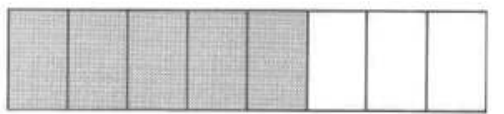

Solution to Exercise 4.10.2 (p. 276)

Numerator, 5; denominator, 9

Solution to Exercise 4.10.3 (p. 276)

$\frac{5}{11}$

Solution to Exercise 4.10.4 (p. 276)

Four fifths 
Solution to Exercise 4.10.5 (p. 276) $\frac{5}{12}$

Solution to Exercise 4.10.6 (p. 276) $\frac{25}{7}$

Solution to Exercise 4.10.7 (p. 276) $3 \frac{1}{5}$

Solution to Exercise 4.10.8 (p. 277) yes

Solution to Exercise 4.10.9 (p. 277) $\frac{3}{5}$

Solution to Exercise 4.10.10 (p. 277) $\frac{5}{17}$

Solution to Exercise 4.10.11 (p. 277) $\frac{13}{60}$

Solution to Exercise 4.10.12 (p. 277) 20

Solution to Exercise 4.10.13 (p. 277) 24

Solution to Exercise 4.10.14 (p. 277) $\frac{3}{20}$

Solution to Exercise 4.10.15 (p. 277) 55

Solution to Exercise 4.10.16 (p. 277) $\frac{5}{6}$

Solution to Exercise 4.10.17 (p. 277) $\frac{3}{4}$

Solution to Exercise 4.10.18 (p. 277)

$\frac{1}{2}$

Solution to Exercise 4.10.19 (p. 277)

$\frac{1}{30}$

Solution to Exercise 4.10.20 (p. 277) $\frac{1}{11}$

Solution to Exercise 4.10.21 (p. 277) $\frac{36}{25}=1 \frac{11}{25}$

Solution to Exercise 4.10.22 (p. 277) $\frac{2}{5}$

Solution to Exercise 4.10.23 (p. 277) $\frac{1}{4}$

Solution to Exercise 4.10.24 (p. 277) $\frac{9}{5}=1 \frac{4}{5}$

Solution to Exercise 4.10.25 (p. 277) $\frac{4}{3}$ or $1 \frac{1}{3}$ 


\section{Chapter 5}

\section{Addition and Subtraction of Fractions, Comparing Fractions, and Complex Fractions}

\subsection{Objectives $^{1}$}

After completing this chapter, you should

Addition and Subtraction of Fractions with Like Denominators (Section 5.2)

- be able to add and subtract fractions with like denominators

Addition and Subtraction of Fractions with Unlike Denominators (Section 5.3)

- be able to add and subtract fractions with unlike denominators

Addition and Subtraction of Mixed Numbers (Section 5.4)

- be able to add and subtract mixed numbers

Comparing Fractions (Section 5.5)

- understand ordering of numbers and be familiar with grouping symbols

- be able to compare two or more fractions

Complex Fractions (Section 5.6)

- be able to distinguish between simple and complex fractions

- be able to convert a complex fraction to a simple fraction

Combinations of Operations with Fractions (Section 5.7)

- gain a further understanding of the order of operations

\footnotetext{
${ }^{1}$ This content is available online at $<$ http://cnx.org/content $/ \mathrm{m} 18893 / 1.3 />$.
} 


\subsection{Addition and Subtraction of Fractions with Like Denominators}

\subsubsection{Section Overview}

- Addition of Fraction With Like Denominators

- Subtraction of Fractions With Like Denominators

\subsubsection{Addition of Fraction With Like Denominators}

Let's examine the following diagram.

\begin{tabular}{|l|l|l|l|l|}
\hline$\frac{1}{5}$ & $\frac{1}{5}$ & $\frac{1}{5}$ & $\frac{1}{5}$ & $\frac{1}{5}$ \\
\hline
\end{tabular}

2 one-fifths and 1 one fifth is shaded.

It is shown in the shaded regions of the diagram that

$(2$ one-fifths $)+(1$ one-fifth $)=(3$ one-fifths $)$

That is,

$\frac{2}{5}+\frac{1}{5}=\frac{3}{5}$

From this observation, we can suggest the following rule.

Method of Adding Fractions Having Like Denominators

To add two or more fractions that have the same denominators, add the numerators and place the resulting sum over the common denominator. Reduce, if necessary.

\subsubsection{Sample Set A}

Find the following sums.

Example 5.1

$\frac{3}{7}+\frac{2}{7}$. The denominators are the same. Add the numerators and place that sum over 7 .

$\frac{3}{7}+\frac{2}{7}=\frac{3+2}{7}=\frac{5}{7}$

Example 5.2

$\frac{1}{8}+\frac{3}{8}$. The denominators are the same. Add the numerators and place the sum over 8 . Reduce.

$\frac{1}{8}+\frac{3}{8}=\frac{1+3}{8}=\frac{4}{8}=\frac{1}{2}$

Example 5.3

$\frac{4}{9}+\frac{5}{9}$. The denominators are the same. Add the numerators and place the sum over 9 .

$\frac{4}{9}+\frac{5}{9}=\frac{4+5}{9}=\frac{9}{9}=1$

Example 5.4

$\frac{7}{8}+\frac{5}{8}$. The denominators are the same. Add the numerators and place the sum over 8 .

$\frac{7}{8}+\frac{5}{8}=\frac{7+5}{8}=\frac{12}{8}=\frac{3}{2}$

\footnotetext{
${ }^{2}$ This content is available online at $<$ http://cnx.org/content $/ \mathrm{m} 34934 / 1.2 />$.
} 


\section{Example 5.5}

To see what happens if we mistakenly add the denominators as well as the numerators, let's add $\frac{1}{2}+\frac{1}{2}$

Adding the numerators and mistakenly adding the denominators produces

$\frac{1}{2}+\frac{1}{2}=\frac{1+1}{2+2}=\frac{2}{4}=\frac{1}{2}$

This means that two $\frac{1}{2}$ 's is the same as one $\frac{1}{2}$. Preposterous! We do not add denominators.

\subsubsection{Practice Set A}

Find the following sums.

Exercise 5.2.1

(Solution on p. 329.)

$\frac{1}{10}+\frac{3}{10}$

Exercise 5.2.2

(Solution on p. 329.)

$\frac{1}{4}+\frac{1}{4}$

Exercise 5.2.3

$\frac{7}{11}+\frac{4}{11}$

Exercise 5.2.4

$\frac{3}{5}+\frac{1}{5}$

(Solution on p. 329.)

(Solution on p. 329.)

Exercise 5.2.5

(Solution on p. 329.)

Show why adding both the numerators and denominators is preposterous by adding $\frac{3}{4}$ and $\frac{3}{4}$ and examining the result.

\subsubsection{Subtraction of Fractions With Like Denominators}

We can picture the concept of subtraction of fractions in much the same way we pictured addition.

\begin{tabular}{|l|l|l|l|l|}
\hline$\frac{1}{5}$ & $\frac{1}{5}$ & $\frac{1}{5}$ & $\frac{1}{5}$ & $\frac{1}{5}$ \\
\hline
\end{tabular}

$\frac{3}{5}$

(3 one-fifths)

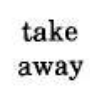

$-$

\begin{tabular}{|l|l|l|l|l|}
\hline$\frac{1}{5}$ & $\frac{1}{5}$ & $\frac{1}{5}$ & $\frac{1}{5}$ & $\frac{1}{5}$ \\
\hline
\end{tabular}

$\frac{1}{5}$

(1 one-fifth)

\begin{tabular}{l|l|l|l|l}
\hline$\frac{1}{5}$ & 15 \\
\hline
\end{tabular}

$=\frac{2}{5}$

From this observation, we can suggest the following rule for subtracting fractions having like denominators:

\section{Subtraction of Fractions with Like Denominators}

To subtract two fractions that have like denominators, subtract the numerators and place the resulting difference over the common denominator. Reduce, if possible.

\subsubsection{Sample Set B}

Find the following differences.

Example 5.6

$\frac{3}{5}-\frac{1}{5}$. The denominators are the same. Subtract the numerators. Place the difference over 5 .

$\frac{3}{5}-\frac{1}{5}=\frac{3-1}{5}=\frac{2}{5}$ 


\section{Example 5.7}

$\frac{8}{6}-\frac{2}{6}$. The denominators are the same. Subtract the numerators. Place the difference over 6 .

$\frac{8}{6}-\frac{2}{6}=\frac{8-2}{6}=\frac{6}{6}=1$

Example 5.8

$\frac{16}{9}-\frac{2}{9}$. The denominators are the same. Subtract numerators and place the difference over 9 .

$\frac{16}{9}-\frac{2}{9}=\frac{16-2}{9}=\frac{14}{9}$

\section{Example 5.9}

To see what happens if we mistakenly subtract the denominators, let's consider

$\frac{7}{15}-\frac{4}{15}=\frac{7-4}{15-15}=\frac{3}{0}$

We get division by zero, which is undefined. We do not subtract denominators.

\subsubsection{Practice Set B}

Find the following differences.

Exercise 5.2.6

$\frac{10}{13}-\frac{8}{13}$

Exercise 5.2.7

$\frac{5}{12}-\frac{1}{12}$

Exercise 5.2.8

$\frac{1}{2}-\frac{1}{2}$

Exercise 5.2.9

$\frac{26}{10}-\frac{14}{10}$

Exercise 5.2.10

(Solution on p. 329.)

(Solution on p. 329.)

(Solution on p. 329.)

(Solution on p. 329.)

Show why subtracting both the numerators and the denominators is in error by performing the subtraction $\frac{5}{9}-\frac{2}{9}$.

\subsubsection{Exercises}

For the following problems, find the sums and differences. Be sure to reduce.

Exercise 5.2.11

$$
\frac{3}{8}+\frac{2}{8}
$$

(Solution on p. 329.)

Exercise 5.2.12

$$
\frac{1}{6}+\frac{2}{6}
$$

Exercise 5.2.13

$$
\frac{9}{10}+\frac{1}{10}
$$

(Solution on p. 329.)

Exercise 5.2.14

$\frac{3}{11}+\frac{4}{11}$

Exercise 5.2.15

$\frac{9}{15}+\frac{4}{15}$

Exercise 5.2.16

$$
\frac{3}{10}+\frac{2}{10}
$$

(Solution on p. 329.) 
Exercise 5.2.17

(Solution on p. 329.)

$\frac{5}{12}+\frac{7}{12}$

Exercise 5.2.18

$\frac{11}{16}-\frac{2}{16}$

Exercise 5.2.19

$\frac{3}{16}-\frac{3}{16}$

(Solution on p. 329.)

Exercise 5.2.20

$\frac{15}{23}-\frac{2}{23}$

Exercise 5.2.21

$\frac{1}{6}-\frac{1}{6}$

(Solution on p. 329.)

Exercise 5.2.22

$\frac{1}{4}+\frac{1}{4}+\frac{1}{4}$

Exercise 5.2.23

$\frac{3}{11}+\frac{1}{11}+\frac{5}{11}$

Exercise 5.2.24

$\frac{16}{20}+\frac{1}{20}+\frac{2}{20}$

Exercise 5.2.25

$\frac{12}{8}+\frac{2}{8}+\frac{1}{8}$

Exercise 5.2.26

$\frac{1}{15}+\frac{8}{15}+\frac{6}{15}$

Exercise 5.2.27

$\frac{3}{8}+\frac{2}{8}-\frac{1}{8}$

(Solution on p. 329.)

Exercise 5.2.28

$\frac{11}{16}+\frac{9}{16}-\frac{5}{16}$

Exercise 5.2.29

$\frac{4}{20}-\frac{1}{20}+\frac{9}{20}$

Exercise 5.2.30

$\frac{7}{10}-\frac{3}{10}+\frac{11}{10}$

Exercise 5.2.31

$\frac{16}{5}-\frac{1}{5}-\frac{2}{5}$

(Solution on p. 329.)

Exercise 5.2.32

$\frac{21}{35}-\frac{17}{35}+\frac{31}{35}$

Exercise 5.2.33

$\frac{5}{2}+\frac{16}{2}-\frac{1}{2}$

Exercise 5.2.34

$\frac{1}{18}+\frac{3}{18}+\frac{1}{18}+\frac{4}{18}-\frac{5}{18}$

Exercise 5.2.35

$\frac{6}{22}-\frac{2}{22}+\frac{4}{22}-\frac{1}{22}+\frac{11}{22}$

(Solution on p. 329.)

(Solution on p. 329.)

(Solution on p. 329.)

(Solution on p. 329.)

(Solution on p. 329.)

The following rule for addition and subtraction of two fractions is preposterous. Show why by performing the operations using the rule for the following two problems.

\section{Preposterous Rule}

To add or subtract two fractions, simply add or subtract the numerators and place this result over the sum or difference of the denominators.

Exercise 5.2.36

$$
\frac{3}{10}-\frac{3}{10}
$$


Exercise 5.2.37

(Solution on p. 329.)

$\frac{8}{15}+\frac{8}{15}$

Exercise 5.2.38

Find the total length of the screw.

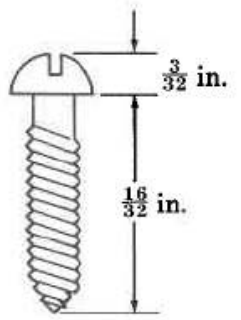

Exercise 5.2.39

(Solution on p. 329.)

Two months ago, a woman paid off $\frac{3}{24}$ of a loan. One month ago, she paid off $\frac{5}{24}$ of the total loan. This month she will again pay off $\frac{5}{24}$ of the total loan. At the end of the month, how much of her total loan will she have paid off?

Exercise 5.2.40

Find the inside diameter of the pipe.

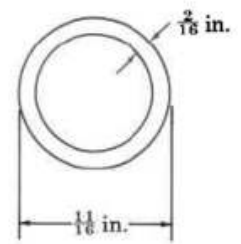

\subsubsection{Exercises for Review}

Exercise 5.2.41

(Solution on p. 329.)

(Section 1.4) Round 2,650 to the nearest hundred.

Exercise 5.2.42

(Section 2.6) Use the numbers 2, 4, and 8 to illustrate the associative property of addition.

Exercise 5.2.43

(Solution on p. 330.)

(Section 3.4) Find the prime factors of 495.

Exercise 5.2.44

(Section 4.5) Find the value of $\frac{3}{4} \cdot \frac{16}{25} \cdot \frac{5}{9}$.

Exercise 5.2.45

(Section 4.7 ) $\frac{8}{3}$ of what number is $1 \frac{7}{9}$ ?

(Solution on p. 330.)

\subsection{Addition and Subtraction of Fractions with Unlike Denominators ${ }^{3}$}

\subsubsection{Section Overview}

- A Basic Rule

- Addition and Subtraction of Fractions

${ }^{3}$ This content is available online at $<\mathrm{http}: / / \mathrm{cnx}$.org/content $/ \mathrm{m} 34935 / 1.2 />$. 


\subsubsection{A Basic Rule}

There is a basic rule that must be followed when adding or subtracting fractions.

A Basic Rule

Fractions can only be added or subtracted conveniently if they have like denominators.

To see why this rule makes sense, let's consider the problem of adding a quarter and a dime.

1 quarter +1 dime $=35$ cents

Now,

1 quarter $\left.=\frac{25}{100}\right\} \quad$ same denominations

1 dime $=\frac{10}{100}$

$$
35,=\frac{35}{100}
$$

$\frac{25}{100}+\frac{10}{100}=\frac{25+10}{100}=\frac{35}{100}$

In order to combine a quarter and a dime to produce $35 \$$, we convert them to quantities of the same denomination.

Same denomination $\rightarrow$ same denominator

\subsubsection{Addition and Subtraction of Fractions}

\section{Least Common Multiple (LCM) and Least Common Denominator (LCD)}

In Section 3.6, we examined the least common multiple (LCM) of a collection of numbers. If these numbers are used as denominators of fractions, we call the least common multiple, the least common denominator (LCD).

\section{Method of Adding or Subtracting Fractions with Unlike Denominators}

To add or subtract fractions having unlike denominators, convert each fraction to an equivalent fraction having as a denominator the least common denominator ( LCD) of the original denominators.

\subsubsection{Sample Set A}

Find the following sums and differences.

\section{Example 5.10}

$\frac{1}{6}+\frac{3}{4}$. The denominators are not the same. Find the LCD of 6 and 4 .

$\left.\begin{array}{l}6=2 \cdot 3 \\ 4=2^{2}\end{array}\right\}$ The $\mathrm{LCD}=2^{2} \cdot 3=4 \cdot 3=12$

Write each of the original fractions as a new, equivalent fraction having the common denominator 12 .

$\frac{1}{6}+\frac{3}{4}=\frac{1}{12}+\frac{1}{12}$

To find a new numerator, we divide the original denominator into the LCD. Since the original denominator is being multiplied by this quotient, we must multiply the original numerator by this quotient. 


\section{Multiply 1 by 2: $\quad 1 \cdot 2=2$. \\ original numerator \\ $12 \div 6=2$ \\ new numerator}

\section{Multiply 3 by $3: \quad 3 \cdot 3=9$. \\ original numerator \\ new numerator}

$12 \div 4=3$

$$
\begin{aligned}
\frac{1}{6}+\frac{3}{4} & =\frac{1 \cdot 2}{12}+\frac{3 \cdot 3}{12} \\
& =\frac{2}{12}+\frac{9}{12} \\
& =\frac{2+9}{12} \\
& =\frac{11}{12}
\end{aligned}
$$

Now the denominators are the same.

Add the numerators and place the sum over the common denominator.

Example 5.11

$\frac{1}{2}+\frac{2}{3}$. The denominators are not the same. Find the LCD of 2 and 3 .

$\mathrm{LCD}=2 \cdot 3=6$

Write each of the original fractions as a new, equivalent fraction having the common denominator 6.

$\frac{1}{2}+\frac{2}{3}=\overline{6}+\overline{6}$

To find a new numerator, we divide the original denominator into the LCD. Since the original denominator is being multiplied by this quotient, we must multiply the original numerator by this quotient.

$6 \div 2=3$ Multiply the numerator 1 by 3 .

$6 \div 2=3$ Multiply the numerator 2 by 2 .

$$
\begin{aligned}
\frac{1}{2}+\frac{2}{3} & =\frac{1 \cdot 3}{6}+\frac{2 \cdot 3}{6} \\
& =\frac{3}{6}+\frac{4}{6} \\
& =\frac{3+4}{6} \\
& =\frac{7}{6} \text { or } 1 \frac{1}{6}
\end{aligned}
$$

Example 5.12

$\frac{5}{9}-\frac{5}{12}$. The denominators are not the same. Find the LCD of 9 and 12 .

$$
\left.\begin{array}{c}
9=3 \cdot 3=3^{2} \\
12=2 \cdot 6=2 \cdot 2 \cdot 3=2^{2} \cdot 3
\end{array}\right\} \quad \mathrm{LCD}=2^{2} \cdot 3^{2}=4 \cdot 9=36
$$


$\frac{5}{9}-\frac{5}{12}=\frac{}{36}-\overline{36}$

$36 \div 9=4$ Multiply the numerator 5 by 4 .

$36 \div 12=3$ Multiply the numerator 5 by 3 .

$$
\begin{aligned}
\frac{5}{9}-\frac{5}{12} & =\frac{5 \cdot 4}{36}-\frac{5 \cdot 3}{36} \\
& =\frac{20}{36}-\frac{15}{36} \\
& =\frac{20-15}{36} \\
& =\frac{5}{36}
\end{aligned}
$$

Example 5.13

$\frac{5}{6}-\frac{1}{8}+\frac{7}{16}$ The denominators are not the same. Find the LCD of 6,8 , and 16

$$
\left.\begin{array}{rlc}
6 & = & 2 \cdot 3 \\
8 & = & 2 \cdot 4=2 \cdot 2 \cdot 2=2^{3} \\
16 & = & 2 \cdot 8=2 \cdot 2 \cdot 4=2 \cdot 2 \cdot 2 \cdot 2=2^{4}
\end{array}\right\} \text { The LCD is } 2^{4} \cdot 3=48
$$

$\frac{5}{6}-\frac{1}{8}+\frac{7}{16}=\frac{}{48}-\frac{}{48}+\overline{48}$

$48 \div 6=8$ Multiply the numerator 5 by 8

$48 \div 8=6$ Multiply the numerator 1 by 6

$48 \div 16=3$ Multiply the numerator 7 by 3

$$
\begin{aligned}
\frac{5}{6}-\frac{1}{8}+\frac{7}{16} & =\frac{5 \cdot 8}{48}-\frac{1 \cdot 6}{48}+\frac{7 \cdot 3}{48} \\
& =\frac{40}{48}-\frac{6}{48}+\frac{21}{48} \\
& =\frac{40-6+21}{48} \\
& =\frac{55}{48} \text { or } 1 \frac{7}{48}
\end{aligned}
$$

\subsubsection{Practice Set A}

Find the following sums and differences.

Exercise 5.3.1

(Solution on p. 330.)

$\frac{3}{4}+\frac{1}{12}$

Exercise 5.3.2

$\frac{1}{2}-\frac{3}{7}$

(Solution on p. 330.)

Exercise 5.3.3

(Solution on p. 330.)

$\frac{7}{10}-\frac{5}{8}$

Exercise 5.3.4

$\frac{15}{16}+\frac{1}{2}-\frac{3}{4}$

Exercise 5.3.5

$\frac{1}{32}-\frac{1}{48}$

(Solution on p. 330.)

(Solution on p. 330.) 


\subsubsection{Exercises}

Exercise 5.3.6

(Solution on p. 330.)

A most basic rule of arithmetic states that two fractions may be added or subtracted conveniently only if they have

For the following problems, find the sums and differences.

Exercise 5.3.7

$$
\frac{1}{2}+\frac{1}{6}
$$

Exercise 5.3.8

$$
\frac{1}{8}+\frac{1}{2}
$$

(Solution on p. 330.)

Exercise 5.3.9

$$
\frac{3}{4}+\frac{1}{3}
$$

Exercise 5.3.10

(Solution on p. 330.)

$$
\frac{5}{8}+\frac{2}{3}
$$

Exercise 5.3.11

$$
\frac{1}{12}+\frac{1}{3}
$$

Exercise 5.3.12 $\frac{6}{7}-\frac{1}{4}$

(Solution on p. 330.)

Exercise 5.3.13

$$
\frac{9}{10}-\frac{2}{5}
$$

Exercise 5.3.14 $\frac{7}{9}-\frac{1}{4}$

(Solution on p. 330.)

Exercise 5.3.15 $\frac{8}{15}-\frac{3}{10}$

Exercise 5.3.16 $\frac{8}{13}-\frac{5}{39}$

Exercise 5.3.17 $\frac{11}{12}-\frac{2}{5}$

Exercise 5.3.18 $\frac{1}{15}+\frac{5}{12}$

Exercise 5.3.19 $\frac{13}{88}-\frac{1}{4}$

Exercise 5.3.20 $\frac{1}{9}-\frac{1}{81}$

(Solution on p. 330.)

Exercise 5.3.21 $\frac{19}{40}+\frac{5}{12}$

Exercise 5.3.22 $\frac{25}{26}-\frac{7}{10}$

(Solution on p. 330.)

Exercise 5.3.23 $\frac{9}{28}-\frac{4}{45}$

Exercise 5.3.24 $\frac{22}{45}-\frac{16}{35}$

(Solution on p. 330.)

Exercise 5.3.25 $\frac{56}{63}+\frac{22}{33}$

Exercise 5.3.26 $\frac{1}{16}+\frac{3}{4}-\frac{3}{8}$

(Solution on p. 330.)

(Solution on p. 330.)

(Solution on p. 330.) 
Exercise 5.3.27 $\frac{5}{12}-\frac{1}{120}+\frac{19}{20}$

Exercise 5.3.28 $\frac{8}{3}-\frac{1}{4}+\frac{7}{36}$

(Solution on p. 330.)

Exercise 5.3.29 $\frac{11}{9}-\frac{1}{7}+\frac{16}{63}$

Exercise 5.3.30 $\frac{12}{5}-\frac{2}{3}+\frac{17}{10}$

(Solution on p. 330.)

Exercise 5.3.31 $\frac{4}{9}+\frac{13}{21}-\frac{9}{14}$

Exercise 5.3.32 $\frac{3}{4}-\frac{3}{22}+\frac{5}{24}$

Exercise 5.3.33 $\frac{25}{48}-\frac{7}{88}+\frac{5}{24}$

Exercise 5.3.34 $\frac{27}{40}+\frac{47}{48}-\frac{119}{126}$

Exercise 5.3.35 $\frac{41}{44}-\frac{5}{99}-\frac{11}{175}$

Exercise 5.3.36 $\frac{5}{12}+\frac{1}{18}+\frac{1}{24}$

Exercise 5.3.37 $\frac{5}{9}+\frac{1}{6}+\frac{7}{15}$

Exercise 5.3.38 $\frac{21}{25}+\frac{1}{6}+\frac{7}{15}$

Exercise 5.3.39 $\frac{5}{18}-\frac{1}{36}+\frac{7}{9}$

Exercise 5.3.40 $\frac{11}{14}-\frac{1}{36}-\frac{1}{32}$

Exercise 5.3.41 $\frac{21}{33}+\frac{12}{22}+\frac{15}{55}$

Exercise 5.3.42 $\frac{5}{51}+\frac{2}{34}+\frac{11}{68}$

Exercise 5.3.43 $\frac{8}{7}-\frac{16}{14}+\frac{19}{21}$

Exercise 5.3.44 $\frac{7}{15}+\frac{3}{10}-\frac{34}{60}$

(Solution on p. 330.)

(Solution on p. 330.)

(Solution on p. 330.)

(Solution on p. 330.)

(Solution on p. 330.)

(Solution on p. 331.)

Exercise 5.3.45 $\frac{14}{15}-\frac{3}{10}-\frac{6}{25}+\frac{7}{20}$

Exercise 5.3.46 $\frac{11}{6}-\frac{5}{12}+\frac{17}{30}+\frac{25}{18}$

(Solution on p. 331.)

Exercise 5.3.47 $\frac{1}{9}+\frac{22}{21}-\frac{5}{18}-\frac{1}{45}$

Exercise 5.3.48 $\frac{7}{26}+\frac{28}{65}-\frac{51}{104}+0$

(Solution on p. 331.)

(Solution on p. 331.) 


\section{Exercise 5.3.49}

A morning trip from San Francisco to Los Angeles took $\frac{13}{12}$ hours. The return trip took $\frac{57}{60}$ hours. How much longer did the morning trip take?

Exercise 5.3.50

(Solution on p. 331.)

At the beginning of the week, Starlight Publishing Company's stock was selling for $\frac{115}{8}$ dollars per share. At the end of the week, analysts had noted that the stock had gone up $\frac{11}{4}$ dollars per share. What was the price of the stock, per share, at the end of the week?

\section{Exercise 5.3.51}

A recipe for fruit punch calls for $\frac{23}{3}$ cups of pineapple juice, $\frac{1}{4}$ cup of lemon juice, $\frac{15}{2}$ cups of orange juice, 2 cups of sugar, 6 cups of water, and 8 cups of carbonated non-cola soft drink. How many cups of ingredients will be in the final mixture?

Exercise 5.3.52

(Solution on p. 331.)

The side of a particular type of box measures $8 \frac{3}{4}$ inches in length. Is it possible to place three such boxes next to each other on a shelf that is $26 \frac{1}{5}$ inches in length? Why or why not?

Exercise 5.3.53

Four resistors, $\frac{3}{8} \mathrm{ohm}, \frac{1}{4} \mathrm{ohm}, \frac{3}{5} \mathrm{ohm}$, and $\frac{7}{8} \mathrm{ohm}$, are connected in series in an electrical circuit. What is the total resistance in the circuit due to these resistors? ("In series" implies addition.)

\section{Exercise 5.3.54}

(Solution on p. 331.)

A copper pipe has an inside diameter of $2 \frac{3}{16}$ inches and an outside diameter of $2 \frac{5}{34}$ inches. How thick is the pipe?

\section{Exercise 5.3.55}

The probability of an event was originally thought to be $\frac{15}{32}$. Additional information decreased the probability by $\frac{3}{14}$. What is the updated probability?

\subsubsection{Exercises for Review}

Exercise 5.3.56

(Solution on p. 331.)

(Section 1.6) Find the difference between 867 and 418.

Exercise 5.3.57

(Section 2.5) Is 81,147 divisible by 3 ?

Exercise 5.3.58

(Solution on p. 331.)

(Section 3.6) Find the LCM of 11, 15, and 20.

Exercise 5.3.59

(Section 4.5) Find $\frac{3}{4}$ of $4 \frac{2}{9}$.

Exercise 5.3.60

(Section 5.2) Find the value of $\frac{8}{15}-\frac{3}{15}+\frac{2}{15}$.

(Solution on p. 331.)

\subsection{Addition and Subtraction of Mixed Numbers ${ }^{4}$}

\subsubsection{Section Overview}

- The Method of Converting to Improper Fractions

To add or subtract mixed numbers, convert each mixed number to an improper fraction, then add or subtract the resulting improper fractions.

\footnotetext{
${ }^{4}$ This content is available online at $<$ http://cnx.org/content/m34936/1.2/>.
} 


\subsubsection{Sample Set A}

Find the following sums and differences.

Example 5.14

$8 \frac{3}{5}+5 \frac{1}{4}$. Convert each mixed number to an improper fraction.

$8 \frac{3}{5}=\frac{5 \cdot 8+3}{5}=\frac{40+3}{5}=\frac{43}{5}$

$5 \frac{1}{4}=\frac{4 \cdot 5+1}{4}=\frac{20+1}{4}=\frac{21}{4}$ Now add the improper fractions $\frac{43}{5}$ and $\frac{21}{4}$.

$\frac{43}{5}+\frac{21}{4}$ The LCD $=20$.

$$
\begin{aligned}
\frac{43}{5}+\frac{21}{4} & =\frac{43 \cdot 4}{20}+\frac{21 \cdot 5}{20} \\
& =\frac{172}{20}+\frac{105}{20} \\
& =\frac{172+105}{20} \\
& =\frac{277}{20} \quad \text { Convert this improper fraction to a mixed number. } \\
& =13 \frac{17}{20}
\end{aligned}
$$

Thus, $8 \frac{3}{5}+5 \frac{1}{4}=13 \frac{17}{20}$.

\section{Example 5.15}

$3 \frac{1}{8}-\frac{5}{6}$. Convert the mixed number to an improper fraction.

$3 \frac{1}{8}=\frac{3 \cdot 8+1}{8}=\frac{24+1}{8}=\frac{25}{8}$

$\frac{25}{8}-\frac{5}{6}$ The $\mathrm{LCD}=24$.

$$
\begin{aligned}
\frac{25}{8}-\frac{5}{6} & =\frac{25 \cdot 3}{24}-\frac{5 \cdot 4}{24} \\
& =\frac{75}{24}-\frac{20}{24} \\
& =\frac{75-20}{24} \\
& =\frac{55}{24} \quad \text { Convert his improper fraction to a mixed number. } \\
& =2 \frac{7}{24}
\end{aligned}
$$

Thus, $3 \frac{1}{8}-\frac{5}{6}=2 \frac{7}{24}$.

\subsubsection{Practice Set A}

Find the following sums and differences.

\section{Exercise 5.4.1}

$$
1 \frac{5}{9}+3 \frac{2}{9}
$$

Exercise 5.4.2 $10 \frac{3}{4}-2 \frac{1}{2}$

Exercise 5.4.3 $2 \frac{7}{8}+5 \frac{1}{4}$

Exercise 5.4.4 


\subsubsection{Exercises}

For the following problems, perform each indicated operation.

Exercise 5.4.6

(Solution on p. 331.)

$3 \frac{1}{8}+4 \frac{3}{8}$

Exercise 5.4.7

$5 \frac{1}{3}+6 \frac{1}{3}$

Exercise 5.4.8

$10 \frac{5}{12}+2 \frac{1}{12}$

(Solution on p. 331.)

Exercise 5.4.9

$15 \frac{1}{5}-11 \frac{3}{5}$

Exercise 5.4.10

$9 \frac{3}{11}+12 \frac{3}{11}$

(Solution on p. 331.)

Exercise 5.4.11

$1 \frac{1}{6}+3 \frac{2}{6}+8 \frac{1}{6}$

Exercise 5.4.12

$5 \frac{3}{8}+1 \frac{1}{8}-2 \frac{5}{8}$

(Solution on p. 331.)

Exercise 5.4.13 $\frac{3}{5}+5 \frac{1}{5}$

Exercise 5.4.14 $2 \frac{2}{9}-\frac{5}{9}$

(Solution on p. 331.)

Exercise 5.4.15 $6+11 \frac{2}{3}$

Exercise 5.4.16 $17-8 \frac{3}{14}$

(Solution on p. 331.)

Exercise 5.4.17 $5 \frac{1}{3}+2 \frac{1}{4}$

Exercise 5.4.18 $6 \frac{2}{7}-1 \frac{1}{3}$

(Solution on p. 331.)

Exercise 5.4.19 $8 \frac{2}{5}+4 \frac{1}{10}$

Exercise 5.4.20 $1 \frac{1}{3}+12 \frac{3}{8}$

(Solution on p. 331.)

Exercise 5.4.21 $3 \frac{1}{4}+1 \frac{1}{3}-2 \frac{1}{2}$

Exercise 5.4.22 $4 \frac{3}{4}-3 \frac{5}{6}+1 \frac{2}{3}$

(Solution on p. 331.)

Exercise 5.4.23 $3 \frac{1}{12}+4 \frac{1}{3}+1 \frac{1}{4}$

Exercise 5.4.24 $5 \frac{1}{15}+8 \frac{3}{10}-5 \frac{4}{5}$

Exercise 5.4.25 $7 \frac{1}{3}+8 \frac{5}{6}-2 \frac{1}{4}$ 
Exercise 5.4.26

(Solution on p. 332.)

$19 \frac{20}{21}+42 \frac{6}{7}-\frac{5}{14}+12 \frac{1}{7}$

Exercise 5.4.27

$\frac{1}{16}+4 \frac{3}{4}+10 \frac{3}{8}-9$

Exercise 5.4.28

$11-\frac{2}{9}+10 \frac{1}{3}-\frac{2}{3}-5 \frac{1}{6}+6 \frac{1}{18}$

(Solution on p. 332.)

Exercise 5.4.29

$\frac{5}{2}+2 \frac{1}{6}+11 \frac{1}{3}-\frac{11}{6}$

Exercise 5.4.30

$1 \frac{1}{8}+\frac{9}{4}-\frac{1}{16}-\frac{1}{32}+\frac{19}{8}$

(Solution on p. 332.)

Exercise 5.4.31

$22 \frac{3}{8}-16 \frac{1}{7}$

Exercise 5.4.32

$15 \frac{4}{9}+4 \frac{9}{16}$

(Solution on p. 332.)

Exercise 5.4.33

$4 \frac{17}{88}+5 \frac{9}{110}$

Exercise 5.4.34

$6 \frac{11}{12}+\frac{2}{3}$

Exercise 5.4.35

$8 \frac{9}{16}-\frac{7}{9}$

Exercise 5.4.36

$5 \frac{2}{11}-\frac{1}{12}$

Exercise 5.4.37

$18 \frac{15}{16}-\frac{33}{34}$

Exercise 5.4.38

$1 \frac{89}{112}-\frac{21}{56}$

Exercise 5.4.39

$11 \frac{11}{24}-7 \frac{13}{18}$

Exercise 5.4.40

$5 \frac{27}{84}-3 \frac{5}{42}+1 \frac{1}{21}$

(Solution on p. 332.)

Exercise 5.4.41

$16 \frac{1}{48}-16 \frac{1}{96}+\frac{1}{144}$

(Solution on p. 332.)

(Solution on p. 332.)

(Solution on p. 332.)

Exercise 5.4.42

(Solution on p. 332.)

A man pours $2 \frac{5}{8}$ gallons of paint from a bucket into a tray. After he finishes pouring, there are $1 \frac{1}{4}$ gallons of paint left in his bucket. How much paint did the man pour into the tray?

HINT: Think about the wording.

Exercise 5.4.43

A particular computer stock opened at $37 \frac{3}{8}$ and closed at $38 \frac{1}{4}$. What was the net gain for this stock?

Exercise 5.4.44

(Solution on p. 332.)

A particular diet program claims that $4 \frac{3}{16}$ pounds can be lost the first month, $3 \frac{1}{4}$ pounds can be lost the second month, and $1 \frac{1}{2}$ pounds can be lost the third month. How many pounds does this diet program claim a person can lose over a 3 -month period?

Exercise 5.4.45

If a person who weighs $145 \frac{3}{4}$ pounds goes on the diet program described in the problem above, how much would he weigh at the end of 3 months? 
Exercise 5.4.46

(Solution on p. 332.)

If the diet program described in the problem above makes the additional claim that from the fourth month on, a person will lose $1 \frac{1}{8}$ pounds a month, how much will a person who begins the program weighing $208 \frac{3}{4}$ pounds weight after 8 months?

\subsubsection{Exercises for Review}

Exercise 5.4.47

(Section 3.2) Use exponents to write $4 \cdot 4 \cdot 4$.

Exercise 5.4.48

(Solution on p. 332.)

(Section 3.5) Find the greatest common factor of 14 and 20.

Exercise 5.4.49

(Section 4.3) Convert $\frac{16}{5}$ to a mixed number.

Exercise 5.4.50

(Section 5.2) Find the sum. $\frac{4}{9}+\frac{1}{9}+\frac{2}{9}$.

(Solution on p. 332.)

Exercise 5.4.51

(Section 5.3) Find the difference. $\frac{15}{26}-\frac{3}{10}$.

\subsection{Comparing Fractions ${ }^{5}$}

\subsubsection{Section Overview}

- Order and the Inequality Symbols

- Comparing Fractions

\subsubsection{Order and the Inequality Symbols}

Our number system is called an ordered number system because the numbers in the system can be placed in order from smaller to larger. This is easily seen on the number line.

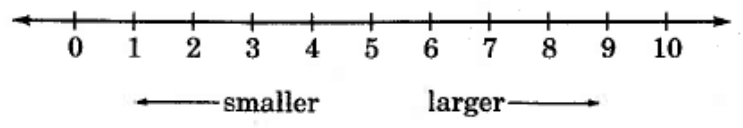

On the number line, a number that appears to the right of another number is larger than that other number. For example, 5 is greater than 2 because 5 is located to the right of 2 on the number line. We may also say that 2 is less than 5 .

To make the inequality phrases "greater than" and "less than" more brief, mathematicians represent them with the symbols $>$ and $<$, respectively.

\section{Symbols for Greater Than > and Less Than <}

$>$ represents the phrase "greater than."

$<$ represents the phrase "less than."

$5>2$ represents " 5 is greater than $2 . "$

$2<5$ represents " 2 is less than $5 . "$

\footnotetext{
${ }^{5}$ This content is available online at $<$ http://cnx.org/content $/ \mathrm{m} 34937 / 1.3 />$.
} 


\subsubsection{Comparing Fractions}

Recall that the fraction $\frac{4}{5}$ indicates that we have 4 of 5 parts of some whole quantity, and the fraction $\frac{3}{5}$ indicates that we have 3 of 5 parts. Since 4 of 5 parts is more than 3 of 5 parts, $\frac{4}{5}$ is greater than $\frac{3}{5}$; that is, $\frac{4}{5}>\frac{3}{5}$

We have just observed that when two fractions have the same denominator, we can determine which is larger by comparing the numerators.

\section{Comparing Fractions}

If two fractions have the same denominators, the fraction with the larger numerator is the larger fraction.

Thus, to compare the sizes of two or more fractions, we need only convert each of them to equivalent fractions that have a common denominator. We then compare the numerators. It is convenient if the common denominator is the LCD. The fraction with the larger numerator is the larger fraction.

\subsubsection{Sample Set A}

\section{Example 5.16}

Compare $\frac{8}{9}$ and $\frac{14}{15}$.

Convert each fraction to an equivalent fraction with the LCD as the denominator. Find the LCD.

$$
\left.\begin{array}{rl}
9 & =3^{2} \\
15 & =3 \cdot 5
\end{array}\right\} \text { The LCD }=3^{2} \cdot 5=9 \cdot 5=45
$$

Since $40<42$,

$\frac{40}{45}<\frac{42}{45}$

Thus $\frac{8}{9}<\frac{14}{15}$.

\section{Example 5.17}

Write $\frac{5}{6}, \frac{7}{10}$, and $\frac{13}{15}$ in order from smallest to largest.

Convert each fraction to an equivalent fraction with the LCD as the denominator.

Find the LCD.

$$
\begin{aligned}
6 & =2 \cdot 3 \\
10 & =2 \cdot 5 \quad\} \text { The LCD }=2 \cdot 3 \cdot 5=30 \\
15 & =3 \cdot 5 \\
\frac{5}{6} & =\frac{5 \cdot 5}{30}=\frac{25}{30} \\
\frac{7}{10} & =\frac{7 \cdot 3}{30}=\frac{21}{30} \\
\frac{13}{15} & =\frac{13 \cdot 2}{30}=\frac{26}{30}
\end{aligned}
$$

Since $21<25<26$, 


$$
\begin{aligned}
& \frac{21}{30}<\frac{25}{30}<\frac{26}{30} \\
& \frac{7}{10}<\frac{5}{6}<\frac{13}{15}
\end{aligned}
$$

Writing these numbers in order from smallest to largest, we get $\frac{7}{10}, \frac{5}{6}, \frac{13}{15}$.

\section{Example 5.18}

Compare $8 \frac{6}{7}$ and $6 \frac{3}{4}$.

To compare mixed numbers that have different whole number parts, we need only compare whole number parts. Since $6<8$,

$6 \frac{3}{4}<8 \frac{6}{7}$

\section{Example 5.19}

Compare $4 \frac{5}{8}$ and $4 \frac{7}{12}$

To compare mixed numbers that have the same whole number parts, we need only compare fractional parts.

$$
\begin{gathered}
8=2^{3} \\
\left.\begin{array}{c}
12=2^{2} \cdot 3
\end{array}\right\} \text { The LCD }=2^{3} \cdot 3=8 \cdot 3=24 \\
\frac{5}{8}=\frac{5 \cdot 3}{24}=\frac{15}{24} \\
\frac{7}{12}=\frac{7 \cdot 2}{24}=\frac{14}{24}
\end{gathered}
$$

Since $14<15$,

$\frac{14}{24}<\frac{15}{24}$

$\frac{7}{12}<\frac{5}{8}$

Hence, $4 \frac{7}{12}<4 \frac{5}{8}$

\subsubsection{Practice Set A}

Exercise 5.5.1

Compare $\frac{3}{4}$ and $\frac{4}{5}$.

(Solution on p. 332.)

Exercise 5.5.2

(Solution on p. 332.)

Compare $\frac{9}{10}$ and $\frac{13}{15}$.

(Solution on p. 332.)

Write $\frac{13}{16}, \frac{17}{20}$, and $\frac{33}{40}$ in order from smallest to largest.

Exercise 5.5.4

(Solution on p. 332.)

Compare $11 \frac{1}{6}$ and $9 \frac{2}{5}$.

Exercise 5.5.5

Compare $1 \frac{9}{14}$ and $1 \frac{11}{16}$.

(Solution on p. 332.) 


\subsubsection{Exercises}

Arrange each collection of numbers in order from smallest to largest.

Exercise 5.5.6

(Solution on p. 332.)

$\frac{3}{5}, \quad \frac{5}{8}$

Exercise 5.5.7

$\frac{1}{6}, \frac{2}{7}$

Exercise 5.5.8

$\frac{3}{4}, \quad \frac{5}{6}$

(Solution on p. 332.)

Exercise 5.5.9

$\frac{7}{9}, \quad \frac{11}{12}$

Exercise 5.5.10 $\frac{3}{8}, \quad \frac{2}{5}$

(Solution on p. 332.)

Exercise 5.5.11 $\frac{1}{2}, \frac{5}{8}, \frac{7}{16}$

Exercise 5.5.12 $\frac{1}{2}, \frac{3}{5}, \frac{4}{7}$

Exercise 5.5.13 $\frac{3}{4}, \quad \frac{2}{3}, \frac{5}{6}$

Exercise 5.5.14 $\frac{3}{4}, \frac{7}{9}, \frac{5}{4}$

Exercise 5.5.15 $\frac{7}{8}, \frac{15}{16}, \frac{11}{12}$

Exercise 5.5.16 $\frac{3}{14}, \frac{2}{7}, \frac{3}{4}$

Exercise 5.5.17 $\frac{17}{32}, \quad \frac{25}{48}, \frac{13}{16}$

Exercise 5.5.18 $5 \frac{3}{5}, \quad 5 \frac{4}{7}$

Exercise 5.5.19 $11 \frac{3}{16}, \quad 11 \frac{1}{12}$

Exercise 5.5.20 $9 \frac{2}{3}, 9 \frac{4}{5}$

(Solution on p. 332.)

Exercise 5.5.21 $7 \frac{2}{3}, \quad 8 \frac{5}{6}$

Exercise 5.5.22 $1 \frac{9}{16}, 2 \frac{1}{20}$

Exercise 5.5.23 $20 \frac{15}{16}, \quad 20 \frac{23}{24}$

Exercise 5.5.24 $2 \frac{2}{9}, \quad 2 \frac{3}{7}$

(Solution on p. 332.)

(Solution on p. 332.)

(Solution on p. 332.)

(Solution on p. 333.)

Exercise 5.5.25

$5 \frac{8}{13}, \quad 5 \frac{9}{20}$ 


\subsubsection{Exercises for Review}

Exercise 5.5.26

(Solution on p. 333.)

(Section 1.4) Round 267,006,428 to the nearest ten million.

Exercise 5.5.27

(Section 2.5 ) Is the number 82,644 divisible by 2 ? by 3 ? by 4 ?

Exercise 5.5.28

(Section 4.3) Convert $3 \frac{2}{7}$ to an improper fraction.

(Solution on p. 333.)

Exercise 5.5.29

(Section 5.3) Find the value of $\frac{5}{6}+\frac{3}{10}-\frac{2}{5}$

Exercise 5.5.30

(Section 5.4) Find the value of $8 \frac{3}{8}+5 \frac{1}{4}$.

(Solution on p. 333.)

\subsection{Complex Fractions ${ }^{6}$}

\subsubsection{Section Overview}

- Simple Fractions and Complex Fractions

- Converting Complex Fractions to Simple Fractions

\subsubsection{Simple Fractions and Complex Fractions}

\section{Simple Fraction}

A simple fraction is any fraction in which the numerator is any whole number and the denominator is any nonzero whole number. Some examples are the following:

$\frac{1}{2}, \frac{4}{3}, \frac{763}{1,000}$

\section{Complex Fraction}

A complex fraction is any fraction in which the numerator and/or the denominator is a fraction; it is a fraction of fractions. Some examples of complex fractions are the following:

$\frac{\frac{3}{4}}{\frac{5}{6}}, \frac{\frac{1}{3}}{2}, \frac{6}{\frac{9}{10}}, \frac{4+\frac{3}{8}}{7-\frac{5}{6}}$

\subsubsection{Converting Complex Fractions to Simple Fractions}

The goal here is to convert a complex fraction to a simple fraction. We can do so by employing the methods of adding, subtracting, multiplying, and dividing fractions. Recall from Section 4.2 that a fraction bar serves as a grouping symbol separating the fractional quantity into two individual groups. We proceed in simplifying a complex fraction to a simple fraction by simplifying the numerator and the denominator of the complex fraction separately. We will simplify the numerator and denominator completely before removing the fraction bar by dividing. This technique is illustrated in problems 3, 4, 5, and 6 of Section 5.6.3.1 (Sample Set A).

\footnotetext{
${ }^{6}$ This content is available online at $<$ http://cnx.org/content $/ \mathrm{m} 34941 / 1.2 />$.
} 


\subsubsection{Sample Set A}

Convert each of the following complex fractions to a simple fraction.

\section{Example 5.20}

$\frac{\frac{3}{8}}{\frac{15}{16}}$

Convert this complex fraction to a simple fraction by performing the indicated division.

$$
\begin{aligned}
\frac{\frac{3}{8}}{\frac{15}{16}} & =\quad \frac{3}{8} \div \frac{15}{16} \quad \text { The divisor is } \frac{15}{16} \text {. Invert } \frac{15}{16} \text { and multiply. } \\
& =\frac{\frac{1}{3}}{\frac{3}{8}} \cdot \frac{\frac{2}{16}}{\frac{16}{15}}=\frac{1 \cdot 2}{1 \cdot 5}=\frac{2}{5}
\end{aligned}
$$

\section{Example 5.21}

$\frac{\frac{4}{9}}{6} \quad$ Write 6 as $\frac{6}{1}$ and divide.

$$
\begin{aligned}
\frac{\frac{4}{9}}{\frac{6}{1}} & = \\
& =\frac{\frac{4}{9} \div \frac{6}{1}}{9} \cdot \frac{1}{\frac{.4}{3}}=\frac{2 \cdot 1}{9 \cdot 3}=\frac{2}{27}
\end{aligned}
$$

\section{Example 5.22}

$\frac{5+\frac{3}{4}}{46} \quad$ Simplify the numerator.

$$
\begin{aligned}
\frac{\frac{4 \cdot 5+3}{4}}{46} & =\frac{\frac{20+3}{4}}{46}=\frac{\frac{23}{4}}{46} \quad \text { Write } 46 \text { as } \frac{46}{1} . \\
\frac{\frac{23}{4}}{\frac{46}{1}}= & \frac{23}{4} \div \frac{46}{1} \\
& =\frac{1}{\frac{23}{4}} \cdot \frac{1}{\frac{46}{26}}=\frac{1 \cdot 1}{4 \cdot 2}=\frac{1}{8}
\end{aligned}
$$

\section{Example 5.23}

$$
\begin{aligned}
& \frac{\frac{1}{4}+\frac{3}{8}}{\frac{1}{2}+\frac{13}{24}}=\frac{\frac{2}{8}+\frac{3}{8}}{\frac{12}{24}+\frac{13}{24}}=\frac{\frac{2+3}{8}}{\frac{12+13}{24}}=\frac{\frac{5}{8}}{\frac{25}{24}}=\frac{5}{8} \div \frac{25}{24} \\
& \frac{5}{8} \div \frac{25}{24}=\frac{\frac{1}{5}}{\frac{5}{18}} \cdot \frac{\frac{3}{24}}{\frac{24}{25}}=\frac{1 \cdot 3}{1 \cdot 5}=\frac{3}{5}
\end{aligned}
$$

\section{Example 5.24}

$$
\begin{aligned}
\frac{4+\frac{5}{6}}{7-\frac{1}{3}}=\frac{\frac{4 \cdot 6+5}{6}}{\frac{7 \cdot 3-1}{3}}=\frac{\frac{29}{6}}{\frac{20}{3}} & =\frac{29}{6} \div \frac{20}{3} \\
& =\frac{29}{76} \cdot \frac{\frac{1}{3}}{20}=\frac{29}{40}
\end{aligned}
$$

Example 5.25

$$
\begin{aligned}
& \frac{11+\frac{3}{10}}{4 \frac{4}{5}}=\frac{\frac{11 \cdot 10+3}{10}}{\frac{4 \cdot 5+4}{5}}=\frac{\frac{110+3}{10}}{\frac{20+4}{5}}=\frac{\frac{113}{10}}{\frac{24}{5}}=\frac{113}{10} \div \frac{24}{5} \\
& \frac{113}{10} \div \frac{24}{5}=\frac{113}{\frac{110}{2}} \cdot \frac{\frac{1}{5}}{24}=\frac{113 \cdot 1}{2 \cdot 24}=\frac{113}{48}=2 \frac{17}{48}
\end{aligned}
$$




\subsubsection{Practice Set A}

Convert each of the following complex fractions to a simple fraction.

Exercise 5.6.1<smiles>[13CH3][131In]</smiles>

Exercise 5.6.2

$\frac{7}{10}$

Exercise 5.6.3 $\frac{5+\frac{2}{5}}{3+\frac{3}{5}}$

Exercise 5.6.4 $\frac{\frac{1}{8}+\frac{7}{8}}{6-\frac{3}{10}}$

Exercise 5.6.5 $\frac{\frac{1}{6}+\frac{5}{8}}{\frac{5}{9}-\frac{1}{4}}$

Exercise 5.6.6 $\frac{16-10 \frac{2}{3}}{11 \frac{5}{6}-7 \frac{7}{6}}$

(Solution on p. 333.)

(Solution on p. 333.)

(Solution on p. 333.)

(Solution on p. 333.)

(Solution on p. 333.)

(Solution on p. 333.)

\subsubsection{Exercises}

Simplify each fraction.

Exercise 5.6.7 $\frac{\frac{3}{5}}{\frac{9}{15}}$

(Solution on p. 333.)

Exercise 5.6.8<smiles>[13CH3]</smiles>

Exercise 5.6.9<smiles>[131In]</smiles>

(Solution on p. 333.)

Exercise 5.6.10<smiles>[131In]</smiles>

Exercise 5.6.11 $\frac{6+\frac{1}{4}}{11+\frac{1}{4}}$

(Solution on p. 333.)

Exercise 5.6.12 $\frac{2+\frac{1}{2}}{7+\frac{1}{2}}$

Exercise 5.6.13 $\frac{5+\frac{1}{3}}{2+\frac{2}{15}}$

(Solution on p. 333.)

Exercise 5.6.14 $\frac{9+\frac{1}{2}}{1+\frac{8}{11}}$

Exercise 5.6.15 $\frac{4+\frac{10}{13}}{\frac{12}{39}}$

(Solution on p. 333.)

Exercise 5.6.16 $\frac{\frac{1}{3}+\frac{2}{7}}{\frac{26}{21}}$

Exercise 5.6.17 $\frac{\frac{5}{6}-\frac{1}{4}}{\frac{1}{12}}$

(Solution on p. 333.) 
Exercise 5.6.18

$$
\frac{\frac{3}{10}+\frac{4}{12}}{\frac{19}{90}}
$$

Exercise 5.6.19

(Solution on p. 333.)

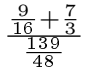

Exercise 5.6.20 $\frac{\frac{1}{288}}{\frac{8}{9}-\frac{3}{16}}$

Exercise 5.6.21

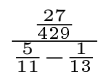

(Solution on p. 333.)

Exercise 5.6.22 $\frac{\frac{1}{3}+\frac{2}{5}}{\frac{3}{5}+\frac{17}{45}}$

Exercise 5.6.23 $\frac{\frac{9}{70}+\frac{5}{42}}{\frac{13}{30}-\frac{1}{21}}$

(Solution on p. 333.)

Exercise 5.6.24 $\frac{\frac{1}{16}+\frac{1}{14}}{\frac{2}{3}-\frac{13}{60}}$

Exercise 5.6.25 $\frac{\frac{3}{20}+\frac{11}{12}}{\frac{19}{7}-1 \frac{11}{35}}$

Exercise 5.6.26 $\frac{2 \frac{2}{3}-1 \frac{1}{2}}{\frac{1}{4}+1 \frac{1}{16}}$

Exercise 5.6.27 $\frac{3 \frac{1}{5}+3 \frac{1}{3}}{\frac{6}{5}-\frac{15}{63}}$

(Solution on p. 333.)

Exercise 5.6.28

$$
\frac{\frac{1 \frac{1}{2}+15}{5 \frac{1}{4}-3 \frac{5}{12}}}{\frac{8 \frac{1}{3}-4 \frac{1}{2}}{11 \frac{2}{3}-5 \frac{11}{12}}}
$$

Exercise 5.6.29

$$
\frac{\frac{5 \frac{3}{4}+3 \frac{1}{5}}{2 \frac{1}{5}+15 \frac{7}{10}}}{\frac{9 \frac{1}{2}-4 \frac{1}{6}}{\frac{1}{8}+2 \frac{1}{120}}}
$$

(Solution on p. 333.)

(Solution on p. 333.)

\subsubsection{Exercises for Review}

Exercise 5.6.30

(Section 3.4) Find the prime factorization of 882 .

Exercise 5.6.31

(Section 4.3) Convert $\frac{62}{7}$ to a mixed number.

(Solution on p. 333.)

Exercise 5.6.32

(Section 4.4) Reduce $\frac{114}{342}$ to lowest terms.

Exercise 5.6.33

(Section 5.4) Find the value of $6 \frac{3}{8}-4 \frac{5}{6}$.

(Solution on p. 334.)

Exercise 5.6.34

(Section 5.5) Arrange from smallest to largest: $\frac{1}{2}, \frac{3}{5}, \frac{4}{7}$. 


\subsection{Combinations of Operations with Fractions ${ }^{7}$}

\subsubsection{Section Overview}

- The Order of Operations

\subsubsection{The Order of Operations}

To determine the value of a quantity such as

$\frac{1}{2}+\frac{5}{8} \cdot \frac{2}{15}$

where we have a combination of operations (more than one operation occurs), we must use the accepted order of operations.

\section{The Order of Operations:}

1. In the order (2), (3), (4) described below, perform all operations inside grouping symbols: ( ), [ ], ( ), — . Work from the innermost set to the outermost set.

2. Perform exponential and root operations.

3. Perform all multiplications and divisions moving left to right.

4. Perform all additions and subtractions moving left to right.

\subsubsection{Sample Set A}

Determine the value of each of the following quantities.

Example 5.26

$\frac{1}{4}+\frac{5}{8} \cdot \frac{2}{15}$

(a) Multiply first.

$$
\frac{1}{4}+\frac{\frac{1}{55}}{\frac{5}{8}} \cdot \frac{\frac{1}{2}}{\frac{2}{15}}=\frac{1}{4}+\frac{1 \cdot 1}{4 \cdot 3}=\frac{1}{4}+\frac{1}{12}
$$

(b) Now perform this addition. Find the LCD.

$$
\begin{aligned}
& \left.\begin{array}{l}
4=2^{2} \\
12=2^{2} \cdot 3^{2}
\end{array}\right\} \text { The LCD }=2^{2} \cdot 3=12 . \\
& \begin{aligned}
\frac{1}{4}+\frac{1}{12} & =\frac{1 \cdot 3}{12}+\frac{1}{12}=\frac{3}{12}+\frac{1}{12} \\
& =\quad \frac{3+1}{12}=\frac{4}{12}=\frac{1}{3}
\end{aligned}
\end{aligned}
$$

Thus, $\frac{1}{4}+\frac{5}{8} \cdot \frac{2}{15}=\frac{1}{3}$

\section{Example 5.27}

$\frac{3}{5}+\frac{9}{44}\left(\frac{5}{9}-\frac{1}{4}\right)$

(a) Operate within the parentheses first, $\left(\frac{5}{9}-\frac{1}{4}\right)$.

$$
\left.\begin{array}{l}
9=3^{2} \\
4=2^{2}
\end{array}\right\} \text { The LCD }=2^{2} \cdot 3^{2}=4 \cdot 9=36 .
$$

\footnotetext{
${ }^{7}$ This content is available online at $<$ http://cnx.org/content/m34942/1.3/>.
} 
$\frac{5 \cdot 4}{36}-\frac{1 \cdot 9}{36}=\frac{20}{36}-\frac{9}{36}=\frac{20-9}{36}=\frac{11}{36}$

Now we have

$\frac{3}{5}+\frac{9}{44}\left(\frac{11}{36}\right)$

(b) Perform the multiplication.

$\frac{3}{5}+\frac{\frac{1}{99}}{\overline{\frac{14}{44}}} \cdot \frac{\frac{1}{\sqrt{11}}}{\overline{36}}=\frac{3}{5}+\frac{1 \cdot 1}{4 \cdot 4}=\frac{3}{5}+\frac{1}{16}$

(c) Now perform the addition. The $\mathrm{LCD}=80$.

$\frac{3}{5}+\frac{1}{16}=\frac{3 \cdot 16}{80}+\frac{1 \cdot 5}{80}=\frac{48}{80}+\frac{5}{80}=\frac{48+5}{80}=\frac{53}{80}$

Thus, $\frac{3}{5}+\frac{9}{44}\left(\frac{5}{9}-\frac{1}{4}\right)=\frac{53}{80}$

\section{Example 5.28}

$8-\frac{15}{426}\left(2-1 \frac{4}{15}\right)\left(3 \frac{1}{5}+2 \frac{1}{8}\right)$

(a) Work within each set of parentheses individually.

$$
\begin{array}{rlc}
2-1 \frac{4}{15} & = & 2 \frac{1 \cdot 15+4}{15}=2-\frac{19}{15} \\
& = & \frac{30}{15}-\frac{19}{15}=\frac{30-19}{15}=\frac{11}{15} \\
3 \frac{1}{5}+2 \frac{1}{8} & = & \frac{3 \cdot 5+1}{5}+\frac{2 \cdot 8+1}{8} \\
& = & \frac{16}{5}+\frac{17}{8} \mathrm{LCD}=40 \\
& = & \frac{16 \cdot 8}{40}+\frac{17 \cdot 5}{40} \\
& = & \frac{128}{40}+\frac{85}{40} \\
& = & \frac{128+85}{40} \\
& = & \frac{213}{40}
\end{array}
$$

Now we have

$8-\frac{15}{426}\left(\frac{11}{15}\right)\left(\frac{213}{40}\right)$

(b) Now multiply.

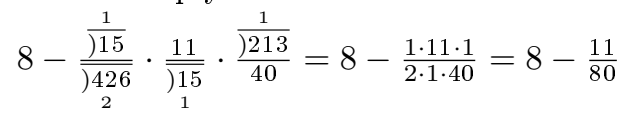

(c) Now subtract.

$8-\frac{11}{80}=\frac{80 \cdot 8}{80}-\frac{11}{80}=\frac{640}{80}-\frac{11}{80}=\frac{640-11}{80}=\frac{629}{80}$ or $7 \frac{69}{80}$

Thus, $8-\frac{15}{426}\left(2-1 \frac{4}{15}\right)^{80}\left(3 \frac{1}{5}+2 \frac{1}{8}\right) \stackrel{80}{=} 7 \frac{69}{80}$

\section{Example 5.29}

$\left(\frac{3}{4}\right)^{2} \cdot \frac{8}{9}-\frac{5}{12}$

(a) Square $\frac{3}{4}$.

$\left(\frac{3}{4}\right)^{2}=\frac{3}{4} \cdot \frac{3}{4}=\frac{3 \cdot 3}{4 \cdot 4}=\frac{9}{16}$

Now we have

$$
\frac{9}{16} \cdot \frac{8}{9}-\frac{5}{12}
$$

(b) Perform the multiplication.

$\frac{\frac{1}{9}}{\overline{\frac{9}{16}}} \cdot \frac{\frac{1}{8}}{\frac{\frac{8}{9}}{1}}-\frac{5}{12}=\frac{1 \cdot 1}{2 \cdot 1}-\frac{5}{12}=\frac{1}{2}-\frac{5}{12}$

(c) Now perform the subtraction.

$\frac{1}{2}-\frac{5}{12}=\frac{6}{12}-\frac{5}{12}=\frac{6-5}{12}=\frac{1}{12}$

Thus, $\left(\frac{4}{3}\right)^{2} \cdot \frac{8}{9}-\frac{5}{12}=\frac{1}{12}$ 
Example 5.30

$2 \frac{7}{8}+\sqrt{\frac{25}{36}} \div\left(2 \frac{1}{2}-1 \frac{1}{3}\right)$

(a) Begin by operating inside the parentheses.

$$
\begin{aligned}
2 \frac{1}{2}-1 \frac{1}{3} & =\frac{2 \cdot 2+1}{2}-\frac{1 \cdot 3+1}{3}=\frac{5}{2}-\frac{4}{3} \\
& =\frac{15}{6}-\frac{8}{6}=\frac{15-8}{6}=\frac{7}{6}
\end{aligned}
$$

(b) Now simplify the square root.

$$
\sqrt{\frac{25}{36}}=\frac{5}{6}\left(\text { since }\left(\frac{5}{6}\right)^{2}=\frac{25}{36}\right)
$$

Now we have

$$
2 \frac{7}{8}+\frac{5}{6} \div \frac{7}{6}
$$

(c) Perform the division.

$$
2 \frac{7}{8}+\frac{5}{\frac{5}{6}} \cdot \frac{\frac{1}{66}}{7}=2 \frac{7}{8}+\frac{5 \cdot 1}{1 \cdot 7}=2 \frac{7}{8}+\frac{5}{7}
$$

(d) Now perform the addition.

$$
\begin{array}{rlcc}
2 \frac{7}{8}+\frac{5}{7} & = & \frac{2 \cdot 8+7}{8}+\frac{5}{7}=\frac{23}{8}+\frac{5}{7} & \text { LCD }=56 . \\
& = & \frac{23 \cdot 7}{56}+\frac{5 \cdot 8}{56}=\frac{161}{56}+\frac{40}{56} \\
& = & \frac{161+40}{56}=\frac{201}{56} \text { or } 3 \frac{33}{56}
\end{array}
$$

Thus, $2 \frac{7}{8}+\sqrt{\frac{25}{36}} \div\left(2 \frac{1}{2}-1 \frac{1}{3}\right)=3 \frac{33}{56}$

\subsubsection{Practice Set A}

Find the value of each of the following quantities.

Exercise 5.7.1

$\frac{5}{16} \cdot \frac{1}{10}-\frac{1}{32}$

Exercise 5.7.2

$$
\frac{6}{7} \cdot \frac{21}{40} \div \frac{9}{10}+5 \frac{1}{3}
$$

Exercise 5.7.3

$$
8 \frac{7}{10}-2\left(4 \frac{1}{2}-3 \frac{2}{3}\right)
$$

Exercise 5.7.4

$$
\frac{17}{18}-\frac{58}{30}\left(\frac{1}{4}-\frac{3}{32}\right)\left(1-\frac{13}{29}\right)
$$

Exercise 5.7.5

$$
\left(\frac{1}{10}+1 \frac{1}{2}\right) \div\left(1 \frac{4}{5}-1 \frac{6}{25}\right)
$$

(Solution on p. 334.)

(Solution on p. 334.)

Exercise 5.7.6

$$
\frac{\frac{2}{3}-\frac{3}{8} \cdot \frac{4}{9}}{\frac{7}{16} \cdot 1 \frac{1}{3}+1 \frac{1}{4}}
$$

Exercise 5.7.7

$$
\left(\frac{3}{8}\right)^{2}+\frac{3}{4} \cdot \frac{1}{8}
$$

Exercise 5.7.8 


\subsubsection{Exercises}

Find each value.

Exercise 5.7.9 $\frac{4}{3}-\frac{1}{6} \cdot \frac{1}{2}$

(Solution on p. 334.)

Exercise 5.7.10 $\frac{7}{9}-\frac{4}{5} \cdot \frac{5}{36}$

Exercise 5.7.11 $2 \frac{2}{7}+\frac{5}{8} \div \frac{5}{16}$

(Solution on p. 334.)

Exercise 5.7.12 $\frac{3}{16} \div \frac{9}{14} \cdot \frac{12}{21}+\frac{5}{6}$

Exercise 5.7.13 $\frac{4}{25} \div \frac{8}{15}-\frac{7}{20} \div 2 \frac{1}{10}$

(Solution on p. 334.)

Exercise 5.7.14 $\frac{2}{5} \cdot\left(\frac{1}{19}+\frac{3}{38}\right)$

Exercise 5.7.15 $\frac{3}{7} \cdot\left(\frac{3}{10}-\frac{1}{15}\right)$

Exercise 5.7.16 $\frac{10}{11} \cdot\left(\frac{8}{9}-\frac{2}{5}\right)+\frac{3}{25} \cdot\left(\frac{5}{3}+\frac{1}{4}\right)$

Exercise 5.7.17 $\frac{2}{7} \cdot\left(\frac{6}{7}-\frac{3}{28}\right)+5 \frac{1}{3} \cdot\left(1 \frac{1}{4}-\frac{1}{8}\right)$

(Solution on p. 334.)

Exercise 5.7.18 $\frac{\left(\frac{6}{11}-\frac{1}{3}\right) \cdot\left(\frac{1}{21}+2 \frac{13}{42}\right)}{1 \frac{1}{5}+\frac{7}{40}}$

Exercise 5.7.19 $\left(\frac{1}{2}\right)^{2}+\frac{1}{8}$

(Solution on p. 334.)

Exercise 5.7.20 $\left(\frac{3}{5}\right)^{2}-\frac{3}{10}$

Exercise 5.7.21 $\sqrt{\frac{36}{81}}+\frac{1}{3} \cdot \frac{2}{9}$

(Solution on p. 334.)

Exercise 5.7.22 $\sqrt{\frac{49}{64}}-\sqrt{\frac{9}{4}}$

Exercise 5.7.23 $\frac{2}{3} \cdot \sqrt{\frac{9}{4}}-\frac{15}{4} \cdot \sqrt{\frac{16}{225}}$

(Solution on p. 334.)

Exercise 5.7.24

$$
\left(\frac{3}{4}\right)^{2}+\sqrt{\frac{25}{16}}
$$

Exercise 5.7.25

$$
\left(\frac{1}{3}\right)^{2} \cdot \sqrt{\frac{81}{25}}+\frac{1}{40} \div \frac{1}{8}
$$

(Solution on p. 334.)

Exercise 5.7.26

$$
\left(\sqrt{\frac{4}{49}}\right)^{2}+\frac{3}{7} \div 1 \frac{3}{4}
$$

Exercise 5.7.27

$$
\left(\sqrt{\frac{100}{121}}\right)^{2}+\frac{21}{(11)^{2}}
$$

(Solution on p. 334.)

Exercise 5.7.28

$$
\sqrt{\frac{3}{8}+\frac{1}{64}}-\frac{1}{2} \div 1 \frac{1}{3}
$$

(Solution on p. 334.) 
Exercise 5.7.29

(Solution on p. 334.)

$\sqrt{\frac{1}{4}} \cdot\left(\frac{5}{6}\right)^{2}+\frac{9}{14} \cdot 2 \frac{1}{3}-\sqrt{\frac{1}{81}}$

Exercise 5.7.30

$\sqrt{\frac{1}{9}} \cdot \sqrt{\frac{6 \frac{3}{8}+2 \frac{5}{8}}{16}}+7 \frac{7}{10}$

Exercise 5.7.31

$\frac{3 \frac{3}{4}+\frac{4}{5} \cdot\left(\frac{1}{2}\right)^{3}}{\frac{67}{240}+\left(\frac{1}{3}\right)^{4} \cdot\left(\frac{9}{10}\right)}$

(Solution on p. 334.)

Exercise 5.7.32

$\sqrt{\sqrt{\frac{16}{81}}}+\frac{1}{4} \cdot 6$

Exercise 5.7.33

$\sqrt{\sqrt{\frac{81}{256}}}-\frac{3}{32} \cdot 1 \frac{1}{8}$

(Solution on p. 334.)

\subsubsection{Exercises for Review}

Exercise 5.7.34

(Section 1.2) True or false: Our number system, the Hindu-Arabic number system, is a positional number system with base ten.

Exercise 5.7.35

(Solution on p. 334.)

(Section 2.6) The fact that 1 times any whole number $=$ that particular whole number illustrates which property of multiplication?

Exercise 5.7.36

(Section 4.3) Convert $8 \frac{6}{7}$ to an improper fraction.

Exercise 5.7.37

(Section 5.3) Find the sum. $\frac{3}{8}+\frac{4}{5}+\frac{5}{6}$.

(Solution on p. 334.)

Exercise 5.7.38

(Section 5.6) Simplify $\frac{6+\frac{1}{8}}{6-\frac{1}{8}}$. 


\subsection{Summary of Key Concepts ${ }^{8}$}

\subsubsection{Summary of Key Concepts}

Addition and Subtraction of Fractions with Like Denominators (Section 5.2)

To add or subtract two fractions that have the same denominators, add or subtract the numerators and place the resulting sum or difference over the common denominator. Reduce, if necessary. Do not add or subtract the denominators.

$\frac{1}{8}+\frac{5}{8}=\frac{1+5}{8}=\frac{6}{8}=\frac{3}{4}$

Basic Rule for Adding and Subtracting Fractions (Section 5.3)

Fractions can be added or subtracted conveniently only if they have like denominators.

Addition and Subtraction of Fractions with Unlike Denominators (Section 5.3)

To add or subtract fractions having unlike denominators, convert each fraction to an equivalent fraction having as denominator the LCD of the original denominators.

Addition and Subtraction of Mixed Numbers (Section 5.4)

1. To add or subtract mixed numbers, convert each mixed number to an improper fraction, then add or subtract the fractions.

Ordered Number System (Section 5.5)

Our number system is ordered because the numbers in the system can be placed in order from smaller to larger.

Inequality Symbols (Section 5.5)

$>$ represents the phrase "greater than."

$<$ represents the phrase "less than."

Comparing Fractions (Section 5.5)

If two fractions have the same denominators, the fraction with the larger numerator is the larger fraction.

$\frac{5}{8}>\frac{3}{8}$

Simple Fractions (Section 5.6)

A simple fraction is any fraction in which the numerator is any whole number and the denominator is any nonzero whole number.

Complex Fractions (Section 5.6)

A complex fraction is any fraction in which the numerator and/or the denominator is a fraction.

Complex fractions can be converted to simple fractions by employing the methods of adding, subtracting, multiplying, and dividing fractions.

\subsection{Exercise Supplement ${ }^{9}$}

\subsubsection{Exercise Supplement}

5.9.1.1 Addition and Subtractions of Fractions with Like and Unlike Denominators, and Addition and Subtraction of Mixed Numbers (Section 5.2, Section 5.3, Section 5.4)

For problems 1-53, perform each indicated operation and write the result in simplest form.

\footnotetext{
${ }^{8}$ This content is available online at $<$ http://cnx.org/content $/ \mathrm{m} 34943 / 1.2 />$.

${ }^{9}$ This content is available online at $<\mathrm{http}: / / \mathrm{cnx} .0 \mathrm{rg} /$ content $/ \mathrm{m} 34944 / 1.2 />$.
} 
Exercise 5.9.1

(Solution on p. 334.)

$\frac{3}{4}+\frac{5}{8}$

Exercise 5.9.2 $\frac{9}{16}+\frac{1}{4}$

Exercise 5.9.3 $\frac{1}{8}+\frac{3}{8}$

(Solution on p. 335.)

Exercise 5.9.4 $\frac{5}{7}+\frac{1}{14}+\frac{5}{21}$

Exercise 5.9.5 $\frac{5}{6}+\frac{1}{3}+\frac{5}{21}$

(Solution on p. 335.)

Exercise 5.9.6 $\frac{2}{5}+\frac{1}{8}$

Exercise 5.9.7 $\frac{1}{4}+\frac{1}{8}+\frac{1}{4}$

Exercise 5.9.8 $\frac{1}{16}+\frac{1}{10}$

Exercise 5.9.9 $\frac{2}{7}+\frac{1}{3}$

Exercise 5.9.10 $2 \frac{1}{3}+\frac{1}{6}$

Exercise 5.9.11 $3 \frac{11}{16}+\frac{3}{4}$

Exercise 5.9.12 $5 \frac{1}{12}+3 \frac{1}{8}$

Exercise 5.9.13 $16 \frac{2}{5}+8 \frac{1}{4}$

(Solution on p. 335.)

Exercise 5.9.14 $1 \frac{1}{7}+2 \frac{4}{7}$

Exercise 5.9.15 $1 \frac{3}{8}+0$

(Solution on p. 335.)

Exercise 5.9.16 $3 \frac{1}{10}+4$

Exercise 5.9.17 $18 \frac{2}{3}+6$

(Solution on p. 335.)

Exercise 5.9.18 $1 \frac{4}{3}+5 \frac{5}{4}$

Exercise 5.9.19 $\frac{21}{4}+\frac{2}{3}$

(Solution on p. 335.)

Exercise 5.9.20 $\frac{15}{16}-\frac{1}{8}$

Exercise 5.9.21 $\frac{9}{11}-\frac{5}{22}$

Exercise 5.9.22 $6 \frac{2}{15}-1 \frac{3}{10}$

Exercise 5.9.23 $5 \frac{2}{3}+8 \frac{1}{5}-2 \frac{1}{4}$

(Solution on p. 335.)

(Solution on p. 335.) 
Exercise 5.9.24

$$
8 \frac{3}{10}-4 \frac{5}{6}-3 \frac{1}{15}
$$

Exercise 5.9.25

$\frac{11}{12}+\frac{1}{9}-\frac{1}{16}$

(Solution on p. 335.)

Exercise 5.9.26

$7 \frac{2}{9}-5 \frac{5}{6}-1 \frac{1}{3}$

Exercise 5.9.27

$16 \frac{2}{5}-8 \frac{1}{6}-3 \frac{2}{15}$

(Solution on p. 335.)

Exercise 5.9.28

$4 \frac{1}{8}+0-\frac{32}{8}$

Exercise 5.9.29

$4 \frac{1}{8}+0-\frac{32}{8}$

(Solution on p. 335.)

Exercise 5.9.30 $8-2 \frac{1}{3}$

Exercise 5.9.31 $4-3 \frac{5}{16}$

(Solution on p. 335.)

Exercise 5.9.32 $6 \frac{3}{7}+4$

Exercise 5.9.33 $11 \frac{2}{11}-3$

(Solution on p. 335.)

Exercise 5.9.34 $21 \frac{5}{8}-\frac{5}{8}$

Exercise 5.9.35 $\frac{3}{4}+\frac{5}{16} \cdot \frac{4}{5}$

Exercise 5.9.36 $\frac{11}{12}+\frac{15}{16} \div 2 \frac{1}{2}$

Exercise 5.9.37 $1 \frac{3}{10}+2 \frac{2}{3} \div \frac{4}{9}$

Exercise 5.9.38 $8 \frac{3}{5}-1 \frac{1}{14} \cdot \frac{3}{7}$

Exercise 5.9.39 $2 \frac{3}{8} \div 3 \frac{9}{16}-\frac{1}{9}$

(Solution on p. 335.)

Exercise 5.9.40 $15 \frac{2}{5} \div 50-\frac{1}{10}$

(Solution on p. 335.)

(Solution on p. 335.)

5.9.1.2 Complex Fractions and Combinations of Operations with Fractions (Section 5.5,Section 5.6)

Exercise 5.9.41

(Solution on p. 335.)<smiles>[13CH]=[131In]</smiles>

Exercise 5.9.42

$\frac{\frac{10}{21}}{\frac{11}{14}}$

Exercise 5.9.43

$\frac{1 \frac{7}{9}}{1 \frac{5}{27}}$

(Solution on p. 335.) 
Exercise 5.9.44

$\frac{\frac{15}{17}}{\frac{50}{51}}$

Exercise 5.9.45

(Solution on p. 335.)

$\frac{1 \frac{9}{16}}{2 \frac{11}{12}}$

Exercise 5.9.46

$\frac{8 \frac{4}{15}}{3}$

Exercise 5.9.47 $\frac{9 \frac{1}{18}}{6}$

(Solution on p. 335.)

Exercise 5.9.48 $\frac{3 \frac{1}{4}+2 \frac{1}{8}}{5 \frac{1}{6}}$

Exercise 5.9.49 $\frac{3+2 \frac{1}{2}}{\frac{1}{4}+\frac{5}{6}}$

Exercise 5.9.50 $\frac{4+1 \frac{7}{10}}{9-2 \frac{1}{5}}$

Exercise 5.9.51 $\frac{1 \frac{2}{5}}{9-\frac{2}{2}}$

Exercise 5.9.52 $\frac{1 \frac{2}{3} \cdot\left(\frac{1}{4}+\frac{1}{5}\right)}{1 \frac{1}{2}}$

Exercise 5.9.53 $\frac{\frac{10}{23} \cdot\left(\frac{5}{6}+2\right)}{\frac{8}{9}}$

(Solution on p. 335.)

(Solution on p. 335.)

(Solution on p. 336.)

\subsubsection{Comparing Fractions (Section 5.5)}

For problems 54-65, place each collection in order from smallest to largest.

Exercise 5.9.54 $\frac{1}{8}, \frac{3}{16}$

Exercise 5.9.55 $\frac{3}{32}, \frac{1}{8}$

Exercise 5.9.56 $\frac{5}{16}, \frac{3}{24}$

Exercise 5.9.57 $\frac{3}{10}, \frac{5}{6}$

Exercise 5.9.58 $\frac{2}{9}, \frac{1}{3}, \frac{1}{6}$

Exercise 5.9.59 $\frac{3}{8}, \frac{8}{3}, \frac{19}{6}$

(Solution on p. 336.)

Exercise 5.9.60

$\frac{3}{5}, \frac{2}{10}, \frac{7}{20}$

Exercise 5.9.61 $\frac{4}{7}, \frac{5}{9}$

Exercise 5.9.62 $\frac{4}{5}, \frac{5}{7}$

(Solution on p. 336.)

(Solution on p. 336.)

(Solution on p. 336.) 
Exercise 5.9.63

(Solution on p. 336.) $\frac{5}{12}, \frac{4}{9}, \frac{7}{15}$

Exercise 5.9.64 $\frac{7}{36}, \frac{1}{24}, \frac{5}{12}$

Exercise 5.9.65 $\frac{5}{8}, \frac{13}{16}, \frac{3}{4}$

(Solution on p. 336.)

\subsection{Proficiency Exam ${ }^{10}$}

\subsubsection{Proficiency Exam}

For problems 1-12, perform each indicated operation and write the result in simplest form.

Exercise 5.10.1

(Section 5.3) $\frac{3}{16}+\frac{1}{8}$

Exercise 5.10.2

(Section 5.4) $2 \frac{2}{3}+5 \frac{1}{6}$

Exercise 5.10.3

(Section 5.7) $\frac{7}{15} \cdot \frac{20}{21}+\frac{5}{9}$

Exercise 5.10.4

(Section 5.2) $\frac{3}{11}+\frac{5}{11}$

Exercise 5.10.5

(Section 5.7) $6 \frac{2}{9} \cdot 1 \frac{17}{28}-\left(3 \frac{4}{17}-\frac{21}{17}\right)$

Exercise 5.10.6

(Section 5.4) $5 \frac{1}{8}-2 \frac{4}{5}$

Exercise 5.10.7

(Section 5.6) $\frac{\frac{7}{12}}{\frac{8}{21}}$

Exercise 5.10.8

(Section 5.6) $\frac{\frac{1}{8}+\frac{3}{4}}{1 \frac{7}{8}}$

Exercise 5.10.9

(Section 5.4) $4 \frac{5}{16}+1 \frac{1}{3}-2 \frac{5}{24}$

Exercise 5.10.10

(Section 5.7) $\frac{5}{18} \cdot\left(\frac{15}{16}-\frac{3}{8}\right)$

Exercise 5.10.11

(Section 5.4) $4+2 \frac{1}{3}$

Exercise 5.10.12

(Section 5.4) $8 \frac{3}{7}-5$
(Solution on p. 336.)

(Solution on p. 336.)

(Solution on p. 336.)

(Solution on p. 336.)

(Solution on p. 336.)

(Solution on p. 336.)

(Solution on p. 336.)

(Solution on p. 336.)

(Solution on p. 336.)

(Solution on p. 336.)

(Solution on p. 336.)

(Solution on p. 336.)

For problems 13-15, specify the fractions that are equivalent.

Exercise 5.10.13

(Section 5.5) $\frac{4}{5}, \frac{12}{15}$

Exercise 5.10.14

(Section 5.5) $\frac{5}{8}, \frac{24}{40}$

Exercise 5.10.15

(Section 5.5) $\frac{5}{12}, \frac{80}{192}$
(Solution on p. 336.)

(Solution on p. 336.)

(Solution on p. 336.)

${ }^{10}$ This content is available online at $<$ http://cnx.org/content $/ \mathrm{m} 34948 / 1.2 />$. 
For problems 16-20, place each collection of fractions in order from smallest to largest.

Exercise 5.10.16

(Section 5.5) $\frac{8}{9}, \frac{6}{7}$

Exercise 5.10.17

(Section 5.5) $\frac{5}{8}, \frac{7}{9}$

Exercise 5.10.18

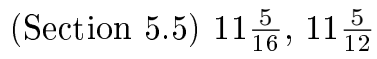

Exercise 5.10.19

(Section 5.5) $\frac{2}{15}, \frac{3}{10}, \frac{1}{6}$

Exercise 5.10.20

(Section 5.5) $\frac{19}{32}, \frac{9}{16}, \frac{5}{8}$
(Solution on p. 336.)

(Solution on p. 336.)

(Solution on p. 336.)

(Solution on p. 337.)

(Solution on p. 337.) 


\section{Solutions to Exercises in Chapter 5}

Solution to Exercise 5.2.1 (p. 297)

$\frac{2}{5}$

Solution to Exercise 5.2.2 (p. 297)

$\frac{1}{2}$

Solution to Exercise 5.2.3 (p. 297)

1

Solution to Exercise 5.2.4 (p. 297)

$\frac{4}{5}$

Solution to Exercise 5.2.5 (p. 297)

$\frac{3}{4}+\frac{3}{4}=\frac{3+3}{4+4}=\frac{6}{8}=\frac{3}{4}$, so two $\frac{3}{4}$ 's $=$ one $\frac{3}{4}$ which is preposterous.

Solution to Exercise 5.2.6 (p. 298)

$\frac{2}{13}$

Solution to Exercise 5.2.7 (p. 298)

$\frac{1}{3}$

Solution to Exercise 5.2.8 (p. 298)

0

Solution to Exercise 5.2.9 (p. 298)

$\frac{6}{5}$

Solution to Exercise 5.2.10 (p. 298)

$\frac{5}{9}-\frac{2}{9}=\frac{5-2}{9-9}=\frac{3}{0}$, which is undefined

Solution to Exercise 5.2.11 (p. 298)

$\frac{5}{8}$

Solution to Exercise 5.2.13 (p. 298)

1

Solution to Exercise 5.2.15 (p. 298)

$\frac{13}{15}$

Solution to Exercise 5.2.17 (p. 299)

1

Solution to Exercise 5.2.19 (p. 299)

0

Solution to Exercise 5.2.21 (p. 299)

0

Solution to Exercise 5.2.23 (p. 299)

$\frac{9}{11}$

Solution to Exercise 5.2.25 (p. 299)

$\frac{15}{8}$

Solution to Exercise 5.2.27 (p. 299)

$\frac{1}{2}$

Solution to Exercise 5.2.29 (p. 299)

$\frac{3}{5}$

Solution to Exercise 5.2.31 (p. 299)

$\frac{13}{5}$

Solution to Exercise 5.2.33 (p. 299)

10

Solution to Exercise 5.2.35 (p. 299)

$\frac{9}{11}$

Solution to Exercise 5.2.37 (p. 300)

$\frac{16}{30}=\frac{8}{5}$ (using the preposterous rule)

Solution to Exercise 5.2.39 (p. 300)

$\frac{13}{24}$ 
Solution to Exercise 5.2.41 (p. 300) 2700

Solution to Exercise 5.2.43 (p. 300) $3^{2} \cdot 5 \cdot 11$

Solution to Exercise 5.2.45 (p. 300)

$\frac{2}{3}$

Solution to Exercise 5.3.1 (p. 303)

$\frac{5}{6}$

Solution to Exercise 5.3.2 (p. 303)

$\frac{1}{14}$

Solution to Exercise 5.3.3 (p. 303)

$\frac{3}{40}$

Solution to Exercise 5.3.4 (p. 303) $\frac{11}{16}$

Solution to Exercise 5.3.5 (p. 303)

$\frac{1}{96}$

Solution to Exercise 5.3.6 (p. 304)

The same denominator

Solution to Exercise 5.3.8 (p. 304)

$\frac{5}{8}$

Solution to Exercise 5.3.10 (p. 304)

$\frac{31}{24}$

Solution to Exercise 5.3.12 (p. 304)

$\frac{17}{28}$

Solution to Exercise 5.3.14 (p. 304)

$\frac{19}{36}$

Solution to Exercise 5.3.16 (p. 304)

$\frac{19}{39}$

Solution to Exercise 5.3.18 (p. 304)

$\frac{29}{60}$

Solution to Exercise 5.3.20 (p. 304)

$\frac{8}{81}$

Solution to Exercise 5.3.22 (p. 304)

$\frac{17}{65}$

Solution to Exercise 5.3.24 (p. 304)

$\frac{2}{63}$

Solution to Exercise 5.3.26 (p. 304)

$\frac{7}{16}$

Solution to Exercise 5.3.28 (p. 305)

$\frac{47}{18}$

Solution to Exercise 5.3.30 (p. 305)

$\frac{103}{30}$

Solution to Exercise 5.3.32 (p. 305)

$\frac{217}{264}$

Solution to Exercise 5.3.34 (p. 305)

$\frac{511}{720}$

Solution to Exercise 5.3.36 (p. 305)

$\frac{37}{72}$

Solution to Exercise 5.3.38 (p. 305)

$\frac{221}{150}$ 
Solution to Exercise 5.3.40 (p. 305)

$\frac{1,465}{2,016}$

Solution to Exercise 5.3.42 (p. 305)

$\frac{65}{204}$

Solution to Exercise 5.3.44 (p. 305)

$\frac{1}{5}$

Solution to Exercise 5.3.46 (p. 305)

$\frac{607}{180}$

Solution to Exercise 5.3.48 (p. 305)

$\frac{109}{520}$

Solution to Exercise 5.3.50 (p. 306) $\$ \frac{137}{8}$ or $\$ 17 \frac{1}{8}$

Solution to Exercise 5.3.52 (p. 306)

No; 3 boxes add up to $26 \frac{1^{\prime \prime}}{4}$, which is larger than $25 \frac{1^{\prime \prime}}{5}$.

Solution to Exercise 5.3.54 (p. 306)

No pipe at all; inside diameter is greater than outside diameter

Solution to Exercise 5.3.56 (p. 306)

449

Solution to Exercise 5.3.58 (p. 306)

660

Solution to Exercise 5.3.60 (p. 306)

$\frac{7}{15}$

Solution to Exercise 5.4.1 (p. 307)

$4 \frac{7}{9}$

Solution to Exercise 5.4.2 (p. 307)

$8 \frac{1}{4}$

Solution to Exercise 5.4.3 (p. 307)

$8 \frac{1}{8}$

Solution to Exercise 5.4.4 (p. 307)

$8 \frac{3}{10}$

Solution to Exercise 5.4.5 (p. 307)

$18 \frac{9}{16}$

Solution to Exercise 5.4.6 (p. 308)

$7 \frac{1}{2}$

Solution to Exercise 5.4.8 (p. 308)

$12 \frac{1}{2}$

Solution to Exercise 5.4.10 (p. 308)

$21 \frac{6}{11}$

Solution to Exercise 5.4.12 (p. 308)

$3 \frac{7}{8}$

Solution to Exercise 5.4.14 (p. 308)

$1 \frac{2}{3}$

Solution to Exercise 5.4.16 (p. 308)

$8 \frac{11}{14}$

Solution to Exercise 5.4.18 (p. 308)

$4 \frac{20}{21}$

Solution to Exercise 5.4.20 (p. 308)

$13 \frac{17}{24}$

Solution to Exercise 5.4.22 (p. 308)

$2 \frac{7}{12}$ 
Solution to Exercise 5.4.24 (p. 308)

$7 \frac{17}{30}$

Solution to Exercise 5.4.26 (p. 309)

$74 \frac{25}{42}$

Solution to Exercise 5.4.28 (p. 309)

$21 \frac{1}{3}$

Solution to Exercise 5.4.30 (p. 309)

$5 \frac{21}{32}$

Solution to Exercise 5.4.32 (p. 309)

$20 \frac{1}{144}$

Solution to Exercise 5.4.34 (p. 309)

$7 \frac{7}{12}$

Solution to Exercise 5.4.36 (p. 309)

$5 \frac{13}{132}$

Solution to Exercise 5.4.38 (p. 309)

$1 \frac{47}{212}$

Solution to Exercise 5.4.40 (p. 309)

$3 \frac{1}{4}$

Solution to Exercise 5.4.42 (p. 309)

$2 \frac{5}{8}$ gallons

Solution to Exercise 5.4.44 (p. 309)

$8 \frac{15}{16}$ pounds

Solution to Exercise 5.4.46 (p. 310) $194 \frac{3}{16}$ pounds

Solution to Exercise 5.4.48 (p. 310) 2

Solution to Exercise 5.4.50 (p. 310)

$\frac{7}{9}$

Solution to Exercise 5.5.1 (p. 312)

$\frac{3}{4}<\frac{4}{5}$

Solution to Exercise 5.5.2 (p. 312)

$\frac{13}{15}<\frac{9}{10}$

Solution to Exercise 5.5.3 (p. 312)

$\frac{13}{16}, \frac{33}{40}, \frac{17}{20}$

Solution to Exercise 5.5.4 (p. 312)

$9 \frac{2}{5}<11 \frac{1}{6}$

Solution to Exercise 5.5.5 (p. 312)

$1 \frac{9}{14}<1 \frac{11}{16}$

Solution to Exercise 5.5.6 (p. 313)

$\frac{3}{5}<\frac{5}{8}$

Solution to Exercise 5.5.8 (p. 313)

$\frac{3}{4}<\frac{5}{6}$

Solution to Exercise 5.5.10 (p. 313)

$\frac{3}{8}<\frac{2}{5}$

Solution to Exercise 5.5.12 (p. 313)

$\frac{1}{2}<\frac{4}{7}<\frac{3}{5}$

Solution to Exercise 5.5.14 (p. 313)

$\frac{3}{4}<\frac{7}{9}<\frac{5}{4}$

Solution to Exercise 5.5.16 (p. 313)

$\frac{3}{14}<\frac{2}{7}<\frac{3}{4}$ 
Solution to Exercise 5.5.18 (p. 313) $5 \frac{4}{7}<5 \frac{3}{5}$

Solution to Exercise 5.5.20 (p. 313) $9 \frac{2}{3}<9 \frac{4}{5}$

Solution to Exercise 5.5.22 (p. 313) $1 \frac{9}{16}<2 \frac{1}{20}$

Solution to Exercise 5.5.24 (p. 313) $2 \frac{2}{9}<2 \frac{3}{7}$

Solution to Exercise 5.5.26 (p. 314) $270,000,000$

Solution to Exercise 5.5.28 (p. 314) $\frac{23}{7}$

Solution to Exercise 5.5.30 (p. 314) $13 \frac{5}{8}$ or $\frac{109}{8}$

Solution to Exercise 5.6.1 (p. 316) $\frac{5}{6}$

$\stackrel{6}{6}$ Solution to Exercise 5.6.2 (p. 316)

$\frac{1}{40}$

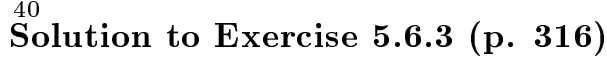

$\frac{3}{2}$

Solution to Exercise 5.6.4 (p. 316)

$\frac{10}{57}$

Solution to Exercise 5.6.5 (p. 316)

$2 \frac{13}{22}$

Solution to Exercise 5.6.6 (p. 316)

$1 \frac{5}{11}$

Solution to Exercise 5.6.7 (p. 316)

1

Solution to Exercise 5.6.9 (p. 316)

$\frac{3}{5}$

Solution to Exercise 5.6.11 (p. 316)

$\frac{5}{9}$

Solution to Exercise 5.6.13 (p. 316)

$\frac{5}{2}$

Solution to Exercise 5.6.15 (p. 316)

$\frac{31}{2}$

Solution to Exercise 5.6.17 (p. 316)

7

Solution to Exercise 5.6.19 (p. 317)

1

Solution to Exercise 5.6.21 (p. 317)

$\frac{1}{6}$

Solution to Exercise 5.6.23 (p. 317)

$\frac{52}{81}$

Solution to Exercise 5.6.25 (p. 317)

$\frac{16}{21}$

Solution to Exercise 5.6.27 (p. 317)

$\frac{686}{101}$

Solution to Exercise 5.6.29 (p. 317) $\frac{1}{3}$ 
Solution to Exercise 5.6.31 (p. 317) $8 \frac{6}{7}$

Solution to Exercise 5.6.33 (p. 317) $1 \frac{13}{24}$ or $\frac{37}{24}$

Solution to Exercise 5.7.1 (p. 320)

0

Solution to Exercise 5.7.2 (p. 320)

$\frac{35}{6}$ or $5 \frac{5}{6}$

Solution to Exercise 5.7.3 (p. 320)

$\frac{211}{30}$ or $7 \frac{1}{30}$

Solution to Exercise 5.7.4 (p. 320)

$\frac{7}{9}$

Solution to Exercise 5.7.5 (p. 320)

$2 \frac{6}{7}$

Solution to Exercise 5.7.6 (p. 320)

$\frac{3}{11}$

Solution to Exercise 5.7.7 (p. 320)

$\frac{15}{64}$

Solution to Exercise 5.7.8 (p. 320)

$\frac{11}{10}$

Solution to Exercise 5.7.9 (p. 321)

$\frac{5}{4}$

Solution to Exercise 5.7.11 (p. 321)

$4 \frac{2}{7}$

Solution to Exercise 5.7.13 (p. 321)

$\frac{2}{15}$

Solution to Exercise 5.7.15 (p. 321)

$\frac{1}{10}$

Solution to Exercise 5.7.17 (p. 321)

$6 \frac{3}{14}$

Solution to Exercise 5.7.19 (p. 321)

$\frac{3}{8}$

$\stackrel{8}{8}$ Solution to Exercise 5.7.21 (p. 321)

$\frac{20}{27}$

Solution to Exercise 5.7.23 (p. 321)

0

Solution to Exercise 5.7.25 (p. 321)

$\frac{2}{5}$

Solution to Exercise 5.7.27 (p. 321)

1

Solution to Exercise 5.7.29 (p. 322)

$\frac{125}{72}$

Solution to Exercise 5.7.31 (p. 322)

$\frac{252}{19}$

Solution to Exercise 5.7.33 (p. 322)

$\frac{165}{256}$

Solution to Exercise 5.7.35 (p. 322)

multiplicative identity

Solution to Exercise 5.7.37 (p. 322)

$\frac{241}{120}$ or $2 \frac{1}{120}$ 
Solution to Exercise 5.9.1 (p. 324) $\frac{11}{8}$ or $1 \frac{3}{8}$

Solution to Exercise 5.9.3 (p. 324)

$\frac{1}{2}$

Solution to Exercise 5.9.5 (p. 324)

$\frac{59}{42}=1 \frac{17}{42}$

Solution to Exercise 5.9.7 (p. 324)

$\frac{5}{8}$

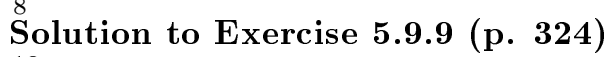

$\frac{13}{21}$

Solution to Exercise 5.9.11 (p. 324)

$4 \frac{7}{16}$

Solution to Exercise 5.9.13 (p. 324)

$8 \frac{3}{20}$

Solution to Exercise 5.9.15 (p. 324)

$1 \frac{3}{8}$

Solution to Exercise 5.9.17 (p. 324)

$24 \frac{2}{3}$

Solution to Exercise 5.9.19 (p. 324)

$\frac{71}{12}=5 \frac{11}{12}$

Solution to Exercise 5.9.21 (p. 324)

$\frac{13}{22}$

Solution to Exercise 5.9.23 (p. 324)

$11 \frac{37}{60}$

Solution to Exercise 5.9.25 (p. 325)

$\frac{139}{144}$

Solution to Exercise 5.9.27 (p. 325)

$5 \frac{1}{10}$

Solution to Exercise 5.9.29 (p. 325)

$\frac{1}{8}$

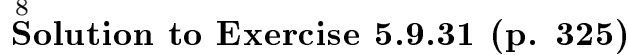

$\frac{11}{16}$

Solution to Exercise 5.9.33 (p. 325)

$8 \frac{2}{11}$

Solution to Exercise 5.9.35 (p. 325) 1

Solution to Exercise 5.9.37 (p. 325)

$7 \frac{3}{10}$

Solution to Exercise 5.9.39 (p. 325)

$\frac{5}{9}$

Solution to Exercise 5.9.41 (p. 325)

$\frac{6}{7}$

Solution to Exercise 5.9.43 (p. 325)

$\frac{3}{2}$ or $1 \frac{1}{2}$

Solution to Exercise 5.9.45 (p. 326)

$\frac{15}{28}$

Solution to Exercise 5.9.47 (p. 326)

$\frac{163}{108}$ or $1 \frac{55}{108}$

Solution to Exercise 5.9.49 (p. 326)

$\frac{66}{13}$ or $5 \frac{1}{13}$ 
Solution to Exercise 5.9.51 (p. 326)

$\frac{7}{40}$

Solution to Exercise 5.9.53 (p. 326)

$\frac{255}{184}$ or $1 \frac{71}{184}$

Solution to Exercise 5.9.55 (p. 326)

$\frac{3}{32}, \frac{1}{8}$

Solution to Exercise 5.9.57 (p. 326)

$\frac{3}{10}, \frac{5}{6}$

Solution to Exercise 5.9.59 (p. 326)

$\frac{3}{8}, \frac{8}{3}, \frac{19}{6}$

Solution to Exercise 5.9.61 (p. 326)

$\frac{5}{9}, \frac{4}{7}$

Solution to Exercise 5.9.63 (p. 327) $\frac{5}{12}, \frac{4}{9}, \frac{7}{15}$

Solution to Exercise 5.9.65 (p. 327) $\frac{5}{8}, \frac{3}{4}, \frac{13}{16}$

Solution to Exercise 5.10.1 (p. 327) $\frac{5}{16}$

Solution to Exercise 5.10.2 (p. 327) $7 \frac{5}{6}$

Solution to Exercise 5.10.3 (p. 327) 1

Solution to Exercise 5.10.4 (p. 327) $\frac{8}{11}$

Solution to Exercise 5.10.5 (p. 327) 8

Solution to Exercise 5.10.6 (p. 327) $2 \frac{13}{40}$

Solution to Exercise 5.10.7 (p. 327) $\frac{49}{32}$ or $\frac{17}{32}$

Solution to Exercise 5.10.8 (p. 327) $\frac{7}{15}$

Solution to Exercise 5.10.9 (p. 327)

$3 \frac{7}{16}$

Solution to Exercise 5.10.10 (p. 327)

$\frac{5}{32}$

Solution to Exercise 5.10.11 (p. 327)

$6 \frac{1}{3}$ or $\frac{19}{3}$

Solution to Exercise 5.10.12 (p. 327)

$3 \frac{3}{7}$

Solution to Exercise 5.10.13 (p. 327)

equivalent

Solution to Exercise 5.10.14 (p. 327) not equivalent

Solution to Exercise 5.10.15 (p. 327)

equivalent

Solution to Exercise 5.10.16 (p. 328)

$\frac{6}{7}, \frac{8}{9}$

Solution to Exercise 5.10.17 (p. 328)

$\frac{5}{8}, \frac{7}{9}$ 
Solution to Exercise 5.10.18 (p. 328) $11 \frac{5}{16}, 11 \frac{5}{12}$

Solution to Exercise 5.10.19 (p. 328)

$\frac{2}{15}, \frac{1}{6}, \frac{3}{10}$

Solution to Exercise 5.10.20 (p. 328)

$\frac{9}{16}, \frac{19}{32}, \frac{5}{8}$ 


\section{Chapter 6}

\section{Decimals}

\subsection{Objectives ${ }^{1}$}

After completing this chapter, you should

Reading and Writing Decimals (Section 6.2)

- understand the meaning of digits occurring to the right of the ones position

- be familiar with the meaning of decimal fractions

- be able to read and write a decimal fraction

\section{Converting a Decimal to a Fraction (Section 6.3)}

- be able to convert an ordinary decimal and a complex decimal to a fraction

\section{Rounding Decimals (Section 6.4)}

- be able to round a decimal number to a specified position

\section{Addition and Subtraction of Decimals (Section 6.5)}

- understand the method used for adding and subtracting decimals

- be able to add and subtract decimals

- be able to use the calculator to add and subtract decimals

\section{Multiplication of Decimals (Section 6.6)}

- understand the method used for multiplying decimals

- be able to multiply decimals

- be able to simplify a multiplication of a decimal by a power of 10

- understand how to use the word "of" in multiplication

\section{Division of Decimals (Section 6.7)}

- understand the method used for dividing decimals

- be able to divide a decimal number by a nonzero whole number and by another, nonzero, decimal number

- be able to simplify a division of a decimal by a power of 10

\section{Nonterminating Divisions (Section 6.8)}

\footnotetext{
${ }^{1}$ This content is available online at $<$ http://cnx.org/content $/ \mathrm{m} 18894 / 1.3 />$. Available for free at Connexions <http://cnx.org/content/col10615/1.4>
} 
- understand the meaning of a nonterminating division

- be able to recognize a nonterminating number by its notation

\section{Converting a Fraction to a Decimal (Section 6.9)}

- be able to convert a fraction to a decimal

Combinations of Operations with Decimals and Fractions (Section 6.10)

- be able to combine operations with decimals

\subsection{Reading and Writing Decimals ${ }^{2}$}

\subsubsection{Section Overview}

- Digits to the Right of the Ones Position

- Decimal Fractions

- Reading Decimal Fractions

- Writing Decimal Fractions

\subsubsection{Digits to the Right of the Ones Position}

We began our study of arithmetic (Section 1.2) by noting that our number system is called a positional number system with base ten. We also noted that each position has a particular value. We observed that each position has ten times the value of the position to its right.

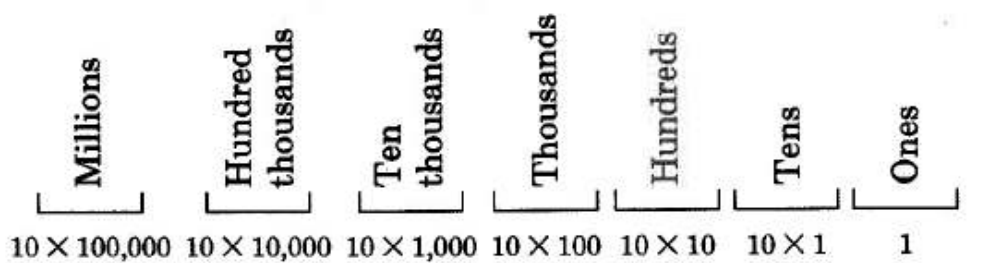

This means that each position has $\frac{1}{10}$ the value of the position to its left.

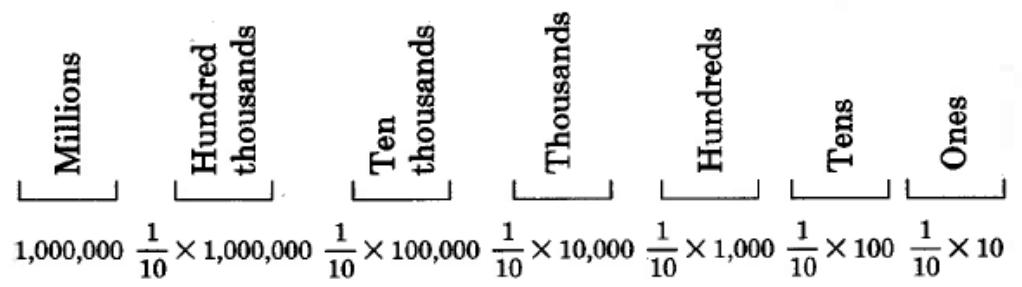

Thus, a digit written to the right of the units position must have a value of $\frac{1}{10}$ of 1 . Recalling that the word "of" translates to multiplication $(\cdot)$, we can see that the value of the first position to the right of the units digit is $\frac{1}{10}$ of 1 , or

$\frac{1}{10} \cdot 1=\frac{1}{10}$

The value of the second position to the right of the units digit is $\frac{1}{10}$ of $\frac{1}{10}$, or

$\frac{1}{10} \cdot \frac{1}{10}=\frac{1}{10^{2}}=\frac{1}{100}$

\footnotetext{
${ }^{2}$ This content is available online at $<$ http://cnx.org/content $/ \mathrm{m} 34957 / 1.2 />$.
} 
The value of the third position to the right of the units digit is $\frac{1}{10}$ of $\frac{1}{100}$, or

$\frac{1}{10} \cdot \frac{1}{100}=\frac{1}{10^{3}}=\frac{1}{1000}$

This pattern continues.

We can now see that if we were to write digits in positions to the right of the units positions, those positions have values that are fractions. Not only do the positions have fractional values, but the fractional values are all powers of $10\left(10,10^{2}, 10^{3}, \ldots\right)$.

\subsubsection{Decimal Fractions}

\section{Decimal Point, Decimal}

If we are to write numbers with digits appearing to the right of the units digit, we must have a way of denoting where the whole number part ends and the fractional part begins. Mathematicians denote the separation point of the units digit and the tenths digit by writing a decimal point. The word decimal comes from the Latin prefix "deci" which means ten, and we use it because we use a base ten number system. Numbers written in this form are called decimal fractions, or more simply, decimals.

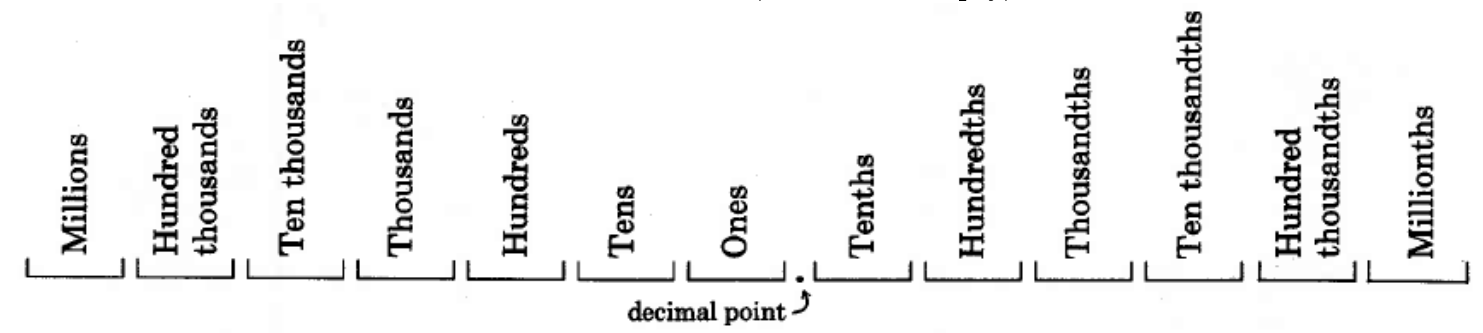

Notice that decimal numbers have the suffix "th."

\section{Decimal Fraction}

A decimal fraction is a fraction in which the denominator is a power of 10 .

The following numbers are examples of decimals.

1. 42.6

The 6 is in the tenths position.

$42.6=42 \frac{6}{10}$

2. 9.8014

The 8 is in the tenths position.

The 0 is in the hundredths position.

The 1 is in the thousandths position.

The 4 is in the ten thousandths position.

3. 0.93

$9.8014=9 \frac{8014}{10,000}$

The 9 is in the tenths position.

The 3 is in the hundredths position.

$0.93=\frac{93}{100}$

NOTE: Quite often a zero is inserted in front of a decimal point (in the units position) of a decimal fraction that has a value less than one. This zero helps keep us from overlooking the decimal point. 
4. 0.7

The 7 is in the tenths position.

$0.7=\frac{7}{10}$

NOTE: We can insert zeros to the right of the right-most digit in a decimal fraction without changing the value of the number. $\frac{7}{10}=0.7=0.70=\frac{70}{100}=\frac{7}{10}$

\subsubsection{Reading Decimal Fractions}

\section{Reading a Decimal Fraction}

To read a decimal fraction,

1. Read the whole number part as usual. (If the whole number is less than 1, omit steps 1 and 2.)

2. Read the decimal point as the word "and."

3. Read the number to the right of the decimal point as if it were a whole number.

4. Say the name of the position of the last digit.

\subsubsection{Sample Set A}

Read the following numbers.

\section{Example 6.1}

6.8

6. 8

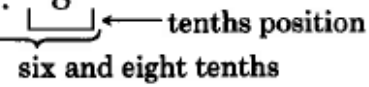

NOTE: Some people read this as "six point eight." This phrasing gets the message across, but technically, "six and eight tenths" is the correct phrasing.

\section{Example 6.2}

14.116

\section{$14.11,6$} fourteen and one hundred sixteen thousandths

\section{Example 6.3}

0.0019

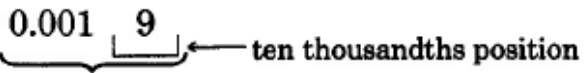 nineteen ten thousandths}

\section{Example 6.4}

81

\section{Eighty-one}

In this problem, the indication is that any whole number is a decimal fraction. Whole numbers are often called decimal numbers.

$81=81.0$ 


\subsubsection{Practice Set A}

Read the following decimal fractions.

Exercise 6.2.1

(Solution on p. 404.)

12.9

Exercise 6.2.2

4.86

(Solution on p. 404.)

Exercise 6.2.3

7.00002

Exercise 6.2.4

0.030405

(Solution on p. 404.)

(Solution on p. 404.)

\subsubsection{Writing Decimal Fractions}

\section{Writing a Decimal Fraction}

To write a decimal fraction,

1. Write the whole number part.

2. Write a decimal point for the word "and."

3. Write the decimal part of the number so that the right-most digit appears in the position indicated in the word name. If necessary, insert zeros to the right of the decimal point in order that the right-most digit appears in the correct position.

\subsubsection{Sample Set B}

Write each number.

Example 6.5

Thirty-one and twelve hundredths.

The decimal position indicated is the hundredths position.

31.12

Example 6.6

Two and three hundred-thousandths.

The decimal position indicated is the hundred thousandths. We'll need to insert enough zeros to the immediate right of the decimal point in order to locate the 3 in the correct position.

2.00003

\section{Example 6.7}

Six thousand twenty-seven and one hundred four millionths.

The decimal position indicated is the millionths position. We'll need to insert enough zeros to the immediate right of the decimal point in order to locate the 4 in the correct position.

$6,027.000104$

\section{Example 6.8}

Seventeen hundredths.

The decimal position indicated is the hundredths position. 
0.17

\subsubsection{Practice Set B}

Write each decimal fraction.

Exercise 6.2.5

(Solution on p. 404.)

Three hundred six and forty-nine hundredths.

Exercise 6.2.6

(Solution on p. 404.)

Nine and four thousandths.

(Solution on p. 404.)

Exercise 6.2.7

Sixty-one millionths.

\subsubsection{Exercises}

For the following three problems, give the decimal name of the position of the given number in each decimal fraction.

Exercise 6.2.8

(Solution on p. 404.)

1. 3.941

9 is in the

4 is in the ion.

1 is in the position. position.

Exercise 6.2.9

17.1085

1 is in the position.

0 is in the position.

8 is in the position.

5 is in the position.

Exercise 6.2.10

(Solution on p. 404.) 652.3561927

9 is in the

7 is in the position. position.

For the following 7 problems, read each decimal fraction by writing it.

Exercise 6.2.11

9.2

Exercise 6.2.12

(Solution on p. 404.)

8.1

Exercise 6.2.13

10.15

Exercise 6.2.14

(Solution on p. 404.) 55.06

Exercise 6.2.15

0.78

Exercise 6.2.16

(Solution on p. 404.)

1.904

Exercise 6.2.17

10.00011 
For the following 10 problems, write each decimal fraction.

Exercise 6.2.18

(Solution on p. 404.)

Three and twenty one-hundredths.

Exercise 6.2.19

Fourteen and sixty seven-hundredths.

Exercise 6.2.20

(Solution on p. 404.)

One and eight tenths.

Exercise 6.2.21

Sixty-one and five tenths.

Exercise 6.2.22

(Solution on p. 404.)

Five hundred eleven and four thousandths.

Exercise 6.2.23

Thirty-three and twelve ten-thousandths.

Exercise 6.2.24

(Solution on p. 404.)

Nine hundred forty-seven thousandths.

Exercise 6.2.25

Two millionths.

Exercise 6.2.26

Seventy-one hundred-thousandths.

(Solution on p. 404.)

Exercise 6.2.27

One and ten ten-millionths.

Calculator Problems

For the following 10 problems, perform each division using a calculator. Then write the resulting decimal using words.

Exercise 6.2.28

(Solution on p. 404.)

$3 \div 4$

Exercise 6.2.29

$1 \div 8$

Exercise 6.2.30

(Solution on p. 404.)

$4 \div 10$

Exercise 6.2.31

$2 \div 5$

Exercise 6.2.32

$4 \div 25$

(Solution on p. 404.)

Exercise 6.2.33

$1 \div 50$

Exercise 6.2.34

(Solution on p. 404.)

$3 \div 16$

Exercise 6.2.35

$15 \div 8$

Exercise 6.2.36

$11 \div 20$

(Solution on p. 404.)

Exercise 6.2.37

$9 \div 40$ 


\subsubsection{Exercises for Review}

Exercise 6.2.38

(Solution on p. 404.)

(Section 1.4) Round 2,614 to the nearest ten.

Exercise 6.2.39

(Section 2.5) Is $691,428,471$ divisible by 3 ?

Exercise 6.2.40

(Solution on p. 404.)

(Section 4.4) Determine the missing numerator.

$\frac{3}{14}=\frac{?}{56}$

Exercise 6.2.41

(Section 4.7) Find $\frac{3}{16}$ of $\frac{32}{39}$

Exercise 6.2.42

(Section 5.7) Find the value of $\sqrt{\frac{25}{81}}+\left(\frac{2}{3}\right)^{2}+\frac{1}{9}$

(Solution on p. 404.)

\subsection{Converting a Decimal to a Fraction ${ }^{3}$}

\subsubsection{Section Overview}

- Converting an Ordinary Decimal to a Fraction

- Converting a Complex Decimal to a Fraction

\subsubsection{Converting an Ordinary Decimal to a Fraction}

We can convert a decimal fraction to a fraction, essentially, by saying it in words, then writing what we say. We may have to reduce that fraction.

\subsubsection{Sample Set A}

Convert each decimal fraction to a proper fraction or a mixed number.

\section{Example 6.9}

0.6

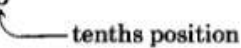

Reading: six tenths $\rightarrow \frac{6}{10}$.

Reduce: $\frac{3}{5}$.

Example 6.10

0.903

— thousandths position

Reading: nine hundred three thousands $\rightarrow \frac{903}{1000}$.

Example 6.11

18.61

\section{hundredths position}

\footnotetext{
${ }^{3}$ This content is available online at $<\mathrm{http}: / / \mathrm{cnx}$. org/content $/ \mathrm{m} 34958 / 1.2 />$.
} 
Reading: eighteen and sixty-one hundredths $\rightarrow 18 \frac{61}{100}$.

Example 6.12

508.0005

ten thousandths position

Reading: five hundred eight and five ten thousandths $\rightarrow 508 \frac{5}{10,000}$.

Reduce: $508 \frac{1}{2,000}$.

\subsubsection{Practice Set A}

Convert the following decimals to fractions or mixed numbers. Be sure to reduce.

Exercise 6.3.1

16.84

Exercise 6.3.2

0.513

Exercise 6.3.3

$6,646.0107$

Exercise 6.3.4

1.1
(Solution on p. 404.)

(Solution on p. 405.)

(Solution on p. 405.)

(Solution on p. 405.)

\subsubsection{Converting A Complex Decimal to a Fraction}

\section{Complex Decimals}

Numbers such as $0.11 \frac{2}{3}$ are called complex decimals. We can also convert complex decimals to fractions.

\subsubsection{Sample Set B}

Convert the following complex decimals to fractions.

\section{Example 6.13}

$0.11 \frac{2}{3}$

The $\frac{2}{3}$ appears to occur in the thousands position, but it is referring to $\frac{2}{3}$ of a hundredth. So, we read $0.11 \frac{2}{3}$ as "eleven and two-thirds hundredths."

$$
\begin{aligned}
0.11 \frac{2}{3}=\frac{11 \frac{2}{3}}{100} & =\frac{\frac{11 \cdot 3+2}{3}}{100} \\
& =\frac{\frac{35}{3}}{\frac{100}{1}} \\
& =\frac{35}{3} \div \frac{100}{1} \\
& =\frac{7}{3} \cdot \frac{1}{\overline{100}} \\
& =\frac{7}{60}
\end{aligned}
$$

Example 6.14

$4.006 \frac{1}{4}$

Note that $4.006 \frac{1}{4}=4+.006 \frac{1}{4}$ 


$$
\begin{aligned}
4+.006 \frac{1}{4} & = & 4+\frac{6 \frac{1}{4}}{1000} \\
& = & 4+\frac{\frac{25}{4}}{\frac{100}{1}} \\
& = & 4+\frac{\frac{1}{25}}{4} \cdot \frac{1}{\frac{11000}{1000}} \\
& = & 4+\frac{1 \cdot 1}{4 \cdot 40} \\
& = & 4+\frac{1}{160} \\
& = & 4 \frac{1}{160}
\end{aligned}
$$

\subsubsection{Practice Set B}

Convert each complex decimal to a fraction or mixed number. Be sure to reduce.

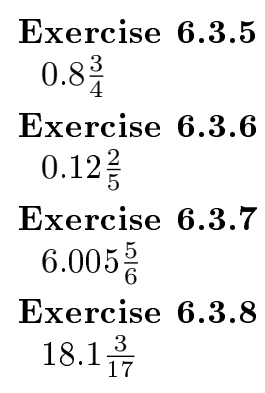

(Solution on p. 405.)

(Solution on p. 405.)

(Solution on p. 405.)

(Solution on p. 405.)

\subsubsection{Exercises}

For the following 20 problems, convert each decimal fraction to a proper fraction or a mixed number. Be sure to reduce.

Exercise 6.3.9

(Solution on p. 405.)

0.7

Exercise 6.3.10

0.1

Exercise 6.3.11

(Solution on p. 405.)

0.53

Exercise 6.3.12

0.71

Exercise 6.3.13

(Solution on p. 405.)

0.219

Exercise 6.3.14

0.811

Exercise 6.3.15

(Solution on p. 405.)

4.8

Exercise 6.3.16

2.6

Exercise 6.3.17

16.12

(Solution on p. 405.)

Exercise 6.3.18

25.88 
Exercise 6.3.19

(Solution on p. 405.)

6.0005

Exercise 6.3.20

1.355

Exercise 6.3.21

(Solution on p. 405.)

16.125

Exercise 6.3.22

0.375

Exercise 6.3.23

3.04

(Solution on p. 405.)

Exercise 6.3.24

21.1875

Exercise 6.3.25

8.225

(Solution on p. 405.)

Exercise 6.3.26

1.0055

Exercise 6.3.27

9.99995

(Solution on p. 405.)

Exercise 6.3.28

22.110

For the following 10 problems, convert each complex decimal to a fraction.

Exercise 6.3.29 $0.7 \frac{1}{2}$

(Solution on p. 405.)

Exercise 6.3.30

$0.012 \frac{1}{2}$

Exercise 6.3.31 $2.16 \frac{1}{4}$

(Solution on p. 405.)

Exercise 6.3.32 $5.18 \frac{2}{3}$

Exercise 6.3.33 $14.112 \frac{1}{3}$

Exercise 6.3.34 $80.0011 \frac{3}{7}$

Exercise 6.3.35 $1.40 \frac{5}{16}$

(Solution on p. 405.)

Exercise 6.3.36 $0.8 \frac{5}{3}$

Exercise 6.3.37 $1.9 \frac{7}{5}$

Exercise 6.3.38 $1.7 \frac{37}{9}$

(Solution on p. 405.)

(Solution on p. 405.) 


\subsubsection{Exercises for Review}

Exercise 6.3.39

(Solution on p. 405.)

(Section 3.6) Find the greatest common factor of 70, 182, and 154.

Exercise 6.3.40

(Section 3.6) Find the greatest common multiple of 14, 26, and 60.

Exercise 6.3.41

(Section 4.5) Find the value of $\frac{3}{5} \cdot \frac{15}{18} \div \frac{5}{9}$.

(Solution on p. 405.)

Exercise 6.3.42

(Section 5.4) Find the value of $5 \frac{2}{3}+8 \frac{1}{12}$.

Exercise 6.3.43

(Section 6.2) In the decimal number 26.10742, the digit 7 is in what position?

\subsection{Rounding Decimals ${ }^{4}$}

\subsubsection{Section Overview}

- Rounding Decimal Numbers

\subsubsection{Rounding Decimal Numbers}

We first considered the concept of rounding numbers in Section 1.4 where our concern with rounding was related to whole numbers only. With a few minor changes, we can apply the same rules of rounding to decimals.

To round a decimal to a particular position:

1. Mark the position of the round-off digit (with an arrow or check).

2. Note whether the digit to the immediate right of the marked digit is

(a) less than 5. If so, leave the round-off digit unchanged.

(b) 5 or greater. If so, add 1 to the round-off digit.

3. If the round-off digit is

(a) to the right of the decimal point, eliminate all the digits to its right.

(b) to the left of the decimal point, replace all the digits between it and the decimal point with zeros and eliminate the decimal point and all the decimal digits.

\subsubsection{Sample Set A}

Round each decimal to the specified position. (The numbers in parentheses indicate which step is being used.)

Example 6.15

Round 32.116 to the nearest hundredth.

\subsection{6}

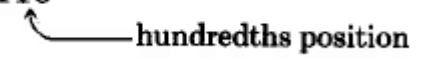

\footnotetext{
${ }^{4}$ This content is available online at <http://cnx.org/content/m34959/1.2/>.
} 
(2b) The digit immediately to the right is 6 , and $6>5$, so we add 1 to the round-off digit: $1+1=2$

(3a) The round-off digit is to the right of the decimal point, so we eliminate all digits to its right. 32.12

The number 32.116 rounded to the nearest hundredth is 32.12 .

\section{Example 6.16}

Round 633.14216 to the nearest hundred.

633.14216

(1)

(2a) The digit immediately to the right is 3 , and $3<5$ so we leave the round-off digit unchanged.

(3b) The round-off digit is to the left of 0 , so we replace all the digits between it and the decimal point with zeros and eliminate the decimal point and all the decimal digits. 600

The number 633.14216 rounded to the nearest hundred is 600 .

\section{Example 6.17}

$1,729.63$ rounded to the nearest ten is 1,730 .

Example 6.18

1.0144 rounded to the nearest tenth is 1.0 .

\section{Example 6.19}

60.98 rounded to the nearest one is 61 .

Sometimes we hear a phrase such as "round to three decimal places." This phrase means that the round-off digit is the third decimal digit (the digit in the thousandths position).

\section{Example 6.20}

67.129 rounded to the second decimal place is 67.13 .

\section{Example 6.21}

67.129558 rounded to 3 decimal places is 67.130 .

\subsubsection{Practice Set A}

Round each decimal to the specified position.

Exercise 6.4.1

(Solution on p. 406.)

4.816 to the nearest hundredth.

Exercise 6.4.2

(Solution on p. 406.)

0.35928 to the nearest ten thousandths.

Exercise 6.4.3

(Solution on p. 406.)

82.1 to the nearest one.

Exercise 6.4.4

(Solution on p. 406.)

753.98 to the nearest hundred.

Exercise 6.4.5

(Solution on p. 406.)

Round 43.99446 to three decimal places.

Exercise 6.4.6

(Solution on p. 406.)

Round 105.019997 to four decimal places.

Exercise 6.4.7

(Solution on p. 406.)

Round 99.9999 to two decimal places. 


\subsubsection{Exercises}

For the first 10 problems, complete the chart by rounding each decimal to the indicated positions.

Exercise 6.4.8 20.01071

(Solution on p. 406.)

\begin{tabular}{|l|l|l|l|}
\hline Tenth & Hundredth & Thousandth & Ten Thousandth \\
\hline & & & \\
\hline
\end{tabular}

Table 6.1

Exercise 6.4.9

3.52612

\begin{tabular}{|l|l|l|l|}
\hline Tenth & Hundredth & Thousandth & Ten Thousandth \\
\hline & 3.53 & & \\
\hline
\end{tabular}

Table 6.2

Exercise 6.4.10

531.21878

(Solution on p. 406.)

\begin{tabular}{|l|l|l|l|}
\hline Tenth & Hundredth & Thousandth & Ten Thousandth \\
\hline & & & \\
\hline
\end{tabular}

Table 6.3

Exercise 6.4.11

36.109053

\begin{tabular}{|l|l|l|l|}
\hline Tenth & Hundredth & Thousandth & Ten Thousandth \\
\hline 36.1 & & & \\
\hline
\end{tabular}

Table 6.4

Exercise 6.4.12 1.999994

(Solution on p. 406.)

\begin{tabular}{|l|l|l|l|}
\hline Tenth & Hundredth & Thousandth & Ten Thousandth \\
\hline & & & \\
\hline
\end{tabular}

Table 6.5

Exercise 6.4.13

7.4141998 


\begin{tabular}{|l|l|l|l|}
\hline Tenth & Hundredth & Thousandth & Ten Thousandth \\
\hline & & 7.414 & \\
\hline
\end{tabular}

Table 6.6

Exercise 6.4.14 0.000007

(Solution on p. 406.)

\begin{tabular}{|l|l|l|l|}
\hline Tenth & Hundredth & Thousandth & Ten Thousandth \\
\hline & & & \\
\hline
\end{tabular}

Table 6.7

Exercise 6.4.15

0.00008

\begin{tabular}{|l|l|l|l|}
\hline Tenth & Hundredth & Thousandth & Ten Thousandth \\
\hline & & & 0.0001 \\
\hline
\end{tabular}

Table 6.8

Exercise 6.4.16

9.19191919

(Solution on p. 406.)

\begin{tabular}{|l|l|l|l|}
\hline Tenth & Hundredth & Thousandth & Ten Thousandth \\
\hline & & & \\
\hline
\end{tabular}

Table 6.9

Exercise 6.4.17

0.0876543

\begin{tabular}{|l|l|l|l|}
\hline Tenth & Hundredth & Thousandth & Ten Thousandth \\
\hline & & & \\
\hline
\end{tabular}

Table 6.10

\section{Calculator Problems}

For the following 5 problems, round 18.4168095 to the indicated place.

Exercise 6.4.18

(Solution on p. 407.)

3 decimal places.

Exercise 6.4.19

1 decimal place.

Exercise 6.4.20

5 decimal places.

(Solution on p. 407.) 
Exercise 6.4.21

6 decimal places.

Exercise 6.4.22

(Solution on p. 407.)

2 decimal places.

Calculator Problems

For the following problems, perform each division using a calculator.

Exercise 6.4.23

$4 \div 3$ and round to 2 decimal places.

Exercise 6.4.24

$1 \div 8$ and round to 1 decimal place.

(Solution on p. 407.)

Exercise 6.4.25

$1 \div 27$ and round to 6 decimal places.

Exercise 6.4.26

$51 \div 61$ and round to 5 decimal places.

(Solution on p. 407.)

Exercise 6.4.27

$3 \div 16$ and round to 3 decimal places.

Exercise 6.4.28

$16 \div 3$ and round to 3 decimal places.

(Solution on p. 407.)

Exercise 6.4.29

$26 \div 7$ and round to 5 decimal places.

\subsubsection{Exercises for Review}

Exercise 6.4.30

(Solution on p. 407.)

(Section 1.2) What is the value of 2 in the number $421,916,017$ ?

Exercise 6.4.31

(Section 2.4) Perform the division: $378 \div 29$.

Exercise 6.4.32

(Solution on p. 407.)

(Section 3.2) Find the value of $4^{4}$.

Exercise 6.4.33

(Section 4.3) Convert $\frac{11}{3}$ to a mixed number.

Exercise 6.4.34

(Section 6.3) Convert 3.16 to a mixed number fraction.

(Solution on p. 407.)

\subsection{Addition and Subtraction of Decimals ${ }^{5}$}

\subsubsection{Section Overview}

- The Logic Behind the Method

- The Method of Adding and Subtracting Decimals

- Calculators

\footnotetext{
${ }^{5}$ This content is available online at $<$ http://cnx.org/content $/ \mathrm{m} 34960 / 1.5 />$.
} 


\subsubsection{The Logic Behind the Method}

Consider the sum of 4.37 and 3.22. Changing each decimal to a fraction, we have

$4 \frac{37}{100}+3 \frac{22}{100}$ Performing the addition, we get

$$
\begin{array}{clc}
4.37+3.22=4 \frac{37}{100}+3 \frac{22}{100} & = & \frac{4 \cdot 100+37}{100}+\frac{3 \cdot 100+22}{100} \\
& = & \frac{437}{100}+\frac{322}{100} \\
& = & \frac{437+322}{100} \\
& = & \frac{759}{100} \\
& = & 7 \frac{59}{100} \\
& = & \text { seven and fifty-nine hundredths } \\
& = &
\end{array}
$$

Thus, $4.37+3.22=7.59$.

\subsubsection{The Method of Adding and Subtracting Decimals}

When writing the previous addition, we could have written the numbers in columns.

7.59

This agrees with our previous result. From this observation, we can suggest a method for adding and subtracting decimal numbers.

\section{Method of Adding and Subtracting Decimals}

To add or subtract decimals:

1. Align the numbers vertically so that the decimal points line up under each other and the corresponding decimal positions are in the same column.

2. Add or subtract the numbers as if they were whole numbers.

3. Place a decimal point in the resulting sum or difference directly under the other decimal points.

\subsubsection{Sample Set A}

Find the following sums and differences.

Example 6.22

$9.813+2.140$

9.813 The decimal points are aligned in the same column.

$+2.140$

11.953

Example 6.23

$841.0056+47.016+19.058$ 
841.0056

47.016

$+19.058$

To insure that the columns align properly, we can write a 0 in the position at the end of the numbers 47.016 and 19.058 without changing their values.

11.1

841.0056

47.0160

$+19.0580$

907.0796

\section{Example 6.24}

$1.314-0.58$

1.314

-0.58 Write a 0 in the thousandths position.

12

$0 \$ 11$

1.314

$-0.580$

0.734

Example 6.25

$16.01-7.053$

16.01

-7.053 Write a 0 in the thousandths position.

1591010

$16 . \phi 16$

$-7.053$

8.957

Example 6.26

Find the sum of 6.88106 and 3.5219 and round it to three decimal places.

6.88106

+3.5219 Write a 0 in the ten thousandths position.

11

6.88106

$\begin{array}{r}3.52190 \\ \hline\end{array}$

10.40296

We need to round the sum to the thousandths position. Since the digit in the position immediately to the right is 9 , and $9>5$, we get

10.403

Example 6.27

Wendy has $\$ 643.12$ in her checking account. She writes a check for $\$ 16.92$. How much is her new account balance? 
To find the new account balance, we need to find the difference between 643.12 and 16.92 . We will subtract 16.92 from 643.12 .

31211

643.12

$-16.92$

626.20

After writing a check for $\$ 16.92$, Wendy now has a balance of $\$ 626.20$ in her checking account.

\subsubsection{Practice Set A}

Find the following sums and differences.

Exercise 6.5.1

(Solution on p. 407.)

$3.187+2.992$

Exercise 6.5.2

(Solution on p. 407.)

14.987 - 5.341

Exercise 6.5.3

(Solution on p. 407.)

$0.5261+1.0783$

(Solution on p. 407.)

Exercise 6.5.4

(Solution on p. 407.)

Exercise 6.5.5

$16,521.07+9,256.15$

(Solution on p. 407.)

Exercise 6.5.6

Find the sum of 11.6128 and 14.07353 , and round it to two decimal places.

\subsubsection{Calculators}

The calculator can be useful for finding sums and differences of decimal numbers. However, calculators with an eight-digit display cannot be used when working with decimal numbers that contain more than eight digits, or when the sum results in more than eight digits. In practice, an eight-place decimal will seldom be encountered. There are some inexpensive calculators that can handle 13 decimal places.

\subsubsection{Sample Set B}

Use a calculator to find each sum or difference.

Example 6.28

$42.0638+126.551$

\begin{tabular}{|l|l|l|}
\hline & & Display Reads \\
\hline Type & 42.0638 & 42.0638 \\
\hline Press & + & 42.0638 \\
\hline Type & 126.551 & 126.551 \\
\hline Press & $=$ & 168.6148 \\
\hline
\end{tabular}

Table 6.11 
The sum is 168.6148 .

Example 6.29

Find the difference between 305.0627 and 14.29667.

\begin{tabular}{|l|l|l|}
\hline & & Display Reads \\
\hline Type & 305.0627 & 305.0627 \\
\hline Press & - & 305.0627 \\
\hline Type & 14.29667 & 14.29667 \\
\hline Press & $=$ & 290.76603 \\
\hline
\end{tabular}

Table 6.12

The difference is 290.76603

Example 6.30

$51.07+3,891.001786$

Since 3,891.001786 contains more than eight digits, we will be unable to use an eight-digit display calculator to perform this addition. We can, however, find the sum by hand.

51.070000

$\underline{3891.001786}$

3942.071786

The sum is $3,942.071786$.

\subsubsection{Practice Set B}

Use a calculator to perform each operation.

Exercise 6.5.7

(Solution on p. 407.)

$4.286+8.97$

Exercise 6.5.8

(Solution on p. 407.)

$452.0092-392.558$

Exercise 6.5.9

(Solution on p. 407.)

Find the sum of 0.095 and 0.001862

Exercise 6.5.10

(Solution on p. 407.)

Find the difference between 0.5 and 0.025

Exercise 6.5.11

Find the sum of 2,776.00019 and 2,009.00012.

(Solution on p. 407.) 


\subsubsection{Exercises}

For the following 15 problems, perform each addition or subtraction. Use a calculator to check each result.

Exercise 6.5.12

(Solution on p. 407.)

$1.84+7.11$

Exercise 6.5.13

$15.015-6.527$

Exercise 6.5.14

$11.842+28.004$

(Solution on p. 407.)

Exercise 6.5.15

$3.16-2.52$

Exercise 6.5.16

(Solution on p. 407.)

$3.55267+8.19664$

Exercise 6.5.17

$0.9162-0.0872$

Exercise 6.5.18

$65.512-8.3005$

(Solution on p. 408.)

Exercise 6.5.19 $761.0808-53.198$

Exercise 6.5.20

$4.305+2.119-3.817$

(Solution on p. 408.)

Exercise 6.5.21

$19.1161+27.8014+39.3161$

Exercise 6.5.22

$0.41276-0.0018-0.00011$

Exercise 6.5.23

$2.181+6.05+1.167+8.101$

Exercise 6.5.24

$1.0031+6.013106+0.00018+0.0092+2.11$

(Solution on p. 408.)

Exercise 6.5.25

$27+42+9.16-0.1761+81.6$

Exercise 6.5.26

(Solution on p. 408.)

$10.28+11.111+0.86+5.1$

For the following 10 problems, solve as directed. A calculator may be useful.

Exercise 6.5.27

Add 6.1121 and 4.916 and round to 2 decimal places.

Exercise 6.5.28

Add 21.66418 and 18.00184 and round to 4 decimal places.

(Solution on p. 408.)

Exercise 6.5.29

Subtract 5.2121 from 9.6341 and round to 1 decimal place.

Exercise 6.5.30

(Solution on p. 408.)

Subtract 0.918 from 12.006 and round to 2 decimal places.

(Solution on p. 408.)

Exercise 6.5.31

Subtract 7.01884 from the sum of 13.11848 and 2.108 and round to 4 decimal places. 
Exercise 6.5.32

(Solution on p. 408.)

A checking account has a balance of $\$ 42.51$. A check is written for $\$ 19.28$. What is the new balance?

Exercise 6.5.33

A checking account has a balance of $\$ 82.97$. One check is written for $\$ 6.49$ and another for $\$ 39.95$. What is the new balance?

Exercise 6.5.34

(Solution on p. 408.)

A person buys $\$ 4.29$ worth of hamburger and pays for it with a $\$ 10$ bill. How much change does this person get?

Exercise 6.5.35

A man buys $\$ 6.43$ worth of stationary and pays for it with a $\$ 20$ bill. After receiving his change, he realizes he forgot to buy a pen. If the total price of the pen is $\$ 2.12$, and he buys it, how much of the $\$ 20$ bill is left?

Exercise 6.5.36

(Solution on p. 408.)

A woman starts recording a movie on her video cassette recorder with the tape counter set at 21.93. The movie runs 847.44 tape counter units. What is the final tape counter reading?

\subsubsection{Exercises for Review}

Exercise 6.5.37

(Section 1.6) Find the difference between 11,206 and 10,884.

Exercise 6.5.38

(Solution on p. 408.)

(Section 2.2) Find the product, $820 \cdot 10,000$.

Exercise 6.5.39

(Section 3.3) Find the value of $\sqrt{121}-\sqrt{25}+8^{2}+16 \div 2^{2}$.

Exercise 6.5.40

(Section 4.6) Find the value of $8 \frac{1}{3} \cdot \frac{36}{75} \div 2 \frac{2}{5}$.

(Solution on p. 408.)

Exercise 6.5.41

(Section 6.4) Round 1.08196 to the nearest hundredth.

\subsection{Multiplication of Decimals ${ }^{6}$}

\subsubsection{Section Overview}

- The Logic Behind the Method

- The Method of Multiplying Decimals

- Calculators

- Multiplying Decimals By Powers of 10

- Multiplication in Terms of "Of"

\footnotetext{
${ }^{6}$ This content is available online at $<\mathrm{http}: / / \mathrm{cnx} . o r g /$ content $/ \mathrm{m} 34963 / 1.2 />$.
} 


\subsubsection{The Logic Behind the Method}

Consider the product of 3.2 and 1.46. Changing each decimal to a fraction, we have

$$
\begin{array}{rlr}
(3.2)(1.46) & = & 3 \frac{2}{10} \cdot 1 \frac{46}{100} \\
& = & \frac{32}{10} \cdot \frac{146}{100} \\
& = & \frac{32 \cdot 146}{10 \cdot 100} \\
& = & \frac{4672}{1000} \\
& = & 4 \frac{672}{1000} \\
& = & \text { four and six hundred seventy-two thousandths } \\
& = & 4.672
\end{array}
$$

Thus, $(3.2)(1.46)=4.672$.

Notice that the factor

3.2 has 1 decimal place,

1.46 has 2 decimal places, $\quad\} 1+2=3$

and the product

4.672 has 3 decimal places.

Using this observation, we can suggest that the sum of the number of decimal places in the factors equals the number of decimal places in the product.

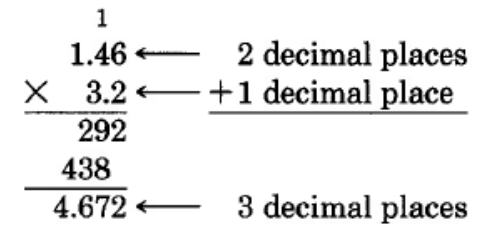

\subsubsection{The Method of Multiplying Decimals}

\section{Method of Multiplying Decimals}

To multiply decimals,

1. Multiply the numbers as if they were whole numbers.

2. Find the sum of the number of decimal places in the factors.

3 . The number of decimal places in the product is the sum found in step 2 .

\subsubsection{Sample Set A}

Find the following products.

\section{Example 6.31}

$6.5 \cdot 4.3$

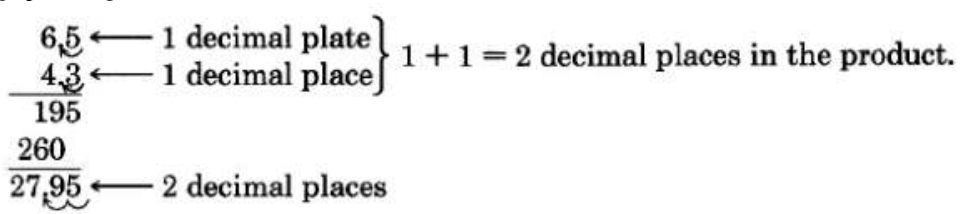


Thus, $6.5 \cdot 4.3=27.95$.

Example 6.32

$23.4 \cdot 1.96$

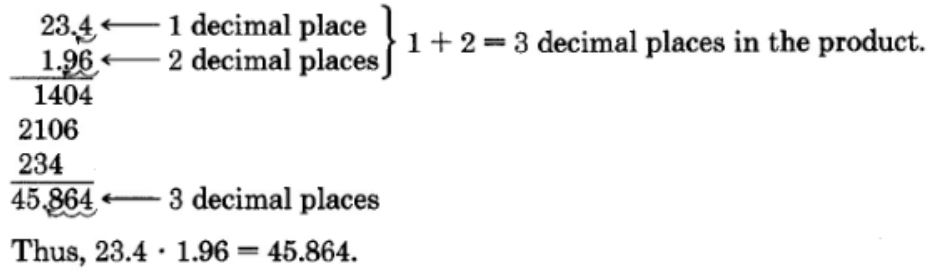

Thus, $23.4 \cdot 1.96=45.864$.

Example 6.33

Find the product of 0.251 and 0.00113 and round to three decimal places.

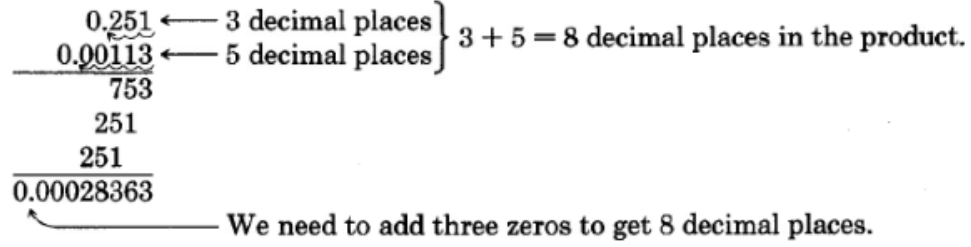

Now, rounding to three decimal places, we get $0.251 \cdot 0.00113=0.000$,

\section{to three decimal places.}

\subsubsection{Practice Set A}

Find the following products.

Exercise 6.6.1

(Solution on p. 408.)

$5.3 \cdot 8.6$

Exercise 6.6.2

(Solution on p. 408.)

$2.12 \cdot 4.9$

Exercise 6.6.3

(Solution on p. 408.)

$1.054 \cdot 0.16$

Exercise 6.6.4

(Solution on p. 408.)

$0.00031 \cdot 0.002$

(Solution on p. 408.)

Find the product of 2.33 and 4.01 and round to one decimal place.

Exercise 6.6.6

$10 \cdot 5.394$

Exercise 6.6.7

$100 \cdot 5.394$

Exercise 6.6.8

(Solution on p. 408.)

$1000 \cdot 5.394$

Exercise 6.6.9

$10,000 \cdot 5.394$

(Solution on p. 408.)

(Solution on p. 408.)

(Solution on p. 408.) 


\subsubsection{Calculators}

Calculators can be used to find products of decimal numbers. However, a calculator that has only an eightdigit display may not be able to handle numbers or products that result in more than eight digits. But there are plenty of inexpensive $(\$ 50-\$ 75)$ calculators with more than eight-digit displays.

\subsubsection{Sample Set B}

Find the following products, if possible, using a calculator.

\section{Example 6.34}

$2.58 \cdot 8.61$

\begin{tabular}{|l|l|l|}
\hline & & Display Reads \\
\hline Type & 2.58 & 2.58 \\
\hline Press & $\times$ & 2.58 \\
\hline Type & 8.61 & 8.61 \\
\hline Press & $=$ & 22.2138 \\
\hline
\end{tabular}

Table 6.13

The product is 22.2138 .

Example 6.35

$0.006 \cdot 0.0042$

\begin{tabular}{|l|l|l|}
\hline & & Display Reads \\
\hline Type & .006 & .006 \\
\hline Press & $\times$ & .006 \\
\hline Type & .0042 & 0.0042 \\
\hline Press & $=$ & 0.0000252 \\
\hline
\end{tabular}

Table 6.14

We know that there will be seven decimal places in the product (since $3+4=7$ ). Since the display shows 7 decimal places, we can assume the product is correct. Thus, the product is 0.0000252 .

\section{Example 6.36}

$0.0026 \cdot 0.11976$

Since we expect $4+5=9$ decimal places in the product, we know that an eight-digit display calculator will not be able to provide us with the exact value. To obtain the exact value, we must use "hand technology." Suppose, however, that we agree to round off this product to three decimal places. We then need only four decimal places on the display.

\begin{tabular}{|l|l|l|}
\hline & & Display Reads \\
\hline Type & .0026 & .0026 \\
\hline Press & $\times$ & .0026 \\
\hline Type & .11976 & 0.11976 \\
\hline Press & $=$ & 0.0003114 \\
\hline
\end{tabular}


Table 6.15

Rounding 0.0003114 to three decimal places we get 0.000 . Thus, $0.0026 \cdot 0.11976=0.000$ to three decimal places.

\subsubsection{Practice Set B}

Use a calculator to find each product. If the calculator will not provide the exact product, round the result to four decimal places.

Exercise 6.6.10

(Solution on p. 408.)

$5.126 \cdot 4.08$

Exercise 6.6.11

(Solution on p. 408.)

$0.00165 \cdot 0.04$

Exercise 6.6.12

(Solution on p. 408.)

$0.5598 \cdot 0.4281$

Exercise 6.6.13

$0.000002 \cdot 0.06$

(Solution on p. 408.)

\subsubsection{Multiplying Decimals by Powers of 10}

There is an interesting feature of multiplying decimals by powers of 10 . Consider the following multiplications.

\begin{tabular}{|l|l|l|}
\hline Multiplication & $\begin{array}{l}\text { Number of Zeros in the Power of } \\
10\end{array}$ & $\begin{array}{l}\text { Number of Positions the Decimal } \\
\text { Point Has Been Moved to the } \\
\text { Right }\end{array}$ \\
\hline $10 \cdot 8.315274=83.15274$ & 1 & 1 \\
\hline $100 \cdot 8.315274=831.5274$ & 2 & 2 \\
\hline $1,000 \cdot 8.315274=8,315.274$ & 3 & 3 \\
\hline $10,000 \cdot 8.315274=83,152.74$ & 4 & 4 \\
\hline
\end{tabular}

Table 6.16

\section{Multiplying a Decimal by a Power of 10}

To multiply a decimal by a power of 10 , move the decimal place to the right of its current position as many places as there are zeros in the power of 10 . Add zeros if necessary.

\subsubsection{Sample Set C}

Find the following products.

Example 6.37

$100 \cdot 34.876$. Since there are 2 zeros in 100 , Move the decimal point in 34.876 two places to the right.

$$
\begin{aligned}
100 \cdot 34.876 & =3487.6 \\
& =3,487.6
\end{aligned}
$$




\section{Example 6.38}

$1,000 \cdot 4.8058$. Since there are 3 zeros in 1,000 , move the decimal point in 4.8058 three places to the right.

$$
\begin{aligned}
1,000 \cdot 4.8058 & =\underbrace{4805.8} \\
& =4,805.8
\end{aligned}
$$

\section{Example 6.39}

$10,000 \cdot 56.82$. Since there are 4 zeros in 10,000 , move the decimal point in 56.82 four places to the right. We will have to add two zeros in order to obtain the four places.

$$
\begin{aligned}
10,000 \cdot 56.82 & =568200 \\
& =568,200
\end{aligned}
$$

Since there is no fractional part, we can drop the decimal point.

Example 6.40

$$
\begin{aligned}
(1,000,000)(2.57) & =2 \underbrace{570000} . \\
& =2,570,000
\end{aligned}
$$

Example 6.41

$$
\begin{aligned}
(1,000)(0.0000029) & =0 \underbrace{000.0029} \\
& =0.0029
\end{aligned}
$$

\subsubsection{Practice Set C}

Find the following products.

Exercise 6.6.14

(Solution on p. 409.)

$100 \cdot 4.27$

Exercise 6.6.15

(Solution on p. 409.)

$10,000 \cdot 16.52187$

(Solution on p. 409.)

(10) (0.0188)

Exercise 6.6.17

$(10,000,000,000)(52.7)$

(Solution on p. 409.)

\subsubsection{Multiplication in Terms of "Of"}

Recalling that the word "of" translates to the arithmetic operation of multiplication, let's observe the following multiplications. 


\subsubsection{Sample Set D}

Example 6.42

Find 4.1 of 3.8 .

Translating "of" to " $\times$ ", we get

$$
\begin{array}{r}
4.1 \\
\times 3.8 \\
\hline 328 \\
123 \\
\hline 15.58
\end{array}
$$

Thus, 4.1 of 3.8 is 15.58 .

\section{Example 6.43}

Find 0.95 of the sum of 2.6 and 0.8 .

We first find the sum of 2.6 and 0.8 .

$$
\begin{array}{r}
2.6 \\
+0.8 \\
\hline 3.4
\end{array}
$$

Now find 0.95 of 3.4

$$
\begin{array}{r}
3.4 \\
\times 0.95 \\
\hline 170 \\
\hline 306 \\
\hline 3.230
\end{array}
$$

Thus, 0.95 of $(2.6+0.8)$ is 3.230 .

\subsubsection{Practice Set D}

Exercise 6.6.18

(Solution on p. 409.)

Find 2.8 of 6.4 .

Exercise 6.6.19

Find 0.1 of 1.3 .

Exercise 6.6.20

Find 1.01 of 3.6.

Exercise 6.6.21

(Solution on p. 409.)

(Solution on p. 409.)

Find 0.004 of 0.0009 .

Exercise 6.6.22

Find 0.83 of 12 .

Exercise 6.6.23

Find 1.1 of the sum of 8.6 and 4.2 .

(Solution on p. 409.)

(Solution on p. 409.)

(Solution on p. 409.) 


\subsubsection{Exercises}

For the following 30 problems, find each product and check each result with a calculator.

Exercise 6.6.24

(Solution on p. 409.)

$3.4 \cdot 9.2$

Exercise 6.6.25

$4.5 \cdot 6.1$

Exercise 6.6.26

(Solution on p. 409.)

$8.0 \cdot 5.9$

Exercise 6.6.27

$6.1 \cdot 7$

Exercise 6.6.28

(0.1) (1.52)

(Solution on p. 409.)

Exercise 6.6.29

(1.99) (0.05)

Exercise 6.6.30

(Solution on p. 409.)

(12.52) (0.37)

Exercise 6.6.31

(5.116) (1.21)

Exercise 6.6.32

(Solution on p. 409.) (31.82) (0.1)

Exercise 6.6.33

(16.527) (9.16)

Exercise 6.6.34

$0.0021 \cdot 0.013$

(Solution on p. 409.)

Exercise 6.6.35

$1.0037 \cdot 1.00037$

Exercise 6.6.36

(Solution on p. 409.) (1.6) (1.6)

Exercise 6.6.37 (4.2) (4.2)

Exercise 6.6.38

$0.9 \cdot 0.9$

(Solution on p. 409.)

Exercise 6.6.39

$1.11 \cdot 1.11$

Exercise 6.6.40

$6.815 \cdot 4.3$

(Solution on p. 409.)

Exercise 6.6.41

$9.0168 \cdot 1.2$

Exercise 6.6.42

(Solution on p. 409.) (3.5162) (0.0000003)

Exercise 6.6.43

$(0.000001)(0.01)$ 
Exercise 6.6.44

(Solution on p. 409.)

(10) (4.96)

Exercise 6.6.45

(10) (36.17)

Exercise 6.6.46

(Solution on p. 409.)

$10 \cdot 421.8842$

Exercise 6.6.47

$10 \cdot 8.0107$

Exercise 6.6.48

$100 \cdot 0.19621$

(Solution on p. 409.)

Exercise 6.6.49

$100 \cdot 0.779$

Exercise 6.6.50

$1000 \cdot 3.596168$

(Solution on p. 409.)

Exercise 6.6.51

$1000 \cdot 42.7125571$

Exercise 6.6.52

$1000 \cdot 25.01$

Exercise 6.6.53

$100,000 \cdot 9.923$

Exercise 6.6.54

(4.6) (6.17)

\begin{tabular}{|l|l|l|l|}
\hline Actual product & Tenths & Hundreds & Thousandths \\
\hline & & & \\
\hline
\end{tabular}

(Solution on p. 410.)

Table 6.17

Exercise 6.6.55

$(8.09)(7.1)$

\begin{tabular}{|l|l|l|l|}
\hline Actual product & Tenths & Hundreds & Thousandths \\
\hline & & & \\
\hline
\end{tabular}

Table 6.18

Exercise 6.6.56

(11.1106) (12.08)

(Solution on p. 410.)

\begin{tabular}{|l|l|l|l|}
\hline Actual product & Tenths & Hundreds & Thousandths \\
\hline & & & \\
\hline
\end{tabular}

Table 6.19

Exercise 6.6.57

$0.0083 \cdot 1.090901$

Available for free at Connexions $<\mathrm{http}: / / \operatorname{cnx} . o r g /$ content/col10615/1.4 $>$ 


\begin{tabular}{|l|l|l|l|}
\hline Actual product & Tenths & Hundreds & Thousandths \\
\hline & & & \\
\hline
\end{tabular}

Table 6.20

Exercise 6.6.58

(Solution on p. 410.)

$7 \cdot 26.518$

\begin{tabular}{|l|l|l|l|}
\hline Actual product & Tenths & Hundreds & Thousandths \\
\hline & & & \\
\hline
\end{tabular}

Table 6.21

For the following 15 problems, perform the indicated operations

Exercise 6.6.59

Find 5.2 of 3.7 .

Exercise 6.6.60

(Solution on p. 410.)

Find 12.03 of 10.1

Exercise 6.6.61

Find 16 of 1.04

Exercise 6.6.62

(Solution on p. 410.)

Find 12 of 0.1

Exercise 6.6.63

Find 0.09 of 0.003

Exercise 6.6.64

(Solution on p. 410.)

Find 1.02 of 0.9801

Exercise 6.6.65

Find 0.01 of the sum of 3.6 and 12.18

Exercise 6.6.66

Find 0.2 of the sum of 0.194 and 1.07

(Solution on p. 410.)

Exercise 6.6.67

Find the difference of 6.1 of 2.7 and 2.7 of 4.03

Exercise 6.6.68

Find the difference of 0.071 of 42 and 0.003 of 9.2

(Solution on p. 410.)

Exercise 6.6.69

If a person earns $\$ 8.55$ an hour, how much does he earn in twenty-five hundredths of an hour?

Exercise 6.6.70

A man buys 14 items at $\$ 1.16$ each. What is the total cost?

(Solution on p. 410.)

Exercise 6.6.71

In the problem above, how much is the total cost if 0.065 sales tax is added?

Exercise 6.6.72

(Solution on p. 410.) A river rafting trip is supposed to last for 10 days and each day 6 miles is to be rafted. On the third day a person falls out of the raft after only $\frac{2}{5}$ of that day's mileage. If this person gets discouraged and quits, what fraction of the entire trip did he complete? 


\section{Exercise 6.6.73}

A woman starts the day with $\$ 42.28$. She buys one item for $\$ 8.95$ and another for $\$ 6.68$. She then buys another item for sixty two-hundredths of the remaining amount. How much money does she have left?

\section{Calculator Problems}

For the following 10 problems, use a calculator to determine each product. If the calculator will not provide the exact product, round the results to five decimal places.

Exercise 6.6.74

(Solution on p. 410.)

$0.019 \cdot 0.321$

Exercise 6.6.75

$0.261 \cdot 1.96$

Exercise 6.6.76

(Solution on p. 410.)

$4.826 \cdot 4.827$

Exercise 6.6.77 $(9.46)^{2}$

Exercise 6.6.78

Exercise 6.6.79

$0.00037 \cdot 0.0065$

Exercise 6.6.80

$0.002 \cdot 0.0009$

(Solution on p. 410.)

Exercise 6.6.81

$0.1286 \cdot 0.7699$

Exercise 6.6.82

$0.01 \cdot 0.00000471$

(Solution on p. 410.)

Exercise 6.6.83

$0.00198709 \cdot 0.03$

\subsubsection{Exercises for Review}

Exercise 6.6.84

(Solution on p. 410.)

(Section 2.3) Find the value, if it exists, of $0 \div 15$.

(Solution on p. 410.)

Exercise 6.6.85

(Section 3.5) Find the greatest common factor of 210, 231, and 357.

Exercise 6.6.86

(Section 4.4) Reduce $\frac{280}{2,156}$ to lowest terms.

(Solution on p. 411.)

Exercise 6.6.87

(Section 6.2) Write "fourteen and one hundred twenty-one ten-thousandths, using digits."

Exercise 6.6.88

(Solution on p. 411.)

(Section 6.5) Subtract 6.882 from 8.661 and round the result to two decimal places. 


\subsection{Division of Decimals ${ }^{7}$}

\subsubsection{Section Overview}

- The Logic Behind the Method

- A Method of Dividing a Decimal By a Nonzero Whole Number

- A Method of Dividing a Decimal by a Nonzero Decimal

- Dividing Decimals by Powers of 10

\subsubsection{The Logic Behind the Method}

As we have done with addition, subtraction, and multiplication of decimals, we will study a method of division of decimals by converting them to fractions, then we will make a general rule.

We will proceed by using this example: Divide 196.8 by 6 .

$\frac{32}{196.8}$
$\frac{18}{16}$
$\frac{12}{4}$

We have, up to this point, divided 196.8 by 6 and have gotten a quotient of 32 with a remainder of 4 . If we follow our intuition and bring down the .8 , we have the division $4.8 \div 6$.

$$
\begin{aligned}
4.8 \div 6 & =4 \frac{8}{10} \div 6 \\
& =\frac{48}{10} \div \frac{6}{1} \\
& =\frac{8}{\frac{48}{10}} \cdot \frac{1}{\frac{1}{16}} \\
& =\frac{8}{10}
\end{aligned}
$$

Thus, $4.8 \div 6=.8$.

Now, our intuition and experience with division direct us to place the .8 immediately to the right of 32 .

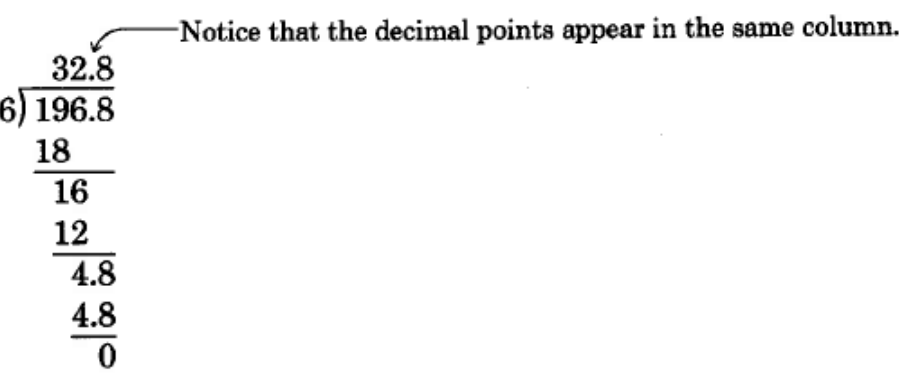

From these observations, we suggest the following method of division.

\footnotetext{
${ }^{7}$ This content is available online at $<$ http://cnx.org/content $/ \mathrm{m} 34968 / 1.2 />$.
} 


\subsubsection{A Method of Dividing a Decimal by a Nonzero Whole Number}

\section{Method of Dividing a Decimal by a Nonzero Whole Number}

To divide a decimal by a nonzero whole number:

1. Write a decimal point above the division line and directly over the decimal point of the dividend.

2. Proceed to divide as if both numbers were whole numbers.

3. If, in the quotient, the first nonzero digit occurs to the right of the decimal point, but not in the tenths position, place a zero in each position between the decimal point and the first nonzero digit of the quotient.

\subsubsection{Sample Set A}

Find the decimal representations of the following quotients.

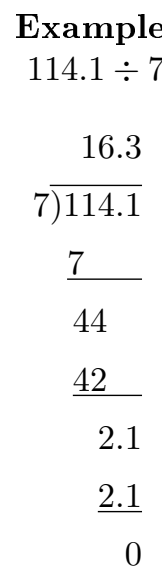

Thus, $114.1 \div 7=16.3$.

Check: If $114.1 \div 7=16.3$, then $7 \cdot 16.3$ should equal 114.1 .

$$
\begin{array}{rr}
\begin{array}{l}
42 \\
16.3 \\
7
\end{array} \\
\text { True. }
\end{array}
$$

Example 6.45

$0.02068 \div 4$

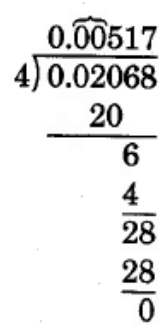

Place zeros in the tenths and hundredths positions. (See Step 3.)

Thus, $0.02068 \div 4=0.00517$. 


\subsubsection{Practice Set A}

Find the following quotients.

Exercise 6.7.1

(Solution on p. 411.)

$184.5 \div 3$

Exercise 6.7.2

$16.956 \div 9$

Exercise 6.7.3

$0.2964 \div 4$

Exercise 6.7.4

$0.000496 \div 8$

(Solution on p. 411.)

(Solution on p. 411.)

(Solution on p. 411.)

\subsubsection{A Method of Dividing a Decimal By a Nonzero Decimal}

Now that we can divide decimals by nonzero whole numbers, we are in a position to divide decimals by a nonzero decimal. We will do so by converting a division by a decimal into a division by a whole number, a process with which we are already familiar. We'll illustrate the method using this example: Divide 4.32 by 1.8 .

Let's look at this problem as $4 \frac{32}{100} \div 1 \frac{8}{10}$.

$$
\begin{aligned}
& 4 \frac{32}{100} \div 1 \frac{8}{10}=\frac{4 \frac{32}{100}}{1 \frac{8}{10}} \\
&=\frac{432}{100} \\
& \frac{18}{10}
\end{aligned}
$$

The divisor is $\frac{18}{10}$. We can convert $\frac{18}{10}$ into a whole number if we multiply it by 10 .

$\frac{18}{10} \cdot 10=\frac{18}{\frac{18}{10}} \cdot \frac{\frac{1}{10}}{1}=18$

But, we know from our experience with fractions, that if we multiply the denominator of a fraction by a nonzero whole number, we must multiply the numerator by that same nonzero whole number. Thus, when converting $\frac{18}{10}$ to a whole number by multiplying it by 10 , we must also multiply the numerator $\frac{432}{100}$ by 10 .

$$
\begin{aligned}
\frac{432}{100} \cdot 10=\frac{432}{\frac{430}{100}} \cdot \frac{\frac{1}{10}}{10} & = & \frac{432 \cdot 1}{10 \cdot 1}=\frac{432}{10} \\
& = & 43 \frac{2}{10} \\
& = & 43.2
\end{aligned}
$$

We have converted the division $4.32 \div 1.8$ into the division $43.2 \div 18$, that is,

$1 . 8 \longdiv { 4 . 3 2 } \rightarrow 1 8 \overline { 4 3 . 2 }$

Notice what has occurred.

\section{$1 . 8 \longdiv { 4 . 3 2 } \longrightarrow 1 8 . \longdiv { 4 { } _ { i } ^ { 3 . 2 } }$}

If we "move" the decimal point of the divisor one digit to the right, we must also "move" the decimal point of the dividend one place to the right. The word "move" actually indicates the process of multiplication by a power of 10 . 


\section{Method of Dividing a Decimal by a Decimal Number}

To divide a decimal by a nonzero decimal,

1. Convert the divisor to a whole number by moving the decimal point to the position immediately to the right of the divisor's last digit.

2. Move the decimal point of the dividend to the right the same number of digits it was moved in the divisor.

3. Set the decimal point in the quotient by placing a decimal point directly above the newly located decimal point in the dividend.

4. Divide as usual.

\subsubsection{Sample Set B}

Find the following quotients.

Example 6.46

$32.66 \div 7.1$

$7.1 \overline{32.66}$

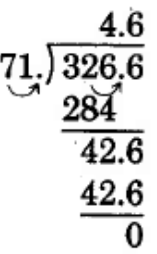

The divisor has one decimal place.

Move the decimal point of both the divisor and the dividend 1 place to the right.

Set the decimal point.

Divide as usual.

Thus, $32.66 \div 7.1=4.6$.

Check: $32.66 \div 7.1=4.6$ if $4.6 \times 7.1=32.66$

4.6

$\times 7.1$

46

322

32.66 True.

Example 6.47

$1.0773 \div 0.513$

2.1

$. 5 1 3 \longdiv { 1 . 0 7 7 3 }$

1026

513

513

The divisor has 3 decimal places.

Available for free at Connexions $<$ http://cnx.org/content/col10615/1.4 $>$ 
Move the decimal point of both the divisor and the dividend 3 places to the right.

Set the decimal place and divide.

Thus, $1.0773 \div 0.513=2.1$.

Checking by multiplying 2.1 and 0.513 will convince us that we have obtained the correct result. (Try it.)

Example 6.48

$12 \div 0.00032$

$0 . 0 0 0 3 2 \longdiv { 1 2 . 0 0 0 0 0 }$

The divisor has 5 decimal places.

Move the decimal point of both the divisor and the dividend 5 places to the right. We will need to add 5 zeros to 12 .

Set the decimal place and divide.

\section{$0 . 0 0 0 3 2 \longdiv { 1 2 . 0 0 0 0 0 }$}

This is now the same as the division of whole numbers.

37500.

$3 2 \longdiv { 1 2 0 0 0 0 0 . }$

$\underline{96}$

240

224

160

$\underline{160}$

000

Checking assures us that $12 \div 0.00032=37,500$.

\subsubsection{Practice Set B}

Find the decimal representation of each quotient.

Exercise 6.7.5

$9.176 \div 3.1$

Exercise 6.7.6

$5.0838 \div 1.11$

Exercise 6.7.7

$16 \div 0.0004$

Exercise 6.7.8

$8,162.41 \div 10$

(Solution on p. 411.)

(Solution on p. 411.)

(Solution on p. 411.)

(Solution on p. 411.)

Exercise 6.7.9

(Solution on p. 411.)

$8,162.41 \div 100$

(Solution on p. 411.)

Exercise 6.7.10

$8,162.41 \div 1,000$ 
Exercise 6.7.11

(Solution on p. 411.)

$8,162.41 \div 10,000$

\subsubsection{Calculators}

Calculators can be useful for finding quotients of decimal numbers. As we have seen with the other calculator operations, we can sometimes expect only approximate results. We are alerted to approximate results when the calculator display is filled with digits. We know it is possible that the operation may produce more digits than the calculator has the ability to show. For example, the multiplication

$\underbrace{0.12345}_{\begin{array}{c}5 \text { decimal } \\ \text { places }\end{array}} \times \underbrace{0.4567}_{\begin{array}{c}4 \text { decimal } \\ \text { places }\end{array}}$

produces $5+4=9$ decimal places. An eight-digit display calculator only has the ability to show eight digits, and an approximation results. The way to recognize a possible approximation is illustrated in problem 3 of the next sample set.

\subsubsection{Sample Set C}

Find each quotient using a calculator. If the result is an approximation, round to five decimal places.

Example 6.49

$12.596 \div 4.7$

\begin{tabular}{|l|l|l|}
\hline & & Display Reads \\
\hline Type & 12.596 & 12.596 \\
\hline Press & $\div$ & 12.596 \\
\hline Type & 4.7 & 4.7 \\
\hline Press & $=$ & 2.68 \\
\hline
\end{tabular}

Table 6.22

Since the display is not filled, we expect this to be an accurate result.

Example 6.50

$0.5696376 \div 0.00123$

\begin{tabular}{|l|l|l|}
\hline & & Display Reads \\
\hline Type & .5696376 & 0.5696376 \\
\hline Press & $\div$ & 0.5696376 \\
\hline Type & .00123 & 0.00123 \\
\hline Press & $=$ & 463.12 \\
\hline
\end{tabular}

Table 6.23

Since the display is not filled, we expect this result to be accurate.

Example 6.51

$0.8215199 \div 4.113$

Available for free at Connexions $<$ http://cnx.org/content/col10615/1.4 $>$ 


\begin{tabular}{|l|l|l|}
\hline & & Display Reads \\
\hline Type & .8215199 & 0.8215199 \\
\hline Press & $\div$ & 0.8215199 \\
\hline Type & 4.113 & 4.113 \\
\hline Press & $=$ & 0.1997373 \\
\hline
\end{tabular}

Table 6.24

There are EIGHT DIGITS - DISPLAY FILLED! BE AWARE OF POSSIBLE APPROXIMATIONS.

We can check for a possible approximation in the following way. Since the division $4 \longdiv { 3 } \frac { 3 } { 1 2 }$ can be checked by multiplying 4 and 3 , we can check our division by performing the multiplication

$$
\underbrace{4.113}_{\begin{array}{c}
3 \text { decimal } \\
\text { places }
\end{array}} \times \underbrace{0.1997373}_{\begin{array}{c}
7 \text { decimal } \\
\text { places }
\end{array}}
$$

This multiplication produces $3+7=10$ decimal digits. But our suspected quotient contains only 8 decimal digits. We conclude that the answer is an approximation. Then, rounding to five decimal places, we get 0.19974 .

\subsubsection{Practice Set C}

Find each quotient using a calculator. If the result is an approximation, round to four decimal places.

Exercise 6.7.12 $42.49778 \div 14.261$

Exercise 6.7.13

$0.001455 \div 0.291$

Exercise 6.7.14

$7.459085 \div 2.1192$
(Solution on p. 411.)

(Solution on p. 411.)

(Solution on p. 411.)

\subsubsection{Dividing Decimals By Powers of 10}

In problems 4 and 5 of Section 6.7.4.2 (Practice Set B), we found the decimal representations of $8,162.41 \div 10$ and $8,162.41 \div 100$. Let's look at each of these again and then, from these observations, make a general statement regarding division of a decimal number by a power of 10 . 
816.241

$1 0 \longdiv { 8 1 6 2 . 4 1 0 }$

$\underline{80}$

16

10

62

$\underline{60}$

24

20

41

$\underline{40}$

10

$\underline{10}$

0

Thus, $8,162.41 \div 10=816.241$.

Notice that the divisor 10 is composed of one 0 and that the quotient 816.241 can be obtained from the dividend $8,162.41$ by moving the decimal point one place to the left.

\begin{tabular}{r}
$\frac{81.6241}{1 0 0 \longdiv { 8 1 6 2 . 4 1 0 0 }}$ \\
\hline 800 \\
162 \\
$\underline{100}$ \\
624 \\
$\underline{600}$ \\
241 \\
$\underline{200}$ \\
410 \\
$\underline{400}$ \\
100 \\
$\underline{100}$ \\
0
\end{tabular}

Thus, $8,162.41 \div 100=81.6241$.

Notice that the divisor 100 is composed of two 0's and that the quotient 81.6241 can be obtained from the dividend by moving the decimal point two places to the left.

Using these observations, we can suggest the following method for dividing decimal numbers by powers of 10. 


\section{Dividing a Decimal Fraction by a Power of 10}

To divide a decimal fraction by a power of 10 , move the decimal point of the decimal fraction to the left as many places as there are zeros in the power of 10 . Add zeros if necessary.

\subsubsection{Sample Set $D$}

Find each quotient.

\section{Example 6.52}

$9,248.6 \div 100$

Since there are 2 zeros in this power of 10 , we move the decimal point 2 places to the left.

$$
92.48 .6 \div 100=92.486
$$

\section{Example 6.53}

$3.28 \div 10,000$

Since there are 4 zeros in this power of 10 , we move the decimal point 4 places to the left. To do so, we need to add three zeros.

$$
0003.28 \div 10,000=0.000328
$$

\subsubsection{Practice Set D}

Find the decimal representation of each quotient.

Exercise 6.7.15

(Solution on p. 411.)

$182.5 \div 10$

Exercise 6.7.16

$182.5 \div 100$

Exercise 6.7.17

$182.5 \div 1,000$

Exercise 6.7.18

(Solution on p. 411.)

$182.5 \div 10,000$

(Solution on p. 411.)

(Solution on p. 411.)

Exercise 6.7.19

(Solution on p. 411.)

$646.18 \div 100$

Exercise 6.7.20

$21.926 \div 1,000$

(Solution on p. 411.)

\subsubsection{Exercises}

For the following 30 problems, find the decimal representation of each quotient. Use a calculator to check each result.

Exercise 6.7.21

(Solution on p. 411.)

$4.8 \div 3$

Exercise 6.7.22

$16.8 \div 8$

Exercise 6.7.23

$18.5 \div 5$

(Solution on p. 411.) 
Exercise 6.7.24

$12.33 \div 3$

Exercise 6.7.25

(Solution on p. 411.)

$54.36 \div 9$

Exercise 6.7.26

$73.56 \div 12$

Exercise 6.7.27

$159.46 \div 17$

(Solution on p. 412.)

Exercise 6.7.28

$12.16 \div 64$

Exercise 6.7.29

(Solution on p. 412.)

$37.26 \div 81$

Exercise 6.7.30 $439.35 \div 435$

Exercise 6.7.31 $36.98 \div 4.3$

(Solution on p. 412.)

Exercise 6.7.32 $46.41 \div 9.1$

Exercise 6.7.33 $3.6 \div 1.5$

(Solution on p. 412.)

Exercise 6.7.34 $0.68 \div 1.7$

Exercise 6.7.35 $50.301 \div 8.1$

(Solution on p. 412.)

Exercise 6.7.36 $2.832 \div 0.4$

Exercise 6.7.37 $4.7524 \div 2.18$

(Solution on p. 412.)

Exercise 6.7.38 $16.2409 \div 4.03$

Exercise 6.7.39 $1.002001 \div 1.001$

(Solution on p. 412.)

Exercise 6.7.40 $25.050025 \div 5.005$

Exercise 6.7.41 $12.4 \div 3.1$

(Solution on p. 412.)

Exercise 6.7.42 $0.48 \div 0.08$

Exercise 6.7.43 $30.24 \div 2.16$

(Solution on p. 412.)

Exercise 6.7.44 $48.87 \div 0.87$

Exercise 6.7.45 $12.321 \div 0.111$

(Solution on p. 412.)

Exercise 6.7.46 $64,351.006 \div 10$ 
Exercise 6.7.47

(Solution on p. 412.)

$64,351.006 \div 100$

Exercise 6.7.48

$64,351.006 \div 1,000$

Exercise 6.7.49

(Solution on p. 412.)

$64,351.006 \div 1,000,000$

Exercise 6.7.50

$0.43 \div 100$

For the following 5 problems, find each quotient. Round to the specified position. A calculator may be used.

Exercise 6.7.51

(Solution on p. 412.)

$11.2944 \div 6.24$

\begin{tabular}{|l|l|l|l|}
\hline Actual Quotient & Tenths & Hundredths & Thousandths \\
\hline & & & \\
\hline
\end{tabular}

Table 6.25

Exercise 6.7.52

$45.32931 \div 9.01$

\begin{tabular}{|l|l|l|l|}
\hline Actual Quotient & Tenths & Hundredths & Thousandths \\
\hline & & & \\
\hline
\end{tabular}

Table 6.26

Exercise 6.7.53

(Solution on p. 412.)

$3.18186 \div 0.66$

\begin{tabular}{|l|l|l|l|}
\hline Actual Quotient & Tenths & Hundredths & Thousandths \\
\hline & & & \\
\hline
\end{tabular}

Table 6.27

Exercise 6.7.54

$4.3636 \div 4$

\begin{tabular}{|l|l|l|l|}
\hline Actual Quotient & Tenths & Hundredths & Thousandths \\
\hline & & & \\
\hline
\end{tabular}

Table 6.28

Exercise 6.7.55

(Solution on p. 412.)

$0.00006318 \div 0.018$

\begin{tabular}{|l|l|l|l|}
\hline Actual Quotient & Tenths & Hundredths & Thousandths \\
\hline & & & \\
\hline
\end{tabular}

Available for free at Connexions $<$ http://cnx.org/content/col10615/1.4 $>$ 
Table 6.29

For the following 9 problems, find each solution.

Exercise 6.7.56

Divide the product of 7.4 and 4.1 by 2.6 .

Exercise 6.7.57

(Solution on p. 413.)

Divide the product of 11.01 and 0.003 by 2.56 and round to two decimal places.

Exercise 6.7.58

Divide the difference of the products of 2.1 and 9.3 , and 4.6 and 0.8 by 0.07 and round to one decimal place.

Exercise 6.7.59

(Solution on p. 413.)

A ring costing $\$ 567.08$ is to be paid off in equal monthly payments of $\$ 46.84$. In how many months will the ring be paid off?

Exercise 6.7.60

Six cans of cola cost $\$ 2.58$. What is the price of one can?

Exercise 6.7.61

(Solution on p. 413.)

A family traveled 538.56 miles in their car in one day on their vacation. If their car used 19.8 gallons of gas, how many miles per gallon did it get?

Exercise 6.7.62

Three college students decide to rent an apartment together. The rent is $\$ 812.50$ per month. How much must each person contribute toward the rent?

Exercise 6.7.63

(Solution on p. 413.)

A woman notices that on slow speed her video cassette recorder runs through 296.80 tape units in 10 minutes and at fast speed through 1098.16 tape units. How many times faster is fast speed than slow speed?

Exercise 6.7.64

A class of 34 first semester business law students pay a total of $\$ 1,354.90$, disregarding sales tax, for their law textbooks. What is the cost of each book?

Calculator Problems

For the following problems, use calculator to find the quotients. If the result is approximate (see Sample Set C Example 6.51) round the result to three decimal places.

Exercise 6.7.65

$3.8994 \div 2.01$

(Solution on p. 413.)

Exercise 6.7.66

$0.067444 \div 0.052$

Exercise 6.7.67

$14,115.628 \div 484.74$

(Solution on p. 413.)

Exercise 6.7.68

$219,709.36 \div 9941.6$

Exercise 6.7.69

(Solution on p. 413.)

$0.0852092 \div 0.49271$

Exercise 6.7.70

$2.4858225 \div 1.11611$

Exercise 6.7.71

(Solution on p. 413.)

$0.123432 \div 0.1111$

Exercise 6.7.72

$2.102838 \div 1.0305$ 


\subsubsection{Exercises for Review}

Exercise 6.7.73

(Solution on p. 413.)

(Section 4.3) Convert $4 \frac{7}{8}$ to an improper fraction.

Exercise 6.7.74

(Section 4.7$) \frac{2}{7}$ of what number is $\frac{4}{5}$ ?

Exercise 6.7.75

(Section 5.3) Find the sum. $\frac{4}{15}+\frac{7}{10}+\frac{3}{5}$.

(Solution on p. 413.)

Exercise 6.7.76

(Section 6.4) Round 0.01628 to the nearest ten-thousandths.

Exercise 6.7.77

(Solution on p. 413.)

(Section 6.6) Find the product (2.06)(1.39)

\subsection{Nonterminating Divisions ${ }^{8}$}

\subsubsection{Section Overview}

- Nonterminating Divisions

- Denoting Nonterminating Quotients

\subsubsection{Nonterminating Divisions}

Let's consider two divisions:

1. $9.8 \div 3.5$

2. $4 \div 3$

\section{Terminating Divisions}

Previously, we have considered divisions like example 1, which is an example of a terminating division. A terminating division is a division in which the quotient terminates after several divisions (the remainder is zero).

$$
\begin{array}{r}
3 . 5 \longdiv { 9 . 8 0 } \\
\frac{70}{280} \\
\frac{280}{0}
\end{array}
$$

Exact Divisions

The quotient in this problem terminates in the tenths position. Terminating divisions are also called exact divisions.

\section{Nonterminating Division}

The division in example 2 is an example of a nonterminating division. A non-terminating division is a division that, regardless of how far we carry it out, always has a remainder.

\footnotetext{
${ }^{8}$ This content is available online at $<$ http://cnx.org/content $/ \mathrm{m} 34969 / 1.2 />$.
} 


$$
\begin{aligned}
& 1.333 \\
& 3 \longdiv { 4 . 0 0 0 0 0 } \\
& 3 \\
& 10 \\
& 9 \\
& 10 \\
& 9 \\
& 10 \\
& \frac{9}{10} \\
& \frac{9}{10}
\end{aligned}
$$

\section{Repeating Decimal}

We can see that the pattern in the brace is repeated endlessly. Such a decimal quotient is called a repeating decimal.

\subsubsection{Denoting Nonterminating Quotients}

We use three dots at the end of a number to indicate that a pattern repeats itself endlessly.

$4 \div 3=1.333 \ldots$

Another way, aside from using three dots, of denoting an endlessly repeating pattern is to write a bar $\left(^{-}\right.$) above the repeating sequence of digits.

$4 \div 3=1 . \overline{3}$

The bar indicates the repeated pattern of 3 .

Repeating patterns in a division can be discovered in two ways:

1. As the division process progresses, should the remainder ever be the same as the dividend, it can be concluded that the division is nonterminating and that the pattern in the quotient repeats. This fact is illustrated in Example 6.54 of Section 6.8.3.1 (Sample Set A).

2. As the division process progresses, should the "product, difference" pattern ever repeat two consecutive times, it can be concluded that the division is nonterminating and that the pattern in the quotient repeats. This fact is illustrated in Example 6.55 and 4 of Section 6.8.3.1 (Sample Set A).

\subsubsection{Sample Set A}

Carry out each division until the repeating pattern can be determined.

\section{Example 6.54}

$100 \div 27$ 
3.70370

$2 7 \longdiv { 1 0 0 . 0 0 0 0 0 }$

$\underline{81}$

190

189

100

81

190

189

When the remainder is identical to the dividend, the division is nonterminating. This implies that the pattern in the quotient repeats.

$100 \div 27=3.70370370 \ldots$ The repeating block is 703 .

$100 \div 27=3 . \overline{703}$

Example 6.55

$1 \div 9$

.111

$9 \longdiv { 1 . 0 0 0 }$

$\left.\begin{array}{c}\frac{9}{10} \\ \frac{9}{10} \\ \frac{9}{1}\end{array}\right\}$

We see that this "product, difference"pattern repeats. We can conclude that the division is nonterminating and that the quotient repeats.

$1 \div 9=0.111 \ldots$ The repeating block is 1 .

$1 \div 9=0 . \overline{1}$

\section{Example 6.56}

Divide 2 by 11 and round to 3 decimal places.

Since we wish to round the quotient to three decimal places, we'll carry out the division so that the quotient has four decimal places. 


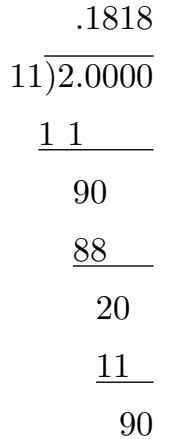

The number .1818 rounded to three decimal places is .182 . Thus, correct to three decimal places,

$2 \div 11=0.182$

Example 6.57

Divide 1 by 6 .

$$
\begin{aligned}
& \text { 6) } \lcm{.166} \\
& \left.\begin{array}{l}
6 \\
\hline \frac{30}{36} \\
\frac{36}{4}
\end{array}\right\}
\end{aligned}
$$

We see that this "product, difference" pattern repeats. We can conclude that the division is nonterminating and that the quotient repeats at the 6.

$1 \div 6=0.1 \overline{6}$

\subsubsection{Practice Set A}

Carry out the following divisions until the repeating pattern can be determined.

Exercise 6.8.1

$1 \div 3$

Exercise 6.8.2

$$
5 \div 6
$$

Exercise 6.8.3

$11 \div 9$

Exercise 6.8.4

$17 \div 9$

Exercise 6.8.5

Divide 7 by 6 and round to 2 decimal places.

Exercise 6.8.6

Divide 400 by 11 and round to 4 decimal places.
(Solution on p. 413.)

(Solution on p. 413.)

(Solution on p. 413.)

(Solution on p. 413.)

(Solution on p. 413.)

(Solution on p. 413.) 


\subsubsection{Exercises}

For the following 20 problems, carry out each division until the repeating pattern is determined. If a repeating pattern is not apparent, round the quotient to three decimal places.

Exercise 6.8.7

(Solution on p. 413.)

$4 \div 9$

Exercise 6.8.8

$8 \div 11$

Exercise 6.8.9

(Solution on p. 413.)

$4 \div 25$

Exercise 6.8.10

$5 \div 6$

Exercise 6.8.11

(Solution on p. 413.)

$1 \div 7$

Exercise 6.8.12

$3 \div 1.1$

Exercise 6.8.13

$20 \div 1.9$

(Solution on p. 413.)

Exercise 6.8.14

$10 \div 2.7$

Exercise 6.8.15

$1.11 \div 9.9$

(Solution on p. 413.)

Exercise 6.8.16

$8.08 \div 3.1$

Exercise 6.8.17

$51 \div 8.2$

(Solution on p. 413.)

Exercise 6.8.18

$0.213 \div 0.31$

Exercise 6.8.19

$0.009 \div 1.1$

(Solution on p. 413.)

Exercise 6.8.20

$6.03 \div 1.9$

Exercise 6.8.21

(Solution on p. 413.)

$0.518 \div 0.62$

Exercise 6.8.22

$1.55 \div 0.27$

Exercise 6.8.23

$0.333 \div 0.999$

(Solution on p. 413.)

Exercise 6.8.24

$0.444 \div 0.999$

Exercise 6.8.25

$0.555 \div 0.27$

(Solution on p. 414.)

Exercise 6.8.26

$3.8 \div 0.99$

Calculator Problems

For the following 10 problems, use a calculator to perform each division. 
Exercise 6.8.27

(Solution on p. 414.)

$7 \div 9$

Exercise 6.8.28

$8 \div 11$

Exercise 6.8.29

(Solution on p. 414.)

$14 \div 27$

Exercise 6.8.30

$1 \div 44$

Exercise 6.8.31

(Solution on p. 414.)

$2 \div 44$

Exercise 6.8.32

$0.7 \div 0.9$ (Compare this with Exercise 6.8.27.)

Exercise 6.8.33

$80 \div 110$ (Compare this with Exercise 6.8.28.)

(Solution on p. 414.)

Exercise 6.8.34

$0.0707 \div 0.7070$

Exercise 6.8.35

$0.1414 \div 0.2020$

(Solution on p. 414.)

Exercise 6.8.36

$1 \div 0.9999999$

\subsubsection{Exercise for Review}

Exercise 6.8.37

(Solution on p. 414.)

(Section 1.2) In the number 411,105, how many ten thousands are there?

Exercise 6.8.38

(Section 2.3) Find the quotient, if it exists. $17 \div 0$.

Exercise 6.8.39

(Solution on p. 414.)

(Section 3.6) Find the least common multiple of 45, 63, and 98.

Exercise 6.8.40

(Section 6.5) Subtract 8.01629 from 9.00187 and round the result to three decimal places.

Exercise 6.8.41

(Solution on p. 414.)

(Section 6.7) Find the quotient. $104.06 \div 12.1$.

\subsection{Converting a Fraction to a Decimal ${ }^{9}$}

Now that we have studied and practiced dividing with decimals, we are also able to convert a fraction to a decimal. To do so we need only recall that a fraction bar can also be a division symbol. Thus, $\frac{3}{4}$ not only means " 3 objects out of 4, " but can also mean "3 divided by $4 . "$

\footnotetext{
${ }^{9}$ This content is available online at $<$ http://cnx.org/content $/ \mathrm{m} 34970 / 1.2 />$.
} 


\subsubsection{Sample Set A}

Convert the following fractions to decimals. If the division is nonterminating, round to two decimal places.

Example 6.58

$\frac{3}{4}$. Divide 3 by 4 .

\begin{tabular}{r}
.75 \\
$4 \longdiv { 3 . 0 0 }$ \\
2.8 \\
\hline 20 \\
$\underline{20}$ \\
0
\end{tabular}

Thus, $\frac{3}{4}=0.75$.

Example 6.59

$\frac{1}{5}$ Divide 1 by 5 .

$5 \longdiv { 1 . 0 }$

$\underline{1.0}$

0

Thus, $\frac{1}{5}=0.2$

Example 6.60

$\frac{5}{6}$. Divide 5 by 6 .

.833

48

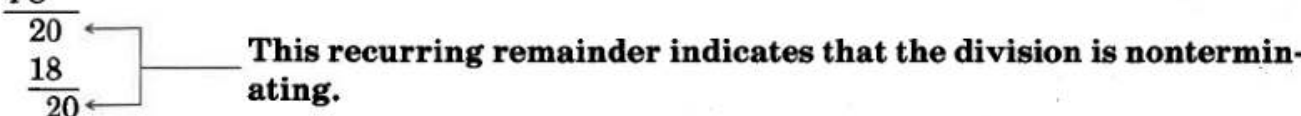

$\frac{5}{6}=0.833 \cdots$ We are to round to two decimal places.

Thus, $\frac{5}{6}=0.83$ to two decimal places.

Example 6.61

$5 \frac{1}{8}$. Note that $5 \frac{1}{8}=5+\frac{1}{8}$.

Convert $\frac{1}{8}$ to a decimal. 
$8 \longdiv { 1 . 0 0 0 }$

8

20

$\underline{16}$

40

$\underline{40}$

0

$\frac{1}{8}=.125$

Thus, $5 \frac{1}{8}=5+\frac{1}{8}=5+.125=5.125$.

\section{Example 6.62}

$0.16 \frac{1}{4}$. This is a complex decimal.

Note that the 6 is in the hundredths position.

The number $0.16 \frac{1}{4}$ is read as "sixteen and one-fourth hundredths."

$0.16 \frac{1}{4}=\frac{16 \frac{1}{4}}{100}=\frac{\frac{16 \cdot 4+1}{4}}{100}=\frac{\frac{65}{4}}{\frac{100}{1}}=\frac{\frac{13}{265}}{4} \cdot \frac{1}{\frac{100}{100}}=\frac{13 \cdot 1}{4 \cdot 20}=\frac{13}{80}$

Now, convert $\frac{13}{80}$ to a decimal.

.1625

$8 0 \longdiv { 1 3 . 0 0 0 0 }$

$\underline{80}$

500

$\underline{480}$

200

$\underline{160}$

400

$\underline{400}$

0

Thus, $0.16 \frac{1}{4}=0.1625$.

\subsubsection{Practice Set A}

Convert the following fractions and complex decimals to decimals (in which no proper fractions appear). If the divison is nonterminating, round to two decimal places.

Exercise 6.9.1

$\frac{1}{4}$

(Solution on p. 414.) 
Exercise 6.9.2

$\frac{1}{25}$

Exercise 6.9.3

$\frac{1}{6}$

Exercise 6.9.4

$\frac{15}{16}$

Exercise 6.9.5

$0.9 \frac{1}{2}$

Exercise 6.9.6

$8.0126 \frac{3}{8}$
(Solution on p. 414.)

(Solution on p. 414.)

(Solution on p. 414.)

(Solution on p. 414.)

(Solution on p. 414.)

\subsubsection{Exercises}

For the following 30 problems, convert each fraction or complex decimal number to a decimal (in which no proper fractions appear).

Exercise 6.9.7

$\frac{1}{2}$

Exercise 6.9.8

$\frac{4}{5}$

Exercise 6.9.9

$\frac{7}{8}$

Exercise 6.9.10

$\frac{5}{8}$

Exercise 6.9.11

$\frac{3}{5}$

Exercise 6.9.12

$\frac{2}{5}$

Exercise 6.9.13

$\frac{1}{25}$

Exercise 6.9.14

$\frac{3}{25}$

Exercise 6.9.15

$\frac{1}{20}$

Exercise 6.9.16

$\frac{1}{15}$

Exercise 6.9.17

$\frac{1}{50}$

Exercise 6.9.18

$\frac{1}{75}$

Exercise 6.9.19

$\frac{1}{3}$

Exercise 6.9.20

$\frac{5}{6}$

Exercise 6.9.21

$\frac{3}{16}$
(Solution on p. 414.)

(Solution on p. 414.)

(Solution on p. 414.)

(Solution on p. 414.)

(Solution on p. 414.)

(Solution on p. 414.)

(Solution on p. 414.)

(Solution on p. 414.) 
Exercise 6.9.22 $\frac{9}{16}$

Exercise 6.9.23 $\frac{1}{27}$

(Solution on p. 414.)

Exercise 6.9.24 $\frac{5}{27}$

Exercise 6.9.25 $\frac{7}{13}$

(Solution on p. 414.)

Exercise 6.9.26 $\frac{9}{14}$

Exercise 6.9.27 $7 \frac{2}{3}$

(Solution on p. 415.)

Exercise 6.9.28 $8 \frac{5}{16}$

Exercise 6.9.29 $1 \frac{2}{15}$

Exercise 6.9.30 $65 \frac{5}{22}$

Exercise 6.9.31 $101 \frac{6}{25}$

Exercise 6.9.32 $0.1 \frac{1}{2}$

Exercise 6.9.33 $0.24 \frac{1}{8}$

Exercise 6.9.34 $5.66 \frac{2}{3}$

Exercise 6.9.35 $810.3106 \frac{5}{16}$

(Solution on p. 415.)

Exercise 6.9.36 $4.1 \frac{1}{9}$

For the following 18 problems, convert each fraction to a decimal. Round to five decimal places.

Exercise 6.9.37

(Solution on p. 415.) $\frac{1}{9}$

Exercise 6.9.38 $\frac{2}{9}$

Exercise 6.9.39 $\frac{3}{9}$ (Solution on p. 415.)

Exercise 6.9.40 $\frac{4}{9}$

Exercise 6.9.41 $\frac{5}{9}$

Exercise 6.9.42 $\frac{6}{9}$

Exercise 6.9.43 $\frac{7}{9}$

(Solution on p. 415.)

(Solution on p. 415.)

(Solution on p. 415.)

(Solution on p. 415.)

(Solution on p. 415.) 
Exercise 6.9.44

$\frac{8}{9}$

Exercise 6.9.45

$\frac{1}{11}$

(Solution on p. 415.)

Exercise 6.9.46

$\frac{2}{11}$

Exercise 6.9.47

$\frac{3}{11}$

Exercise 6.9.48

$\frac{4}{11}$

Exercise 6.9.49

$\frac{5}{11}$

Exercise 6.9.50

$\frac{6}{11}$

Exercise 6.9.51

$\frac{7}{11}$

Exercise 6.9.52

$\frac{8}{11}$

Exercise 6.9.53

$\frac{9}{11}$

Exercise 6.9.54

$\frac{10}{11}$

Calculator Problems

For the following problems, use a calculator to convert each fraction to a decimal. If no repeating pattern seems to exist, round to four decimal places.

Exercise 6.9.55

$\frac{16}{125}$

(Solution on p. 415.)

Exercise 6.9.56

$\frac{85}{311}$

Exercise 6.9.57

$\frac{192}{197}$

(Solution on p. 415.)

Exercise 6.9.58

$\frac{1}{1469}$

Exercise 6.9.59

$\frac{4}{21,015}$

(Solution on p. 415.)

(Solution on p. 415.)

(Solution on p. 415.)

Exercise 6.9.60

$\frac{81,426}{106,001}$

Exercise 6.9.61

$\frac{16,501}{426}$

(Solution on p. 415.) 


\subsubsection{Exercises for Review}

Exercise 6.9.62

(Section 1.4) Round 2,105,106 to the nearest hundred thousand.

Exercise 6.9.63

(Section 4.7 ) $\frac{8}{5}$ of what number is $\frac{3}{2}$ ?

(Solution on p. 415.)

Exercise 6.9.64

(Section 5.5) Arrange $1 \frac{9}{16}, 1 \frac{5}{8}$, and $1 \frac{7}{12}$ in increasing order.

Exercise 6.9.65

(Section 6.3) Convert the complex decimal $3.6 \frac{5}{4}$ to a fraction.

(Solution on p. 415.)

Exercise 6.9.66

(Section 6.8) Find the quotient. $30 \div 1.1$.

\subsection{Combinations of Operations with Decimals and Fractions ${ }^{10}$}

Having considered operations with decimals and fractions, we now consider operations that involve both decimals and fractions.

\subsubsection{Sample Set A}

Perform the following operations.

Example 6.63

$0.38 \cdot \frac{1}{4}$. Convert both numbers to decimals or both numbers to fractions. We'll convert to decimals.

$\frac{.25}{4 \longdiv { 1 . 0 0 }}$
$\frac{8}{20}$
$\underline{20}$
0

To convert $\frac{1}{4}$ to a decimal, divide 1 by 4 .

Now multiply 0.38 and .25 .

1
4
3

.38

$\underline{\times .25}$

190

$\underline{76}$

.0950

Thus, $0.38 \cdot \frac{1}{4}=0.095$.

${ }^{10}$ This content is available online at $<$ http://cnx.org/content $/ \mathrm{m} 34971 / 1.2 />$. 
In the problems that follow, the conversions from fraction to decimal, or decimal to fraction, and some of the additions, subtraction, multiplications, and divisions will be left to you.

Example 6.64

$1.85+\frac{3}{8} \cdot 4.1$ Convert $\frac{3}{8}$ to a decimal.

$1.85+0.375 \cdot 4.1$ Multiply before adding.

$1.85+1.5375$ Now add.

3.3875

Example 6.65

$\frac{5}{13}\left(\frac{4}{5}-0.28\right)$ Convert 0.28 to a fraction.

$$
\begin{aligned}
& \frac{5}{13}\left(\frac{4}{5}-\frac{28}{100}\right)=\frac{5}{13}\left(\frac{4}{5}-\frac{7}{25}\right) \\
& =\frac{5}{13}\left(\frac{20}{25}-\frac{7}{25}\right) \\
& =\quad \frac{\frac{1}{55}}{\frac{15}{13}} \cdot \frac{\frac{1}{13}}{\frac{13}{25}} \\
& =\frac{1}{5}
\end{aligned}
$$

\title{
Example 6.66
}

$$
\begin{aligned}
& \frac{0.125}{1 \frac{1}{3}}+\frac{1}{16}-0.1211= \\
& =\quad \frac{\frac{3}{8}}{\frac{8}{3}}+\frac{1}{16}-0.1211 \\
& =\quad \frac{1}{8} \cdot \frac{3}{4}+\frac{1}{16}-0.1211 \\
& =\quad \frac{3}{32}+\frac{1}{16}-0.1211 \\
& =\frac{3}{32}+\frac{2}{32}-0.1211=\frac{5}{32}-0.1211 \\
& =0.15625-0.1211 \\
& =0.03515 \quad \text { Convert this to fraction form } \\
& =\quad \frac{3515}{100,000} \\
& =\frac{703}{20,000}
\end{aligned}
$$

\subsubsection{Practice Set A}

Perform the following operations.

Exercise 6.10.1

(Solution on p. 415.)

$\frac{3}{5}+1.6$

Exercise 6.10.2

$8.91+\frac{1}{5} \cdot 1.6$

Exercise 6.10.3

$1 \frac{9}{16}\left(6.12+\frac{7}{25}\right)$

Exercise 6.10.4

$\frac{0.156}{1 \frac{115}{15}}-0.05$

\author{
(Solution on p. 415.) \\ (Solution on p. 415.) \\ (Solution on p. 415.)
}




\subsubsection{Exercises}

Exercise 6.10.5

(Solution on p. 415.) $\frac{3}{10}+0.7$

Exercise 6.10.6 $\frac{1}{5}+0.1$

Exercise 6.10.7

(Solution on p. 416.) $\frac{5}{8}-0.513$

Exercise 6.10.8 $0.418-\frac{67}{200}$

Exercise 6.10.9 $0.22 \cdot \frac{1}{4}$

(Solution on p. 416.)

Exercise 6.10.10 $\frac{3}{5} \cdot 8.4$

Exercise 6.10.11 $\frac{1}{25} \cdot 3.19$

(Solution on p. 416.)

Exercise 6.10.12 $\frac{3}{20} \div 0.05$

Exercise 6.10.13 $\frac{7}{40} \div 0.25$

(Solution on p. 416.)

Exercise 6.10.14 $1 \frac{1}{15} \div 0.9 \cdot 0.12$

Exercise 6.10.15 $9.26+\frac{1}{4} \cdot 0.81$

(Solution on p. 416.)

Exercise 6.10.16 $0.588+\frac{1}{40} \cdot 0.24$

Exercise 6.10.17 $\frac{1}{20}+3.62 \cdot \frac{3}{8}$

(Solution on p. 416.)

Exercise 6.10.18

$7+0.15 \div \frac{3}{30}$

Exercise 6.10.19 $\frac{15}{16} \cdot\left(\frac{7}{10}-0.5\right)$

Exercise 6.10.20 $0.2 \cdot\left(\frac{7}{20}+1.1143\right)$

Exercise 6.10.21 $\frac{3}{4} \cdot\left(0.875+\frac{1}{8}\right)$

Exercise 6.10.22 $5.198-0.26 \cdot\left(\frac{14}{250}+0.119\right)$

Exercise 6.10.23 $0.5 \frac{1}{4}+(0.3)^{2}$

(Solution on p. 416.)

Exercise 6.10.24 $(1.4)^{2}-1.6 \frac{1}{2}$

Exercise 6.10.25 $\left(\frac{3}{8}\right)^{2}-0.000625+(1.1)^{2}$

(Solution on p. 416.)

(Solution on p. 416.)

Exercise 6.10.26

$(0.6)^{2} \cdot\left(\frac{1}{20}-\frac{1}{25}\right)$ 
Exercise 6.10.27

(Solution on p. 416.)

$\left(\frac{1}{2}\right)^{2}-0.125$

Exercise 6.10.28

$\frac{0.75}{4 \frac{1}{2}}+\frac{5}{12}$

Exercise 6.10.29

$\left(\frac{0.375}{2 \frac{1}{16}}-\frac{1}{33}\right)$

Exercise 6.10.30

$8 \frac{1}{3} \cdot\left(\frac{1 \frac{1}{4}}{2.25}+\frac{9}{25}\right)$

Exercise 6.10.31

$\frac{0.32}{\frac{12}{35}}$

$\frac{35}{0.35}$

Exercise 6.10.32

$\frac{\left(\sqrt{\frac{49}{64}}-5\right) 0.125}{1.375}$

(Solution on p. 416.)

(Solution on p. 416.)

\subsubsection{Exercises for Review}

Exercise 6.10.33

(Solution on p. 416.)

(Section 2.5) Is 21,480 divisible by 3 ?

Exercise 6.10.34

(Section 3.2) Expand 14 . Do not find the actual value.

Exercise 6.10.35

(Solution on p. 416.)

(Section 3.3) Find the prime factorization of 15,400 .

Exercise 6.10.36

(Section 6.3) Convert 8.016 to a fraction.

Exercise 6.10.37

(Section 6.9) Find the quotient. $16 \div 27$.

(Solution on p. 416.) 


\subsection{Summary of Key Concepts ${ }^{11}$}

\subsubsection{Summary of Key Concepts}

Decimal Point (Section 6.2)

A decimal point is a point that separates the units digit from the tenths digit.

\section{Decimal or Decimal Fraction (Section 6.2)}

A decimal fraction is a fraction whose denominator is a power of ten.

\section{Converting a Decimal to a Fraction (Section 6.3)}

Decimals can be converted to fractions by saying the decimal number in words, then writing what was said.

\section{Rounding Decimals (Section 6.4)}

Decimals are rounded in much the same way whole numbers are rounded.

\section{Addition and Subtraction of Decimals (Section 6.5)}

To add or subtract decimals,

1. Align the numbers vertically so that the decimal points line up under each other and the corresponding decimal positions are in the same column.

2. Add or subtract the numbers as if they were whole numbers.

3. Place a decimal point in the resulting sum directly under the other decimal points.

\section{Multiplication of Decimals (Section 6.6)}

To multiply two decimals,

1. Multiply the numbers as if they were whole numbers.

2. Find the sum of the number of decimal places in the factors.

3. The number of decimal places in the product is the number found in step 2 .

\section{Multiplying Decimals by Powers of 10 (Section 6.6)}

To multiply a decimal by a power of 10 , move the decimal point to the right as many places as there are zeros in the power of ten. Add zeros if necessary.

Division of a Decimal by a Decimal (Section 6.7)

To divide a decimal by a nonzero decimal,

1. Convert the divisor to a whole number by moving the decimal point until it appears to the right of the divisor's last digit.

2. Move the decimal point of the dividend to the right the same number of digits it was moved in the divisor.

3. Proceed to divide.

4. Locate the decimal in the answer by bringing it straight up from the dividend.

\section{Dividing Decimals by Powers of 10 (Section 6.7)}

To divide a decimal by a power of 10 , move the decimal point to the left as many places as there are zeros in the power of ten. Add zeros if necessary.

\section{Terminating Divisions (Section 6.8)}

A terminating division is a division in which the quotient terminates after several divisions. Terminating divisions are also called exact divisions.

\footnotetext{
${ }^{11}$ This content is available online at $<$ http://cnx.org/content/m34972/1.2/>.
} 


\section{Nonterminating Divisions (Section 6.8)}

A nonterminating division is a division that, regardless of how far it is carried out, always has a remainder. Nonterminating divisions are also called nonexact divisions.

\section{Converting Fractions to Decimals (Section 6.9)}

A fraction can be converted to a decimal by dividing the numerator by the denominator.

\subsection{Exercise Supplement ${ }^{12}$}

\subsubsection{Exercise Supplement}

\subsubsection{Reading and Writing Decimals (Section 6.2)}

Exercise 6.12.1

(Solution on p. 416.)

The decimal digit that appears two places to the right of the decimal point is in the position.

\section{Exercise 6.12.2}

The decimal digit that appears four places to the right of the decimal point is in the position.

For problems 3-8, read each decimal by writing it in words.

Exercise 6.12.3

7.2

(Solution on p. 416.)

Exercise 6.12.4

8.105

Exercise 6.12.5

(Solution on p. 416.)

16.52

Exercise 6.12.6

5.9271

Exercise 6.12.7

(Solution on p. 416.)

0.005

Exercise 6.12.8

4.01701

For problems 9-13, write each decimal using digits.

Exercise 6.12.9

(Solution on p. 416.)

Nine and twelve-hundredths.

Exercise 6.12.10

Two and one hundred seventy-seven thousandths.

Exercise 6.12.11

(Solution on p. 416.)

Fifty-six and thirty-five ten-thousandths.

Exercise 6.12.12

Four tenths.

Exercise 6.12.13

Four thousand eighty-one millionths.

(Solution on p. 416.)

\footnotetext{
${ }^{12}$ This content is available online at $<\mathrm{http}: / / \mathrm{cnx}$. org/content $/ \mathrm{m} 34978 / 1.2 />$.
} 


\subsubsection{Converting a Decimal to a Fraction (Section 6.3)}

For problem 14-20, convert each decimal to a proper fraction or a mixed number.

Exercise 6.12.14

1.07

Exercise 6.12.15

(Solution on p. 416.)

58.63

Exercise 6.12.16

0.05

Exercise 6.12.17

$0.14 \frac{2}{3}$

Exercise 6.12.18

$1.09 \frac{1}{8}$

Exercise 6.12.19

$4.01 \frac{1}{27}$

Exercise 6.12.20

$9.11 \frac{1}{9}$

\subsubsection{Rounding Decimals (Section 6.4)}

(Solution on p. 416.)

(Solution on p. 417.)

For problems 21-25, round each decimal to the specified position.

Exercise 6.12.21

(Solution on p. 417.)

4.087 to the nearest hundredth.

Exercise 6.12.22

4.087 to the nearest tenth.

Exercise 6.12.23

(Solution on p. 417.)

16.5218 to the nearest one.

Exercise 6.12.24

817.42 to the nearest ten.

Exercise 6.12.25

(Solution on p. 417.)

0.9811602 to the nearest one.

6.12.1.4 Addition, Subtraction, Multiplication and Division of Decimals, and Nonterminating Divisions (Section 6.5,Section 6.6,Section 6.7,Section 6.8)

For problem 26-45, perform each operation and simplify.

Exercise 6.12.26

$7.10+2.98$

Exercise 6.12.27

$14.007-5.061$

(Solution on p. 417.)

Exercise 6.12.28

$1.2 \cdot 8.6$

Exercise 6.12.29

(Solution on p. 417.)

$41.8 \cdot 0.19$

Exercise 6.12.30

$57.51 \div 2.7$ 
Exercise 6.12.31

(Solution on p. 417.)

$0.54003 \div 18.001$

Exercise 6.12.32

$32,051.3585 \div 23,006.9999$

Exercise 6.12.33

(Solution on p. 417.)

$100 \cdot 1,816.001$

Exercise 6.12.34

$1,000 \cdot 1,816.001$

Exercise 6.12.35

$10.000 \cdot 0.14$

(Solution on p. 417.)

Exercise 6.12.36

$0.135888 \div 16.986$

Exercise 6.12.37

$150.79 \div 100$

(Solution on p. 417.)

Exercise 6.12.38

$4.119 \div 10,000$

Exercise 6.12.39

(Solution on p. 417.)

$42.7 \div 18$

Exercise 6.12.40

$6.9 \div 12$

Exercise 6.12.41

$0.014 \div 47.6$. Round to three decimal places.

(Solution on p. 417.)

Exercise 6.12.42

$8.8 \div 19$. Round to one decimal place.

Exercise 6.12.43

(Solution on p. 417.)

$1.1 \div 9$

Exercise 6.12.44

$1.1 \div 9.9$

Exercise 6.12.45

$30 \div 11.1$

(Solution on p. 417.)

\subsubsection{Converting a Fraction to a Decimal (Section 6.9)}

For problems $46-55$, convert each fraction to a decimal.

Exercise 6.12.46

$\frac{3}{8}$

Exercise 6.12.47

$\frac{43}{100}$

(Solution on p. 417.)

Exercise 6.12.48

$\frac{82}{1000}$

Exercise 6.12.49

$9 \frac{4}{7}$

(Solution on p. 417.)

Exercise 6.12.50

$8 \frac{5}{16}$ 
Exercise 6.12.51

(Solution on p. 417.) $1.3 \frac{1}{3}$

Exercise 6.12.52 $25.6 \frac{2}{3}$

Exercise 6.12.53

(Solution on p. 417.) $125.125 \frac{1}{8}$

Exercise 6.12.54 $9.11 \frac{1}{9}$

Exercise 6.12.55 $0.0 \frac{5}{6}$

(Solution on p. 417.)

6.12.1.6 Combinations of Operations with Decimals and Fractions (Section 6.10)

For problems 56-62, perform each operation.

Exercise 6.12.56

$\frac{5}{8} \cdot 0.25$

Exercise 6.12.57

$\frac{3}{16} \cdot 1.36$

(Solution on p. 417.)

Exercise 6.12.58

$\frac{3}{5} \cdot\left(\frac{1}{2}+1.75\right)$

Exercise 6.12.59

$\frac{7}{2} \cdot\left(\frac{5}{4}+0.30\right)$

Exercise 6.12.60

$19.375 \div\left(4.375-1 \frac{1}{16}\right)$

Exercise 6.12.61

$\frac{15}{602} \cdot\left(2 . \overline{6}+3 \frac{1}{4}\right)$

Exercise 6.12.62

$4 \frac{13}{18} \div\left(5 \frac{3}{14}+3 \frac{5}{21}\right)$

\subsection{Proficiency Exam ${ }^{13}$}

\subsubsection{Proficiency Exam}

Exercise 6.13.1

(Solution on p. 417.)

(Section 6.2) The decimal digit that appears three places to the right of the decimal point is in the position.

Exercise 6.13.2

(Solution on p. 417.)

(Section 6.2) Write, using words, 15.036.

(Solution on p. 417.)

(Solution on p. 417.)

Exercise 6.13.3

(Solution on p. 417.)

(Section 6.2) Write eighty-one and twelve hundredths using digits. 81.12

Exercise 6.13.4

(Solution on p. 418.)

(Section 6.2) Write three thousand seventeen millionths using digits.

(Solution on p. 418.)

Exercise 6.13.5

(Section 6.3) Convert 0.78 to a fraction. Reduce.

${ }^{13}$ This content is available online at $<$ http://cnx.org/content/m34979/1.2/>. 
Exercise 6.13.6

(Solution on p. 418.)

(Section 6.3) Convert 0.875 to a fraction. Reduce.

Exercise 6.13.7

(Solution on p. 418.)

(Section 6.4) Round 4.8063 to the nearest tenth.

Exercise 6.13.8

(Solution on p. 418.)

(Section 6.4) Round 187.51 to the nearest hundred.

Exercise 6.13.9

(Solution on p. 418.)

(Section 6.4) Round 0.0652 to the nearest hundredth.

For problems 10-20, perform each operation.

Exercise 6.13.10

(Solution on p. 418.)

(Section 6.5) $15.026+5.971$

Exercise 6.13.11

(Solution on p. 418.)

(Section 6.5) 72.15 - 26.585

Exercise 6.13.12

(Solution on p. 418.)

(Section 6.6) 16.2 4.8

Exercise 6.13.13

(Section 6.6) 10,000 0.016

Exercise 6.13.14

(Solution on p. 418.)

(Section 6.7) $44.64 \div 18.6$

Exercise 6.13.15

(Section 6.7) $0.21387 \div 0.19$

Exercise 6.13.16

(Section 6.8) $0 . \overline{27}-\frac{3}{11}$

Exercise 6.13.17

(Section 6.9) Convert $6 \frac{2}{11}$ to a decimal.

Exercise 6.13.18

(Section 6.9) Convert $0.5 \frac{9}{16}$ to a decimal.

Exercise 6.13.19

(Section 6.10) $3 \frac{1}{8}+2.325$

Exercise 6.13.20

(Section 6.10) $\frac{3}{8} \times 0.5625$

(Solution on p. 418.)

(Solution on p. 418.)

(Solution on p. 418.)

(Solution on p. 418.)

(Solution on p. 418.)

(Solution on p. 418.)

(Solution on p. 418.) 


\section{Solutions to Exercises in Chapter 6}

Solution to Exercise 6.2.1 (p. 343)

twelve and nine tenths

Solution to Exercise 6.2.2 (p. 343)

four and eighty-six hundredths

Solution to Exercise 6.2.3 (p. 343)

seven and two hundred thousandths

Solution to Exercise 6.2.4 (p. 343)

thirty thousand four hundred five millionths

Solution to Exercise 6.2.5 (p. 344)

306.49

Solution to Exercise 6.2.6 (p. 344)

9.004

Solution to Exercise 6.2.7 (p. 344)

0.000061

Solution to Exercise 6.2.8 (p. 344)

Tenths; hundredths, thousandths

Solution to Exercise 6.2.10 (p. 344)

Hundred thousandths; ten millionths

Solution to Exercise 6.2.12 (p. 344)

eight and one tenth

Solution to Exercise 6.2.14 (p. 344)

fifty-five and six hundredths

Solution to Exercise 6.2.16 (p. 344)

one and nine hundred four thousandths

Solution to Exercise 6.2.18 (p. 345)

3.20

Solution to Exercise 6.2.20 (p. 345)

1.8

Solution to Exercise 6.2.22 (p. 345)

511.004

Solution to Exercise 6.2.24 (p. 345)

0.947

Solution to Exercise 6.2.26 (p. 345)

0.00071

Solution to Exercise 6.2.28 (p. 345)

seventy-five hundredths

Solution to Exercise 6.2.30 (p. 345)

four tenths

Solution to Exercise 6.2.32 (p. 345)

sixteen hundredths

Solution to Exercise 6.2.34 (p. 345)

one thousand eight hundred seventy-five ten thousandths

Solution to Exercise 6.2.36 (p. 345)

fifty-five hundredths

Solution to Exercise 6.2.38 (p. 346)

2610

Solution to Exercise 6.2.40 (p. 346)

12

Solution to Exercise 6.2.42 (p. 346)

$\frac{10}{9}$ or $1 \frac{1}{9}$ 
Solution to Exercise 6.3.1 (p. 347) $16 \frac{21}{25}$

Solution to Exercise 6.3.2 (p. 347) $\frac{513}{1,000}$

Solution to Exercise 6.3.3 (p. 347) $6,646 \frac{107}{10,000}$

Solution to Exercise 6.3.4 (p. 347) $1 \frac{1}{10}$

Solution to Exercise 6.3.5 (p. 348) $\frac{7}{8}$

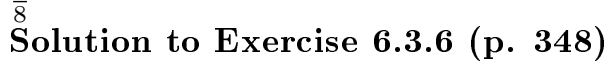

$\frac{31}{250}$

Solution to Exercise 6.3.7 (p. 348)

$6 \frac{7}{1,200}$

Solution to Exercise 6.3.8 (p. 348)

$18 \frac{2}{17}$

Solution to Exercise 6.3.9 (p. 348) $\frac{7}{10}$

Solution to Exercise 6.3.11 (p. 348)

$\frac{53}{100}$

Solution to Exercise 6.3.13 (p. 348)

$\frac{219}{1,000}$

Solution to Exercise 6.3.15 (p. 348) $4 \frac{4}{5}$

Solution to Exercise 6.3.17 (p. 348) $16 \frac{3}{25}$

Solution to Exercise 6.3.19 (p. 349) $6 \frac{1}{2,000}$

Solution to Exercise 6.3.21 (p. 349) $16 \frac{1}{8}$

Solution to Exercise 6.3.23 (p. 349) $3 \frac{1}{25}$

Solution to Exercise 6.3.25 (p. 349) $8 \frac{9}{40}$

Solution to Exercise 6.3.27 (p. 349) $9 \frac{19,999}{20,000}$

Solution to Exercise 6.3.29 (p. 349) $\frac{3}{4}$

Solution to Exercise 6.3.31 (p. 349) $2 \frac{13}{80}$

Solution to Exercise 6.3.33 (p. 349) $14 \frac{337}{3,000}$

Solution to Exercise 6.3.35 (p. 349) $1 \frac{129}{320}$

Solution to Exercise 6.3.37 (p. 349)

$2 \frac{1}{25}$

Solution to Exercise 6.3.39 (p. 350) 14

Solution to Exercise 6.3.41 (p. 350) $\frac{9}{10}$ 
Solution to Exercise 6.3.43 (p. 350) thousandths

Solution to Exercise 6.4.1 (p. 351)

4.82

Solution to Exercise 6.4.2 (p. 351)

0.3593

Solution to Exercise 6.4.3 (p. 351)

82

Solution to Exercise 6.4.4 (p. 351)

800

Solution to Exercise 6.4.5 (p. 351)

43.994

Solution to Exercise 6.4.6 (p. 351)

105.0200

Solution to Exercise 6.4.7 (p. 351)

100.00

Solution to Exercise 6.4.8 (p. 352)

\begin{tabular}{|l|l|l|l|}
\hline Tenth & Hundredth & Thousandth & Ten Thousandth \\
\hline 20.0 & 20.01 & 20.011 & 20.0107 \\
\hline
\end{tabular}

Table 6.30

Solution to Exercise 6.4.10 (p. 352)

\begin{tabular}{|l|l|l|l|}
\hline Tenth & Hundredth & Thousandth & Ten Thousandth \\
\hline 531.2 & 531.22 & 531.219 & 531.2188 \\
\hline
\end{tabular}

Table 6.31

Solution to Exercise 6.4.12 (p. 352)

\begin{tabular}{|l|l|l|l|}
\hline Tenth & Hundredth & Thousandth & Ten Thousandth \\
\hline 2.0 & 2.00 & 2.000 & 2.0000 \\
\hline
\end{tabular}

Table 6.32

Solution to Exercise 6.4.14 (p. 353)

\begin{tabular}{|l|l|l|l|}
\hline Tenth & Hundredth & Thousandth & Ten Thousandth \\
\hline 0.0 & 0.00 & 0.000 & 0.0000 \\
\hline
\end{tabular}

Table 6.33

Solution to Exercise 6.4.16 (p. 353) 


\begin{tabular}{|l|l|l|l|}
\hline Tenth & Hundredth & Thousandth & Ten Thousandth \\
\hline 9.2 & 9.19 & 9.192 & 9.1919 \\
\hline
\end{tabular}

Table 6.34

Solution to Exercise 6.4.18 (p. 353)

18.417

Solution to Exercise 6.4.20 (p. 353)

18.41681

Solution to Exercise 6.4.22 (p. 354)

18.42

Solution to Exercise 6.4.24 (p. 354)

0.1

Solution to Exercise 6.4.26 (p. 354)

0.83607

Solution to Exercise 6.4.28 (p. 354)

5.333

Solution to Exercise 6.4.30 (p. 354)

Ten million

Solution to Exercise 6.4.32 (p. 354)

256

Solution to Exercise 6.4.34 (p. 354)

$3 \frac{4}{25}$

Solution to Exercise 6.5.1 (p. 357)

6.179

Solution to Exercise 6.5.2 (p. 357)

9.646

Solution to Exercise 6.5.3 (p. 357)

1.6044

Solution to Exercise 6.5.4 (p. 357)

0.0065

Solution to Exercise 6.5.5 (p. 357)

$25,777.22$

Solution to Exercise 6.5.6 (p. 357)

25.69

Solution to Exercise 6.5.7 (p. 358)

13.256

Solution to Exercise 6.5.8 (p. 358)

59.4512

Solution to Exercise 6.5.9 (p. 358)

0.096862

Solution to Exercise 6.5.10 (p. 358)

0.475

Solution to Exercise 6.5.11 (p. 358)

Since each number contains more than eight digits, using some calculators may not be helpful. Adding these by "hand technology," we get $4,785.00031$

Solution to Exercise 6.5.12 (p. 359)

8.95

Solution to Exercise 6.5.14 (p. 359)

39.846 
Solution to Exercise 6.5.16 (p. 359) 11.74931

Solution to Exercise 6.5.18 (p. 359) 57.2115

Solution to Exercise 6.5.20 (p. 359) 2.607

Solution to Exercise 6.5.22 (p. 359) 0.41085

Solution to Exercise 6.5.24 (p. 359) 9.135586

Solution to Exercise 6.5.26 (p. 359) 27.351

Solution to Exercise 6.5.28 (p. 359) 39.6660

Solution to Exercise 6.5.30 (p. 359) 11.09

Solution to Exercise 6.5.32 (p. 360) $\$ 23.23$

Solution to Exercise 6.5.34 (p. 360) $\$ 5.71$

Solution to Exercise 6.5.36 (p. 360) 869.37

Solution to Exercise 6.5.38 (p. 360) $8,200,000$

Solution to Exercise 6.5.40 (p. 360) $\frac{20}{9}=\frac{5}{3}$ or $2 \frac{2}{9}$

Solution to Exercise 6.6.1 (p. 362) 45.58

Solution to Exercise 6.6.2 (p. 362) 10.388

Solution to Exercise 6.6.3 (p. 362) 0.16864

Solution to Exercise 6.6.4 (p. 362) 0.00000062

Solution to Exercise 6.6.5 (p. 362) 9.3

Solution to Exercise 6.6.6 (p. 362) 53.94

Solution to Exercise 6.6.7 (p. 362) 539.4

Solution to Exercise 6.6.8 (p. 362) 5,394

Solution to Exercise 6.6.9 (p. 362) 59,340

Solution to Exercise 6.6.10 (p. 364) 20.91408

Solution to Exercise 6.6.11 (p. 364) 0.000066

Solution to Exercise 6.6.12 (p. 364) 0.2397 
Solution to Exercise 6.6.13 (p. 364) 0.0000

Solution to Exercise 6.6.14 (p. 365)

427

Solution to Exercise 6.6.15 (p. 365)

$165,218.7$

Solution to Exercise 6.6.16 (p. 365) 0.188

Solution to Exercise 6.6.17 (p. 365) $527,000,000,000$

Solution to Exercise 6.6.18 (p. 366) 17.92

Solution to Exercise 6.6.19 (p. 366) 0.13

Solution to Exercise 6.6.20 (p. 366) 3.636

Solution to Exercise 6.6.21 (p. 366) 0.0000036

Solution to Exercise 6.6.22 (p. 366) 9.96

Solution to Exercise 6.6.23 (p. 366) 14.08

Solution to Exercise 6.6.24 (p. 367) 31.28

Solution to Exercise 6.6.26 (p. 367) 47.20

Solution to Exercise 6.6.28 (p. 367)

0.152

Solution to Exercise 6.6.30 (p. 367) 4.6324

Solution to Exercise 6.6.32 (p. 367) 3.182

Solution to Exercise 6.6.34 (p. 367) 0.0000273

Solution to Exercise 6.6.36 (p. 367) 2.56

Solution to Exercise 6.6.38 (p. 367) 0.81

Solution to Exercise 6.6.40 (p. 367) 29.3045

Solution to Exercise 6.6.42 (p. 367) 0.00000105486

Solution to Exercise 6.6.44 (p. 368) 49.6

Solution to Exercise 6.6.46 (p. 368) $4,218.842$

Solution to Exercise 6.6.48 (p. 368) 19.621

Solution to Exercise 6.6.50 (p. 368) $3,596.168$ 
Solution to Exercise 6.6.52 (p. 368)

25,010

Solution to Exercise 6.6.54 (p. 368)

\begin{tabular}{|l|l|l|l|}
\hline Actual product & Tenths & Hundreds & Thousandths \\
\hline 28.382 & 28.4 & 28.38 & 28.382 \\
\hline
\end{tabular}

Table 6.35

Solution to Exercise 6.6.56 (p. 368)

\begin{tabular}{|l|l|l|l|}
\hline Actual product & Tenths & Hundreds & Thousandths \\
\hline 134.216048 & 134.2 & 134.22 & 134.216 \\
\hline
\end{tabular}

Table 6.36

Solution to Exercise 6.6.58 (p. 369)

\begin{tabular}{|l|l|l|l|}
\hline Actual product & Tenths & Hundreds & Thousandths \\
\hline 185.626 & 185.6 & 185.63 & 185.626 \\
\hline
\end{tabular}

Table 6.37

Solution to Exercise 6.6.60 (p. 369)

121.503

Solution to Exercise 6.6.62 (p. 369)

1.2

Solution to Exercise 6.6.64 (p. 369) 0.999702

Solution to Exercise 6.6.66 (p. 369)

0.2528

Solution to Exercise 6.6.68 (p. 369)

2.9544

Solution to Exercise 6.6.70 (p. 369)

$\$ 16.24$

Solution to Exercise 6.6.72 (p. 369)

0.24

Solution to Exercise 6.6.74 (p. 370) 0.006099

Solution to Exercise 6.6.76 (p. 370) 23.295102

Solution to Exercise 6.6.78 (p. 370)

0.000144

Solution to Exercise 6.6.80 (p. 370) 0.0000018

Solution to Exercise 6.6.82 (p. 370) 0.0000000471 
Solution to Exercise 6.6.84 (p. 370)

0

Solution to Exercise 6.6.86 (p. 370)

$\frac{10}{77}$

Solution to Exercise 6.6.88 (p. 370)

1.78

Solution to Exercise 6.7.1 (p. 373)

61.5

Solution to Exercise 6.7.2 (p. 373) 1.884

Solution to Exercise 6.7.3 (p. 373) 0.0741

Solution to Exercise 6.7.4 (p. 373) 0.000062

Solution to Exercise 6.7.5 (p. 375)

2.96

Solution to Exercise 6.7.6 (p. 375)

4.58

Solution to Exercise 6.7.7 (p. 375)

40,000

Solution to Exercise 6.7.8 (p. 375)

816.241

Solution to Exercise 6.7.9 (p. 375)

81.6241

Solution to Exercise 6.7.10 (p. 375)

8.16241

Solution to Exercise 6.7.11 (p. 376)

0.816241

Solution to Exercise 6.7.12 (p. 377)

2.98

Solution to Exercise 6.7.13 (p. 377)

0.005

Solution to Exercise 6.7.14 (p. 377)

3.5197645 is an approximate result. Rounding to four decimal places, we get 3.5198

Solution to Exercise 6.7.15 (p. 379)

18.25

Solution to Exercise 6.7.16 (p. 379)

1.825

Solution to Exercise 6.7.17 (p. 379)

0.1825

Solution to Exercise 6.7.18 (p. 379)

0.01825

Solution to Exercise 6.7.19 (p. 379)

6.4618

Solution to Exercise 6.7.20 (p. 379)

0.021926

Solution to Exercise 6.7.21 (p. 379)

1.6

Solution to Exercise 6.7.23 (p. 379)

3.7 
Solution to Exercise 6.7.25 (p. 380) 6.04

Solution to Exercise 6.7.27 (p. 380) 9.38

Solution to Exercise 6.7.29 (p. 380)

0.46

Solution to Exercise 6.7.31 (p. 380)

8.6

Solution to Exercise 6.7.33 (p. 380)

2.4

Solution to Exercise 6.7.35 (p. 380)

6.21

Solution to Exercise 6.7.37 (p. 380) 2.18

Solution to Exercise 6.7.39 (p. 380)

1.001

Solution to Exercise 6.7.41 (p. 380)

4

Solution to Exercise 6.7.43 (p. 380)

14

Solution to Exercise 6.7.45 (p. 380)

111

Solution to Exercise 6.7.47 (p. 381)

643.51006

Solution to Exercise 6.7.49 (p. 381)

0.064351006

Solution to Exercise 6.7.51 (p. 381)

\begin{tabular}{|l|l|l|l|}
\hline Actual Quotient & Tenths & Hundredths & Thousandths \\
\hline 1.81 & 1.8 & 1.81 & 1.810 \\
\hline
\end{tabular}

Table 6.38

Solution to Exercise 6.7.53 (p. 381)

\begin{tabular}{|l|l|l|l|}
\hline Actual Quotient & Tenths & Hundredths & Thousandths \\
\hline 4.821 & 4.8 & 4.82 & 4.821 \\
\hline
\end{tabular}

Table 6.39

Solution to Exercise 6.7.55 (p. 381)

\begin{tabular}{|l|l|l|l|}
\hline Actual Quotient & Tenths & Hundredths & Thousandths \\
\hline 0.00351 & 0.0 & 0.00 & 0.004 \\
\hline
\end{tabular}

Table 6.40 
Solution to Exercise 6.7.57 (p. 382)

0.01

Solution to Exercise 6.7.59 (p. 382)

12.11 months

Solution to Exercise 6.7.61 (p. 382)

27.2 miles per gallon

Solution to Exercise 6.7.63 (p. 382)

3.7

Solution to Exercise 6.7.65 (p. 382)

1.94

Solution to Exercise 6.7.67 (p. 382)

29.120

Solution to Exercise 6.7.69 (p. 382)

0.173

Solution to Exercise 6.7.71 (p. 382)

1.111

Solution to Exercise 6.7.73 (p. 383)

$\frac{39}{8}$

Solution to Exercise 6.7.75 (p. 383)

$\frac{47}{30}$ or $1 \frac{17}{30}$

Solution to Exercise 6.7.77 (p. 383)

2.8634

Solution to Exercise 6.8.1 (p. 386)

$0 . \overline{3}$

Solution to Exercise 6.8.2 (p. 386)

$0.8 \overline{3}$

Solution to Exercise 6.8.3 (p. 386)

$1 . \overline{2}$

Solution to Exercise 6.8.4 (p. 386)

$1 . \overline{8}$

Solution to Exercise 6.8.5 (p. 386)

1.17

Solution to Exercise 6.8.6 (p. 386)

36.3636

Solution to Exercise 6.8.7 (p. 387)

$0 . \overline{4}$

Solution to Exercise 6.8.9 (p. 387)

0.16

Solution to Exercise 6.8.11 (p. 387)

$0 . \overline{142857}$

Solution to Exercise 6.8.13 (p. 387)

10.526

Solution to Exercise 6.8.15 (p. 387)

$0.1 \overline{12}$

Solution to Exercise 6.8.17 (p. 387)

$6 . \overline{21951}$

Solution to Exercise 6.8.19 (p. 387)

$0.00 \overline{81}$

Solution to Exercise 6.8.21 (p. 387)

0.835 
Solution to Exercise 6.8.23 (p. 387)

$0 . \overline{3}$

Solution to Exercise 6.8.25 (p. 387)

$2.0 \overline{5}$

Solution to Exercise 6.8.27 (p. 387)

$0 . \overline{7}$

Solution to Exercise 6.8.29 (p. 388)

$0 . \overline{518}$

Solution to Exercise 6.8.31 (p. 388) $0.0 \overline{45}$

Solution to Exercise 6.8.33 (p. 388) $0 . \overline{72}$

Solution to Exercise 6.8.35 (p. 388)

0.7

Solution to Exercise 6.8.37 (p. 388)

1

Solution to Exercise 6.8.39 (p. 388)

4410

Solution to Exercise 6.8.41 (p. 388)

8.6

Solution to Exercise 6.9.1 (p. 390)

0.25

Solution to Exercise 6.9.2 (p. 391)

0.04

Solution to Exercise 6.9.3 (p. 391)

0.17

Solution to Exercise 6.9.4 (p. 391)

0.9375

Solution to Exercise 6.9.5 (p. 391)

0.95

Solution to Exercise 6.9.6 (p. 391)

8.0126375

Solution to Exercise 6.9.7 (p. 391)

0.5

Solution to Exercise 6.9.9 (p. 391)

0.875

Solution to Exercise 6.9.11 (p. 391)

0.6

Solution to Exercise 6.9.13 (p. 391)

0.04

Solution to Exercise 6.9.15 (p. 391)

0.05

Solution to Exercise 6.9.17 (p. 391)

0.02

Solution to Exercise 6.9.19 (p. 391)

$0 . \overline{3}$

Solution to Exercise 6.9.21 (p. 391)

0.1875

Solution to Exercise 6.9.23 (p. 392) $0.0 \overline{37}$ 
Solution to Exercise 6.9.25 (p. 392) $0 . \overline{538461}$

Solution to Exercise 6.9.27 (p. 392)

$7 . \overline{6}$

Solution to Exercise 6.9.29 (p. 392)

$1.1 \overline{3}$

Solution to Exercise 6.9.31 (p. 392) 101.24

Solution to Exercise 6.9.33 (p. 392) 0.24125

Solution to Exercise 6.9.35 (p. 392) 810.31063125

Solution to Exercise 6.9.37 (p. 392) 0.11111

Solution to Exercise 6.9.39 (p. 392) 0.33333

Solution to Exercise 6.9.41 (p. 392) 0.55556

Solution to Exercise 6.9.43 (p. 392) 0.77778

Solution to Exercise 6.9.45 (p. 393) 0.09091

Solution to Exercise 6.9.47 (p. 393) 0.27273

Solution to Exercise 6.9.49 (p. 393) 0.45455

Solution to Exercise 6.9.51 (p. 393) 0.63636

Solution to Exercise 6.9.53 (p. 393) 0.81818

Solution to Exercise 6.9.55 (p. 393) 0.128

Solution to Exercise 6.9.57 (p. 393) 0.9746

Solution to Exercise 6.9.59 (p. 393) 0.0002

Solution to Exercise 6.9.61 (p. 393) 38.7347

Solution to Exercise 6.9.63 (p. 394) $\frac{15}{16}$

Solution to Exercise 6.9.65 (p. 394) $3 \frac{29}{40}$ or 3.725

Solution to Exercise 6.10.1 (p. 395) 2.2 or $2 \frac{1}{5}$

Solution to Exercise 6.10.2 (p. 395) 9.23

Solution to Exercise 6.10.3 (p. 395) 10

Solution to Exercise 6.10.4 (p. 395) $\frac{1}{25}$ or 0.04 
Solution to Exercise 6.10.5 (p. 396) 1

Solution to Exercise 6.10.7 (p. 396)

0.112

Solution to Exercise 6.10.9 (p. 396)

0.055

Solution to Exercise 6.10.11 (p. 396)

0.1276

Solution to Exercise 6.10.13 (p. 396)

0.7

Solution to Exercise 6.10.15 (p. 396)

9.4625

Solution to Exercise 6.10.17 (p. 396)

1.4075

Solution to Exercise 6.10.19 (p. 396)

0.1875

Solution to Exercise 6.10.21 (p. 396)

0.75

Solution to Exercise 6.10.23 (p. 396)

0.615

Solution to Exercise 6.10.25 (p. 396)

1.35

Solution to Exercise 6.10.27 (p. 397)

0.125

Solution to Exercise 6.10.29 (p. 397)

$0 . \overline{15}$

Solution to Exercise 6.10.31 (p. 397)

$2 . \overline{6}$

Solution to Exercise 6.10.33 (p. 397)

yes

Solution to Exercise 6.10.35 (p. 397)

$2^{3} \cdot 5^{2} \cdot 7 \cdot 11$

Solution to Exercise 6.10.37 (p. 397)

$0 . \overline{592}$

Solution to Exercise 6.12.1 (p. 399) hundredths

Solution to Exercise 6.12.3 (p. 399) seven and two tenths

Solution to Exercise 6.12.5 (p. 399)

sixteen and fifty-two hundredths

Solution to Exercise 6.12.7 (p. 399)

five thousandths

Solution to Exercise 6.12.9 (p. 399)

9.12

Solution to Exercise 6.12.11 (p. 399)

56.0035

Solution to Exercise 6.12.13 (p. 399)

0.004081

Solution to Exercise 6.12.15 (p. 400) $85 \frac{63}{100}$ 
Solution to Exercise 6.12.17 (p. 400) $\frac{11}{75}$

Solution to Exercise 6.12.19 (p. 400)

$4 \frac{7}{675}$

Solution to Exercise 6.12.21 (p. 400)

4.09

Solution to Exercise 6.12.23 (p. 400)

17

Solution to Exercise 6.12.25 (p. 400)

1

Solution to Exercise 6.12.27 (p. 400)

8.946

Solution to Exercise 6.12.29 (p. 400)

7.942

Solution to Exercise 6.12.31 (p. 401)

0.03

Solution to Exercise 6.12.33 (p. 401)

$181,600.1$

Solution to Exercise 6.12.35 (p. 401)

1.4

Solution to Exercise 6.12.37 (p. 401)

1.5079

Solution to Exercise 6.12.39 (p. 401)

$2.37 \overline{2}$

Solution to Exercise 6.12.41 (p. 401)

0.000

Solution to Exercise 6.12.43 (p. 401)

$0.1 \overline{2}$

Solution to Exercise 6.12.45 (p. 401)

$2 . \overline{702}$

Solution to Exercise 6.12.47 (p. 401)

0.43

Solution to Exercise 6.12.49 (p. 401)

$9 . \overline{571428}$

Solution to Exercise 6.12.51 (p. 402)

$1 . \overline{3}$

Solution to Exercise 6.12.53 (p. 402)

125.125125 (not repeating)

Solution to Exercise 6.12.55 (p. 402)

$0.08 \overline{3}$

Solution to Exercise 6.12.57 (p. 402)

0.255

Solution to Exercise 6.12.59 (p. 402)

5.425

Solution to Exercise 6.12.61 (p. 402)

0.09343

Solution to Exercise 6.13.1 (p. 402)

thousandth

Solution to Exercise 6.13.2 (p. 402)

fifteen and thirty-six thousandths 
Solution to Exercise 6.13.3 (p. 402)

81.12

Solution to Exercise 6.13.4 (p. 402)

0.003017

Solution to Exercise 6.13.5 (p. 402)

$\frac{39}{50}$

Solution to Exercise 6.13.6 (p. 403)

$\frac{7}{8}$

Solution to Exercise 6.13.7 (p. 403)

4.8

Solution to Exercise 6.13.8 (p. 403)

200

Solution to Exercise 6.13.9 (p. 403)

0.07

Solution to Exercise 6.13.10 (p. 403)

20.997

Solution to Exercise 6.13.11 (p. 403)

45.565

Solution to Exercise 6.13.12 (p. 403)

77.76

Solution to Exercise 6.13.13 (p. 403)

16

Solution to Exercise 6.13.14 (p. 403)

2.4

Solution to Exercise 6.13.15 (p. 403)

1.1256

Solution to Exercise 6.13.16 (p. 403)

0

Solution to Exercise 6.13.17 (p. 403)

$6 . \overline{18}$

Solution to Exercise 6.13.18 (p. 403)

0.055625

Solution to Exercise 6.13.19 (p. 403)

5.45

Solution to Exercise 6.13.20 (p. 403)

$\frac{27}{128}$ or 0.2109375 


\section{Chapter 7}

\section{Ratios and Rates}

\subsection{Objectives ${ }^{1}$}

After completing this chapter, you should

Ratios and Rates (Section 7.2)

- be able to distinguish between denominate and pure numbers and between ratios and rates

\section{Proportions (Section 7.3)}

- be able to describe proportions and find the missing factor in a proportion

- be able to work with proportions involving rates

\section{Applications of Proportions (Section 7.4)}

- solve proportion problems using the five-step method

\section{Percent (Section 7.5)}

- understand the relationship between ratios and percents

- be able to make conversions between fractions, decimals, and percents

\section{Fractions of One Percent (Section 7.6)}

- understand the meaning of a fraction of one percent

- be able to make conversions involving fractions of one percent

\section{Applications of Percents (Section 7.7)}

- be able to distinguish between base, percent, and percentage

- be able to find the percentage, the percent, and the base

\subsection{Ratios and Rates ${ }^{2}$}

\subsubsection{Section Overview}

- Denominate Numbers and Pure Numbers

- Ratios and Rates

\footnotetext{
${ }^{1}$ This content is available online at $<$ http://cnx.org/content $/ \mathrm{m} 18895 / 1.3 />$.

${ }^{2}$ This content is available online at $<$ http://cnx.org/content $/ \mathrm{m} 34980 / 1.2 />$.
}

Available for free at Connexions $<$ http://cnx.org/content/col10615/1.4> 


\subsubsection{Denominate Numbers and Pure Numbers}

\section{Denominate Numbers, Like and Unlike Denominate Numbers}

It is often necessary or convenient to compare two quantities. Denominate numbers are numbers together with some specified unit. If the units being compared are alike, the denominate numbers are called like denominate numbers. If units are not alike, the numbers are called unlike denominate numbers. Examples of denominate numbers are shown in the diagram:

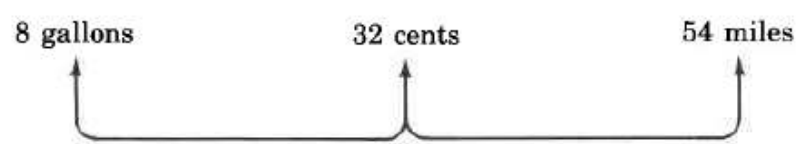

The denominations

\section{Pure Numbers}

Numbers that exist purely as numbers and do not represent amounts of quantities are called pure numbers. Examples of pure numbers are 8, 254, 0, 21 $\frac{5}{8}, \frac{2}{5}$, and 0.07 .

Numbers can be compared in two ways: subtraction and division.

Comparing Numbers by Subtraction and Division

Comparison of two numbers by subtraction indicates how much more one number is than another. Comparison by division indicates how many times larger or smaller one number is than another.

\section{Comparing Pure or Like Denominate Numbers by Subtraction}

Numbers can be compared by subtraction if and only if they both are like denominate numbers or both pure numbers.

\subsubsection{Sample Set A}

\section{Example 7.1}

Compare 8 miles and 3 miles by subtraction.

8 mile -3 miles $=5$ miles

This means that 8 miles is 5 miles more than 3 miles.

Examples of use: I can now jog 8 miles whereas I used to jog only 3 miles. So, I can now jog 5 miles more than I used to.

\section{Example 7.2}

Compare 12 and 5 by subtraction.

$12-5=7$

This means that 12 is 7 more than 5 .

\section{Example 7.3}

Comparing 8 miles and 5 gallons by subtraction makes no sense.

8 miles -5 gallons $=$ ?

\section{Example 7.4}

Compare 36 and 4 by division.

$36 \div 4=9$

This means that 36 is 9 times as large as 4 . Recall that $36 \div 4=9$ can be expressed as $\frac{36}{4}=9$. 


\section{Example 7.5}

Compare 8 miles and 2 miles by division.

$\frac{8 \text { miles }}{2 \text { miles }}=4$

This means that 8 miles is 4 times as large as 2 miles.

Example of use: I can jog 8 miles to your 2 miles. Or, for every 2 miles that you jog, I jog 8 . So, I jog 4 times as many miles as you jog.

Notice that when like quantities are being compared by division, we drop the units. Another way of looking at this is that the units divide out (cancel).

\section{Example 7.6}

Compare 30 miles and 2 gallons by division.

$\frac{30 \text { miles }}{2 \text { gallons }}=\frac{15 \text { miles }}{1 \text { gallon }}$

Example of use: A particular car goes 30 miles on 2 gallons of gasoline. This is the same as getting 15 miles to 1 gallon of gasoline.

Notice that when the quantities being compared by division are unlike quantities, we do not drop the units.

\subsubsection{Practice Set A}

Make the following comparisons and interpret each one.

Exercise 7.2.1

Compare 10 diskettes to 2 diskettes by

(Solution on p. 466.)

(a) subtraction:

(b) division:

Exercise 7.2.2

Compare, if possible, 16 bananas and 2 bags by

(Solution on p. 466.)
(a) subtraction:
(b) division:

\subsubsection{Ratios and Rates}

\section{Ratio}

A comparison, by division, of two pure numbers or two like denominate numbers is a ratio.

The comparison by division of the pure numbers $\frac{36}{4}$ and the like denominate numbers $\frac{8 \text { miles }}{2 \text { miles }}$ are examples of ratios.

\section{Rate}

A comparison, by division, of two unlike denominate numbers is a rate.

The comparison by division of two unlike denominate numbers, such as

$\frac{55 \text { miles }}{1 \text { gallon }}$ and $\frac{40 \text { dollars }}{5 \text { tickets }}$ 
are examples of rates.

Let's agree to represent two numbers (pure or denominate) with the letters $a$ and $b$. This means that we're letting $a$ represent some number and $b$ represent some, perhaps different, number. With this agreement, we can write the ratio of the two numbers $a$ and $b$ as

$\frac{a}{b}$ or $\frac{b}{a}$

The ratio $\frac{a}{b}$ is read as " $a$ to $b . "$

The ratio $\frac{b}{a}$ is read as " $b$ to $a . "$

Since a ratio or a rate can be expressed as a fraction, it may be reducible.

\subsubsection{Sample Set B}

\section{Example 7.7}

The ratio 30 to 2 can be expressed as $\frac{30}{2}$. Reducing, we get $\frac{15}{1}$.

The ratio 30 to 2 is equivalent to the ratio 15 to 1 .

Example 7.8

The rate " 4 televisions to 12 people" can be expressed as $\frac{4 \text { televisions }}{12 \text { people }}$. The meaning of this rate is that "for every 4 televisions, there are 12 people."

Reducing, we get $\frac{1 \text { television }}{3 \text { people }}$ The meaning of this rate is that "for every 1 television, there are 3 people."

Thus, the rate of " 4 televisions to 12 people" is the same as the rate of " 1 television to 3 people."

\subsubsection{Practice Set B}

Write the following ratios and rates as fractions.

Exercise 7.2.3

(Solution on p. 466.)

3 to 2

Exercise 7.2.4

(Solution on p. 466.)

1 to 9

Exercise 7.2.5

(Solution on p. 466.)

5 books to 4 people

Exercise 7.2.6

(Solution on p. 466.)

120 miles to 2 hours

Exercise 7.2.7

(Solution on p. 466.)

8 liters to 3 liters

Write the following ratios and rates in the form " $a$ to $b . "$ Reduce when necessary.

Exercise 7.2.8

(Solution on p. 466.) $\frac{9}{5}$

Exercise 7.2.9

$\frac{1}{3}$

(Solution on p. 466.)

Exercise 7.2.10

$\frac{25 \text { miles }}{2 \text { gallons }}$

(Solution on p. 466.) 
Exercise 7.2.11

(Solution on p. 466.) $\frac{2 \text { mechanics }}{4 \text { wrenches }}$

Exercise 7.2.12

$\frac{15 \text { video tapes }}{18 \text { video tapes }}$

(Solution on p. 466.)

\subsubsection{Exercises}

For the following 9 problems, complete the statements.

Exercise 7.2.13

(Solution on p. 466.)

Two numbers can be compared by subtraction if and only if

Exercise 7.2.14

A comparison, by division, of two pure numbers or two like denominate numbers is called a

Exercise 7.2.15

(Solution on p. 466.)

A comparison, by division, of two unlike denominate numbers is called a

Exercise 7.2.16

$\frac{6}{11}$ is an example of a . (ratio/rate)

Exercise 7.2.17

$\frac{5}{12}$ is an example of a . (ratio/rate)

(Solution on p. 466.)

Exercise 7.2.18

$\frac{7 \text { erasers }}{12 \text { pencils }}$ is an example of a - (ratio/rate)

Exercise 7.2.19

$\frac{20 \text { silver coins }}{35 \text { gold coins }}$ is an example of a .(ratio/rate)

Exercise 7.2.20

$\frac{3 \text { sprinklers }}{5 \text { sprinklers }}$ is an example of a - (ratio/rate)

Exercise 7.2.21

$\frac{18 \text { exhaust valves }}{11 \text { exhaust valves }}$ is an example of a .(ratio/rate)

(Solution on p. 466.)

For the following 7 problems, write each ratio or rate as a verbal phrase.

Exercise 7.2.22

$\frac{8}{3}$

Exercise 7.2.23

$\frac{2}{5}$

(Solution on p. 466.)

Exercise 7.2.24

$\frac{8 \text { feet }}{3 \text { seconds }}$

Exercise 7.2.25

29 miles

Exercise 7.2.26

30,000 stars

300 stars

Exercise 7.2.27

$\frac{5 \text { yards }}{2 \text { yards }}$

Exercise 7.2.28

$\frac{164 \text { trees }}{28 \text { trees }}$

(Solution on p. 466.)

he following problems, write the simplified fractional form of each ratio or rate.

Exercise 7.2.29

(Solution on p. 466.)

12 to 5 
Exercise 7.2.30

81 to 19

Exercise 7.2.31

(Solution on p. 466.)

42 plants to 5 homes

Exercise 7.2.32

8 books to 7 desks

Exercise 7.2.33

(Solution on p. 466.)

16 pints to 1 quart

Exercise 7.2.34

4 quarts to 1 gallon

Exercise 7.2.35

(Solution on p. 467.)

$2.54 \mathrm{~cm}$ to 1 in

Exercise 7.2.36

80 tables to 18 tables

Exercise 7.2.37

25 cars to 10 cars

Exercise 7.2.38

37 wins to 16 losses

Exercise 7.2.39

(Solution on p. 467.)

105 hits to 315 at bats

(Solution on p. 467.)

Exercise 7.2.40

510 miles to 22 gallons

Exercise 7.2.41

(Solution on p. 467.)

1,042 characters to 1 page

Exercise 7.2.42

1,245 pages to 2 books

\subsubsection{Exercises for Review}

Exercise 7.2.43

(Section 4.3) Convert $\frac{16}{3}$ to a mixed number.

(Solution on p. 467.)

Exercise 7.2.44

(Section 4.7) $1 \frac{5}{9}$ of $2 \frac{4}{7}$ is what number?

Exercise 7.2.45

(Section 5.3) Find the difference. $\frac{11}{28}-\frac{7}{45}$.

(Solution on p. 467.)

Exercise 7.2.46

(Section 6.8) Perform the division. If no repeating patterns seems to exist, round the quotient to three decimal places: $22.35 \div 17$

Exercise 7.2.47

(Section 6.10) Find the value of $1.85+\frac{3}{8} \cdot 4.1$

(Solution on p. 467.) 


\subsection{Proportions ${ }^{3}$}

\subsubsection{Section Overview}

- Ratios, Rates, and Proportions

- Finding the Missing Factor in a Proportion

- Proportions Involving Rates

\subsubsection{Ratios, Rates, and Proportions}

\section{Ratio, Rate}

We have defined a ratio as a comparison, by division, of two pure numbers or two like denominate numbers. We have defined a rate as a comparison, by division, of two unlike denominate numbers.

\section{Proportion}

A proportion is a statement that two ratios or rates are equal. The following two examples show how to read proportions.
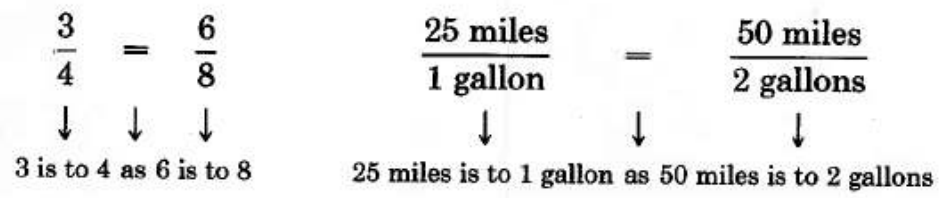

\subsubsection{Sample Set A}

Write or read each proportion.

\section{Example 7.9}

$\frac{3}{5}=\frac{12}{20}$

3 is to 5 as 12 is to 20

\section{Example 7.10}

$\frac{10 \text { items }}{5 \text { dollars }}=\frac{2 \text { items }}{1 \text { dollar }}$

10 items is to 5 dollars as 2 items is to 1 dollar

\section{Example 7.11}

8 is to 12 as 16 is to 24 .

$\frac{8}{12}=\frac{16}{24}$

\section{Example 7.12}

50 milligrams of vitamin $\mathrm{C}$ is to 1 tablet as 300 milligrams of vitamin $\mathrm{C}$ is to 6 tablets.

$$
\frac{50}{1}=\frac{300}{6}
$$

\footnotetext{
${ }^{3}$ This content is available online at $<$ http://cnx.org/content $/ \mathrm{m} 34981 / 1.2 />$.
} 


\subsubsection{Practice Set A}

Write or read each proportion.

\section{Exercise 7.3.1}

(Solution on p. 467.)

$\frac{3}{8}=\frac{6}{16}$

Exercise 7.3.2

(Solution on p. 467.)

$\frac{2 \text { people }}{1 \text { window }}=\frac{10 \text { people }}{5 \text { windows }}$

Exercise 7.3.3

(Solution on p. 467.)

15 is to 4 as 75 is to 20 .

Exercise 7.3.4

(Solution on p. 467.)

2 plates are to 1 tray as 20 plates are to 10 trays.

\subsubsection{Finding the Missing Factor in a Proportion}

Many practical problems can be solved by writing the given information as proportions. Such proportions will be composed of three specified numbers and one unknown number. It is customary to let a letter, such as $x$, represent the unknown number. An example of such a proportion is

$\frac{x}{4}=\frac{20}{16}$

This proportion is read as " $x$ is to 4 as 20 is to $16 . "$

There is a method of solving these proportions that is based on the equality of fractions. Recall that two fractions are equivalent if and only if their cross products are equal. For example,

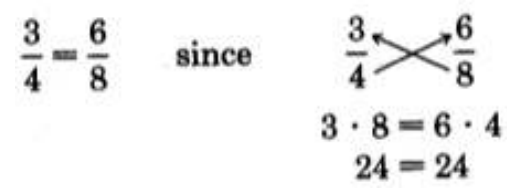

Notice that in a proportion that contains three specified numbers and a letter representing an unknown quantity, that regardless of where the letter appears, the following situation always occurs.

$\underbrace{(\text { number }) \cdot(\text { letter })=(\text { number }) \cdot(\text { number })}$

We recognize this as a multiplication statement. Specifically, it is a missing factor statement. (See Section 4.7 for a discussion of multiplication statements.) For example,

$$
\begin{aligned}
& \frac{x}{4}=\frac{20}{16} \quad \text { means that } \quad 16 \cdot x=4 \cdot 20 \\
& \frac{4}{x}=\frac{16}{20} \quad \text { means that } 4 \cdot 20=16 \cdot x \\
& \frac{5}{4}=\frac{x}{16} \quad \text { means that } 5 \cdot 16=4 \cdot x \\
& \frac{5}{4}=\frac{20}{x} \quad \text { means that } \quad 5 \cdot x=4 \cdot 20
\end{aligned}
$$

Each of these statements is a multiplication statement. Specifically, each is a missing factor statement. (The letter used here is $x$, whereas $M$ was used in Section 4.7.)

\section{Finding the Missing Factor in a Proportion}

The missing factor in a missing factor statement can be determined by dividing the product by the known factor, that is, if $x$ represents the missing factor, then

$x=$ (product) $\div$ (known factor $)$ 


\subsubsection{Sample Set B}

Find the unknown number in each proportion.

\section{Example 7.13}

$\frac{x}{4}=\frac{20}{16}$. Find the cross product.

$$
\begin{array}{rlr}
16 \cdot x & =20 \cdot 4 & \\
16 \cdot x & =80 & \text { Divide the product } 80 \text { by the known factor } 16 . \\
x & =\frac{80}{16} & \\
x & =5 & \text { The unknown number is } 5 .
\end{array}
$$

This mean that $\frac{5}{4}=\frac{20}{16}$, or 5 is to 4 as 20 is to 16 .

\section{Example 7.14}

$\frac{5}{x}=\frac{20}{16}$. Find the cross product.

$$
\begin{aligned}
5 \cdot 16 & =20 \cdot x & \\
80 & =20 \cdot x & \text { Divide the product } 80 \text { by the known factor } 20 . \\
\frac{80}{20} & =x & \\
4 & =x & \text { The unknown number is } 4 .
\end{aligned}
$$

This means that $\frac{5}{4}=\frac{20}{16}$, or, 5 is to 4 as 20 is to 6 .

\section{Example 7.15}

$\frac{16}{3}=\frac{64}{x}$ Find the cross product.

$$
\begin{aligned}
16 \cdot x & =64 \cdot 3 \\
16 \cdot x & =192 \quad \text { Divide } 192 \text { by } 16 . \\
x & =\frac{192}{16} \\
x & =12 \quad \text { The unknown number is } 12 .
\end{aligned}
$$

The means that $\frac{16}{3}=\frac{64}{12}$, or, 16 is to 3 as 64 is to 12 .

\section{Example 7.16}

$\frac{9}{8}=\frac{x}{40}$ Find the cross products.

$$
\begin{array}{rlr}
9 \cdot 40 & =8 \cdot x & \\
360 & =8 \cdot x \quad \text { Divide } 360 \text { by } 8 . \\
\frac{360}{8} & =x \\
45 & =x \quad \text { The unknown number is } 45 .
\end{array}
$$

\subsubsection{Practice Set B}

Find the unknown number in each proportion.

\section{Exercise 7.3.5} $\frac{x}{8}=\frac{12}{32}$ 
Exercise 7.3.6 $\frac{7}{x}=\frac{14}{10}$

Exercise 7.3.7 $\frac{9}{11}=\frac{x}{55}$

Exercise 7.3.8 $\frac{1}{6}=\frac{8}{x}$
(Solution on p. 467.)

(Solution on p. 467.)

(Solution on p. 467.)

\subsubsection{Proportions Involving Rates}

Recall that a rate is a comparison, by division, of unlike denominate numbers. We must be careful when setting up proportions that involve rates. The form is important. For example, if a rate involves two types of units, say unit type 1 and unit type 2, we can write

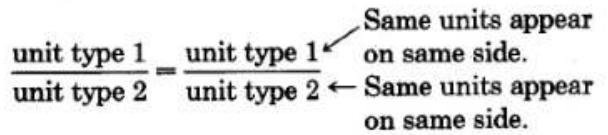

or

unit type $1=$ unit type 2

$\overline{\text { unit type } 1}=\overline{\text { unit type } 2}$

$\uparrow \uparrow$

Same units Same units

appear on appear on

same side. same side.

Both cross products produce a statement of the type

$($ unit type 1$) \cdot($ unit type 2$)=($ unit type 1$) \cdot($ unit type 2$)$

which we take to mean the comparison

(unit type 1) is to (unit type 2)

Comparison of type 1 with type 2

as (unit type 1) is to (unit type 2)

Comparison of type 1 with type 2

Same overall type

Examples of correctly expressed proportions are the following:

1. $\frac{\mathrm{mi}}{\mathrm{hr}}=\frac{\mathrm{mi}}{\mathrm{hr}}$ Same units appear on the same side

2. $\frac{\mathrm{mi}}{\mathrm{mi}}=\frac{\mathrm{hr}}{\mathrm{hr}}$

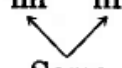

Same

units

appear on

the same

side.

However, if we write the same type of units on different sides, such as,

$\frac{\text { unit type } 1}{\text { unit type } 2}=\frac{\text { unit type } 2}{\text { unit type } 1}$

the cross product produces a statement of the form 


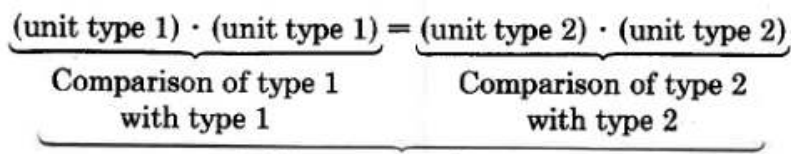

\section{Different overall types}

We can see that this is an incorrect comparison by observing the following example: It is incorrect to write

$\frac{2 \text { hooks }}{3 \text { poles }}=\frac{6 \text { poles }}{4 \text { hooks }}$

for two reason.

1. The cross product is numerically wrong: $(2 \cdot 4 \neq 3 \cdot 6)$.

2. The cross product produces the statement "hooks are to hooks as poles are to poles," which makes no sense.

\subsubsection{Exercises}

Exercise 7.3.9

(Solution on p. 467.)

A statement that two ratios or are equal is called a

For the following 9 problems, write each proportion in fractional form.

Exercise 7.3.10

3 is to 7 as 18 is to 42 .

Exercise 7.3.11

(Solution on p. 467.)

1 is to 11 as 3 is to 33 .

Exercise 7.3.12

9 is to 14 as 27 is to 42 .

Exercise 7.3.13

(Solution on p. 467.)

6 is to 90 as 3 is to 45 .

Exercise 7.3.14

5 liters is to 1 bottle as 20 liters is to 4 bottles.

Exercise 7.3.15

(Solution on p. 467.)

18 grams of cobalt is to 10 grams of silver as 36 grams of cobalt is to 20 grams of silver.

Exercise 7.3.16

4 cups of water is to 1 cup of sugar as 32 cups of water is to 8 cups of sugar.

Exercise 7.3.17

(Solution on p. 467.)

3 people absent is to 31 people present as 15 people absent is to 155 people present.

Exercise 7.3.18

6 dollars is to 1 hour as 90 dollars is to 15 hours.

For the following 10 problems, write each proportion as a sentence.

Exercise 7.3.19

$$
\frac{3}{4}=\frac{15}{20}
$$

(Solution on p. 467.)

Exercise 7.3.20

$$
\frac{1}{8}=\frac{5}{40}
$$

Exercise 7.3.21

$\frac{3 \text { joggers }}{100 \text { feet }}=\frac{6 \text { joggers }}{200 \text { feet }}$

(Solution on p. 467.)

Exercise 7.3.22

$\frac{12 \text { marshmallows }}{3 \text { sticks }}=\frac{36 \text { marshmallows }}{9 \text { sticks }}$ 
Exercise 7.3.23

(Solution on p. 467.)

$\frac{40 \text { miles }}{80 \text { miles }}=\frac{2 \text { gallons }}{4 \text { gallons }}$

Exercise 7.3.24

$\frac{4 \text { couches }}{10 \text { couches }}=\frac{2 \text { houses }}{5 \text { houses }}$

Exercise 7.3.25

$\frac{1 \text { person }}{1 \text { job }}=\frac{8 \text { people }}{8 \text { jobs }}$

(Solution on p. 467.)

Exercise 7.3.26

$\frac{1 \text { popsicle }}{2 \text { children }}=\frac{\frac{1}{2} \text { popsicle }}{1 \text { child }}$

Exercise 7.3.27

$\frac{2,000 \text { pounds }}{1 \text { ton }}=\frac{60,000 \text { pounds }}{30 \text { tons }}$

(Solution on p. 467.)

Exercise 7.3.28

$\frac{1 \text { table }}{5 \text { tables }}=\frac{2 \text { people }}{10 \text { people }}$

For the following 10 problems, solve each proportion.

Exercise 7.3.29

$\frac{x}{5}=\frac{6}{15}$

(Solution on p. 468.)

Exercise 7.3.30

$\frac{x}{10}=\frac{28}{40}$

Exercise 7.3.31

$\frac{5}{x}=\frac{10}{16}$

(Solution on p. 468.)

Exercise 7.3.32

$\frac{13}{x}=\frac{39}{60}$

Exercise 7.3.33

$\frac{1}{3}=\frac{x}{24}$

Exercise 7.3.34

$\frac{7}{12}=\frac{x}{60}$

Exercise 7.3.35

$\frac{8}{3}=\frac{72}{x}$

Exercise 7.3.36

$\frac{16}{1}=\frac{48}{x}$

Exercise 7.3.37

$\frac{x}{25}=\frac{200}{125}$

Exercise 7.3.38

$\frac{65}{30}=\frac{x}{60}$

For the following 5 problems, express each sentence as a proportion then solve the proportion.

Exercise 7.3.39

(Solution on p. 468.)

5 hats are to 4 coats as $x$ hats are to 24 coats.

Exercise 7.3.40

$x$ cushions are to 2 sofas as 24 cushions are to 16 sofas.

Exercise 7.3.41

(Solution on p. 468.)

1 spacecraft is to 7 astronauts as 5 spacecraft are to $x$ astronauts.

Exercise 7.3.42

56 microchips are to $\mathrm{x}$ circuit boards as 168 microchips are to 3 circuit boards.

Exercise 7.3.43

(Solution on p. 468.)

18 calculators are to 90 calculators as $x$ students are to 150 students.

Exercise 7.3.44

$x$ dollars are to $\$ 40,000$ as 2 sacks are to 1 sack. 
Indicate whether the proportion is true or false.

Exercise 7.3.45

$\frac{3}{16}=\frac{12}{64}$

(Solution on p. 468.)

Exercise 7.3.46

$\frac{2}{15}=\frac{10}{75}$

Exercise 7.3.47

$\frac{1}{9}=\frac{3}{30}$

Exercise 7.3.48

$\frac{6 \text { knives }}{7 \text { forks }}=\frac{12 \text { knives }}{15 \text { forks }}$

Exercise 7.3.49

$\frac{33 \text { miles }}{1 \text { gallon }}=\frac{99 \text { miles }}{3 \text { gallons }}$

Exercise 7.3.50

$\frac{320 \text { feet }}{5 \text { seconds }}=\frac{65 \text { feet }}{1 \text { second }}$

Exercise 7.3.51

$\frac{35 \text { students }}{70 \text { students }}=\frac{1 \text { class }}{2 \text { classes }}$

Exercise 7.3.52

$\frac{9 \mathrm{ml} \text { chloride }}{45 \mathrm{ml} \text { chloride }}=\frac{1 \text { test tube }}{7 \text { test tubes }}$

(Solution on p. 468.)

$\frac{9 \mathrm{ml} \text { chloride }}{45}=\frac{1}{7}$ test tubes

(Solution on p. 468.)

(Solution on p. 468.)

\subsubsection{Exercises for Review}

Exercise 7.3.53

(Solution on p. 468.)

(Section 1.7) Use the number 5 and 7 to illustrate the commutative property of addition.

Exercise 7.3.54

(Section 2.7) Use the numbers 5 and 7 to illustrate the commutative property of multiplication.

Exercise 7.3.55

(Section 5.3) Find the difference. $\frac{5}{14}-\frac{3}{22}$.

(Solution on p. 468.)

Exercise 7.3.56

(Section 6.6) Find the product. 8.06129 1 1,000.

Exercise 7.3.57

(Solution on p. 468.)

(Section 7.2) Write the simplified fractional form of the rate "sixteen sentences to two paragraphs."

\subsection{Applications of Proportions ${ }^{4}$}

\subsubsection{Section Overview}

- The Five-Step Method

- Problem Solving

\footnotetext{
${ }^{4}$ This content is available online at $<$ http://cnx.org/content $/ \mathrm{m} 34982 / 1.2 />$.
} 


\subsubsection{The Five-Step Method}

In Section 7.3 we noted that many practical problems can be solved by writing the given information as proportions. Such proportions will be composed of three specified numbers and one unknown number represented by a letter.

The first and most important part of solving a proportion problem is to determine, by careful reading, what the unknown quantity is and to represent it with some letter.

\section{The Five-Step Method}

The five-step method for solving proportion problems:

1. By careful reading, determine what the unknown quantity is and represent it with some letter. There will be only one unknown in a problem.

2. Identify the three specified numbers.

3. Determine which comparisons are to be made and set up the proportion.

4. Solve the proportion (using the methods of Section 7.3).

5. Interpret and write a conclusion in a sentence with the appropriate units of measure.

Step 1 is extremely important. Many problems go unsolved because time is not taken to establish what quantity is to be found.

When solving an applied problem, always begin by determining the unknown quantity and representing it with a letter.

\subsubsection{Problem Solving}

\subsubsection{Sample Set A}

\section{Example 7.17}

On a map, 2 inches represents 25 miles. How many miles are represented by 8 inches?

Step 1: The unknown quantity is miles.

Let $x=$ number of miles represented by 8 inches

Step 2: The three specified numbers are

2 inches

25 miles

8 inches

Step 3: The comparisons are

2 inches to 25 miles $\rightarrow \frac{2 \text { inches }}{25 \text { miles }}$

8 inches to $\mathrm{x}$ miles $\rightarrow \frac{8 \text { inches }}{\mathrm{x} \text { miles }}$

Proportions involving ratios and rates are more readily solved by suspending the units while doing the computations.

$\frac{2}{25}=\frac{8}{x}$

Step 4: $\frac{2}{25}=\frac{8}{x} \quad$ Perform the cross multiplication.

$2 \cdot x=8 \cdot 25$

$2 \cdot x=200 \quad$ Divide 200 by 2 .

$x=\frac{200}{2}$

$x=100$

In step 1 , we let $x$ represent the number of miles. So, $x$ represents 100 miles.

Step 5: If 2 inches represents 25 miles, then 8 inches represents 100 miles.

Try Exercise 7.4.1 in Section 7.4.3.2 (Practice Set A). 


\section{Example 7.18}

An acid solution is composed of 7 parts water to 2 parts acid. How many parts of water are there in a solution composed of 20 parts acid?

Step 1: The unknown quantity is the number of parts of water.

Let $n=$ number of parts of water.

Step 2: The three specified numbers are

7 parts water

2 parts acid

20 parts acid

Step 3: The comparisons are

7 parts water to 2 parts acid $\rightarrow \frac{7}{2}$

$n$ parts water to 20 parts acid $\rightarrow \frac{n}{20}$

$\frac{7}{2}=\frac{n}{20}$

Step 4: $\frac{7}{2}=\frac{n}{20} \quad$ Perform the cross multiplication.

$7 \cdot 20=2 \cdot n$

$140=2 \cdot n \quad$ Divide 140 by 2 .

$\frac{140}{2}=n$

$70=n$

In step 1 we let $n$ represent the number of parts of water. So, $n$ represents 70 parts of water.

Step 5: 7 parts water to 2 parts acid indicates 70 parts water to 20 parts acid.

Try Problem 7.4.2 in Section 7.4.3.2 (Practice Set A).

\section{Example 7.19}

A 5 -foot girl casts a $3 \frac{1}{3}$-foot shadow at a particular time of the day. How tall is a person who casts a 3 -foot shadow at the same time of the day?

Step 1: The unknown quantity is the height of the person.

Let $h=$ height of the person.

Step 2: The three specified numbers are

5 feet ( height of girl)

$3 \frac{1}{3}$ feet (length of shadow)

3 feet (length of shadow)

Step 3: The comparisons are

5 -foot girl is to $3 \frac{1}{3}$ foot shadow $\rightarrow \frac{5}{3 \frac{1}{3}}$

$h$-foot person is to 3 -foot shadow $\rightarrow \frac{h}{3}$

$\frac{5}{3 \frac{1}{3}}=\frac{h}{3}$

Step 4: $\frac{5}{3 \frac{1}{3}}=\frac{h}{3}$

$$
\begin{aligned}
5 \cdot 3 & =3 \frac{1}{3} \cdot h \\
15 & =\frac{10}{3} \cdot h \quad \text { Divide } 15 \text { by } \frac{10}{3} \\
\frac{15}{\frac{10}{3}} & =h \\
\frac{3}{\frac{15}{1} \cdot \frac{3}{\sqrt{10}}} & =h \\
\frac{9}{2} & =h \\
h & =4 \frac{1}{2}
\end{aligned}
$$

Step 5: A person who casts a 3 -foot shadow at this particular time of the day is $4 \frac{1}{2}$ feet tall.

Try Exercise 7.4.3 in Section 7.4.3.2 (Practice Set A). 


\section{Example 7.20}

The ratio of men to women in a particular town is 3 to 5 . How many women are there in the town if there are 19,200 men in town?

Step 1: The unknown quantity is the number of women in town.

Let $x=$ number of women in town.

Step 2: The three specified numbers are

3

5

19,200

Step 3: The comparisons are 3 men to 5 women $\rightarrow \frac{3}{5}$

19,200 men to $x$ women $\rightarrow \frac{19,200}{x}$

$\frac{3}{5}=\frac{19,200}{x}$

Step 4: $\frac{3}{5}=\frac{19,200}{x}$

$$
\begin{aligned}
3 \cdot x & =19,200 \cdot 5 \\
3 \cdot x & =96,000 \\
x & =\frac{96,000}{3} \\
x & =32,000
\end{aligned}
$$

Step 5: There are 32,000 women in town.

\section{Example 7.21}

The rate of wins to losses of a particular baseball team is $\frac{9}{2}$. How many games did this team lose if they won 63 games?

Step 1: The unknown quantity is the number of games lost.

Let $n=$ number of games lost.

Step 2: Since $\frac{9}{2} \rightarrow$ means 9 wins to 2 losses, the three specified numbers are

9 (wins)

2 (losses)

63 (wins)

Step 3: The comparisons are

9 wins to 2 losses $\rightarrow \frac{9}{2}$

63 wins to $n$ losses $\rightarrow \frac{63}{n}$

$\frac{9}{2}=\frac{63}{n}$

Step 4: $\frac{9}{2}=\frac{63}{n}$

$9 \cdot n=2 \cdot 63$

$9 \cdot n=126$

$n=\frac{126}{9}$

$n=14$

Step 5: This team had 14 losses.

Try Exercise 7.4.4 in Section 7.4.3.2 (Practice Set A).

\subsubsection{Practice Set A}

Solve each problem.

Exercise 7.4.1

(Solution on p. 468.)

On a map, 3 inches represents 100 miles. How many miles are represented by 15 inches? 


\section{Step 1: \\ Step 2: \\ Step 3: \\ Step 4: \\ Step 5:}

Exercise 7.4.2

(Solution on p. 468.)

An alcohol solution is composed of 14 parts water to 3 parts alcohol. How many parts of alcohol are in a solution that is composed of 112 parts water?

\section{Step 1:}

Step 2:

Step 3:

Step 4:

Step 5:

Exercise 7.4.3

(Solution on p. 468.)

A $5 \frac{1}{2}$-foot woman casts a 7 -foot shadow at a particular time of the day. How long of a shadow does a 3 -foot boy cast at that same time of day?

Step 1:

Step 2:

Step 3:

Step 4:

Step 5:

Exercise 7.4.4

(Solution on p. 468.)

The rate of houseplants to outside plants at a nursery is 4 to 9 . If there are 384 houseplants in the nursery, how many outside plants are there?

\section{Step 1: \\ Step 2: \\ Step 3: \\ Step 4: \\ Step 5:}

Exercise 7.4.5

(Solution on p. 468.)

The odds for a particular event occurring are 11 to 2. (For every 11 times the event does occur, it will not occur 2 times.) How many times does the event occur if it does not occur 18 times?

\section{Step 1:}

Step 2:

Step 3:

Step 4:

Step 5:

Exercise 7.4.6

(Solution on p. 468.)

The rate of passing grades to failing grades in a particular chemistry class is $\frac{7}{2}$. If there are 21 passing grades, how many failing grades are there?

\section{Step 1:}

Step 2:

Step 3:

Step 4:

Step 5: 


\subsubsection{Exercises}

For the following 20 problems, use the five-step method to solve each problem.

Exercise 7.4.7

On a map, 4 inches represents 50 miles. How many inches represent 300 miles?

(Solution on p. 468.)

Exercise 7.4.8

On a blueprint for a house, 2 inches represents 3 feet. How many inches represent 10 feet?

Exercise 7.4.9

(Solution on p. 468.)

A model is built to $\frac{2}{15}$ scale. If a particular part of the model measures 6 inches, how long is the actual structure?

Exercise 7.4.10

An acid solution is composed of 5 parts acid to 9 parts of water. How many parts of acid are there in a solution that contains 108 parts of water?

Exercise 7.4.11

(Solution on p. 468.)

An alloy contains 3 parts of nickel to 4 parts of silver. How much nickel is in an alloy that contains 44 parts of silver?

Exercise 7.4.12

The ratio of water to salt in a test tube is 5 to 2 . How much salt is in a test tube that contains 35 $\mathrm{ml}$ of water?

Exercise 7.4.13

(Solution on p. 469.)

The ratio of sulfur to air in a container is $\frac{4}{45}$. How many $\mathrm{ml}$ of air are there in a container that contains $207 \mathrm{ml}$ of sulfur?

Exercise 7.4.14

A 6 -foot man casts a 4 -foot shadow at a particular time of the day. How tall is a person that casts a 3 -foot shadow at that same time of the day?

Exercise 7.4.15

(Solution on p. 469.)

A $5 \frac{1}{2}$-foot woman casts a $1 \frac{1}{2}$-foot shadow at a particular time of the day. How long a shadow does her $3 \frac{1}{2}$-foot niece cast at the same time of the day?

Exercise 7.4.16

A man, who is 6 feet tall, casts a 7 -foot shadow at a particular time of the day. How tall is a tree that casts an 84-foot shadow at that same time of the day?

Exercise 7.4.17

(Solution on p. 469.)

The ratio of books to shelves in a bookstore is 350 to 3 . How many books are there in a store that has 105 shelves?

Exercise 7.4.18

The ratio of algebra classes to geometry classes at a particular community college is 13 to 2 . How many geometry classes does this college offer if it offers 13 algebra classes?

Exercise 7.4.19

(Solution on p. 469.)

The odds for a particular event to occur are 16 to 3 . If this event occurs 64 times, how many times would you predict it does not occur?

Exercise 7.4.20

The odds against a particular event occurring are 8 to 3 . If this event does occur 64 times, how many times would you predict it does not occur?

Exercise 7.4.21

(Solution on p. 469.)

The owner of a stationery store knows that a 1-inch stack of paper contains 300 sheets. The owner wishes to stack the paper in units of 550 sheets. How many inches tall should each stack be? 


\section{Exercise 7.4.22}

A recipe that requires 6 cups of sugar for 15 servings is to be used to make 45 servings. How much sugar will be needed?

\section{Exercise 7.4.23}

(Solution on p. 469.)

A pond loses $7 \frac{1}{2}$ gallons of water every 2 days due to evaporation. How many gallons of water are lost, due to evaporation, in $\frac{1}{2}$ day?

\section{Exercise 7.4.24}

A photograph that measures 3 inches wide and $4 \frac{1}{2}$ inches high is to be enlarged so that it is 5 inches wide. How high will it be?

Exercise 7.4.25

(Solution on p. 469.)

If 25 pounds of fertilizer covers 400 square feet of grass, how many pounds will it take to cover 500 square feet of grass?

Exercise 7.4.26

Every $1 \frac{1}{2}$ teaspoons of a particular multiple vitamin, in granular form, contains 0.65 the minimum daily requirement of vitamin C. How many teaspoons of this vitamin are required to supply 1.25 the minimum daily requirement?

\subsubsection{Exercises for Review}

Exercise 7.4.27

(Solution on p. 469.)

(Section 2.2) Find the product, $818 \cdot 0$.

Exercise 7.4.28

(Section 4.4) Determine the missing numerator: $\frac{8}{15}=\frac{N}{90}$.

Exercise 7.4.29

(Solution on p. 469.)

(Section 5.6) Find the value of $\frac{\frac{3}{10}+\frac{4}{12}}{\frac{19}{20}}$.

Exercise 7.4.30

(Section 6.5) Subtract 0.249 from the sum of 0.344 and 0.612 .

Exercise 7.4.31

(Section 7.3) Solve the proportion: $\frac{6}{x}=\frac{36}{30}$.

(Solution on p. 469.)

\subsection{Percent $^{5}$}

\subsubsection{Section Overview}

- Ratios and Percents

- The Relationship Between Fractions, Decimals, and Percents - Making Conversions

\footnotetext{
${ }^{5}$ This content is available online at $<$ http://cnx.org/content $/ \mathrm{m} 34983 / 1.2 />$.
} 


\subsubsection{Ratios and Percents}

\section{Ratio, Percent}

We defined a ratio as a comparison, by division, of two pure numbers or two like denominate numbers. A most convenient number to compare numbers to is 100 . Ratios in which one number is compared to 100 are called percents. The word percent comes from the Latin word "per centum." The word "per" means "for each" or "for every," and the word "centum" means "hundred." Thus, we have the following definition.

Percent means "for each hundred," or "for every hundred."

The symbol \% is used to represent the word percent.

\subsubsection{Sample Set A}

\section{Example 7.22}

The ratio 26 to 100 can be written as $26 \%$. We read $26 \%$ as "twenty-six percent."

\section{Example 7.23}

The ratio $\frac{165}{100}$ can be written as $165 \%$.

We read $165 \%$ as "one hundred sixty-five percent."

\section{Example 7.24}

The percent $38 \%$ can be written as the fraction $\frac{38}{100}$.

\section{Example 7.25}

The percent $210 \%$ can be written as the fraction $\frac{210}{100}$ or the mixed number $2 \frac{10}{100}$ or 2.1 .

Example 7.26

Since one dollar is 100 cents, 25 cents is $\frac{25}{100}$ of a dollar. This implies that 25 cents is $25 \%$ of one dollar.

\subsubsection{Practice Set A}

Exercise 7.5.1

Write the ratio 16 to 100 as a percent.

Exercise 7.5.2

Write the ratio 195 to 100 as a percent.

Exercise 7.5.3

Write the percent $83 \%$ as a ratio in fractional form.

Exercise 7.5.4

Write the percent $362 \%$ as a ratio in fractional form.
(Solution on p. 469.)

(Solution on p. 469.)

(Solution on p. 469.)

(Solution on p. 469.)

\subsubsection{The Relationship Between Fractions, Decimals, and Percents - Making Conversions}

Since a percent is a ratio, and a ratio can be written as a fraction, and a fraction can be written as a decimal, any of these forms can be converted to any other.

Before we proceed to the problems in Section 7.5.3.1 (Sample Set B) and Section 7.5.3.2 (Practice Set B), let's summarize the conversion techniques. 
Conversion Techniques - Fractions, Decimals, Percents 


\begin{tabular}{|l|l|l|}
\hline To Convert a Fraction & To Convert a Decimal & To Convert a Percent \\
\hline $\begin{array}{l}\text { To a decimal: Divide the numer- } \\
\text { ator by the denominator }\end{array}$ & $\begin{array}{l}\text { To a fraction: Read the decimal } \\
\text { and reduce the resulting fraction }\end{array}$ & $\begin{array}{l}\text { To a decimal: Move the decimal } \\
\text { point 2 places to the left and drop } \\
\text { the \% symbol }\end{array}$ \\
\hline $\begin{array}{l}\text { To a percent: Convert the frac- } \\
\text { tion first to a decimal, then move } \\
\text { the decimal point } 2 \text { places to the } \\
\text { right and affix the } \% \text { symbol. }\end{array}$ & $\begin{array}{l}\text { To a percent: Move the decimal } \\
\text { point 2 places to the right and af- } \\
\text { fix the } \% \text { symbol }\end{array}$ & $\begin{array}{l}\text { To a fraction: Drop the \% sign } \\
\text { and write the number "over" 100. } \\
\text { Reduce, if possible. }\end{array}$ \\
\hline
\end{tabular}

Table 7.1

\subsubsection{Sample Set B}

\section{Example 7.27}

Convert $12 \%$ to a decimal.

$12 \%=\frac{12}{100}=0.12$

Note that

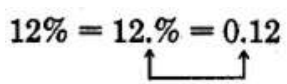

The $\%$ symbol is dropped, and the decimal point moves 2 places to the left.

\section{Example 7.28}

Convert 0.75 to a percent.

$0.75=\frac{75}{100}=75 \%$

Note that $\stackrel{0.75=75 \%=75 . \%}{\imath}$

The $\%$ symbol is affixed, and the decimal point moves 2 units to the right.

\section{Example 7.29}

Convert $\frac{3}{5}$ to a percent.

We see in Example 7.28 that we can convert a decimal to a percent. We also know that we can convert a fraction to a decimal. Thus, we can see that if we first convert the fraction to a decimal, we can then convert the decimal to a percent.

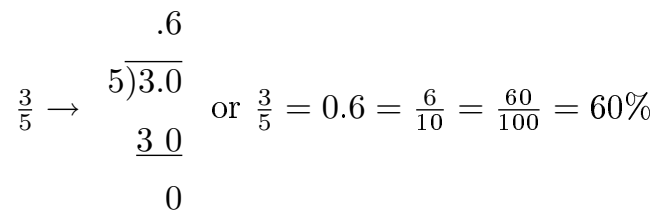

\section{Example 7.30}

Convert $42 \%$ to a fraction.

$42 \%=\frac{42}{100}=\frac{21}{50}$

or 
$42 \%=0.42=\frac{42}{100}=\frac{21}{50}$

\subsubsection{Practice Set $B$}

Exercise 7.5.5

(Solution on p. 469.)

Convert $21 \%$ to a decimal.

(Solution on p. 469.)

Convert $461 \%$ to a decimal.

(Solution on p. 469.)

Convert 0.55 to a percent.

Exercise 7.5.8

(Solution on p. 469.)

Convert 5.64 to a percent.

(Solution on p. 469.)

Exercise 7.5.9

Convert $\frac{3}{20}$ to a percent.

Exercise 7.5.10

Convert $\frac{11}{8}$ to a percent

(Solution on p. 469.)

Exercise 7.5.11

Convert $\frac{3}{11}$ to a percent.

(Solution on p. 469.)

\subsubsection{Exercises}

For the following 12 problems, convert each decimal to a percent.

Exercise 7.5.12

(Solution on p. 469.)

0.25

Exercise 7.5.13

0.36

Exercise 7.5.14

0.48

(Solution on p. 469.)

Exercise 7.5.15

0.343

Exercise 7.5.16

0.771

(Solution on p. 469.)

Exercise 7.5.17

1.42

Exercise 7.5.18

2.58

(Solution on p. 469.)

Exercise 7.5.19

4.976

Exercise 7.5.20

16.1814

(Solution on p. 470.)

Exercise 7.5.21

533.01

Exercise 7.5.22

2

(Solution on p. 470.) 
Exercise 7.5.23

14

For the following 10 problems, convert each percent to a decimal.

Exercise 7.5.24

(Solution on p. 470.)

$15 \%$

Exercise 7.5.25

$43 \%$

Exercise 7.5.26

(Solution on p. 470.)

$16.2 \%$

Exercise 7.5.27

$53.8 \%$

Exercise 7.5.28

$5.05 \%$

(Solution on p. 470.)

Exercise 7.5.29

$6.11 \%$

Exercise 7.5.30

(Solution on p. 470.)

$0.78 \%$

Exercise 7.5.31

$0.88 \%$

Exercise 7.5.32

$0.09 \%$

(Solution on p. 470.)

Exercise 7.5.33

$0.001 \%$

For the following 14 problems, convert each fraction to a percent.

Exercise 7.5.34

(Solution on p. 470.)

$\frac{1}{5}$

Exercise 7.5.35

$\frac{3}{5}$

Exercise 7.5.36

$\frac{5}{8}$

(Solution on p. 470.)

Exercise 7.5.37 $\frac{1}{16}$

Exercise 7.5.38

$\frac{7}{25}$

Exercise 7.5.39

$\frac{16}{45}$

Exercise 7.5.40 $\frac{27}{55}$

Exercise 7.5.41 $\frac{15}{8}$

Exercise 7.5.42

$\frac{41}{25}$

Exercise 7.5.43

$6 \frac{4}{5}$

Exercise 7.5.44

$9 \frac{9}{20}$

(Solution on p. 470.)

(Solution on p. 470.)

(Solution on p. 470.)

Available for free at Connexions $<$ http://cnx.org/content/col10615/1.4 $>$ 
Exercise 7.5.45

$\frac{1}{200}$

Exercise 7.5.46

$\frac{6}{11}$

(Solution on p. 470.)

Exercise 7.5.47

$\frac{35}{27}$

For the following 14 problems, convert each percent to a fraction.

Exercise 7.5.48

(Solution on p. 470.)

$80 \%$

Exercise 7.5.49

$60 \%$

Exercise 7.5.50

$25 \%$

(Solution on p. 470.)

Exercise 7.5.51

$75 \%$

Exercise 7.5.52

$65 \%$

(Solution on p. 470.)

Exercise 7.5.53

$18 \%$

Exercise 7.5.54

$12.5 \%$

(Solution on p. 470.)

Exercise 7.5.55

$37.5 \%$

Exercise 7.5.56

(Solution on p. 470.)

$512.5 \%$

Exercise 7.5.57

$937.5 \%$

Exercise 7.5.58

(Solution on p. 470.)

9. $\overline{9} \%$

Exercise 7.5.59

$55 . \overline{5} \%$

Exercise 7.5.60

22. $\overline{2} \%$

(Solution on p. 470.)

Exercise 7.5.61

63. $\overline{6} \%$

\subsubsection{Exercises for Review}

Exercise 7.5.62

(Section 4.6) Find the quotient. $\frac{40}{54} \div 8 \frac{7}{21}$.

(Solution on p. 470.)

Exercise 7.5.63

(Section 4.7 ) $\frac{3}{8}$ of what number is $2 \frac{2}{3}$ ?

Exercise 7.5.64

(Section 5.3) Find the value of $\frac{28}{15}+\frac{7}{10}-\frac{5}{12}$.

(Solution on p. 470.) 
Exercise 7.5.65

(Section 6.4) Round 6.99997 to the nearest ten thousandths.

Exercise 7.5.66

(Solution on p. 470.)

(Section 7.4) On a map, 3 inches represent 40 miles. How many inches represent 480 miles?

\subsection{Fractions of One Percent ${ }^{6}$}

\subsubsection{Section Overview}

- Conversions Involving Fractions of One Percent

- Conversions Involving Nonterminating Fractions

\subsubsection{Conversions Involving Fractions of One Percent}

Percents such as $\frac{1}{2} \%, \frac{3}{5} \%, \frac{5}{8} \%$, and $\frac{7}{11} \%$, where $1 \%$ has not been attained, are fractions of $1 \%$. This implies that

$\frac{1}{2} \%=\frac{1}{2}$ of $1 \%$

$\frac{3}{5} \%=\frac{3}{5}$ of $1 \%$

$\frac{5}{8} \%=\frac{5}{8}$ of $1 \%$

$\frac{7}{11} \%=\frac{7}{11}$ of $1 \%$

Since "percent" means "for each hundred," and "of" means "times," we have

$\frac{1}{2} \%=\frac{1}{2}$ of $1 \%=\frac{1}{2} \cdot \frac{1}{100}=\frac{1}{200}$

$\frac{3}{5} \%=\frac{3}{5}$ of $1 \%=\frac{3}{5} \cdot \frac{1}{100}=\frac{3}{500}$

$\frac{5}{8} \%=\frac{5}{8}$ of $1 \%=\frac{5}{8} \cdot \frac{1}{100}=\frac{5}{800}$

$\frac{7}{11} \%=\frac{7}{11}$ of $1 \%=\frac{7}{11} \cdot \frac{1}{100}=\frac{7}{1100}$

\subsubsection{Sample Set A}

Example 7.31

Convert $\frac{2}{3} \%$ to a fraction.

$$
\begin{aligned}
\frac{2}{3} \%=\frac{2}{3} \text { of } 1 \% & =\frac{\frac{1}{2}}{3} \cdot \frac{1}{\overline{500}} \\
& =\frac{1 \cdot 1}{3 \cdot 50} \\
& =\frac{1}{150}
\end{aligned}
$$

\section{Example 7.32}

Convert $\frac{5}{8} \%$ to a decimal.

\footnotetext{
${ }^{6}$ This content is available online at $<$ http://cnx.org/content $/ \mathrm{m} 34997 / 1.2 />$.
} 


$$
\begin{aligned}
\frac{5}{8} \%=\frac{5}{8} \text { of } 1 \% & \frac{5}{8} \cdot \frac{1}{100} \\
& =0.625 \cdot 0.01 \\
& =0.00625
\end{aligned}
$$

\subsubsection{Practice Set A}

Exercise 7.6.1

(Solution on p. 470.)

Convert $\frac{1}{4} \%$ to a fraction.

Exercise 7.6.2

(Solution on p. 471.)

Convert $\frac{3}{8} \%$ to a fraction.

Exercise 7.6.3

(Solution on p. 471.)

Convert $3 \frac{1}{3} \%$ to a fraction.

\subsubsection{Conversions Involving Nonterminating Fractions}

We must be careful when changing a fraction of $1 \%$ to a decimal. The number $\frac{2}{3}$, as we know, has a nonterminating decimal representation. Therefore, it cannot be expressed exactly as a decimal.

When converting nonterminating fractions of $1 \%$ to decimals, it is customary to express the fraction as a rounded decimal with at least three decimal places.

\section{Converting a Nonterminating Fraction to a Decimal}

To convert a nonterminating fraction of $1 \%$ to a decimal:

1. Convert the fraction as a rounded decimal.

2. Move the decimal point two digits to the left and remove the percent sign.

\subsubsection{Sample Set B}

\section{Example 7.33}

Convert $\frac{2}{3} \%$ to a three-place decimal.

1. Convert $\frac{2}{3}$ to a decimal.

Since we wish the resulting decimal to have three decimal digits, and removing the percent sign will account for two of them, we need to round $\frac{2}{3}$ to one place $(2+1=3)$.

$\frac{2}{3} \%=0.7 \%$ to one decimal place. $\left(\frac{2}{3}=0.6666 \ldots\right)$

2. Move the decimal point two digits to the left and remove the $\%$ sign. We'll need to add zeros to locate the decimal point in the correct location.

$\frac{2}{3} \%=0.007$ to 3 decimal places

\section{Example $\mathbf{7 . 3 4}$}

Convert $5 \frac{4}{11} \%$ to a four-place decimal.

1. Since we wish the resulting decimal to have four decimal places, and removing the percent sign will account for two, we to round $\frac{4}{11}$ to two places. $5 \frac{4}{11} \%=5.36 \%$ to two decimal places. $\left(\frac{4}{11}=0.3636 \ldots\right)$

2. Move the decimal point two places to the left and drop the percent sign. $5 \frac{4}{11} \%=0.0536$ to four decimal places. 


\section{Example 7.35}

Convert $28 \frac{5}{9} \%$ to a decimal rounded to ten thousandths.

1. Since we wish the resulting decimal to be rounded to ten thousandths (four decimal places), and removing the percent sign will account for two, we need to round $\frac{5}{9}$ to two places. $28 \frac{5}{9} \%=28.56 \%$ to two decimal places. $\left(\frac{5}{9}=0.5555 \ldots\right)$

2. Move the decimal point to the left two places and drop the percent sign. $28 \frac{5}{9} \%=0.2856$ correct to ten thousandths.

\subsubsection{Practice Set B}

Exercise 7.6.4

(Solution on p. 471.)

Convert $\frac{7}{9} \%$ to a three-place decimal.

Exercise 7.6.5

Convert $51 \frac{5}{11} \%$ to a decimal rounded to ten thousandths.

(Solution on p. 471.)

\subsubsection{Exercises}

Make the conversions as indicated.

Exercise 7.6.6

(Solution on p. 471.)

Convert $\frac{3}{4} \%$ to a fraction.

Exercise 7.6.7

Convert $\frac{5}{6} \%$ to a fraction.

Exercise 7.6.8

Convert $\frac{1}{9} \%$ to a fraction.

(Solution on p. 471.)

Exercise 7.6.9

Convert $\frac{15}{19} \%$ to a fraction.

Exercise 7.6.10

(Solution on p. 471.)

Convert $\frac{5}{4} \%$ to a fraction.

Exercise 7.6.11

Convert $\frac{7}{3} \%$ to a fraction.

Exercise 7.6.12

Convert $1 \frac{6}{7} \%$ to a fraction.

(Solution on p. 471.)

Exercise 7.6.13

Convert $2 \frac{5}{16} \%$ to a fraction.

Exercise 7.6.14

Convert $25 \frac{1}{4} \%$ to a fraction.

(Solution on p. 471.)

Exercise 7.6.15

Convert $50 \frac{1}{2} \%$ to a fraction.

Exercise 7.6.16

Convert $72 \frac{3}{5} \%$ to a fraction.

(Solution on p. 471.)

Exercise 7.6.17

Convert $99 \frac{1}{8} \%$ to a fraction.

Exercise 7.6.18

Convert $136 \frac{2}{3} \%$ to a fraction.

(Solution on p. 471.) 
Exercise 7.6.19

Convert $521 \frac{3}{4} \%$ to a fraction.

Exercise 7.6.20

(Solution on p. 471.)

Convert $10 \frac{1}{5} \%$ to a decimal.

Exercise 7.6.21

Convert $12 \frac{3}{4} \%$ to a decimal.

Exercise 7.6.22

(Solution on p. 471.)

Convert $3 \frac{7}{8} \%$ to a decimal.

Exercise 7.6.23

Convert $7 \frac{1}{16} \%$ to a decimal.

Exercise 7.6.24

(Solution on p. 471.)

Convert $\frac{3}{7} \%$ to a three-place decimal.

Exercise 7.6.25

Convert $\frac{1}{9} \%$ to a three-place decimal.

Exercise 7.6.26

(Solution on p. 471.)

Convert $6 \frac{3}{11} \%$ to a four-place decimal.

Exercise 7.6.27

Convert $9 \frac{2}{7} \%$ to a four-place decimal.

Exercise 7.6.28

(Solution on p. 471.)

Convert $24 \frac{5}{21} \%$ to a three-place decimal.

Exercise 7.6.29

Convert $45 \frac{8}{27} \%$ to a three-place decimal.

Exercise 7.6.30

Convert $11 \frac{16}{17} \%$ to a four-place decimal.

(Solution on p. 471.)

Exercise 7.6.31

Convert $5 \frac{1}{7} \%$ to a three-place decimal.

\subsubsection{Exercises for Review}

Exercise 7.6.32

(Solution on p. 471.)

(Section 3.2) Write $8 \cdot 8 \cdot 8 \cdot 8 \cdot 8$ using exponents.

Exercise 7.6.33

(Section 4.3) Convert $4 \frac{7}{8}$ to an improper fraction.

Exercise 7.6.34

(Solution on p. 471.)

(Section 5.4) Find the sum. $\frac{7}{10}+\frac{2}{21}+\frac{1}{7}$.

Exercise 7.6.35

(Section 6.6) Find the product. (4.21) (0.006).

Exercise 7.6.36

(Solution on p. 471.)

(Section 7.5) Convert 8.062 to a percent. 


\subsection{Applications of Percents ${ }^{7}$}

\subsubsection{Section Overview}

- Base, Percent, and Percentage

- Finding the Percentage

- Finding the Percent

- Finding the Base

\subsubsection{Base, Percent, and Percentage}

There are three basic types of percent problems. Each type involves a base, a percent, and a percentage, and when they are translated from words to mathematical symbols each becomes a multiplication statement. Examples of these types of problems are the following:

1. What number is $30 \%$ of 50 ? (Missing product statement.)

2. 15 is what percent of 50 ? (Missing factor statement.)

3. 15 is $30 \%$ of what number? (Missing factor statement.)

In problem 1 (list, p. 448), the product is missing. To solve the problem, we represent the missing product with $P$.

$P=30 \% \cdot 50$

\section{Percentage}

The missing product $P$ is called the percentage. Percentage means part, or portion. In $P=30 \% \cdot 50, P$ represents a particular part of 50 .

In problem 2 (list, p. 448), one of the factors is missing. Here we represent the missing factor with $Q$.

$15=Q \cdot 50$

\section{Percent}

The missing factor is the percent. Percent, we know, means per 100 , or part of 100 . In $15=Q \cdot 50, Q$ indicates what part of 50 is being taken or considered. Specifically, $15=Q \cdot 50$ means that if 50 was to be divided into 100 equal parts, then $Q$ indicates 15 are being considered.

In problem 3 (list, p. 448), one of the factors is missing. Represent the missing factor with $B$.

$15=30 \% \cdot B$

Base

The missing factor is the base. Some meanings of base are a source of supply, or a starting place. In $15=30 \% \cdot B, B$ indicates the amount of supply. Specifically, $15=30 \% \cdot B$ indicates that 15 represents $30 \%$ of the total supply.

Each of these three types of problems is of the form

$($ percentage $)=($ percent $) \cdot($ base $)$

We can determine any one of the three values given the other two using the methods discussed in Section 4.7.

\footnotetext{
${ }^{7}$ This content is available online at $<$ http://cnx.org/content/m35007/1.2/>.
} 


\subsubsection{Finding the Percentage}

\subsubsection{Sample Set A}

\section{Example 7.36}

\begin{tabular}{|c|c|c|c|c|c|}
\hline$\underbrace{\text { What number }}$ & is & $30 \%$ & of & $50 ?$ & Missing product statement. \\
\hline (percentage) & $=$ & (percent) & & (base) & \\
\hline$\downarrow$ & $\downarrow$ & $\downarrow$ & $\downarrow$ & $\downarrow$ & \\
\hline$P$ & $=$ & $30 \%$ & . & 50 & Convert $30 \%$ to a decimal. \\
\hline$P$ & $=$ & .30 & & 50 & Multiply. \\
\hline$P$ & $=$ & 15 & & & \\
\hline
\end{tabular}

Thus, 15 is $30 \%$ of 50 .

Do Section 7.7.3.2 (Practice Set A), Exercise 7.7.1.

\section{Example 7.37}

\begin{tabular}{|c|c|c|c|c|c|}
\hline$\underbrace{\text { What number }}$ & is & $\underset{\downarrow}{36 \%}$ & of & $\underset{\downarrow}{95 ?}$ & Missing product statement. \\
\hline (percentage) & $\overline{\bar{\downarrow}}$ & $\begin{array}{c}\text { (percent) } \\
\downarrow\end{array}$ & $\downarrow$ & $\begin{array}{c}\text { (base) } \\
\downarrow\end{array}$ & \\
\hline$P$ & $=$ & $36 \%$ & . & 95 & Convert $36 \%$ to a decimal. \\
\hline$P$ & $=$ & .36 & . & 95 & Multiply \\
\hline$P$ & 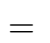 & 34.2 & & & \\
\hline
\end{tabular}

Thus, 34.2 is $36 \%$ of 95 .

Do Section 7.7.3.2 (Practice Set A), Exercise 7.7.1.

\section{Example 7.38}

A salesperson, who gets a commission of $12 \%$ of each sale she makes, makes a sale of $\$ 8,400.00$. How much is her commission?

We need to determine what part of $\$ 8,400.00$ is to be taken. What part indicates percentage.

$$
\begin{aligned}
& \underbrace{\text { What number }} \text { is } \quad \begin{array}{l}
\downarrow \\
\downarrow
\end{array} \quad \text { of } \quad 8,400.00 \text { ? Missing product statement. } \\
& \text { (percentage) } \bar{\downarrow} \text { (percent) } i \quad \text { (base) } \\
& P \quad=12 \% \quad 8,400.00 \quad \text { Convert to decimals. }
\end{aligned}
$$

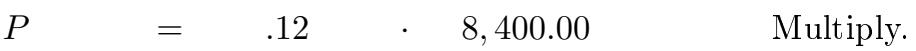

$$
\begin{aligned}
& P \quad=1008.00
\end{aligned}
$$

Thus, the salesperson's commission is $\$ 1,008.00$.

Do Section 7.7.3.2 (Practice Set A), Exercise 7.7.2.

\section{Example 7.39}

A girl, by practicing typing on her home computer, has been able to increase her typing speed by $110 \%$. If she originally typed 16 words per minute, by how many words per minute was she able to increase her speed?

We need to determine what part of 16 has been taken. What part indicates percentage. 


\begin{tabular}{|c|c|c|c|c|c|}
\hline$\underbrace{\text { What number }}$ & is & $110 \%$ & of & $16 ?$ & Missing product statement \\
\hline (percentage) & $=$ & (percent) & & (base) & \\
\hline$\downarrow$ & $\downarrow$ & $\downarrow$ & $\downarrow$ & $\downarrow$ & \\
\hline$P$ & $=$ & $110 \%$ & . & 16 & Convert to decimals. \\
\hline$P$ & $=$ & 1.10 & . & 16 & Multiply. \\
\hline$P$ & $=$ & 17.6 & & & \\
\hline
\end{tabular}

Thus, the girl has increased her typing speed by 17.6 words per minute. Her new speed is $16+17.6=$ 33.6 words per minute.

Do Section 7.7.3.2 (Practice Set A), Exercise 7.7.3.

\section{Example 7.40}

A student who makes $\$ 125$ a month working part-time receives a $4 \%$ salary raise. What is the student's new monthly salary?

With a $4 \%$ raise, this student will make $100 \%$ of the original salary $+4 \%$ of the original salary. This means the new salary will be $104 \%$ of the original salary. We need to determine what part of $\$ 125$ is to be taken. What part indicates percentage.

\begin{tabular}{|c|c|c|c|c|c|}
\hline$\underbrace{}_{\text {What number }}$ & is & $104 \%$ & of & 125 & Missing product statement. \\
\hline (percentage) & $\overline{\bar{\downarrow}}$ & $\underset{\downarrow}{\text { (percent) }}$ & $i$ & (base) & \\
\hline$P$ & $=$ & $104 \%$ & . & 125 & Convert to decimals. \\
\hline$P$ & - & 1.04 & . & 125 & Multiply. \\
\hline$P$ & & 130 & & & \\
\hline
\end{tabular}

Thus, this student's new monthly salary is $\$ 130$.

Do Section 7.7.3.2 (Practice Set A), Exercise 7.7.4.

\section{Example 7.41}

An article of clothing is on sale at $15 \%$ off the marked price. If the marked price is $\$ 24.95$, what is the sale price?

Since the item is discounted $15 \%$, the new price will be $100 \%-15 \%=85 \%$ of the marked price. We need to determine what part of 24.95 is to be taken. What part indicates percentage.

\begin{tabular}{|c|c|c|c|c|c|}
\hline$\underbrace{\text { What number }}$ & is & $85 \%$ & of & $\$ 24.95$. & Missing product statement. \\
\hline (percentage) & $=$ & (percent) & i & (base) & \\
\hline$\downarrow$ & $\downarrow$ & $\downarrow$ & $\downarrow$ & $\downarrow$ & \\
\hline$P$ & $=$ & $85 \%$ & . & 24.95 & Convert to decimals. \\
\hline$P$ & $=$ & .85 & . & 24.95 & Multiply. \\
\hline$P$ & $=$ & 21.2075 & & & $\begin{array}{l}\text { Since this number represents money, } \\
\text { we'll round to } 2 \text { decimal places }\end{array}$ \\
\hline$P$ & $=$ & 21.21 & & & \\
\hline
\end{tabular}

Thus, the sale price of the item is $\$ 21.21$. 


\subsubsection{Practice Set A}

Exercise 7.7.1

(Solution on p. 471.)

What number is $42 \%$ of 85 ?

(Solution on p. 471.)

Exercise 7.7.2 A sales person makes a commission of $16 \%$ on each sale he makes. How much is his commission if he makes a sale of $\$ 8,500$ ?

Exercise 7.7.3

(Solution on p. 471.)

An assembly line worker can assemble 14 parts of a product in one hour. If he can increase his assembly speed by $35 \%$, by how many parts per hour would he increase his assembly of products?

Exercise 7.7.4

(Solution on p. 471.)

A computer scientist in the Silicon Valley makes $\$ 42,000$ annually. What would this scientist's new annual salary be if she were to receive an $8 \%$ raise?

\subsubsection{Finding the Percent}

\subsubsection{Sample Set B}

Example 7.42

\begin{tabular}{|c|c|c|c|c|c|}
\hline 15 & is & what percent & of & $50 ?$ & Missing factor statement. \\
\hline percentage) & $=$ & (percent) & & (base) & {$[($ product $)=($ factor $) \cdot($ factor $)]$} \\
\hline$\downarrow$ & $\downarrow$ & $\downarrow$ & $\downarrow$ & $\downarrow$ & \\
\hline 15 & $=$ & $Q$ & & 50 & \\
\hline
\end{tabular}

Recall that $($ missing factor $)=$ (product $) \div($ known factor $)$.

$$
\begin{array}{lccc}
Q= & 15 \div 50 & \text { Divide. } \\
Q & =0.3 & \text { Convert to a percent. } \\
Q & =30 \% &
\end{array}
$$

Thus, 15 is $30 \%$ of 50 .

Do Section 7.7.4.2 (Practice Set B), Exercise 7.7.5.

\section{Example 7.43}

$$
\begin{aligned}
& 4.32 \text { is } \underbrace{\text { what percent }} \text { of }{ }_{\downarrow}^{7} \underset{\downarrow}{7} \text { ? Missing factor statement. }
\end{aligned}
$$

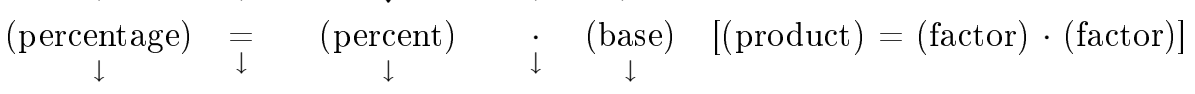

$$
\begin{aligned}
& 4.32=Q \quad \cdot \quad 72 \\
& Q=4.32 \div 72 \quad \text { Divide. } \\
& Q=0.06 \quad \text { Convert to a percent. } \\
& Q=6 \%
\end{aligned}
$$

Thus, 4.32 is $6 \%$ of 72 . 
Do Section 7.7.4.2 (Practice Set B), Exercise 7.7.5.

\section{Example 7.44}

On a 160 question exam, a student got 125 correct answers. What percent is this? Round the result to two decimal places.

We need to determine the percent.

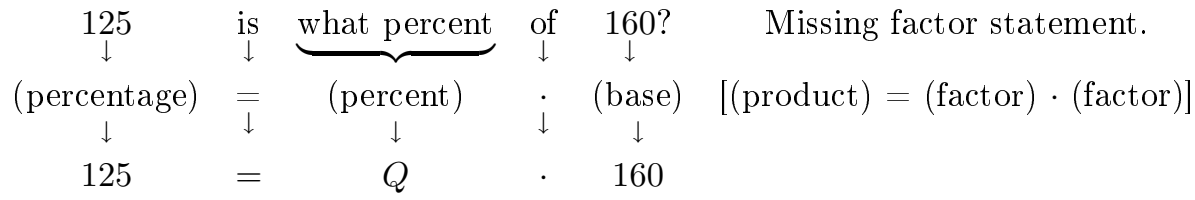

$$
\begin{aligned}
& Q=125 \div 160 \quad \text { Divide } \\
& Q=0.78125 \text { Round to two decimal places. } \\
& Q=.78
\end{aligned}
$$

Thus, this student received a $78 \%$ on the exam.

Do Section 7.7.4.2 (Practice Set B), Exercise 7.7.6.

\section{Example 7.45}

A bottle contains 80 milliliters of hydrochloric acid $(\mathrm{HCl})$ and 30 milliliters of water. What percent of $\mathrm{HCl}$ does the bottle contain? Round the result to two decimal places.

We need to determine the percent. The total amount of liquid in the bottle is

80 milliliters +30 milliliters $=110$ milliliters.

$$
\begin{aligned}
& \left.\begin{array}{cccccc}
\begin{array}{c}
80 \\
\downarrow \\
\text { (percentage) } \\
\downarrow
\end{array} & \begin{array}{c}
\text { is } \\
\downarrow
\end{array} & \begin{array}{c}
\text { what percent } \\
\text { (percent) } \\
\downarrow
\end{array} & \begin{array}{c}
\text { of } \\
\downarrow
\end{array} & \begin{array}{c}
110 \text { (base) } \\
\downarrow
\end{array} & \text { Missing factor statement. }
\end{array}\right] \\
& 80 \quad=\quad Q \quad \cdot 110 \\
& Q=80 \div 110 \quad \text { Divide } \\
& Q=0.727272 \ldots \quad \text { Round to two decimal places. } \\
& Q \approx 73 \% \quad \text { The symbol " } \approx \text { " is read as "approximately." }
\end{aligned}
$$

Thus, this bottle contains approximately $73 \% \mathrm{HCl}$.

Do Section 7.7.4.2 (Practice Set B), Exercise 7.7.7.

\section{Example 7.46}

Five years ago a woman had an annual income of $\$ 19,200$. She presently earns $\$ 42,000$ annually. By what percent has her salary increased? Round the result to two decimal places.

We need to determine the percent. 


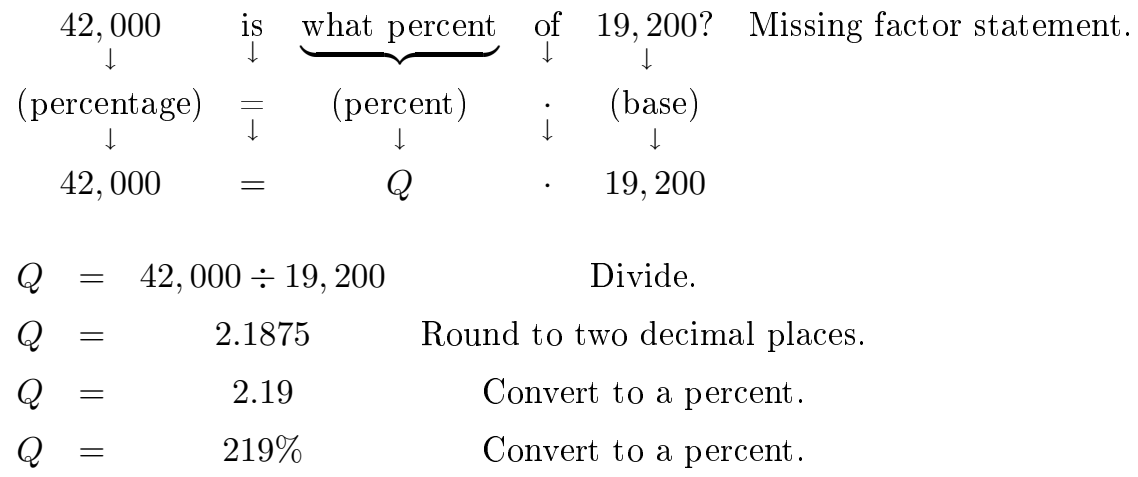

Thus, this woman's annual salary has increased $219 \%$.

\subsubsection{Practice Set B}

\section{Exercise 7.7.5}

(Solution on p. 471.)

99.13 is what percent of 431 ?

Exercise 7.7.6

(Solution on p. 472.)

On an 80 question exam, a student got 72 correct answers. What percent did the student get on the exam?

Exercise 7.7.7

(Solution on p. 472.)

A bottle contains 45 milliliters of sugar and 67 milliliters of water. What fraction of sugar does the bottle contain? Round the result to two decimal places (then express as a percent).

\subsubsection{Finding the Base}

\subsubsection{Sample Set C}

\section{Example 7.47}

\begin{tabular}{|c|c|c|c|c|c|}
\hline $\begin{array}{l}15 \\
\downarrow\end{array}$ & is & $30 \%$ & of & $\underbrace{\text { what number? }}$ & Missing factor statement. \\
\hline (percentage) & $=$ & (percent) & & (base) & {$[($ percentage $)=($ factor $) \cdot($ factor $)]$} \\
\hline$\downarrow$ & $\downarrow$ & $\downarrow$ & $\downarrow$ & $\downarrow$ & \\
\hline 15 & $=$ & $30 \%$ & . & $B$ & Convert to decimals. \\
\hline 15 & $=$ & .30 & . & $B$ & {$[($ missing factor $)=($ product $) \div($ known factor $)]$} \\
\hline \multicolumn{6}{|c|}{$B=15 \div .30$} \\
\hline \multicolumn{6}{|l|}{$B=50$} \\
\hline
\end{tabular}

Thus, 15 is $30 \%$ of 50 .

Try Exercise 7.7.8 in Section 7.7.5.2 (Practice Set C). 


\section{Example 7.48}

$\begin{array}{ccccccc}56.43 & \text { is } & 33 \% & \text { of } & \text { what number? } & \text { Missing factor statement. } \\ \downarrow & \downarrow & \downarrow & \downarrow & \underbrace{}_{\text {(base) }} & \\ \text { (percentage) } & = & \text { (percent) } & \downarrow & \downarrow & \downarrow & \\ \downarrow & \downarrow & \downarrow & \downarrow & \downarrow & B & \text { Convert to decimals. } \\ 56.43 & = & 33 \% & \cdot & B & \text { Divide. } \\ 56.43 & = & .33 & \cdot & & & \\ B= & 56.43 \div .33 & & & & \\ B= & 171\end{array}$

Thus, 56.43 is $33 \%$ of 171 .

Try Exercise 7.7.8 in Section 7.7.5.2 (Practice Set C).

Example 7.49

Fifteen milliliters of water represents $2 \%$ of a hydrochloric acid $(\mathrm{HCl})$ solution. How many milliliters of solution are there?

We need to determine the total supply. The word supply indicates base.

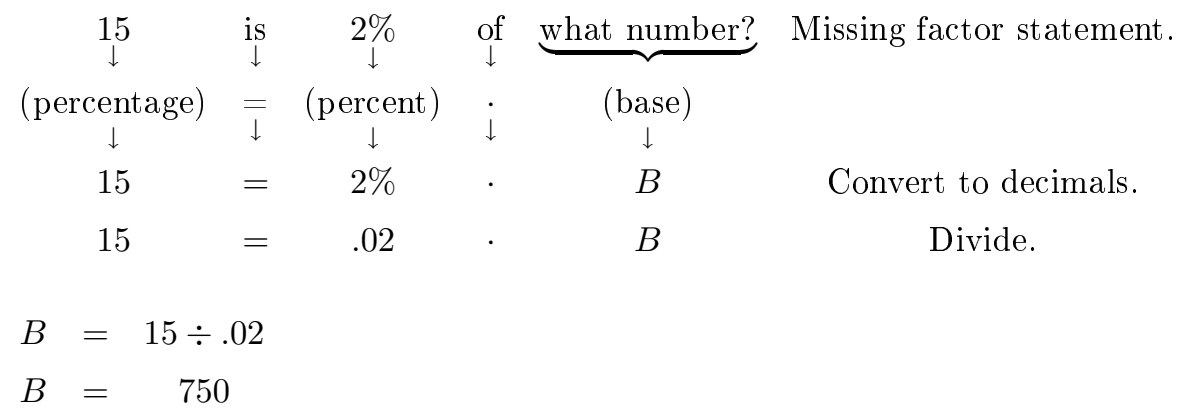

Thus, there are 750 milliliters of solution in the bottle.

Try Exercise 7.7.9 in Section 7.7.5.2 (Practice Set C).

\section{Example $\mathbf{7 . 5 0}$}

In a particular city, a sales tax of $6 \frac{1}{2} \%$ is charged on items purchased in local stores. If the tax on an item is $\$ 2.99$, what is the price of the item?

We need to determine the price of the item. We can think of price as the starting place. Starting place indicates base. We need to determine the base.

\begin{tabular}{|c|c|c|c|c|c|}
\hline 2.99 & is & $\begin{array}{c}6 \frac{1}{2} \% \\
\downarrow\end{array}$ & of & $\underbrace{\text { what number? }}$ & Missing factor statement. \\
\hline $\begin{array}{c}\text { (percentage) } \\
\downarrow\end{array}$ & $\overline{\bar{\downarrow}}$ & $\begin{array}{c}\text { (percent) } \\
\downarrow\end{array}$ & $i$ & $\begin{array}{c}\text { (base) } \\
\downarrow\end{array}$ & \\
\hline 2.99 & $=$ & $6 \frac{1}{2} \%$ & . & $B$ & Convert to decimals. \\
\hline 2.99 & $=$ & $6.5 \%$ & . & $B$ & \\
\hline 2.99 & $=$ & .065 & . & $B$ & {$[($ missing factor $)=($ product $) \div($ known factor $)]$} \\
\hline
\end{tabular}




$$
\begin{array}{llll}
B & = & 2.99 \div .065 & \text { Divide } \\
B & = & 46
\end{array}
$$

Thus, the price of the item is $\$ 46.00$.

Try Exercise 7.7.10 in Section 7.7.5.2 (Practice Set C).

\section{Example 7.51}

A clothing item is priced at $\$ 20.40$. This marked price includes a $15 \%$ discount. What is the original price?

We need to determine the original price. We can think of the original price as the starting place. Starting place indicates base. We need to determine the base. The new price, $\$ 20.40$, represents $100 \%-15 \%=85 \%$ of the original price.

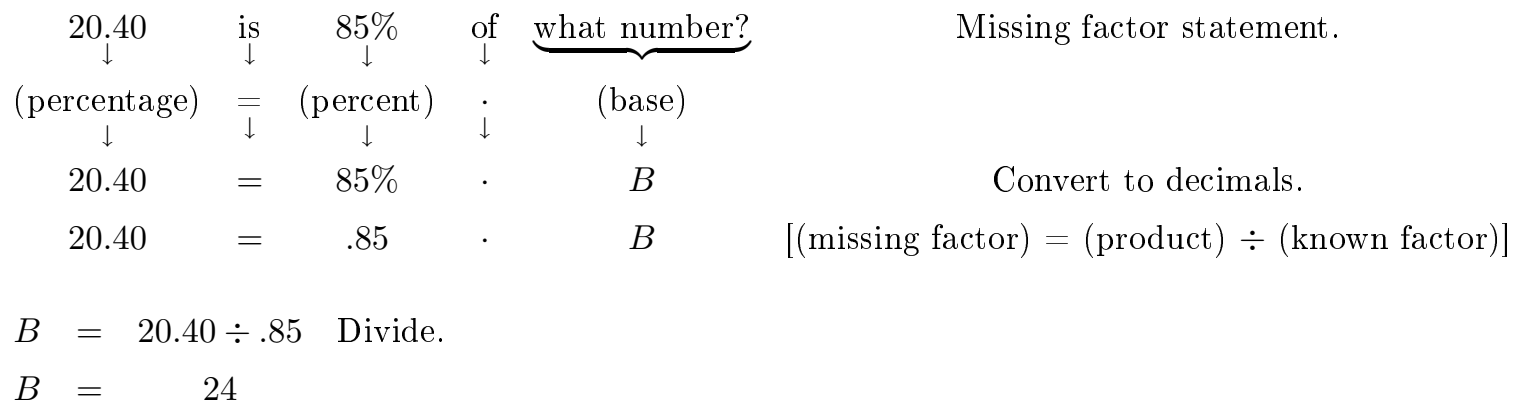

Thus, the original price of the item is $\$ 24.00$.

Try Exercise 7.7.11 in Section 7.7.5.2 (Practice Set C).

\subsubsection{Practice Set C}

Exercise 7.7.8

(Solution on p. 472.)

1.98 is $2 \%$ of what number?

(Solution on p. 472.)

Exercise 7.7.9

3.3 milliliters of $\mathrm{HCl}$ represents $25 \%$ of an $\mathrm{HCl}$ solution. How many milliliters of solution are there?

Exercise 7.7.10

(Solution on p. 472.)

A salesman, who makes a commission of $18 \frac{1}{4} \%$ on each sale, makes a commission of $\$ 152.39$ on a particular sale. Rounded to the nearest dollar, what is the amount of the sale?

Exercise 7.7.11

(Solution on p. 472.)

At "super-long play," $2 \frac{1}{2}$ hours of play of a video cassette recorder represents $31.25 \%$ of the total playing time. What is the total playing time?

\subsubsection{Exercises}

For the following 25 problems, find each indicated quantity.

Exercise 7.7.12

What is $21 \%$ of 104 ?

(Solution on p. 472.) 
Exercise 7.7.13

What is $8 \%$ of 36 ?

Exercise 7.7.14

(Solution on p. 472.)

What is $98 \%$ of 545 ?

Exercise 7.7.15

What is $143 \%$ of 33 ?

Exercise 7.7.16

(Solution on p. 472.)

What is $10 \frac{1}{2} \%$ of $20 ?$

Exercise 7.7.17

3.25 is what percent of 88 ?

Exercise 7.7.18

(Solution on p. 472.)

22.44 is what percent of 44 ?

Exercise 7.7.19

0.0036 is what percent of 0.03 ?

Exercise 7.7.20

(Solution on p. 472.)

31.2 is what percent of $26 ?$

Exercise 7.7.21

266.4 is what percent of 74 ?

Exercise 7.7.22

(Solution on p. 472.)

0.0101 is what percent of 0.0505 ?

Exercise 7.7.23

2.4 is $24 \%$ of what number?

Exercise 7.7.24

24.19 is $41 \%$ of what number?

(Solution on p. 472.)

Exercise 7.7.25

61.12 is $16 \%$ of what number?

Exercise 7.7.26

(Solution on p. 472.)

82.81 is $91 \%$ of what number?

Exercise 7.7.27

115.5 is $20 \%$ of what number?

Exercise 7.7.28

43.92 is $480 \%$ of what number?

(Solution on p. 472.)

Exercise 7.7.29

What is $85 \%$ of 62 ?

Exercise 7.7.30

(Solution on p. 472.)

29.14 is what percent of 5.13 ?

Exercise 7.7.31

0.6156 is what percent of 5.13 ?

Exercise 7.7.32

What is $0.41 \%$ of 291.1 ?

(Solution on p. 472.)

Exercise 7.7.33

26.136 is $121 \%$ of what number?

Exercise 7.7.34

(Solution on p. 472.) $1,937.5$ is what percent of 775 ?

Exercise 7.7.35

1 is what percent of 2,000 ? 
Exercise 7.7.36

(Solution on p. 472.)

0 is what percent of 59 ?

\section{Exercise 7.7.37}

An item of clothing is on sale for $10 \%$ off the marked price. If the marked price is $\$ 14.95$, what is the sale price? (Round to two decimal places.)

Exercise 7.7.38

(Solution on p. 472.)

A grocery clerk, who makes $\$ 365$ per month, receives a $7 \%$ raise. How much is her new monthly salary?

Exercise 7.7.39

An item of clothing which originally sells for $\$ 55.00$ is marked down to $\$ 46.75$. What percent has it been marked down?

Exercise 7.7.40

(Solution on p. 472.)

On a 25 question exam, a student gets 21 correct. What percent is this?

Exercise 7.7.41

On a 45 question exam, a student gets $40 \%$. How many questions did this student get correct?

Exercise 7.7.42

(Solution on p. 472.)

A vitamin tablet, which weighs 250 milligrams, contains 35 milligrams of vitamin $\mathrm{C}$. What percent of the weight of this tablet is vitamin $\mathrm{C}$ ?

Exercise 7.7.43

Five years ago a secretary made $\$ 11,200$ annually. The secretary now makes $\$ 17,920$ annually. By what percent has this secretary's salary been increased?

Exercise 7.7.44

(Solution on p. 472.)

A baseball team wins $48 \frac{3}{4} \%$ of all their games. If they won 78 games, how many games did they play?

\section{Exercise 7.7.45}

A typist was able to increase his speed by $120 \%$ to 42 words per minute. What was his original typing speed?

Exercise 7.7.46

(Solution on p. 472.)

A salesperson makes a commission of $12 \%$ on the total amount of each sale. If, in one month, she makes a total of $\$ 8,520$ in sales, how much has she made in commission?

Exercise 7.7.47

A salesperson receives a salary of $\$ 850$ per month plus a commission of $8 \frac{1}{2} \%$ of her sales. If, in a particular month, she sells $\$ 22,800$ worth of merchandise, what will be her monthly earnings?

Exercise 7.7.48

(Solution on p. 472.)

A man borrows $\$ 1150.00$ from a loan company. If he makes 12 equal monthly payments of $\$ 130.60$, what percent of the loan is he paying in interest?

\section{Exercise 7.7.49}

The distance from the sun to the earth is approximately 93,000,000 miles. The distance from the sun to Pluto is approximately $860.2 \%$ of the distance from the sun to the Earth. Approximately, how many miles is Pluto from the sun?

Exercise 7.7.50

(Solution on p. 473.)

The number of people on food stamps in Maine in 1975 was 151,000. By 1980, the number had decreased to 59,200 . By what percent did the number of people on food stamps decrease? (Round the result to the nearest percent.)

\section{Exercise 7.7.51}

In Nebraska, in 1960, there were 734,000 motor-vehicle registrations. By 1979, the total had increased by about $165.6 \%$. About how many motor-vehicle registrations were there in Nebraska in 1979 ? 
Exercise 7.7.52

(Solution on p. 473.)

From 1973 to 1979 , in the United States, there was an increase of $166.6 \%$ of Ph.D. social scientists to 52,000 . How many were there in 1973 ?

\section{Exercise 7.7.53}

In 1950, in the United States, there were 1,894 daily newspapers. That number decreased to 1,747 by 1981 . What percent did the number of daily newspapers decrease?

Exercise 7.7.54

(Solution on p. 473.)

A particular alloy is $27 \%$ copper. How many pounds of copper are there in 55 pounds of the alloy?

Exercise 7.7.55

A bottle containing a solution of hydrochloric acid $(\mathrm{HCl})$ is marked $15 \%$ (meaning that $15 \%$ of the $\mathrm{HCl}$ solution is acid). If a bottle contains 65 milliliters of solution, how many milliliters of water does it contain?

Exercise 7.7.56

(Solution on p. 473.)

A bottle containing a solution of $\mathrm{HCl}$ is marked $45 \%$. A test shows that 36 of the 80 milliliters contained in the bottle are hydrochloric acid. Is the bottle marked correctly? If not, how should it be remarked?

\subsubsection{Exercises For Review}

\section{Exercise 7.7.57}

(Section 2.6) Use the numbers 4 and 7 to illustrate the commutative property of multiplication.

Exercise 7.7.58

(Section 4.3) Convert $\frac{14}{5}$ to a mixed number.

(Solution on p. 473.)

Exercise 7.7.59

(Section 5.5) Arrange the numbers $\frac{7}{12}, \frac{5}{9}$ and $\frac{4}{7}$ in increasing order.

Exercise 7.7.60

(Solution on p. 473.)

(Section 6.3) Convert 4.006 to a mixed number.

Exercise 7.7.61

(Section 7.6) Convert $\frac{7}{8} \%$ to a fraction. 


\subsection{Summary of Key Concepts ${ }^{8}$}

\subsubsection{Summary of Key Concepts}

Denominate Numbers (Section 7.2)

Numbers that appear along with units are denominate numbers. The amounts 6 dollars and 4 pints are examples of denominate numbers.

Like and Unlike Denominate Numbers (Section 7.2)

Like denominate numbers are denominate numbers with like units. If the units are not the same, the numbers are unlike denominate numbers.

Pure Numbers (Section 7.2)

Numbers appearing without a unit are pure numbers.

Comparing Numbers by Subtraction and Division (Section 7.2)

Comparison of two numbers by subtraction indicates how much more one number is than another. Comparison by division indicates how many times larger or smaller one number is than another.

Comparing Pure or Like Denominate Numbers by Subtraction (Section 7.2)

Numbers can be compared by subtraction if and only if they are pure numbers or like denominate numbers.

Ratio Rate (Section 7.2)

A comparison, by division, of two like denominate numbers is a ratio. A comparison, by division, of two unlike denominate numbers is a rate.

Proportion (Section 7.3)

A proportion is a statement that two ratios or rates are equal.

$\frac{3 \text { people }}{2 \text { jobs }}=\frac{6 \text { people }}{4 \text { jobs }}$ is a proportion.

Solving a Proportion (Section 7.3)

To solve a proportion that contains three known numbers and a letter that represents an unknown quantity, perform the cross multiplication, then divide the product of the two numbers by the number that multiplies the letter.

Proportions Involving Rates (Section 7.3)

When writing a proportion involving rates it is very important to write it so that the same type of units appears on the same side of either the equal sign or the fraction bar.

$\frac{\text { unit type } 1}{\text { unit type } 2}=\frac{\text { unit type } 1}{\text { unit type } 2}$ or $\frac{\text { unit type } 1}{\text { unit type } 1}=\frac{\text { unit type } 2}{\text { unit type } 2}$

\section{Five-Step Method for Solving Proportions (Section 7.4)}

1. By careful reading, determine what the unknown quantity is and represent it with some letter. There will be only one unknown in a problem.

2. Identify the three specified numbers.

3. Determine which comparisons are to be made and set up the proportion.

4. Solve the proportion.

5. Interpret and write a conclusion.

When solving applied problems, ALWAYS begin by determining the unknown quantity and representing it with a letter.

\footnotetext{
${ }^{8}$ This content is available online at $<$ http://cnx.org/content $/ \mathrm{m} 35008 / 1.2 />$.
} 
Percents (Section 7.5)

A ratio in which one number is compared to 100 is a percent. Percent means "for each hundred."

Conversion of Fractions, Decimals, and Percents (Section 7.5)

It is possible to convert decimals to percents, fractions to percents, percents to decimals, and percents to fractions.

Applications of Percents:

The three basic types of percent problems involve a base, a percentage, and a percent.

Base (Section 7.7)

The base is the number used for comparison.

Percentage (Section 7.7)

The percentage is the number being compared to the base.

Percent (Section 7.7)

By its definition, percent means part of.

Solving Problems (Section 7.7)

Percentage $=($ percent $) \times($ base $)$

Percent $=\frac{\text { percentage }}{\text { base }}$

Base $=\frac{\text { percentage }}{\text { percent }}$

\subsection{Exercise Supplement ${ }^{9}$}

\subsubsection{Exercise Supplement}

\subsubsection{Ratios and Rates (Section 7.2)}

Exercise 7.9.1

(Solution on p. 473.)

Compare 250 watts to 100 watts by subtraction.

Exercise 7.9.2

Compare 126 and 48 by subtraction.

Exercise 7.9.3

(Solution on p. 473.)

Compare 98 radishes to 41 radishes by division.

Exercise 7.9.4

Compare 144 to 9 by division.

Exercise 7.9.5

(Solution on p. 473.)

Compare 100 tents to 5 tents by division.

Exercise 7.9.6

Compare 28 feet to 7 feet by division.

Exercise 7.9.7

(Solution on p. 473.)

Comparison, by division, of two pure numbers or two like denominate numbers is called a

Exercise 7.9.8

A comparison, by division, of two unlike denominate numbers is called a

For problems 9-12, express each ratio or rate as a fraction.

\footnotetext{
${ }^{9}$ This content is available online at $<$ http://cnx.org/content $/ \mathrm{m} 35009 / 1.2 />$.
} 
Exercise 7.9.9

(Solution on p. 473.)

15 to 5

Exercise 7.9.10

72 to 12

Exercise 7.9.11

(Solution on p. 473.)

8 millimeters to 5 milliliters

Exercise 7.9.12

106 tablets to 52 tablets

For problems 13-16, write each ratio in the form " $a$ to $b$ ".

Exercise 7.9.13

$\frac{9}{16}$

(Solution on p. 473.)

Exercise 7.9.14

$\frac{5}{11}$

Exercise 7.9.15

1 diskette

Exercise 7.9.16

$\frac{5 \text { papers }}{3 \text { pens }}$

For problems 17-21, write each ratio or rate using words.

Exercise 7.9.17

$\frac{9}{16}=\frac{18}{32}$

(Solution on p. 473.)

Exercise 7.9.18

$\frac{1}{4}=\frac{12}{48}$

Exercise 7.9.19

$\frac{8 \text { items }}{4 \text { dollars }}=\frac{2 \text { items }}{1 \text { dollar }}$

Exercise 7.9.20

150 milligrams of niacin is to 2 tablets as 300 milligrams of niacin is to 4 tablets.

Exercise 7.9.21

(Solution on p. 473.)

20 people is to 4 seats as 5 people is to 1 seat.

(Solution on p. 473.)

\subsubsection{Proportions (Section 7.3)}

For problems 22-27, determine the missing number in each proportion.

Exercise 7.9.22

$\frac{x}{3}=\frac{24}{9}$

Exercise 7.9.23

$\frac{15}{7}=\frac{60}{x}$

(Solution on p. 473.)

Exercise 7.9.24

$\frac{1}{1}=\frac{x}{44}$

Exercise 7.9.25

$\frac{3}{x}=\frac{15}{50}$

Exercise 7.9.26

$\frac{15 \text { bats }}{16 \text { balls }}=\frac{x \text { bats }}{128 \text { balls }}$

Exercise 7.9.27

$\frac{36 \text { rooms }}{29 \text { fans }}=\frac{504 \text { rooms }}{x \text { fans }}$

(Solution on p. 473.)

(Solution on p. 473.) 


\subsubsection{Applications of Proportions (Section 7.4)}

Exercise 7.9.28

On a map, 3 inches represents 20 miles. How many miles does 27 inches represent?

Exercise 7.9.29

(Solution on p. 473.)

A salt solution is composed of 8 parts of salt to 5 parts of water. How many parts of salt are there in a solution that contains 50 parts of water?

Exercise 7.9.30

A model is built to $\frac{4}{15}$ scale. If a particular part of the model measures 8 inches in length, how long is the actual structure?

Exercise 7.9.31

(Solution on p. 473.)

The ratio of ammonia to air in a container is $\frac{3}{40}$ How many milliliters of air should be in a container that contains 8 milliliters of ammonia?

Exercise 7.9.32

A 4 -foot girl casts a 9-foot shadow at a particular time of the day. How tall is a pole that casts a 144 -foot shadow at the same time of the day?

Exercise 7.9.33

(Solution on p. 473.)

The odds that a particular event will occur are 11 to 2 . If this event occurs 55 times, how many times would you predict it does not occur?

Exercise 7.9.34

Every $1 \frac{3}{4}$ teaspoon of a multiple vitamin, in granular form, contains 0.85 the minimum daily requirement of vitamin A. How many teaspoons of this vitamin are required to supply 2.25 the minimum daily requirement?

\subsubsection{Percent and Fractions of One Percent (Section 7.5,Section 7.6)}

For problems 35-39, convert each decimal to a percent.

Exercise 7.9.35

(Solution on p. 473.)

0.16

Exercise 7.9.36

0.818

Exercise 7.9.37

5.3536

(Solution on p. 473.)

Exercise 7.9.38

0.50

Exercise 7.9.39

3

(Solution on p. 474.)

For problems 40-48, convert each percent to a decimal.

Exercise 7.9.40

$62 \%$

Exercise 7.9.41

$1.58 \%$

(Solution on p. 474.)

Exercise 7.9.42

$9.15 \%$

Exercise 7.9.43

$0.06 \%$

(Solution on p. 474.) 
Exercise 7.9.44

$0.003 \%$

Exercise 7.9.45

(Solution on p. 474.)

$5 \frac{3}{11} \%$ to a three-place decimal

Exercise 7.9.46

$\frac{9}{13} \%$ to a three-place decimal

Exercise 7.9.47

$82 \frac{25}{29} \%$ to a four-place decimal

(Solution on p. 474.)

Exercise 7.9.48

$18 \frac{1}{7} \%$ to a four-place decimal

For problems 49-55, convert each fraction or mixed number to a percent.

Exercise 7.9.49

$\frac{3}{5}$

(Solution on p. 474.)

Exercise 7.9.50

$\frac{2}{10}$

Exercise 7.9.51

$\frac{5}{16}$

Exercise 7.9.52

$\frac{35}{8}$

Exercise 7.9.53

$\frac{105}{16}$

Exercise 7.9.54

$45 \frac{1}{11}$

Exercise 7.9.55

$6 \frac{278}{9}$

(Solution on p. 474.)

For problems 56-64, convert each percent to a fraction or mixed number.

Exercise 7.9.56

$95 \%$

Exercise 7.9.57

$12 \%$

(Solution on p. 474.)

Exercise 7.9.58

$83 \%$

Exercise 7.9.59

$38.125 \%$

(Solution on p. 474.)

(Solution on p. 474.)

Exercise 7.9.60

61. $\overline{2} \%$

Exercise 7.9.61

$\frac{5}{8} \%$

(Solution on p. 474.)

Exercise 7.9.62

$6 \frac{9}{20} \%$

Exercise 7.9.63

$15 \frac{3}{22} \%$

(Solution on p. 474.)

Exercise 7.9.64

$106 \frac{19}{45} \%$

(Solution on p. 474.) 


\subsubsection{Applications of Percents (Section 7.7)}

For problems 65-72, find each solution.

Exercise 7.9.65

(Solution on p. 474.)

What is $16 \%$ of 40 ?

Exercise 7.9.66

29.4 is what percent of 105 ?

Exercise 7.9.67

(Solution on p. 474.)

$3 \frac{21}{50}$ is $547.2 \%$ of what number?

Exercise 7.9.68

0.09378 is what percent of 52.1 ?

Exercise 7.9.69

(Solution on p. 474.)

What is $680 \%$ of 1.41 ?

Exercise 7.9.70

A kitchen knife is on sale for $15 \%$ off the marked price. If the marked price is $\$ 39.50$, what is the sale price?

Exercise 7.9.71

(Solution on p. 474.)

On an 80 question geology exam, a student gets 68 correct. What percent is correct?

Exercise 7.9.72

A salesperson makes a commission of $18 \%$ of her monthly sales total. She also receives a monthly salary of $\$ 1,600.00$. If, in a particular month, she sells $\$ 4,000.00$ worth of merchandise, how much will she make that month?

\subsection{Proficiency Exam ${ }^{10}$}

\subsubsection{Proficiency Exam}

Exercise 7.10.1

(Solution on p. 474.)

(Section 7.2) Compare 4 cassette tapes to 7 dollars.

(Solution on p. 474.)

Exercise 7.10.2

(Section 7.2) What do we call a comparison, by division, of two unlike denominate numbers?

For problems 3 and 4, express each ratio or rate as a fraction.

Exercise 7.10.3

(Solution on p. 474.)

(Section 7.2) 11 to 9

Exercise 7.10.4

(Solution on p. 474.)

(Section 7.2) 5 televisions to 2 radios

For problems 5 and 6 , write each ratio or rate in the form " $a$ to $b . "$

Exercise 7.10.5

(Solution on p. 474.)

(Section 7.2) $\frac{8 \text { maps }}{3 \text { people }}$

Exercise 7.10.6

(Solution on p. 474.)

(Section 7.2) $\frac{2 \text { psychologists }}{75 \text { people }}$

For problems 7-9, solve each proportion.

Exercise 7.10.7

(Section 7.3) $\frac{8}{x}=\frac{48}{90}$

(Solution on p. 474.)

\footnotetext{
${ }^{10}$ This content is available online at $<\mathrm{http}: / / \mathrm{cnx} .0 \mathrm{rg} /$ content $/ \mathrm{m} 35010 / 1.2 />$.
} 
Exercise 7.10.8

(Solution on p. 474.)

(Section 7.3) $\frac{x}{7}=\frac{4}{28}$

Exercise 7.10.9

(Section 7.3$) \frac{3 \text { computers }}{8 \text { students }}=\frac{24 \text { computers }}{x \text { students }}$

(Solution on p. 475.)

Exercise 7.10.10

(Solution on p. 475.)

(Section 7.4) On a map, 4 inches represents 50 miles. How many miles does 3 inches represent?

Exercise 7.10.11

(Solution on p. 475.)

(Section 7.4) An acid solution is composed of 6 milliliters of acid to 10 milliliters of water. How many milliliters of acid are there in an acid solution that is composed of 3 milliliters of water?

Exercise 7.10.12

(Solution on p. 475.)

(Section 7.4) The odds that a particular event will occur are 9 to 7 . If the event occurs 27 times, how many times would you predict it will it not occur?

For problems 13 and 14, convert each decimal to a percent.

Exercise 7.10.13

(Solution on p. 475.)

(Section 7.5) 0.82

Exercise 7.10.14

(Solution on p. 475.)

(Section 7.5) 3. $\overline{7}$

For problems 15 and 16, convert each percent to a decimal.

Exercise 7.10.15

(Solution on p. 475.)

(Section 7.5 ) $2.813 \%$

Exercise 7.10.16

(Solution on p. 475.)

(Section 7.5 ) $0.006 \%$

For problems 17-19, convert each fraction to a percent.

Exercise 7.10.17

(Section 7.5) $\frac{42}{5}$

Exercise 7.10.18

(Section 7.5) $\frac{1}{8}$

Exercise 7.10.19

(Section 7.5) $\frac{800}{80}$

(Solution on p. 475.)

(Solution on p. 475.)

(Solution on p. 475.)

For problems 20 and 21, convert each percent to a fraction.

Exercise 7.10.20

(Solution on p. 475.)

(Section 7.5) 15\%

Exercise 7.10.21

(Section 7.5) $\frac{4}{27} \%$

(Solution on p. 475.)

For problems 22-25, find each indicated quantity.

Exercise 7.10.22

(Solution on p. 475.)

(Section 7.7) What is $18 \%$ of 26 ?

Exercise 7.10.23

(Solution on p. 475.)

(Section 7.7) 0.618 is what percent of 0.3 ?

Exercise 7.10.24

(Section 7.7 ) 0.1 is $1.1 \%$ of what number?

Exercise 7.10.25

(Solution on p. 475.)

(Solution on p. 475.)

(Section 7.7) A salesperson makes a monthly salary of $\$ 1,000.00$. He also gets a commission of $12 \%$ of his total monthly sales. If, in a particular month, he sells $\$ 5,500.00$ worth of merchandise, what is his income that month? 


\section{Solutions to Exercises in Chapter 7}

Solution to Exercise 7.2.1 (p. 421)

(a) 8 diskettes; 10 diskettes is 8 diskettes more than 2 diskettes.

(b) 5; 10 diskettes is 5 times as many diskettes as 2 diskettes.

Solution to Exercise 7.2.2 (p. 421)

(a) Comparison by subtraction makes no sense.

(b) $\frac{16 \text { bananas }}{2 \text { bags }}=\frac{8 \text { bananas }}{\text { bag }}, 8$ bananas per bag.

Solution to Exercise 7.2.3 (p. 422)

$\frac{3}{2}$

Solution to Exercise 7.2.4 (p. 422)

$\frac{1}{9}$

Solution to Exercise 7.2.5 (p. 422)

$\frac{5 \text { books }}{4 \text { people }}$

Solution to Exercise 7.2.6 (p. 422)

$\frac{60 \text { miles }}{1 \text { hour }}$

Solution to Exercise 7.2.7 (p. 422)

$\frac{8}{3}$

Solution to Exercise 7.2.8 (p. 422)

9 to 5

Solution to Exercise 7.2.9 (p. 422)

1 to 3

Solution to Exercise 7.2.10 (p. 422)

25 miles to 2 gallons

Solution to Exercise 7.2.11 (p. 423)

1 mechanic to 2 wrenches

Solution to Exercise 7.2.12 (p. 423)

5 to 6

Solution to Exercise 7.2.13 (p. 423)

They are pure numbers or like denominate numbers.

Solution to Exercise 7.2.15 (p. 423)

rate

Solution to Exercise 7.2.17 (p. 423)

ratio

Solution to Exercise 7.2.19 (p. 423)

rate

Solution to Exercise 7.2.21 (p. 423)

ratio

Solution to Exercise 7.2.23 (p. 423)

two to five

Solution to Exercise 7.2.25 (p. 423)

29 mile per 2 gallons or $14 \frac{1}{2}$ miles per 1 gallon

Solution to Exercise 7.2.27 (p. 423)

5 to 2

Solution to Exercise 7.2.29 (p. 423)

$\frac{12}{5}$

Solution to Exercise 7.2.31 (p. 424)

$\frac{42 \text { plants }}{5 \text { homes }}$ 
Solution to Exercise 7.2.33 (p. 424)

$\frac{16 \text { pints }}{1 \text { quart }}$

Solution to Exercise 7.2.35 (p. 424)

$\frac{2.54 \mathrm{~cm}}{1 \text { inch }}$

Solution to Exercise 7.2.37 (p. 424)

$\frac{5}{2}$

Solution to Exercise 7.2.39 (p. 424)

$\frac{1 \text { hit }}{3 \text { at bats }}$

Solution to Exercise 7.2.41 (p. 424)

$\frac{1,042 \text { characters }}{1 \text { page }}$

Solution to Exercise 7.2.43 (p. 424)

$5 \frac{1}{3}$

Solution to Exercise 7.2.45 (p. 424)

$\frac{299}{1260}$

Solution to Exercise 7.2.47 (p. 424)

3.3875

Solution to Exercise 7.3.1 (p. 426)

3 is to 8 as 6 is to 16

Solution to Exercise 7.3.2 (p. 426)

2 people are to 1 window as 10 people are to 5 windows

Solution to Exercise 7.3.3 (p. 426)

$\frac{15}{4}=\frac{75}{20}$

Solution to Exercise 7.3.4 (p. 426)

$\frac{2 \text { plates }}{1 \text { tray }}=\frac{20 \text { plates }}{10 \text { trays }}$

Solution to Exercise 7.3.5 (p. 427)

$x=3$

Solution to Exercise 7.3.6 (p. 428)

$x=5$

Solution to Exercise 7.3.7 (p. 428)

$x=45$

Solution to Exercise 7.3.8 (p. 428)

$x=48$

Solution to Exercise 7.3.9 (p. 429)

rates, proportion

Solution to Exercise 7.3.11 (p. 429)

$\frac{1}{11}=\frac{3}{33}$

Solution to Exercise 7.3.13 (p. 429)

$\frac{6}{90}=\frac{3}{45}$

Solution to Exercise 7.3.15 (p. 429)

$\frac{18 \mathrm{gr} \text { cobalt }}{10 \mathrm{gr} \text { silver }}=\frac{36 \mathrm{gr} \text { cobalt }}{20 \mathrm{gr} \mathrm{silver}}$

Solution to Exercise 7.3.17 (p. 429)

$\frac{3 \text { people absent }}{31 \text { people present }}=\frac{15 \text { people absent }}{155 \text { people present }}$

Solution to Exercise 7.3.19 (p. 429)

3 is to 4 as 15 is to 20

Solution to Exercise 7.3.21 (p. 429)

3 joggers are to 100 feet as 6 joggers are to 200 feet

Solution to Exercise 7.3.23 (p. 430)

40 miles are to 80 miles as 2 gallons are to 4 gallons

Solution to Exercise 7.3.25 (p. 430)

1 person is to 1 job as 8 people are to 8 jobs 
Solution to Exercise 7.3.27 (p. 430)

2,000 pounds are to 1 ton as 60,000 pounds are to 30 tons

Solution to Exercise 7.3.29 (p. 430)

$x=2$

Solution to Exercise 7.3.31 (p. 430)

$x=8$

Solution to Exercise 7.3.33 (p. 430)

$x=8$

Solution to Exercise 7.3.35 (p. 430)

$x=27$

Solution to Exercise 7.3.37 (p. 430)

$x=40$

Solution to Exercise 7.3.39 (p. 430)

$x=30$

Solution to Exercise 7.3.41 (p. 430)

$x=35$

Solution to Exercise 7.3.43 (p. 430)

$x=30$

Solution to Exercise 7.3.45 (p. 431)

true

Solution to Exercise 7.3.47 (p. 431)

false

Solution to Exercise 7.3.49 (p. 431)

true

Solution to Exercise 7.3.51 (p. 431)

true

Solution to Exercise 7.3.53 (p. 431)

$5+7=12$

$7+5=12$

Solution to Exercise 7.3.55 (p. 431)

$\frac{17}{77}$

Solution to Exercise 7.3.57 (p. 431)

$\frac{8 \text { sentences }}{1 \text { paragraph }}$

Solution to Exercise 7.4.1 (p. 434)

500 miles

Solution to Exercise 7.4.2 (p. 435)

24 parts of alcohol

Solution to Exercise 7.4.3 (p. 435)

$3 \frac{9}{11}$ feet

Solution to Exercise 7.4.4 (p. 435)

864 outside plants

Solution to Exercise 7.4.5 (p. 435)

The event occurs 99 times.

Solution to Exercise 7.4.6 (p. 435)

6 failing grades

Solution to Exercise 7.4.7 (p. 436)

24

Solution to Exercise 7.4.9 (p. 436)

45 inches 
Solution to Exercise 7.4.11 (p. 436) 33 parts

Solution to Exercise 7.4.13 (p. 436) 2328.75

Solution to Exercise 7.4.15 (p. 436)

$\frac{21}{22}$ feet

Solution to Exercise 7.4.17 (p. 436) 12,250

Solution to Exercise 7.4.19 (p. 436) 12

Solution to Exercise 7.4.21 (p. 436) $1 \frac{5}{6}$

Solution to Exercise 7.4.23 (p. 437) $1 \frac{7}{8}$

Solution to Exercise 7.4.25 (p. 437) $31 \frac{1}{4}$

Solution to Exercise 7.4.27 (p. 437) 0

Solution to Exercise 7.4.29 (p. 437) $\frac{2}{3}$

Solution to Exercise 7.4.31 (p. 437) 5

Solution to Exercise 7.5.1 (p. 438) $16 \%$

Solution to Exercise 7.5.2 (p. 438) $195 \%$

Solution to Exercise 7.5.3 (p. 438) $\frac{83}{100}$

Solution to Exercise 7.5.4 (p. 438) $\frac{362}{100}$ or $\frac{181}{50}$

Solution to Exercise 7.5.5 (p. 441) 0.21

Solution to Exercise 7.5.6 (p. 441) 4.61

Solution to Exercise 7.5.7 (p. 441) $55 \%$

Solution to Exercise 7.5.8 (p. 441) $564 \%$

Solution to Exercise 7.5.9 (p. 441) $15 \%$

Solution to Exercise 7.5.10 (p. 441) $137.5 \%$

Solution to Exercise 7.5.11 (p. 441) $27 . \overline{27 \%}$

Solution to Exercise 7.5.12 (p. 441) $25 \%$

Solution to Exercise 7.5.14 (p. 441) $48 \%$

Solution to Exercise 7.5.16 (p. 441) $77.1 \%$ 
Solution to Exercise 7.5.18 (p. 441) $258 \%$

Solution to Exercise 7.5.20 (p. 441) $1,618.14 \%$

Solution to Exercise 7.5.22 (p. 441) $200 \%$

Solution to Exercise 7.5.24 (p. 442) 0.15

Solution to Exercise 7.5.26 (p. 442) 0.162

Solution to Exercise 7.5.28 (p. 442) 0.0505

Solution to Exercise 7.5.30 (p. 442) 0.0078

Solution to Exercise 7.5.32 (p. 442) 0.0009

Solution to Exercise 7.5.34 (p. 442) $20 \%$

Solution to Exercise 7.5.36 (p. 442) $62.5 \%$

Solution to Exercise 7.5.38 (p. 442) $28 \%$

Solution to Exercise 7.5.40 (p. 442) $49 . \overline{09} \%$

Solution to Exercise 7.5.42 (p. 442) $164 \%$

Solution to Exercise 7.5.44 (p. 442) $945 \%$

Solution to Exercise 7.5.46 (p. 443) $54 . \overline{54} \%$

Solution to Exercise 7.5.48 (p. 443)

$\frac{4}{5}$

Solution to Exercise 7.5.50 (p. 443)

$\frac{1}{4}$

Solution to Exercise 7.5.52 (p. 443)

$\frac{13}{20}$

Solution to Exercise 7.5.54 (p. 443) $\frac{1}{8}$

Solution to Exercise 7.5.56 (p. 443)

$\frac{41}{8}$ or $5 \frac{1}{8}$

Solution to Exercise 7.5.58 (p. 443)

$\frac{1}{10}$

Solution to Exercise 7.5.60 (p. 443)

$\frac{2}{9}$

Solution to Exercise 7.5.62 (p. 443)

$\frac{4}{45}$

Solution to Exercise 7.5.64 (p. 443)

$\frac{129}{60}$ or $2 \frac{9}{60}=2 \frac{3}{20}$

Solution to Exercise 7.5.66 (p. 444)

36 inches 
Solution to Exercise 7.6.1 (p. 445) $\frac{1}{400}$

Solution to Exercise 7.6.2 (p. 445) $\frac{3}{800}$

Solution to Exercise 7.6.3 (p. 445)

$\frac{1}{30}$

Solution to Exercise 7.6.4 (p. 446) 0.008

Solution to Exercise 7.6.5 (p. 446) 0.5145

Solution to Exercise 7.6.6 (p. 446) $\frac{3}{400}$

Solution to Exercise 7.6.8 (p. 446) $\frac{1}{900}$

Solution to Exercise 7.6.10 (p. 446) $\frac{5}{400}$ or $\frac{1}{80}$

Solution to Exercise 7.6.12 (p. 446) $\frac{13}{700}$

Solution to Exercise 7.6.14 (p. 446) $\frac{101}{400}$

Solution to Exercise 7.6.16 (p. 446) $\frac{363}{500}$

Solution to Exercise 7.6.18 (p. 446) $\frac{41}{30}$

Solution to Exercise 7.6.20 (p. 447) $\frac{51}{500}=0.102$

Solution to Exercise 7.6.22 (p. 447) $\frac{31}{800}=0.03875$

Solution to Exercise 7.6.24 (p. 447) 0.004

Solution to Exercise 7.6.26 (p. 447) 0.0627

Solution to Exercise 7.6.28 (p. 447) 0.242

Solution to Exercise 7.6.30 (p. 447) 0.1194

Solution to Exercise 7.6.32 (p. 447) $8^{5}$

Solution to Exercise 7.6.34 (p. 447) $\frac{197}{210}$

Solution to Exercise 7.6.36 (p. 447) $806.2 \%$

Solution to Exercise 7.7.1 (p. 451) 35.7

Solution to Exercise 7.7.2 (p. 451) $\$ 1,360$

Solution to Exercise 7.7.3 (p. 451) 4.9

Solution to Exercise 7.7.4 (p. 451) $\$ 45,360$ 
Solution to Exercise 7.7.5 (p. 453) $23 \%$

Solution to Exercise 7.7.6 (p. 453) $90 \%$

Solution to Exercise 7.7.7 (p. 453) $40 \%$

Solution to Exercise 7.7.8 (p. 455) 99

Solution to Exercise 7.7.9 (p. 455) $13.2 \mathrm{ml}$

Solution to Exercise 7.7.10 (p. 455) $\$ 835$

Solution to Exercise 7.7.11 (p. 455) 8 hours

Solution to Exercise 7.7.12 (p. 455) 21.84

Solution to Exercise 7.7.14 (p. 456) 534.1

Solution to Exercise 7.7.16 (p. 456) 2.1

Solution to Exercise 7.7.18 (p. 456) 51

Solution to Exercise 7.7.20 (p. 456) 120

Solution to Exercise 7.7.22 (p. 456) 20

Solution to Exercise 7.7.24 (p. 456) 59

Solution to Exercise 7.7.26 (p. 456) 91

Solution to Exercise 7.7.28 (p. 456) 9.15

Solution to Exercise 7.7.30 (p. 456) 568

Solution to Exercise 7.7.32 (p. 456) 1.19351

Solution to Exercise 7.7.34 (p. 456) 250

Solution to Exercise 7.7.36 (p. 457) 0

Solution to Exercise 7.7.38 (p. 457) 390.55

Solution to Exercise 7.7.40 (p. 457) 84

Solution to Exercise 7.7.42 (p. 457) 14

Solution to Exercise 7.7.44 (p. 457) 160

Solution to Exercise 7.7.46 (p. 457) $\$ 1,022.40$ 
Solution to Exercise 7.7.48 (p. 457) $36.28 \%$

Solution to Exercise 7.7.50 (p. 457)

61

Solution to Exercise 7.7.52 (p. 458)

19,500

Solution to Exercise 7.7.54 (p. 458)

14.85

Solution to Exercise 7.7.56 (p. 458)

Marked correctly

Solution to Exercise 7.7.58 (p. 458)

$2 \frac{4}{5}$

Solution to Exercise 7.7.60 (p. 458) $4 \frac{3}{500}$

Solution to Exercise 7.9.1 (p. 460)

250 watts are 150 watts more than 100 watts

Solution to Exercise 7.9.3 (p. 460)

98 radishes are 2.39 times as many radishes as 41 radishes

Solution to Exercise 7.9.5 (p. 460)

100 tents are 20 times as many tents as 5 tents

Solution to Exercise 7.9.7 (p. 460)

ratio

Solution to Exercise 7.9.9 (p. 460)

$\frac{3}{1}$

Solution to Exercise 7.9.11 (p. 461)

$\frac{8 \mathrm{ml}}{5 \mathrm{ml}}$

Solution to Exercise 7.9.13 (p. 461)

9 to 16

Solution to Exercise 7.9.15 (p. 461)

1 diskette to 8 diskettes

Solution to Exercise 7.9.17 (p. 461)

9 is to 16 as 18 is to 32

Solution to Exercise 7.9.19 (p. 461)

8 items are to 4 dollars as 2 items are to 1 dollar

Solution to Exercise 7.9.21 (p. 461)

$\frac{20}{4}=\frac{5}{1}$

20 people are to 4 seats as 5 people are to 1 seat

Solution to Exercise 7.9.23 (p. 461)

28

Solution to Exercise 7.9.25 (p. 461)

10

Solution to Exercise 7.9.27 (p. 461)

406

Solution to Exercise 7.9.29 (p. 462)

80

Solution to Exercise 7.9.31 (p. 462)

$\frac{320}{3}$ or $106 \frac{2}{3}$

Solution to Exercise 7.9.33 (p. 462)

10

Solution to Exercise 7.9.35 (p. 462)

$16 \%$ 
Solution to Exercise 7.9.37 (p. 462) $535.36 \%$

Solution to Exercise 7.9.39 (p. 462) $300 \%$

Solution to Exercise 7.9.41 (p. 462)

0.0158

Solution to Exercise 7.9.43 (p. 462) 0.0006

Solution to Exercise 7.9.45 (p. 463) 0.053

Solution to Exercise 7.9 .47 (p. 463) 0.8286

Solution to Exercise 7.9.49 (p. 463) $60 \%$

Solution to Exercise 7.9.51 (p. 463) $31.25 \%$

Solution to Exercise 7.9.53 (p. 463) $656.25 \%$

Solution to Exercise 7.9.55 (p. 463) 3688. $\overline{8} \%$

Solution to Exercise 7.9.57 (p. 463)

$\frac{3}{25}$

Solution to Exercise 7.9.59 (p. 463) $\frac{61}{160}$

Solution to Exercise 7.9.61 (p. 463) $\frac{1}{160}$

Solution to Exercise 7.9.63 (p. 463) $\frac{2977}{19800}$

Solution to Exercise 7.9.65 (p. 464)

6.4

Solution to Exercise 7.9.67 (p. 464) 0.625 or $\frac{5}{8}$

Solution to Exercise 7.9.69 (p. 464) 9.588

Solution to Exercise 7.9.71 (p. 464) 85

Solution to Exercise 7.10.1 (p. 464) $\frac{4 \text { cassette tapes }}{7 \text { dollars }}$

Solution to Exercise 7.10.2 (p. 464) Rate

Solution to Exercise 7.10.3 (p. 464) $\frac{11}{9}$

Solution to Exercise 7.10.4 (p. 464) $\frac{5 \text { televisions }}{2 \text { radios }}$

Solution to Exercise 7.10.5 (p. 464)

8 maps to 3 people

Solution to Exercise 7.10.6 (p. 464) two psychologists to seventy-five people Solution to Exercise 7.10.7 (p. 464) 15 
Solution to Exercise 7.10.8 (p. 465)

1

Solution to Exercise 7.10.9 (p. 465)

64

Solution to Exercise 7.10.10 (p. 465)

$37 \frac{1}{2}$

Solution to Exercise 7.10.11 (p. 465)

1.8

Solution to Exercise 7.10.12 (p. 465)

21

Solution to Exercise 7.10.13 (p. 465) $82 \%$

Solution to Exercise 7.10.14 (p. 465) $377 \frac{7}{9} \%$

Solution to Exercise 7.10.15 (p. 465)

0.02813

Solution to Exercise 7.10.16 (p. 465)

0.00006

Solution to Exercise 7.10.17 (p. 465)

$840 \%$

Solution to Exercise 7.10.18 (p. 465)

$12.5 \%$

Solution to Exercise 7.10.19 (p. 465)

$1,000 \%$

Solution to Exercise 7.10.20 (p. 465)

$\frac{3}{20}$

Solution to Exercise 7.10.21 (p. 465)

$\frac{4}{2,700}$ or $\frac{1}{675}$

Solution to Exercise 7.10.22 (p. 465)

4.68

Solution to Exercise 7.10.23 (p. 465)

206

Solution to Exercise 7.10.24 (p. 465)

$9 . \overline{09}$

Solution to Exercise 7.10.25 (p. 465)

$\$ 1,660$ 


\section{Chapter 8}

\section{Techniques of Estimation}

\subsection{Objectives ${ }^{1}$}

After completing this chapter, you should

Estimation by Rounding (Section 8.2)

- understand the reason for estimation

- be able to estimate the result of an addition, multiplication, subtraction, or division using the rounding technique

\section{Estimation by Clustering (Section 8.3)}

- understand the concept of clustering

- be able to estimate the result of adding more than two numbers when clustering occurs using the clustering technique

Mental Arithmetic-Using the Distributive Property (Section 8.4)

- understand the distributive property

- be able to obtain the exact result of a multiplication using the distributive property

Estimation by Rounding Fractions (Section 8.5)

- be able to estimate the sum of two or more fractions using the technique of rounding fractions

\subsection{Estimation by Rounding ${ }^{2}$}

\subsubsection{Section Overview}

- Estimation By Rounding

When beginning a computation, it is valuable to have an idea of what value to expect for the result. When a computation is completed, it is valuable to know if the result is reasonable.

In the rounding process, it is important to note two facts:

\footnotetext{
${ }^{1}$ This content is available online at $<$ http://cnx.org/content $/ \mathrm{m} 18896 / 1.3 />$.

${ }^{2}$ This content is available online at $<$ http://cnx.org/content $/ \mathrm{m} 35011 / 1.2 />$. 
1. The rounding that is done in estimation does not always follow the rules of rounding discussed in Section 1.4 (Rounding Whole Numbers). Since estimation is concerned with the expected value of a computation, rounding is done using convenience as the guide rather than using hard-and-fast rounding rules. For example, if we wish to estimate the result of the division $80 \div 26$, we might round 26 to 20 rather than to 30 since 80 is more conveniently divided by 20 than by 30 .

2. Since rounding may occur out of convenience, and different people have different ideas of what may be convenient, results of an estimation done by rounding may vary. For a particular computation, different people may get different estimated results. Results may vary.

\section{Estimation}

Estimation is the process of determining an expected value of a computation.

Common words used in estimation are about, near, and between.

\subsubsection{Estimation by Rounding}

The rounding technique estimates the result of a computation by rounding the numbers involved in the computation to one or two nonzero digits.

\subsubsection{Sample Set A}

\section{Example 8.1}

Estimate the sum: $2,357+6,106$.

Notice that 2,357 is near $\underbrace{2,400,}_{\begin{array}{c}\text { two nonzero } \\ \text { digits }\end{array}}$ and that 6,106 is near $\underbrace{6,100 .}_{\begin{array}{c}\text { two nonzero } \\ \text { digits }\end{array}}$

The sum can be estimated by $2,400+6,100=8,500$. (It is quick and easy to add 24 and 61 .)

Thus, $2,357+6,106$ is about 8,400 . In fact, $2,357+6,106=8,463$.

\subsubsection{Practice Set A}

Exercise 8.2.1

Estimate the sum: $4,216+3,942$.

Exercise 8.2.2

Estimate the sum: $812+514$.

Exercise 8.2.3

Estimate the sum: $43,892+92,106$.
(Solution on p. 505.)

(Solution on p. 505.)

(Solution on p. 505.)

\subsubsection{Sample Set B}

\section{Example 8.2}

Estimate the difference: 5, $203-3,015$.

Notice that 5,203 is near $\underbrace{5,200,}_{\begin{array}{c}\text { two nonzero } \\ \text { digits }\end{array}}$ and that 3,015 is near $\underbrace{3,000 \text {. }}_{\begin{array}{c}\text { one nonzero } \\ \text { digit }\end{array}}$

The difference can be estimated by $5,200-3,000=2,200$. 
Thus, 5, $203-3,015$ is about 2,200 . In fact, $5,203-3,015=2,188$.

We could make a less accurate estimation by observing that 5,203 is near 5,000. The number 5,000 has only one nonzero digit rather than two (as does 5,200). This fact makes the estimation quicker (but a little less accurate). We then estimate the difference by $5,000-3,000=2,000$, and conclude that $5,203-3,015$ is about 2,000 . This is why we say "answers may vary."

\subsubsection{Practice Set B}

Exercise 8.2.4

(Solution on p. 505.)

Estimate the difference: $628-413$.

Exercise 8.2.5

(Solution on p. 505.)

Estimate the difference: $7,842-5,209$.

Exercise 8.2.6

Estimate the difference: $73,812-28,492$.

(Solution on p. 505.)

\subsubsection{Sample Set C}

\section{Example 8.3}

Estimate the product: $73 \cdot 46$.

Notice that 73 is near $\underbrace{70,}_{\begin{array}{c}\text { one nonzero } \\ \text { digit }\end{array}}$ and that 46 is near $\underbrace{50 .}_{\begin{array}{c}\text { one nonzero } \\ \text { digit }\end{array}}$

The product can be estimated by $70 \cdot 50=3,500$. (Recall that to multiply numbers ending in zeros, we multiply the nonzero digits and affix to this product the total number of ending zeros in the factors. See Section 2.2 for a review of this technique.)

Thus, $73 \cdot 46$ is about 3,500 . In fact, $73 \cdot 46=3,358$.

\section{Example 8.4}

Estimate the product: $87 \cdot 4,316$.

Notice that 87 is close to $\underbrace{90,}_{\begin{array}{c}\text { one nonzero } \\ \text { digit }\end{array}}$ and that 4,316 is close to $\underbrace{4,000}_{\begin{array}{c}\text { one nonzero } \\ \text { digit }\end{array}}$

The product can be estimated by $90 \cdot 4,000=360,000$.

Thus, $87 \cdot 4,316$ is about 360,000 . In fact, $87 \cdot 4,316=375,492$.

\subsubsection{Practice Set C}

Exercise 8.2.7

(Solution on p. 505.)

Estimate the product: $31 \cdot 87$.

Exercise 8.2.8

(Solution on p. 505.)

Estimate the product: $18 \cdot 42$.

Exercise 8.2.9

(Solution on p. 505.)

Estimate the product: $16 \cdot 94$. 


\subsubsection{Sample Set D}

\section{Example 8.5}

Estimate the quotient: $153 \div 17$.

Notice that 153 is close to $\underbrace{150,}_{\begin{array}{c}\text { two nonzero } \\ \text { digits }\end{array}}$ and that 17 is close to $\underbrace{15 .}_{\begin{array}{c}\text { two nonzero } \\ \text { digits }\end{array}}$

The quotient can be estimated by $150 \div 15=10$.

Thus, $153 \div 17$ is about 10 . In fact, $153 \div 17=9$.

\section{Example 8.6}

Estimate the quotient: $742,000 \div 2,400$.

Notice that 742,000 is close to $\underbrace{700,000}_{\text {one nonzero }}$, and that 2,400 is close to $\underbrace{2,000}_{\text {one nonzero }}$ $\begin{gathered}\text { one nonz } \\ \text { digit } \\ \text { digit }\end{gathered}$
digit

The quotient can be estimated by $700,000 \div 2,000=350$.

Thus, $742,000 \div 2,400$ is about 350 . In fact, $742,000 \div 2,400=309.1 \overline{6}$.

\subsubsection{Practice Set D}

Exercise 8.2.10

(Solution on p. 505.)

Estimate the quotient: $221 \div 18$.

Exercise 8.2.11

(Solution on p. 505.)

Estimate the quotient: $4,079 \div 381$.

Exercise 8.2.12

(Solution on p. 505.)

Estimate the quotient: $609,000 \div 16,000$.

\subsubsection{Sample Set E}

\section{Example 8.7}

Estimate the sum: $53.82+41.6$.

Notice that 53.82 is close to $\underbrace{54,}_{\begin{array}{c}\text { two nonzero } \\ \text { digits }\end{array}}$ and that 41.6 is close to $\underbrace{42 .}_{\begin{array}{c}\text { two nonzero } \\ \text { digits }\end{array}}$

The sum can be estimated by $54+42=96$.

Thus, $53.82+41.6$ is about 96. In fact, $53.82+41.6=95.42$. 


\subsubsection{Practice Set E}

Exercise 8.2.13

(Solution on p. 505.)

Estimate the sum: $61.02+26.8$.

Exercise 8.2.14

(Solution on p. 505.)

Estimate the sum: $109.12+137.88$.

\subsubsection{Sample Set F}

\section{Example 8.8}

Estimate the product: (31.28) (14.2).

Notice that 31.28 is close to $\underbrace{30,}_{\begin{array}{c}\text { one nonzero } \\ \text { digit }\end{array}}$ and that 14.2 is close to $\underbrace{15 .}_{\begin{array}{c}\text { two nonzero } \\ \text { digits }\end{array}}$

The product can be estimated by $30 \cdot 15=450 .(3 \cdot 15=45$, then affix one zero.)

Thus, (31.28) (14.2) is about 450. In fact, (31.28) (14.2) $=444.176$.

Example 8.9

Estimate $21 \%$ of 5.42 .

Notice that $21 \%=.21$ as a decimal, and that .21 is close to

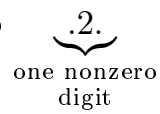

Notice also that 5.42 is close to

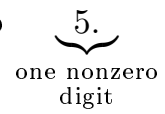

Then, $21 \%$ of 5.42 can be estimated by $(.2)(5)=1$.

Thus, $21 \%$ of 5.42 is about 1 . In fact, $21 \%$ of 5.42 is 1.1382 .

\subsubsection{Practice Set F}

Exercise 8.2.15

(Solution on p. 505.)

Estimate the product: (47.8) (21.1).

Exercise 8.2.16

Estimate $32 \%$ of 14.88 .

(Solution on p. 505.)

\subsubsection{Exercises}

Estimate each calculation using the method of rounding. After you have made an estimate, find the exact value and compare this to the estimated result to see if your estimated value is reasonable. Results may vary.

Exercise 8.2.17

(Solution on p. 505.)

$1,402+2,198$

Exercise 8.2.18

$3,481+4,216$ 
Exercise 8.2.19

(Solution on p. 505.)

$921+796$

Exercise 8.2.20

$611+806$

Exercise 8.2.21

(Solution on p. 505.)

$4,681+9,325$

Exercise 8.2.22

$6,476+7,814$

Exercise 8.2.23

$7,805-4,266$

(Solution on p. 505.)

Exercise 8.2.24

$8,427-5,342$

Exercise 8.2.25

$14,106-8,412$

Exercise 8.2.26

$26,486-18,931$

Exercise 8.2.27 $32 \cdot 53$

Exercise 8.2.28 $67 \cdot 42$

Exercise 8.2.29 $628 \cdot 891$

(Solution on p. 505.)

Exercise 8.2.30 $426 \cdot 741$

Exercise 8.2.31 $18,012 \cdot 32,416$

(Solution on p. 505.)

(Solution on p. 505.)

Exercise 8.2.32

$22,481 \cdot 51,076$

Exercise 8.2.33

$287 \div 19$

(Solution on p. 505.)

Exercise 8.2.34

$884 \div 33$

Exercise 8.2.35

$1,254 \div 57$

Exercise 8.2.36

$2,189 \div 42$

Exercise 8.2.37

$8,092 \div 239$

(Solution on p. 506.)

Exercise 8.2.38

$2,688 \div 48$

Exercise 8.2.39

$72.14+21.08$

Exercise 8.2.40 $43.016+47.58$

Exercise 8.2.41 $96.53-26.91$

(Solution on p. 506.)

(Solution on p. 506.) 
Exercise 8.2.42

$115.0012-25.018$

Exercise 8.2.43

(Solution on p. 506.)

$206.19+142.38$

Exercise 8.2.44

$592.131+211.6$

Exercise 8.2.45

(32.12) (48.7)

(Solution on p. 506.)

Exercise 8.2.46

(87.013) (21.07)

Exercise 8.2.47

(3.003) (16.52)

(Solution on p. 506.)

Exercise 8.2.48

(6.032) (14.091)

Exercise 8.2.49

(114.06) (384.3)

(Solution on p. 506.)

Exercise 8.2.50

$(5,137.118)(263.56)$

Exercise 8.2.51

(6.92) (0.88)

(Solution on p. 506.)

Exercise 8.2.52

(83.04) (1.03)

Exercise 8.2.53

(17.31) (.003)

(Solution on p. 506.)

Exercise 8.2.54

(14.016) (.016)

Exercise 8.2.55

$93 \%$ of 7.01

(Solution on p. 506.)

Exercise 8.2.56

$107 \%$ of 12.6

Exercise 8.2.57

$32 \%$ of 15.3

(Solution on p. 506.)

Exercise 8.2.58

$74 \%$ of 21.93

Exercise 8.2.59

$18 \%$ of 4.118

(Solution on p. 506.)

Exercise 8.2.60

$4 \%$ of .863

Exercise 8.2.61

$2 \%$ of .0039

(Solution on p. 506.) 


\subsubsection{Exercises for Review}

Exercise 8.2.62

(Section 5.3) Find the difference: $\frac{7}{10}-\frac{5}{16}$.

Exercise 8.2.63

(Solution on p. 506.)

(Section 5.6) Find the value $\frac{6-\frac{1}{4}}{6+\frac{1}{4}}$.

Exercise 8.2.64

(Section 6.3) Convert the complex decimal $1.11 \frac{1}{4}$ to a decimal.

Exercise 8.2.65

(Solution on p. 506.)

(Section 7.4) A woman 5 foot tall casts an 8-foot shadow at a particular time of the day. How tall is a tree that casts a 96 -foot shadow at the same time of the day?

Exercise 8.2.66

(Section 7.7 ) 11.62 is $83 \%$ of what number?

\subsection{Estimation by Clustering ${ }^{3}$}

\subsubsection{Section Overview}

- Estimation by Clustering

\section{Cluster}

When more than two numbers are to be added, the sum may be estimated using the clustering technique. The rounding technique could also be used, but if several of the numbers are seen to cluster (are seen to be close to) one particular number, the clustering technique provides a quicker estimate. Consider a sum such as

$32+68+29+73$

Notice two things:

1. There are more than two numbers to be added.

2. Clustering occurs.

(a) Both 68 and 73 cluster around 70 , so $68+73$ is close to $80+70=2(70)=140$.

$$
\stackrel{32+68+29+71}{\longleftarrow}
$$

(b) Both 32 and 29 cluster around 30 , so $32+29$ is close to $30+30=2(30)=60$.

The sum may be estimated by

$$
\begin{aligned}
(2 \cdot 30)+(2 \cdot 70) & =6+140 \\
& =\quad 200
\end{aligned}
$$

In fact, $32+68+29+73=202$.

\footnotetext{
${ }^{3}$ This content is available online at $<$ http://cnx.org/content/m35012/1.2/>.
} 


\subsubsection{Sample Set A}

Estimate each sum. Results may vary.

Example 8.10

$27+48+31+52$.

27 and 31 cluster near 30 . Their sum is about $2 \cdot 30=60$.

48 and 52 cluster near 50 . Their sum is about $2 \cdot 50=100$. Thus, $27+48+31+52$ is about $\begin{array}{rlc}(2 \cdot 30)+(2 \cdot 50) & = & 60+100 \\ & = & 160\end{array}$

In fact, $27+48+31+52=158$.

Example 8.11

$88+21+19+91$.

88 and 91 cluster near 90 . Their sum is about $2 \cdot 90=180$.

21 and 19 cluster near 20 . Their sum is about $2 \cdot 20=40$.

Thus, $88+21+19+91$ is about $\begin{array}{rlc}(2 \cdot 90)+(2 \cdot 20) & = & 180+40 \\ & = & 220\end{array}$

In fact, $88+21+19+91=219$.

Example 8.12

$17+21+48+18$.

17,21 , and 18 cluster near 20 . Their sum is about $3 \cdot 20=60$.

48 is about 50 .

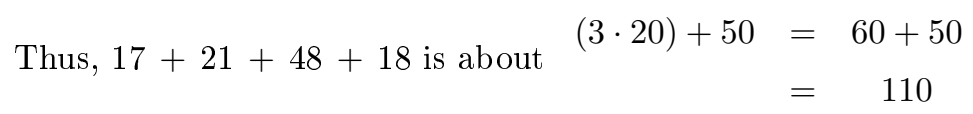

In fact, $17+21+48+18=104$.

Example 8.13

$61+48+49+57+52$.

61 and 57 cluster near 60 . Their sum is about $2 \cdot 60=120$.

48, 49, and 52 cluster near 50 . Their sum is about $3 \cdot 50=150$.

Thus, $61+48+49+57+52$ is about $\begin{array}{rlc}(2 \cdot 60)+(3 \cdot 50) & = & 120+150 \\ & = & 270\end{array}$

In fact, $61+48+49+57+52=267$.

Example 8.14

$706+321+293+684$.

706 and 684 cluster near 700 . Their sum is about $2 \cdot 700=1,400$. 
321 and 293 cluster near 300 . Their sum is about $2 \cdot 300=600$.

Thus, $706+321+293+684$ is about $(2 \cdot 700)+(2 \cdot 300)=1,400+600$

$$
=2,000
$$

In fact, $706+321+293+684=2,004$.

\subsubsection{Practice Set A}

Use the clustering method to estimate each sum.

Exercise 8.3.1

(Solution on p. 506.)

$28+51+31+47$

Exercise 8.3.2

(Solution on p. 506.)

$42+39+68+41$

Exercise 8.3.3

$37+39+83+42+79$

(Solution on p. 506.)

Exercise 8.3.4

$612+585+830+794$

(Solution on p. 506.)

\subsubsection{Exercises}

Use the clustering method to estimate each sum. Results may vary.

Exercise 8.3.5

(Solution on p. 506.)

$28+51+31+47$

Exercise 8.3.6

$42+19+39+23$

Exercise 8.3.7

$88+62+59+90$

(Solution on p. 506.)

Exercise 8.3.8

$76+29+33+82$

Exercise 8.3.9

$19+23+87+21$

(Solution on p. 506.)

Exercise 8.3.10

$41+28+42+37$

Exercise 8.3.11

$89+32+89+93$

(Solution on p. 506.)

Exercise 8.3.12

$73+72+27+71$

Exercise 8.3.13

$43+62+61+55$

(Solution on p. 506.)

Exercise 8.3.14

$31+77+31+27$

Exercise 8.3.15

(Solution on p. 507.)

$57+34+28+61+62$ 
Exercise 8.3.16

$94+18+23+91+19$

Exercise 8.3.17

(Solution on p. 507.)

$103+72+66+97+99$

Exercise 8.3.18

$42+121+119+124+41$

Exercise 8.3.19

$19+24+87+23+91+93$

(Solution on p. 507.)

Exercise 8.3.20

$108+61+63+96+57+99$

Exercise 8.3.21

(Solution on p. 507.)

$518+721+493+689$

Exercise 8.3.22

$981+1208+1214+1006$

Exercise 8.3.23

$23+81+77+79+19+81$

(Solution on p. 507.)

Exercise 8.3.24

$94+68+66+101+106+71+110$

\subsubsection{Exercises for Review}

Exercise 8.3.25

(Solution on p. 507.)

(Section 1.2) Specify all the digits greater than 6 .

Exercise 8.3.26

(Section 4.5) Find the product: $\frac{2}{3} \cdot \frac{9}{14} \cdot \frac{7}{12}$.

Exercise 8.3.27

(Solution on p. 507.)

(Section 6.3) Convert 0.06 to a fraction.

Exercise 8.3.28

(Section 7.3) Write the proportion in fractional form: "5 is to 8 as 25 is to 40."

Exercise 8.3.29

(Solution on p. 507.)

(Section 8.2) Estimate the sum using the method of rounding: 4,882 +2,704.

\subsection{Mental Arithmetic-Using the Distributive Property ${ }^{4}$}

\subsubsection{Section Overview}

- The Distributive Property

- Estimation Using the Distributive Property

\footnotetext{
${ }^{4}$ This content is available online at $<$ http://cnx.org/content/m35013/1.2/>.
} 


\subsubsection{The Distributive Property}

\section{Distributive Property}

The distributive property is a characteristic of numbers that involves both addition and multiplication. It is used often in algebra, and we can use it now to obtain exact results for a multiplication.

Suppose we wish to compute $3(2+5)$. We can proceed in either of two ways, one way which is known to us already (the order of operations), and a new way (the distributive property).

1. Compute $3(2+5)$ using the order of operations.

$$
3(2+5)
$$

Operate inside the parentheses first: $2+5=7$.

$3(2+5)=3 \cdot 7$

Now multiply 3 and 7 .

$3(2+5)=3 \cdot 7=21$

Thus, $3(2+5)=21$.

2. Compute $3(2+5)$ using the distributive property.

We know that multiplication describes repeated addition. Thus,

$$
\begin{array}{rlrl}
3(2+5) & =\underbrace{2+5+2+5+2+5}_{2+5 \text { appears } 3 \text { times }} \\
& =2+2+2+5+5+5 & \text { (by the commutative property of addition) } \\
& = & 3 \cdot 2+3 \cdot 5 & \text { (since multiplication describes repeated addition) } \\
& = & 6+15 &
\end{array}
$$

Thus, $3(2+5)=21$.

Let's look again at this use of the distributive property.

$$
\begin{aligned}
& 3(2+5)=\underbrace{2+5+2+5+2+5}_{2+5 \text { appears } 3 \text { times }} \\
& 3(2+5)=\underbrace{2+2+2}_{2 \text { appears } 3 \text { times }}+\underbrace{5+5+5}_{5 \text { appears } 3 \text { times }} \\
& \underbrace{3(2+5)}_{3 \text { times } 2}=3 \text { times } 5
\end{aligned}
$$

The 3 has been distributed to the 2 and 5 .

This is the distributive property. We distribute the factor to each addend in the parentheses. The distributive property works for both sums and differences. 


\subsubsection{Sample Set A}

Example 8.15

$$
\begin{aligned}
\underbrace{4(6+2)} & =4 \cdot 6+4 \cdot 2 \\
& =24+8 \\
& =32
\end{aligned}
$$

Using the order of operations, we get

$$
\begin{aligned}
4(6+2) & =4 \cdot 8 \\
& =32
\end{aligned}
$$

Example 8.16

$$
\begin{aligned}
8(9+6) & =8 \cdot 9+8 \cdot 6 \\
& =72+48 \\
& =120
\end{aligned}
$$

Using the order of operations, we get

$$
\begin{aligned}
8(9+6) & =8 \cdot 15 \\
& =120
\end{aligned}
$$

Example 8.17

$$
\begin{aligned}
\underbrace{(9-5)} & =4 \cdot 9-4 \cdot 5 \\
& =36-20 \\
& =16
\end{aligned}
$$

Example 8.18

$$
\begin{aligned}
25(20-3) & =25 \cdot 20-25 \cdot 3 \\
& =500-75 \\
& =425
\end{aligned}
$$

\subsubsection{Practice Set A}

Use the distributive property to compute each value.

Exercise 8.4.1

(Solution on p. 507.)

$6(8+4)$

Exercise 8.4.2

$4(4+7)$

Exercise 8.4.3

$8(2+9)$

Exercise 8.4.4

$12(10+3)$

Exercise 8.4.5

(Solution on p. 507.)

(Solution on p. 507.)

$6(11-3)$

Exercise 8.4.6

$8(9-7)$

(Solution on p. 507.)

(Solution on p. 507.)

(Solution on p. 507.)

Exercise 8.4.7 $15(30-8)$ 


\subsubsection{Estimation Using the Distributive Property}

We can use the distributive property to obtain exact results for products such as $25 \cdot 23$. The distributive property works best for products when one of the factors ends in 0 or 5 . We shall restrict our attention to only such products.

\subsubsection{Sample Set B}

Use the distributive property to compute each value.

\section{Example 8.19}

$25 \cdot 23$

Notice that $23=20+3$. We now write

$$
\begin{aligned}
25 \cdot 23 & =25(20+3) \\
& =25 \cdot 20+25 \cdot 3 \\
& =500+75 \\
& =575
\end{aligned}
$$

Thus, $25 \cdot 23=575$

We could have proceeded by writing 23 as $30-7$.

$$
\begin{aligned}
25 \cdot 23 & =25(30-7) \\
& =25 \cdot 30-25 \cdot 7 \\
& =750-175 \\
& =575
\end{aligned}
$$

\section{Example 8.20}

$$
15 \cdot 37
$$

Notice that $37=30+7$. We now write

$$
\begin{aligned}
15 \cdot 37 & =\underbrace{15(30+7)} \\
& =15 \cdot 30+15 \cdot 7 \\
& =450+105 \\
& =555
\end{aligned}
$$

Thus, $15 \cdot 37=555$

We could have proceeded by writing 37 as $40-3$.

$$
\begin{aligned}
15 \cdot 37 & =15(40-3) \\
& =15 \cdot 40-15 \cdot 3 \\
& =600-45 \\
& =555
\end{aligned}
$$

\section{Example 8.21}

$15 \cdot 86$

Notice that $86=80+6$. We now write 


$$
\begin{aligned}
15 \cdot 86 & =15(80+6) \\
& =15 \cdot 80+15 \cdot 6 \\
& =1,200+90 \\
& =1,290
\end{aligned}
$$

We could have proceeded by writing 86 as $90-4$.

$$
\begin{aligned}
15 \cdot 86 & =15(90-4) \\
& =15 \cdot 90-15 \cdot 4 \\
& =1,350-60 \\
& =1,290
\end{aligned}
$$

\subsubsection{Practice Set B}

Use the distributive property to compute each value.

Exercise 8.4.8

(Solution on p. 507.)

$25 \cdot 12$

Exercise 8.4.9

(Solution on p. 507.)

$35 \cdot 14$

Exercise 8.4.10

(Solution on p. 507.)

$80 \cdot 58$

Exercise 8.4.11

$65 \cdot 62$

(Solution on p. 507.)

\subsubsection{Exercises}

Use the distributive property to compute each product.

Exercise 8.4.12

(Solution on p. 507.)

$15 \cdot 13$

Exercise 8.4.13

$15 \cdot 14$

Exercise 8.4.14

(Solution on p. 507.)

$25 \cdot 11$

Exercise 8.4.15

$25 \cdot 16$

Exercise 8.4.16

$15 \cdot 16$

(Solution on p. 507.)

Exercise 8.4.17

$35 \cdot 12$

Exercise 8.4.18

(Solution on p. 507.)

$45 \cdot 83$

Exercise 8.4.19

$45 \cdot 38$

Exercise 8.4.20

$25 \cdot 38$

(Solution on p. 507.) 
Exercise 8.4.21

$25 \cdot 96$

Exercise 8.4.22

(Solution on p. 507.)

$75 \cdot 14$

Exercise 8.4.23

$85 \cdot 34$

Exercise 8.4.24

(Solution on p. 508.)

$65 \cdot 26$

Exercise 8.4.25

$55 \cdot 51$

Exercise 8.4.26

(Solution on p. 508.) $15 \cdot 107$

Exercise 8.4.27 $25 \cdot 208$

Exercise 8.4.28 $35 \cdot 402$

(Solution on p. 508.)

Exercise 8.4.29 $85 \cdot 110$

Exercise 8.4.30

(Solution on p. 508.) $95 \cdot 12$

Exercise 8.4.31 $65 \cdot 40$

Exercise 8.4.32 $80 \cdot 32$

(Solution on p. 508.)

Exercise 8.4.33 $30 \cdot 47$

Exercise 8.4.34 $50 \cdot 63$

(Solution on p. 508.)

Exercise 8.4.35 $90 \cdot 78$

Exercise 8.4.36 $40 \cdot 89$

(Solution on p. 508.)

\subsubsection{Exercises for Review}

Exercise 8.4.37

(Section 3.5) Find the greatest common factor of 360 and 3,780.

Exercise 8.4.38

(Section 4.5) Reduce $\frac{594}{5,148}$ to lowest terms.

(Solution on p. 508.)

Exercise 8.4.39

(Section 4.7 ) $1 \frac{5}{9}$ of $2 \frac{4}{7}$ is what number?

Exercise 8.4.40

(Section 7.3) Solve the proportion: $\frac{7}{15}=\frac{x}{90}$.

(Solution on p. 508.)

Exercise 8.4.41

(Section 8.3) Use the clustering method to estimate the sum: $88+106+91+114$. 


\subsection{Estimation by Rounding Fractions ${ }^{5}$}

\subsubsection{Section Overview}

- Estimation by Rounding Fractions

Estimation by rounding fractions is a useful technique for estimating the result of a computation involving

fractions. Fractions are commonly rounded to $\frac{1}{4}, \frac{1}{2}, \frac{3}{4}, 0$, and 1 . Remember that rounding may cause estimates to vary.

\subsubsection{Sample Set A}

Make each estimate remembering that results may vary.

\section{Example 8.22}

Estimate $\frac{3}{5}+\frac{5}{12}$.

Notice that $\frac{3}{5}$ is about $\frac{1}{2}$, and that $\frac{5}{12}$ is about $\frac{1}{2}$.

Thus, $\frac{3}{5}+\frac{5}{12}$ is about $\frac{1}{2}+\frac{1}{2}=1$. In fact, $\frac{3}{5}+\frac{5}{12}=\frac{61}{60}$, a little more than 1 .

Example 8.23

Estimate $5 \frac{3}{8}+4 \frac{9}{10}+11 \frac{1}{5}$.

Adding the whole number parts, we get 20 . Notice that $\frac{3}{8}$ is close to $\frac{1}{4}, \frac{9}{10}$ is close to 1 , and $\frac{1}{5}$ is close to $\frac{1}{4}$. Then $\frac{3}{8}+\frac{9}{10}+\frac{1}{5}$ is close to $\frac{1}{4}+1+\frac{1}{4}=1 \frac{1}{2}$.

Thus, $5 \frac{3}{8}+4 \frac{9}{10}+11 \frac{1}{5}$ is close to $20+1 \frac{1}{2}=21 \frac{1}{2}$.

In fact, $5 \frac{3}{8}+4 \frac{9}{10}+11 \frac{1}{5}=21 \frac{19}{40}$, a little less than $21 \frac{1}{2}$.

\subsubsection{Practice Set A}

Use the method of rounding fractions to estimate the result of each computation. Results may vary.

Exercise 8.5.1

$$
\frac{5}{8}+\frac{5}{12}
$$

Exercise 8.5.2

$$
\frac{7}{9}+\frac{3}{5}
$$

Exercise 8.5.3

$$
8 \frac{4}{15}+3 \frac{7}{10}
$$

Exercise 8.5.4

$$
16 \frac{1}{20}+4 \frac{7}{8}
$$

(Solution on p. 508.)

(Solution on p. 508.)

(Solution on p. 508.)

(Solution on p. 508.)

\footnotetext{
${ }^{5}$ This content is available online at $<$ http://cnx.org/content $/ \mathrm{m} 35014 / 1.2 />$.
} 


\subsubsection{Exercises}

Estimate each sum or difference using the method of rounding. After you have made an estimate, find the exact value of the sum or difference and compare this result to the estimated value. Result may vary.

Exercise 8.5.5

(Solution on p. 508.)

$$
\frac{5}{6}+\frac{7}{8}
$$

Exercise 8.5.6

$$
\frac{3}{8}+\frac{11}{12}
$$

Exercise 8.5.7

$$
\frac{9}{10}+\frac{3}{5}
$$

(Solution on p. 508.)

Exercise 8.5.8

$$
\frac{13}{15}+\frac{1}{20}
$$

Exercise 8.5.9

$$
\frac{3}{20}+\frac{6}{25}
$$

(Solution on p. 508.)

Exercise 8.5.10

$$
\frac{1}{12}+\frac{4}{5}
$$

Exercise 8.5.11 $\frac{15}{16}+\frac{1}{12}$

Exercise 8.5.12 $\frac{29}{30}+\frac{11}{20}$

Exercise 8.5.13 $\frac{5}{12}+6 \frac{4}{11}$

(Solution on p. 508.)

Exercise 8.5.14 $\frac{3}{7}+8 \frac{4}{15}$

Exercise 8.5.15 $\frac{9}{10}+2 \frac{3}{8}$

(Solution on p. 508.)

Exercise 8.5.16 $\frac{19}{20}+15 \frac{5}{9}$

Exercise 8.5.17 $8 \frac{3}{5}+4 \frac{1}{20}$

Exercise 8.5.18 $5 \frac{3}{20}+2 \frac{8}{15}$

Exercise 8.5.19 $9 \frac{1}{15}+6 \frac{4}{5}$

(Solution on p. 508.)

Exercise 8.5.20 $7 \frac{5}{12}+10 \frac{1}{16}$

Exercise 8.5.21 $3 \frac{11}{20}+2 \frac{13}{25}+1 \frac{7}{8}$

(Solution on p. 508.)

Exercise 8.5.22 $6 \frac{1}{12}+1 \frac{1}{10}+5 \frac{5}{6}$

Exercise 8.5.23 $\frac{15}{16}-\frac{7}{8}$

(Solution on p. 508.)

Exercise 8.5.24 $\frac{12}{25}-\frac{9}{20}$ 


\subsubsection{Exercises for Review}

Exercise 8.5.25

(Section 2.6) The fact that

(a first number - a second number)

(a second number · a third number)

is an example of which property of multiplication?

Exercise 8.5.26

(Section 4.6) Find the quotient: $\frac{14}{15} \div \frac{4}{45}$.

Exercise 8.5.27

(Section 5.4) Find the difference: $3 \frac{5}{9}-2 \frac{2}{3}$.

Exercise 8.5.28

(Section 6.8) Find the quotient: $4.6 \div 0.11$.

Exercise 8.5.29

(Section 8.4) Use the distributive property to compute the product: $25 \cdot 37$.
(Solution on p. 508.)

a first number

(Solution on p. 508.)

(Solution on p. 509.)

Available for free at Connexions $<$ http://cnx.org/content/col10615/1.4 $>$ 


\subsection{Summary of Key Concepts ${ }^{6}$}

\subsubsection{Summary of Key Concepts}

Estimation (Section 8.2)

Estimation is the process of determining an expected value of a computation.

\section{Estimation By Rounding (Section 8.2)}

The rounding technique estimates the result of a computation by rounding the numbers involved in the computation to one or two nonzero digits. For example, $512+896$ can be estimated by $500+900=1,400$.

\section{Cluster (Section 8.3)}

When several numbers are close to one particular number, they are said to cluster near that particular number.

\section{Estimation By Clustering (Section 8.3)}

The clustering technique of estimation can be used when

1. there are more than two numbers to be added, and

2. clustering occurs.

For example, $31+62+28+59$ can be estimated by $(2 \cdot 30)+(2 \cdot 60)=60+120=180$

Distributive Property (Section 8.4)

The distributive property is a characteristic of numbers that involves both addition and multiplication. For example,

$3(4+6)=3 \cdot 4+3 \cdot 6=12+18=30$

Estimation Using the Distributive Property (Section 8.4)

The distributive property can be used to obtain exact results for a multiplication.

For example,

$15 \cdot 23=15 \cdot(20+3)=15 \cdot 20+15 \cdot 3=300+45=345$

Estimation by Rounding Fractions (Section 8.5)

Estimation by rounding fractions commonly rounds fractions to $\frac{1}{4}, \frac{1}{2}, \frac{3}{4}, 0$, and 1 .

For example,

$\frac{5}{12}+\frac{5}{16}$ can be estimated by $\frac{1}{2}+\frac{1}{4}=\frac{3}{4}$

\subsection{Exercise Supplement ${ }^{7}$}

\subsubsection{Exercise Supplement}

\subsubsection{Estimation by Rounding (Section 8.2)}

For problems 1-70, estimate each value using the method of rounding. After you have made an estimate, find the exact value. Compare the exact and estimated values. Results may vary.

Exercise 8.7.1

(Solution on p. 509.)

$286+312$

Exercise 8.7.2

$$
419+582
$$

\footnotetext{
${ }^{6}$ This content is available online at $<\mathrm{http}: / / \mathrm{cnx} .0 \mathrm{rg} /$ content $/ \mathrm{m} 35015 / 1.2 />$.

${ }^{7}$ This content is available online at $<$ http://cnx.org/content $/ \mathrm{m} 35016 / 1.2 />$.
} 
Exercise 8.7.3

(Solution on p. 509.)

$689+511$

Exercise 8.7.4 $926+1,105$

Exercise 8.7.5

(Solution on p. 509.)

$1,927+3,017$

Exercise 8.7.6

$5,026+2,814$

Exercise 8.7.7

$1,408+2,352$

(Solution on p. 509.)

Exercise 8.7.8 $1,186+4,228$

Exercise 8.7.9

$5,771+246$

(Solution on p. 509.)

Exercise 8.7.10

$8,305+484$

Exercise 8.7.11 $3,812+2,906$

(Solution on p. 509.)

Exercise 8.7.12 $5,293+8,007$

Exercise 8.7.13 $28,481+32,856$

(Solution on p. 509.)

Exercise 8.7.14 $92,512+26,071$

Exercise 8.7.15 $87,612+2,106$

(Solution on p. 509.)

Exercise 8.7.16 $42,612+4,861$

Exercise 8.7.17 $212,413+609$

(Solution on p. 509.)

Exercise 8.7.18 $487,235+494$

Exercise 8.7.19 $2,409+1,526$

Exercise 8.7.20 $3,704+4,704$

Exercise 8.7.21 $41 \cdot 63$

Exercise 8.7.22 $38 \cdot 81$

Exercise 8.7.23 $18 \cdot 28$

(Solution on p. 509.)

Exercise 8.7.24 $52 \cdot 21$

Exercise 8.7.25 $307 \cdot 489$

(Solution on p. 509.)

(Solution on p. 509.)

(Solution on p. 509.) 
Exercise 8.7.26

$412 \cdot 807$

Exercise 8.7.27

(Solution on p. 509.) $77 \cdot 614$

Exercise 8.7.28 $62 \cdot 596$

Exercise 8.7.29

(Solution on p. 509.) $27 \cdot 473$

Exercise 8.7.30 $92 \cdot 336$

Exercise 8.7.31

(Solution on p. 509.) $12 \cdot 814$

Exercise 8.7.32 $8 \cdot 2,106$

Exercise 8.7.33 $192 \cdot 452$

(Solution on p. 509.)

Exercise 8.7.34 $374 \cdot 816$

Exercise 8.7.35 $88 \cdot 4,392$

(Solution on p. 509.)

Exercise 8.7.36 $126 \cdot 2,834$

Exercise 8.7.37 $3,896 \cdot 413$

Exercise 8.7.38 $5,794 \cdot 837$

Exercise 8.7.39 $6,311 \cdot 3,512$

(Solution on p. 509.)

Exercise 8.7.40 $7,471 \cdot 5,782$

Exercise 8.7.41 $180 \div 12$

(Solution on p. 509.)

Exercise 8.7.42 $309 \div 16$

Exercise 8.7.43 $286 \div 22$

(Solution on p. 509.)

Exercise 8.7.44 $527 \div 17$

Exercise 8.7.45 $1,007 \div 19$

(Solution on p. 509.)

Exercise 8.7.46 $1,728 \div 36$

Exercise 8.7.47 $2,703 \div 53$

(Solution on p. 509.)

Exercise 8.7.48

$2,562 \div 61$ 
Exercise 8.7.49

(Solution on p. 510.)

$1,260 \div 12$

Exercise 8.7.50

$3,618 \div 18$

Exercise 8.7.51

(Solution on p. 510.)

$3,344 \div 76$

Exercise 8.7.52

$7,476 \div 356$

Exercise 8.7.53

$20,984 \div 488$

(Solution on p. 510.)

Exercise 8.7.54

$43,776 \div 608$

Exercise 8.7.55

$7,196 \div 514$

(Solution on p. 510.)

Exercise 8.7.56

$51,492 \div 514$

Exercise 8.7.57

$26,962 \div 442$

(Solution on p. 510.)

Exercise 8.7.58

$33,712 \div 112$

Exercise 8.7.59

$105,152 \div 106$

(Solution on p. 510.)

Exercise 8.7.60 $176,978 \div 214$

Exercise 8.7.61 $48.06+23.11$

(Solution on p. 510.)

Exercise 8.7.62 $73.73+72.9$

Exercise 8.7.63 $62.91+56.4$

(Solution on p. 510.)

Exercise 8.7.64 $87.865+46.772$

Exercise 8.7.65 $174.6+97.2$

Exercise 8.7.66 (48.3) (29.6)

Exercise 8.7.67 (87.11) (23.2)

Exercise 8.7.68 (107.02) (48.7)

Exercise 8.7.69 (0.76) (5.21)

Exercise 8.7.70 (1.07) (13.89) 


\subsubsection{Estimation by Clustering (Section 8.3)}

For problems 71-90, estimate each value using the method of clustering. After you have made an estimate, find the exact value. Compare the exact and estimated values. Results may vary.

Exercise 8.7.71

(Solution on p. 510.)

$38+51+41+48$

Exercise 8.7.72

$19+73+23+71$

Exercise 8.7.73

$27+62+59+31$

(Solution on p. 510.)

Exercise 8.7.74

$18+73+69+19$

Exercise 8.7.75

$83+49+79+52$

(Solution on p. 510.)

Exercise 8.7.76

$67+71+84+81$

Exercise 8.7.77

$16+13+24+26$

(Solution on p. 510.)

Exercise 8.7.78

$34+56+36+55$

Exercise 8.7.79

$14+17+83+87$

(Solution on p. 510.)

Exercise 8.7.80

$93+108+96+111$

Exercise 8.7.81

$18+20+31+29+24+38$

(Solution on p. 510.)

Exercise 8.7.82

$32+27+48+51+72+69$

Exercise 8.7.83

$64+17+27+59+31+21$

(Solution on p. 510.)

Exercise 8.7.84

$81+41+92+38+88+80$

Exercise 8.7.85

$87+22+91$

(Solution on p. 510.)

Exercise 8.7.86

$44+38+87$

Exercise 8.7.87

$19+18+39+22+42$

(Solution on p. 510.)

Exercise 8.7.88

$31+28+49+29$

Exercise 8.7.89

$88+86+27+91+29$

(Solution on p. 510.)

Exercise 8.7.90

$57+62+18+23+61+21$ 
8.7.1.3 Mental Arithmetic- Using the Distributive Property (Section 8.4)

For problems 91-110, compute each product using the distributive property.

Exercise 8.7.91

(Solution on p. 510.) $15 \cdot 33$

Exercise 8.7.92 $15 \cdot 42$

Exercise 8.7.93 $35 \cdot 36$

(Solution on p. 510.)

Exercise 8.7.94 $35 \cdot 28$

Exercise 8.7.95 $85 \cdot 23$

(Solution on p. 510.)

Exercise 8.7.96 $95 \cdot 11$

Exercise 8.7.97 $30 \cdot 14$

(Solution on p. 510.)

Exercise 8.7.98 $60 \cdot 18$

Exercise 8.7.99 $75 \cdot 23$

(Solution on p. 511.)

Exercise 8.7.100 $65 \cdot 31$

Exercise 8.7.101 $17 \cdot 15$

Exercise 8.7.102 $38 \cdot 25$

Exercise 8.7.103 $14 \cdot 65$

(Solution on p. 511.)

Exercise 8.7.104 $19 \cdot 85$

Exercise 8.7.105 $42 \cdot 60$

(Solution on p. 511.)

Exercise 8.7.106 $81 \cdot 40$

Exercise 8.7.107 $15 \cdot 105$

Exercise 8.7.108 $35 \cdot 202$

Exercise 8.7.109 $45 \cdot 306$

(Solution on p. 511.)

Exercise 8.7.110 $85 \cdot 97$ 


\subsubsection{Estimation by Rounding Fractions (Section 8.5)}

For problems 111-125, estimate each sum using the method of rounding fractions. After you have made an estimate, find the exact value. Compare the exact and estimated values. Results may vary.

Exercise 8.7.111

(Solution on p. 511.) $\frac{3}{8}+\frac{5}{6}$

Exercise 8.7.112 $\frac{7}{16}+\frac{1}{24}$

Exercise 8.7.113 $\frac{7}{15}+\frac{13}{30}$

(Solution on p. 511.)

Exercise 8.7.114 $\frac{14}{15}+\frac{19}{20}$

Exercise 8.7.115 $\frac{13}{25}+\frac{7}{30}$

(Solution on p. 511.)

Exercise 8.7.116 $\frac{11}{12}+\frac{7}{8}$

Exercise 8.7.117 $\frac{9}{32}+\frac{15}{16}$

Exercise 8.7.118 $\frac{5}{8}+\frac{1}{32}$

Exercise 8.7.119 $2 \frac{3}{4}+6 \frac{3}{5}$

(Solution on p. 511.)

Exercise 8.7.120 $4 \frac{5}{9}+8 \frac{1}{27}$

Exercise 8.7.121 $11 \frac{5}{18}+7 \frac{22}{45}$

Exercise 8.7.122 $14 \frac{19}{36}+2 \frac{7}{18}$

Exercise 8.7.123 $6 \frac{1}{20}+2 \frac{1}{10}+8 \frac{13}{60}$

(Solution on p. 511.)

Exercise 8.7.124 $5 \frac{7}{8}+1 \frac{1}{4}+12 \frac{5}{12}$

Exercise 8.7.125 $10 \frac{1}{2}+6 \frac{15}{16}+8 \frac{19}{80}$

(Solution on p. 511.)

(Solution on p. 511.)

(Solution on p. 511.)

\subsection{Proficiency Exam ${ }^{8}$}

\subsubsection{Proficiency Exam}

For problems 1 - 16, estimate each value. After you have made an estimate, find the exact value. Results may vary.

Exercise 8.8.1

(Solution on p. 511.)

(Section 8.2) 3,716 + 6,789

Exercise 8.8.2

(Solution on p. 511.)

(Section 8.2) 8,821 + 9,217

${ }^{8}$ This content is available online at $<$ http://cnx.org/content/m35017/1.2/>. 
Exercise 8.8.3

(Solution on p. 511.)

(Section 8.2) 7,316-2,305

Exercise 8.8.4

(Solution on p. 511.)

(Section 8.2) 110, 812 - 83, 406

Exercise 8.8.5

(Solution on p. 511.)

(Section 8.2) $82 \cdot 38$

Exercise 8.8.6

(Solution on p. 511.)

(Section 8.2) $51 \cdot 92$

Exercise 8.8.7

(Section 8.2) $48 \cdot 6,012$

Exercise 8.8.8

(Section 8.2) $238 \div 17$

Exercise 8.8.9

(Section 8.2 ) $2,660 \div 28$

Exercise 8.8.10

(Section 8.2) $43.06+37.94$

Exercise 8.8.11

(Section 8.2) $307.006+198.0005$

Exercise 8.8.12

(Section 8.2) (47.2) (92.8)

Exercise 8.8.13

$($ Section 8.3$) 58+91+61+88$

Exercise 8.8.14

(Section 8.3) $43+39+89+92$

Exercise 8.8.15

(Section 8.3) $81+78+27+79$

Exercise 8.8.16

(Section 8.3) $804+612+801+795+606$

(Solution on p. 511.)

(Solution on p. 511.)

(Solution on p. 511.)

(Solution on p. 511.)

(Solution on p. 511.)

(Solution on p. 512.)

(Solution on p. 512.)

(Solution on p. 512.)

(Solution on p. 512.)

(Solution on p. 512.)

For problems 17-21, use the distributive property to obtain the exact result.

Exercise 8.8.17

(Solution on p. 512.)

(Section 8.4) 25 14

Exercise 8.8.18

(Solution on p. 512.)

(Section 8.4) $15 \cdot 83$

Exercise 8.8.19

(Solution on p. 512.)

(Section 8.4) $65 \cdot 98$

Exercise 8.8.20

(Solution on p. 512.)

(Section 8.4) $80 \cdot 107$

Exercise 8.8.21

(Solution on p. 512.)

(Section 8.4) $400 \cdot 215$

For problems 22-25, estimate each value. After you have made an estimate, find the exact value. Results may vary.

Exercise 8.8.22

(Section 8.5) $\frac{15}{16}+\frac{5}{8}$

Exercise 8.8.23

(Solution on p. 512.)

(Section 8.5$) \frac{1}{25}+\frac{11}{20}+\frac{17}{30}$

(Solution on p. 512.) 
Exercise 8.8.24

(Solution on p. 512.)

(Section 8.5) $8 \frac{9}{16}+14 \frac{1}{12}$

Exercise 8.8.25

(Section 8.5) $5 \frac{4}{9}+1 \frac{17}{36}+6 \frac{5}{12}$

(Solution on p. 512.) 


\section{Solutions to Exercises in Chapter 8}

Solution to Exercise 8.2.1 (p. 478)

$4,216+3,942: 4,200+3,900$. About 8,100. In fact, 8,158.

Solution to Exercise 8.2.2 (p. 478)

$812+514: 800+500$. About 1,300. In fact, 1,326.

Solution to Exercise 8.2.3 (p. 478)

43, $892+92,106: 44,000+92,000$. About 136,000. In fact, 135,998.

Solution to Exercise 8.2.4 (p. 479)

$628-413: 600-400$. About 200. In fact, 215.

Solution to Exercise 8.2.5 (p. 479)

7, $842-5,209: 7,800-5,200$. About 2,600. In fact, 2,633.

Solution to Exercise 8.2.6 (p. 479)

73,812 - 28, 492 : 74, $000-28,000$. About 46,000. In fact, 45,320.

Solution to Exercise 8.2.7 (p. 479)

$31 \cdot 87: 30 \cdot 90$. About 2,700. In fact, 2,697.

Solution to Exercise 8.2.8 (p. 479)

$18 \cdot 42: 20 \cdot 40$. About 800 . In fact, 756 .

Solution to Exercise 8.2.9 (p. 479)

$16 \cdot 94: 15 \cdot 100$. About 1,500. In fact, 1,504.

Solution to Exercise 8.2.10 (p. 480)

$221 \div 18: 200 \div 20$. About 10 . In fact, 12.27 .

Solution to Exercise 8.2.11 (p. 480)

$4,079 \div 381: 4,000 \div 400$. About 10 . In fact, $10.70603675 \ldots$

Solution to Exercise 8.2.12 (p. 480)

$609,000 \div 16,000: 600,000 \div 15,000$. About 40. In fact, 38.0625 .

Solution to Exercise 8.2.13 (p. 481)

$61.02+26.8: 61+27$. About 88. In fact, 87.82.

Solution to Exercise 8.2.14 (p. 481)

$109.12+137.88: 110+138$. About 248. In fact, 247 . We could have estimated 137.88 with 140 . Then

$110+140$ is an easy mental addition. We would conclude then that $109.12+137.88$ is about 250 .

Solution to Exercise 8.2.15 (p. 481)

(47.8) (21.1) : (50) (20). About 1,000. In fact, 1,008.58.

Solution to Exercise 8.2.16 (p. 481)

$32 \%$ of $14.88:(.3)(15)$. About 4.5. In fact, 4.7616 .

Solution to Exercise 8.2.17 (p. 481)

about 3,600 ; in fact 3,600

Solution to Exercise 8.2.19 (p. 482)

about 1,700 ; in fact 1,717

Solution to Exercise 8.2.21 (p. 482)

about 14,000; in fact 14,006

Solution to Exercise 8.2.23 (p. 482)

about 3,500 ; in fact 3,539

Solution to Exercise 8.2.25 (p. 482)

about 5,700; in fact 5,694

Solution to Exercise 8.2.27 (p. 482)

about 1,500 ; in fact 1,696

Solution to Exercise 8.2.29 (p. 482)

about 540,000 ; in fact 559,548

Solution to Exercise 8.2.31 (p. 482)

about $583,200,000$; in fact $583,876,992$ 
Solution to Exercise 8.2.33 (p. 482) about 15; in fact 15.11

Solution to Exercise 8.2.35 (p. 482) about 20; in fact 22

Solution to Exercise 8.2.37 (p. 482) about 33 ; in fact 33.86

Solution to Exercise 8.2.39 (p. 482) about 93.2 ; in fact 93.22

Solution to Exercise 8.2.41 (p. 482) about 70 ; in fact 69.62

Solution to Exercise 8.2.43 (p. 483) about 348.6 ; in fact 348.57

Solution to Exercise 8.2.45 (p. 483) about 1,568.0; in fact 1,564.244

Solution to Exercise 8.2.47 (p. 483) about 49.5 ; in fact 49.60956

Solution to Exercise 8.2.49 (p. 483) about 43,776 ; in fact $43,833.258$

Solution to Exercise 8.2.51 (p. 483) about 6.21 ; in fact 6.0896

Solution to Exercise 8.2.53 (p. 483) about 0.0519 ; in fact 0.05193

Solution to Exercise 8.2.55 (p. 483) about 6.3 ; in fact 6.5193

Solution to Exercise 8.2.57 (p. 483) about 4.5 ; in fact 4.896

Solution to Exercise 8.2.59 (p. 483) about 0.8 ; in fact 0.74124

Solution to Exercise 8.2.61 (p. 483) about 0.00008 ; in fact 0.000078

Solution to Exercise 8.2.63 (p. 484) $\frac{23}{25}$

Solution to Exercise 8.2.65 (p. 484) 60 feet tall

Solution to Exercise 8.3.1 (p. 486)

$(2 \cdot 30)+(2 \cdot 50)=60+100=160$

Solution to Exercise 8.3.2 (p. 486)

$(3 \cdot 40)+70=120+70=190$

Solution to Exercise 8.3.3 (p. 486)

$(3 \cdot 40)+(2 \cdot 80)=120+160=280$

Solution to Exercise 8.3.4 (p. 486)

$(2 \cdot 600)+(2 \cdot 800)=1,200+1,600=2,800$

Solution to Exercise 8.3.5 (p. 486)

$2(30)+2(50)=160 \quad(157)$

Solution to Exercise 8.3.7 (p. 486)

$2(90)+2(60)=300 \quad(299)$

Solution to Exercise 8.3.9 (p. 486)

$3(20)+90=150 \quad(150)$

Solution to Exercise 8.3.11 (p. 486)

$3(90)+30=300 \quad(303)$ 
Solution to Exercise 8.3.13 (p. 486) $40+3(60)=220 \quad(221)$

Solution to Exercise 8.3.15 (p. 486) $3(60)+2(30)=240 \quad(242)$

Solution to Exercise 8.3.17 (p. 487) $3(100)+2(70)=440 \quad(437)$

Solution to Exercise 8.3.19 (p. 487) $3(20)+3(90)=330 \quad(337)$

Solution to Exercise 8.3.21 (p. 487) $2(500)+2(700)=2,400 \quad(2,421)$

Solution to Exercise 8.3.23 (p. 487) $2(20)+4(80)=360 \quad(360)$

Solution to Exercise 8.3.25 (p. 487) $7,8,9$

Solution to Exercise 8.3.27 (p. 487) $\frac{3}{50}$

Solution to Exercise 8.3.29 (p. 487) $4,900+2,700=7,600 \quad(7,586)$

Solution to Exercise 8.4.1 (p. 489) $6 \cdot 8+6 \cdot 4=48+24=72$

Solution to Exercise 8.4.2 (p. 489) $4 \cdot 4+4 \cdot 7=16+28=44$

Solution to Exercise 8.4.3 (p. 489) $8 \cdot 2+8 \cdot 9=16+72=88$

Solution to Exercise 8.4.4 (p. 489) $12 \cdot 10+12 \cdot 3=120+36=156$

Solution to Exercise 8.4.5 (p. 489) $6 \cdot 11-6 \cdot 3=66-18=48$

Solution to Exercise 8.4.6 (p. 489) $8 \cdot 9--8 \cdot 7=72--56=16$

Solution to Exercise 8.4.7 (p. 489) $15 \cdot 30-15 \cdot 8=450-120=330$

Solution to Exercise 8.4.8 (p. 491) $25(10+2)=25 \cdot 10+25 \cdot 2=250+50=300$

Solution to Exercise 8.4.9 (p. 491)

$35(10+4)=35 \cdot 10+35 \cdot 4=350+140=490$

Solution to Exercise 8.4.10 (p. 491)

$80(50+8)=80 \cdot 50+80 \cdot 8=4,000+640=4,640$

Solution to Exercise 8.4.11 (p. 491)

$65(60+2)=65 \cdot 60+65 \cdot 2=3,900+130=4,030$

Solution to Exercise 8.4.12 (p. 491)

$15(10+3)=150+45=195$

Solution to Exercise 8.4.14 (p. 491)

$25(10+1)=250+25=275$

Solution to Exercise 8.4.16 (p. 491)

$15(20-4)=300-60=240$

Solution to Exercise 8.4.18 (p. 491)

$45(80+3)=3600+135=3735$

Solution to Exercise 8.4.20 (p. 491) $25(40-2)=1,000-50=950$ 
Solution to Exercise 8.4.22 (p. 492)

$75(10+4)=750+300=1,050$

Solution to Exercise 8.4.24 (p. 492)

$65(20+6)=1,300+390=1,690$ or $65(30-4)=1,950-260=1,690$

Solution to Exercise 8.4.26 (p. 492)

$15(100+7)=1,500+105=1,605$

Solution to Exercise 8.4.28 (p. 492)

$35(400+2)=14,000+70=14,070$

Solution to Exercise 8.4.30 (p. 492)

$95(10+2)=950+190=1,140$

Solution to Exercise 8.4.32 (p. 492)

$80(30+2)=2,400+160=2,560$

Solution to Exercise 8.4.34 (p. 492)

$50(60+3)=3,000+150=3,150$

Solution to Exercise 8.4.36 (p. 492)

$40(90-1)=3,600-40=3,560$

Solution to Exercise 8.4.38 (p. 492)

$\frac{3}{26}$

Solution to Exercise 8.4.40 (p. 492)

$x=42$

Solution to Exercise 8.5.1 (p. 493)

Results may vary. $\frac{1}{2}+\frac{1}{2}=1$. In fact, $\frac{5}{8}+\frac{5}{12}=\frac{25}{24}=1 \frac{1}{24}$

Solution to Exercise 8.5.2 (p. 493)

Results may vary. $1+\frac{1}{2}=1 \frac{1}{2}$. In fact, $\frac{7}{9}+\frac{3}{5}=1 \frac{17}{45}$

Solution to Exercise 8.5.3 (p. 493)

Results may vary. $8 \frac{1}{4}+3 \frac{3}{4}=11+1=12$. In fact, $8 \frac{4}{15}+3 \frac{7}{10}=11 \frac{29}{30}$

Solution to Exercise 8.5.4 (p. 493)

Results may vary. $(16+0)+(4+1)=16+5=21$. In fact, $16 \frac{1}{20}+4 \frac{7}{8}=20 \frac{37}{40}$

Solution to Exercise 8.5.5 (p. 494)

$1+1=2\left(1 \frac{17}{24}\right)$

Solution to Exercise 8.5.7 (p. 494)

$1+\frac{1}{2}=1 \frac{1}{2}\left(1 \frac{1}{2}\right)$

Solution to Exercise 8.5.9 (p. 494)

$\frac{1}{4}+\frac{1}{4}=\frac{1}{2}\left(\frac{39}{100}\right)$

Solution to Exercise 8.5.11 (p. 494)

$1+0=1\left(1 \frac{1}{48}\right)$

Solution to Exercise 8.5.13 (p. 494)

$\frac{1}{2}+6 \frac{1}{2}=7\left(6 \frac{103}{132}\right)$

Solution to Exercise 8.5.15 (p. 494)

$1+2 \frac{1}{2}=3 \frac{1}{2}\left(3 \frac{11}{40}\right)$

Solution to Exercise 8.5.17 (p. 494)

$8 \frac{1}{2}+4=12 \frac{1}{2}\left(12 \frac{13}{20}\right)$

Solution to Exercise 8.5.19 (p. 494)

$9+7=16 \quad\left(15 \frac{13}{15}\right)$

Solution to Exercise 8.5.21 (p. 494)

$3 \frac{1}{2}+2 \frac{1}{2}+2=8\left(7 \frac{189}{200}\right)$

Solution to Exercise 8.5.23 (p. 494)

$1-1=0\left(\frac{1}{16}\right)$

Solution to Exercise 8.5.25 (p. 495)

associative 
Solution to Exercise 8.5.27 (p. 495) $\frac{8}{9}$

Solution to Exercise 8.5.29 (p. 495)

$25(40-3)=1000-75=925$

Solution to Exercise 8.7.1 (p. 496)

600 (598)

Solution to Exercise 8.7.3 (p. 497) $(1,200)$

Solution to Exercise 8.7.5 (p. 497) $4,900(4,944)$

Solution to Exercise 8.7.7 (p. 497) $3,800(3,760)$

Solution to Exercise 8.7.9 (p. 497) $6,050(6,017)$

Solution to Exercise 8.7.11 (p. 497) $6,700(6,718)$

Solution to Exercise 8.7.13 (p. 497) 61,400 (61,337)

Solution to Exercise 8.7.15 (p. 497) $89,700(89,718)$

Solution to Exercise 8.7.17 (p. 497) 213,000 (213,022)

Solution to Exercise 8.7.19 (p. 497) $3,900(3,935)$

Solution to Exercise 8.7.21 (p. 497) $2,400(2,583)$

Solution to Exercise 8.7.23 (p. 497) 600 (504)

Solution to Exercise 8.7.25 (p. 497) 150,123 147,000 $(150,123)$

Solution to Exercise 8.7.27 (p. 498) 47,278 48,000 (47,278)

Solution to Exercise 8.7.29 (p. 498) 12,771 14,100 (12,711)

Solution to Exercise 8.7.31 (p. 498) $8,100(9,768)$

Solution to Exercise 8.7.33 (p. 498) $90,000(86,784)$

Solution to Exercise 8.7.35 (p. 498) $396,000(386,496)$

Solution to Exercise 8.7.37 (p. 498) 1,609,048 1,560,000 (1,609,048)

Solution to Exercise 8.7.39 (p. 498) $22,050,000(22,164,232)$

Solution to Exercise 8.7.41 (p. 498) 18 (15)

Solution to Exercise 8.7.43 (p. 498) $14 \frac{1}{2}$ (13)

Solution to Exercise 8.7.45 (p. 498) $50(53)$ 
Solution to Exercise 8.7.47 (p. 498) $54(51)$

Solution to Exercise 8.7.49 (p. 499) 130 (105)

Solution to Exercise 8.7.51 (p. 499) 41.25 (44)

Solution to Exercise 8.7.53 (p. 499) $42(43)$

Solution to Exercise 8.7.55 (p. 499) $14.4(14)$

Solution to Exercise 8.7.57 (p. 499) $60(61)$

Solution to Exercise 8.7.59 (p. 499) $1,000(992)$

Solution to Exercise 8.7.61 (p. 499) 71.1 (71.17)

Solution to Exercise 8.7.63 (p. 499) 119.4 (119.31)

Solution to Exercise 8.7.65 (p. 499) $272(271.8)$

Solution to Exercise 8.7.67 (p. 499) 2,001 (2,020.952)

Solution to Exercise 8.7.69 (p. 499) 4.16 (3.9596)

Solution to Exercise 8.7.71 (p. 500) $2(40)+2(50)=180(178)$

Solution to Exercise 8.7.73 (p. 500) $2(30)+2(60)=180(179)$

Solution to Exercise 8.7.75 (p. 500) $2(80)+2(50)=260(263)$

Solution to Exercise 8.7.77 (p. 500) $3(20)+1(10)=70(79)$

Solution to Exercise 8.7.79 (p. 500) $2(15)+2(80)=190(201)$

Solution to Exercise 8.7.81 (p. 500) $3(20)+2(30)+40=160(160)$

Solution to Exercise 8.7.83 (p. 500) $2(60)+2(20)+2(30)=220(219)$

Solution to Exercise 8.7.85 (p. 500) $2(90)+20=200(200)$

Solution to Exercise 8.7.87 (p. 500) $3(20)+2(40)=140(140)$

Solution to Exercise 8.7.89 (p. 500) $3(90)+2(30)=330(321)$

Solution to Exercise 8.7.91 (p. 501) $15(30+3)=450+45=495$

Solution to Exercise 8.7.93 (p. 501) $35(40-4)=1400-140=1,260$

Solution to Exercise 8.7.95 (p. 501) $85(20+3)=1,700+225=1,955$ 
Solution to Exercise 8.7.97 (p. 501)

$30(10+4)=300+120=420$

Solution to Exercise 8.7.99 (p. 501)

$75(20+3)=1,500+225=1,725$

Solution to Exercise 8.7.101 (p. 501)

$15(20-3)=300-45=255$

Solution to Exercise 8.7.103 (p. 501)

$65(10+4)=650+260=910$

Solution to Exercise 8.7.105 (p. 501)

$60(40+2)=2,400+120=2,520$

Solution to Exercise 8.7.107 (p. 501)

$15(100+5)=1,500+75=1,575$

Solution to Exercise 8.7.109 (p. 501)

$45(300+6)=13,500+270=13,770$

Solution to Exercise 8.7.111 (p. 502)

$\frac{1}{2}+1=1 \frac{1}{2}\left(1 \frac{5}{24}\right)$

Solution to Exercise 8.7.113 (p. 502)

$\frac{1}{2}+\frac{1}{2}=1\left(\frac{27}{30}\right.$ or $\left.\frac{9}{10}\right)$

Solution to Exercise 8.7.115 (p. 502)

$\frac{1}{2}+\frac{1}{4}=\frac{3}{4}\left(\frac{113}{150}\right)$

Solution to Exercise 8.7.117 (p. 502)

$\frac{1}{4}+1=1 \frac{1}{4}\left(\frac{39}{32}\right.$ or $\left.1 \frac{7}{32}\right)$

Solution to Exercise 8.7.119 (p. 502)

$2 \frac{3}{4}+6 \frac{1}{2}=9 \frac{1}{4}\left(9 \frac{7}{20}\right)$

Solution to Exercise 8.7.121 (p. 502)

$11 \frac{1}{4}+7 \frac{1}{2}=18 \frac{3}{4}\left(18 \frac{23}{30}\right)$

Solution to Exercise 8.7.123 (p. 502)

$6+2+8 \frac{1}{4}=16 \frac{1}{4}\left(16 \frac{11}{30}\right)$

Solution to Exercise 8.7.125 (p. 502)

$10 \frac{1}{2}+7+8 \frac{1}{4}=25 \frac{3}{4}\left(25 \frac{27}{40}\right)$

Solution to Exercise 8.8.1 (p. 502)

$10,500(10,505)$

Solution to Exercise 8.8.2 (p. 502)

$18,000(18,038)$

Solution to Exercise 8.8.3 (p. 503) $5,000(5,011)$

Solution to Exercise 8.8.4 (p. 503) $28,000(27,406)$

Solution to Exercise 8.8.5 (p. 503)

$3,200(3,116)$

Solution to Exercise 8.8.6 (p. 503) 4,500 (4,692)

Solution to Exercise 8.8.7 (p. 503) $300,000(288,576)$

Solution to Exercise 8.8.8 (p. 503) $12(14)$

Solution to Exercise 8.8.9 (p. 503) $90(95)$

Solution to Exercise 8.8.10 (p. 503)

81 (81.00) 
Solution to Exercise 8.8.11 (p. 503) 505 (505.0065)

Solution to Exercise 8.8.12 (p. 503) $4,371(4,380.16)$

Solution to Exercise 8.8.13 (p. 503) $2(60)+2(90)=300(298)$

Solution to Exercise 8.8.14 (p. 503) $2(40)+2(90)=260(263)$

Solution to Exercise 8.8.15 (p. 503) $30+3(80)=270(265)$

Solution to Exercise 8.8.16 (p. 503) $3(800)+2(600)=3,600(3,618)$

Solution to Exercise 8.8.17 (p. 503) $25(10+4)=250+100=350$

Solution to Exercise 8.8.18 (p. 503) $15(80+3)=1,200+45=1,245$

Solution to Exercise 8.8.19 (p. 503) $65(100-2)=6,500-130=6,370$

Solution to Exercise 8.8.20 (p. 503) $80(100+7)=8,000+560=8,560$

Solution to Exercise 8.8.21 (p. 503) $400(200+15)=80,000+6,000=86,000$

Solution to Exercise 8.8.22 (p. 503) $1+\frac{1}{2}=1 \frac{1}{2}\left(1 \frac{9}{16}\right)$

Solution to Exercise 8.8.23 (p. 503) $0+\frac{1}{2}+\frac{1}{2}=1\left(1 \frac{47}{300}\right)$

Solution to Exercise 8.8.24 (p. 504) $8 \frac{1}{2}+14=22 \frac{1}{2}\left(22 \frac{31}{48}\right)$

Solution to Exercise 8.8.25 (p. 504) $5 \frac{1}{2}+1 \frac{1}{2}+6 \frac{1}{2}=13 \frac{1}{2}\left(13 \frac{1}{3}\right)$ 


\section{Chapter 9}

\section{Measurement and Geometry}

\subsection{Objectives ${ }^{1}$}

After completing this chapter, you should

Measurement and the United States System (Section 9.2)

- know what the word measurement means

- be familiar with United States system of measurement

- be able to convert from one unit of measure in the United States system to another unit of measure

\section{The Metric System of Measurement (Section 9.3)}

- be more familiar with some of the advantages of the base ten number system

- know the prefixes of the metric measures

- be familiar with the metric system of measurement

- be able to convert from one unit of measure in the metric system to another unit of measure

\section{Simplification of Denominate Numbers (Section 9.4)}

- be able to convert an unsimplified unit of measure to a simplified unit of measure

- be able to add and subtract denominate numbers

- be able to multiply and divide a denominate number by a whole number

\section{Perimeter and Circumference of Geometric Figures (Section 9.5)}

- know what a polygon is

- know what perimeter is and how to find it

- know what the circumference, diameter, and radius of a circle is and how to find each one

- know the meaning of the symbol $\pi$ and its approximating value

- know what a formula is and four versions of the circumference formula of a circle

\section{Area and Volume of Geometric Figures and Objects (Section 9.6)}

- know the meaning and notation for area

- know the area formulas for some common geometric figures

- be able to find the areas of some common geometric figures

- know the meaning and notation for volume

- know the volume formulas for some common geometric objects

- be able to find the volume of some common geometric objects

\footnotetext{
${ }^{1}$ This content is available online at $<$ http://cnx.org/content $/ \mathrm{m} 18897 / 1.3 />$.
}

Available for free at Connexions $<$ http://cnx.org/content/col10615/1.4 $>$ 


\subsection{Measurement and the United States System²}

\subsubsection{Section Overview}

- Measurement

- The United States System of Measurement

- Conversions in the United States System

\subsubsection{Measurement}

There are two major systems of measurement in use today. They are the United States system and the metric system. Before we describe these systems, let's gain a clear understanding of the concept of measurement.

\section{Measurement}

Measurement is comparison to some standard.

\section{Standard Unit of Measure}

The concept of measurement is based on the idea of direct comparison. This means that measurement is the result of the comparison of two quantities. The quantity that is used for comparison is called the standard unit of measure.

Over the years, standards have changed. Quite some time in the past, the standard unit of measure was determined by a king. For example,

1 inch was the distance between the tip of the thumb and the knuckle of the king.

1 inch was also the length of 16 barley grains placed end to end.

Today, standard units of measure rarely change. Standard units of measure are the responsibility of the Bureau of Standards in Washington D.C.

Some desirable properties of a standard are the following:

1. Accessibility. We should have access to the standard so we can make comparisons.

2. Invariance. We should be confident that the standard is not subject to change.

3. Reproducibility. We should be able to reproduce the standard so that measurements are convenient and accessible to many people.

\subsubsection{The United States System of Measurement}

Some of the common units (along with their abbreviations) for the United States system of measurement are listed in the following table.

\begin{tabular}{|l|l|}
\hline Unit Conversion Table \\
\hline Length & $\begin{array}{l}1 \text { foot }(\mathrm{ft})=12 \text { inches (in.) } \\
1 \text { yard }(\mathrm{yd})=3 \text { feet }(\mathrm{ft}) \\
1 \text { mile }(\mathrm{mi})=5,280 \text { feet }\end{array}$ \\
\hline
\end{tabular}

continued on next page

\footnotetext{
${ }^{2}$ This content is available online at $<$ http://cnx.org/content $/ \mathrm{m} 35018 / 1.2 />$.
} 


\begin{tabular}{|l|l|}
\hline Weight & 1 pound $(\mathrm{lb})=16$ ounces $(\mathrm{oz})$ \\
& 1 ton $(\mathrm{T})=2,000$ pounds \\
\hline Liquid Volume & 1 tablespoon $($ tbsp $)=3$ teaspoons $(\mathrm{tsp})$ \\
& 1 fluid ounce $(\mathrm{fl}$ oz $)=2$ tablespoons \\
& 1 cup $(\mathrm{c})=8$ fluid ounces \\
& 1 pint $(\mathrm{pt})=2$ cups \\
& 1 quart $(\mathrm{qt})=2$ pints \\
& 1 gallon $(\mathrm{gal})=4$ quarts \\
\hline Time & 1 minute $(\mathrm{min})=60$ seconds $(\mathrm{sec})$ \\
& 1 hour $(\mathrm{hr})=60$ minutes \\
& 1 day $(\mathrm{da})=24$ hours \\
& 1 week $(\mathrm{wk})=7$ days \\
\hline
\end{tabular}

Table 9.1

\subsubsection{Conversions in the United States System}

It is often convenient or necessary to convert from one unit of measure to another. For example, it may be convenient to convert a measurement of length that is given in feet to one that is given in inches. Such conversions can be made using unit fractions.

\section{Unit Fraction}

A unit fraction is a fraction with a value of 1 .

Unit fractions are formed by using two equal measurements. One measurement is placed in the numerator of the fraction, and the other in the denominator. Placement depends on the desired conversion.

\section{Placement of Units}

Place the unit being converted to in the numerator.

Place the unit being converted from in the denominator.

For example,

\begin{tabular}{|l|l|}
\hline Equal Measurements & Unit Fraction \\
\hline $1 \mathrm{ft}=12 \mathrm{in}$. & $\frac{1 \mathrm{ft}}{12 \mathrm{in} .}$ or $\frac{12 \mathrm{in} .}{1 \mathrm{ft}}$ \\
\hline $1 \mathrm{pt}=16 \mathrm{fl} \mathrm{oz}$ & $\frac{1 \mathrm{pt}}{16 \mathrm{fl} \mathrm{oz}}$ or $\frac{16 \mathrm{fl} \mathrm{oz}}{1 \mathrm{pt}}$ \\
\hline $1 \mathrm{wk}=7 \mathrm{da}$ & $\frac{7 \mathrm{da}}{1 \mathrm{wk}}$ or $\frac{1 \mathrm{wk}}{7 \mathrm{da}}$ \\
\hline
\end{tabular}

Table 9.2

\subsubsection{Sample Set A}

Make the following conversions. If a fraction occurs, convert it to a decimal rounded to two decimal places.

\section{Example 9.1}

Convert 11 yards to feet.

Looking in the unit conversion table under length, we see that $1 \mathrm{yd}=3 \mathrm{ft}$. There are two corresponding unit fractions, $\frac{1 \mathrm{yd}}{3 \mathrm{ft}}$ and $\frac{3 \mathrm{ft}}{1 \mathrm{yd}}$. Which one should we use? Look to see which unit we wish to convert to. Choose the unit fraction with this unit in the numerator. We will choose $\frac{3 \mathrm{ft}}{1 \mathrm{yd}}$ since 
this unit fraction has feet in the numerator. Now, multiply $11 \mathrm{yd}$ by the unit fraction. Notice that since the unit fraction has the value of 1 , multiplying by it does not change the value of $11 \mathrm{yd}$.

$$
\begin{aligned}
11 \mathrm{yd} & =\frac{11 \mathrm{yd}}{1} \cdot \frac{3 \mathrm{ft}}{1 \mathrm{yd}} \\
& =\frac{11) \mathrm{yd}}{1} \cdot \frac{3 \mathrm{ft}}{1 \mathrm{yd}} \quad \text { (Units can be added, subtracted, multiplied, and divided, just as numbers can.) } \\
& =\frac{11 \cdot 3 \mathrm{ft}}{1} \\
& =33 \mathrm{ft}
\end{aligned}
$$

Thus, $11 \mathrm{yd}=33 \mathrm{ft}$.

Example 9.2

Convert $36 \mathrm{fl} \mathrm{oz}$ to pints.

Looking in the unit conversion table under liquid volume, we see that $1 \mathrm{pt}=16 \mathrm{fl}$ oz. Since we are to convert to pints, we will construct a unit fraction with pints in the numerator.

$$
\begin{aligned}
& 36 \mathrm{fl} \mathrm{oz}=\frac{36 \mathrm{floz}}{1} \cdot \frac{1 \mathrm{pt}}{16 \mathrm{f} \mathrm{oz}} \quad \text { Divide out common units. } \\
& =\frac{36) \overline{\mathrm{floz}}}{1} \cdot \frac{1 \mathrm{pt}}{16) \overline{\mathrm{floz}}} \\
& =\frac{36 \cdot 1 \mathrm{pt}}{16} \\
& =\quad \frac{36 \mathrm{pt}}{16} \quad \text { Reduce. } \\
& =\quad \frac{9}{4} \mathrm{pt} \quad \text { Convert to decimals: } \frac{9}{4}=2.25 \text {. }
\end{aligned}
$$

Thus, $36 \mathrm{fl} \mathrm{oz}=2.25 \mathrm{pt}$.

\section{Example 9.3}

Convert 2,016 hr to weeks.

Looking in the unit conversion table under time, we see that $1 \mathrm{wk}=7 \mathrm{da}$ and that $1 \mathrm{da}=24 \mathrm{hr}$. To convert from hours to weeks, we must first convert from hours to days and then from days to weeks. We need two unit fractions.

The unit fraction needed for converting from hours to days is $\frac{1 \mathrm{da}}{24 \mathrm{hr}}$. The unit fraction needed for converting from days to weeks is $\frac{1 \mathrm{wk}}{7 \mathrm{da}}$.

$$
\begin{aligned}
& 2,016 \mathrm{hr}=\frac{2,016 \mathrm{hr}}{1} \cdot \frac{1 \mathrm{da}}{24 \mathrm{hr}} \cdot \frac{1 \mathrm{wk}}{7 \mathrm{da}} \quad \text { Divide out common units. } \\
& =\frac{2,016 \overline{\mathrm{hr}}}{1} \cdot \frac{1 \overline{\mathrm{da}}}{24 \overline{\mathrm{hr}}} \cdot \frac{1 \mathrm{wk}}{7 \overline{\mathrm{da}}} \\
& =\quad \frac{2,016 \cdot 1 \mathrm{wk}}{24 \cdot 7} \quad \text { Reduce. } \\
& =\quad 12 \mathrm{wk}
\end{aligned}
$$

Thus, $2,016 \mathrm{hr}=12 \mathrm{wk}$.

\subsubsection{Practice Set A}

Make the following conversions. If a fraction occurs, convert it to a decimal rounded to two decimal places.

Exercise 9.2.1

(Solution on p. 569.)

Convert $18 \mathrm{ft}$ to yards. 
Exercise 9.2.2

(Solution on p. 569.)

Convert $2 \mathrm{mi}$ to feet.

Exercise 9.2.3

(Solution on p. 569.)

Convert $26 \mathrm{ft}$ to yards.

(Solution on p. 569.)

Convert 9 qt to pints.

Exercise 9.2.5

(Solution on p. 569.)

Convert 52 min to hours.

Exercise 9.2.6

Convert $412 \mathrm{hr}$ to weeks.

(Solution on p. 569.)

\subsubsection{Exercises}

Make each conversion using unit fractions. If fractions occur, convert them to decimals rounded to two decimal places.

Exercise 9.2.7

(Solution on p. 569.)

$14 \mathrm{yd}$ to feet

Exercise 9.2.8

3 mi to yards

Exercise 9.2.9

$8 \mathrm{mi}$ to inches

(Solution on p. 569.)

Exercise 9.2.10

$2 \mathrm{mi}$ to inches

Exercise 9.2.11

18 in. to feet

(Solution on p. 569.)

Exercise 9.2.12

84 in. to yards

Exercise 9.2.13

5 in. to yards

Exercise 9.2.14

$106 \mathrm{ft}$ to miles

Exercise 9.2.15

62 in. to miles

(Solution on p. 569.)

Exercise 9.2.16

0.4 in. to yards

Exercise 9.2.17

3 qt to pints

Exercise 9.2.18

$5 \mathrm{lb}$ to ounces

Exercise 9.2.19

$6 \mathrm{~T}$ to ounces

(Solution on p. 569.)

Exercise 9.2.20

4 oz to pounds

Exercise 9.2.21

15,000 oz to pounds

(Solution on p. 569.)

(Solution on p. 569.)

(Solution on p. 569.) 
Exercise 9.2.22

15,000 oz to tons

Exercise 9.2.23

(Solution on p. 569.)

9 tbsp to teaspoons

Exercise 9.2.24

$3 \mathrm{c}$ to tablespoons

Exercise 9.2.25

(Solution on p. 569.)

$5 \mathrm{pt}$ to fluid ounces

Exercise 9.2.26

16 tsp to cups

Exercise 9.2.27

$5 \mathrm{fl} \mathrm{oz}$ to quarts

Exercise 9.2.28

3 qt to gallons

Exercise 9.2.29

$5 \mathrm{pt}$ to teaspoons

Exercise 9.2.30

3 qt to tablespoons

Exercise 9.2.31

(Solution on p. 569.)

$18 \mathrm{~min}$ to seconds

Exercise 9.2.32

4 days to hours

Exercise 9.2.33

$3 \mathrm{hr}$ to days

Exercise 9.2.34

$\frac{1}{2} \mathrm{hr}$ to days

Exercise 9.2.35

$\frac{1}{2}$ da to weeks

Exercise 9.2.36

$3 \frac{1}{7}$ wk to seconds

(Solution on p. 569.)

(Solution on p. 569.)

(Solution on p. 569.)

(Solution on p. 569.)

\subsubsection{Exercises for Review}

Exercise 9.2.37

(Solution on p. 569.)

(Section 2.5) Specify the digits by which 23,840 is divisible.

Exercise 9.2.38

(Section 4.5) Find $2 \frac{4}{5}$ of $5 \frac{5}{6}$ of $7 \frac{5}{7}$.

Exercise 9.2.39

() Convert $0.3 \frac{2}{3}$ to a fraction.

(Solution on p. 569.)

Exercise 9.2.40

(Section 8.3) Use the clustering method to estimate the sum: $53+82+79+49$.

Exercise 9.2.41

(Solution on p. 569.)

(Section 8.4) Use the distributive property to compute the product: $60 \cdot 46$. 


\subsection{The Metric System of Measurement ${ }^{3}$}

\subsubsection{Section Overview}

- The Advantages of the Base Ten Number System

- Prefixes

- Conversion from One Unit to Another Unit

- Conversion Table

\subsubsection{The Advantages of the Base Ten Number System}

The metric system of measurement takes advantage of our base ten number system. The advantage of the metric system over the United States system is that in the metric system it is possible to convert from one unit of measure to another simply by multiplying or dividing the given number by a power of 10 . This means we can make a conversion simply by moving the decimal point to the right or the left.

\subsubsection{Prefixes}

Common units of measure in the metric system are the meter (for length), the liter (for volume), and the gram (for mass). To each of the units can be attached a prefix. The metric prefixes along with their meaning are listed below.

\section{Metric Prefixes}

$$
\begin{aligned}
& \text { kilo - thousand } \\
& \text { deci - tenth } \\
& \text { hecto - hundred } \\
& \text { centi - hundredth } \\
& \text { deka - ten } \\
& \text { milli - thousandth }
\end{aligned}
$$

For example, if length is being measured,

1 kilometer is equivalent to 1000 meters.

1 centimeter is equivalent to one hundredth of a meter.

1 millimeter is equivalent to one thousandth of a meter.

\subsubsection{Conversion from One Unit to Another Unit}

Let's note three characteristics of the metric system that occur in the metric table of measurements.

1. In each category, the prefixes are the same.

2. We can move from a larger to a smaller unit of measure by moving the decimal point to the right.

3. We can move from a smaller to a larger unit of measure by moving the decimal point to the left.

The following table provides a summary of the relationship between the basic unit of measure (meter, gram, liter) and each prefix, and how many places the decimal point is moved and in what direction.

kilo hecto deka unit deci centi milli

\footnotetext{
${ }^{3}$ This content is available online at $<$ http://cnx.org/content/m35019/1.2/>.
} 


\begin{tabular}{|l|l|l|}
\hline Basic Unit to Prefix & & Move the Decimal Point \\
\hline unit to deka & 1 to 10 & 1 place to the left \\
\hline unit to hector & 1 to 100 & 2 places to the left \\
\hline unit to kilo & 1 to 1,000 & 3 places to the left \\
\hline unit to deci & 1 to 0.1 & 1 place to the right \\
\hline unit to centi & 1 to 0.01 & 2 places to the right \\
\hline unit to milli & 1 to 0.001 & 3 places to the right \\
\hline
\end{tabular}

Table 9.3

\subsubsection{Conversion Table}

Listed below, in the unit conversion table, are some of the common metric units of measure.

\begin{tabular}{|c|c|c|}
\hline \multicolumn{3}{|c|}{ Unit Conversion Table } \\
\hline \multirow{7}{*}{ Length } & $\begin{array}{l}1 \quad \text { kilometer }(\mathrm{km})= \\
1,000 \text { meters }(m)\end{array}$ & $1,000 \times 1 \mathrm{~m}$ \\
\hline & $\begin{array}{l}1 \text { hectometer }(\mathrm{hm})= \\
100 \text { meters }\end{array}$ & $100 \times 1 \mathrm{~m}$ \\
\hline & $\begin{array}{l}1 \text { dekameter }(\text { dam })= \\
10 \text { meters }\end{array}$ & $10 \times 1 \mathrm{~m}$ \\
\hline & 1 meter $(\mathrm{m})$ & $1 \times 1 \mathrm{~m}$ \\
\hline & 1 decimeter $(\mathrm{dm})=\frac{1}{10}$ meter & $.1 \times 1 \mathrm{~m}$ \\
\hline & 1 centimeter $(\mathrm{cm})=\frac{1}{100}$ meter & $.01 \times 1 \mathrm{~m}$ \\
\hline & $\begin{array}{l}1 \text { millimeter } \quad(\mathrm{mm})= \\
\frac{1}{1,000} \text { meter }\end{array}$ & $.001 \times 1 \mathrm{~m}$ \\
\hline \multirow{5}{*}{ Mass } & $\begin{array}{lrl}1 \quad \text { kilogram } & (\mathrm{kg}) \\
1,000 \text { grams } & (g)\end{array}$ & $1,000 \times 1 \mathrm{~g}$ \\
\hline & 1 hectogram $(\mathrm{hg})=100$ grams & $100 \times 1 \mathrm{~g}$ \\
\hline & 1 dekagram $($ dag $)=10$ grams & $10 \times 1 \mathrm{~g}$ \\
\hline & 1 gram (g) & $1 \times 1 \mathrm{~g}$ \\
\hline & $1 \quad$ decigram $\quad(\mathrm{dg})=\frac{1}{10} \quad$ gram & $.1 \times 1 \mathrm{~g}$ \\
\hline
\end{tabular}




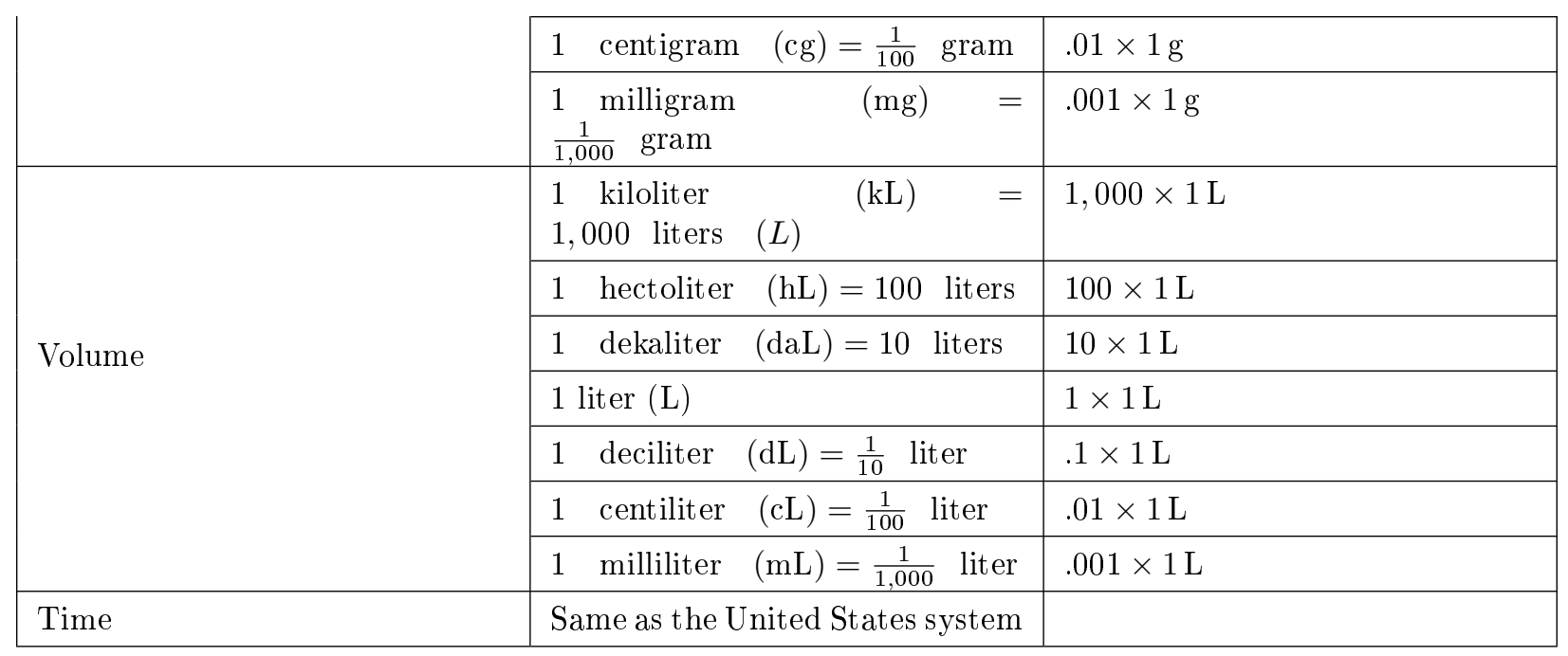

Table 9.4

\section{Distinction Between Mass and Weight}

There is a distinction between mass and weight. The weight of a body is related to gravity whereas the mass of a body is not. For example, your weight on the earth is different than it is on the moon, but your mass is the same in both places. Mass is a measure of a body's resistance to motion. The more massive a body, the more resistant it is to motion. Also, more massive bodies weigh more than less massive bodies.

\section{Converting Metric Units}

To convert from one metric unit to another metric unit:

1. Determine the location of the original number on the metric scale (pictured in each of the following examples).

2. Move the decimal point of the original number in the same direction and same number of places as is necessary to move to the metric unit you wish to go to.

We can also convert from one metric unit to another using unit fractions. Both methods are shown in Example 9.4 of Section 9.3.5.1 (Sample Set A).

\subsubsection{Sample Set A}

\section{Example 9.4}

Convert 3 kilograms to grams.

(a) $3 \mathrm{~kg}$ can be written as $3.0 \mathrm{~kg}$. Then,

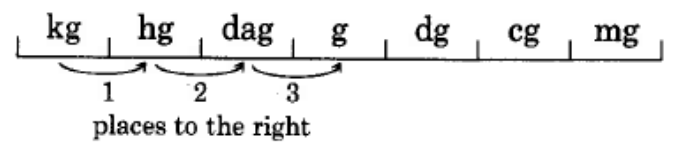

\section{$3.0 \mathrm{~kg}=3000, \mathrm{~g}$ \\ 123}

Thus, $3 \mathrm{~kg}=3,000 \mathrm{~g}$.

(b) We can also use unit fractions to make this conversion. Since we are converting to grams, and $1,000 \mathrm{~g}=1 \mathrm{~kg}$, we choose the unit fraction $\frac{1,000 \mathrm{~g}}{1 \mathrm{~kg}}$ since grams is in the numerator. 


$$
\begin{aligned}
3 \mathrm{~kg} & =3 \mathrm{~kg} \cdot \frac{1,000 \mathrm{~g}}{1 \mathrm{~kg}} \\
& =3 \overline{\mathrm{kg}} \cdot \frac{1,000 \mathrm{~g}}{1 \overline{\mathrm{kg}}} \\
& =3 \cdot 1,000 \mathrm{~g} \\
& =3,000 \mathrm{~g}
\end{aligned}
$$

\section{Example 9.5}

Convert 67.2 hectoliters to milliliters.

$\mathrm{kL}, \mathrm{hL}, \underbrace{\mathrm{daL}}_{1}, \underbrace{\frac{\mathrm{s}}{3} \underbrace{}_{5}, \mathrm{dL}, \mathrm{mL}}_{\begin{array}{c}2 \\ \text { places to the right }\end{array}}$

$67.2 \mathrm{hL}=6720000 \mathrm{~mL}$

$$
12345
$$

Thus, $67.2 \mathrm{hL}=6,720,000 \mathrm{~mL}$.

\section{Example 9.6}

Convert 100.07 centimeters to meters.

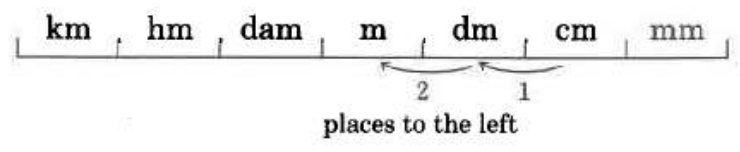

$$
100.07 \mathrm{~cm}=\underset{21}{1.0007} \mathrm{~m}
$$

Thus, $100.07 \mathrm{~cm}=1.0007 \mathrm{~m}$.

Example 9.7

Convert 0.16 milligrams to grams.

$\mathrm{kg}, \mathrm{hg}, \mathrm{dg}, \underbrace{\mathrm{g}, \mathrm{dg}, \mathrm{cg}, \mathrm{mg}}_{\begin{array}{c}3 \\ \text { places to the left }\end{array}}$

$$
0.16 \mathrm{mg}=\underbrace{0.000}_{321} 16 \mathrm{~g}
$$

Thus, $0.16 \mathrm{mg}=0.00016 \mathrm{~g}$.

\subsubsection{Practice Set A}

Exercise 9.3.1

(Solution on p. 569.)

Convert 411 kilograms to grams.

Exercise 9.3.2

(Solution on p. 569.)

Convert 5.626 liters to centiliters.

Exercise 9.3.3

(Solution on p. 570.)

Convert 80 milliliters to kiloliters.

Exercise 9.3.4

(Solution on p. 570.)

Convert 150 milligrams to centigrams.

Exercise 9.3.5

(Solution on p. 570.) 


\subsubsection{Exercises}

Make each conversion.

Exercise 9.3.6

(Solution on p. 570.)

$87 \mathrm{~m}$ to $\mathrm{cm}$

Exercise 9.3.7

$905 \mathrm{~L}$ to $\mathrm{mL}$

Exercise 9.3.8

(Solution on p. 570.)

$16,005 \mathrm{mg}$ to $\mathrm{g}$

Exercise 9.3.9

$48.66 \mathrm{~L}$ to $\mathrm{dL}$

Exercise 9.3.10

(Solution on p. 570.)

$11.161 \mathrm{~kL}$ to $\mathrm{L}$

Exercise 9.3.11

$521.85 \mathrm{~cm}$ to $\mathrm{mm}$

Exercise 9.3.12

$1.26 \mathrm{dag}$ to $\mathrm{dg}$

Exercise 9.3.13

99.04 dam to $\mathrm{cm}$

Exercise 9.3.14

(Solution on p. 570.)

$0.51 \mathrm{~kL}$ to daL

Exercise 9.3.15

$0.17 \mathrm{~kL}$ to daL

Exercise 9.3.16

(Solution on p. 570.)

$0.05 \mathrm{~m}$ to $\mathrm{dm}$

Exercise 9.3.17

$0.001 \mathrm{~km}$ to $\mathrm{mm}$

Exercise 9.3.18

(Solution on p. 570.)

$8.106 \mathrm{hg}$ to $\mathrm{cg}$

Exercise 9.3.19

$17.0186 \mathrm{~kL}$ to $\mathrm{mL}$

Exercise 9.3.20

(Solution on p. 570.)

$3 \mathrm{~cm}$ to $\mathrm{m}$

(Solution on p. 570.)

Exercise 9.3.21

$9 \mathrm{~mm}$ to $\mathrm{m}$

Exercise 9.3.22

(Solution on p. 570.)

$4 \mathrm{~g}$ to $\mathrm{mg}$

Exercise 9.3.23

$2 \mathrm{~L}$ to $\mathrm{kL}$

Exercise 9.3.24

$6 \mathrm{~kg}$ to $\mathrm{mg}$

(Solution on p. 570.)

Exercise 9.3.25

7 daL to $\mathrm{mL}$ 


\subsubsection{Exercises for Review}

Exercise 9.3.26

(Solution on p. 570.)

(Section 5.3) Find the value of $\frac{5}{8}-\frac{1}{3}+\frac{3}{4}$.

Exercise 9.3.27

(Section 7.3) Solve the proportion: $\frac{9}{x}=\frac{27}{60}$.

Exercise 9.3.28

(Solution on p. 570.)

(Section 8.2) Use the method of rounding to estimate the sum: $8,226+4,118$.

Exercise 9.3.29

(Section 8.3) Use the clustering method to estimate the sum: $87+121+118+91+92$.

Exercise 9.3.30

(Section 9.2) Convert 3 in. to yd.

(Solution on p. 570.)

\subsection{Simplification of Denominate Numbers ${ }^{4}$}

\subsubsection{Section Overview}

- Converting to Multiple Units

- Adding and Subtracting Denominate Numbers

- Multiplying a Denominate Number by a Whole Number

- Dividing a Denominate Number by a Whole Number

\subsubsection{Converting to Multiple Units}

\section{Denominate Numbers}

Numbers that have units of measure associated with them are called denominate numbers. It is often convenient, or even necessary, to simplify a denominate number.

\section{Simplified Denominate Number}

A denominate number is simplified when the number of standard units of measure associated With it does not exceed the next higher type of unit.

The denominate number $55 \mathrm{~min}$ is simplified since it is smaller than the next higher type of unit, $1 \mathrm{hr}$. The denominate number $65 \mathrm{~min}$ is not simplified since it is not smaller than the next higher type of unit, $1 \mathrm{hr}$. The denominate number $65 \mathrm{~min}$ can be simplified to $1 \mathrm{hr} 5 \mathrm{~min}$. The denominate number $1 \mathrm{hr} 5 \mathrm{~min}$ is simplified since the next higher type of unit is day, and $1 \mathrm{hr}$ does not exceed 1 day.

\subsubsection{Sample Set A}

\section{Example 9.8}

Simplify 19 in.

Since 12 in. $=1 \mathrm{ft}$, and $19=12+7$,

$$
\begin{aligned}
19 \mathrm{in.} & =12 \mathrm{in.}+7 \mathrm{in} . \\
& =1 \mathrm{ft}+7 \mathrm{in} . \\
& =1 \mathrm{ft} 7 \mathrm{in} .
\end{aligned}
$$

\footnotetext{
${ }^{4}$ This content is available online at $<$ http://cnx.org/content/m35021/1.2/ $>$.
} 


\section{Example 9.9}

Simplify 4 gal 5 qt.

Since 4 qt $=1$ gal, and $5=4+1$,

$$
\begin{aligned}
4 \mathrm{gal} 5 \mathrm{qt} & = & 4 \mathrm{gal}+4 \mathrm{qt}+1 \mathrm{qt} \\
& = & 4 \mathrm{gal}+1 \mathrm{gal}+1 \mathrm{qt} \\
& = & 5 \mathrm{gal}+1 \mathrm{qt} \\
& = & 5 \mathrm{gal} 1 \mathrm{qt}
\end{aligned}
$$

\section{Example 9.10}

Simplify 2 hr 75 min.

Since $60 \mathrm{~min}=1 \mathrm{hr}$, and $75=60+15$,

$$
\begin{aligned}
& 2 \mathrm{hr} 75 \mathrm{~min}=2 \mathrm{hr}+60 \mathrm{~min}+15 \mathrm{~min} \\
& =2 \mathrm{hr}+1 \mathrm{hr}+15 \mathrm{~min} \\
& =\quad 3 \mathrm{hr}+15 \mathrm{~min} \\
& =\quad 3 \mathrm{hr} 15 \mathrm{~min}
\end{aligned}
$$

\section{Example 9.11}

Simplify $43 \mathrm{fl}$ oz.

Since $8 \mathrm{fl} \mathrm{oz}=1 \mathrm{c}(1 \mathrm{cup})$, and $43 \div 8=5 \mathrm{R} 3$,

$$
\begin{aligned}
43 \mathrm{fl} \mathrm{oz} & =40 \mathrm{fl} \mathrm{oz}+3 \mathrm{floz} \\
& =5 \cdot 8 \mathrm{floz}+3 \mathrm{floz} \\
& =5 \cdot 1 \mathrm{c}+3 \mathrm{floz} \\
& =\quad 5 \mathrm{c}+3 \mathrm{floz}
\end{aligned}
$$

But, $2 \mathrm{c}=1 \mathrm{pt}$ and $5 \div 2=2 \mathrm{R} 1$. So,

$$
\begin{aligned}
5 \mathrm{c}+3 \mathrm{fl} \mathrm{oz} & =2 \cdot 2 \mathrm{c}+1 \mathrm{c}+3 \mathrm{fl} \mathrm{oz} \\
& =2 \cdot 1 \mathrm{pt}+1 \mathrm{c}+3 \mathrm{floz} \\
& =2 \mathrm{pt}+1 \mathrm{c}+3 \mathrm{fl} \mathrm{oz}
\end{aligned}
$$

But, $2 \mathrm{pt}=1 \mathrm{qt}$, so

$2 \mathrm{pt}+1 \mathrm{c}+3 \mathrm{floz}=1 \mathrm{qt} 1 \mathrm{c} 3 \mathrm{floz}$

\subsubsection{Practice Set A}

Simplify each denominate number. Refer to the conversion tables given in Section 9.2, if necessary.

Exercise 9.4.1

(Solution on p. 570.)

18 in.

Exercise 9.4.2

8 gal 9 qt

(Solution on p. 570.) 
Exercise 9.4.3

(Solution on p. 570.)

$5 \mathrm{hr} 80 \mathrm{~min}$

Exercise 9.4.4

(Solution on p. 570.)

8 wk $11 \mathrm{da}$

Exercise 9.4.5

(Solution on p. 570.)

86 da

\subsubsection{Adding and Subtracting Denominate Numbers}

\section{Adding and Subtracting Denominate Numbers}

Denominate numbers can be added or subtracted by:

1. writing the numbers vertically so that the like units appear in the same column.

2. adding or subtracting the number parts, carrying along the unit.

3. simplifying the sum or difference.

\subsubsection{Sample Set B}

Example 9.12

Add $6 \mathrm{ft} 8$ in. to $2 \mathrm{ft} 9$ in.

$6 \mathrm{ft} 8$ in.

$+2 \mathrm{ft} 9 \mathrm{in}$.

$8 \mathrm{ft} 17$ in. Simplify this denominate number.

Since 12 in. $=1 \mathrm{ft}$,

$$
\begin{aligned}
8 \mathrm{ft}+12 \text { in. }+5 \text { in. } & =8 \mathrm{ft}+1 \mathrm{ft}+5 \mathrm{in} . \\
& =9 \mathrm{ft}+5 \mathrm{in} . \\
& =\quad 9 \mathrm{ft} 5 \mathrm{in} .
\end{aligned}
$$

\section{Example 9.13}

Subtract 5 da 3 hr from 8 da 11 hr.

8 da 11 hr

$-5 \mathrm{da} 3 \mathrm{hr}$

3 da $8 \mathrm{hr}$

\section{Example 9.14}

Subtract $3 \mathrm{lb} 14$ oz from $5 \mathrm{lb} 3$ oz.

$$
\begin{array}{r}
5 \mathrm{lb} \quad 3 \mathrm{oz} \\
-3 \mathrm{lb} 14 \mathrm{oz} \\
\hline
\end{array}
$$

We cannot directly subtract $14 \mathrm{oz}$ from $3 \mathrm{oz}$, so we must borrow $16 \mathrm{oz}$ from the pounds. 


$$
\begin{array}{rlrl}
5 \mathrm{lb} 3 \mathrm{oz} & = & 5 \mathrm{lb}+3 \mathrm{oz} \\
& = & 4 \mathrm{lb}+1 \mathrm{lb}+3 \mathrm{oz} & \\
& = & 4 \mathrm{lb}+16 \mathrm{oz}+3 \mathrm{oz} \quad & \text { (Since } 1 \mathrm{lb}=16 \mathrm{oz} .) \\
& = & 4 \mathrm{lb}+19 \mathrm{oz} \\
& = & 4 \mathrm{lb} 19 \mathrm{oz}
\end{array}
$$

$4 \mathrm{lb} 19 \mathrm{oz}$

$-3 \mathrm{lb} 14 \mathrm{oz}$

$1 \mathrm{lb} \quad 5 \mathrm{oz}$

\section{Example 9.15}

Subtract 4 da 9 hr $21 \mathrm{~min}$ from 7 da $10 \mathrm{~min}$.

7 da 0 hr 10 min

-4 da 9 hr $21 \mathrm{~min}$

Borrow 1 da from the 7 da.

6 da 24 hr 10 min

-4 da 9 hr $21 \mathrm{~min}$

Borrow $1 \mathrm{hr}$ from the $24 \mathrm{hr}$.

6 da 23 hr 70 min

-4 da $9 \mathrm{hr} 21 \mathrm{~min}$

2 da 14 hr 49 min

\subsubsection{Practice Set B}

Perform each operation. Simplify when possible.

Exercise 9.4.6

(Solution on p. 570.)

Add 4 gal 3 qt to 1 gal 2 qt.

Exercise 9.4.7

Add 9 hr 48 min to 4 hr $26 \mathrm{~min}$.

(Solution on p. 570.)

Exercise 9.4.8

(Solution on p. 570.)

Subtract $2 \mathrm{ft} 5$ in. from $8 \mathrm{ft} 7$ in.

Exercise 9.4.9

Subtract $15 \mathrm{~km} 460 \mathrm{~m}$ from $27 \mathrm{~km} 800 \mathrm{~m}$.

(Solution on p. 570.)

Exercise 9.4.10

Subtract 8 min $35 \mathrm{sec}$ from $12 \mathrm{~min} 10 \mathrm{sec}$.

(Solution on p. 571.)

Exercise 9.4.11

(Solution on p. 571.)

Add 4 yd $2 \mathrm{ft} 7$ in. to 9 yd $2 \mathrm{ft} 8 \mathrm{in}$.

Exercise 9.4.12

(Solution on p. 571.)

Subtract $11 \mathrm{~min} 55 \mathrm{sec}$ from $25 \mathrm{~min} 8 \mathrm{sec}$. 


\subsubsection{Multiplying a Denominate Number by a Whole Number}

Let's examine the repeated sum

$\underbrace{4 \mathrm{ft} 9 \mathrm{in} .+4 \mathrm{ft} 9 \mathrm{in} .+4 \mathrm{ft} 9 \mathrm{in} .}_{3 \text { times }}=12 \mathrm{ft} 27 \mathrm{in}$.

Recalling that multiplication is a description of repeated addition, by the distributive property we have

$$
\begin{aligned}
3(4 \mathrm{ft} 9 \mathrm{in.}) & =3(4 \mathrm{ft}+9 \mathrm{in} .) \\
& =\quad 3 \cdot 4 \mathrm{ft}+3 \cdot 9 \mathrm{in} . \\
& =\quad 12 \mathrm{ft}+27 \mathrm{in} . \quad \text { Now, } 27 \mathrm{in.}=2 \mathrm{ft} 3 \mathrm{in} . \\
& =12 \mathrm{ft}+2 \mathrm{ft}+3 \mathrm{in} . \\
& =\quad 14 \mathrm{ft}+3 \mathrm{in} . \\
& =14 \mathrm{ft} 3 \mathrm{in} .
\end{aligned}
$$

From these observations, we can suggest the following rule.

\section{Multiplying a Denominate Number by a Whole Number}

To multiply a denominate number by a whole number, multiply the number part of each unit by the whole number and affix the unit to this product.

\subsubsection{Sample Set C}

Perform the following multiplications. Simplify if necessary.

\section{Example 9.16}

$$
\begin{aligned}
6 \cdot(2 \mathrm{ft} 4 \mathrm{in} .) & =6 \cdot 2 \mathrm{ft}+6 \cdot 4 \mathrm{in} . \\
& =12 \mathrm{ft}+24 \mathrm{in} .
\end{aligned}
$$

Since $3 \mathrm{ft}=1 \mathrm{yd}$ and 12 in. $=1 \mathrm{ft}$,

$$
\begin{aligned}
12 \mathrm{ft}+24 \mathrm{in} . & =4 \mathrm{yd}+2 \mathrm{ft} \\
& =4 \mathrm{yd} 2 \mathrm{ft}
\end{aligned}
$$

\section{Example 9.17}

$$
\begin{aligned}
& 8 \cdot(5 \mathrm{hr} 21 \mathrm{~min} 55 \mathrm{sec})=8 \cdot 5 \mathrm{hr}+8 \cdot 21 \mathrm{~min}+8 \cdot 55 \mathrm{sec} \\
& =40 \mathrm{hr}+168 \mathrm{~min}+440 \mathrm{sec} \\
& =40 \mathrm{hr}+168 \mathrm{~min}+7 \mathrm{~min}+20 \mathrm{sec} \\
& =40 \mathrm{hr}+175 \mathrm{~min}+20 \mathrm{sec} \\
& =40 \mathrm{hr}+2 \mathrm{hr}+55 \mathrm{~min}+20 \mathrm{sec} \\
& =\quad 42 \mathrm{hr}+55 \mathrm{~min}+20 \mathrm{sec} \\
& =24 \mathrm{hr}+18 \mathrm{hr}+55 \mathrm{~min}+20 \mathrm{sec} \\
& =1 \mathrm{da}+18 \mathrm{hr}+55 \mathrm{~min}+20 \mathrm{sec} \\
& =\quad 1 \mathrm{da} 18 \mathrm{hr} 55 \mathrm{~min} 20 \mathrm{sec}
\end{aligned}
$$




\subsubsection{Practice Set C}

Perform the following multiplications. Simplify.

Exercise 9.4.13

(Solution on p. 571.)

$2 \cdot(10 \mathrm{~min})$

Exercise 9.4.14

(Solution on p. 571.)

$5 \cdot(3 \mathrm{qt})$

Exercise 9.4.15

$4 \cdot(5 \mathrm{ft} 8$ in. $)$

Exercise 9.4.16

$10 \cdot(2 \mathrm{hr} 15 \mathrm{~min} 40 \mathrm{sec})$

(Solution on p. 571.)

(Solution on p. 571.)

\subsubsection{Dividing a Denominate Number by a Whole Number}

\section{Dividing a Denominate Number by a Whole Number}

To divide a denominate number by a whole number, divide the number part of each unit by the whole number beginning with the largest unit. Affix the unit to this quotient. Carry any remainder to the next unit.

\subsubsection{Sample Set D}

Perform the following divisions. Simplify if necessary.

Example 9.18

$(12 \min 40 \mathrm{sec}) \div 4$

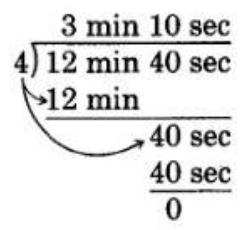

Thus $(12 \mathrm{~min} 40 \mathrm{sec}) \div 4=3 \mathrm{~min} 10 \mathrm{sec}$

Example 9.19

(5 yd 2 ft 9 in. $) \div 3$

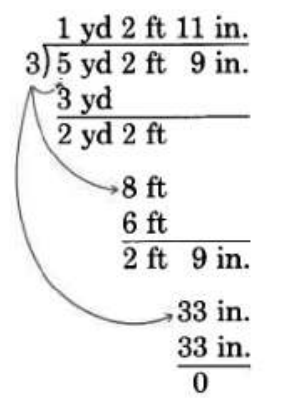

Convert to feet: $2 \mathrm{yd} 2 \mathrm{ft}=8 \mathrm{ft}$.

Convert to inches: $2 \mathrm{ft} 9 \mathrm{in} .=33$ in .

Thus ( 5 yd 2 ft 9 in. $) \div 3=1$ yd 2 ft 11 in. 


\subsubsection{Practice Set D}

Perform the following divisions. Simplify if necessary.

Exercise 9.4.17

(Solution on p. 571.)

$(18 \mathrm{hr} 36 \mathrm{~min}) \div 9$

Exercise 9.4.18

(Solution on p. 571.)

(34 hr 8 min. $) \div 8$

Exercise 9.4.19

(13 yd 7 in.) $\div 5$

(Solution on p. 571.)

Exercise 9.4.20

(Solution on p. 571.)

(47 gal 2 qt 1 pt $) \div 3$

\subsubsection{Exercises}

For the following 15 problems, simplify the denominate numbers.

Exercise 9.4.21

(Solution on p. 571.)

16 in.

Exercise 9.4.22

$19 \mathrm{ft}$

Exercise 9.4.23

(Solution on p. 571.)

$85 \mathrm{~min}$

Exercise 9.4.24

$90 \mathrm{~min}$

Exercise 9.4.25

(Solution on p. 571.)

17 da

Exercise 9.4.26

$25 \mathrm{oz}$

Exercise 9.4.27

(Solution on p. 571.)

$240 \mathrm{oz}$

Exercise 9.4.28

$3,500 \mathrm{lb}$

Exercise 9.4.29

$26 \mathrm{qt}$

Exercise 9.4.30

$300 \mathrm{sec}$

Exercise 9.4.31

(Solution on p. 571.)

$135 \mathrm{oz}$

Exercise 9.4.32

14 tsp

Exercise 9.4.33

$18 \mathrm{pt}$

(Solution on p. 571.)

Exercise 9.4.34

$3,500 \mathrm{~m}$

Exercise 9.4.35

$16,300 \mathrm{~mL}$

(Solution on p. 571.)

For the following 15 problems, perform the indicated operations and simplify the answers if possible. 
Exercise 9.4.36

Add 6 min $12 \mathrm{sec}$ to 5 min $15 \mathrm{sec}$.

Exercise 9.4.37

(Solution on p. 571.)

Add 14 da $6 \mathrm{hr}$ to 1 da $5 \mathrm{hr}$.

Exercise 9.4.38

Add 9 gal 3 qt to 2 gal 3 qt.

Exercise 9.4.39

(Solution on p. 571.)

Add $16 \mathrm{lb} 10 \mathrm{oz}$ to $42 \mathrm{lb} 15 \mathrm{oz}$.

Exercise 9.4.40

Subtract 3 gal 1 qt from 8 gal 3 qt.

Exercise 9.4.41

(Solution on p. 571.)

Subtract $3 \mathrm{ft} 10 \mathrm{in}$. from $5 \mathrm{ft} 8 \mathrm{in}$.

Exercise 9.4.42

Subtract $5 \mathrm{lb} 9 \mathrm{oz}$ from $12 \mathrm{lb} 5$ oz.

Exercise 9.4.43

(Solution on p. 571.)

Subtract $10 \mathrm{hr} 10 \mathrm{~min}$ from $11 \mathrm{hr} 28 \mathrm{~min}$.

Exercise 9.4.44

Add $3 \mathrm{fl} \mathrm{oz} 1$ tbsp 2 tsp to $5 \mathrm{fl} \mathrm{oz} 1$ tbsp 2 tsp.

Exercise 9.4.45

(Solution on p. 571.)

Add 4 da 7 hr 12 min to 1 da $8 \mathrm{hr} 53 \mathrm{~min}$.

Exercise 9.4.46

Subtract 5 hr $21 \mathrm{sec}$ from $11 \mathrm{hr} 2 \mathrm{~min} 14 \mathrm{sec}$.

Exercise 9.4.47

(Solution on p. 571.)

Subtract $6 \mathrm{~T} 1,300 \mathrm{lb} 10 \mathrm{oz}$ from $8 \mathrm{~T} 400 \mathrm{lb} 10 \mathrm{oz}$.

Exercise 9.4.48

Subtract $15 \mathrm{mi} 10 \mathrm{in}$. from $27 \mathrm{mi} 800 \mathrm{ft} 7 \mathrm{in}$.

Exercise 9.4.49

Subtract 3 wk 5 da 50 min 12 sec from 5 wk 6 da 20 min 5 sec.

(Solution on p. 572.)

Exercise 9.4.50

Subtract 3 gal 3 qt 1 pt 1 oz from 10 gal 2 qt 2 oz.

\subsubsection{Exercises for Review}

Exercise 9.4.51

(Section 4.6) Find the value: $\left(\frac{5}{8}\right)^{2}+\frac{39}{64}$.

(Solution on p. 572.)

Exercise 9.4.52

(Section 5.4) Find the sum: $8+6 \frac{3}{5}$.

Exercise 9.4.53

(Section 6.3) Convert 2.05 $\frac{1}{11}$ to a fraction.

(Solution on p. 572.)

Exercise 9.4.54

(Section 7.4) An acid solution is composed of 3 parts acid to 7 parts water. How many parts of acid are there in a solution that contains 126 parts water?

Exercise 9.4.55

(Solution on p. 572.)

(Section 9.3) Convert $126 \mathrm{~kg}$ to grams. 


\subsection{Perimeter and Circumference of Geometric Figures ${ }^{5}$}

\subsubsection{Section Overview}

- Polygons

- Perimeter

- Circumference/Diameter/Radius

- The Number $\pi$

- Formulas

\subsubsection{Polygons}

We can make use of conversion skills with denominate numbers to make measurements of geometric figures such as rectangles, triangles, and circles. To make these measurements we need to be familiar with several definitions.

\section{Polygon}

A polygon is a closed plane (flat) figure whose sides are line segments (portions of straight lines).

\section{Polygons}
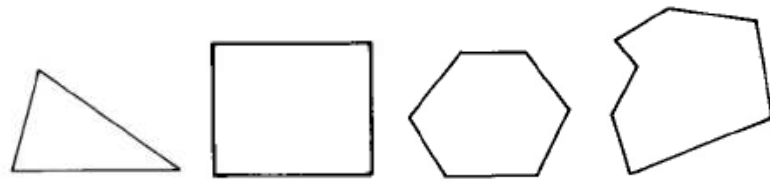

Not polygons
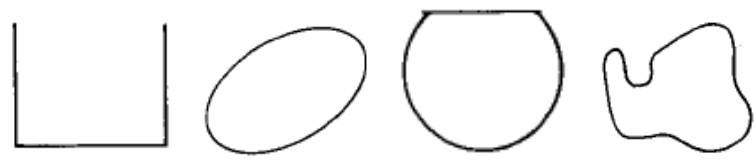

\subsubsection{Perimeter}

\section{Perimeter}

The perimeter of a polygon is the distance around the polygon.

To find the perimeter of a polygon, we simply add up the lengths of all the sides.

\subsubsection{Sample Set A}

Find the perimeter of each polygon.

\section{Example 9.20}

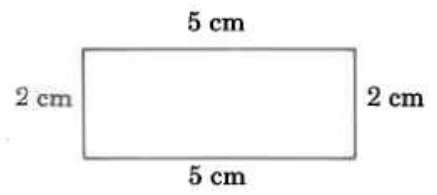

\footnotetext{
${ }^{5}$ This content is available online at $<$ http://cnx.org/content $/ \mathrm{m} 35022 / 1.2 />$.
} 


$$
\begin{array}{rlc}
\text { Perimeter } & =2 \mathrm{~cm}+5 \mathrm{~cm}+2 \mathrm{~cm}+5 \mathrm{~cm} \\
& = & 14 \mathrm{~cm}
\end{array}
$$

\section{Example 9.21}
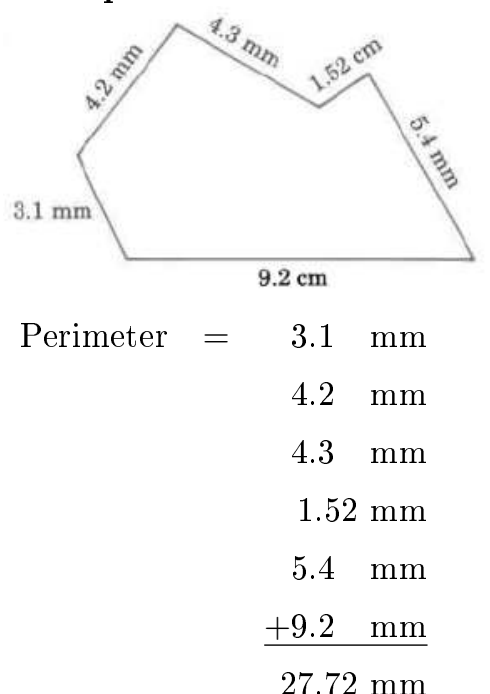

\section{Example 9.22}

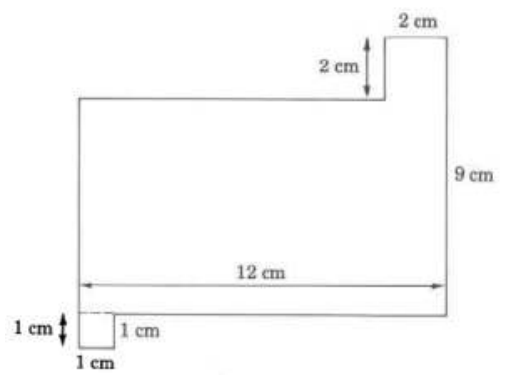

Our first observation is that three of the dimensions are missing. However, we can determine the missing measurements using the following process. Let $\mathrm{A}, \mathrm{B}$, and $\mathrm{C}$ represent the missing measurements. Visualize

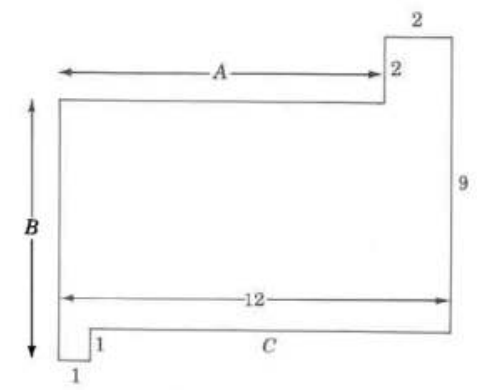

$A=12 \mathrm{~m}-2 \mathrm{~m}=10 \mathrm{~m}$

$B=9 \mathrm{~m}+1 \mathrm{~m}-2 \mathrm{~m}=8 \mathrm{~m}$

$C=12 \mathrm{~m}-1 \mathrm{~m}=11 \mathrm{~m}$ 


$$
\begin{aligned}
& \text { Perimeter }=8 \mathrm{~m} \\
& 10 \mathrm{~m} \\
& 2 \mathrm{~m} \\
& 2 \mathrm{~m} \\
& 9 \mathrm{~m} \\
& 11 \mathrm{~m} \\
& 1 \mathrm{~m} \\
& \begin{array}{r}
+1 \mathrm{~m} \\
\hline 44 \mathrm{~m}
\end{array}
\end{aligned}
$$

\subsubsection{Practice Set A}

Find the perimeter of each polygon.

Exercise 9.5.1

(Solution on p. 572.)

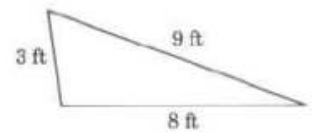

\section{Exercise 9.5.2}

(Solution on p. 572.)

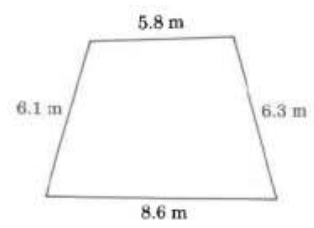

Exercise 9.5.3

(Solution on p. 572.)

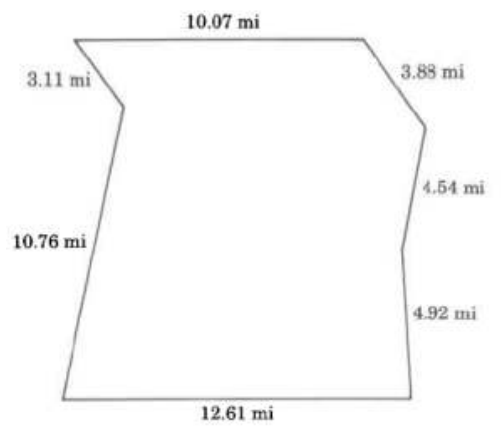

\subsubsection{Circumference/Diameter/Radius}

\section{Circumference}

The circumference of a circle is the distance around the circle.

\section{Diameter}

A diameter of a circle is any line segment that passes through the center of the circle and has its endpoints on the circle. 


\section{Radius}

A radius of a circle is any line segment having as its endpoints the center of the circle and a point on the circle.

The radius is one half the diameter.

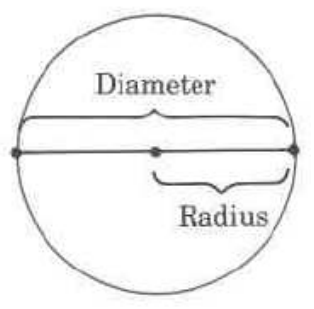

\subsubsection{The Number $\pi$}

The symbol $\pi$, read "pi," represents the nonterminating, nonrepeating decimal number $3.14159 \ldots$.. This number has been computed to millions of decimal places without the appearance of a repeating block of digits.

For computational purposes, $\pi$ is often approximated as 3.14 . We will write $\pi \approx 3.14$ to denote that $\pi$ is approximately equal to 3.14 . The symbol " $\approx$ " means "approximately equal to."

\subsubsection{Formulas}

To find the circumference of a circle, we need only know its diameter or radius. We then use a formula for computing the circumference of the circle.

\section{Formula}

A formula is a rule or method for performing a task. In mathematics, a formula is a rule that directs us in computations.

Formulas are usually composed of letters that represent important, but possibly unknown, quantities.

If $C, d$, and $r$ represent, respectively, the circumference, diameter, and radius of a circle, then the following two formulas give us directions for computing the circumference of the circle.

\section{Circumference Formulas}

1. $C=\pi d$ or $C \approx(3.14) d$

2. $C=2 \pi r$ or $C \approx 2(3.14) r$

\subsubsection{Sample Set B}

\section{Example 9.23}

Find the exact circumference of the circle.

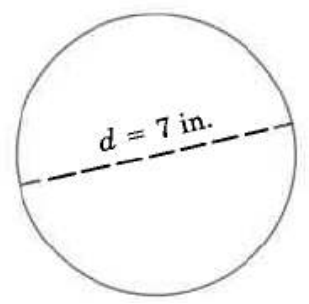

Use the formula $C=\pi d$. 
$C=\pi \cdot 7$ in

By commutativity of multiplication,

$C=7$ in. $\cdot \pi$

$C=7 \pi$ in., exactly

This result is exact since $\pi$ has not been approximated.

Example 9.24

Find the approximate circumference of the circle.

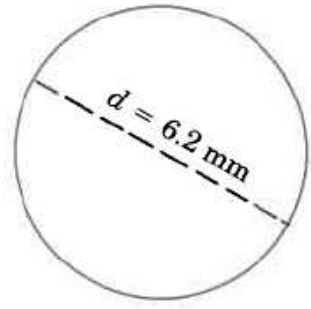

Use the formula $C=\pi d$.

$C \approx(3.14)(6.2)$

$C \approx 19.648 \mathrm{~mm}$

This result is approximate since $\pi$ has been approximated by 3.14 .

\section{Example 9.25}

Find the approximate circumference of a circle with radius 18 inches.

Since we're given that the radius, $r$, is 18 in., we'll use the formula $C=2 \pi r$.

$C \approx(2)(3.14)(18$ in. $)$

$C \approx 113.04$ in.

\section{Example 9.26}

Find the approximate perimeter of the figure.

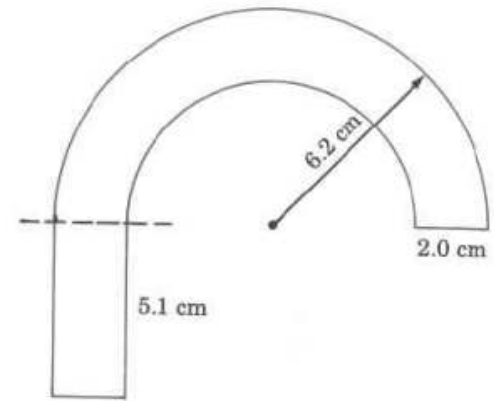

We notice that we have two semicircles (half circles).

The larger radius is $6.2 \mathrm{~cm}$.

The smaller radius is $6.2 \mathrm{~cm}-2.0 \mathrm{~cm}=4.2 \mathrm{~cm}$.

The width of the bottom part of the rectangle is $2.0 \mathrm{~cm}$. 


$$
\begin{aligned}
& \text { Perimeter }= \\
& 2.0 \mathrm{~cm} \\
& (0.5) \cdot(2) \cdot(3.14) \cdot(6.2 \mathrm{~cm}) \quad \text { Circumference of outer semicircle. } \\
& \text { Perimeter } \approx 2.0 \mathrm{~cm} \\
& 5.1 \mathrm{~cm} \\
& 2.0 \mathrm{~cm} \\
& 5.1 \quad \mathrm{~cm} \\
& 19.468 \mathrm{~cm} \\
& +13.188 \mathrm{~cm} \\
& 48.856 \mathrm{~cm}
\end{aligned}
$$$$
5.1 \mathrm{~cm}
$$$$
2.0 \mathrm{~cm}
$$$$
5.1 \mathrm{~cm}
$$$$
+(0.5) \cdot(2) \cdot(3.14) \cdot(4.2 \mathrm{~cm}) \quad \text { Circumference of inner semicircle }
$$$$
6.2 \mathrm{~cm}-2.0 \mathrm{~cm}=4.2 \mathrm{~cm}
$$

The 0.5 appears because we want the perimeter of only half a circle.

\subsubsection{Practice Set B}

Exercise 9.5.4

(Solution on p. 572.)

Find the exact circumference of the circle.

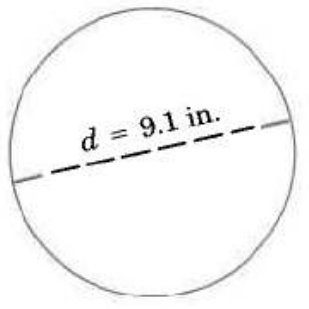

Exercise 9.5.5

(Solution on p. 572.)

Find the approximate circumference of the circle.

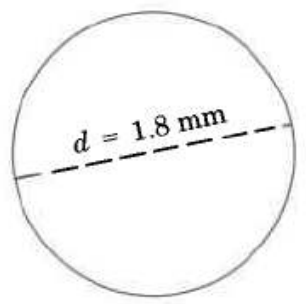

Exercise 9.5.6

(Solution on p. 572.)

Find the approximate circumference of the circle with radius $20.1 \mathrm{~m}$.

Exercise 9.5.7

(Solution on p. 572.)

Find the approximate outside perimeter of 


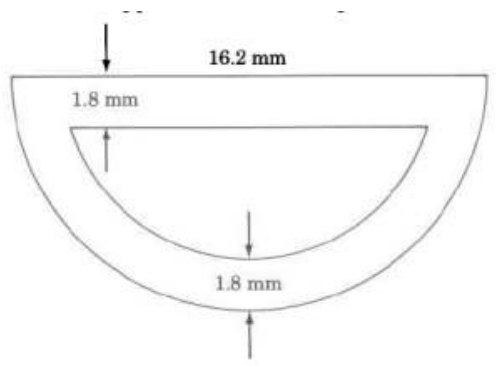

\subsubsection{Exercises}

Find each perimeter or approximate circumference. Use $\pi=3.14$.

Exercise 9.5.8

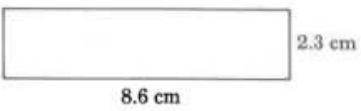

Exercise 9.5.9

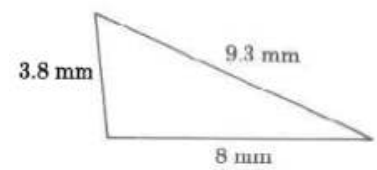

Exercise 9.5.10

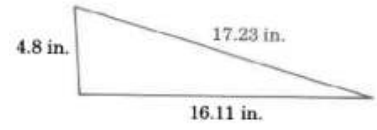

Exercise 9.5.11

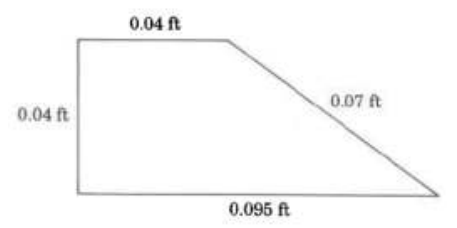

Exercise 9.5.12

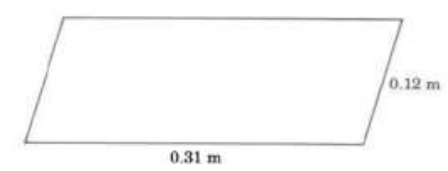

Exercise 9.5.13

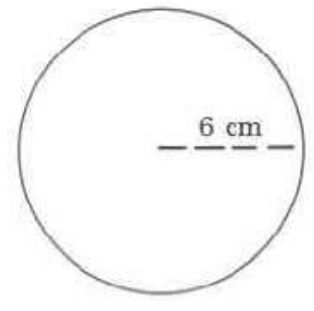

(Solution on p. 572.)

(Solution on p. 572.)

(Solution on p. 572.) 
Exercise 9.5.14

(Solution on p. 572.)

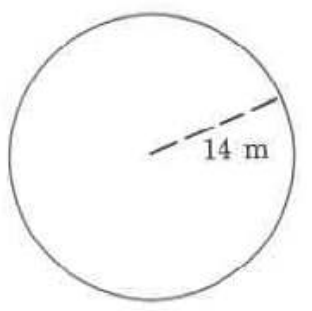

Exercise 9.5.15

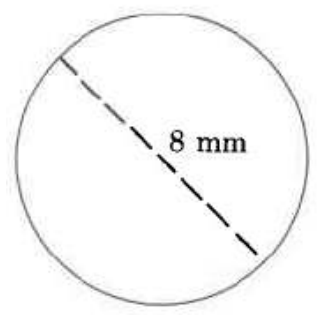

Exercise 9.5.16

(Solution on p. 572.)

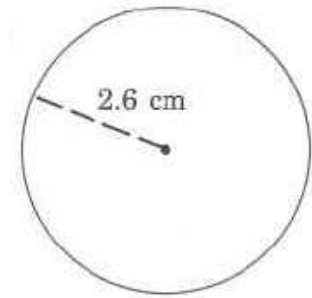

Exercise 9.5.17

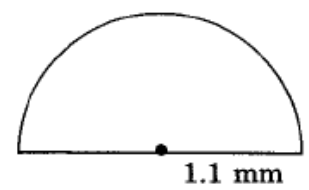

Exercise 9.5.18

(Solution on p. 572.)

$0.03 \mathrm{~cm}$

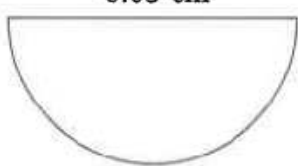

Exercise 9.5.19

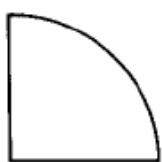

5 in. 
Exercise 9.5.20

(Solution on p. 572.)

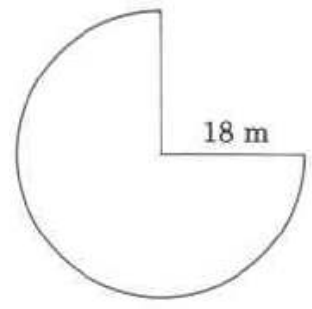

Exercise 9.5.21

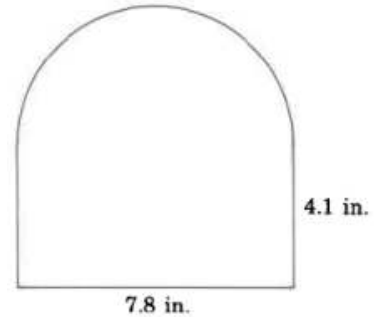

Exercise 9.5.22

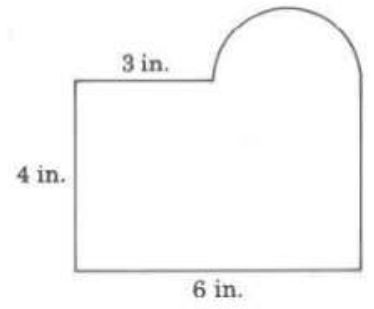

(Solution on p. 572.)

Exercise 9.5.23

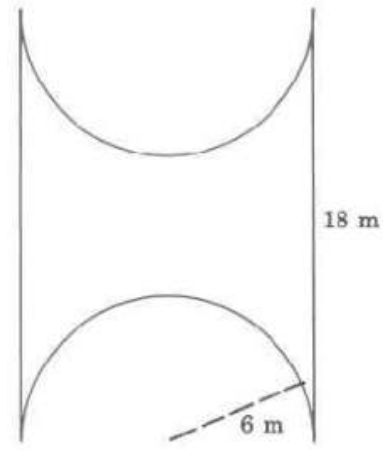


Exercise 9.5.24

(Solution on p. 572.)

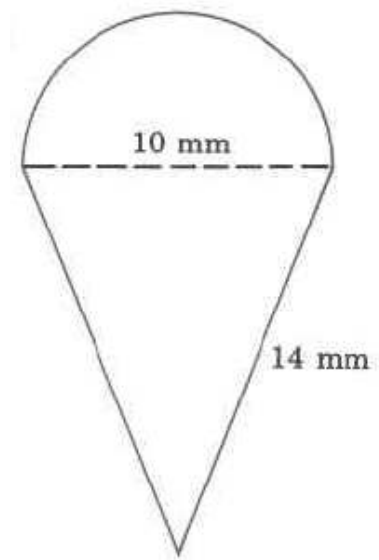

Exercise 9.5.25

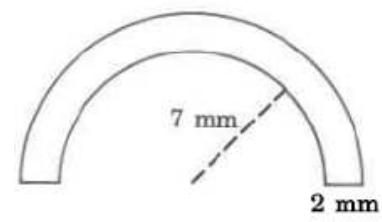

Exercise 9.5.26

(Solution on p. 572.)

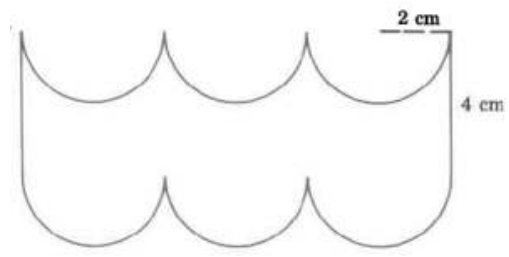

Exercise 9.5.27

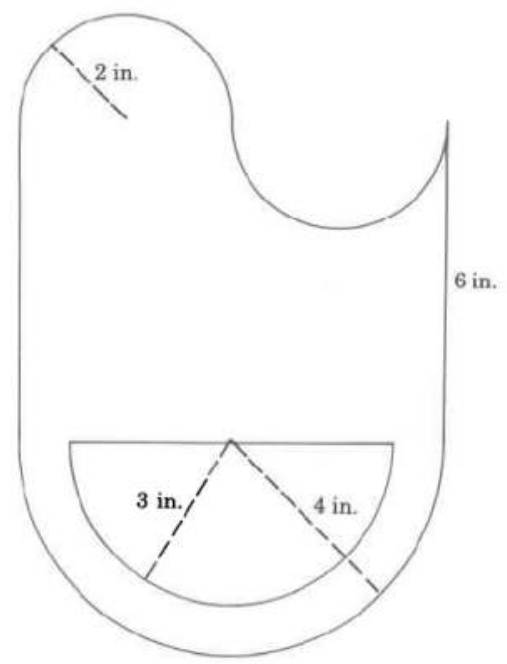




\subsubsection{Exercises for Review}

Exercise 9.5.28

(Solution on p. 572.)

(Section 4.5) Find the value of $2 \frac{8}{13} \cdot \sqrt{10 \frac{9}{16}}$.

Exercise 9.5.29

(Section 5.3) Find the value of $\frac{8}{15}+\frac{7}{10}+\frac{21}{60}$.

Exercise 9.5.30

(Solution on p. 572.)

(Section 6.9) Convert $\frac{7}{8}$ to a decimal.

Exercise 9.5.31

(Section 9.2) What is the name given to a quantity that is used as a comparison to determine the measure of another quantity?

Exercise 9.5.32

(Solution on p. 572.)

(Section 9.4) Add $42 \mathrm{~min} 26 \mathrm{sec}$ to $53 \mathrm{~min} 40 \mathrm{sec}$ and simplify the result.

\subsection{Area and Volume of Geometric Figures and Objects ${ }^{6}$}

\subsubsection{Section Overview}

- The Meaning and Notation for Area

- Area Formulas

- Finding Areas of Some Common Geometric Figures

- The Meaning and Notation for Volume

- Volume Formulas

- Finding Volumes of Some Common Geometric Objects

Quite often it is necessary to multiply one denominate number by another. To do so, we multiply the number parts together and the unit parts together. For example,

$$
\begin{aligned}
& 8 \text { in. } \cdot 8 \text { in. }=8 \cdot 8 \cdot \text { in. } \cdot \text { in. } \\
& =64 \mathrm{in}^{2} \\
& 4 \mathrm{~mm} \cdot 4 \mathrm{~mm} \cdot 4 \mathrm{~mm}=4 \cdot 4 \cdot 4 \cdot \mathrm{mm} \cdot \mathrm{mm} \cdot \mathrm{mm} \\
& =64 \mathrm{~mm}^{3}
\end{aligned}
$$

Sometimes the product of units has a physical meaning. In this section, we will examine the meaning of the products (length unit) $)^{2}$ and (length unit) ${ }^{3}$.

\subsubsection{The Meaning and Notation for Area}

The product (length unit) ( (length unit) $=\left(\right.$ length unit) ${ }^{2}$, or, square length unit (sq length unit), can be interpreted physically as the area of a surface.

Area

The area of a surface is the amount of square length units contained in the surface.

For example, $3 \mathrm{sq}$ in. means that 3 squares, 1 inch on each side, can be placed precisely on some surface. (The squares may have to be cut and rearranged so they match the shape of the surface.)

\footnotetext{
${ }^{6}$ This content is available online at $<$ http://cnx.org/content $/ \mathrm{m} 35023 / 1.2 />$.
} 
We will examine the area of the following geometric figures.

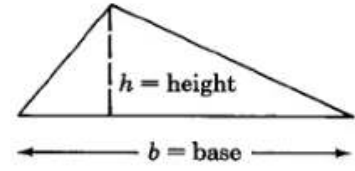

Triangles

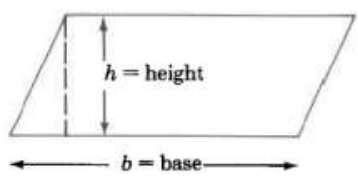

Parallelograms

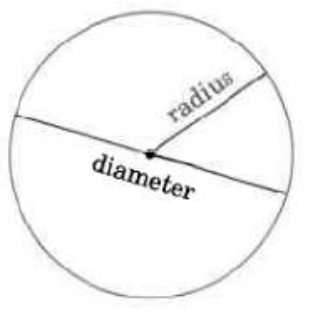

Circles

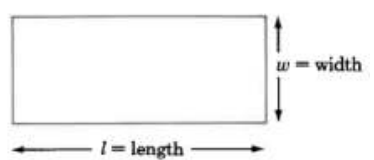

Rectangles

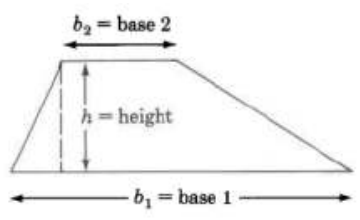

Trapezoids

\subsubsection{Area Formulas}

We can determine the areas of these geometric figures using the following formulas.

\begin{tabular}{|l|l|l|l|}
\hline & Figure & Area Formula & Statement \\
\hline & Triangle & $A_{T}=\frac{1}{2} \cdot b \cdot h$ & $\begin{array}{l}\text { Area of a triangle is one } \\
\text { half the base times the } \\
\text { height. }\end{array}$ \\
\hline & Rectangle & $A_{R}=l \cdot w$ & $\begin{array}{l}\text { Area of a rectangle is } \\
\text { the length times the } \\
\text { width. }\end{array}$ \\
\hline & & \begin{tabular}{l} 
continued on next page \\
\hline
\end{tabular} &
\end{tabular}




\begin{tabular}{|l|l|l|l|}
\hline & Parallelogram & $A_{P}=b \cdot h$ & $\begin{array}{l}\text { Area of a parallelogram } \\
\text { is base times the height. }\end{array}$ \\
\hline & Trapezoid & $A_{\text {Trap }}=\frac{1}{2} \cdot\left(b_{1}+b_{2}\right) \cdot h$ & $\begin{array}{l}\text { Area of a trapezoid is } \\
\text { one half the sum of } \\
\text { the two bases times the } \\
\text { height. }\end{array}$ \\
\hline & Circle & $A_{C}=\pi r^{2}$ & $\begin{array}{l}\text { Area of a circle is } \pi \\
\text { times the square of the } \\
\text { radius. }\end{array}$ \\
\hline
\end{tabular}

Table 9.5

\subsubsection{Finding Areas of Some Common Geometric Figures}

\subsubsection{Sample Set A}

\section{Example 9.27}

Find the area of the triangle.

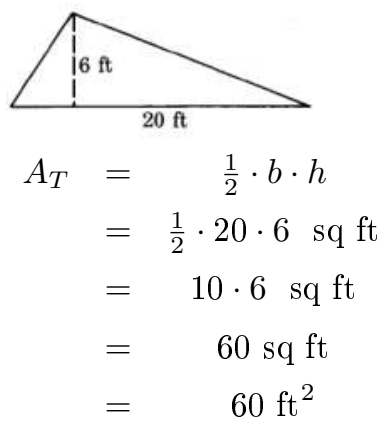

The area of this triangle is $60 \mathrm{sq} \mathrm{ft}$, which is often written as $60 \mathrm{ft}^{2}$.

Example 9.28

Find the area of the rectangle.

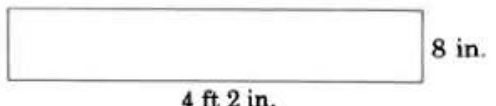

Let's first convert $4 \mathrm{ft} 2 \mathrm{in}$. to inches. Since we wish to convert to inches, we'll use the unit fraction $\frac{12 \mathrm{in} .}{1 \mathrm{ft}}$ since it has inches in the numerator. Then,

$$
\begin{aligned}
4 \mathrm{ft} & =\frac{4 \mathrm{ft}}{\frac{1}{1}} \cdot \frac{12 \mathrm{in} .}{1 \mathrm{ft}} \\
& =\frac{4) \mathrm{ft}}{1} \cdot \frac{12 \mathrm{in} .}{1) \mathrm{ft}} \\
& =48 \mathrm{in} .
\end{aligned}
$$


Thus, 4 ft 2 in. $=48$ in. +2 in. $=50$ in.

$$
\begin{aligned}
A_{R} & =l \cdot w \\
& =50 \mathrm{in.} \cdot 8 \mathrm{in} . \\
& =\quad 400 \mathrm{sq} \mathrm{in} .
\end{aligned}
$$

The area of this rectangle is $400 \mathrm{sq}$ in.

\section{Example 9.29}

Find the area of the parallelogram.

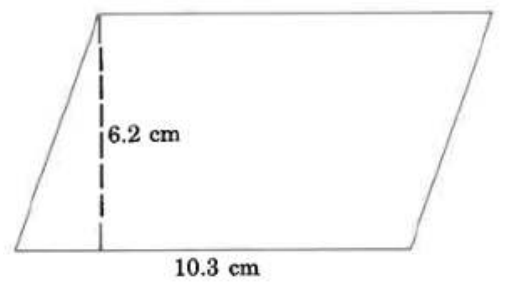

$$
\begin{array}{rlc}
A_{P} & = & b \cdot h \\
& = & 10.3 \mathrm{~cm} \cdot 6.2 \mathrm{~cm} \\
& = & 63.86 \mathrm{sq} \mathrm{cm}
\end{array}
$$

The area of this parallelogram is $63.86 \mathrm{sq} \mathrm{cm}$.

\section{Example 9.30}

Find the area of the trapezoid.

$$
\begin{array}{rlc}
A_{\text {Trap }} & = & \\
& 20.4 \mathrm{~mm} & \frac{1}{2} \cdot\left(b_{1}+b_{2}\right) \cdot h \\
& = & \frac{1}{2} \cdot(14.5 \mathrm{~mm},+, 20.4 \mathrm{~mm}) \cdot(4.1 \mathrm{~mm}) \\
= & \frac{1}{2} \cdot(34.9 \mathrm{~mm}) \cdot(4.1 \mathrm{~mm}) \\
= & \frac{1}{2} \cdot(143.09 \mathrm{sq} \mathrm{mm}) \\
= & 71.545 \mathrm{sq} \mathrm{mm}
\end{array}
$$

The area of this trapezoid is $71.545 \mathrm{sq} \mathrm{mm}$.

\section{Example 9.31}

Find the approximate area of the circle.

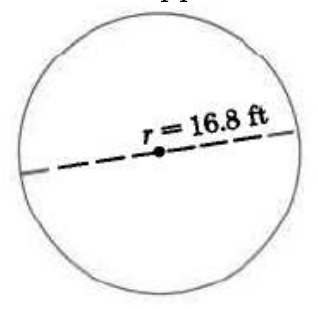




$$
\begin{array}{rlc}
A_{c} & = & \pi \cdot r^{2} \\
& \approx & (3.14) \cdot(16.8 \mathrm{ft})^{2} \\
& \approx & (3.14) \cdot(282.24 \mathrm{sq} \mathrm{ft}) \\
& \approx & 888.23 \mathrm{sq} \mathrm{ft}
\end{array}
$$

The area of this circle is approximately $886.23 \mathrm{sq} \mathrm{ft}$.

\subsubsection{Practice Set A}

Find the area of each of the following geometric figures.

Exercise 9.6.1

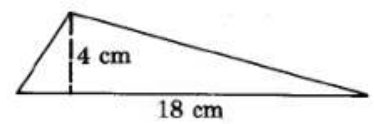

Exercise 9.6.2

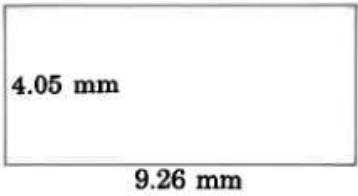

Exercise 9.6.3

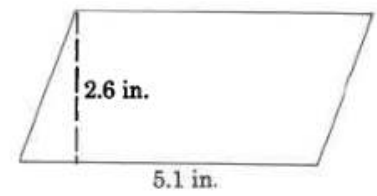

Exercise 9.6.4

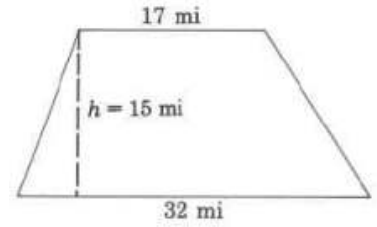

Exercise 9.6.5

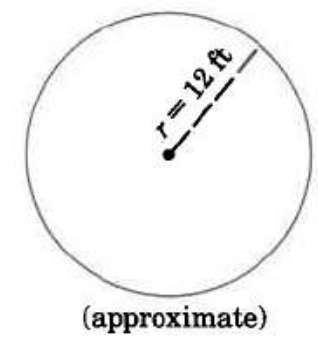

(Solution on p. 572.)

(Solution on p. 573.)

(Solution on p. 573.)

(Solution on p. 573.)

(Solution on p. 573.) 
Exercise 9.6.6

(Solution on p. 573.)

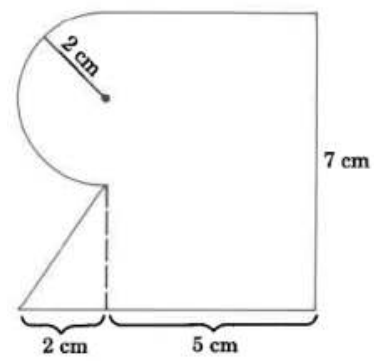

\subsubsection{The Meaning and Notation for Volume}

The product (length unit) (length unit) (length unit) $=(\text { length unit })^{3}$, or cubic length unit (cu length unit), can be interpreted physically as the volume of a three-dimensional object.

\section{Volume}

The volume of an object is the amount of cubic length units contained in the object.

For example, $4 \mathrm{cu} \mathrm{mm}$ means that 4 cubes, $1 \mathrm{~mm}$ on each side, would precisely fill some three-dimensional object. (The cubes may have to be cut and rearranged so they match the shape of the object.)

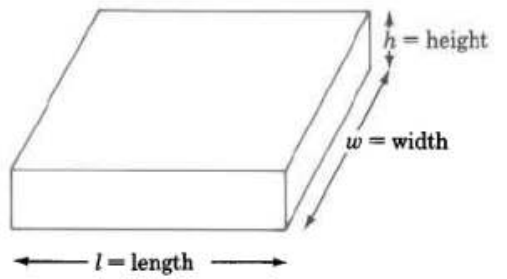

Rectangular solid

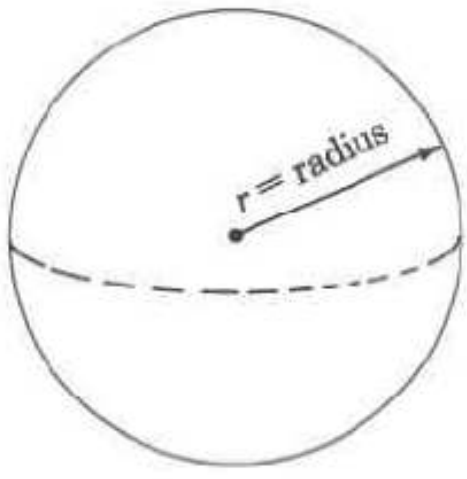

Sphere 

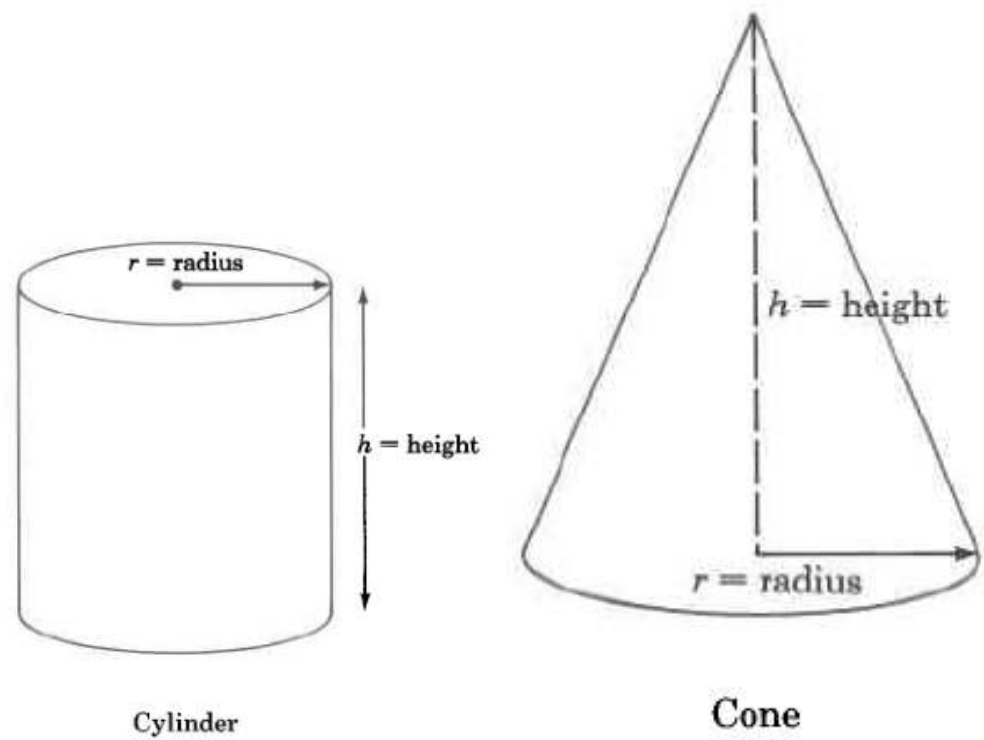

Cone

\subsubsection{Volume Formulas}

\begin{tabular}{|l|l|l|l|}
\hline & Figure & Volume Formula & Statement \\
\hline & Rectangular solid & $\begin{array}{r}V_{R}=l \cdot w \cdot h \\
=\quad(\text { area of base) }\end{array}$ & $\begin{array}{l}\text { The volume of a rectan- } \\
\text { (beirht } \\
\text { times the width times } \\
\text { the height. }\end{array}$ \\
\hline & Sphere & $V_{S}=\frac{4}{3} \cdot \pi \cdot r^{3}$ & $\begin{array}{l}\text { The volume of a sphere } \\
\text { is } \frac{4}{3} \text { times } \pi \text { times the } \\
\text { cube of the radius. }\end{array}$ \\
\hline
\end{tabular}




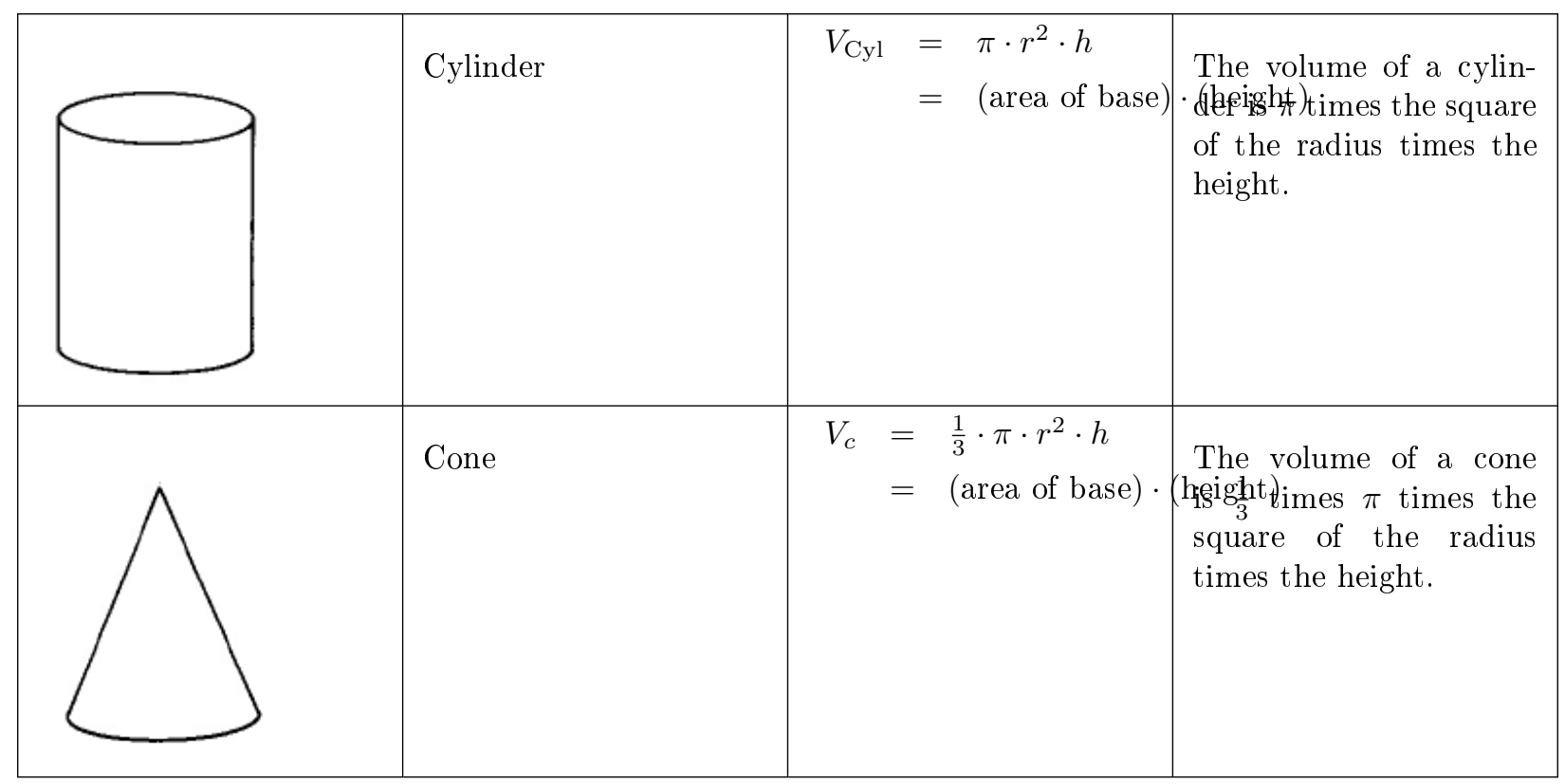

Table 9.6

\subsubsection{Finding Volumes of Some Common Geometric Objects}

\subsubsection{Sample Set B}

Example 9.32

Find the volume of the rectangular solid.

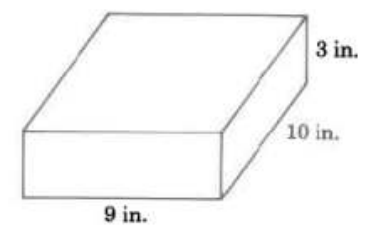

$$
\begin{array}{rcc}
V_{R} & = & l \cdot w \cdot h \\
& = & 9 \mathrm{in} . \cdot 10 \mathrm{in.} \cdot 3 \mathrm{in} . \\
& = & 270 \mathrm{cu} \mathrm{in.} \\
& = & 270 \mathrm{in}^{3}
\end{array}
$$

The volume of this rectangular solid is $270 \mathrm{cu}$ in.

Example 9.33

Find the approximate volume of the sphere.

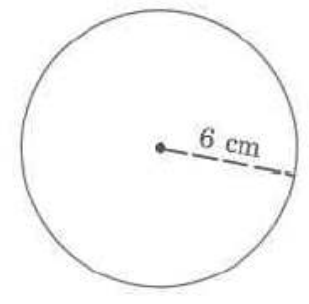




$$
\begin{array}{rlc}
V_{S} & = & \frac{4}{3} \cdot \pi \cdot r^{3} \\
& \approx & \left(\frac{4}{3}\right) \cdot(3.14) \cdot(6 \mathrm{~cm})^{3} \\
& \approx & \left(\frac{4}{3}\right) \cdot(3.14) \cdot(216 \mathrm{cu} \mathrm{cm}) \\
& \approx & 904.32 \mathrm{cu} \mathrm{cm}
\end{array}
$$

The approximate volume of this sphere is $904.32 \mathrm{cu} \mathrm{cm}$, which is often written as $904.32 \mathrm{~cm}^{3}$.

\section{Example 9.34}

Find the approximate volume of the cylinder.

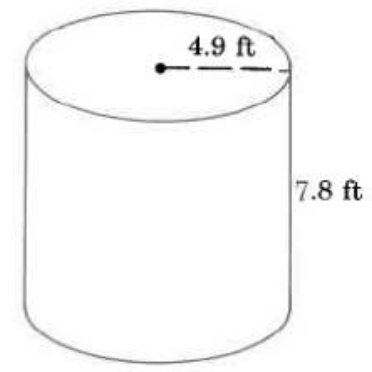

$$
\begin{array}{rlc}
V_{\mathrm{Cyl}} & = & \pi \cdot r^{2} \cdot h \\
& \approx & (3.14) \cdot(4.9 \mathrm{ft})^{2} \cdot(7.8 \mathrm{ft}) \\
& \approx & (3.14) \cdot(24.01 \mathrm{sq} \mathrm{ft}) \cdot(7.8 \mathrm{ft}) \\
& \approx & (3.14) \cdot(187.278 \mathrm{cu} \mathrm{ft}) \\
& \approx & 588.05292 \mathrm{cu} \mathrm{ft}
\end{array}
$$

The volume of this cylinder is approximately $588.05292 \mathrm{cu} \mathrm{ft}$. The volume is approximate because we approximated $\pi$ with 3.14 .

\section{Example 9.35}

Find the approximate volume of the cone. Round to two decimal places.

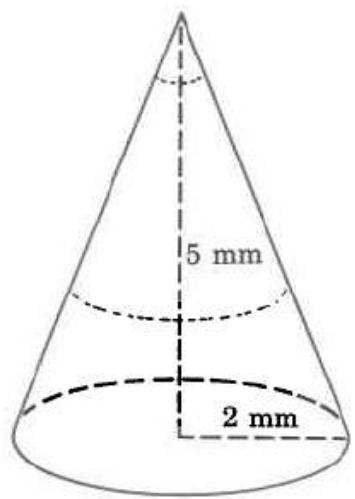

$$
\begin{aligned}
& V_{c}=\quad \frac{1}{3} \cdot \pi \cdot r^{2} \cdot h \\
& \approx\left(\frac{1}{3}\right) \cdot(3.14) \cdot(2 \mathrm{~mm})^{2} \cdot(5 \mathrm{~mm}) \\
& \approx\left(\frac{1}{3}\right) \cdot(3.14) \cdot(4 \mathrm{sq} \mathrm{mm}) \cdot(5 \mathrm{~mm}) \\
& \approx \quad\left(\frac{1}{3}\right) \cdot(3.14) \cdot(20 \mathrm{cu} \mathrm{mm}) \\
& \approx \quad 20.9 \overline{3} \mathrm{cu} \mathrm{mm} \\
& \approx \quad 20.93 \mathrm{cu} \mathrm{mm}
\end{aligned}
$$


The volume of this cone is approximately $20.93 \mathrm{cu} \mathrm{mm}$. The volume is approximate because we approximated $\pi$ with 3.14 .

\subsubsection{Practice Set B}

Find the volume of each geometric object. If $\pi$ is required, approximate it with 3.14 and find the approximate volume.

Exercise 9.6.7

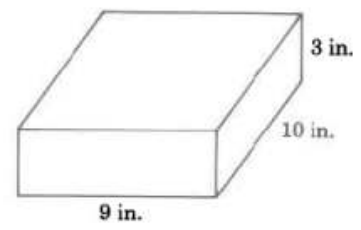

Exercise 9.6.8

Sphere

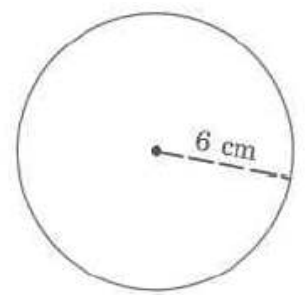

Exercise 9.6.9

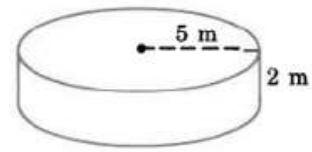

Exercise 9.6.10

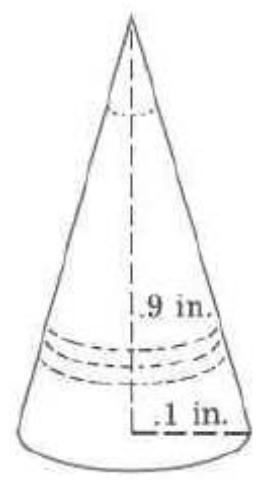

(Solution on p. 573.)

(Solution on p. 573.)

(Solution on p. 573.)

(Solution on p. 573.)

\subsubsection{Exercises}

Find each indicated measurement. 
Exercise 9.6.11

(Solution on p. 573.)

Area

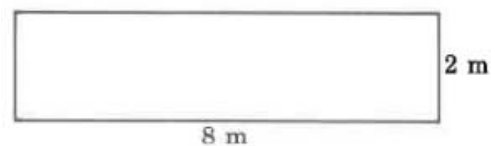

Exercise 9.6.12

Area

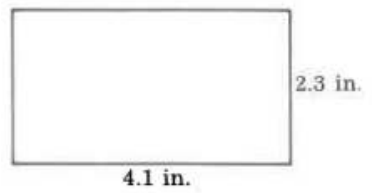

Exercise 9.6.13

(Solution on p. 573.)

Area

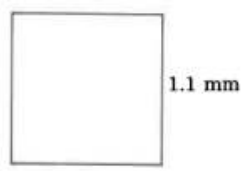

Exercise 9.6.14

Area

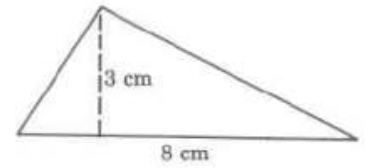

Exercise 9.6.15

Area

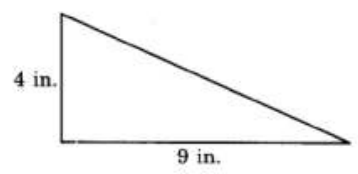

Exercise 9.6.16

Area

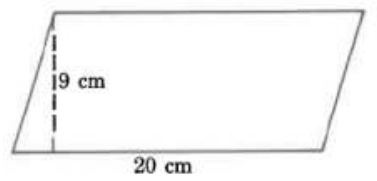

(Solution on p. 573.)

Exercise 9.6.17

Exact area

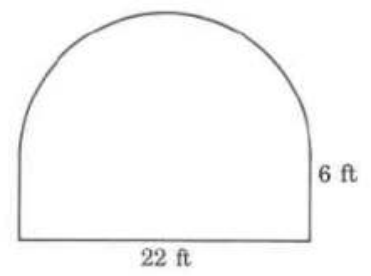

(Solution on p. 573.) 
Exercise 9.6.18

Approximate area

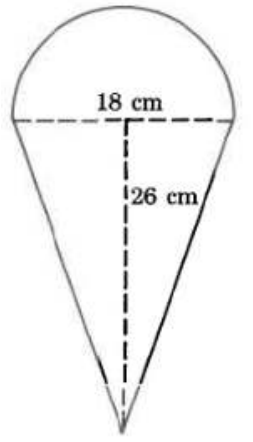

Exercise 9.6.19

(Solution on p. 573.)

Area

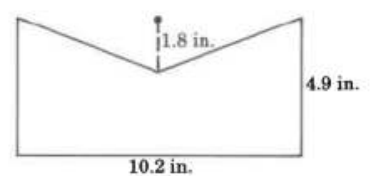

Exercise 9.6.20

Area

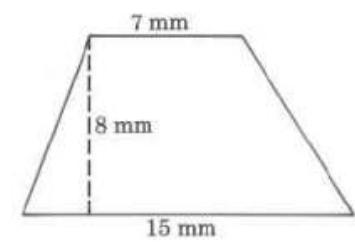

Exercise 9.6.21

Approximate area

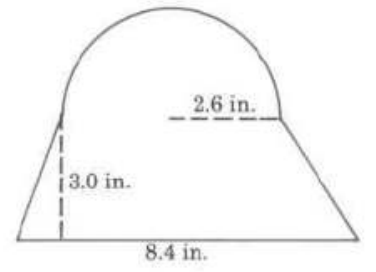

Exercise 9.6.22

Exact area

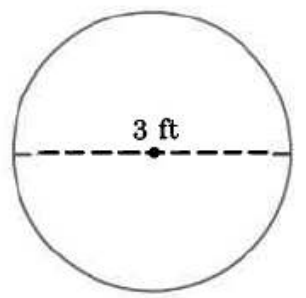

Exercise 9.6.23

(Solution on p. 573.)

Approximate area 


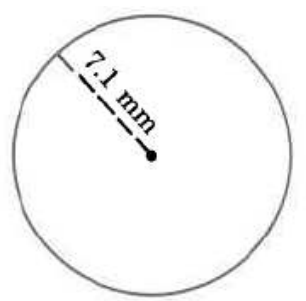

Exercise 9.6.24

Exact area

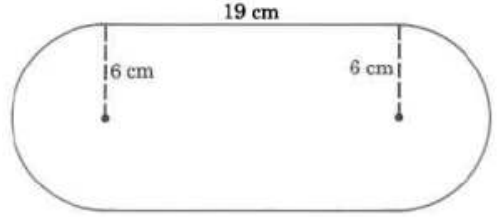

Exercise 9.6.25

(Solution on p. 573.)

Approximate area

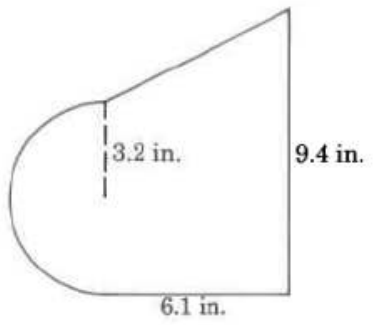

Exercise 9.6.26

Area

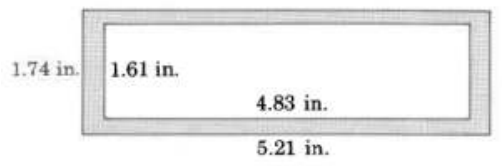

Exercise 9.6.27

(Solution on p. 573.)

Approximate area

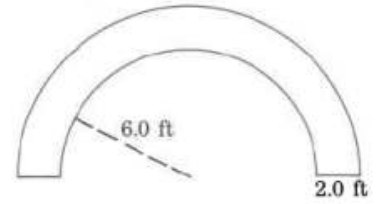

Exercise 9.6.28

Volume

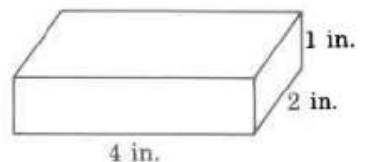

Exercise 9.6.29

(Solution on p. 573.)

Volume 


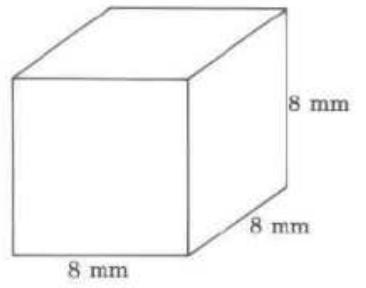

Exercise 9.6.30

Exact volume

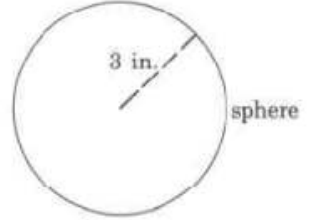

Exercise 9.6.31

(Solution on p. 573.)

Approximate volume

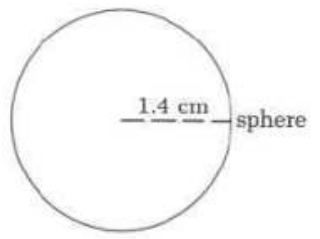

Exercise 9.6.32

Approximate volume

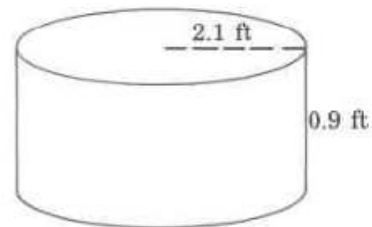

Exercise 9.6.33

(Solution on p. 573.)

Exact volume

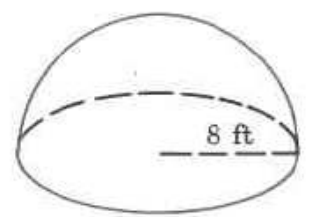

Exercise 9.6.34

Approximate volume

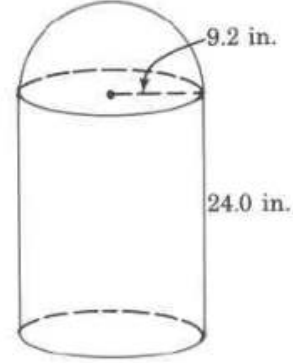


Exercise 9.6.35

(Solution on p. 573.)

Approximate volume

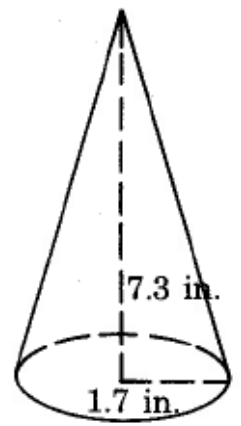

\section{Exercise 9.6.36}

Approximate volume

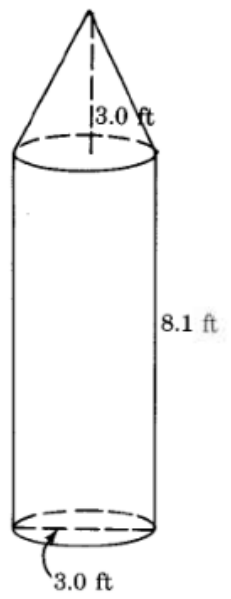

\subsubsection{Exercises for Review}

Exercise 9.6.37

(Solution on p. 573.)

(Section 1.2) In the number 23,426, how many hundreds are there?

Exercise 9.6.38

(Section 3.4) List all the factors of 32.

Exercise 9.6.39

(Section 5.4) Find the value of $4 \frac{3}{4}-3 \frac{5}{6}+1 \frac{2}{3}$.

(Solution on p. 573.)

Exercise 9.6.40

(Section 5.6) Find the value of $\frac{5+\frac{1}{3}}{2+\frac{2}{15}}$.

Exercise 9.6.41

(Solution on p. 573.)

(Section 9.5) Find the perimeter.

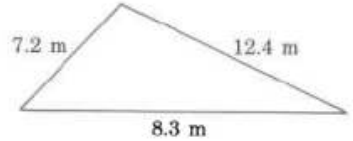




\subsection{Summary of Key Concepts ${ }^{7}$}

\subsubsection{Summary of Key Concepts}

Measurement (Section 9.2)

Measurement is comparison to some standard.

Standard Unit of Measure (Section 9.2)

A quantity that is used for comparison is called a standard unit of measure.

Two Types of Measurement Systems (Section 9.2)

There are two major types of measurement systems in use today. They are the United States system and the metric system.

Unit Fraction (Section 9.2)

A unit fraction is a fraction that has a value of 1 . Unit fractions can be used to convert from one unit of measure to another.

Meter, Liter, Gram, and associated prefixes (Section 9.3)

Common units of measure in the metric system are the meter $(\mathrm{m})$, for length, the liter (L), for volume, and the gram (g), for mass. To each of these units, a prefix can be attached.

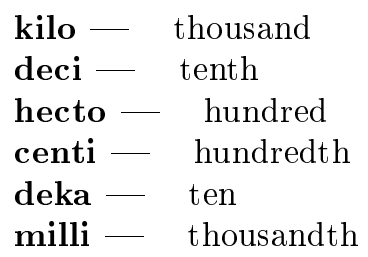

Metric Conversions (Section 9.3)

To convert from one metric unit to another:

1. Determine the location of the original number on the metric scale.

2. Move the decimal point of the original number in the same direction and the same number of places as is necessary to move to the metric unit you wish to convert to.

\section{Denominate Numbers (Section 9.4)}

Numbers that have units of measure associated with them are denominate numbers. The number $25 \mathrm{mg}$ is a denominate number since the $\mathrm{mg}$ unit is associated with the pure number 25 . The number 82 is not a denominate number since it has no unit of measure associated with it.

Simplified Denominate Number (Section 9.4)

A denominate number is simplified when the number of standard units of measure associated with it does not exceed the next higher type of unit. $55 \mathrm{~min}$ is simplified, whereas $65 \mathrm{~min}$ is not simplified

Addition and Subtraction of Denominate Numbers (Section 9.4) Denominate numbers can be added or subtracted by

1. writing the numbers vertically so that the like units appear in the same column.

2. adding or subtracting the number parts, carrying along the unit.

3. simplifying the sum or difference.

\footnotetext{
${ }^{7}$ This content is available online at $<\mathrm{http}: / / \mathrm{cnx} . o r g /$ content $/ \mathrm{m} 35024 / 1.2 />$.
} 
Multiplying a Denominate Number by a Whole Number (Section 9.4)

To multiply a denominate number by a whole number, multiply the number part of each unit by the whole number and affix the unit to the product.

Dividing a Denominate Number by a Whole Number (Section 9.4)

To divide a denominate number by a whole number, divide the number part of each unit by the whole number beginning with the largest unit. Affix the unit to this quotient. Carry the remainder to the next unit.

Polygon (Section 9.5)

A polygon is a closed plane (flat) figure whose sides are line segments (portions of straight lines).

Perimeter (Section 9.5)

The perimeter of a polygon is the distance around the polygon.

Circumference, Diameter, Radius (Section 9.5)

The circumference of a circle is the distance around the circle. The diameter of a circle is any line segment that passes through the center of the circle and has its endpoints on the circle. The radius of a circle is one half the diameter of the circle.

The number $\pi$ (Section 9.5)

The symbol $\pi$, read "pi," represents the nonterminating, nonrepeating decimal number 3.14159... . For computational purposes, $\pi$ is often approximated by the number 3.14 .

\section{Formula (Section 9.5)}

A formula is a rule for performing a task. In mathematics, a formula is a rule that directs us in computations.

Circumference Formulas (Section 9.5)

$C=\pi \cdot d \quad C \approx(3.14) d$

$C=2 \cdot \pi \cdot r \quad C \approx 2(3.14) r$

Area (Section 9.6)

The area of a surface is the amount of square length units contained in the surface.

Volume (Section 9.6)

The volume of an object is a measure of the amount of cubic length units contained in the object.

Area Formulas (Section 9.6)

Triangle: $A=\frac{1}{2} \cdot b \cdot h$

Rectangle: $A=l \cdot w$

Parallelogram: $A=b \cdot h$

Trapezoid: $A=\frac{1}{2} \cdot\left(b_{1}+b_{2}\right) \cdot h$
Circle: $A=\pi \cdot r^{2}$

Volume Formulas (Section 9.6)

Rectangle solid: $V=l \cdot w \cdot h$

Sphere: $V=\frac{4}{3} \cdot \pi \cdot r^{3}$

Cylinder: $V=\pi \cdot r^{2} \cdot h$

Cone: $V=\frac{1}{3} \cdot \pi \cdot r^{2} \cdot h$ 


\subsection{Exercise Supplement ${ }^{8}$}

\subsubsection{Exercise Supplement}

9.8.1.1 Measurement and the United States System (Section 9.2)

Exercise 9.8.1

(Solution on p. 574.)

What is measurement?

For problems 2-6, make each conversion. Use the conversion table given in Section 9.1.

Exercise 9.8.2

$9 \mathrm{ft}=$ yd

Exercise 9.8.3

$32 \mathrm{oz}=$ $\mathrm{lb}$

(Solution on p. 574.)

Exercise 9.8.4

$1,500 \mathrm{mg}=$ g

Exercise 9.8.5

$12,000 \mathrm{lb}=$ $\mathrm{T}$

(Solution on p. 574.)

Exercise 9.8.6

$5,280 \mathrm{ft}=$ mi

For problems 7-23, make each conversion.

Exercise 9.8.7

(Solution on p. 574.)

$23 \mathrm{yd}$ to $\mathrm{ft}$

Exercise 9.8.8

$2 \frac{1}{2} \mathrm{mi}$ to $\mathrm{yd}$

Exercise 9.8.9

8 in. to $\mathrm{ft}$

(Solution on p. 574.)

Exercise 9.8.10

51 in. to $\mathrm{mi}$

Exercise 9.8.11

(Solution on p. 574.)

3 qt to pt

Exercise 9.8.12

$8 \mathrm{lb}$ to oz

Exercise 9.8.13

5 cups to tbsp

(Solution on p. 574.)

Exercise 9.8.14

9 da to hr

Exercise 9.8.15

$3 \frac{1}{2}$ min to sec

(Solution on p. 574.)

Exercise 9.8.16

$\frac{3}{4}$ wk to $\min$

\footnotetext{
${ }^{8}$ This content is available online at $<$ http://cnx.org/content/m35025/1.2/>.
} 
9.8.1.2 The Metric System of Measurement (Section 9.3)

Exercise 9.8.17

(Solution on p. 574.)

$250 \mathrm{~mL}$ to $\mathrm{L}$

Exercise 9.8.18

$18.57 \mathrm{~cm}$ to $\mathrm{m}$

Exercise 9.8.19

(Solution on p. 574.)

$0.01961 \mathrm{~kg}$ to $\mathrm{mg}$

Exercise 9.8.20

$52,211 \mathrm{mg}$ to $\mathrm{kg}$

Exercise 9.8.21

54.006 dag to $\mathrm{g}$

(Solution on p. 574.)

Exercise 9.8.22

$1.181 \mathrm{hg}$ to $\mathrm{mg}$

Exercise 9.8.23

(Solution on p. 574.)

$3.5 \mathrm{~kL}$ to $\mathrm{mL}$

\subsubsection{Simplification of Denominate Numbers (Section 9.4)}

For problems 24-31, perform the indicated operations. Simplify, if possible.

Exercise 9.8.24

Add 8 min $50 \mathrm{sec}$ to 5 min $25 \mathrm{sec}$.

Exercise 9.8.25

(Solution on p. 574.)

Add 3 wk 3 da to 2 wk 5 da

Exercise 9.8.26

Subtract 4 gal 3 qt from 5 gal 2 qt.

Exercise 9.8.27

Subtract 2 gal 3 qt 1 pt from 8 gal 2 qt.

(Solution on p. 574.)

Exercise 9.8.28

Subtract 5 wk 4 da $21 \mathrm{hr}$ from 12 wk 3 da $14 \mathrm{hr}$.

Exercise 9.8.29

(Solution on p. 574.)

Subtract 2 T 1,850 lb from $10 \mathrm{~T} 1,700 \mathrm{lb}$.

Exercise 9.8.30

Subtract the sum of 2 wk 3 da $15 \mathrm{hr}$ and 5 wk 2 da 9 hr from 10 wk.

Exercise 9.8.31

(Solution on p. 574.)

Subtract the sum of $20 \mathrm{hr} 15 \mathrm{~min}$ and $18 \mathrm{hr} 18 \mathrm{~min}$ from the sum of 8 da $1 \mathrm{hr} 16 \mathrm{~min} 5 \mathrm{sec}$.

For problems $32-43$, simplify, if necessary.

Exercise 9.8.32

18 in.

Exercise 9.8.33

(Solution on p. 574.)

$4 \mathrm{ft}$

Exercise 9.8.34

$23 \mathrm{da}$

Exercise 9.8.35

$3,100 \mathrm{lb}$

(Solution on p. 574.) 
Exercise 9.8.36

$135 \mathrm{~min}$

Exercise 9.8.37

(Solution on p. 574.)

4 tsp

Exercise 9.8.38

$10 \mathrm{fl} \mathrm{oz}$

Exercise 9.8.39

(Solution on p. 574.)

$7 \mathrm{pt}$

Exercise 9.8.40

$9 \mathrm{qt}$

Exercise 9.8.41

(Solution on p. 574.)

$2,300 \mathrm{~mm}$

Exercise 9.8.42

$14,780 \mathrm{~mL}$

Exercise 9.8.43

$1,050 \mathrm{~m}$

(Solution on p. 574.)

9.8.1.4 Perimeter, Circumference, Area and Volume of Geometric Figures and Objects (Section 9.5,Section 9.6)

For problems 44-58, find the perimeter, circumference, area or volume.

Exercise 9.8.44

Perimeter, area

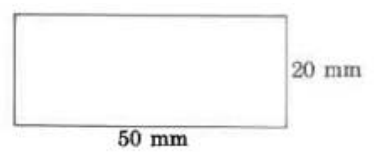

Exercise 9.8.45

(Solution on p. 574.)

Approximate circumference

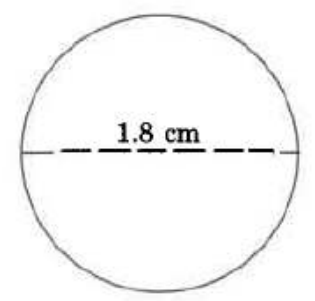

Exercise 9.8.46

Approximate volume 


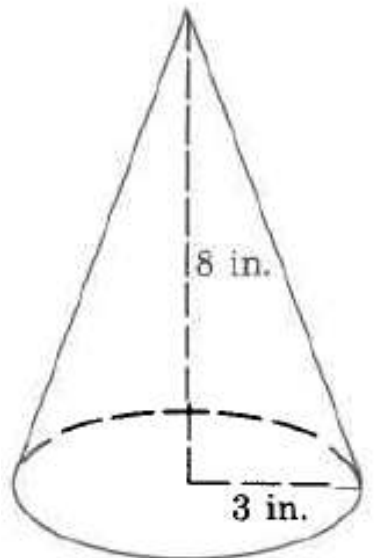

Exercise 9.8.47

(Solution on p. 574.)

Approximate volume

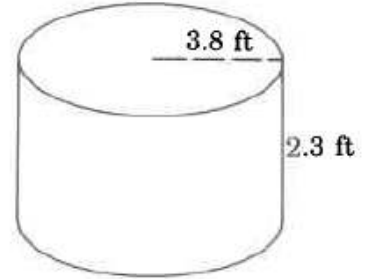

Exercise 9.8.48

Exact area

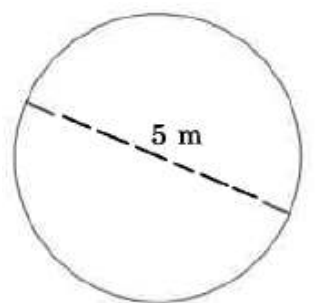

Exercise 9.8.49

Exact area

$0.6 \mathrm{in}$.

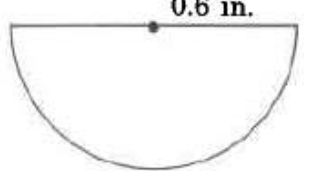

Exercise 9.8.50

Exact volume 


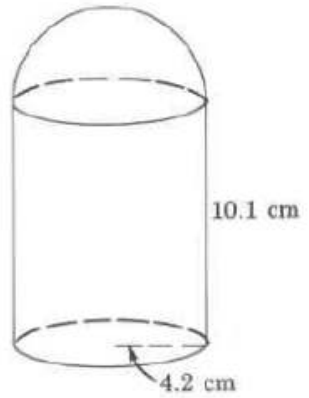

Exercise 9.8.51

(Solution on p. 575.)

Approximate volume

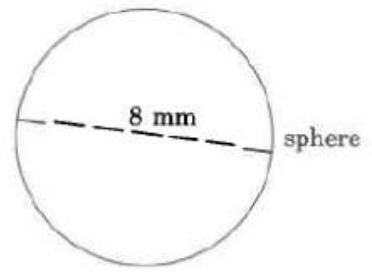

Exercise 9.8.52

Area

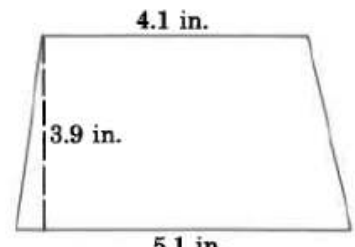

5.1 in.

Exercise 9.8.53

Volume

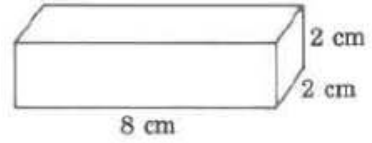

Exercise 9.8.54

Exact area

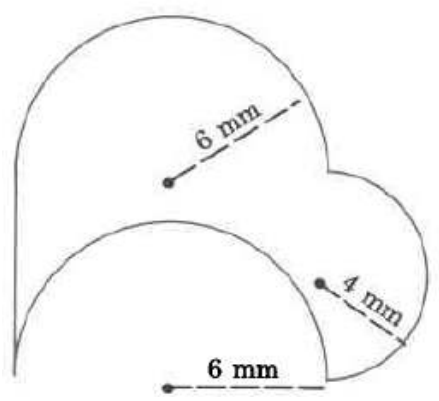

Exercise 9.8.55

(Solution on p. 575.)

Approximate area 


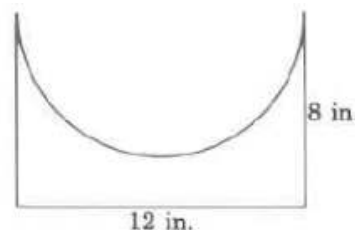

Exercise 9.8.56

Exact area

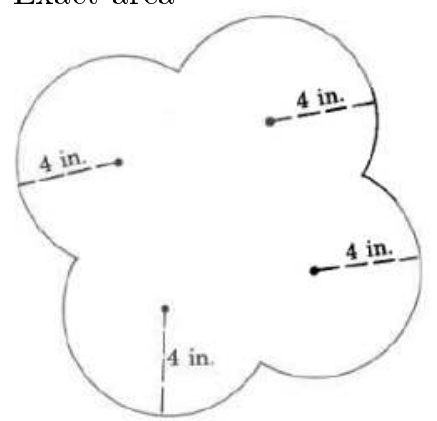

Exercise 9.8.57

(Solution on p. 575.)

Approximate area

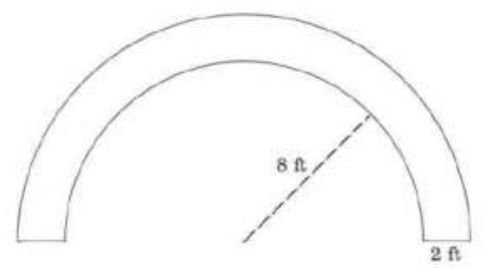

Exercise 9.8.58

Approximate area

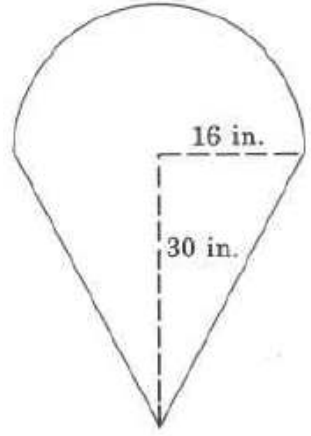

\subsection{Proficiency Exam ${ }^{9}$}

\subsubsection{Proficiency Exam}

Exercise 9.9.1

(Solution on p. 575.)

(Section 9.2) The process of determining, by comparison to some standard, the size of something is called

\footnotetext{
${ }^{9}$ This content is available online at $<$ http://cnx.org/content $/ \mathrm{m} 35026 / 1.2 />$.
} 
For problems 2-9, make each conversion.

Exercise 9.9.2

(Solution on p. 575.)

(Section 9.2) 14 yards to feet

Exercise 9.9.3

(Solution on p. 575.)

(Section 9.2) 51 feet to inches

Exercise 9.9.4

(Solution on p. 575.)

(Section 9.2) $\frac{1}{3}$ yard to feet

Exercise 9.9.5

(Section 9.2) $2 \frac{1}{4}$ minutes to seconds

(Solution on p. 575.)

Exercise 9.9.6

(Solution on p. 575.)

(Section 9.3) 8,500 $\mathrm{mg}$ to $\mathrm{cg}$

Exercise 9.9.7

(Section 9.3) 5.8623 L to kL

Exercise 9.9.8

(Section 9.3) $213.1062 \mathrm{~mm}$ to $\mathrm{m}$

Exercise 9.9.9

(Section 9.3) 100,001 kL to $\mathrm{mL}$

(Solution on p. 575.)

(Solution on p. 575.)

(Solution on p. 575.)

For problems 10-13, simplify each number.

Exercise 9.9.10

(Solution on p. 575.)

(Section 9.4) 23 da

Exercise 9.9.11

(Solution on p. 575.)

(Section 9.4) $88 \mathrm{ft}$

Exercise 9.9.12

(Solution on p. 575.)

(Section 9.4) $4216 \mathrm{lb}$

Exercise 9.9.13

(Section 9.4) $7 \mathrm{qt}$

(Solution on p. 575.)

For problems 14-18, perform the indicated operations. Simplify answers if possible.

Exercise 9.9.14

(Section 9.4) Add 6 wk 3 da to 2 wk 2 da.

Exercise 9.9.15

(Section 9.4) Add 9 gal 3 qt to 4 gal 3 qt.

Exercise 9.9.16

(Section 9.4) Subtract $3 \mathrm{yd} 2 \mathrm{ft} 5$ in. from $5 \mathrm{yd} 8 \mathrm{ft} 2 \mathrm{in}$.

Exercise 9.9.17

(Section 9.4) Subtract $2 \mathrm{hr} 50 \mathrm{~min} 12 \mathrm{sec}$ from $3 \mathrm{hr} 20 \mathrm{~min} 8 \mathrm{sec}$.

Exercise 9.9.18

(Solution on p. 575.)

(Solution on p. 575.)

(Solution on p. 575.)

(Section 9.4) Subtract the sum of 3 wk 6 da and 2 wk 3 da from 10 wk.

(Solution on p. 575.)

(Solution on p. 575.)

For problems 19-30, find either the perimeter, circumference, area, or volume.

Exercise 9.9.19

(Solution on p. 575.)

(Section 9.5) Perimeter

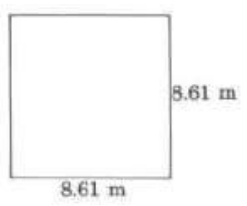


Exercise 9.9.20

(Solution on p. 575.)

(Section 9.5) Perimeter

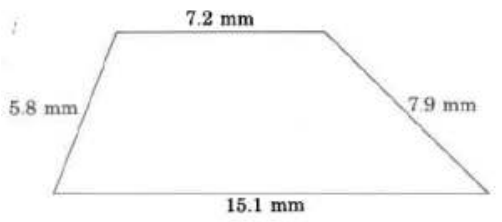

Exercise 9.9.21

(Solution on p. 575.)

(Section 9.5) Approximate circumference

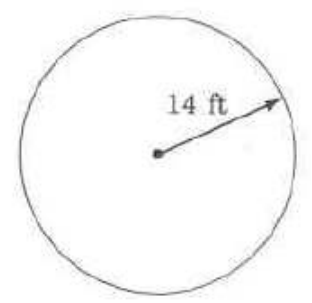

Exercise 9.9.22

(Solution on p. 576.)

(Section 9.5) Approximate perimeter

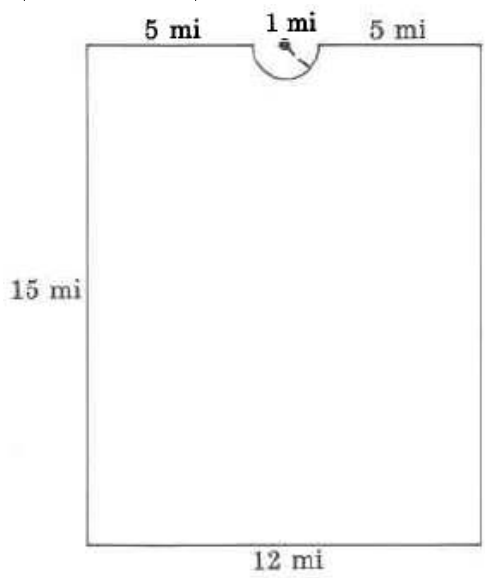

Exercise 9.9.23

(Solution on p. 576.)

(Section 9.6) Area

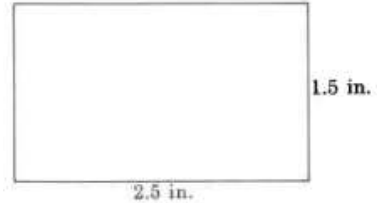

Exercise 9.9.24

(Solution on p. 576.)

(Section 9.6) Approximate area 


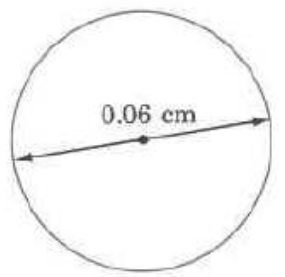

Exercise 9.9.25

(Solution on p. 576.)

(Section 9.6) Approximate area

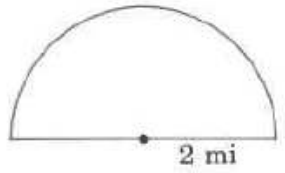

Exercise 9.9.26

(Solution on p. 576.)

(Section 9.6) Area

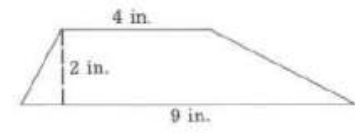

Exercise 9.9.27

(Solution on p. 576.)

(Section 9.6) Exact area

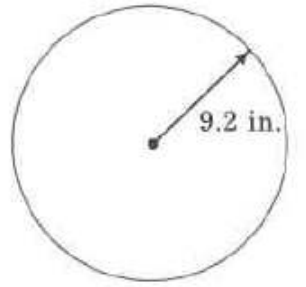

Exercise 9.9.28

(Solution on p. 576.)

(Section 9.6) Approximate volume

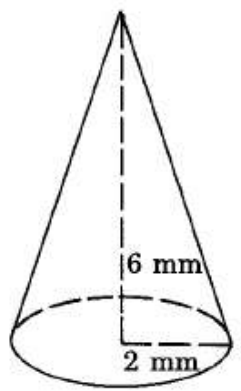

Exercise 9.9.29

(Section 9.6) Exact volume

(Solution on p. 576.)

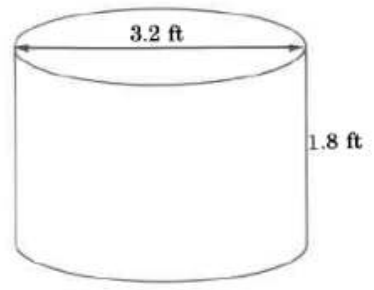


Exercise 9.9.30

(Solution on p. 576.)

(Section 9.6) Approximate volume

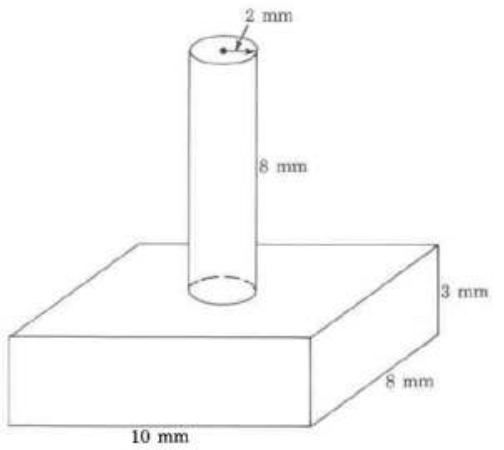




\section{Solutions to Exercises in Chapter 9}

Solution to Exercise 9.2.1 (p. 516)

$6 \mathrm{yd}$

Solution to Exercise 9.2.2 (p. 517)

$10,560 \mathrm{ft}$

Solution to Exercise 9.2.3 (p. 517)

$8.67 \mathrm{yd}$

Solution to Exercise 9.2.4 (p. 517)

$18 \mathrm{pt}$

Solution to Exercise 9.2.5 (p. 517)

$0.87 \mathrm{hr}$

Solution to Exercise 9.2.6 (p. 517)

$2.45 \mathrm{wk}$

Solution to Exercise 9.2.7 (p. 517)

42 feet

Solution to Exercise 9.2.9 (p. 517)

506,880 inches

Solution to Exercise 9.2.11 (p. 517)

1.5 feet

Solution to Exercise 9.2.13 (p. 517)

0.14 yard

Solution to Exercise 9.2.15 (p. 517)

0.00 miles (to two decimal places)

Solution to Exercise 9.2.17 (p. 517)

6 pints

Solution to Exercise 9.2.19 (p. 517) 192,000 ounces

Solution to Exercise 9.2.21 (p. 517) 937.5 pounds

Solution to Exercise 9.2.23 (p. 518)

27 teaspoons

Solution to Exercise 9.2.25 (p. 518)

80 fluid ounces

Solution to Exercise 9.2.27 (p. 518)

0.16 quart

Solution to Exercise 9.2.29 (p. 518)

480 teaspoons

Solution to Exercise 9.2.31 (p. 518)

1,080 seconds

Solution to Exercise 9.2.33 (p. 518)

$\frac{1}{8}=0.125$ day

Solution to Exercise 9.2.35 (p. 518)

$\frac{1}{14}=0.0714$ week

Solution to Exercise 9.2.37 (p. 518)

$1,2,4,5,8$

Solution to Exercise 9.2.39 (p. 518)

$\frac{11}{30}$

Solution to Exercise 9.2.41 (p. 518)

$60(50-4)=3,000-240=2,760$

Solution to Exercise 9.3.1 (p. 522)

$411,000 \mathrm{~g}$ 
Solution to Exercise 9.3.2 (p. 522) $562.6 \mathrm{cL}$

Solution to Exercise 9.3.3 (p. 522) $0.00008 \mathrm{~kL}$

Solution to Exercise 9.3.4 (p. 522)

$15 \mathrm{cg}$

Solution to Exercise 9.3.5 (p. 522) $0.025 \mathrm{~m}$

Solution to Exercise 9.3.6 (p. 523) $8,700 \mathrm{~cm}$

Solution to Exercise 9.3.8 (p. 523) $16.005 \mathrm{~g}$

Solution to Exercise 9.3.10 (p. 523) $11,161 \mathrm{~L}$

Solution to Exercise 9.3.12 (p. 523) $126 \mathrm{dg}$

Solution to Exercise 9.3.14 (p. 523) $5.1 \mathrm{daL}$

Solution to Exercise 9.3.16 (p. 523) $0.5 \mathrm{dm}$

Solution to Exercise 9.3.18 (p. 523) $81,060 \mathrm{cg}$

Solution to Exercise 9.3.20 (p. 523) $0.03 \mathrm{~m}$

Solution to Exercise 9.3.22 (p. 523) $4,000 \mathrm{mg}$

Solution to Exercise 9.3.24 (p. 523) $6,000,000 \mathrm{mg}$

Solution to Exercise 9.3.26 (p. 524) $\frac{25}{24}=1 \frac{1}{24}$

Solution to Exercise 9.3.28 (p. 524) $12,300(12,344)$

Solution to Exercise 9.3.30 (p. 524) $0.08 \overline{3}$ yard

Solution to Exercise 9.4.1 (p. 525) $1 \mathrm{ft} 6$ in.

Solution to Exercise 9.4.2 (p. 525) 10 gal $1 \mathrm{qt}$

Solution to Exercise 9.4.3 (p. 526)

6 hr 20 min

Solution to Exercise 9.4.4 (p. 526) 9 wk 4 da

Solution to Exercise 9.4.5 (p. 526) 12 wk 2 da

Solution to Exercise 9.4.6 (p. 527) 6 gal $1 \mathrm{qt}$

Solution to Exercise 9.4.7 (p. 527) $14 \mathrm{hr} 14 \mathrm{~min}$

Solution to Exercise 9.4.8 (p. 527) $6 \mathrm{ft} 2$ in. 
Solution to Exercise 9.4.9 (p. 527)

$12 \mathrm{~km} 340 \mathrm{~m}$

Solution to Exercise 9.4.10 (p. 527)

3 min $35 \mathrm{sec}$

Solution to Exercise 9.4.11 (p. 527)

14 yd $2 \mathrm{ft} 3$ in

Solution to Exercise 9.4.12 (p. 527)

$13 \mathrm{~min} 13 \mathrm{sec}$

Solution to Exercise 9.4.13 (p. 529)

20 min

Solution to Exercise 9.4.14 (p. 529)

$15 \mathrm{qt}=3$ gal $3 \mathrm{qt}$

Solution to Exercise 9.4.15 (p. 529)

$20 \mathrm{ft} 32 \mathrm{in.}=7 \mathrm{yd} 1 \mathrm{ft} 8 \mathrm{in}$.

Solution to Exercise 9.4.16 (p. 529)

20 hr $150 \mathrm{~min} 400 \mathrm{sec}=22 \mathrm{hr} 36 \mathrm{~min} 40 \mathrm{sec}$

Solution to Exercise 9.4.17 (p. 530)

$2 \mathrm{hr} 4 \mathrm{~min}$

Solution to Exercise 9.4.18 (p. 530)

$4 \mathrm{hr} 16 \mathrm{~min}$

Solution to Exercise 9.4.19 (p. 530)

2 yd $1 \mathrm{ft} 11$ in

Solution to Exercise 9.4.20 (p. 530)

15 gal 3 qt 1 pt

Solution to Exercise 9.4.21 (p. 530)

1 foot 4 inches

Solution to Exercise 9.4.23 (p. 530)

1 hour 25 minutes

Solution to Exercise 9.4.25 (p. 530)

2 weeks 3 days

Solution to Exercise 9.4.27 (p. 530)

15 pounds

Solution to Exercise 9.4.29 (p. 530)

6 gallons 2 quarts

Solution to Exercise 9.4.31 (p. 530)

8 pounds 7 ounces

Solution to Exercise 9.4.33 (p. 530)

2 gallons 1 quart

Solution to Exercise 9.4.35 (p. 530)

16 liters 300 milliliters (or 1daL 6 L 3dL)

Solution to Exercise 9.4.37 (p. 531)

15 days 11 hours

Solution to Exercise 9.4.39 (p. 531)

59 pounds 9 ounces

Solution to Exercise 9.4.41 (p. 531)

1 foot 10 inches

Solution to Exercise 9.4.43 (p. 531)

1 hour 18 minutes

Solution to Exercise 9.4.45 (p. 531)

5 days 16 hours 5 minutes 
Solution to Exercise 9.4.47 (p. 531) 1 ton 1,100 pounds (or 1T $1,100 \mathrm{lb}$ )

Solution to Exercise 9.4.49 (p. 531) 2 weeks 23 hours 29 minutes 53 seconds Solution to Exercise 9.4.51 (p. 531) 1

Solution to Exercise 9.4.53 (p. 531) $2 \frac{14}{275}$

Solution to Exercise 9.4.55 (p. 531) $126,000 \mathrm{~g}$

Solution to Exercise 9.5.1 (p. 534) $20 \mathrm{ft}$

Solution to Exercise 9.5.2 (p. 534) $26.8 \mathrm{~m}$

Solution to Exercise 9.5.3 (p. 534) $49.89 \mathrm{mi}$

Solution to Exercise 9.5.4 (p. 537) $9.1 \pi$ in.

Solution to Exercise 9.5.5 (p. 537) $5.652 \mathrm{~mm}$

Solution to Exercise 9.5.6 (p. 537) $126.228 \mathrm{~m}$

Solution to Exercise 9.5.7 (p. 537) $41.634 \mathrm{~mm}$

Solution to Exercise 9.5.8 (p. 538) $21.8 \mathrm{~cm}$

Solution to Exercise 9.5.10 (p. 538) 38.14 inches

Solution to Exercise 9.5.12 (p. 538) $0.86 \mathrm{~m}$

Solution to Exercise 9.5.14 (p. 539) $87.92 \mathrm{~m}$

Solution to Exercise 9.5.16 (p. 539) $16.328 \mathrm{~cm}$

Solution to Exercise 9.5.18 (p. 539) $0.0771 \mathrm{~cm}$

Solution to Exercise 9.5.20 (p. 540) $120.78 \mathrm{~m}$

Solution to Exercise 9.5.22 (p. 540)

21.71 inches

Solution to Exercise 9.5.24 (p. 541) $43.7 \mathrm{~mm}$

Solution to Exercise 9.5.26 (p. 541) $45.68 \mathrm{~cm}$

Solution to Exercise 9.5.28 (p. 542) 8.5 or $\frac{17}{2}$ or $8 \frac{1}{2}$

Solution to Exercise 9.5.30 (p. 542) 0.875

Solution to Exercise 9.5.32 (p. 542) 1 hour 36 minutes 6 seconds 
Solution to Exercise 9.6.1 (p. 546) $36 \mathrm{sq} \mathrm{cm}$

Solution to Exercise 9.6.2 (p. 546)

$37.503 \mathrm{sq} \mathrm{mm}$

Solution to Exercise 9.6.3 (p. 546)

$13.26 \mathrm{sq}$ in.

Solution to Exercise 9.6.4 (p. 546)

$367.5 \mathrm{sq} \mathrm{mi}$

Solution to Exercise 9.6.5 (p. 546) $452.16 \mathrm{sq} \mathrm{ft}$

Solution to Exercise 9.6.6 (p. 547) $44.28 \mathrm{sq} \mathrm{cm}$

Solution to Exercise 9.6.7 (p. 551) $21 \mathrm{cu}$ in.

Solution to Exercise 9.6.8 (p. 551)

$904.32 \mathrm{cu} \mathrm{ft}$

Solution to Exercise 9.6.9 (p. 551)

$157 \mathrm{cu} \mathrm{m}$

Solution to Exercise 9.6.10 (p. 551) $0.00942 \mathrm{cu}$ in.

Solution to Exercise 9.6.11 (p. 551)

$16 \mathrm{sq} \mathrm{m}$

Solution to Exercise 9.6.13 (p. 552)

$1.21 \mathrm{sq} \mathrm{mm}$

Solution to Exercise 9.6.15 (p. 552)

$18 \mathrm{sq}$ in.

Solution to Exercise 9.6.17 (p. 552)

$(60.5 \pi+132) \quad \mathrm{sq} \mathrm{ft}$

Solution to Exercise 9.6.19 (p. 553) $40.8 \mathrm{sq}$ in.

Solution to Exercise 9.6.21 (p. 553) $31.0132 \mathrm{sq}$ in.

Solution to Exercise 9.6.23 (p. 553)

$158.2874 \mathrm{sq} \mathrm{mm}$

Solution to Exercise 9.6.25 (p. 554) $64.2668 \mathrm{sq}$ in.

Solution to Exercise 9.6.27 (p. 554) $43.96 \mathrm{sq} \mathrm{ft}$

Solution to Exercise 9.6.29 (p. 554)

$512 \mathrm{cu} \mathrm{cm}$

Solution to Exercise 9.6.31 (p. 555)

$11.49 \mathrm{cu} \mathrm{cm}$

Solution to Exercise 9.6.33 (p. 555) $\frac{1024}{3} \pi \mathrm{cu} \mathrm{ft}$

Solution to Exercise 9.6.35 (p. 556) $22.08 \mathrm{cu}$ in.

Solution to Exercise 9.6.37 (p. 556) 4

Solution to Exercise 9.6.39 (p. 556)

$\frac{31}{12}=2 \frac{7}{12}=2.58$ 
Solution to Exercise 9.6.41 (p. 556)

$27.9 \mathrm{~m}$

Solution to Exercise 9.8.1 (p. 559)

Measurement is comparison to a standard (unit of measure).

Solution to Exercise 9.8.3 (p. 559)

2 pounds

Solution to Exercise 9.8.5 (p. 559)

6 tons

Solution to Exercise 9.8.7 (p. 559)

69 feet

Solution to Exercise 9.8.9 (p. 559)

$\frac{2}{3}=0.66 \overline{6}$ feet

Solution to Exercise 9.8.11 (p. 559)

6 pints

Solution to Exercise 9.8.13 (p. 559)

80 tablespoons

Solution to Exercise 9.8.15 (p. 559)

210 seconds

Solution to Exercise 9.8.17 (p. 560)

$\frac{1}{4}=0.25 \mathrm{~L}$

Solution to Exercise 9.8.19 (p. 560)

$19,610 \mathrm{mg}$

Solution to Exercise 9.8.21 (p. 560) $540.06 \mathrm{~g}$

Solution to Exercise 9.8.23 (p. 560)

$3,500,000 \mathrm{~mL}$

Solution to Exercise 9.8.25 (p. 560)

6 weeks 1 day

Solution to Exercise 9.8.27 (p. 560)

5 gallons 2 quarts 1 pint

Solution to Exercise 9.8.29 (p. 560)

7 T 1,850 pounds

Solution to Exercise 9.8.31 (p. 560)

7 days, 11 hours, 56 minutes, 7 seconds

Solution to Exercise 9.8.33 (p. 560)

1 yard 1 foot

Solution to Exercise 9.8.35 (p. 560)

1 ton 1,100 pounds

Solution to Exercise 9.8.37 (p. 561)

1 tablespoon 1 teaspoon

Solution to Exercise 9.8.39 (p. 561)

3 quarts 1 pint

Solution to Exercise 9.8.41 (p. 561)

2.3 meters

Solution to Exercise 9.8.43 (p. 561)

$1.05 \mathrm{~km}$

Solution to Exercise 9.8.45 (p. 561)

$5.652 \mathrm{sq} \mathrm{cm}$

Solution to Exercise 9.8.47 (p. 562)

$104.28568 \mathrm{cu} \mathrm{ft}$ 
Solution to Exercise 9.8.49 (p. 562)

$0.18 \pi \mathrm{sq}$ in.

Solution to Exercise 9.8.51 (p. 563)

$267.94667 \mathrm{cu} \mathrm{mm}$

Solution to Exercise 9.8.53 (p. 563)

$32 \mathrm{cu} \mathrm{cm}$

Solution to Exercise 9.8.55 (p. 563)

$39.48 \mathrm{sq}$ in.

Solution to Exercise 9.8.57 (p. 564)

$56.52 \mathrm{sq} \mathrm{ft}$

Solution to Exercise 9.9.1 (p. 564)

measurement

Solution to Exercise 9.9.2 (p. 565)

42 feet

Solution to Exercise 9.9.3 (p. 565)

612 inches

Solution to Exercise 9.9.4 (p. 565)

1 foot

Solution to Exercise 9.9.5 (p. 565)

135 seconds

Solution to Exercise 9.9.6 (p. 565)

$850 \mathrm{cg}$

Solution to Exercise 9.9.7 (p. 565)

$0.0058623 \mathrm{~kL}$

Solution to Exercise 9.9.8 (p. 565)

$0.2132062 \mathrm{~m}$

Solution to Exercise 9.9.9 (p. 565)

100,001,000,000 mL

Solution to Exercise 9.9.10 (p. 565)

3 weeks 2 days

Solution to Exercise 9.9.11 (p. 565)

29 yards 1 foot

Solution to Exercise 9.9.12 (p. 565)

2 tons 216 pounds

Solution to Exercise 9.9.13 (p. 565)

1 gallon 3 quarts

Solution to Exercise 9.9.14 (p. 565)

8 weeks 5 days

Solution to Exercise 9.9.15 (p. 565)

14 gallons 2 quarts

Solution to Exercise 9.9.16 (p. 565)

2 yards 5 feet 9 inches

Solution to Exercise 9.9.17 (p. 565)

29 minutes 56 seconds

Solution to Exercise 9.9.18 (p. 565)

3 weeks 5 days

Solution to Exercise 9.9.19 (p. 565)

$34.44 \mathrm{~m}$

Solution to Exercise 9.9.20 (p. 566)

$36 \mathrm{~mm}$ 
Solution to Exercise 9.9.21 (p. 566) 87.92 feet

Solution to Exercise 9.9.22 (p. 566) 55.14 miles

Solution to Exercise 9.9.23 (p. 566) $3.75 \mathrm{sq}$ in.

Solution to Exercise 9.9.24 (p. 566) $6.002826 \mathrm{sq} \mathrm{cm}$

Solution to Exercise 9.9.25 (p. 567) $6.28 \mathrm{sq}$ miles

Solution to Exercise 9.9.26 (p. 567) $13 \mathrm{sq}$ in.

Solution to Exercise 9.9.27 (p. 567) $84.64 \pi \mathrm{sq}$ in.

Solution to Exercise 9.9 .28 (p. 567) $25.12 \mathrm{cu} \mathrm{mm}$

Solution to Exercise 9.9.29 (p. 567) $4.608 \pi \mathrm{cu} \mathrm{ft}$

Solution to Exercise 9.9.30 (p. 568) $340.48 \mathrm{cu} \mathrm{mm}$ 


\section{Chapter 10}

\section{Signed Numbers}

\subsection{Objectives ${ }^{1}$}

After completing this chapter, you should

Variables, Constants, and Real Numbers (Section 10.2)

- be able to distinguish between variables and constants

- be able to recognize a real number and particular subsets of the real numbers

- understand the ordering of the real numbers

\section{Signed Numbers (Section 10.3)}

- be able to distinguish between positive and negative real numbers

- be able to read signed numbers

- understand the origin and use of the double-negative product property

\section{Absolute Value (Section 10.4)}

- understand the geometric and algebraic definitions of absolute value

\section{Addition of Signed Numbers (Section 10.5)}

- be able to add numbers with like signs and with unlike signs

- be able to use the calculator for addition of signed numbers

\section{Subtraction of Signed Numbers (Section 10.6)}

- understand the definition of subtraction

- be able to subtract signed numbers

- be able to use a calculator to subtract signed numbers

\section{Multiplication and Division of Signed Numbers (Section 10.7)}

- be able to multiply and divide signed numbers

- be able to multiply and divide signed numbers using a calculator

\footnotetext{
${ }^{1}$ This content is available online at $<\mathrm{http}: / / \mathrm{cnx} . o r g /$ content $/ \mathrm{m} 18898 / 1.3 />$.
} 


\subsection{Variables, Constants, and Real Numbers ${ }^{2}$}

\subsubsection{Section Overview}

- Variables and Constants

- Real Numbers

- Subsets of Real Numbers

- Ordering Real Numbers

\subsubsection{Variables and Constants}

A basic distinction between algebra and arithmetic is the use of symbols (usually letters) in algebra to represent numbers. So, algebra is a generalization of arithmetic. Let us look at two examples of situations in which letters are substituted for numbers:

1. Suppose that a student is taking four college classes, and each class can have at most 1 exam per week. In any 1 -week period, the student may have $0,1,2,3$, or 4 exams. In algebra, we can let the letter $x$ represent the number of exams this student may have in a 1-week period. The letter $x$ may assume any of the various values $0,1,2,3,4$.

2. Suppose that in writing a term paper for a biology class a student needs to specify the average lifetime, in days, of a male housefly. If she does not know this number off the top of her head, she might represent it (at least temporarily) on her paper with the letter $t$ (which reminds her of time). Later, she could look up the average time in a reference book and find it to be 17 days. The letter $t$ can assume only the one value, 17 , and no other values. The value $t$ is constant.

\section{Variable, Constant}

1. A letter or symbol that represents any member of a collection of two or more numbers is called a variable.

2. A letter or symbol that represents one specific number, known or unknown, is called a constant.

In example 1 , the letter $x$ is a variable since it can represent any of the numbers $0,1,2,3,4$. The letter $t$ example 2 is a constant since it can only have the value 17 .

\subsubsection{Real Numbers}

\section{Real Number Line}

The study of mathematics requires the use of several collections of numbers. The real number line allows us to visually display (graph) the numbers in which we are interested.

A line is composed of infinitely many points. To each point we can associate a unique number, and with each number, we can associate a particular point.

\section{Coordinate}

The number associated with a point on the number line is called the coordinate of the point.

\section{Graph}

The point on a number line that is associated with a particular number is called the graph of that number.

\section{Constructing a Real Number Line}

We construct a real number line as follows:

\footnotetext{
${ }^{2}$ This content is available online at $<$ http://cnx.org/content $/ \mathrm{m} 35027 / 1.2 />$.
} 
1. Draw a horizontal line.

2. Origin

Choose any point on the line and label it 0 . This point is called the origin.

\section{0}

3. Choose a convenient length. Starting at 0 , mark this length off in both directions, being careful to have the lengths look like they are about the same.

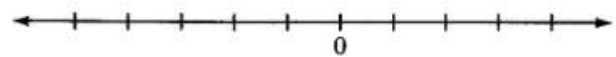

We now define a real number.

\section{Real Number}

A real number is any number that is the coordinate of a point on the real number line.

\section{Positive Numbers, Negative Numbers}

Real numbers whose graphs are to the right of 0 are called positive real numbers, or more simply, positive numbers. Real numbers whose graphs appear to the left of 0 are called negative real numbers, or more simply, negative numbers.

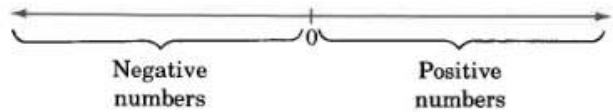

The number 0 is neither positive nor negative.

\subsubsection{Subsets of Real Numbers}

The set of real numbers has many subsets. Some of the subsets that are of interest in the study of algebra are listed below along with their notations and graphs.

Natural Numbers, Counting Numbers

The natural or counting numbers $(N): 1,2,3,4, \ldots$ Read "and so on."

\section{Whole Numbers}

The whole numbers $(W): 0,1,2,3,4, \ldots$.

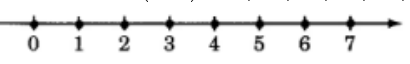

Notice that every natural number is a whole number.

\section{Integers}

The integers $(Z): \ldots-3,-2,-1,0,1,2,3, \ldots$.

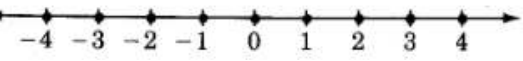

Notice that every whole number is an integer.

\section{Rational Numbers (Fractions)}

The rational numbers $(Q)$ : Rational numbers are sometimes called fractions. They are numbers that can be written as the quotient of two integers. They have decimal representations that either terminate or do not terminate but contain a repeating block of digits. Some examples are

$$
\underbrace{\frac{-3}{4}=-0.75}_{\text {Terminating }} \quad \underbrace{8 \frac{11}{27}=8.407407407 \ldots}_{\text {Nonterminating, but repeating }}
$$


Some rational numbers are graphed below.

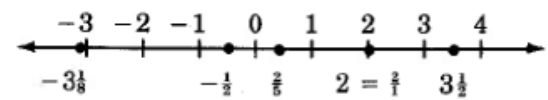

Notice that every integer is a rational number.

Notice that there are still a great many points on the number line that have not yet been assigned a type of number. We will not examine these other types of numbers in this text. They are examined in detail in algebra. An example of these numbers is the number $\pi$, whose decimal representation does not terminate nor contain a repeating block of digits. An approximation for $\pi$ is 3.14 .

\subsubsection{Sample Set A}

\section{Example 10.1}

Is every whole number a natural number?

No. The number 0 is a whole number but it is not a natural number.

\section{Example 10.2}

Is there an integer that is not a natural number?

Yes. Some examples are $0,-1,-2,-3$, and -4 .

\section{Example 10.3}

Is there an integer that is a whole number?

Yes. In fact, every whole number is an integer.

\subsubsection{Practice Set A}

Exercise 10.2.1

(Solution on p. 620.)

Is every natural number a whole number?

Exercise 10.2.2

(Solution on p. 620.)

Is every whole number an integer?

Exercise 10.2.3

(Solution on p. 620.)

Is every integer a real number?

Exercise 10.2.4

(Solution on p. 620.)

Is there an integer that is a whole number?

Exercise 10.2.5

Is there an integer that is not a natural number?

(Solution on p. 620.)

\subsubsection{Ordering Real Numbers}

\section{Ordering Real Numbers}

A real number $b$ is said to be greater than a real number $a$, denoted $b>a$, if $b$ is to the right of $a$ on the number line. Thus, as we would expect, $5>2$ since 5 is to the right of 2 on the number line. Also, $-2>-5$ since -2 is to the right of -5 on the number line.

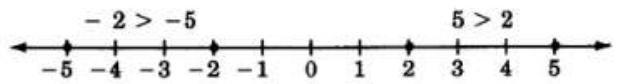

If we let $a$ and $b$ represent two numbers, then $a$ and $b$ are related in exactly one of three ways: Either 


\section{Equality Symbol}

$$
a=b \quad a \text { and } b \text { are equal } \quad(8=8)
$$

\section{Inequality Symbols}

$$
\begin{array}{ccc}
a>b & a \text { is greater than } b & (8>5) \\
a<b & a \text { is less than } b & (5<8) \\
\left\{\begin{array}{cc}
\text { Some variations of these symbols are } \\
a \neq b
\end{array}\right. & a \text { is not equal to } b & (8 \neq 5) \\
a \geq b & a \text { is greater than or equal to } b & (a \geq 8) \\
a \leq b & a \text { is less than or equal to } b & (a \leq 8)
\end{array}
$$

\subsubsection{Sample Set B}

\section{Example 10.4}

What integers can replace $x$ so that the following statement is true?

$$
-3 \leq x<2
$$

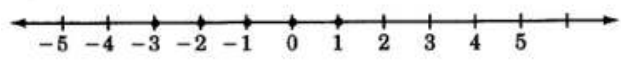

The integers are $-3,-2,-1,0,1$.

\section{Example 10.5}

Draw a number line that extends from -3 to 5 . Place points at all whole numbers between and including -1 and 3 .

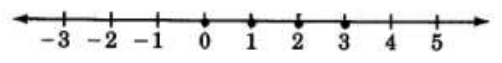

-1 is not a whole number

\subsubsection{Practice Set B}

Exercise 10.2.6

(Solution on p. 620.) What integers can replace $x$ so that the following statement is true? $-5 \leq x<2$

\section{Exercise 10.2.7}

(Solution on p. 620.) Draw a number line that extends from -4 to 3 . Place points at all natural numbers between, but not including, -2 to 2 .

\subsubsection{Exercises}

For the following 8problems, next to each real number, note all collections to which it belongs by writing $N$ for natural number, $W$ for whole number, or $Z$ for integer. Some numbers may belong to more than one collection.

Exercise 10.2.8

(Solution on p. 620.)

6

Exercise 10.2.9

12 
Exercise 10.2.10

(Solution on p. 620.)

0

Exercise 10.2.11

1

Exercise 10.2.12

$-3$

(Solution on p. 620.)

Exercise 10.2.13

$-7$

Exercise 10.2.14

(Solution on p. 620.)

$-805$

Exercise 10.2.15

$-900$

Exercise 10.2.16

Is the number 0 a positive number, a negative number, neither, or both?

(Solution on p. 620.)

Exercise 10.2.17

An integer is an even integer if it is evenly divisible by 2. Draw a number line that extends from -5 to 5 and place points at all negative even integers and all positive odd integers.

Exercise 10.2.18

(Solution on p. 620.)

Draw a number line that extends from -5 to 5 . Place points at all integers that satisfy $-3 \leq x<4$.

Exercise 10.2.19

Is there a largest two digit number? If so, what is it?

Exercise 10.2.20

Is there a smallest two digit number? If so, what is it?

(Solution on p. 620.)

For the pairs of real numbers in the following 5 problems, write the appropriate symbol $(<,>,=)$ in place of the $\square$.

Exercise 10.2.21

$-7 \square-2$

Exercise 10.2.22

(Solution on p. 620.)

$-5 \square 0$

Exercise 10.2.23

$-1 \square 4$

Exercise 10.2.24

(Solution on p. 620.)

$6 \square-1$

Exercise 10.2.25

$10 \square 10$

For the following 5 problems, what numbers can replace $m$ so that the following statements are true?

Exercise 10.2.26

(Solution on p. 620.)

$-1 \leq m \leq-5, m$ an integer.

Exercise 10.2.27

$-7<m<-1, m$ an integer.

Exercise 10.2.28

(Solution on p. 620.)

$-3 \leq m<2, m$ a natural number.

Exercise 10.2.29

$-15<m \leq-1, m$ a natural number.

Exercise 10.2.30

(Solution on p. 620.)

$-5 \leq m<5, m$ a whole number. 
For the following 10 problems, on the number line, how many units are there between the given pair of numbers?

Exercise 10.2.31

0 and 3

Exercise 10.2.32

-4 and 0

(Solution on p. 620.)

Exercise 10.2.33

-1 and 6

Exercise 10.2.34

-6 and 2

(Solution on p. 620.)

Exercise 10.2.35

-3 and 3

Exercise 10.2.36

(Solution on p. 620.)

Are all positive numbers greater than zero?

Exercise 10.2.37

Are all positive numbers greater than all negative numbers?

Exercise 10.2.38

Is 0 greater than all negative number?

(Solution on p. 620.)

Exercise 10.2.39

Is there a largest natural number?

Exercise 10.2.40

(Solution on p. 620.)

Is there a largest negative integer?

\subsubsection{Exercises for Review}

Exercise 10.2.41

(Section 4.3) Convert $6 \frac{5}{8}$ to an improper fraction.

Exercise 10.2.42

(Solution on p. 621.)

(Section 4.5) Find the value: $\frac{3}{11}$ of $\frac{33}{5}$.

Exercise 10.2.43

(Section 5.3) Find the sum of $\frac{4}{5}+\frac{3}{8}$.

Exercise 10.2.44

(Solution on p. 621.)

(Section 9.3) Convert $30.06 \mathrm{~cm}$ to $\mathrm{m}$.

Exercise 10.2.45

(Section 9.6) Find the area of the triangle.

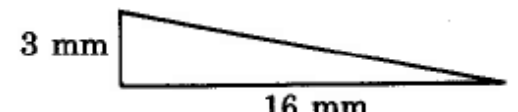

$16 \mathrm{~mm}$ 


\subsection{Signed Numbers ${ }^{3}$}

\subsubsection{Section Overview}

- Positive and Negative Numbers

- Reading Signed Numbers

- Opposites

- The Double-Negative Property

\subsubsection{Positive and Negative Numbers}

\section{Positive and Negative Numbers}

Each real number other than zero has a sign associated with it. A real number is said to be a positive number if it is to the right of 0 on the number line and negative if it is to the left of 0 on the number line.

\section{NOTE: + and - Notation}

A number is denoted as positive if it is directly preceded by a plus sign or no sign at all.

A number is denoted as negative if it is directly preceded by a minus sign.

\subsubsection{Reading Signed Numbers}

The plus and minus signs now have two meanings:

The plus sign can denote the operation of addition or a positive number.

The minus sign can denote the operation of subtraction or a negative number.

To avoid any confusion between "sign" and "operation," it is preferable to read the sign of a number as "positive" or "negative." When "+" is used as an operation sign, it is read as "plus." When "-" is used as an operation sign, it is read as "minus."

\subsubsection{Sample Set A}

Read each expression so as to avoid confusion between "operation" and "sign."

Example 10.6

-8 should be read as "negative eight" rather than "minus eight."

\section{Example 10.7}

$4+(-2)$ should be read as "four plus negative two" rather than "four plus minus two."

\section{Example 10.8}

$-6+(-3)$ should be read as "negative six plus negative three" rather than "minus six plus minus three."

\section{Example 10.9}

$-15-(-6)$ should be read as "negative fifteen minus negative six" rather than "minus fifteen minus minus six."

\section{Example 10.10}

$-5+7$ should be read as "negative five plus seven" rather than "minus five plus seven."

\section{Example 10.11}

$0-2$ should be read as "zero minus two."

\footnotetext{
${ }^{3}$ This content is available online at $<$ http://cnx.org/content $/ \mathrm{m} 35029 / 1.3 />$.
} 


\subsubsection{Practice Set A}

Write each expression in words.

Exercise 10.3.1

(Solution on p. 621.)

$6+1$

Exercise 10.3.2

(Solution on p. 621.)

$2+(-8)$

Exercise 10.3.3

(Solution on p. 621.)

$-7+5$

Exercise 10.3.4

(Solution on p. 621.)

$-10-(+3)$

Exercise 10.3.5

(Solution on p. 621.)

$-1-(-8)$

Exercise 10.3.6

$0+(-11)$

(Solution on p. 621.)

\subsubsection{Opposites}

\section{Opposites}

On the number line, each real number, other than zero, has an image on the opposite side of 0 . For this reason, we say that each real number has an opposite. Opposites are the same distance from zero but have opposite signs.

The opposite of a real number is denoted by placing a negative sign directly in front of the number. Thus, if $a$ is any real number, then $-a$ is its opposite.

Note: The letter " $a$ " is a variable. Thus, " $a$ " need not be positive, and " $-a$ " need not be negative.

If $a$ is any real number, $-a$ is opposite $a$ on the number line.

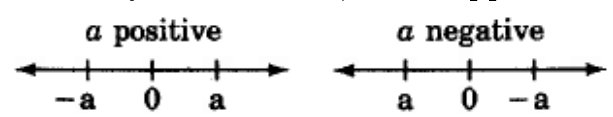

\subsubsection{The Double-Negative Property}

The number $a$ is opposite $-a$ on the number line. Therefore, $-(-a)$ is opposite $-a$ on the number line. This means that

$-(-a)=a$

From this property of opposites, we can suggest the double-negative property for real numbers.

Double-Negative Property: $-(-a)=a$

If $a$ is a real number, then

$-(-a)=a$ 


\subsubsection{Sample Set B}

Find the opposite of each number.

\section{Example 10.12}

If $a=2$, then $-a=-2$. Also, $-(-a)=-(-2)=2$.

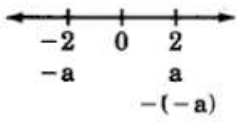

\section{Example 10.13}

If $a=-4$, then $-a=-(-4)=4$. Also, $-(-a)=a=-4$.

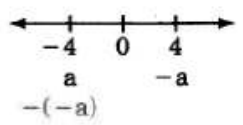

\subsubsection{Practice Set B}

Find the opposite of each number.

Exercise 10.3.7

(Solution on p. 621.)

8

Exercise 10.3.8

(Solution on p. 621.)

17

Exercise 10.3.9

(Solution on p. 621.)

$-6$

(Solution on p. 621.)

Exercise 10.3.10

(Solution on p. 621.)

Exercise 10.3.11

$-(-1)$

Exercise 10.3.12

(Solution on p. 621.)

$-[-(-7)]$

Exercise 10.3.13

(Solution on p. 621.)

Suppose $a$ is a positive number. Is $-a$ positive or negative?

(Solution on p. 621.)

Exercise 10.3.14

Suppose $a$ is a negative number. Is $-a$ positive or negative?

(Solution on p. 621.)

Exercise 10.3.15

Suppose we do not know the sign of the number $k$. Is $-k$ positive, negative, or do we not know?

\subsubsection{Exercises}

Exercise 10.3.16

(Solution on p. 621.)

A number is denoted as positive if it is directly preceded by

Exercise 10.3.17

A number is denoted as negative if it is directly preceded by

How should the number in the following 6 problems be read? (Write in words.)

Exercise 10.3.18 $-7$

(Solution on p. 621.) 
Exercise 10.3.19

$-5$

Exercise 10.3.20

(Solution on p. 621.)

15

Exercise 10.3.21

11

Exercise 10.3.22

$-(-1)$

(Solution on p. 621.)

Exercise 10.3.23

$-(-5)$

For the following 6 problems, write each expression in words.

Exercise 10.3.24

$5+3$

(Solution on p. 621.)

Exercise 10.3.25

$3+8$

Exercise 10.3.26

(Solution on p. 621.) $15+(-3)$

Exercise 10.3.27 $1+(-9)$

Exercise 10.3.28

$$
-7-(-2)
$$

(Solution on p. 621.)

Exercise 10.3.29

$0-(-12)$

For the following 6 problems, rewrite each number in simpler form.

Exercise 10.3.30 $-(-2)$

(Solution on p. 621.)

Exercise 10.3.31 $-(-16)$

Exercise 10.3.32 $-[-(-8)]$

(Solution on p. 622.)

Exercise 10.3.33 $-[-(-20)]$

Exercise 10.3.34 $7-(-3)$

(Solution on p. 622.)

Exercise 10.3.35

$6-(-4)$ 


\subsubsection{Exercises for Review}

Exercise 10.3.36

(Solution on p. 622.)

(Section 6.7) Find the quotient; $8 \div 27$.

Exercise 10.3.37

(Section 7.3) Solve the proportion: $\frac{5}{9}=\frac{60}{x}$

Exercise 10.3.38

(Solution on p. 622.)

(Section 8.2) Use the method of rounding to estimate the sum: $5829+8767$

Exercise 10.3.39

(Section 9.2) Use a unit fraction to convert 4 yd to feet.

Exercise 10.3.40

(Section 9.3) Convert $25 \mathrm{~cm}$ to $\mathrm{hm}$.

(Solution on p. 622.)

\subsection{Absolute Value ${ }^{4}$}

\subsubsection{Section Overview}

- Geometric Definition of Absolute Value

- Algebraic Definition of Absolute Value

\subsubsection{Geometric Definition of Absolute Value}

\section{Absolute Value-Geometric Approach}

Geometric definition of absolute value:

The absolute value of a number $a$, denoted $|a|$, is the distance from a to 0 on the number line.

Absolute value answers the question of "how far," and not "which way." The phrase "how far" implies "length" and length is always a nonnegative quantity. Thus, the absolute value of a number is a nonnegative number.

\subsubsection{Sample Set A}

Determine each value.

Example 10.14

$|4|=4$

4 units in length

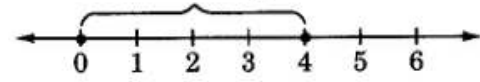

Example 10.15

$|-4|=4$

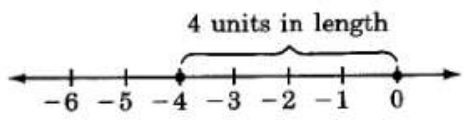

Example 10.16

$|0|=0$

${ }^{4}$ This content is available online at $<$ http://cnx.org/content $/ \mathrm{m} 35030 / 1.3 />$. 


\section{Example 10.17}

$-|5|=-5$. The quantity on the left side of the equal sign is read as "negative the absolute value of $5 . "$ The absolute value of 5 is 5 . Hence, negative the absolute value of 5 is -5 .

\section{Example 10.18}

$-|-3|=-3$. The quantity on the left side of the equal sign is read as "negative the absolute value of -3 ." The absolute value of -3 is 3 . Hence, negative the absolute value of -3 is $-(3)=-3$.

\subsubsection{Practice Set A}

By reasoning geometrically, determine each absolute value.

Exercise 10.4.1

| 7 |

Exercise 10.4.2

$$
|-3|
$$

Exercise 10.4.3

| 12 |

Exercise 10.4.4

$|0|$

Exercise 10.4.5

$-|9|$

Exercise 10.4.6

$-|-6|$
(Solution on p. 622.)

(Solution on p. 622.)

(Solution on p. 622.)

(Solution on p. 622.)

(Solution on p. 622.)

(Solution on p. 622.)

\subsubsection{Algebraic Definition of Absolute Value}

From the problems in Section 10.4.2.1 (Sample Set A), we can suggest the following algebraic definition of absolute value. Note that the definition has two parts.

\section{Absolute Value - Algebraic Approach}

Algebraic definition of absolute value

The absolute value of a number $a$ is

$|a|=\left\{\begin{array}{cc}a, & \text { if } a \geq 0 \\ -a, & \text { if }<0\end{array}\right.$

The algebraic definition takes into account the fact that the number $a$ could be either positive or zero $(a \geq 0)$ or negative $(a<0)$.

1. If the number $a$ is positive or zero $(a \geq 0)$, the upper part of the definition applies. The upper part of the definition tells us that if the number enclosed in the absolute value bars is a nonnegative number, the absolute value of the number is the number itself.

2. The lower part of the definition tells us that if the number enclosed within the absolute value bars is a negative number, the absolute value of the number is the opposite of the number. The opposite of a negative number is a positive number.

NOTE: The definition says that the vertical absolute value lines may be eliminated only if we know whether the number inside is positive or negative. 


\title{
10.4.3.1 Sample Set B
}

Use the algebraic definition of absolute value to find the following values.

\section{Example 10.19}

$|8|$. The number enclosed within the absolute value bars is a nonnegative number, so the upper part of the definition applies. This part says that the absolute value of 8 is 8 itself.

$|8|=8$

\section{Example 10.20}

$|-3|$. The number enclosed within absolute value bars is a negative number, so the lower part of the definition applies. This part says that the absolute value of -3 is the opposite of -3 , which is $-(-3)$. By the definition of absolute value and the double-negative property,

$|-3|=-(-3)=3$

\subsubsection{Practice Set $B$}

Use the algebraic definition of absolute value to find the following values.

Exercise 10.4.7

$$
|7|
$$

Exercise 10.4.8

$|9|$

Exercise 10.4.9

$|-12|$

Exercise 10.4.10

$$
|-5|
$$

Exercise 10.4.11

$-|8|$

Exercise 10.4.12

$$
-|1|
$$

Exercise 10.4.13

$$
-|-52|
$$

Exercise 10.4.14

$-|-31|$

\author{
(Solution on p. 622.) \\ (Solution on p. 622.) \\ (Solution on p. 622.) \\ (Solution on p. 622.) \\ (Solution on p. 622.) \\ (Solution on p. 622.) \\ (Solution on p. 622.) \\ (Solution on p. 622.)
}

\subsubsection{Exercises}

Determine each of the values.

Exercise 10.4.15

(Solution on p. 622.)

Exercise 10.4.16

$$
|3|
$$

Exercise 10.4.17

Exercise 10.4.18

$$
|-9|
$$


Exercise 10.4.19

(Solution on p. 622.)

$|-1|$

Exercise 10.4.20

$|-4|$

Exercise 10.4.21

(Solution on p. 622.)

$-|3|$

Exercise 10.4.22

$-|7|$

Exercise 10.4.23

$-|-14|$

(Solution on p. 622.)

Exercise 10.4.24

$|0|$

Exercise 10.4.25

$|-26|$

(Solution on p. 622.)

Exercise 10.4.26

$-|-26|$

Exercise 10.4.27

$-(-|4|)$

Exercise 10.4.28

$-(-|2|)$

Exercise 10.4.29

$-(-|-6|)$

(Solution on p. 623.)

Exercise 10.4.30

$-(-|-42|)$

Exercise 10.4.31

$|5|-|-2|$

(Solution on p. 623.)

Exercise 10.4.32 $|-2|^{3}$

Exercise 10.4.33 $|-(2 \cdot 3)|$

(Solution on p. 623.)

Exercise 10.4.34

$|-2|-|-9|$

Exercise 10.4.35

$(|-6|+|4|)^{2}$

(Solution on p. 623.)

Exercise 10.4.36

$$
(|-1|-|1|)^{3}
$$

Exercise 10.4.37

$(|4|+|-6|)^{2}-(|-2|)^{3}$

(Solution on p. 623.)

Exercise 10.4.38

$-[|-10|-6]^{2}$

Exercise 10.4.39

$-\left\{-[-|-4|+|-3|]^{3}\right\}^{2}$

(Solution on p. 623.)

Exercise 10.4.40

A Mission Control Officer at Cape Canaveral makes the statement "lift-off, $T$ minus 50 seconds." How long is it before lift-off? 
Exercise 10.4.41

(Solution on p. 623.)

Due to a slowdown in the industry, a Silicon Valley computer company finds itself in debt $\$ 2,400,000$. Use absolute value notation to describe this company's debt.

Exercise 10.4.42

A particular machine is set correctly if upon action its meter reads 0 . One particular machine has a meter reading of -1.6 upon action. How far is this machine off its correct setting?

\subsubsection{Exercises for Review}

Exercise 10.4.43

(Section 5.3) Find the sum: $\frac{9}{70}+\frac{5}{21}+\frac{8}{15}$.

(Solution on p. 623.)

Exercise 10.4.44

(Section 5.6) Find the value of $\frac{\frac{3}{10}+\frac{4}{12}}{\frac{19}{20}}$.

Exercise 10.4.45

(Section 6.3) Convert $3.2 \frac{3}{5}$ to a fraction.

(Solution on p. 623.)

Exercise 10.4.46

(Section 7.3) The ratio of acid to water in a solution is $\frac{3}{8}$. How many $\mathrm{mL}$ of acid are there in a solution that contain $112 \mathrm{~mL}$ of water?

Exercise 10.4.47

(Solution on p. 623.)

(Section 10.3) Find the value of $-6-(-8)$.

\subsection{Addition of Signed Numbers ${ }^{5}$}

\subsubsection{Section Overview}

- Addition of Numbers with Like Signs

- Addition with Zero

- Addition of Numbers with Unlike Signs

- Calculators

\subsubsection{Addition of Numbers with Like Signs}

The addition of the two positive numbers 2 and 3 is performed on the number line as follows.

Begin at 0 , the origin.

Since 2 is positive, move 2 units to the right.

Since 3 is positive, move 3 more units to the right.

We are now located at 5 .

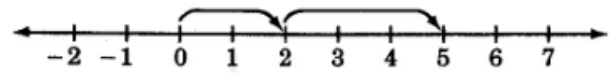

Thus, $2+3=5$.

Summarizing, we have

\footnotetext{
${ }^{5}$ This content is available online at $<$ http://cnx.org/content $/ \mathrm{m} 35031 / 1.2 />$.
} 
$(2$ positive units $)+(3$ positive units $)=(5$ positive units $)$

The addition of the two negative numbers -2 and -3 is performed on the number line as follows.

Begin at 0 , the origin.

Since -2 is negative, move 2 units to the left.

Since -3 is negative, move 3 more units to the left.

We are now located at -5 .

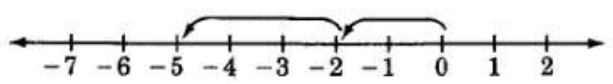

Thus, $(-2)+(-3)=-5$.

Summarizing, we have

( 2 negative units $)+(3$ negative units $)=(5$ negative units $)$

Observing these two examples, we can suggest these relationships:

$($ positive number $)+($ positive number $)=($ positive number $)$

(negative number $)+($ negative number $)=($ negative number $)$

\section{Adding Numbers with the Same Sign}

Addition of numbers with like sign:

To add two real numbers that have the same sign, add the absolute values of the numbers and associate with the sum the common sign.

\subsubsection{Sample Set A}

Find the sums.

\section{Example 10.21}

$3+7$

$$
\left.\begin{array}{l}
|3|=3 \\
|7|=7
\end{array}\right\} \text { Add these absolute values. }
$$

$3+7=10$

The common sign is "+."

Thus, $3+7=+10$, or $3+7=10$.

\section{Example 10.22}

$$
(-4)+(-9)
$$

$$
\left.\begin{array}{l}
|-4|=4 \\
|-9|=9
\end{array}\right\} \text { Add these absolute values. }
$$

$4+9=13$

The common sign is "-." 
Thus, $(-4)+(-9)=-13$.

\subsubsection{Practice Set A}

Find the sums.

Exercise 10.5.1

(Solution on p. 623.)

$8+6$

Exercise 10.5.2

$41+11$

Exercise 10.5.3

$(-4)+(-8)$

Exercise 10.5.4

$(-36)+(-9)$

Exercise 10.5.5

$-14+(-20)$

Exercise 10.5.6

$$
-\frac{2}{3}+\left(-\frac{5}{3}\right)
$$

Exercise 10.5.7

$-2.8+(-4.6)$

Exercise 10.5.8

$0+(-16)$

(Solution on p. 623.)

(Solution on p. 623.)

(Solution on p. 623.)

(Solution on p. 623.)

(Solution on p. 623.)

(Solution on p. 623.)

(Solution on p. 623.)

\subsubsection{Addition With Zero}

\section{Addition with Zero}

Notice that

$(0)+($ a positive number $)=($ that same positive number $)$.

$(0)+($ a negative number $)=($ that same negative number $)$.

The Additive Identity Is Zero

Since adding zero to a real number leaves that number unchanged, zero is called the additive identity.

\subsubsection{Addition of Numbers with Unlike Signs}

The addition $2+(-6)$, two numbers with unlike signs, can also be illustrated using the number line.

Begin at 0 , the origin.

Since 2 is positive, move 2 units to the right.

Since -6 is negative, move, from 2, 6 units to the left.

We are now located at -4 .

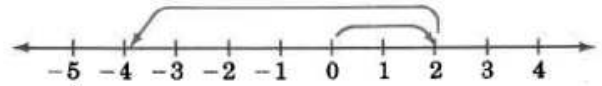

We can suggest a rule for adding two numbers that have unlike signs by noting that if the signs are disregarded, 4 can be obtained by subtracting 2 from 6 . But 2 and 6 are precisely the absolute values of 2 and 
-6. Also, notice that the sign of the number with the larger absolute value is negative and that the sign of the resulting sum is negative.

\section{Adding Numbers with Unlike Signs}

Addition of numbers with unlike signs: To add two real numbers that have unlike signs, subtract the smaller absolute value from the larger absolute value and associate with this difference the sign of the number with the larger absolute value.

\subsubsection{Sample Set B}

Find the following sums.

\section{Example 10.23}

$$
7+(-2)
$$

$$
\underbrace{|7|=7}_{\begin{array}{c}
\text { Larger absolute } \\
\text { value. Sign is positive. }
\end{array}} \quad \underbrace{|-2|=2}_{\begin{array}{c}
\text { Smaller absolute } \\
\text { value. }
\end{array}}
$$

Subtract absolute values: $7-2=5$.

Attach the proper sign: "+."

Thus, $7+(-2)=+5$ or $7+(-2)=5$.

\section{Example 10.24}

$3+(-11)$

$\underbrace{|3|=3}_{\begin{array}{c}\text { Smaller absolute } \\ \text { value. }\end{array}} \quad \underbrace{|-11|=11}_{\begin{array}{c}\text { Larger absolute } \\ \text { value. Sign is negative. }\end{array}}$

Subtract absolute values: $11-3=8$.

Attach the proper sign: "-."

Thus, $3+(-11)=-8$.

\section{Example 10.25}

The morning temperature on a winter's day in Lake Tahoe was -12 degrees. The afternoon temperature was 25 degrees warmer. What was the afternoon temperature?

We need to find $-12+25$.

$$
\underbrace{|-12|=12}_{\begin{array}{c}
\text { Smaller absolute } \\
\text { value. }
\end{array}} \quad \underbrace{|25|=25}_{\begin{array}{c}
\text { Larger absolute } \\
\text { value. Sign is positive. }
\end{array}}
$$

Subtract absolute values: $25-12=16$.

Attach the proper sign: "+."

Thus, $-12+25=13$. 


\subsubsection{Practice Set B}

Find the sums.

Exercise 10.5.9

(Solution on p. 623.)

$4+(-3)$

Exercise 10.5.10

(Solution on p. 623.)

$-3+5$

Exercise 10.5.11

(Solution on p. 623.)

$15+(-18)$

Exercise 10.5.12

(Solution on p. 623.)

$0+(-6)$

Exercise 10.5.13

(Solution on p. 623.)

$-26+12$

Exercise 10.5.14

(Solution on p. 623.)

$35+(-78)$

Exercise 10.5.15

$15+(-10)$

Exercise 10.5.16

$1.5+(-2)$

Exercise 10.5.17

$-8+0$

Exercise 10.5.18

$0+(0.57)$

Exercise 10.5.19

$-879+454$

(Solution on p. 624.)

(Solution on p. 624.)

(Solution on p. 624.)

(Solution on p. 624.)

(Solution on p. 624.)

\subsubsection{Calculators}

Calculators having the $+/-$ key can be used for finding sums of signed numbers.

\subsubsection{Sample Set C}

Use a calculator to find the sum of -147 and 84 .

\begin{tabular}{|l|l|l|l|}
\hline & & Display Reads & \\
\hline Type & 147 & 147 & \\
\hline Press & $+/-$ & -147 & This key changes the sign of a number. It is different than - . \\
\hline Press & + & -147 & \\
\hline Type & 84 & 84 & \\
\hline Press & $=$ & -63 & \\
\hline
\end{tabular}

Table 10.1 


\subsubsection{Practice Set C}

Use a calculator to find each sum.

Exercise 10.5.20

(Solution on p. 624.)

$673+(-721)$

Exercise 10.5.21

(Solution on p. 624.)

$-8,261+2,206$

Exercise 10.5.22

(Solution on p. 624.)

$-1,345.6+(-6,648.1)$

\subsubsection{Exercises}

Find the sums in the following 27 problems. If possible, use a calculator to check each result.

Exercise 10.5.23

(Solution on p. 624.)

$4+12$

Exercise 10.5.24

$8+6$

Exercise 10.5.25

(Solution on p. 624.)

$(-3)+(-12)$

Exercise 10.5.26

$(-6)+(-20)$

Exercise 10.5.27

(Solution on p. 624.)

$$
10+(-2)
$$

Exercise 10.5.28

$$
8+(-15)
$$

Exercise 10.5.29

$$
-16+(-9)
$$

(Solution on p. 624.)

Exercise 10.5.30 $-22+(-1)$

Exercise 10.5.31 $0+(-12)$

(Solution on p. 624.)

Exercise 10.5.32 $0+(-4)$

Exercise 10.5.33 $0+(24)$

(Solution on p. 624.)

Exercise 10.5.34 $-6+1+(-7)$

Exercise 10.5.35 $-5+(-12)+(-4)$

(Solution on p. 624.)

Exercise 10.5.36 $-5+5$

Exercise 10.5.37 $-7+7$

(Solution on p. 624.)

Exercise 10.5.38

$-14+14$ 
Exercise 10.5.39

(Solution on p. 624.)

$4+(-4)$

Exercise 10.5.40

$9+(-9)$

Exercise 10.5.41

(Solution on p. 624.)

$84+(-61)$

Exercise 10.5.42

$13+(-56)$

Exercise 10.5.43

$452+(-124)$

(Solution on p. 624.)

Exercise 10.5.44

$636+(-989)$

Exercise 10.5.45

$1,811+(-935)$

(Solution on p. 624.)

Exercise 10.5.46

$-373+(-14)$

Exercise 10.5.47

(Solution on p. 624.)

$-1,211+(-44)$

Exercise 10.5.48

$-47.03+(-22.71)$

Exercise 10.5.49

(Solution on p. 624.)

$$
-1.998+(-4.086)
$$

Exercise 10.5.50

In order for a small business to break even on a project, it must have sales of $\$ 21,000$. If the amount of sales was $\$ 15,000$, by how much money did this company fall short?

Exercise 10.5.51

(Solution on p. 624.)

Suppose a person has $\$ 56$ in his checking account. He deposits $\$ 100$ into his checking account by using the automatic teller machine. He then writes a check for $\$ 84.50$. If an error causes the deposit not to be listed into this person's account, what is this person's checking balance?

Exercise 10.5.52

A person borrows $\$ 7$ on Monday and then $\$ 12$ on Tuesday. How much has this person borrowed?

Exercise 10.5.53

(Solution on p. 624.)

A person borrows $\$ 11$ on Monday and then pays back $\$ 8$ on Tuesday. How much does this person owe?

\subsubsection{Exercises for Review}

Exercise 10.5.54

(Section 4.6) Find the reciprocal of $8 \frac{5}{6}$.

Exercise 10.5.55

(Section 5.3) Find the value of $\frac{5}{12}+\frac{7}{18}-\frac{1}{3}$.

(Solution on p. 624.)

Exercise 10.5.56

(Section 6.4) Round 0.01628 to the nearest tenth.

Exercise 10.5.57

(Solution on p. 625.)

(Section 7.5) Convert $62 \%$ to a fraction. 
Exercise 10.5.58

(Section 10.4) Find the value of $|-12|$.

\subsection{Subtraction of Signed Numbers ${ }^{6}$}

\subsubsection{Section Overview}

- Definition of Subtraction

- The Process of Subtraction

- Calculators

\subsubsection{Definition of Subtraction}

We know from experience with arithmetic that the subtraction $5-2$ produces 3 , that is $5-2=3$. We can suggest a rule for subtracting signed numbers by illustrating this process on the number line.

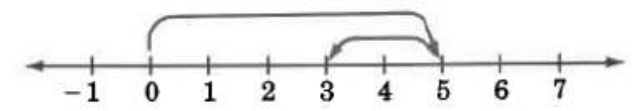

Begin at 0 , the origin.

Since 5 is positive, move 5 units to the right.

Then, move 2 units to the left to get to 6 . (This reminds us of addition with a negative number.)

From this illustration we can see that $5-2$ is the same as $5+(-2)$. This leads us directly to the definition of subtraction.

\section{Definition of Subtraction}

If $a$ and $b$ are real numbers, $a-b$ is the same as $a+(-b)$, where $-b$ is the opposite of $b$.

\subsubsection{The Process of Subtraction}

From this definition, we suggest the following rule for subtracting signed numbers.

\section{Subtraction of Signed Numbers}

To perform the subtraction $a-b$, add the opposite of $b$ to $a$, that is, change the sign of $b$ and add.

\subsubsection{Sample Set A}

Perform the indicated subtractions.

Example 10.26

$5-3=5+(-3)=2$

Example 10.27

$4-9=4+(-9)=-5$

Example 10.28

$$
-4-6=-4+(-6)=-10
$$

Example 10.29

$-3-(-12)=-3+12=9$

\footnotetext{
${ }^{6}$ This content is available online at $<$ http://cnx.org/content $/ \mathrm{m} 35032 / 1.2 />$.
} 
Example 10.30

$0-(-15)=0+15=15$

Example 10.31

The high temperature today in Lake Tahoe was $26^{\circ} \mathrm{F}$. The low temperature tonight is expected to be $-7^{\circ} \mathrm{F}$. How many degrees is the temperature expected to drop?

We need to find the difference between 26 and -7 .

$26-(-7)=26+7=33$

Thus, the expected temperature drop is $33^{\circ} \mathrm{F}$.

Example 10.32

$$
\begin{aligned}
& -6-(-5)-10=-6+5+(-10) \\
& =(-6+5)+(-10) \\
& =-1+(-10) \\
& =-11
\end{aligned}
$$

\subsubsection{Practice Set A}

Perform the indicated subtractions.

Exercise 10.6.1

(Solution on p. 625.)

$9-6$

Exercise 10.6.2

(Solution on p. 625.)

$6-9$

Exercise 10.6.3

$0-7$

Exercise 10.6.4

(Solution on p. 625.)

$1-14$

Exercise 10.6.5

$-8-12$

Exercise 10.6.6

$-21-6$

Exercise 10.6.7

$-6-(-4)$

Exercise 10.6.8

$8-(-10)$

Exercise 10.6.9

$1-(-12)$

Exercise 10.6.10

$86-(-32)$

Exercise 10.6.11

$0-16$

(Solution on p. 625.)

(Solution on p. 625.)

(Solution on p. 625.)

(Solution on p. 625.)

(Solution on p. 625.)

(Solution on p. 625.)

(Solution on p. 625.)

Exercise 10.6.12

(Solution on p. 625.)

$0-(-16)$

Exercise 10.6.13

$0-(8)$ 
Exercise 10.6.14

$5-(-5)$

Exercise 10.6.15

$24-[-(-24)]$
(Solution on p. 625.)

(Solution on p. 625.)

\subsubsection{Calculators}

Calculators can be used for subtraction of signed numbers. The most efficient calculators are those with a $+/-$ key.

\subsubsection{Sample Set B}

Use a calculator to find each difference.

Example 10.33

$3,187-8,719$

\begin{tabular}{|l|l|l|}
\hline \multicolumn{3}{|c|}{ Display Reads } \\
\hline Type & 3187 & 3187 \\
\hline Press & - & 3187 \\
\hline Type & 8719 & 8719 \\
\hline Press & $=$ & -5532 \\
\hline
\end{tabular}

Table 10.2

Thus, $3,187-8,719=-5,532$.

Example 10.34

$-156-(-211)$

Method A:

\begin{tabular}{|c|l|l|}
\hline \multicolumn{3}{|c|}{ Display Reads } \\
\hline Type & 156 & 156 \\
\hline Press & $+/-$ & -156 \\
\hline Type & - & -156 \\
\hline Press & 211 & 211 \\
\hline Type & $+/-$ & -211 \\
\hline Press & $=$ & 55 \\
\hline
\end{tabular}

Table 10.3

Thus, $-156-(-211)=55$.

Method B: 
We manually change the subtraction to an addition and change the sign of the number to be subtracted.

$-156-(-211)$ becomes $-156+211$

\begin{tabular}{|l|l|l|}
\hline \multicolumn{3}{|c|}{ Display Reads } \\
\hline Type & 156 & 156 \\
\hline Press & ++- & -156 \\
\hline Press & + & -156 \\
\hline Type & 211 & 211 \\
\hline Press & $=$ & 55 \\
\hline
\end{tabular}

Table 10.4

\subsubsection{Practice Set $B$}

Use a calculator to find each difference.

Exercise 10.6.16

(Solution on p. 625.)

$44-315$

Exercise 10.6.17

$12.756-15.003$

Exercise 10.6.18

$-31.89-44.17$

Exercise 10.6.19

(Solution on p. 625.)

(Solution on p. 625.)

$-0.797-(-0.615)$

(Solution on p. 625.)

\subsubsection{Exercises}

For the following 18 problems, perform each subtraction. Use a calculator to check each result.

Exercise 10.6.20

(Solution on p. 625.)

$$
8-3
$$

Exercise 10.6.21

$12-7$

Exercise 10.6.22

(Solution on p. 625.)

$$
5-6
$$

Exercise 10.6.23

$14-30$

Exercise 10.6.24

$$
-6-8
$$

(Solution on p. 625.)

Exercise 10.6.25

$$
-1-12
$$

Exercise 10.6.26

$$
-5-(-3)
$$

(Solution on p. 625.) 
Exercise 10.6.27

$-11-(-8)$

Exercise 10.6.28

(Solution on p. 625.)

$0-6$

Exercise 10.6.29

$0-15$

Exercise 10.6.30

(Solution on p. 626.)

$0-(-7)$

Exercise 10.6.31

$0-(-10)$

Exercise 10.6.32

$67-38$

(Solution on p. 626.)

Exercise 10.6.33

$142-85$

Exercise 10.6.34

$816-1140$

(Solution on p. 626.)

Exercise 10.6.35

$105-421$

Exercise 10.6.36

$-550-(-121)$

(Solution on p. 626.)

Exercise 10.6.37

$-15.016-(4.001)$

For the following 4 problems, perform the indicated operations.

Exercise 10.6.38

$-26+7-52$

(Solution on p. 626.)

Exercise 10.6.39

$-15-21-(-2)$

Exercise 10.6.40

$-104-(-216)-(-52)$

(Solution on p. 626.)

Exercise 10.6.41

$-0.012-(-0.111)-(0.035)$

Exercise 10.6.42

(Solution on p. 626.)

When a particular machine is operating properly, its meter will read 34. If a broken bearing in the machine causes the meter reading to drop by 45 units, what is the meter reading?

Exercise 10.6.43

The low temperature today in Denver was $-4^{\circ} \mathrm{F}$ and the high was $-42^{\circ} \mathrm{F}$. What is the temperature difference? 


\subsubsection{Exercises for Review}

Exercise 10.6.44

(Solution on p. 626.)

(Section 6.3) Convert $16.02 \frac{1}{5}$ to a decimal.

Exercise 10.6.45

(Section 6.6) Find 4.01 of 6.2 .

Exercise 10.6.46

(Section 7.5) Convert $\frac{5}{16}$ to a percent.

(Solution on p. 626.)

Exercise 10.6.47

(Section 8.4) Use the distributive property to compute the product: $15 \cdot 82$.

Exercise 10.6.48

(Section 10.5) Find the sum: $16+(-21)$.

(Solution on p. 626.)

\subsection{Multiplication and Division of Signed Numbers ${ }^{7}$}

\subsubsection{Section Overview}

- Multiplication of Signed Numbers

- Division of Signed Numbers

- Calculators

\subsubsection{Multiplication of Signed Numbers}

Let us consider first, the product of two positive numbers. Multiply: $3 \cdot 5$.

$3 \cdot 5$ means $5+5+5=15$

This suggests ${ }^{8}$ that

$($ positive number $) \cdot($ positive number $)=($ positive number $)$

More briefly,

$(+)(+)=(+)$

Now consider the product of a positive number and a negative number. Multiply: $(3)(-5)$.

(3) $(-5)$ means $(-5)+(-5)+(-5)=-15$

This suggests that

$($ positive number $) \cdot($ negative number $)=($ negative number $)$

More briefly,

$(+)(-)=(-)$

By the commutative property of multiplication, we get

\footnotetext{
${ }^{7}$ This content is available online at $<$ http://cnx.org/content $/ \mathrm{m} 35033 / 1.2 />$.

${ }^{8}$ In later mathematics courses, the word "suggests" turns into the word "proof." One example does not prove a claim. Mathematical proofs are constructed to validate a claim for all possible cases.
} 
$($ negative number $) \cdot($ positive number $)=($ negative number $)$

More briefly,

$(-)(+)=(-)$

The sign of the product of two negative numbers can be suggested after observing the following illustration.

Multiply -2 by, respectively, 4, 3, 2, 1, 0, -1, -2, -3, -4.

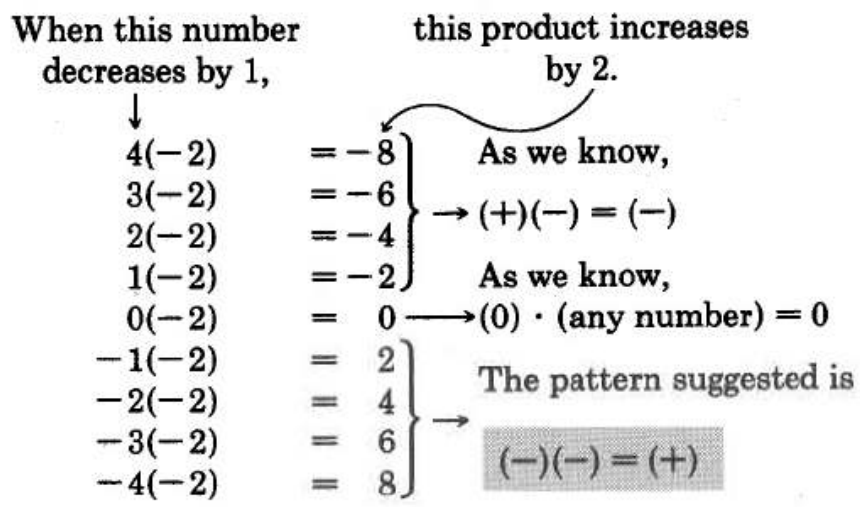

We have the following rules for multiplying signed numbers.

\section{Rules for Multiplying Signed Numbers}

Multiplying signed numbers:

1. To multiply two real numbers that have the same sign, multiply their absolute values. The product is positive.

$(+)(+)=(+)$

$(-)(-)=(+)$

2. To multiply two real numbers that have opposite signs, multiply their absolute values. The product is negative.

$$
\begin{aligned}
& (+)(-)=(-) \\
& (-)(+)=(-)
\end{aligned}
$$

\subsubsection{Sample Set A}

Find the following products.

Example 10.35

$8 \cdot 6$

$$
\begin{aligned}
& |8|=8 \\
& |6|=6
\end{aligned}
$$

$8 \cdot 6=48$

Since the numbers have the same sign, the product is positive.

Thus, $8 \cdot 6=+48$, or $8 \cdot 6=48$.

Example 10.36

$$
(-8)(-6)
$$


$\left.\begin{array}{l}|-8|=8 \\ |-6|=6\end{array}\right\}$ Multiply these absolute values.

$8 \cdot 6=48$

Since the numbers have the same sign, the product is positive.

Thus, $(-8)(-6)=+48$, or $(-8)(-6)=48$.

Example 10.37

$(-4)(7)$

$\left.\begin{array}{c}|-4|=4 \\ |7|=7\end{array}\right\}$ Multiply these absolute values.

$4 \cdot 7=28$

Since the numbers have opposite signs, the product is negative.

Thus, $(-4)(7)=-28$.

Example 10.38

$6(-3)$

$\left.\begin{array}{ccc}|6| & = & 6 \\ |-3| & = & 3\end{array}\right\}$ Multiply these absolute values.

$6 \cdot 3=18$

Since the numbers have opposite signs, the product is negative.

Thus, $6(-3)=-18$.

\subsubsection{Practice Set A}

Find the following products.

Exercise 10.7.1

$$
3(-8)
$$

(Solution on p. 626.)

Exercise 10.7.2

(Solution on p. 626.)

4 (16)

Exercise 10.7.3

$$
(-6)(-5)
$$

Exercise 10.7.4

$$
(-7)(-2)
$$

Exercise 10.7.5

$(-1)(4)$

Exercise 10.7.6

$(-7) 7$ 


\subsubsection{Division of Signed Numbers}

To determine the signs in a division problem, recall that

$\frac{12}{3}=4$ since $12=3 \cdot 4$

This suggests that

$\frac{(+)}{(+)}=(+)$

$\frac{(+)}{(+)}=(+)$ since $(+)=(+)(+)$

What is $\frac{12}{-3}$ ?

$-12=(-3)(-4)$ suggests that $\frac{12}{-3}=-4$. That is,

$\frac{(+)}{(-)}=(-)$

$(+)=(-)(-)$ suggests that $\frac{(+)}{(-)}=(-)$

What is $\frac{-12}{3}$ ?

$-12=(3)(-4)$ suggests that $\frac{-12}{3}=-4$. That is,

$\frac{(-)}{(+)}=(-)$

$(-)=(+)(-)$ suggests that $\frac{(-)}{(+)}=(-)$

What is $\frac{-12}{-3}$ ?

$-12=(-3)(4)$ suggests that $\frac{-12}{-3}=4$. That is,

$\frac{(-)}{(-)}=(+)$

$(-)=(-)(+)$ suggests that $\frac{(-)}{(-)}=(+)$

We have the following rules for dividing signed numbers.

\section{Rules for Dividing Signed Numbers}

Dividing signed numbers:

1. To divide two real numbers that have the same sign, divide their absolute values. The quotient is positive.

$\frac{(+)}{(+)}=(+) \frac{(-)}{(-)}=(+)$

2. To divide two real numbers that have opposite signs, divide their absolute values. The quotient is negative.

$\frac{(-)}{(+)}=(-) \frac{(+)}{(-)}=(-)$

\subsubsection{Sample Set B}

Find the following quotients.

\section{Example 10.39}

$\frac{-10}{2}$ 


$$
\begin{aligned}
& \left.\begin{array}{ccc}
|-10| & =10 \\
|2| & =2
\end{array}\right\} \text { Divide these absolute values. } \\
& \frac{10}{2}=5
\end{aligned}
$$

Since the numbers have opposite signs, the quotient is negative.

Thus $\frac{-10}{2}=-5$.

Example 10.40

$\frac{-35}{-7}$

$\left.\begin{array}{c}|-35|=35 \\ |-7|=7\end{array}\right\}$ Divide these absolute values.

$\frac{35}{7}=5$

Since the numbers have the same signs, the quotient is positive.

Thus, $\frac{-35}{-7}=5$.

\section{Example 10.41}

$\frac{18}{-9}$

$$
\begin{aligned}
& |18|=18 \\
& |-9|=9 \\
& \text { \} Divide these absolute values. } \\
& \frac{18}{9}=2
\end{aligned}
$$

Since the numbers have opposite signs, the quotient is negative.

Thus, $\frac{18}{-9}=2$.

\subsubsection{Practice Set B}

Find the following quotients.

Exercise 10.7.7

(Solution on p. 626.)

$\frac{-24}{-6}$

Exercise 10.7.8

$\frac{30}{-5}$

Exercise 10.7.9

$\frac{-54}{27}$

Exercise 10.7.10

$\frac{51}{17}$

(Solution on p. 626.)

(Solution on p. 626.)

(Solution on p. 626.) 


\subsubsection{Sample Set C}

\section{Example 10.42}

Find the value of $\frac{-6(4-7)-2(8-9)}{-(4+1)+1}$.

Using the order of operations and what we know about signed numbers, we get,

$$
\begin{array}{rlc}
\frac{-6(4-7)-2(8-9)}{-(4+1)+1} & = & \frac{-6(-3)-2(-1)}{-(5)+1} \\
& = & \frac{18+2}{-5+1} \\
& = & \frac{20}{-} \\
& = & -5
\end{array}
$$

\subsubsection{Practice Set C}

\section{Exercise 10.7.11}

Find the value of $\frac{-5(2-6)-4(-8-1)}{2(3-10)-9(-2)}$.

(Solution on p. 626.)

\subsubsection{Calculators}

Calculators with the $+/-$ key can be used for multiplying and dividing signed numbers.

\subsubsection{Sample Set D}

Use a calculator to find each quotient or product.

\section{Example 10.43}

$$
(-186) \cdot(-43)
$$

Since this product involves a (negative) -(negative), we know the result should be a positive number. We'll illustrate this on the calculator.

\begin{tabular}{|l|l|l|}
\hline & & Display Reads \\
\hline Type & 186 & 186 \\
\hline Press & $+/-$ & -186 \\
\hline Press & $\times$ & -186 \\
\hline Type & 43 & 43 \\
\hline Press & $+/-$ & -43 \\
\hline Press & $=$ & 7998 \\
\hline
\end{tabular}

Table 10.5

Thus, $(-186) \cdot(-43)=7,998$.

\section{Example 10.44}

$\frac{158.64}{-54.3}$. Round to one decimal place. 


\begin{tabular}{|l|l|l|}
\hline & & Display Reads \\
\hline Type & 158.64 & 158.64 \\
\hline Press & $\div$ & 158.64 \\
\hline Type & 54.3 & 54.3 \\
\hline Press & $+/-$ & -54.3 \\
\hline Press & $=$ & -2.921546961 \\
\hline
\end{tabular}

Table 10.6

Rounding to one decimal place we get -2.9 .

\subsubsection{Practice Set D}

Use a calculator to find each value.

Exercise 10.7.12

(Solution on p. 626.)

$(-51.3) \cdot(-21.6)$

Exercise 10.7.13

(Solution on p. 626.)

$-2.5746 \div-2.1$

Exercise 10.7.14

$(0.006) \cdot(-0.241)$. Round to three decimal places.

(Solution on p. 626.)

\subsubsection{Exercises}

Find the value of each of the following. Use a calculator to check each result.

Exercise 10.7.15

(Solution on p. 626.) $(-2)(-8)$

Exercise 10.7.16

$(-3)(-9)$

Exercise 10.7.17

(Solution on p. 627.)

$(-4)(-8)$

Exercise 10.7.18

$(-5)(-2)$

Exercise 10.7.19

(Solution on p. 627.)

(3) $(-12)$

Exercise 10.7.20

(4) $(-18)$

Exercise 10.7.21

(10) $(-6)$

(Solution on p. 627.)

Exercise 10.7.22

$(-6)(4)$

Exercise 10.7.23

$(-2)(6)$

(Solution on p. 627.) 
Exercise 10.7.24

$(-8)(7)$

Exercise 10.7.25

$\frac{21}{7}$

(Solution on p. 627.)

Exercise 10.7.26

$\frac{42}{6}$

Exercise 10.7.27 $\frac{-39}{3}$

(Solution on p. 627.)

Exercise 10.7.28

$\frac{-20}{10}$

Exercise 10.7.29 $\frac{-45}{-5}$

(Solution on p. 627.)

Exercise 10.7.30 $\frac{-16}{-8}$

Exercise 10.7.31 $\frac{25}{-5}$

(Solution on p. 627.)

Exercise 10.7.32 $\frac{36}{-4}$

Exercise 10.7.33

$8-(-3)$

(Solution on p. 627.)

Exercise 10.7.34 $14-(-20)$

Exercise 10.7.35 $20-(-8)$

Exercise 10.7.36 $-4-(-1)$

Exercise 10.7.37

$0-4$

(Solution on p. 627.)

Exercise 10.7.38 $0-(-1)$

Exercise 10.7.39

$-6+1-7$

(Solution on p. 627.)

Exercise 10.7.40

$15-12-20$

Exercise 10.7.41

$1-6-7+8$

(Solution on p. 627.)

Exercise 10.7.42

$2+7-10+2$

Exercise 10.7.43

$3(4-6)$

(Solution on p. 627.)

Exercise 10.7.44

$8(5-12)$

Exercise 10.7.45

$-3(1-6)$

Exercise 10.7.46

$-8(4-12)+2$ 
Exercise 10.7.47

(Solution on p. 627.)

$-4(1-8)+3(10-3)$

Exercise 10.7.48

$-9(0-2)+4(8-9)+0(-3)$

Exercise 10.7.49

(Solution on p. 627.)

$6(-2-9)-6(2+9)+4(-1-1)$

Exercise 10.7.50

$\frac{3(4+1)-2(5)}{-2}$

Exercise 10.7.51

$\frac{4(8+1)-3(-2)}{-4-2}$

(Solution on p. 627.)

Exercise 10.7.52

$\frac{-1(3+2)+5}{-1}$

Exercise 10.7.53

$\frac{-3(4-2)+(-3)(-6)}{-4}$

(Solution on p. 627.)

Exercise 10.7.54

$-1(4+2)$

Exercise 10.7.55

$-1(6-1)$

(Solution on p. 627.)

Exercise 10.7.56

$-(8+21)$

Exercise 10.7.57

$-(8-21)$

(Solution on p. 627.)

\subsubsection{Exercises for Review}

\section{Exercise 10.7.58}

(Section 3.3) Use the order of operations to simplify $\left(5^{2}+3^{2}+2\right) \div 2^{2}$.

Exercise 10.7.59

(Section 4.7) Find $\frac{3}{8}$ of $\frac{32}{9}$.

(Solution on p. 627.)

Exercise 10.7.60

(Section 6.2) Write this number in decimal form using digits: "fifty-two three-thousandths"

Exercise 10.7.61

(Solution on p. 627.)

(Section 7.4) The ratio of chlorine to water in a solution is 2 to 7 . How many $\mathrm{mL}$ of water are in a solution that contains $15 \mathrm{~mL}$ of chlorine?

Exercise 10.7.62

(Section 10.6) Perform the subtraction $-8-(-20)$ 


\subsection{Summary of Key Concepts ${ }^{9}$}

\subsubsection{Summary of Key Concepts}

Variables and Constants (Section 10.2)

A variable is a letter or symbol that represents any member of a set of two or more numbers. A constant is a letter or symbol that represents a specific number. For example, the Greek letter $\pi$ (pi) represents the constant 3.14159 . . . .

The Real Number Line (Section 10.2)

The real number line allows us to visually display some of the numbers in which we are interested.

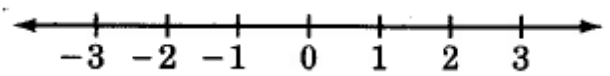

\section{Coordinate and Graph (Section 10.2)}

The number associated with a point on the number line is called the coordinate of the point. The point associated with a number is called the graph of the number.

Real Number (Section 10.2)

A real number is any number that is the coordinate of a point on the real number line.

Types of Real Numbers (Section 10.2)

The set of real numbers has many subsets. The ones of most interest to us are:

The natural numbers: $\{1,2,3,4, \ldots\}$

The whole numbers: $\{0,1,2,3,4, \ldots\}$

The integers: $\{\ldots,-3,-2,-1,0,1,2,3, \ldots\}$

The rational numbers: \{All numbers that can be expressed as the quotient of two integers.\}

Positive and Negative Numbers (Section 10.3)

A number is denoted as positive if it is directly preceded by a plus sign $(+)$ or no sign at all. A number is denoted as negative if it is directly preceded by a minus sign $(-)$.

Opposites (Section 10.3)

Opposites are numbers that are the same distance from zero on the number line but have opposite signs. The numbers $a$ and $-a$ are opposites.

Double-Negative Property (Section 10.3)

$-(-a)=a$

Absolute Value (Geometric) (Section 10.4)

The absolute value of a number $a$, denoted $|a|$, is the distance from $a$ to 0 on the number line.

Absolute Value (Algebraic) (Section 10.4)

$|a|=\left\{\begin{array}{cc}a, & \text { if } a \geq 0 \\ -a, & \text { if } a<0\end{array}\right.$

Addition of Signed Numbers (Section 10.5)

To add two numbers with

1. like signs, add the absolute values of the numbers and associate with the sum the common sign.

2. unlike signs, subtract the smaller absolute value from the larger absolute value and associate with the difference the sign of the larger absolute value.

\footnotetext{
${ }^{9}$ This content is available online at $<$ http://cnx.org/content/m35034/1.2/>.
} 
Addition with Zero (Section 10.5)

$0+($ any number $)=$ that particular number.

Additive Identity (Section 10.5)

Since adding 0 to any real number leaves that number unchanged, 0 is called the additive identity.

Definition of Subtraction (Section 10.6)

$a-b=a+(-b)$

Subtraction of Signed Numbers (Section 10.6)

To perform the subtraction $a-b$, add the opposite of $b$ to $a$, that is, change the sign of $b$ and follow the addition rules (Section 10.5).

Multiplication and Division of Signed Numbers (Section 10.7)

$(+)(+)=(+) \frac{(+)}{(+)}=(+) \frac{(+)}{(-)}=(-)$

$(-)(-)=(+)$

$(+)(-)=(-) \frac{(-)}{(-)}=(+) \frac{(-)}{(+)}=(-)$

$(-)(+)=(-)$

\subsection{Exercise Supplement ${ }^{10}$}

\subsubsection{Exercise Supplement}

\subsubsection{Variables, Constants, and Real Numbers (Section 10.2)}

For problems 1-5, next to each real number, note all subsets of the real numbers to which it belongs by writing $N$ for natural numbers, $W$ for whole numbers, or $Z$ for integers. Some numbers may belong to more than one subset.

\section{Exercise 10.9.1}

(Solution on p. 627.)

61

Exercise 10.9.2

$-14$

Exercise 10.9.3

(Solution on p. 627.)

0

Exercise 10.9.4

1

Exercise 10.9.5

(Solution on p. 628.)

Write all the integers that are strictly between -4 and 3

\section{Exercise 10.9.6}

Write all the integers that are between and including -6 and -1

For each pair of numbers in problems $7-10$, write the appropriate symbol $(<,>,=)$ in place of the $\square$.

Exercise 10.9.7

(Solution on p. 628.)

$-5 \square-1$

Exercise 10.9.8

$0 \square 2$

Exercise 10.9.9

(Solution on p. 628.) $-7 \square 0$

\footnotetext{
${ }^{10}$ This content is available online at $<\mathrm{http}: / / \mathrm{cnx} . o r g /$ content $/ \mathrm{m} 35035 / 1.2 />$.
} 


\section{Exercise 10.9.10}

$-1 \square 0$

For problems $11-15$, what numbers can replace $\mathrm{x}$ so that each statement is true?

Exercise 10.9.11

(Solution on p. 628.)

$-5 \leq x \leq-1, \mathrm{x}$ is an integer

Exercise 10.9.12

$-10<x \leq 0, \mathrm{x}$ is a whole number.

Exercise 10.9.13

(Solution on p. 628.)

$0 \leq x<5, \mathrm{x}$ is a natural number.

Exercise 10.9.14

$-3<x<3, \mathrm{x}$ is a natural number

Exercise 10.9.15

(Solution on p. 628.)

$-8<x \leq-2, x$ is a whole number.

For problems 16-20, how many units are there between the given pair of numbers?

Exercise 10.9.16

0 and 4

Exercise 10.9.17

-1 and 3

(Solution on p. 628.)

Exercise 10.9.18

-7 and -4

Exercise 10.9.19

(Solution on p. 628.)

-6 and 0

Exercise 10.9.20

-1 and 1

Exercise 10.9.21

A number is positive if it is directly preceded by a sign or no sign at all.

Exercise 10.9.22

A number is negative if it is directly preceded by a

\subsubsection{Signed Numbers (Section 10.3)}

For problems 23-26, how should each number be read?

Exercise 10.9.23

(Solution on p. 628.)

$-8$

Exercise 10.9.24

$$
-(-4)
$$

Exercise 10.9.25

$-(-1)$

(Solution on p. 628.)

Exercise 10.9.26

$$
-2
$$

For problems 27-31, write each expression in words.

Exercise 10.9.27

$1+(-7)$

Exercise 10.9.28

$$
-2-(-6)
$$

(Solution on p. 628.) 
Exercise 10.9.29

(Solution on p. 628.)

$-1-(+4)$

Exercise 10.9.30

$-(-(-3))$

Exercise 10.9.31

(Solution on p. 628.)

$0-(-11)$

For problems 32-36, rewrite each expression in simpler form.

Exercise 10.9.32 $-(-4)$

Exercise 10.9.33

(Solution on p. 628.)

$-(-15)$

Exercise 10.9.34

$-[-(-7)]$

Exercise 10.9.35

$1-(-18)$

Exercise 10.9.36

$0-(-1)$

\subsubsection{Absolute Value (Section 10.4)}

For problems 37-52, determine each value.

Exercise 10.9.37

(Solution on p. 628.)

$|9|$

Exercise 10.9.38

$\mid 16$ |

Exercise 10.9.39

$|-5|$

(Solution on p. 628.)

Exercise 10.9.40 $|-8|$

Exercise 10.9.41

$-|-2|$

(Solution on p. 628.)

Exercise 10.9.42

$-|-1|$

Exercise 10.9.43

$-(-|12|)$

(Solution on p. 628.)

Exercise 10.9.44

$-(-|90|)$

Exercise 10.9.45

$-(-|-16|)$

(Solution on p. 628.)

Exercise 10.9.46

$-(-|0|)$

Exercise 10.9.47

$|-4|^{2}$

(Solution on p. 628.)

Exercise 10.9.48

$|-5|^{2}$ 
Exercise 10.9.49

(Solution on p. 628.)

$|-2|^{3}$

Exercise 10.9.50

$|-(3 \cdot 4)|$

Exercise 10.9.51

(Solution on p. 628.)

$$
|-5|+|-2|
$$

Exercise 10.9.52

$|-7|-|-10|$

10.9.1.4 Addition, Subtraction, Multiplication and Division of Signed Numbers (Section 10.5,Section 10.6,Section 10.7)

For problems 53-71, perform each operation.

Exercise 10.9.53

(Solution on p. 628.)

$-6+4$

Exercise 10.9.54

$-10+8$

Exercise 10.9.55

(Solution on p. 629.)

$$
-1-6
$$

Exercise 10.9.56

$$
\text { 8- } 12
$$

Exercise 10.9.57

$$
0-14
$$

(Solution on p. 629.)

Exercise 10.9.58 $5 \cdot(-2)$

Exercise 10.9.59 $-8 \cdot(-6)$

Exercise 10.9.60 $(-3) \cdot(-9)$

Exercise 10.9.61 $14 \cdot(-3)$

Exercise 10.9.62 $5 \cdot(-70)$

Exercise 10.9.63

$-18 \div-6$

Exercise 10.9.64

$72 \div-12$

Exercise 10.9.65

$-16 \div-16$

(Solution on p. 629.)

Exercise 10.9.66

$0 \div-8$

Exercise 10.9.67

$-5 \div 0$

(Solution on p. 629.)

Exercise 10.9.68 $\frac{-15}{-3}$ 
Exercise 10.9.69

(Solution on p. 629.) $\frac{-28}{7}$

Exercise 10.9.70 $\frac{-120}{-|2|}$

Exercise 10.9.71

(Solution on p. 629.) $\frac{|-66|}{-|-3|}$

\subsection{Proficiency Exam ${ }^{11}$}

\subsubsection{Proficiency Exam}

Exercise 10.10.1

(Solution on p. 629.)

(Section 10.2) Write all integers that are strictly between -8 and -3 .

Exercise 10.10.2

(Solution on p. 629.)

(Section 10.2) Write all integers that are between and including -2 and 1.

For problems 3-5, write the appropriate symbol $(<,>,=)$ in place of the $\square$ for each pair of numbers.

Exercise 10.10.3

(Solution on p. 629.)

(Section 10.2) $-1 \square-1$

Exercise 10.10.4

(Solution on p. 629.)

(Section 10.2) $0 \square 3$

Exercise 10.10.5

(Section 10.2) $-1 \square-2$

(Solution on p. 629.)

For problems 6 and 7, what numbers can replace $x$ so that the statement is true?

Exercise 10.10.6

(Solution on p. 629.)

(Section 10.2) $-3 \leq x<0, x$ is an integer.

Exercise 10.10.7

(Solution on p. 629.)

(Section 10.2) $-4 \leq x \leq 0, x$ is a natural number.

Exercise 10.10.8

(Solution on p. 629.)

(Section 10.2) How many units are there between -3 and 2?

For problems 9-20, find each value.

Exercise 10.10.9

(Solution on p. 629.)

(Section 10.4) |-16|

Exercise 10.10.10

(Solution on p. 629.)

(Section 10.4) $-|-2|$

Exercise 10.10.11

(Section 10.4) $-\left(-|-4|^{2}\right)$

Exercise 10.10.12

(Section 10.4) $|-5|+|-10|$

Exercise 10.10.13

(Solution on p. 629.)

(Section 10.5) $-8+6$

Exercise 10.10.14

(Section 10.5) $-3+(-8)$

(Solution on p. 629.)

(Solution on p. 629.)

(Solution on p. 629.)

\footnotetext{
${ }^{11}$ This content is available online at $<\mathrm{http}: / / \mathrm{cnx} . o r g /$ content $/ \mathrm{m} 35036 / 1.2 />$.
} 
Exercise 10.10.15

(Solution on p. 629.)

(Section 10.6) $0-16$

(Solution on p. 629.)

Exercise 10.10.16

(Section 10.7) $(-14) \cdot(-3)$

Exercise 10.10.17

(Section 10.7) $(-5-6)^{2}$

Exercise 10.10.18

(Solution on p. 630.)

(Section 10.7) $(-51) \div(-7)$

(Solution on p. 630.)

Exercise 10.10.19

(Section 10.7) $\frac{-42}{-7}$

Exercise 10.10.20

(Section 10.7) $\left|\frac{-32}{8}-\frac{-15-5}{5}\right|$

(Solution on p. 630.)

(Solution on p. 630.) 


\section{Solutions to Exercises in Chapter 10}

Solution to Exercise 10.2.1 (p. 580)

yes

Solution to Exercise 10.2.2 (p. 580)

yes

Solution to Exercise 10.2.3 (p. 580)

yes

Solution to Exercise 10.2.4 (p. 580)

yes

Solution to Exercise 10.2.5 (p. 580)

yes

Solution to Exercise 10.2.6 (p. 581)

$-5,-4,-3,-2,-1,0$

Solution to Exercise 10.2.7 (p. 581)

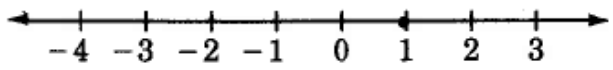

Solution to Exercise 10.2.8 (p. 581)

$N, W, Z$

Solution to Exercise 10.2.10 (p. 582)

$W, Z$

Solution to Exercise 10.2.12 (p. 582)

Z

Solution to Exercise 10.2.14 (p. 582)

Z

Solution to Exercise 10.2.16 (p. 582)

Neither

Solution to Exercise 10.2.18 (p. 582)

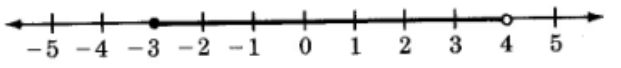

Solution to Exercise 10.2.20 (p. 582)

Yes, 10

Solution to Exercise 10.2.22 (p. 582)

$<$

Solution to Exercise 10.2.24 (p. 582)

$>$

Solution to Exercise 10.2.26 (p. 582)

$\{-1,0,1,2,3,4,5\}$

Solution to Exercise 10.2.28 (p. 582)

$\{1\}$

Solution to Exercise 10.2.30 (p. 582)

$\{0,1,2,3,4\}$

Solution to Exercise 10.2.32 (p. 583)

4

Solution to Exercise 10.2.34 (p. 583) 8

Solution to Exercise 10.2.36 (p. 583)

yes

Solution to Exercise 10.2.38 (p. 583)

yes 
Solution to Exercise 10.2.40 (p. 583) yes, -1

Solution to Exercise 10.2.42 (p. 583) $\frac{9}{5}$ or $1 \frac{4}{5}$ or 1.8

Solution to Exercise 10.2.44 (p. 583)

$0.3006 \mathrm{~m}$

Solution to Exercise 10.3.1 (p. 585)

six plus one

Solution to Exercise 10.3.2 (p. 585)

two plus negative eight

Solution to Exercise 10.3.3 (p. 585)

negative seven plus five

Solution to Exercise 10.3.4 (p. 585)

negative ten minus three

Solution to Exercise 10.3.5 (p. 585)

negative one minus negative eight

Solution to Exercise 10.3.6 (p. 585)

zero plus negative eleven

Solution to Exercise 10.3.7 (p. 586)

$-8$

Solution to Exercise 10.3.8 (p. 586)

$-17$

Solution to Exercise 10.3.9 (p. 586)

6

Solution to Exercise 10.3.10 (p. 586)

15

Solution to Exercise 10.3.11 (p. 586)

$-1$

Solution to Exercise 10.3.12 (p. 586)

7

Solution to Exercise 10.3.13 (p. 586)

$-a$ is negative

Solution to Exercise 10.3.14 (p. 586)

$-a$ is positive

Solution to Exercise 10.3.15 (p. 586)

We must say that we do not know.

Solution to Exercise 10.3.16 (p. 586)

+ (or no sign)

Solution to Exercise 10.3.18 (p. 586)

negative seven

Solution to Exercise 10.3.20 (p. 587)

fifteen

Solution to Exercise 10.3.22 (p. 587)

negative negative one, or opposite negative one

Solution to Exercise 10.3.24 (p. 587)

five plus three

Solution to Exercise 10.3.26 (p. 587)

fifteen plus negative three

Solution to Exercise 10.3.28 (p. 587)

negative seven minus negative two 
Solution to Exercise 10.3.30 (p. 587)

2

Solution to Exercise 10.3.32 (p. 587)

$-8$

Solution to Exercise 10.3.34 (p. 587)

$7+3=10$

Solution to Exercise 10.3.36 (p. 588)

$0 . \overline{296}$

Solution to Exercise 10.3.38 (p. 588)

$6,000+9,000=15,000 \quad(5,829+8,767=14,596) \quad$ or $5,800+8,800=14,600$

Solution to Exercise 10.3.40 (p. 588)

$0.0025 \mathrm{hm}$

Solution to Exercise 10.4.1 (p. 589)

7

Solution to Exercise 10.4.2 (p. 589)

3

Solution to Exercise 10.4.3 (p. 589)

12

Solution to Exercise 10.4.4 (p. 589)

0

Solution to Exercise 10.4.5 (p. 589)

$-9$

Solution to Exercise 10.4.6 (p. 589)

$-6$

Solution to Exercise 10.4.7 (p. 590)

7

Solution to Exercise 10.4.8 (p. 590)

9

Solution to Exercise 10.4.9 (p. 590)

12

Solution to Exercise 10.4.10 (p. 590)

5

Solution to Exercise 10.4.11 (p. 590)

$-8$

Solution to Exercise 10.4.12 (p. 590)

$-1$

Solution to Exercise 10.4.13 (p. 590)

$-52$

Solution to Exercise 10.4.14 (p. 590)

$-31$

Solution to Exercise 10.4.15 (p. 590)

5

Solution to Exercise 10.4.17 (p. 590)

6

Solution to Exercise 10.4.19 (p. 591)

1

Solution to Exercise 10.4.21 (p. 591)

$-3$

Solution to Exercise 10.4.23 (p. 591)

$-14$ 
Solution to Exercise 10.4.25 (p. 591)

26

Solution to Exercise 10.4.27 (p. 591)

4

Solution to Exercise 10.4.29 (p. 591)

6

Solution to Exercise 10.4.31 (p. 591)

3

Solution to Exercise 10.4.33 (p. 591)

6

Solution to Exercise 10.4.35 (p. 591)

100

Solution to Exercise 10.4.37 (p. 591) 92

Solution to Exercise 10.4.39 (p. 591)

$-1$

Solution to Exercise 10.4.41 (p. 592)

$-\$|-2,400,000|$

Solution to Exercise 10.4.43 (p. 592)

$\frac{9}{10}$

Solution to Exercise 10.4.45 (p. 592)

$3 \frac{13}{50}$ or $\frac{163}{50}$

Solution to Exercise 10.4.47 (p. 592)

2

Solution to Exercise 10.5.1 (p. 594)

14

Solution to Exercise 10.5.2 (p. 594)

52

Solution to Exercise 10.5.3 (p. 594)

$-12$

Solution to Exercise 10.5.4 (p. 594)

$-45$

Solution to Exercise 10.5.5 (p. 594)

$-34$

Solution to Exercise 10.5.6 (p. 594)

$-\frac{7}{3}$

Solution to Exercise 10.5.7 (p. 594)

$-7.4$

Solution to Exercise 10.5.8 (p. 594)

$-16$

Solution to Exercise 10.5.9 (p. 596)

1

Solution to Exercise 10.5.10 (p. 596)

2

Solution to Exercise 10.5.11 (p. 596)

$-3$

Solution to Exercise 10.5.12 (p. 596)

$-6$

Solution to Exercise 10.5.13 (p. 596)

$-14$ 
Solution to Exercise 10.5.14 (p. 596) $-43$

Solution to Exercise 10.5.15 (p. 596) 5

Solution to Exercise 10.5.16 (p. 596)

$-0.5$

Solution to Exercise 10.5.17 (p. 596)

$-8$

Solution to Exercise 10.5.18 (p. 596)

0.57

Solution to Exercise 10.5.19 (p. 596) $-425$

Solution to Exercise 10.5.20 (p. 597) $-48$

Solution to Exercise 10.5.21 (p. 597) $-6,085$

Solution to Exercise 10.5.22 (p. 597) $-7,993.7$

Solution to Exercise 10.5.23 (p. 597) 16

Solution to Exercise 10.5.25 (p. 597) $-15$

Solution to Exercise 10.5.27 (p. 597) 8

Solution to Exercise 10.5.29 (p. 597) $-25$

Solution to Exercise 10.5.31 (p. 597)

$-12$

Solution to Exercise 10.5.33 (p. 597) 24

Solution to Exercise 10.5.35 (p. 597) $-21$

Solution to Exercise 10.5.37 (p. 597) 0

Solution to Exercise 10.5.39 (p. 598) 0

Solution to Exercise 10.5.41 (p. 598) 23

Solution to Exercise 10.5.43 (p. 598) 328

Solution to Exercise 10.5.45 (p. 598) 876

Solution to Exercise 10.5.47 (p. 598) $-1,255$

Solution to Exercise 10.5.49 (p. 598) $-6.084$

Solution to Exercise 10.5.51 (p. 598) $-\$ 28.50$

Solution to Exercise 10.5.53 (p. 598) $\$ 3.00$ 
Solution to Exercise 10.5.55 (p. 598)

$\frac{17}{36}$

Solution to Exercise 10.5.57 (p. 598) $\frac{62}{100}=\frac{31}{50}$

Solution to Exercise 10.6.1 (p. 600) 3

Solution to Exercise 10.6.2 (p. 600) $-3$

Solution to Exercise 10.6.3 (p. 600) $-7$

Solution to Exercise 10.6.4 (p. 600) $-13$

Solution to Exercise 10.6.5 (p. 600) $-20$

Solution to Exercise 10.6.6 (p. 600) $-27$

Solution to Exercise 10.6.7 (p. 600) $-2$

Solution to Exercise 10.6.8 (p. 600) 18

Solution to Exercise 10.6.9 (p. 600) 13

Solution to Exercise 10.6.10 (p. 600) 118

Solution to Exercise 10.6.11 (p. 600) $-16$

Solution to Exercise 10.6.12 (p. 600) 16

Solution to Exercise 10.6.13 (p. 600) $-8$

Solution to Exercise 10.6.14 (p. 601) 10

Solution to Exercise 10.6.15 (p. 601) 0

Solution to Exercise 10.6.16 (p. 602) $-271$

Solution to Exercise 10.6.17 (p. 602) $-2.247$

Solution to Exercise 10.6.18 (p. 602) $-76.06$

Solution to Exercise 10.6.19 (p. 602) $-0.182$

Solution to Exercise 10.6.20 (p. 602) 5

Solution to Exercise 10.6.22 (p. 602)

$-1$

Solution to Exercise 10.6.24 (p. 602) $-14$

Solution to Exercise 10.6.26 (p. 602) $-2$ 
Solution to Exercise 10.6.28 (p. 603)

$-6$

Solution to Exercise 10.6.30 (p. 603)

7

Solution to Exercise 10.6.32 (p. 603)

29

Solution to Exercise 10.6.34 (p. 603) $-324$

Solution to Exercise 10.6.36 (p. 603) $-429$

Solution to Exercise 10.6.38 (p. 603) $-71$

Solution to Exercise 10.6.40 (p. 603) 164

Solution to Exercise 10.6.42 (p. 603)

$-11$

Solution to Exercise 10.6.44 (p. 604)

16.022

Solution to Exercise 10.6.46 (p. 604) $31.25 \%$

Solution to Exercise 10.6.48 (p. 604)

$-5$

Solution to Exercise 10.7.1 (p. 606)

$-24$

Solution to Exercise 10.7.2 (p. 606)

64

Solution to Exercise 10.7.3 (p. 606)

30

Solution to Exercise 10.7.4 (p. 606)

14

Solution to Exercise 10.7.5 (p. 606)

$-4$

Solution to Exercise 10.7.6 (p. 606)

$-49$

Solution to Exercise 10.7.7 (p. 608) 4

Solution to Exercise 10.7.8 (p. 608)

$-6$

Solution to Exercise 10.7.9 (p. 608)

$-2$

Solution to Exercise 10.7.10 (p. 608)

3

Solution to Exercise 10.7.11 (p. 609)

14

Solution to Exercise 10.7.12 (p. 610) $1,108.08$

Solution to Exercise 10.7.13 (p. 610) 1.226

Solution to Exercise 10.7.14 (p. 610) $-0.001$ 
Solution to Exercise 10.7.15 (p. 610) 16

Solution to Exercise 10.7.17 (p. 610) 32

Solution to Exercise 10.7.19 (p. 610)

$-36$

Solution to Exercise 10.7.21 (p. 610) $-60$

Solution to Exercise 10.7.23 (p. 610)

$-12$

Solution to Exercise 10.7.25 (p. 611) 3

Solution to Exercise 10.7.27 (p. 611) $-13$

Solution to Exercise 10.7.29 (p. 611) 9

Solution to Exercise 10.7.31 (p. 611)

$-5$

Solution to Exercise 10.7.33 (p. 611)

11

Solution to Exercise 10.7.35 (p. 611) 28

Solution to Exercise 10.7.37 (p. 611) $-4$

Solution to Exercise 10.7.39 (p. 611) $-12$

Solution to Exercise 10.7.41 (p. 611)

$-4$

Solution to Exercise 10.7.43 (p. 611)

$-6$

Solution to Exercise 10.7.45 (p. 611) 15

Solution to Exercise 10.7.47 (p. 612) 49

Solution to Exercise 10.7.49 (p. 612) $-140$

Solution to Exercise 10.7.51 (p. 612) $-7$

Solution to Exercise 10.7.53 (p. 612)

$-3$

Solution to Exercise 10.7.55 (p. 612)

$-5$

Solution to Exercise 10.7.57 (p. 612)

13

Solution to Exercise 10.7.59 (p. 612) $\frac{4}{3}=1 \frac{1}{3}$

Solution to Exercise 10.7.61 (p. 612)

$52 \frac{1}{2}$

Solution to Exercise 10.9.1 (p. 614)

$N, W, Z$ 
Solution to Exercise 10.9.3 (p. 614) $W, Z$

Solution to Exercise 10.9.5 (p. 614) $\{-3,-2,-1,0,1,2\}$

Solution to Exercise 10.9.7 (p. 614)

$<$

Solution to Exercise 10.9.9 (p. 614)

$<$

Solution to Exercise 10.9.11 (p. 615) $\{-5,-4,-3,-2,-1\}$

Solution to Exercise 10.9.13 (p. 615) $\{1,2,3,4\}$

Solution to Exercise 10.9.15 (p. 615) none

Solution to Exercise 10.9.17 (p. 615) 4

Solution to Exercise 10.9.19 (p. 615)

6

Solution to Exercise 10.9.21 (p. 615)

+ (plus)

Solution to Exercise 10.9.23 (p. 615) negative eight

Solution to Exercise 10.9.25 (p. 615)

negative negative one or opposite negative one

Solution to Exercise 10.9.27 (p. 615)

one plus negative seven

Solution to Exercise 10.9.29 (p. 616)

negative one minus four

Solution to Exercise 10.9.31 (p. 616)

zero minus negative eleven

Solution to Exercise 10.9.33 (p. 616)

15

Solution to Exercise 10.9.35 (p. 616)

19 or $1+18$

Solution to Exercise 10.9.37 (p. 616) 9

Solution to Exercise 10.9.39 (p. 616) 5

Solution to Exercise 10.9.41 (p. 616)

$-2$

Solution to Exercise 10.9.43 (p. 616)

12

Solution to Exercise 10.9.45 (p. 616)

16

Solution to Exercise 10.9.47 (p. 616) 16

Solution to Exercise 10.9.49 (p. 617) 8

Solution to Exercise 10.9.51 (p. 617)

7 
Solution to Exercise 10.9.53 (p. 617)

$-2$

Solution to Exercise 10.9.55 (p. 617)

$-7$

Solution to Exercise 10.9.57 (p. 617)

$-14$

Solution to Exercise 10.9.59 (p. 617) 48

Solution to Exercise 10.9.61 (p. 617) $-42$

Solution to Exercise 10.9.63 (p. 617) 3

Solution to Exercise 10.9.65 (p. 617) 1

Solution to Exercise 10.9.67 (p. 617) not defined

Solution to Exercise 10.9.69 (p. 618)

$-4$

Solution to Exercise 10.9.71 (p. 618)

$-22$

Solution to Exercise 10.10.1 (p. 618) $\{-7,-6,-5,-4\}$

Solution to Exercise 10.10.2 (p. 618) $\{-2,-1,0,1\}$

Solution to Exercise 10.10.3 (p. 618)

$=$

Solution to Exercise 10.10.4 (p. 618)

$<$

Solution to Exercise 10.10.5 (p. 618)

$>$

Solution to Exercise 10.10.6 (p. 618) $\{-3,-2,-1\}$

Solution to Exercise 10.10.7 (p. 618) $\{1,2\}$

Solution to Exercise 10.10.8 (p. 618) 5

Solution to Exercise 10.10.9 (p. 618)

16

Solution to Exercise 10.10.10 (p. 618)

$-2$

Solution to Exercise 10.10.11 (p. 618)

16

Solution to Exercise 10.10.12 (p. 618)

15

Solution to Exercise 10.10.13 (p. 618)

$-2$

Solution to Exercise 10.10.14 (p. 618)

$-11$

Solution to Exercise 10.10.15 (p. 619)

$-16$ 
Solution to Exercise 10.10.16 (p. 619) 42

Solution to Exercise 10.10.17 (p. 619)

121

Solution to Exercise 10.10.18 (p. 619)

$\frac{51}{7}$ or $7 \frac{2}{7}$

Solution to Exercise 10.10.19 (p. 619) 6

Solution to Exercise 10.10.20 (p. 619) 0 


\section{Chapter 11}

\section{Algebraic Expressions and Equations}

\subsection{Objectives ${ }^{1}$}

After completing this chapter, you should

\section{Algebraic Expressions (Section 11.2)}

- be able to recognize an algebraic expression

- be able to distinguish between terms and factors

- understand the meaning and function of coefficients

- be able to perform numerical evaluation

Combining Like Terms Using Addition and Subtraction (Section 11.3)

- be able to combine like terms in an algebraic expression

Solving Equations of the Form $x+a=b$ and $x-a=b$ (Section 11.4)

- understand the meaning and function of an equation

- understand what is meant by the solution to an equation

- be able to solve equations of the form $x+a=b$ and $x-a=b$

Solving Equations of the Form $a x=b$ and $\frac{x}{a}=b$ (Section 11.5)

- be familiar with the multiplication/division property of equality

- be able to solve equations of the form $\mathrm{ax}=b$ and $\frac{x}{a}=b$

- be able to use combined techniques to solve equations

\section{Applications I: Translating Words to Mathematical Symbols (Section 11.6)}

- be able to translate phrases and statements to mathematical expressions and equations

\section{Applications II: Solving Problems (Section 11.7)}

- be more familiar with the five-step method for solving applied problems

- be able to use the five-step method to solve number problems and geometry problems

\footnotetext{
${ }^{1}$ This content is available online at $<$ http://cnx.org/content $/ \mathrm{m} 18899 / 1.3 />$.
} 


\subsection{Algebraic Expressions ${ }^{2}$}

\subsubsection{Section Overview}

- Algebraic Expressions

- Terms and Factors

- Coefficients

- Numerical Evaluation

\subsubsection{Algebraic Expressions}

Numerical Expression

In arithmetic, a numerical expression results when numbers are connected by arithmetic operation signs $(+,-, \cdot, \div)$. For example, $8+5,4-9,3 \cdot 8$, and $9 \div 7$ are numerical expressions.

\section{Algebraic Expression}

In algebra, letters are used to represent numbers, and an algebraic expression results when an arithmetic operation sign associates a letter with a number or a letter with a letter. For example, $x+8,4-y, 3 \cdot x$, $x \div 7$, and $x \cdot y$ are algebraic expressions.

\section{Expressions}

Numerical expressions and algebraic expressions are often referred to simply as expressions.

\subsubsection{Terms and Factors}

In algebra, it is extremely important to be able to distinguish between terms and factors.

\section{Distinction Between Terms and Factors}

Terms are parts of sums and are therefore connected by + signs.

Factors are parts of products and are therefore separated by $\cdot$ signs.

NOTE: While making the distinction between sums and products, we must remember that subtraction and division are functions of these operations.

1. In some expressions it will appear that terms are separated by minus signs. We must keep in mind that subtraction is addition of the opposite, that is, $x-y=x+(-y)$

2. In some expressions it will appear that factors are separated by division signs. We must keep in mind that

$\frac{x}{y}=\frac{x}{1} \cdot \frac{1}{y}=x \cdot \frac{1}{y}$

\subsubsection{Sample Set A}

State the number of terms in each expression and name them.

\section{Example 11.1}

$x+4$. In this expression, $\mathrm{x}$ and 4 are connected by a "+" sign. Therefore, they are terms. This expression consists of two terms.

\section{Example 11.2}

$y-8$. The expression $y-8$ can be expressed as $y+(-8)$. We can now see that this expression consists of the two terms $y$ and -8 .

\footnotetext{
${ }^{2}$ This content is available online at $<\mathrm{http}: / / \mathrm{cnx} . o r g /$ content $/ \mathrm{m} 35038 / 1.2 />$. 
Rather than rewriting the expression when a subtraction occurs, we can identify terms more quickly by associating the + or - sign with the individual quantity.

\section{Example 11.3}

$a+7-b-m$. Associating the sign with the individual quantities, we see that this expression consists of the four terms $a, 7,-b,-m$.

\section{Example 11.4}

$5 m-8 n$. This expression consists of the two terms, $5 m$ and $-8 n$. Notice that the term $5 m$ is composed of the two factors 5 and $m$. The term $-8 n$ is composed of the two factors -8 and $n$.

\section{Example 11.5}

$3 x$. This expression consists of one term. Notice that $3 x$ can be expressed as $3 x+0$ or $3 x$. 1 (indicating the connecting signs of arithmetic). Note that no operation sign is necessary for multiplication.

\subsubsection{Practice Set A}

Specify the terms in each expression.

Exercise 11.2.1

(Solution on p. 682.)

$x+7$

Exercise 11.2.2

(Solution on p. 682.)

$3 m-6 n$

Exercise 11.2.3

(Solution on p. 682.)

$5 y$

Exercise 11.2.4

(Solution on p. 682.)

$a+2 b-c$

Exercise 11.2.5

$-3 x-5$

(Solution on p. 682.)

\subsubsection{Coefficients}

We know that multiplication is a description of repeated addition. For example, $5 \cdot 7$ describes $7+7+7+7+7$

Suppose some quantity is represented by the letter $x$. The multiplication $5 x$ describes $x+x+x+x+x$. It is now easy to see that $5 x$ specifies 5 of the quantities represented by $x$. In the expression $5 x, 5$ is called the numerical coefficient, or more simply, the coefficient of $x$.

\section{Coefficient}

The coefficient of a quantity records how many of that quantity there are.

Since constants alone do not record the number of some quantity, they are not usually considered as numerical coefficients. For example, in the expression $7 x+2 y-8 z+12$, the coefficient of

$7 x$ is 7 . (There are $7 \mathrm{x}$ 's.)

$2 y$ is 2 . (There are $2 y^{\prime}$ 's.)

$-8 z$ is -8 . (There are $-8 z$ 's.)

The constant 12 is not considered a numerical coefficient. 
$1 x=x$

When the numerical coefficient of a variable is 1 , we write only the variable and not the coefficient. For example, we write $x$ rather than $1 x$. It is clear just by looking at $x$ that there is only one.

\subsubsection{Numerical Evaluation}

We know that a variable represents an unknown quantity. Therefore, any expression that contains a variable represents an unknown quantity. For example, if the value of $x$ is unknown, then the value of $3 x+5$ is unknown. The value of $3 x+5$ depends on the value of $x$.

Numerical Evaluation

Numerical evaluation is the process of determining the numerical value of an algebraic expression by replacing the variables in the expression with specified numbers.

\subsubsection{Sample Set B}

Find the value of each expression.

\section{Example 11.6}

$2 x+7 y$, if $x=-4$ and $y=2$

Replace $\mathrm{x}$ with -4 and $\mathrm{y}$ with 2 .

$$
\begin{array}{rlc}
2 x+7 y & = & 2(-4)+7(2) \\
& = & -8+14 \\
& = & 6
\end{array}
$$

Thus, when $x=--4$ and $y=2,2 x+7 y=6$.

\section{Example 11.7}

$\frac{5 a}{b}+\frac{8 b}{12}$, if $a=6$ and $b=-3$.

Replace a with 6 and $b$ with -3 .

$$
\begin{aligned}
& \frac{5 a}{b}+\frac{8 b}{12}=\frac{5(6)}{-3}+\frac{8(-3)}{12} \\
& =\quad \frac{30}{-}+\frac{-}{24} \\
& =-10+(-2) \\
& =-12
\end{aligned}
$$

Thus, when $a=6$ and $b=-3, \frac{5 a}{b}+\frac{8 b}{12}=-12$.

Example 11.8

$6(2 a-15 b)$, if $a=-5$ and $b=-1$

Replace $a$ with -5 and $b$ with -1 .

$$
\begin{array}{rlc}
6(2 a-15 b) & = & 6(2(-5)-15(-1)) \\
& = & 6(-10+15) \\
= & 6(5) \\
= & 30
\end{array}
$$

Thus, when $a=--5$ and $b=--1,6(2 a-15 b)=30$. 


\section{Example 11.9}

$3 x^{2}-2 x+1$, if $x=4$

Replace $\mathrm{x}$ with 4 .

$$
\begin{array}{rlc}
3 x^{2}-2 x+1 & = & 3(4)^{2}-2(4)+1 \\
& = & 3 \cdot 16-2(4)+1 \\
& = & 48-8+1 \\
& = & 41
\end{array}
$$

Thus, when $x=4,3 x^{2}-2 x+1=41$.

\section{Example 11.10}

$-x^{2}-4$, if $x=3$

Replace $\mathrm{x}$ with 3 .

$$
\begin{aligned}
-x^{2}-4 & =-{ }^{3}-4 \quad \text { Be careful to square only the } 3 . \text { The exponent } 2 \text { is connected only to } 3, \text { not }-3 \\
& =-9-4 \\
& =-13
\end{aligned}
$$

\section{Example 11.11}

$$
(-x)^{2}-4 \text {, if } x=3 \text {. }
$$

Replace $x$ with 3 .

$$
\begin{aligned}
(-x)^{2}-4 & =(-3)^{2}-4 \text { The exponent is connected to }-3, \text { not } 3 \text { as in problem } 5 \text { above. } \\
& =9-4 \\
& =\quad-5
\end{aligned}
$$

The exponent is connected to -3 , not 3 as in the problem above.

\subsubsection{Practice Set B}

Find the value of each expression.

Exercise 11.2.6

(Solution on p. 682.)

$9 m-2 n$, if $m=-2$ and $n=5$

Exercise 11.2.7

(Solution on p. 682.)

$-3 x-5 y+2 z$, if $x=-4, y=3, z=0$

Exercise 11.2.8

$\frac{10 a}{3 b}+\frac{4 b}{2}$, if $a=-6$, and $b=2$

(Solution on p. 682.)

Exercise 11.2.9

(Solution on p. 682.)

$8(3 m-5 n)$, if $m=-4$ and $n=-5$

Exercise 11.2.10

(Solution on p. 682.)

$3[-40-2(4 a-3 b)]$, if $a=-6$ and $b=0$

(Solution on p. 682.)

Exercise 11.2.11

$5 y^{2}+6 y-11$, if $y=-1$ 
Exercise 11.2.12

(Solution on p. 682.)

$-x^{2}+2 x+7$, if $x=4$

Exercise 11.2.13

(Solution on p. 682.)

$(-x)^{2}+2 x+7$, if $x=4$

\subsubsection{Exercises}

Exercise 11.2.14

In an algebraic expression, terms are separated by by signs.

For the following 8 problems, specify each term.

Exercise 11.2.15

$3 m+7 n$

Exercise 11.2.16

$5 x+18 y$

Exercise 11.2.17

$4 a-6 b+c$

Exercise 11.2.18

$8 s+2 r-7 t$

Exercise 11.2.19

$m-3 n-4 a+7 b$

Exercise 11.2.20

$7 a-2 b-3 c-4 d$

Exercise 11.2.21

$-6 a-5 b$

Exercise 11.2.22

$-x-y$

Exercise 11.2.23

What is the function of a numerical coefficient?

Exercise 11.2.24

Write $1 m$ in a simpler way.

Exercise 11.2.25

Write $1 \mathrm{~s}$ in a simpler way.

Exercise 11.2.26

In the expression 5a, how many a's are indicated?

Exercise 11.2.27

In the expression $-7 c$, how many $c$ 's are indicated?

Find the value of each expression.

Exercise 11.2.28

$2 m-6 n$, if $m=-3$ and $n=4$

Exercise 11.2.29

$5 a+6 b$, if $a=-6$ and $b=5$

Exercise 11.2.30

$2 x-3 y+4 z$, if $x=1, y=-1$, and $z=-2$
(Solution on p. 682.)

(Solution on p. 682.)

(Solution on p. 682.)

(Solution on p. 682.) signs and factors are separated

(Solution on p. 682.)

(Solution on p. 682.)

(Solution on p. 682.)

(Solution on p. 682.) 
Exercise 11.2.31

$9 a+6 b-8 x+4 y$, if $a=-2, b=-1, x=-2$, and $y=0$

Exercise 11.2.32

$\frac{8 x}{3 y}+\frac{18 y}{2 x}$, if $x=9$ and $y=-2$

(Solution on p. 682.)

Exercise 11.2.33

$\frac{-3 m}{2 n}-\frac{-6 n}{m}$, if $m=-6$ and $n=3$

Exercise 11.2.34

$4(3 r+2 s)$, if $r=4$ and $s=1$

(Solution on p. 682.)

Exercise 11.2.35

$3(9 a-6 b)$, if $a=-1$ and $b=-2$

Exercise 11.2.36

(Solution on p. 682.)

$-8(5 m+8 n)$, if $m=0$ and $n=-1$

Exercise 11.2.37

$-2(-6 x+y-2 z)$, if $x=1, y=1$, and $z=2$

Exercise 11.2.38

$-(10 x-2 y+5 z)$ if $x=2, y=8$, and $z=-1$

(Solution on p. 682.)

Exercise 11.2.39

$-(a-3 b+2 c-d)$, if $a=-5, b=2, c=0$, and $d=-1$

Exercise 11.2.40

$3[16-3(a+3 b)]$, if $a=3$ and $b=-2$

(Solution on p. 683.)

Exercise 11.2.41

$-2[5 a+2 b(b-6)]$, if $a=-2$ and $b=3$

Exercise 11.2.42

$-\{6 x+3 y[-2(x+4 y)]\}$, if $x=0$ and $y=1$

(Solution on p. 683.)

Exercise 11.2.43

$-2\{19-6[4-2(a-b-7)]\}$, if $a=10$ and $b=3$

Exercise 11.2.44

(Solution on p. 683.)

$x^{2}+3 x-1$, if $x=5$

Exercise 11.2.45

$m^{2}-2 m+6$, if $m=3$

Exercise 11.2.46

(Solution on p. 683.)

$6 a^{2}+2 a-15$, if $a=-2$

Exercise 11.2.47

$5 s^{2}+6 s+10$, if $x=-1$

Exercise 11.2.48

(Solution on p. 683.)

$16 x^{2}+8 x-7$, if $x=0$

Exercise 11.2.49

$-8 y^{2}+6 y+11$, if $y=0$

Exercise 11.2.50

$(y-6)^{2}+3(y-5)+4$, if $y=5$

(Solution on p. 683.)

Exercise 11.2.51

$(x+8)^{2}+4(x+9)+1$, if $x=-6$ 


\subsubsection{Exercises for Review}

Exercise 11.2.52

(Solution on p. 683.)

(Section 5.3) Perform the addition: $5 \frac{3}{8}+2 \frac{1}{6}$.

Exercise 11.2.53

(Section 5.5) Arrange the numbers in order from smallest to largest: $\frac{11}{32}, \frac{15}{48}$, and $\frac{7}{16}$

Exercise 11.2.54

(Solution on p. 683.)

(Section 5.7) Find the value of $\left(\frac{2}{3}\right)^{2}+\frac{8}{27}$

Exercise 11.2.55

(Section 7.3) Write the proportion in fractional form: "9 is to 8 as $x$ is to 7."

Exercise 11.2.56

(Section 10.7) Find the value of $-3(2-6)-12$

(Solution on p. 683.)

\subsection{Combining Like Terms Using Addition and Subtraction ${ }^{3}$}

\subsubsection{Section Overview}

- Combining Like Terms

\subsubsection{Combining Like Terms}

From our examination of terms in Section 11.2, we know that like terms are terms in which the variable parts are identical. Like terms is an appropriate name since terms with identical variable parts and different numerical coefficients represent different amounts of the same quantity. When we are dealing with quantities of the same type, we may combine them using addition and subtraction.

\section{Simplifying an Algebraic Expression}

An algebraic expression may be simplified by combining like terms.

This concept is illustrated in the following examples.

1. 8 records +5 records $=13$ records.

Eight and 5 of the same type give 13 of that type. We have combined quantities of the same type.

2. 8 records +5 records +3 tapes $=13$ records +3 tapes. Eight and 5 of the same type give 13 of that type. Thus, we have 13 of one type and 3 of another type. We have combined only quantities of the same type.

3. Suppose we let the letter $x$ represent "record." Then, $8 x+5 x=13 x$. The terms $8 x$ and $5 x$ are like terms. So, 8 and 5 of the same type give 13 of that type. We have combined like terms.

4. Suppose we let the letter $x$ represent "record" and $y$ represent "tape." Then, $8 x+5 x+3 y=13 x+5 y$

We have combined only the like terms.

After observing the problems in these examples, we can suggest a method for simplifying an algebraic expression by combining like terms.

\section{Combining Like Terms}

Like terms may be combined by adding or subtracting their coefficients and affixing the result to the common variable.

\footnotetext{
${ }^{3}$ This content is available online at $<$ http://cnx.org/content/m35039/1.2/ $>$.
} 


\subsubsection{Sample Set A}

Simplify each expression by combining like terms.

Example 11.12

$2 m+6 m-4 m$. All three terms are alike. Combine their coefficients and affix this result to $m$ : $2+6-4=4$.

Thus, $2 m+6 m-4 m=4 m$.

\section{Example 11.13}

$5 x+2 y-9 y$. The terms $2 y$ and $-9 y$ are like terms. Combine their coefficients: $2-9=-7$.

Thus, $5 x+2 y-9 y=5 x-7 y$.

\section{Example 11.14}

$-3 a+2 b-5 a+a+6 b$. The like terms are

$\underbrace{-3 a, \quad-5 a, \quad a}_{\substack{-3-5+1=-7 \\-7 a}} \underbrace{2 b, \quad 6 b}_{\substack{2+6=8 \\ 8 b}}$

Thus, $-3 a+2 b-5 a+a+6 b=-7 a+8 b$.

\section{Example 11.15}

$$
\begin{aligned}
& r-2 s+7 s+3 r-4 r-5 s \text {. The like terms are } \\
& \underbrace{r, 3 r,-4 r}_{1+3-4=0} \quad \underbrace{-2 s, 7 s,-5 s}_{-2+7-5=0} \\
& 0 r \quad 0 s \text {, }
\end{aligned}
$$

Thus, $r-2 s+7 s+3 r-4 r-5 s=0$.

\subsubsection{Practice Set A}

Simplify each expression by combining like terms.

Exercise 11.3.1

(Solution on p. 683.)

$4 x+3 x+6 x$

Exercise 11.3.2

$5 a+8 b+6 a-2 b$

(Solution on p. 683.)

Exercise 11.3.3

(Solution on p. 683.)

$10 m-6 n-2 n-m+n$

Exercise 11.3.4

(Solution on p. 683.)

$16 a+6 m+2 r-3 r-18 a+m-7 m$

Exercise 11.3.5

$5 h-8 k+2 h-7 h+3 k+5 k$

(Solution on p. 683.) 


\subsubsection{Exercises}

Simplify each expression by combining like terms.

Exercise 11.3.6

(Solution on p. 683.)

$4 a+7 a$

Exercise 11.3.7

$3 m+5 m$

Exercise 11.3.8

$6 h-2 h$

(Solution on p. 683.)

Exercise 11.3.9

$11 k-8 k$

Exercise 11.3.10

(Solution on p. 683.)

$5 m+3 n-2 m$

Exercise 11.3.11

$7 x-6 x+3 y$

Exercise 11.3.12

$14 s+3 s-8 r+7 r$

(Solution on p. 683.)

Exercise 11.3.13

$-5 m-3 n+2 m+6 n$

Exercise 11.3.14

$7 h+3 a-10 k+6 a-2 h-5 k-3 k$

(Solution on p. 683.)

Exercise 11.3.15

$4 x-8 y-3 z+x-y-z-3 y-2 z$

Exercise 11.3.16

$11 w+3 x-6 w-5 w+8 x-11 x$

(Solution on p. 683.)

Exercise 11.3.17

$15 r-6 s+2 r+8 s-6 r-7 s-s-2 r$

Exercise 11.3.18

$|-7| m+|6| m+|-3| m$

(Solution on p. 683.)

Exercise 11.3.19

$|-2| x+|-8| x+|10| x$

Exercise 11.3.20

$(-4+1) k+(6-3) k+(12-4) h+(5+2) k$

(Solution on p. 683.)

Exercise 11.3.21

$(-5+3) a-(2+5) b-(3+8) b$

Exercise 11.3.22

$5 \star+2 \Delta+3 \Delta-8 \star$

(Solution on p. 683.)

Exercise 11.3.23

$9 \otimes+10 \boxplus-11 \otimes-12 \boxplus$

Exercise 11.3.24

$16 x-12 y+5 x+7-5 x-16-3 y$

(Solution on p. 683.)

Exercise 11.3.25

$$
-3 y+4 z-11-3 z-2 y+5-4(8-3)
$$




\subsubsection{Exercises for Review}

Exercise 11.3.26

(Section 4.3) Convert $\frac{24}{11}$ to a mixed number

(Solution on p. 683.)

Exercise 11.3.27

(Section 4.4) Determine the missing numerator: $\frac{3}{8}=\frac{?}{64}$.

Exercise 11.3.28

(Section 5.6) Simplify $\frac{\frac{5}{6}-\frac{1}{4}}{\frac{1}{12}}$.

(Solution on p. 684.)

Exercise 11.3.29

(Section 7.5) Convert $\frac{5}{16}$ to a percent.

Exercise 11.3.30

(Section 11.2) In the expression $6 k$, how many $k$ 's are there?

(Solution on p. 684.)

\subsection{Solving Equations of the Form $\mathrm{x}+\mathrm{a}=\mathrm{b}$ and $\mathrm{x}-\mathrm{a}=\mathrm{b}^{4}$}

\subsubsection{Section Overview}

- Equations

- Solutions and Equivalent Equations

- Solving Equations

\subsubsection{Equations}

\section{Equation}

An equation is a statement that two algebraic expressions are equal.

The following are examples of equations:

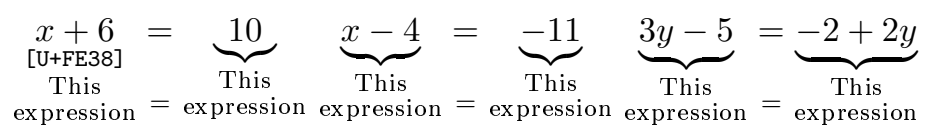

Notice that $x+6, x-4$, and $3 y-5$ are not equations. They are expressions. They are not equations because there is no statement that each of these expressions is equal to another expression.

\subsubsection{Solutions and Equivalent Equations}

\section{Conditional Equations}

The truth of some equations is conditional upon the value chosen for the variable. Such equations are called conditional equations. There are two additional types of equations. They are examined in courses in algebra, so we will not consider them now.

\section{Solutions and Solving an Equation}

The set of values that, when substituted for the variables, make the equation true, are called the solutions of the equation.

An equation has been solved when all its solutions have been found.

\footnotetext{
${ }^{4}$ This content is available online at $<\mathrm{http}: / / \operatorname{cnx} . o r g /$ content $/ \mathrm{m} 35044 / 1.3 />$.
} 


\subsubsection{Sample Set A}

\section{Example 11.16}

Verify that 3 is a solution to $x+7=10$.

When $x=3$,

$$
\begin{aligned}
& x+7=10 \\
& \text { becomes } 3+7=10 \\
& 10=10 \text { which is a true statement, verifying that } \\
& 3 \text { is a solution to } x+7=10
\end{aligned}
$$

\section{Example 11.17}

Verify that -6 is a solution to $5 y+8=-22$

When $y=-6$,

$$
\begin{aligned}
& 5 y+8=-22 \\
& \text { becomes } 5(-6)+8=-22 \\
& -30+8=-22 \\
& -22=-22
\end{aligned}
$$

\section{Example 11.18}

Verify that 5 is not a solution to $a-1=2 a+3$.

When $a=5$,

$$
\begin{aligned}
& a-1=2 a+3 \\
& \text { becomes } 5-1=2 \cdot 5+3 \\
& 5-1=10+3 \\
& 4=13 \quad \text { a false statement, verifying that } 5 \\
& \text { is not a solution to } a-1=2 a+3
\end{aligned}
$$

\section{Example 11.19}

Verify that -2 is a solution to $3 m-2=-4 m-16$.

When $m=-2$,

$$
\begin{aligned}
& 3 m-2=-4 m-16 \\
& \text { becomes } 3(-2)-2=-4(-2)-16 \\
& -6-2=8-16 \\
& \begin{array}{l}
-8=-8 \text { which is a true statement, verifying that } \\
-2 \text { is a solution to } 3 m-2=-4 m-16
\end{array}
\end{aligned}
$$




\subsubsection{Practice Set A}

Exercise 11.4.1

(Solution on p. 684.)

Verify that 5 is a solution to $m+6=11$.

Exercise 11.4.2

(Solution on p. 684.)

Verify that -5 is a solution to $2 m-4=-14$.

Exercise 11.4.3

(Solution on p. 684.)

Verify that 0 is a solution to $5 x+1=1$.

Exercise 11.4.4

(Solution on p. 684.)

Verify that 3 is not a solution to $-3 y+1=4 y+5$.

Exercise 11.4.5

Verify that -1 is a solution to $6 m-5+2 m=7 m-6$.

(Solution on p. 684.)

\section{Equivalent Equations}

Some equations have precisely the same collection of solutions. Such equations are called equivalent equations. For example, $x-5=-1, x+7=11$, and $x=4$ are all equivalent equations since the only solution to each is $x=4$. (Can you verify this?)

\subsubsection{Solving Equations}

We know that the equal sign of an equation indicates that the number represented by the expression on the left side is the same as the number represented by the expression on the right side.

\begin{tabular}{|l|l|l|}
\hline This number & is the same as & this number \\
\hline$\downarrow$ & $\downarrow$ & $\downarrow$ \\
\hline$x$ & $=$ & 4 \\
\hline$x+7$ & $=$ & 11 \\
\hline$x-5$ & $=$ & -1 \\
\hline
\end{tabular}

Table 11.1

\section{Addition/Subtraction Property of Equality}

From this, we can suggest the addition/subtraction property of equality.

Given any equation,

1. We can obtain an equivalent equation by adding the same number to both sides of the equation.

2. We can obtain an equivalent equation by subtracting the same number from both sides of the equation.

\section{The Idea Behind Equation Solving}

The idea behind equation solving is to isolate the variable on one side of the equation. Signs of operation $(+,-, \cdot, \div)$ are used to associate two numbers. For example, in the expression $5+3$, the numbers 5 and 3 are associated by addition. An association can be undone by performing the opposite operation. The addition/subtraction property of equality can be used to undo an association that is made by addition or subtraction.

Subtraction is used to undo an addition.

Addition is used to undo a subtraction.

The procedure is illustrated in the problems of . 


\subsubsection{Sample Set B}

Use the addition/subtraction property of equality to solve each equation.

\section{Example 11.20}

$x+4=6$.

4 is associated with $x$ by addition. Undo the association by subtracting 4 from both sides.

$$
\begin{gathered}
x+4-4=6-4 \\
x+0=2 \\
x=2
\end{gathered}
$$

Check: When $x=2, x+4$ becomes

$2+4 \stackrel{?}{=} 6$

$6 \underline{\underline{ }} 6$.

The solution to $x+4=6$ is $x=2$.

\section{Example 11.21}

$m-8=5.8$ is associated with $m$ by subtraction. Undo the association by adding 8 to both sides.

$$
\begin{gathered}
m-8+8=5+8 \\
m+0=13 \\
m=13
\end{gathered}
$$

Check: When $m=13$,

$$
\begin{aligned}
& \text { becomes } \\
& m-8=5 \\
& 13-8 \stackrel{?}{=} 5 \\
& 5 \underline{\underline{ }} 5
\end{aligned}
$$

a true statement.

The solution to $m-8=5$ is $m=13$.

\section{Example 11.22}

$-3-5=y-2+8$. Before we use the addition/subtraction property, we should simplify as much as possible.

$-3-5=y-2+8$

$-8=y+6$

6 is associated with $y$ by addition. Undo the association by subtracting 6 from both sides.

$$
\begin{gathered}
-8-6=y+6-6 \\
-14=y+0 \\
-14=y
\end{gathered}
$$

This is equivalent to $y=-14$.

Check: When $y=-14$,

$-3-5=y-2+8$ 
becomes

$$
\begin{gathered}
-3-5 \stackrel{ \pm}{=}-14-2+8 \\
-8 \stackrel{ \pm}{=}-16+8 \\
-8 \stackrel{ \pm}{=}-8 .
\end{gathered}
$$

a true statement.

The solution to $-3-5=y-2+8$ is $y=-14$.

\section{Example 11.23}

$-5 a+1+6 a=-2$. Begin by simplifying the left side of the equation.

$\underbrace{-5 a+1+6 a}_{-5+6=1}=-2$

$a+1=-21$ is associated with $a$ by addition. Undo the association by subtracting 1 from both sides.

$$
\begin{gathered}
a+1-1=-2-1 \\
a+0=-3 \\
a=-3
\end{gathered}
$$

Check: When $a=-3$,

$-5 a+1+6 a=-2$

becomes

$-5(-3)+1+6(-3) \geq-2$

$$
\begin{array}{r}
15+1-18 \geq-2 \\
-2 \leq-2,
\end{array}
$$

a true statement.

The solution to $-5 a+1+6 a=-2$ is $a=-3$.

\section{Example 11.24}

$7 k-4=6 k+1$. In this equation, the variable appears on both sides. We need to isolate it on one side. Although we can choose either side, it will be more convenient to choose the side with the larger coefficient. Since 8 is greater than 6 , we'll isolate $k$ on the left side.

$7 k-4=6 k+1$ Since $6 k$ represents $+6 k$, subtract $6 k$ from each side.

$\underbrace{7 k-4-6 k}_{7-6=1}=\underbrace{6 k+1-6 k}_{6-6=0}$

$k-4=14$ is associated with $k$ by subtraction. Undo the association by adding 4 to both sides.

$$
\begin{gathered}
k-4+4=1+4 \\
k=5
\end{gathered}
$$

Check: When $k=5$,

$$
\begin{aligned}
& 7 k-4=6 k+1 \\
& \text { becomes } \\
& 7 \cdot 5-4 \stackrel{2}{=} 6 \cdot 5+1 \\
& 35-4 \stackrel{?}{=} 30+1 \\
& 31 \simeq 31 \text {. }
\end{aligned}
$$

a true statement. 
The solution to $7 k-4=6 k+1$ is $k=5$.

Example 11.25

$-8+x=5 .-8$ is associated with $x$ by addition. Undo the by subtracting -8 from both sides. Subtracting -8 we get $-(-8)=+8$. We actually add 8 to both sides.

$-8+x+8=5+8$

$x=13$

Check: When $x=13$

$-8+x=5$

becomes

$$
\begin{aligned}
-8+13 & \stackrel{?}{=} 5 \\
5 & \cong 5
\end{aligned}
$$

a true statement.

The solution to $-8+x=5$ is $x=13$.

\subsubsection{Practice Set B}

Exercise 11.4.6

(Solution on p. 684.)

$y+9=4$

Exercise 11.4.7

(Solution on p. 684.)

$a-4=11$

Exercise 11.4.8

$-1+7=x+3$

(Solution on p. 684.)

Exercise 11.4.9

(Solution on p. 684.)

$8 m+4-7 m=(-2)(-3)$

Exercise 11.4.10

(Solution on p. 684.)

$12 k-4=9 k-6+2 k$

(Solution on p. 684.)

Exercise 11.4.11

$-3+a=-4$

\subsubsection{Exercises}

For the following 10 problems, verify that each given value is a solution to the given equation.

Exercise 11.4.12

(Solution on p. 684.)

$x-11=5, x=16$

Exercise 11.4.13

$y-4=-6, y=-2$

Exercise 11.4.14

(Solution on p. 684.)

$2 m-1=1, m=1$

Exercise 11.4.15

$5 y+6=-14, y=-4$ 
Exercise 11.4.16

(Solution on p. 685.)

$3 x+2-7 x=-5 x-6, x=-8$

Exercise 11.4.17

$-6 a+3+3 a=4 a+7-3 a, a=-1$

Exercise 11.4.18

(Solution on p. 685.)

$-8+x=-8, x=0$

Exercise 11.4.19

$8 b+6=6-5 b, b=0$

Exercise 11.4.20

$4 x-5=6 x-20, x=\frac{15}{2}$

(Solution on p. 685.)

Exercise 11.4.21

$-3 y+7=2 y-15, y=\frac{22}{5}$

Solve each equation. Be sure to check each result.

Exercise 11.4.22

(Solution on p. 685.)

$y-6=5$

Exercise 11.4.23

$m+8=4$

Exercise 11.4.24

$k-1=4$

(Solution on p. 685.)

Exercise 11.4.25

$h-9=1$

Exercise 11.4.26

(Solution on p. 685.)

$a+5=-4$

Exercise 11.4.27

$b-7=-1$

Exercise 11.4.28

$x+4-9=6$

(Solution on p. 685.)

Exercise 11.4.29

$y-8+10=2$

Exercise 11.4.30

$z+6=6$

(Solution on p. 685.)

Exercise 11.4.31

$w-4=-4$

Exercise 11.4.32

$x+7-9=6$

(Solution on p. 685.)

Exercise 11.4.33

$y-2+5=4$

Exercise 11.4.34

$m+3-8=-6+2$

(Solution on p. 685.)

Exercise 11.4.35

$z+10-8=-8+10$

Exercise 11.4.36

(Solution on p. 685.)

$2+9=k-8$

Exercise 11.4.37

$-5+3=h-4$ 
Exercise 11.4.38

(Solution on p. 685.)

$3 m-4=2 m+6$

Exercise 11.4.39

$5 a+6=4 a-8$

Exercise 11.4.40

(Solution on p. 685.)

$8 b+6+2 b=3 b-7+6 b-8$

Exercise 11.4.41

$12 h-1-3-5 h=2 h+5 h+3(-4)$

Exercise 11.4.42

$-4 a+5-2 a=-3 a-11-2 a$

(Solution on p. 685.)

Exercise 11.4.43

$-9 n-2-6+5 n=3 n-(2)(-5)-6 n$

Calculator Exercises

Exercise 11.4.44

$y-2.161=5.063$

(Solution on p. 685.)

Exercise 11.4.45

$a-44.0014=-21.1625$

Exercise 11.4.46

$-0.362-0.416=5.63 m-4.63 m$

(Solution on p. 685.)

Exercise 11.4.47

$8.078-9.112=2.106 y-1.106 y$

Exercise 11.4.48

$4.23 k+3.18=3.23 k-5.83$

(Solution on p. 685.)

Exercise 11.4.49

$6.1185 x-4.0031=5.1185 x-0.0058$

Exercise 11.4.50

$21.63 y+12.40-5.09 y=6.11 y-15.66+9.43 y$

(Solution on p. 685.)

Exercise 11.4.51

$0.029 a-0.013-0.034-0.057=-0.038+0.56+1.01 a$

\subsubsection{Exercises for Review}

Exercise 11.4.52

(Section 7.2 ) Is $\frac{7 \text { calculators }}{12 \text { students }}$ an example of a ratio or a rate?

(Solution on p. 685.)

Exercise 11.4.53

(Section 7.5) Convert $\frac{3}{8} \%$ to a decimal.

Exercise 11.4.54

(Section 7.6 ) $0.4 \%$ of what number is 0.014 ?

(Solution on p. 685.)

Exercise 11.4.55

(Section 8.3) Use the clustering method to estimate the sum: $89+93+206+198+91$

Exercise 11.4.56

(Solution on p. 686.)

(Section 11.3) Combine like terms: $4 x+8 y+12 y+9 x-2 y$. 


\subsection{Solving Equations of the Form $a x=b$ and $x / a=b^{5}$}

\subsubsection{Section Overview}

- Multiplication/ Division Property of Equality

- Combining Techniques in Equations Solving

\subsubsection{Multiplication/ Division Property of Equality}

Recall that the equal sign of an equation indicates that the number represented by the expression on the left side is the same as the number represented by the expression on the right side. From this, we can suggest the multiplication/division property of equality.

\section{Multiplication/Division Property of Equality}

Given any equation,

1. We can obtain an equivalent equation by multiplying both sides of the equation by the same nonzero number, that is, if $c \neq 0$, then $a=b$ is equivalent to $a \cdot c=b \cdot c$

2. We can obtain an equivalent equation by dividing both sides of the equation by the same nonzero number, that is, if $c \neq 0$, then $a=b$ is equivalent to $\frac{a}{c}=\frac{b}{c}$

The multiplication/division property of equality can be used to undo an association with a number that multiplies or divides the variable.

\subsubsection{Sample Set A}

Use the multiplication / division property of equality to solve each equation.

\section{Example 11.26}

$6 y=54$

6 is associated with y by multiplication. Undo the association by dividing both sides by 6

$$
\begin{aligned}
\frac{6 y}{6} & =\frac{54}{6} \\
\frac{\overline{6} y}{\overline{\gamma 6}} & =\frac{\frac{9}{54}}{\overline{56}} \\
y & =9
\end{aligned}
$$

Check: When $y=9$

$6 y=54$

becomes

$6 \cdot 9 \stackrel{?}{=} 54$

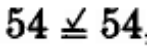

a true statement.

The solution to $6 y=54$ is $y=9$.

\footnotetext{
${ }^{5}$ This content is available online at $<\mathrm{http}: / / \mathrm{cnx} . o r g /$ content $/ \mathrm{m} 35045 / 1.3 />$.
} 


\section{Example 11.27}

$\frac{x}{-2}=27$.

-2 is associated with $x$ by division. Undo the association by multiplying both sides by -2 .

$(-2) \frac{x}{-2}=(-2) 27$

$(\overline{\overline{l-2}}) \frac{x}{\overline{--2}}=(-2) 27$

$x=-54$

Check: When $x=-54$,

$\frac{x}{-2}=27$

becomes

$\frac{-54}{-2} \stackrel{?}{=} 27$

\section{$27 \underline{\underline{ }} 27$}

a true statement.

The solution to $\frac{x}{-2}=27$ is $x=-54$

\section{Example 11.28}

$\frac{3 a}{7}=6$.

We will examine two methods for solving equations such as this one.

Method 1: Use of dividing out common factors.

$\frac{3 a}{7}=6$

7 is associated with $a$ by division. Undo the association by multiplying both sides by 7 .

$7 \cdot \frac{3 a}{7}=7 \cdot 6$

Divide out the 7's.

$\overline{7} \cdot \frac{3 a}{\overline{7}}=42$

$3 a=42$

3 is associated with $a$ by multiplication. Undo the association by dviding both sides by 3 .

$\frac{3 a}{3}=\frac{42}{3}$

$\frac{\overline{3} a}{\overline{\overline{3}}}=14$

$a=14$

Check: When $a=14$,

$\frac{3 a}{7}=6$ 
becomes

$$
\begin{aligned}
& \frac{3 \cdot 14}{7} \stackrel{?}{=} 6 \\
& \frac{42}{7}=6 \\
& 6 \unrhd 6
\end{aligned}
$$

a true statement.

The solution to $\frac{3 a}{7}=6$ is $a=14$.

Method 2: Use of reciprocals

Recall that if the product of two numbers is 1 , the numbers are reciprocals. Thus $\frac{3}{7}$ and $\frac{7}{3}$ are reciprocals.

$$
\begin{aligned}
& \frac{3 a}{7}=6 \\
& \text { Multiply both sides of the equation by } \frac{7}{3} \text {, the reciprocal of } \frac{3}{7} \text {. } \\
& \frac{7}{3} \cdot \frac{3 a}{7}=\frac{7}{3} \cdot 6 \\
& \frac{\frac{1}{\frac{7}{3}} \cdot \frac{\frac{1}{3} a}{\overline{1}}=\frac{7}{\overline{3}} \cdot \frac{\frac{2}{6}}{1}}{1} \\
& \begin{array}{l}
1 \cdot a=14 \\
\quad a=14
\end{array}
\end{aligned}
$$

Notice that we get the same solution using either method.

\section{Example 11.29}

$-8 x=24$

-8 is associated with $x$ by multiplication. Undo the association by dividing both sides by -8 .

$$
\begin{aligned}
& \frac{-8 x}{-8}=\frac{24}{-8} \\
& \frac{-8 x}{-8}=\frac{24}{-8} \\
& x=-3
\end{aligned}
$$

Check: When $x=-3$,

$$
-8 x=24
$$

becomes

\section{$-8(-3) \stackrel{?}{=} 24$}

$24 \unrhd 24$

a true statement.

\section{Example 11.30}

$$
-x=7 \text {. }
$$

Since $--x$ is actually $-1 \cdot x$ and $(-1)(-1)=1$, we can isolate $x$ by multiplying both sides of the 
equation by -1 .

$$
\begin{gathered}
(-1)(-x)=-1 \cdot 7 \\
x=-7
\end{gathered}
$$

Check: When $x=7$,

$$
-x=7
$$

becomes

$-(-7) \stackrel{?}{=} 7$

$7 \unrhd 7$

The solution to $-x=7$ is $x=-7$.

\subsubsection{Practice Set A}

Use the multiplication/division property of equality to solve each equation. Be sure to check each solution.

Exercise 11.5.1

$7 x=21$

Exercise 11.5.2

$-5 x=65$

Exercise 11.5.3

$\frac{x}{4}=-8$

Exercise 11.5.4

$\frac{3 x}{8}=6$

Exercise 11.5.5

$-y=3$

Exercise 11.5.6

$-k=-2$
(Solution on p. 686.)

(Solution on p. 686.)

(Solution on p. 686.)

(Solution on p. 686.)

(Solution on p. 686.)

(Solution on p. 686.)

\subsubsection{Combining Techniques in Equation Solving}

Having examined solving equations using the addition/subtraction and the multiplication/division principles of equality, we can combine these techniques to solve more complicated equations.

When beginning to solve an equation such as $6 x-4=-16$, it is helpful to know which property of equality to use first, addition/subtraction or multiplication/division. Recalling that in equation solving we are trying to isolate the variable (disassociate numbers from it), it is helpful to note the following.

To associate numbers and letters, we use the order of operations.

1. Multiply/divide

2. Add/subtract

To undo an association between numbers and letters, we use the order of operations in reverse.

1. Add/subtract

2. Multiply/divide 


\subsubsection{Sample Set B}

Solve each equation. (In these example problems, we will not show the checks.)

\section{Example 11.31}

$6 x-4=-16$

-4 is associated with $x$ by subtraction. Undo the association by adding 4 to both sides.

$6 x-4+4=-16+4$

$6 x=-12$

6 is associated with $x$ by multiplication. Undo the association by dividing both sides by 6

$\frac{6 x}{6}=\frac{-12}{6}$

$x=-2$

\section{Example 11.32}

$-8 k+3=-45$.

3 is associated with $k$ by addition. Undo the association by subtracting 3 from both sides.

$-8 k+3-3=-45-3$

$-8 k=-48$

-8 is associated with $k$ by multiplication. Undo the association by dividing both sides by -8 .

$\frac{-8 k}{-8}=\frac{-48}{-8}$

$k=6$

\section{Example 11.33}

$5 m-6-4 m=4 m-8+3 m$. Begin by solving this equation by combining like terms.

$m-6=7 m-8$ Choose a side on which to isolate $m$. Since 7 is greater than 1 , we'll isolate $m$ on the right side.

Subtract $m$ from both sides.

$-m-6-m=7 m-8-m$

$-6=6 m-8$

8 is associated with $m$ by subtraction. Undo the association by adding 8 to both sides.

$-6+8=6 m-8+8$

$2=6 m$

6 is associated with $\mathrm{m}$ by multiplication. Undo the association by dividing both sides by 6 .

$\frac{2}{6}=\frac{6 m}{6}$ Reduce.

$\frac{1}{3}=m$

Notice that if we had chosen to isolate $m$ on the left side of the equation rather than the right side, we would have proceeded as follows:

$m-6=7 m-8$

Subtract $7 m$ from both sides.

$m-6-7 m=7 m-8-7 m$ 
$-6 m-6=-8$

Add 6 to both sides,

$-6 m-6+6=-8+6$

$-6 m=-2$

Divide both sides by -6 .

$\frac{-6 m}{-6}=\frac{-2}{-6}$

$m=\frac{1}{3}$

This is the same result as with the previous approach.

\section{Example 11.34}

$\frac{8 x}{7}=-2$

7 is associated with $x$ by division. Undo the association by multiplying both sides by 7 .

$\overline{) 7} \cdot \frac{8 x}{\overline{) 7}}=7(-2)$

$7 \cdot \frac{8 x}{7}=-14$

$8 x=-14$

8 is associated with $x$ by multiplication. Undo the association by dividing both sides by 8 .

$\frac{\overline{8} x}{\overline{8}}=\frac{-7}{4}$

$x=\frac{-7}{4}$

\subsubsection{Practice Set B}

Solve each equation. Be sure to check each solution.

Exercise 11.5.7

(Solution on p. 686.)

$5 m+7=-13$

Exercise 11.5.8

$-3 a-6=9$

Exercise 11.5.9

$2 a+10-3 a=9$

Exercise 11.5.10

$11 x-4-13 x=4 x+14$

Exercise 11.5.11

$-3 m+8=-5 m+1$

Exercise 11.5.12

$5 y+8 y-11=-11$

(Solution on p. 686.)

(Solution on p. 686.)

(Solution on p. 686.)

(Solution on p. 686.)

(Solution on p. 686.) 


\subsubsection{Exercises}

Solve each equation. Be sure to check each result.

Exercise 11.5.13

(Solution on p. 686.)

$7 x=42$

Exercise 11.5.14

$8 x=81$

Exercise 11.5.15

$10 x=120$

(Solution on p. 686.)

Exercise 11.5.16

$11 x=121$

Exercise 11.5.17

$-6 a=48$

(Solution on p. 686.)

Exercise 11.5.18

$-9 y=54$

Exercise 11.5.19

$-3 y=-42$

(Solution on p. 686.)

Exercise 11.5.20

$-5 a=-105$

Exercise 11.5.21

$2 m=-62$

(Solution on p. 686.)

Exercise 11.5.22

$3 m=-54$

Exercise 11.5.23 $\frac{x}{4}=7$

(Solution on p. 686.)

Exercise 11.5.24 $\frac{y}{3}=11$

Exercise 11.5.25 $\frac{-z}{6}=-14$

(Solution on p. 686.)

Exercise 11.5.26 $\frac{-w}{5}=1$

Exercise 11.5.27

$3 m-1=-13$

Exercise 11.5.28

$4 x+7=-17$

Exercise 11.5.29

$2+9 x=-7$

Exercise 11.5.30

$5-11 x=27$

Exercise 11.5.31

$32=4 y+6$

(Solution on p. 686.)

Exercise 11.5.32

$-5+4=-8 m+1$

Exercise 11.5.33

$3 k+6=5 k+10$

(Solution on p. 686.) 
Exercise 11.5.34

$4 a+16=6 a+8 a+6$

Exercise 11.5.35

(Solution on p. 686.)

$6 x+5+2 x-1=9 x-3 x+15$

Exercise 11.5.36

$-9 y-8+3 y+7=-7 y+8 y-5 y+9$

Exercise 11.5.37

(Solution on p. 687.)

$-3 a=a+5$

Exercise 11.5.38

$5 b=-2 b+8 b+1$

Exercise 11.5.39

(Solution on p. 687.)

$-3 m+2-8 m-4=-14 m+m-4$

Exercise 11.5.40

$5 a+3=3$

Exercise 11.5.41

$7 x+3 x=0$

(Solution on p. 687.)

Exercise 11.5.42

$7 g+4-11 g=-4 g+1+g$

Exercise 11.5.43

$\frac{5 a}{7}=10$

(Solution on p. 687.)

Exercise 11.5.44

$\frac{2 m}{9}=4$

Exercise 11.5.45

$\frac{3 x}{4}=\frac{9}{2}$

(Solution on p. 687.)

Exercise 11.5.46

$\frac{8 k}{3}=32$

Exercise 11.5.47 $\frac{3 a}{8}-\frac{3}{2}=0$

Exercise 11.5.48 $\frac{5 m}{6}-\frac{25}{3}=0$

\subsubsection{Exercises for Review}

Exercise 11.5.49

(Solution on p. 687.)

(Section 8.4) Use the distributive property to compute $40 \cdot 28$.

(Solution on p. 687.)

Exercise 11.5.50

(Section 9.5) Approximating $\pi$ by 3.14, find the approximate circumference of the circle.

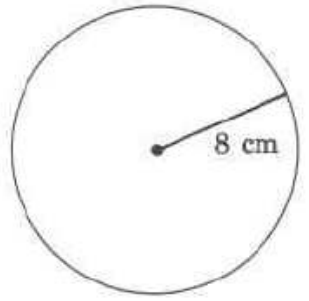

Exercise 11.5.51

(Solution on p. 687.)

(Section 9.6) Find the area of the parallelogram. 


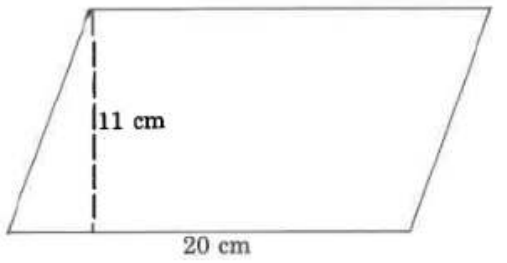

Exercise 11.5.52

(Section 10.7) Find the value of $\frac{-3(4-15)-2}{-5}$.

Exercise 11.5.53

(Solution on p. 687.)

(Section 11.4) Solve the equation $x-14+8=-2$.

\subsection{Applications I: Translating Words to Mathematical Symbols ${ }^{6}$}

\subsubsection{Section Overview}

- Translating Words to Symbols

\subsubsection{Translating Words to Symbols}

Practical problems seldom, if ever, come in equation form. The job of the problem solver is to translate the problem from phrases and statements into mathematical expressions and equations, and then to solve the equations.

As problem solvers, our job is made simpler if we are able to translate verbal phrases to mathematical expressions and if we follow the five-step method of solving applied problems. To help us translate from words to symbols, we can use the following Mathematics Dictionary.

\begin{tabular}{|l|l|}
\hline \multicolumn{2}{|c|}{ MATHEMATICS DICTIONARY } \\
\hline Word or Phrase & Mathematical Operation \\
\hline Sum, sum of, added to, increased by, more than, and, plus & + \\
\hline Difference, minus, subtracted from, decreased by, less, less than & - \\
\hline Product, the product of, of, multiplied by, times, per & $\cdot$ \\
\hline Quotient, divided by, ratio, per & $\div$ \\
\hline Equals, is equal to, is, the result is, becomes & $=$ \\
\hline A number, an unknown quantity, an unknown, a quantity & $x$ (or any symbol) \\
\hline
\end{tabular}

Table 11.2

\footnotetext{
${ }^{6}$ This content is available online at $<$ http://cnx.org/content $/ \mathrm{m} 35046 / 1.2 />$.
} 


\subsubsection{Sample Set A}

Translate each phrase or sentence into a mathematical expression or equation.

Example 11.35

$\underbrace{\text { Nine }}_{9} \underbrace{\text { more than }}_{+} \underbrace{\text { some number. }}_{x}$

Translation: $9+x$.

Example 11.36

$\underbrace{\text { Eighteen }}_{18} \underbrace{\text { minus }}_{-} \underbrace{\text { a number. }}_{x}$

Translation: $18-x$.

Example 11.37

$\underbrace{\text { A quantity }}_{y} \underbrace{\text { less five. }}_{-}$

Translation: $y-5$.

Example 11.38

$\underbrace{\text { Four }}_{4} \underbrace{\text { times }}_{\cdot} \underbrace{\text { a number }}_{x} \underbrace{\text { is }}_{=} \underbrace{\text { sixteen }}_{16}$

Translation: $4 x=16$.

\section{Example 11.39}

$\underbrace{\text { One fifth }}_{\frac{1}{5}} \underbrace{\text { of }}_{n} \underbrace{\text { a number }}_{=} \underbrace{\text { is }}_{30} \underbrace{\text { thirty. }}_{30}$

Translation: $\frac{1}{5} n=30$, or $\frac{n}{5}=30$.

Example 11.40

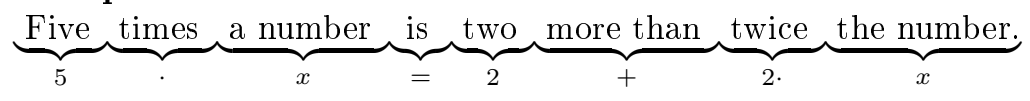

Translation: $5 x=2+2 x$.

\subsubsection{Practice Set A}

Translate each phrase or sentence into a mathematical expression or equation.

Exercise 11.6.1

(Solution on p. 687.)

Twelve more than a number.

Exercise 11.6.2

(Solution on p. 687.)

Eight minus a number.

Exercise 11.6.3

(Solution on p. 687.)

An unknown quantity less fourteen.

Exercise 11.6.4

(Solution on p. 687.)

Six times a number is fifty-four.

Exercise 11.6.5

(Solution on p. 687.)

Two ninths of a number is eleven. 
Exercise 11.6.6

(Solution on p. 687.)

Three more than seven times a number is nine more than five times the number.

Exercise 11.6.7

(Solution on p. 687.)

Twice a number less eight is equal to one more than three times the number.

\subsubsection{Sample Set B}

\section{Example 11.41}

Sometimes the structure of the sentence indicates the use of grouping symbols. We'll be alert for commas. They set off terms.

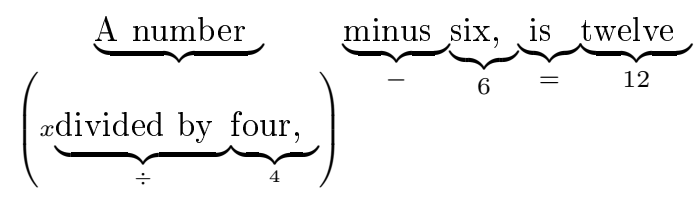

Translation: $\frac{x}{4}-6=12$.

Example 11.42

Some phrases and sentences do not translate directly. We must be careful to read them properly. The word from often appears in such phrases and sentences. The word from means "a point of departure for motion." The following translation will illustrate this use.

\section{Twenty is subtracted from $\underbrace{\text { some number. }}$}

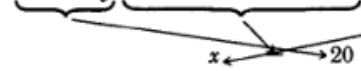

Translation: $x-20$.

The word from indicated the motion (subtraction) is to begin at the point of "some number."

Example 11.43

Ten less than some number. Notice that less than can be replaced by from.

Ten from some number.

Translation: $x-10$.

\subsubsection{Practice Set B}

Translate each phrase or sentence into a mathematical expression or equation.

Exercise 11.6.8

A number divided by eight, plus seven, is fifty.

(Solution on p. 687.)

Exercise 11.6.9

(Solution on p. 687.)

A number divided by three, minus the same number multiplied by six, is one more than the number.

Exercise 11.6.10

(Solution on p. 687.)

Nine from some number is four.

Exercise 11.6.11

(Solution on p. 687.)

Five less than some quantity is eight. 


\subsubsection{Exercises}

Translate each phrase or sentence to a mathematical expression or equation.

Exercise 11.6.12

(Solution on p. 687.)

A quantity less twelve.

Exercise 11.6.13

Six more than an unknown number.

Exercise 11.6.14

A number minus four.

(Solution on p. 687.)

Exercise 11.6.15

A number plus seven.

Exercise 11.6.16

(Solution on p. 687.)

A number increased by one.

Exercise 11.6.17

A number decreased by ten.

Exercise 11.6.18

Negative seven added to some number.

(Solution on p. 687.)

Exercise 11.6.19

Negative nine added to a number.

Exercise 11.6.20

(Solution on p. 687.)

A number plus the opposite of six.

Exercise 11.6.21

A number minus the opposite of five.

Exercise 11.6.22

A number minus the opposite of negative one.

(Solution on p. 688.)

Exercise 11.6.23

A number minus the opposite of negative twelve.

Exercise 11.6.24

(Solution on p. 688.)

Eleven added to three times a number.

Exercise 11.6.25

Six plus five times an unknown number.

Exercise 11.6.26

(Solution on p. 688.)

Twice a number minus seven equals four.

Exercise 11.6.27

Ten times a quantity increased by two is nine.

Exercise 11.6.28

When fourteen is added to two times a number the result is six.

(Solution on p. 688.)

Exercise 11.6.29

Four times a number minus twenty-nine is eleven.

Exercise 11.6.30

(Solution on p. 688.)

Three fifths of a number plus eight is fifty.

Exercise 11.6.31

Two ninths of a number plus one fifth is forty-one.

Exercise 11.6.32

When four thirds of a number is increased by twelve, the result is five.

(Solution on p. 688.) 


\section{Exercise 11.6.33}

When seven times a number is decreased by two times the number, the result is negative one.

Exercise 11.6.34

(Solution on p. 688.)

When eight times a number is increased by five, the result is equal to the original number plus twenty-six.

\section{Exercise 11.6.35}

Five more than some number is three more than four times the number.

Exercise 11.6.36

When a number divided by six is increased by nine, the result is one.

(Solution on p. 688.)

\section{Exercise 11.6.37}

A number is equal to itself minus three times itself.

Exercise 11.6.38

(Solution on p. 688.)

A number divided by seven, plus two, is seventeen.

Exercise 11.6.39

A number divided by nine, minus five times the number, is equal to one more than the number.

Exercise 11.6.40

(Solution on p. 688.)

When two is subtracted from some number, the result is ten.

\section{Exercise 11.6.41}

When four is subtracted from some number, the result is thirty-one.

Exercise 11.6.42

(Solution on p. 688.)

Three less than some number is equal to twice the number minus six.

\section{Exercise 11.6.43}

Thirteen less than some number is equal to three times the number added to eight.

Exercise 11.6.44

(Solution on p. 688.)

When twelve is subtracted from five times some number, the result is two less than the original number.

Exercise 11.6.45

When one is subtracted from three times a number, the result is eight less than six times the original number.

Exercise 11.6.46

(Solution on p. 688.)

When a number is subtracted from six, the result is four more than the original number.

Exercise 11.6.47

When a number is subtracted from twenty-four, the result is six less than twice the number.

Exercise 11.6.48

(Solution on p. 688.)

A number is subtracted from nine. This result is then increased by one. The result is eight more than three times the number.

\section{Exercise 11.6.49}

Five times a number is increased by two. This result is then decreased by three times the number. The result is three more than three times the number.

Exercise 11.6.50

(Solution on p. 688.)

Twice a number is decreased by seven. This result is decreased by four times the number. The result is negative the original number, minus six.

\section{Exercise 11.6.51}

Fifteen times a number is decreased by fifteen. This result is then increased by two times the number. The result is negative five times the original number minus the opposite of ten. 


\subsubsection{Exercises for Review}

Exercise 11.6.52

(Section 4.7 ) $\frac{8}{9}$ of what number is $\frac{2}{3}$ ?

(Solution on p. 688.)

Exercise 11.6.53

(Section 5.3) Find the value of $\frac{21}{40}+\frac{17}{30}$.

Exercise 11.6.54

(Section 5.4) Find the value of $3 \frac{1}{12}+4 \frac{1}{3}+1 \frac{1}{4}$.

(Solution on p. 688.)

Exercise 11.6.55

(Section 6.3) Convert $6.11 \frac{1}{5}$ to a fraction.

Exercise 11.6.56

(Section 11.5) Solve the equation $\frac{3 x}{4}+1=-5$.

(Solution on p. 688.)

\subsection{Applications II: Solving Problems ${ }^{7}$}

\subsubsection{Section Overview}

- The Five-Step Method

- Number Problems

- Geometry Problems

\subsubsection{The Five Step Method}

We are now in a position to solve some applied problems using algebraic methods. The problems we shall solve are intended as logic developers. Although they may not seem to reflect real situations, they do serve as a basis for solving more complex, real situation, applied problems. To solve problems algebraically, we will use the five-step method.

\section{Strategy for Reading Word Problems}

When solving mathematical word problems, you may wish to apply the following "reading strategy." Read the problem quickly to get a feel for the situation. Do not pay close attention to details. At the first reading, too much attention to details may be overwhelming and lead to confusion and discouragement. After the first, brief reading, read the problem carefully in phrases. Reading phrases introduces information more slowly and allows us to absorb and put together important information. We can look for the unknown quantity by reading one phrase at a time.

\section{Five-Step Method for Solving Word Problems}

1. Let $x$ (or some other letter) represent the unknown quantity.

2. Translate the words to mathematical symbols and form an equation. Draw a picture if possible.

3. Solve the equation.

4. Check the solution by substituting the result into the original statement, not equation, of the problem.

5. Write a conclusion.

If it has been your experience that word problems are difficult, then follow the five-step method carefully. Most people have trouble with word problems for two reasons:

1. They are not able to translate the words to mathematical symbols. (See Section 11.5.)

\footnotetext{
${ }^{7}$ This content is available online at $<$ http://cnx.org/content $/ \mathrm{m} 35047 / 1.2 />$.
} 
2. They neglect step 1. After working through the problem phrase by phrase, to become familiar with the situation,

\section{INTRODUCE A VARIABLE}

\subsubsection{Number Problems}

\subsubsection{Sample Set A}

\section{Example 11.44}

What number decreased by six is five?

Step 1: Let $n$ represent the unknown number.

Step 2: Translate the words to mathematical symbols and construct an equation. Read phrases.

What number: $n$

decreased by: -

$\begin{array}{cl}\text { six: } & 6\} n-6=5 \\ \text { is: } & = \\ \text { five: } & 5\end{array}$

Step 3: Solve this equation.

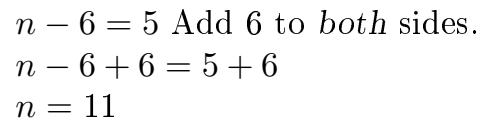

Step 4: Check the result.

When 11 is decreased by 6 , the result is $11-6$, which is equal to 5 . The solution checks.

Step 5: The number is 11.

\section{Example 11.45}

When three times a number is increased by four, the result is eight more than five times the number.

Step 1: Let $x=$ the unknown number.

Step 2: Translate the phrases to mathematical symbols and construct an equation.

When three times a number: $3 x$

$\begin{array}{ll}\text { is increased by: } & + \\ \text { four: } & 4\end{array}$

the result is: $\quad=\quad 33 x+4=5 x+8$

eight: $\quad 8$

more than: $\quad+$

five times the number: $\quad 5 x$

$$
\begin{array}{cc}
3 x+4=5 x+8 . & \text { Subtract } 3 x \text { from both sides. } \\
3 x+4-3 x=5 x+8-3 x & \\
4=2 x+8 & \text { Subtract } 8 \text { from both sides. }
\end{array}
$$

Step 3: $\quad 4-8=2 x+8-8$

$-4=2 x \quad$ Divide both sides by 2 .

$$
-2=x
$$


Step 4: Check this result.

Three times -2 is -6 . Increasing -6 by 4 results in $-6+4=-2$. Now, five times -2 is -10 . Increasing -10 by 8 results in $-10+8=-2$. The results agree, and the solution checks.

Step 5: The number is -2

\section{Example 11.46}

Consecutive integers have the property that if

$$
\begin{aligned}
n & =\text { the smallest integer, then } \\
n+1 & =\text { the next integer, and } \\
n+2 & =\text { the next integer, and so on. }
\end{aligned}
$$

Consecutive odd or even integers have the property that if

$$
\begin{aligned}
n & = & \text { the smallest integer, then } \\
n+2 & = & \text { the next odd or even integer (since odd or even numbers differ by } 2 \text { ), and } \\
n+4 & = & \text { the next odd or even integer, and so on. }
\end{aligned}
$$

The sum of three consecutive odd integers is equal to one less than twice the first odd integer. Find the three integers.

$$
\text { Let } \quad n=\text { the first odd integer. Then, }
$$

Step 1. $\quad n+2=$ the second odd integer, and

$$
n+4=\text { the third odd integer. }
$$

Step 2. Translate the words to mathematical symbols and construct an equation. Read phrases.

The sum of:

three consecutive odd integers:

is equal to:

one less than:

twice the first odd integer: add some numbers

$$
\left.\begin{array}{cl}
n, n+2, n+4 \\
=
\end{array}\right\} n+(n+2)+(n+4)=2 n-1
$$

subtract 1 from

$$
2 n
$$

$$
\begin{array}{cc}
n+n+2+n+4=2 n-1 & \\
3 n+6=2 n-1 & \text { Subtract } 2 n \text { from both sides. } \\
3 n+6-2 n=2 n-1-2 n & \\
n+6=-1 & \text { Subtract } 6 \text { from both sides. }
\end{array}
$$

Step 3.

$$
\begin{array}{cc}
n+6-6=-1-6 & \\
n=-7 & \text { The first integer is }-7 . \\
n+2=-7+2=-5 & \text { The second integer is }-5 . \\
n+4=-7+4=-3 & \text { The third integer is }-3 .
\end{array}
$$

Step 4. Check this result.

The sum of the three integers is $\begin{array}{rlc}-7+(-5)+(-3) & = & -12+(-3) \\ & = & -15\end{array}$

One less than twice the first integer is $2(-7)-1=-14-1=-15$. Since these two results are equal, the solution checks. 
Step 5. The three odd integers are $-7,-5,-3$.

\subsubsection{Practice Set A}

Exercise 11.7.1

(Solution on p. 688.)

When three times a number is decreased by 5 , the result is -23 . Find the number.

Step 1: Let $x=$

Step 2:

Step 3:

Step 4: Check:

Step 5: The number is

Exercise 11.7.2

(Solution on p. 688.)

When five times a number is increased by 7 , the result is five less than seven times the number. Find the number.

Step 1: Let $n=$

Step 2:

Step 3:

Step 4: Check:

Step 5: The number is

Exercise 11.7.3

(Solution on p. 688.)

Two consecutive numbers add to 35 . Find the numbers.

Step 1:

Step 2:

Step 3:

Step 4: Check:

Step 5: The numbers are and

\section{Exercise 11.7.4}

(Solution on p. 688.)

The sum of three consecutive even integers is six more than four times the middle integer. Find the integers.

Step 1: Let $x=$ smallest integer.

$$
=\text { next integer. }
$$

Step 2:

$$
=\text { largest integer. }
$$

Step 3:

Step 4: Check:

Step 5: The integers are , and 


\subsubsection{Geometry Problems}

\subsubsection{Sample Set B}

\section{Example 11.47}

The perimeter (length around) of a rectangle is 20 meters. If the length is 4 meters longer than the width, find the length and width of the rectangle.

Step 1: Let $x=$ the width of the rectangle. Then, $x+4=$ the length of the rectangle.

Step 2: We can draw a picture.

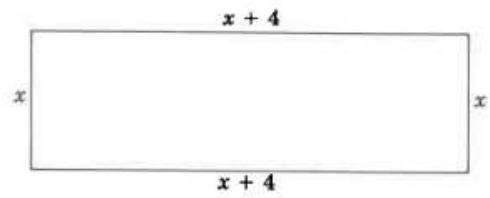

The length around the rectangle is

$$
\begin{aligned}
& \underbrace{x}_{\text {width }}+\underbrace{(x+4)}_{\text {length }}+\underbrace{x}_{\text {width }}+\underbrace{(x+4)}_{\text {length }}=20 \\
& x+x+4+x+x+4=20 \quad \text { Subtract } 8 \text { from both sides. } \\
& 4 x+8=20 \quad
\end{aligned}
$$

Step 3: $\quad 4 x=12 \quad$ Divide both sides by 4 .

$$
x=3 \quad \text { Then, }
$$

$$
x+4=3+4=7
$$

Step 4: Check:

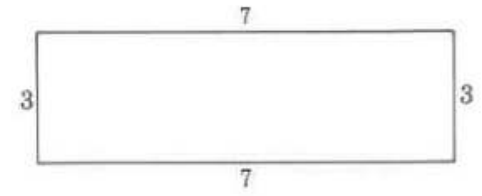

$3+7+3+7 \stackrel{2}{\doteq} 20$

$20 \cong 20$

Step 5: The length of the rectangle is 7 meters.

The width of the rectangle is 3 meters.

\subsubsection{Practice Set $B$}

\section{Exercise 11.7.5}

(Solution on p. 688.)

The perimeter of a triangle is 16 inches. The second leg is 2 inches longer than the first leg, and the third leg is 5 inches longer than the first leg. Find the length of each leg.

Step 1: Let $x=$ length of the first leg.

$=$ length of the second leg.

$=$ length of the third leg.

Step 2: We can draw a picture.

Step 3:

Step 4: Check:

Step 5: The lengths of the legs are , and 


\subsubsection{Exercises}

For the following 17 problems, find each solution using the five-step method.

Exercise 11.7.6

What number decreased by nine is fifteen?

(Solution on p. 688.)

Step 1. Let $n=$ the number.

Step 2.

Step 3.

Step 4. Check:

Step 5. The number is

\section{Exercise 11.7.7}

What number increased by twelve is twenty?

Step 1. Let $n=$ the number.

Step 2.

Step 3.

Step 4. Check:

Step 5. The number is

Exercise 11.7.8

(Solution on p. 688.)

If five more than three times a number is thirty-two, what is the number?

Step 1. Let $x=$ the number.

Step 2.

Step 3.

Step 4. Check:

Step 5. The number is

\section{Exercise 11.7.9}

If four times a number is increased by fifteen, the result is five. What is the number?

Step 1. Let $x=$

Step 2.

Step 3.

Step 4. Check:

Step 5. The number is

Exercise 11.7.10

(Solution on p. 689.)

When three times a quantity is decreased by five times the quantity, the result is negative twenty. What is the quantity?

Step 1. Let $x=$

Step 2.

Step 3.

Step 4. Check:

Step 5. The quantity is

\section{Exercise 11.7.11}

If four times a quantity is decreased by nine times the quantity, the result is ten. What is the quantity? 
Step 1. Let $y=$

Step 2.

Step 3.

Step 4. Check:

Step 5. The quantity is

Exercise 11.7.12

(Solution on p. 689.)

When five is added to three times some number, the result is equal to five times the number decreased by seven. What is the number?

Step 1. Let $n=$

Step 2.

Step 3.

Step 4. Check:

Step 5. The number is

\section{Exercise 11.7.13}

When six times a quantity is decreased by two, the result is six more than seven times the quantity. What is the quantity?

Step 1. Let $x=$

Step 2.

Step 3.

Step 4. Check:

Step 5. The quantity is

Exercise 11.7.14

(Solution on p. 689.)

When four is decreased by three times some number, the result is equal to one less than twice the number. What is the number?

Step 1.

Step 2.

Step 3.

Step 4. Check:

Step 5.

\section{Exercise 11.7.15}

When twice a number is subtracted from one, the result is equal to twenty-one more than the number. What is the number?

Step 1.

Step 2.

Step 3.

Step 4.

Step 5.

Exercise 11.7.16

(Solution on p. 689.)

The perimeter of a rectangle is 36 inches. If the length of the rectangle is 6 inches more than the width, find the length and width of the rectangle.

Step 1. Let $w=$ the width.

$=$ the length. 
Step 2. We can draw a picture.

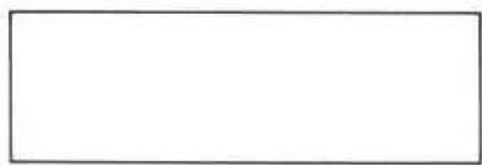

Step 3.

Step 4. Check:

Step 5. The length of the rectangle is inches, and the width is inches.

\section{Exercise 11.7.17}

The perimeter of a rectangle is 48 feet. Find the length and the width of the rectangle if the length is 8 feet more than the width.

Step 1. Let $w=$ the width. $=$ the length.

Step 2. We can draw a picture.

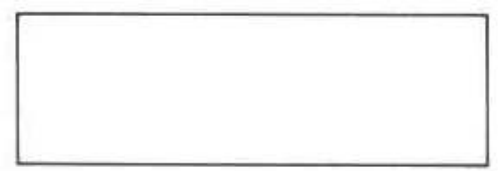

Step 3.

Step 4. Check:

Step 5. The length of the rectangle is feet, and the width is feet.

\section{Exercise 11.7.18}

(Solution on p. 689.)

The sum of three consecutive integers is 48 . What are they?

Step 1. Let $n=$ the smallest integer.

$=$ the next integer.

$=$ the next integer.

Step 2.

Step 3.

Step 4. Check:

Step 5. The three integers are , and

\section{Exercise 11.7.19}

The sum of three consecutive integers is -27 . What are they?

Step 1. Let $n=$ the smallest integer.

$=$ the next integer.

$=$ the next integer.

Step 2.

Step 3.

Step 4. Check:

Step 5. The three integers are , _ and

\section{Exercise 11.7.20}

(Solution on p. 689.)

The sum of five consecutive integers is zero. What are they?

Step 1. Let $n=$

Step 2. 
Step 3.

Step 4.

Step 5. The five integers are

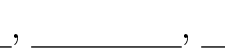
, and

\section{Exercise 11.7.21}

The sum of five consecutive integers is -5 . What are they?

Step 1. Let $n=$

Step 2.

Step 3.

Step 4.

Step 5. The five integers are , and

Continue using the five-step procedure to find the solutions.

Exercise 11.7.22

(Solution on p. 689.)

The perimeter of a rectangle is 18 meters. Find the length and width of the rectangle if the length is 1 meter more than three times the width.

\section{Exercise 11.7.23}

The perimeter of a rectangle is 80 centimeters. Find the length and width of the rectangle if the length is 2 meters less than five times the width.

Exercise 11.7.24

(Solution on p. 689.)

Find the length and width of a rectangle with perimeter 74 inches, if the width of the rectangle is 8 inches less than twice the length.

Exercise 11.7.25

Find the length and width of a rectangle with perimeter 18 feet, if the width of the rectangle is 7 feet less than three times the length.

\section{Exercise 11.7.26}

(Solution on p. 689.)

A person makes a mistake when copying information regarding a particular rectangle. The copied information is as follows: The length of a rectangle is 5 inches less than two times the width. The perimeter of the rectangle is 2 inches. What is the mistake?

\section{Exercise 11.7.27}

A person makes a mistake when copying information regarding a particular triangle. The copied information is as follows: Two sides of a triangle are the same length. The third side is 10 feet less than three times the length of one of the other sides. The perimeter of the triangle is 5 feet. What is the mistake?

\section{Exercise 11.7.28}

(Solution on p. 689.)

The perimeter of a triangle is 75 meters. If each of two legs is exactly twice the length of the shortest leg, how long is the shortest leg?

\section{Exercise 11.7.29}

If five is subtracted from four times some number the result is negative twenty-nine. What is the number?

Exercise 11.7.30

(Solution on p. 689.)

If two is subtracted from ten times some number, the result is negative two. What is the number?

\section{Exercise 11.7.31}

If three less than six times a number is equal to five times the number minus three, what is the number?

Exercise 11.7.32

(Solution on p. 689.)

If one is added to negative four times a number the result is equal to eight less than five times the number. What is the number? 


\section{Exercise 11.7.33}

Find three consecutive integers that add to -57 .

Exercise 11.7.34

(Solution on p. 689.)

Find four consecutive integers that add to negative two.

Exercise 11.7.35

Find three consecutive even integers that add to -24 .

Exercise 11.7.36

(Solution on p. 689.)

Find three consecutive odd integers that add to -99 .

\section{Exercise 11.7.37}

Suppose someone wants to find three consecutive odd integers that add to 120 . Why will that person not be able to do it?

Exercise 11.7.38

(Solution on p. 689.)

Suppose someone wants to find two consecutive even integers that add to 139 . Why will that person not be able to do it?

Exercise 11.7.39

Three numbers add to 35 . The second number is five less than twice the smallest. The third number is exactly twice the smallest. Find the numbers.

Exercise 11.7.40

(Solution on p. 689.)

Three numbers add to 37 . The second number is one less than eight times the smallest. The third number is two less than eleven times the smallest. Find the numbers.

\subsubsection{Exercises for Review}

\section{Exercise 11.7.41}

(Section 6.7) Find the decimal representation of $0.34992 \div 4.32$.

Exercise 11.7.42

(Solution on p. 689.)

(Section 7.4) A 5-foot woman casts a 9-foot shadow at a particular time of the day. How tall is a person that casts a 10.8 -foot shadow at the same time of the day?

Exercise 11.7.43

(Section 8.5) Use the method of rounding to estimate the sum: $4 \frac{5}{12}+15 \frac{1}{25}$.

Exercise 11.7.44

(Solution on p. 689.)

(Section 9.3) Convert $463 \mathrm{mg}$ to $\mathrm{cg}$.

Exercise 11.7.45

(Section 11.6) Twice a number is added to 5. The result is 2 less than three times the number. What is the number? 


\subsection{Summary of Key Concepts ${ }^{8}$}

\subsubsection{Summary of Key Concepts}

Numerical Expression (Section 11.2)

A numerical expression results when numbers are associated by arithmetic operation signs. The expressions $3+5,9-2,5 \cdot 6$ and $8 \div 5$ are numerical expressions.

\section{Algebraic Expressions (Section 11.2)}

When an arithmetic operation sign connects a letter with a number or a letter with a letter, an algebraic expression results. The expressions $4 x+1, x-5,7 x \cdot 6 y$, and $4 x \div 3$ are algebraic expressions.

\section{Terms and Factors (Section 11.2)}

Terms are parts of sums and are therefore separated by addition (or subtraction) signs. In the expression, $5 x-2 y, 5 x$ and $-2 y$ are the terms. Factors are parts of products and are therefore separated by multiplication signs. In the expression $5 a, 5$ and $a$ are the factors.

\section{Coefficients (Section 11.2)}

The coefficient of a quantity records how many of that quantity there are. In the expression $7 x$, the coefficient 7 indicates that there are seven $x$ 's.

\section{Numerical Evaluation (Section 11.2)}

Numerical evaluation is the process of determining the value of an algebraic expression by replacing the variables in the expression with specified values.

\section{Combining Like Terms (Section 11.3)}

An algebraic expression may be simplified by combining like terms. To combine like terms, we simply add or subtract their coefficients then affix the variable. For example $4 x+9 x=(4+9) x=13 x$.

\section{Equation (Section 11.4)}

An equation is a statement that two expressions are equal. The statements $5 x+1=3$ and $\frac{4 x}{5}+4=\frac{2}{5}$ are equations. The expressions represent the same quantities.

\section{Conditional Equation (Section 11.4)}

A conditional equation is an equation whose truth depends on the value selected for the variable. The equation $3 x=9$ is a conditional equation since it is only true on the condition that 3 is selected for $x$.

\section{Solutions and Solving an Equation (Section 11.4)}

The values that when substituted for the variables make the equation true are called the solutions of the equation.

An equation has been solved when all its solutions have been found.

\section{Equivalent Equations (Section 11.4)}

Equations that have precisely the same solutions are called equivalent equations. The equations $6 y=18$ and $y=3$ are equivalent equations.

\section{Addition/Subtraction Property of Equality (Section 11.4)}

Given any equation, we can obtain an equivalent equation by

1. adding the same number to both sides, or

2. subtracting the same number from both sides.

\footnotetext{
${ }^{8}$ This content is available online at $<$ http://cnx.org/content/m35049/1.2/>.
} 
Solving $x+a=b$ and $x-a=b$ (Section 11.4)

To solve $x+a=b$, subtract $a$ from both sides.

$$
\begin{gathered}
x+a=b \\
x+a-a=b-a \\
x=b-a
\end{gathered}
$$

To solve $x-a=b$, add $a$ to both sides.

$$
\begin{gathered}
x-a=b \\
x-a+a=b+a \\
x=b+a
\end{gathered}
$$

Multiplication/Division Property of Equality (Section 11.5)

Given any equation, we can obtain an equivalent equation by

1. multiplying both sides by the same nonzero number, that is, if $c \neq 0, a=b$ and $a \cdot c=b \cdot c$ are equivalent.

2. dividing both sides by the same nonzero number, that is, if $c \neq 0, a=b$ and $\frac{a}{c}=\frac{b}{c}$ are equivalent.

Solving $a x=b$ and $\frac{x}{a}=b$ (Section 11.5)

To solve $\mathrm{ax}=b, a \neq 0$, divide both sides by $a$.

$$
\begin{aligned}
a x & =b \\
\frac{a x}{a} & =\frac{b}{a} \\
\frac{\bar{a} x}{\bar{a}} & =\frac{b}{a} \\
x & =\frac{b}{a}
\end{aligned}
$$

To solve $\frac{x}{a}=b, a \neq 0$, multiply both sides by $a$.

$$
\begin{aligned}
\frac{x}{a} & =b \\
a \cdot \frac{x}{a} & =a \cdot b \\
\overline{) a} \cdot \frac{x}{\bar{a}} & =a \cdot b \\
x & =a \cdot b
\end{aligned}
$$

\section{Translating Words to Mathematics (Section 11.6)}

In solving applied problems, it is important to be able to translate phrases and sentences to mathematical expressions and equations.

\section{The Five-Step Method for Solving Applied Problems (Section 11.7)}

To solve problems algebraically, it is a good idea to use the following five-step procedure.

After working your way through the problem carefully, phrase by phrase:

1. Let $x$ (or some other letter) represent the unknown quantity.

2. Translate the phrases and sentences to mathematical symbols and form an equation. Draw a picture if possible.

3. Solve this equation.

4. Check the solution by substituting the result into the original statement of the problem.

5. Write a conclusion. 


\subsection{Exercise Supplement ${ }^{9}$}

\subsubsection{Exercise Supplement}

11.9.1.1 Algebraic Expressions (Section 11.2)

For problems 1-10, specify each term.

Exercise 11.9.1

(Solution on p. 689.)

$6 a-2 b+5 c$

Exercise 11.9.2

$9 x-6 y+1$

Exercise 11.9.3

(Solution on p. 689.)

$7 m-3 n$

Exercise 11.9.4

$-5 h+2 k-8+4 m$

Exercise 11.9.5

(Solution on p. 689.)

$x+2 n-z$

Exercise 11.9.6

$y-5$

Exercise 11.9.7

$-y-3 z$

(Solution on p. 689.)

Exercise 11.9.8

$-a-b-c-1$

Exercise 11.9.9

$-4$

(Solution on p. 689.)

Exercise 11.9.10

$-6$

Exercise 11.9.11

(Solution on p. 689.)

Write $1 k$ in a simpler way.

Exercise 11.9.12

Write $1 x$ in a simpler way.

Exercise 11.9.13

In the expression $7 r$, how many $r$ 's are indicated?

(Solution on p. 689.)

Exercise 11.9.14

In the expression $12 m$, how many $m$ 's are indicated?

Exercise 11.9.15

In the expression $-5 n$, how many $n$ 's are indicated?

(Solution on p. 690.)

Exercise 11.9.16

In the expression $-10 y$, how many $y$ 's are indicated?

For problems 17-46, find the value of each expression.

Exercise 11.9.17

(Solution on p. 690.)

$5 a-2 s$, if $a=-5$ and $s=1$

Exercise 11.9.18

$7 n-3 r$, if $n=-6$ and $r=2$

Exercise 11.9.19

$9 x+2 y-3 s$, if $x=-2, y=5$, and $s=-3$

(Solution on p. 690.)

${ }^{9}$ This content is available online at $<\mathrm{http}: / / \mathrm{cnx} . o r g /$ content $/ \mathrm{m} 35050 / 1.2 />$. 
Exercise 11.9.20

$10 a-2 b+5 c$, if $a=0, b=-6$, and $c=8$

Exercise 11.9.21

$-5 s-2 t+1$, if $s=2$ and $t=-2$

(Solution on p. 690.)

Exercise 11.9.22

$-3 m-4 n+5$, if $m=-1$ and $n=-1$

Exercise 11.9.23

(Solution on p. 690.)

$m-4$, if $m=4$

Exercise 11.9.24

$n=2$, if $n=2$

Exercise 11.9.25

$-x+2 y$, if $x=-7$ and $y=-1$

(Solution on p. 690.)

Exercise 11.9.26

$-a+3 b-6$, if $a=-3$ and $b=0$

Exercise 11.9.27

$5 x-4 y-7 y+y-7 x$, if $x=1$ and $y=-2$

(Solution on p. 690.)

Exercise 11.9.28

$2 a-6 b-3 a-a+2 b$, if $a=4$ and $b=-2$

Exercise 11.9.29

(Solution on p. 690.)

$a^{2}-6 a+4$, if $a=-2$

Exercise 11.9.30

$m^{2}-8 m-6$, if $m=-5$

Exercise 11.9.31

$4 y^{2}+3 y+1$, if $y=-2$

Exercise 11.9.32

$5 a^{2}-6 a+11$, if $a=0$

Exercise 11.9.33

$-k^{2}-k-1$, if $k=-1$

(Solution on p. 690.)

Exercise 11.9.34

$-h^{2}-2 h-3$, if $h=-4$

Exercise 11.9.35

$\frac{m}{6}+5 m$, if $m=-18$

Exercise 11.9.36

$\frac{a}{8}-2 a+1$, if $a=24$

Exercise 11.9.37

$\frac{5 x}{7}+3 x-7$, if $x=14$

(Solution on p. 690.)

Exercise 11.9.38

$\frac{3 k}{4}-5 k+18$, if $k=16$

Exercise 11.9.39

$\frac{-6 a}{5}+3 a+10$, if $a=25$

(Solution on p. 690.)

Exercise 11.9.40

$\frac{-7 h}{9}-7 h-7$, if $h=-18$

Exercise 11.9.41

$5(3 a+4 b)$, if $a=-2$ and $b=2$

(Solution on p. 690.)

Exercise 11.9.42

$7(2 y-x)$, if $x=-1$ and $y=2$ 
Exercise 11.9.43

(Solution on p. 690.)

$-(a-b)$, if $a=0$ and $b=-6$

Exercise 11.9.44

$-(x-x-y)$, if $x=4$ and $y=-4$

Exercise 11.9.45

$(y+2)^{2}-6(y+2)-6$, if $y=2$

Exercise 11.9.46

$(a-7)^{2}-2(a-7)-2$, if $a=7$

(Solution on p. 690.)

11.9.1.2 Combining Like Terms Using Addition and Subtraction (Section 11.3)

For problems 47-56, simplify each expression by combining like terms.

Exercise 11.9.47

(Solution on p. 690.)

$4 a+5-2 a+1$

Exercise 11.9.48

$7 x+3 x-14 x$

Exercise 11.9.49

(Solution on p. 690.)

$-7 b+4 m-3+3 n$

Exercise 11.9.50

$-9 k-8 h-k+6 h$

Exercise 11.9.51

(Solution on p. 690.)

$-x+5 y-8 x-6 x+7 y$

Exercise 11.9.52

$6 n-2 n+6-2-n$

Exercise 11.9.53

(Solution on p. 690.)

$0 m+3 k-5 s+2 m-s$

Exercise 11.9.54

$|-8| a+|2| b-|-4| a$

Exercise 11.9.55

$|6| h-|-7| k+|-12| h+|4| \cdot|-5| h$

(Solution on p. 690.)

Exercise 11.9.56

$|0| a-0 a+0$

11.9.1.3 Equations of the Form $a x=b$ and $\frac{x}{a}=b$, Translating Words to Mathematical Symbols , and Solving Problems (Section 11.5,Section 11.6,Section 11.7)

For problems 57-140, solve each equation.

Exercise 11.9.57

(Solution on p. 690.)

$x+1=5$

Exercise 11.9.58

$y-3=-7$

Exercise 11.9.59

(Solution on p. 690.)

$x+12=10$

Exercise 11.9.60

$x-4=-6$ 
Exercise 11.9.61

(Solution on p. 690.)

$5 x=25$

Exercise 11.9.62

$3 x=17$

Exercise 11.9.63

(Solution on p. 690.)

$\frac{x}{2}=6$

Exercise 11.9.64

$\frac{x}{-8}=3$

Exercise 11.9.65

$\frac{x}{15}=-1$

(Solution on p. 691.)

Exercise 11.9.66 $\frac{x}{-4}=-3$

Exercise 11.9.67

$-3 x=9$

(Solution on p. 691.)

Exercise 11.9.68

$-2 x=5$

Exercise 11.9.69

(Solution on p. 691.)

$-5 x=-5$

Exercise 11.9.70

$-3 x=-1$

Exercise 11.9.71

$\frac{x}{-3}=9$

(Solution on p. 691.)

Exercise 11.9.72

$\frac{a}{-5}=2$

Exercise 11.9.73

$-7=3 y$

(Solution on p. 691.)

Exercise 11.9.74

$-7=\frac{x}{3}$

Exercise 11.9.75

$\frac{m}{4}=\frac{-2}{5}$

Exercise 11.9.76

$4 y=\frac{1}{2}$

Exercise 11.9.77 $\frac{-1}{3}=-5 x$

Exercise 11.9.78 $\frac{-1}{9}=\frac{k}{3}$

Exercise 11.9.79 $\frac{-1}{6}=\frac{s}{-6}$

(Solution on p. 691.)

Exercise 11.9.80

$\frac{0}{4}=4 \mathrm{~s}$

Exercise 11.9.81

$x+2=-1$

(Solution on p. 691.)

(Solution on p. 691.)

Exercise 11.9.82

$x-5=-6$

Exercise 11.9.83

$\frac{-3}{2} x=6$

(Solution on p. 691.) 
Exercise 11.9.84

$3 x+2=7$

Exercise 11.9.85

(Solution on p. 691.)

$-4 x-5=-3$

Exercise 11.9.86

$\frac{x}{6}+1=4$

Exercise 11.9.87

$\frac{a}{-5}-3=-2$

(Solution on p. 691.)

Exercise 11.9.88

$\frac{4 x}{3}=7$

Exercise 11.9.89

$\frac{2 x}{5}+2=8$

(Solution on p. 691.)

Exercise 11.9.90 $\frac{3 y}{2}-4=6$

Exercise 11.9.91

$m+3=8$

(Solution on p. 691.)

Exercise 11.9.92 $\frac{1 x}{2}=2$

Exercise 11.9.93 $\frac{2 a}{3}=5$

(Solution on p. 691.)

Exercise 11.9.94 $\frac{-3 x}{7}-4=4$

Exercise 11.9.95 $\frac{5 x}{-2}-6=-10$

Exercise 11.9.96 $-4 k-6=7$

Exercise 11.9.97 $\frac{-3 x}{-2}+1=4$

Exercise 11.9.98 $\frac{-6 x}{4}=2$

Exercise 11.9.99

$x+9=14$

(Solution on p. 691.)

Exercise 11.9.100

$y+5=21$

Exercise 11.9.101

(Solution on p. 691.)

$y+5=-7$

Exercise 11.9.102

$4 x=24$

Exercise 11.9.103

(Solution on p. 691.)

$4 w=37$

Exercise 11.9.104

$6 y-11=13$

Exercise 11.9.105

(Solution on p. 691.)

$-3 x+8=-7$

(Solution on p. 691.)

Exercise 11.9.106

$3 z+9=-51$ 
Exercise 11.9.107

(Solution on p. 691.)

$\frac{x}{-3}=8$

Exercise 11.9.108

$\frac{6 y}{7}=5$

Exercise 11.9.109

(Solution on p. 691.)

$\frac{w}{2}-15=4$

Exercise 11.9.110

$\frac{x}{-2}-23=-10$

Exercise 11.9.111

$\frac{2 x}{3}-5=8$

(Solution on p. 691.)

Exercise 11.9.112 $\frac{3 z}{4}=\frac{-7}{8}$

Exercise 11.9.113

$-2-\frac{2 x}{7}=3$

(Solution on p. 691.)

Exercise 11.9.114

$3-x=4$

Exercise 11.9.115

(Solution on p. 692.)

$-5-y=-2$

Exercise 11.9.116

$3-z=-2$

Exercise 11.9.117

$3 x+2 x=6$

Exercise 11.9.118

$4 x+1+6 x=10$

Exercise 11.9.119

(Solution on p. 692.)

$6 y-6=-4+3 y$

Exercise 11.9.120

$3=4 a-2 a+a$

Exercise 11.9.121

$3 m+4=2 m+1$

Exercise 11.9.122

$5 w-6=4+2 w$

Exercise 11.9.123

$8-3 a=32-2 a$

(Solution on p. 692.)

Exercise 11.9.124

$5 x-2 x+6 x=13$

Exercise 11.9.125

(Solution on p. 692.)

$x+2=3-x$

Exercise 11.9.126

$5 y+2 y-1=6 y$

Exercise 11.9.127

$x=32$

(Solution on p. 692.)

Exercise 11.9.128

$k=-4$

Exercise 11.9.129

$\frac{3 x}{2}+4=\frac{5 x}{2}=6$

(Solution on p. 692.) 
Exercise 11.9.130

$\frac{x}{3}+\frac{3 x}{3}-2=16$

Exercise 11.9.131

(Solution on p. 692.)

$x-2=6-x$

Exercise 11.9.132

$\frac{-5 x}{7}=\frac{2 x}{7}$

Exercise 11.9.133

(Solution on p. 692.)

$\frac{2 x}{3}+1=5$

Exercise 11.9.134

$\frac{-3 x}{5}+3=\frac{2 x}{5}+2$

Exercise 11.9.135

$\frac{3 x}{4}+5=\frac{-3 x}{4}-11$

(Solution on p. 692.)

Exercise 11.9.136

$\frac{3 x}{7}=\frac{-3 x}{7}+12$

Exercise 11.9.137

$\frac{5 y}{13}-4=\frac{7 y}{26}+1$

(Solution on p. 692.)

Exercise 11.9.138

$\frac{-3 m}{5}=\frac{6 m}{10}-2$

Exercise 11.9.139

$\frac{-3 m}{2}+1=5 m$

(Solution on p. 692.)

Exercise 11.9.140

$-3 z=\frac{2 z}{5}$

\subsection{Proficiency Exam ${ }^{10}$}

\subsubsection{Proficiency Exam}

For problems 1 and 2 specify each term.

Exercise 11.10.1

(Solution on p. 692.)

(Section 11.2) $5 x+6 y+3 z$

Exercise 11.10.2

(Section 11.2) $8 m-2 n-4$

Exercise 11.10.3

(Section 11.2) In the expression $-9 a$, how many $a$ 's are indicated?

(Solution on p. 692.)

For problems 4-9, find the value of each expression.

Exercise 11.10.4

(Section 11.2) $6 a-3 b$, if $a=-2$, and $b=-1$.

(Solution on p. 692.)

Exercise 11.10.5

(Section 11.2) $-5 m+2 n-6$, if $m=-1$ and $n=4$.

(Solution on p. 692.)

Exercise 11.10.6

(Solution on p. 692.)

(Section 11.2) $-x^{2}+3 x-5$, if $x=-2$.

Exercise 11.10.7

(Section 11.2) $y^{2}+9 y+1$, if $y=0$.

(Solution on p. 692.)

\footnotetext{
${ }^{10}$ This content is available online at $<$ http://cnx.org/content $/ \mathrm{m} 35052 / 1.2 />$.
} 
Exercise 11.10.8

(Solution on p. 692.)

(Section 11.2) $-a^{2}+3 a+4$, if $a=4$.

(Solution on p. 692.)

Exercise 11.10.9

(Section 11.2) $-(5-x)^{2}+7(m-x)+x-2 m$, if $x=5$ and $m=5$.

For problems 10-12, simplify each expression by combining like terms.

Exercise 11.10.10

(Solution on p. 692.)

(Section 11.3) $6 y+5-2 y+1$

Exercise 11.10.11

(Section 11.3) $14 a-3 b+5 b-6 a-b$

Exercise 11.10.12

(Section 11.3) $9 x+5 y-7+4 x-6 y+3(-2)$

(Solution on p. 692.)

(Solution on p. 692.)

For problems 13-22, solve each equation.

Exercise 11.10.13

(Solution on p. 693.)

(Section 11.4) $x+7=15$

Exercise 11.10.14

(Section 11.4) $y-6=2$

Exercise 11.10.15

(Section 11.4) $m+8=-1$

Exercise 11.10.16

(Section 11.4) $-5+a=-4$

Exercise 11.10.17

(Section 11.5) $4 x=104$

Exercise 11.10.18

(Section 11.5) $6 y+3=-21$

Exercise 11.10.19

(Section 11.5) $\frac{5 m}{6}=\frac{10}{3}$

Exercise 11.10.20

(Section 11.5) $\frac{7 y}{8}+\frac{1}{4}=\frac{-13}{4}$

Exercise 11.10.21

(Section 11.5) $6 x+5=4 x-11$

Exercise 11.10.22

(Section 11.5) $4 y-8-6 y=3 y+1$

(Solution on p. 693.)

(Solution on p. 693.)

(Solution on p. 693.)

(Solution on p. 693.)

(Solution on p. 693.)

(Solution on p. 693.)

Exercise 11.10.23

(Solution on p. 693.)

(Solution on p. 693.)

(Solution on p. 693.)

(Section 11.6 and Section 11.7) Three consecutive even integers add to -36 . What are they?

Exercise 11.10.24

(Solution on p. 693.)

(Section 11.6 and Section 11.7) The perimeter of a rectangle is 38 feet. Find the length and width of the rectangle if the length is 5 feet less than three times the width.

Exercise 11.10.25

(Solution on p. 693.)

(Section 11.6 and Section 11.7) Four numbers add to -2. The second number is three more than twice the negative of the first number. The third number is six less than the first number. The fourth number is eleven less than twice the first number. Find the numbers. 


\section{Solutions to Exercises in Chapter 11}

Solution to Exercise 11.2.1 (p. 633)

$x, 7$

Solution to Exercise 11.2.2 (p. 633)

$3 m-6 n$

Solution to Exercise 11.2.3 (p. 633)

$5 y$

Solution to Exercise 11.2.4 (p. 633) $a, 2 b,-c$

Solution to Exercise 11.2.5 (p. 633)

$-3 x,-5$

Solution to Exercise 11.2.6 (p. 635)

$-28$

Solution to Exercise 11.2.7 (p. 635)

$-3$

Solution to Exercise 11.2.8 (p. 635)

$-6$

Solution to Exercise 11.2.9 (p. 635)

104

Solution to Exercise 11.2.10 (p. 635)

24

Solution to Exercise 11.2.11 (p. 635)

$-12$

Solution to Exercise 11.2.12 (p. 636)

$-1$

Solution to Exercise 11.2.13 (p. 636)

31

Solution to Exercise 11.2.14 (p. 636)

Addition; multiplication

Solution to Exercise 11.2.16 (p. 636)

$5 x, 18 y$

Solution to Exercise 11.2.18 (p. 636)

$8 s, 2 r,-7 t$

Solution to Exercise 11.2.20 (p. 636)

$7 a,-2 b,-3 c,-4 d$

Solution to Exercise 11.2.22 (p. 636)

$-x,-y$

Solution to Exercise 11.2.24 (p. 636)

$m$

Solution to Exercise 11.2.26 (p. 636) 5

Solution to Exercise 11.2.28 (p. 636) $-30$

Solution to Exercise 11.2.30 (p. 636)

$-3$

Solution to Exercise 11.2.32 (p. 637)

$-14$

Solution to Exercise 11.2.34 (p. 637)

56

Solution to Exercise 11.2.36 (p. 637)

64 
Solution to Exercise 11.2.38 (p. 637) 1

Solution to Exercise 11.2.40 (p. 637)

75

Solution to Exercise 11.2.42 (p. 637)

24

Solution to Exercise 11.2.44 (p. 637) 39

Solution to Exercise 11.2.46 (p. 637) 5

Solution to Exercise 11.2.48 (p. 637) $-7$

Solution to Exercise 11.2.50 (p. 637) 5

Solution to Exercise 11.2.52 (p. 638) $\frac{181}{24}=7 \frac{13}{24}$

Solution to Exercise 11.2.54 (p. 638) $\frac{20}{27}$

Solution to Exercise 11.2.56 (p. 638) 0

Solution to Exercise 11.3.1 (p. 639) $13 x$

Solution to Exercise 11.3.2 (p. 639) $11 a+6 b$

Solution to Exercise 11.3.3 (p. 639) $9 m-7 n$

Solution to Exercise 11.3.4 (p. 639)

$-2 a-r$

Solution to Exercise 11.3.5 (p. 639) 0

Solution to Exercise 11.3.6 (p. 640)

$11 a$

Solution to Exercise 11.3.8 (p. 640)

$4 h$

Solution to Exercise 11.3.10 (p. 640) $3 m+3 n$

Solution to Exercise 11.3.12 (p. 640) $17 s-r$

Solution to Exercise 11.3.14 (p. 640) $5 h+9 a-18 k$

Solution to Exercise 11.3.16 (p. 640) 0

Solution to Exercise 11.3.18 (p. 640) $16 m$

Solution to Exercise 11.3.20 (p. 640) $8 h+7 k$

Solution to Exercise 11.3.22 (p. 640) $5 \Delta-3 \star$

Solution to Exercise 11.3.24 (p. 640) $16 x-15 y-9$ 
Solution to Exercise 11.3.26 (p. 641)

$2 \frac{2}{11}$

Solution to Exercise 11.3.28 (p. 641)

7

Solution to Exercise 11.3.30 (p. 641)

6

Solution to Exercise 11.4.1 (p. 643)

$5+6 \stackrel{?}{=} 11$

Substitute 5 into $m+6=11 . \quad \mathbf{1 1} \cong \mathbf{1 1}$ Thus, 5 is a solution.

Solution to Exercise 11.4.2 (p. 643)

$$
\begin{array}{r}
2(-5)-4 \stackrel{?}{=}-14 \\
-10-4 \stackrel{?}{=}-14
\end{array}
$$

Substitute -5 into $2 m-4=-14$. $\quad-\mathbf{1 4} \stackrel{\underline{\Perp}-14}{ }$ Thus, -5 is a solution.

Solution to Exercise 11.4.3 (p. 643)

$$
\begin{aligned}
5(0)+1 & \stackrel{?}{=} 1 \\
0+1 & \stackrel{?}{=} 1
\end{aligned}
$$

Substitute 0 into $5 x+1=1$. $1 \cong 1$ Thus, 0 is a solution.

Solution to Exercise 11.4.4 (p. 643)

Substitute 3 into $-3 y+1=4 y+5$.

$$
\begin{aligned}
-3(3)+1 & \stackrel{?}{=} 4(3)+5 \\
-9+1 & \stackrel{?}{=} 12+5 \\
-8 & \neq 17
\end{aligned}
$$

Solution to Exercise 11.4.5 (p. 643)

$$
\begin{aligned}
6(-1)-5+2(-1) & \stackrel{?}{=} 7(-1)-6 \\
-6-5-2 & \stackrel{?}{=}-7-6 \\
-13 & \stackrel{ }{=}-13
\end{aligned}
$$

Substitute -1 into $6 m-5+2 m=7 m-6$.

solution.

Solution to Exercise 11.4.6 (p. 646)

$y=-5$

Solution to Exercise 11.4.7 (p. 646)

$a=15$

Solution to Exercise 11.4 .8 (p. 646)

$x=3$

Solution to Exercise 11.4 .9 (p. 646)

$m=2$

Solution to Exercise 11.4.10 (p. 646)

$k=-2$

Solution to Exercise 11.4.11 (p. 646)

$a=-1$

Solution to Exercise 11.4.12 (p. 646)

Substitute $x=4$ into the equation $4 x-11=5$.

$16-11=5$

$5=5$

$x=4$ is a solution.

Solution to Exercise 11.4.14 (p. 646)

Substitute $m=1$ into the equation $2 m-1=1$. 
$2-1 \stackrel{?}{\stackrel{1}{1}}$

$1 \cong 1$

$m=1$ is a solution.

Solution to Exercise 11.4.16 (p. 647)

Substitute $x=-8$ into the equation $3 x+2-7=-5 x-6$.

$x=-8$ is a solution.

Solution to Exercise 11.4.18 (p. 647)

Substitute $x=0$ into the equation $-8+x=-8$.

$-8+0 \stackrel{?}{=}-8$

$-8 \underline{\underline{\perp}}-8$

$x=0$ is a solution.

Solution to Exercise 11.4.20 (p. 647)

Substitute $x=\frac{15}{2}$ into the equation $4 x-5=6 x-20$.

$30-5 \stackrel{?}{=} 45-20$

$25 \underline{25}$

$x=\frac{15}{2}$ is a solution.

Solution to Exercise 11.4.22 (p. 647)

$y=11$

Solution to Exercise 11.4.24 (p. 647)

$k=5$

Solution to Exercise 11.4.26 (p. 647)

$a=-9$

Solution to Exercise 11.4.28 (p. 647)

$x=11$

Solution to Exercise 11.4.30 (p. 647)

$z=0$

Solution to Exercise 11.4.32 (p. 647)

$x=8$

Solution to Exercise 11.4.34 (p. 647)

$m=1$

Solution to Exercise 11.4.36 (p. 647)

$k=19$

Solution to Exercise 11.4.38 (p. 648)

$m=10$

Solution to Exercise 11.4.40 (p. 648)

$b=-21$

Solution to Exercise 11.4.42 (p. 648)

$a=16$

Solution to Exercise 11.4.44 (p. 648)

$y=7.224$

Solution to Exercise 11.4.46 (p. 648)

$m=-0.778$

Solution to Exercise 11.4.48 (p. 648)

$k=-9.01$

Solution to Exercise 11.4.50 (p. 648)

$y=-28.06$

Solution to Exercise 11.4.52 (p. 648)

rate 
Solution to Exercise 11.4.54 (p. 648)

3.5

Solution to Exercise 11.4.56 (p. 648)

$13 x+18 y$

Solution to Exercise 11.5.1 (p. 652)

$x=3$

Solution to Exercise 11.5.2 (p. 652)

$x=-13$

Solution to Exercise 11.5.3 (p. 652)

$x=-32$

Solution to Exercise 11.5.4 (p. 652)

$x=16$

Solution to Exercise 11.5.5 (p. 652)

$y=-3$

Solution to Exercise 11.5.6 (p. 652)

$k=2$

Solution to Exercise 11.5.7 (p. 654)

$m=-4$

Solution to Exercise 11.5.8 (p. 654)

$a=-5$

Solution to Exercise 11.5.9 (p. 654)

$a=1$

Solution to Exercise 11.5.10 (p. 654)

$x=-3$

Solution to Exercise 11.5.11 (p. 654)

$m=-\frac{7}{2}$

Solution to Exercise 11.5.12 (p. 654)

$y=0$

Solution to Exercise 11.5.13 (p. 655)

$x=6$

Solution to Exercise 11.5.15 (p. 655)

$x=12$

Solution to Exercise 11.5.17 (p. 655)

$a=-8$

Solution to Exercise 11.5.19 (p. 655)

$y=14$

Solution to Exercise 11.5.21 (p. 655)

$m=-31$

Solution to Exercise 11.5.23 (p. 655)

$x=28$

Solution to Exercise 11.5.25 (p. 655)

$z=84$

Solution to Exercise 11.5.27 (p. 655)

$m=-4$

Solution to Exercise 11.5.29 (p. 655)

$x=-1$

Solution to Exercise 11.5.31 (p. 655) $y=\frac{13}{2}$

Solution to Exercise 11.5.33 (p. 655)

$k=-2$ 
Solution to Exercise 11.5.35 (p. 656) $x=\frac{11}{2}$ or $5 \frac{1}{2}$

Solution to Exercise 11.5.37 (p. 656) $a=-\frac{5}{4}$

Solution to Exercise 11.5.39 (p. 656)

$m=-1$

Solution to Exercise 11.5.41 (p. 656)

$x=0$

Solution to Exercise 11.5.43 (p. 656)

$a=14$

Solution to Exercise 11.5.45 (p. 656)

$x=6$

Solution to Exercise 11.5.47 (p. 656)

$a=4$

Solution to Exercise 11.5.49 (p. 656)

$40(30-2)=1200-80=1120$

Solution to Exercise 11.5.51 (p. 656)

$220 \mathrm{sq} \mathrm{cm}$

Solution to Exercise 11.5.53 (p. 657)

$x=4$

Solution to Exercise 11.6.1 (p. 658)

$12+x$

Solution to Exercise 11.6.2 (p. 658)

$8-x$

Solution to Exercise 11.6.3 (p. 658)

$x-14$

Solution to Exercise 11.6.4 (p. 658)

$6 x=54$

Solution to Exercise 11.6.5 (p. 658)

$\frac{2}{9} x=11$

Solution to Exercise 11.6.6 (p. 659)

$3+7 x=9+5 x$

Solution to Exercise 11.6.7 (p. 659)

$2 x-8=3 x+1$ or $2 x-8=1+3 x$

Solution to Exercise 11.6.8 (p. 659)

$\frac{x}{8}+7=50$

Solution to Exercise 11.6.9 (p. 659)

$\frac{2}{3}-6 x=x+1$

Solution to Exercise 11.6.10 (p. 659)

$x-9=4$

Solution to Exercise 11.6.11 (p. 659)

$x-5=8$

Solution to Exercise 11.6.12 (p. 660)

$x-12$

Solution to Exercise 11.6.14 (p. 660)

$x-4$

Solution to Exercise 11.6.16 (p. 660)

$x+1$

Solution to Exercise 11.6.18 (p. 660)

$-7+x$ 
Solution to Exercise 11.6.20 (p. 660) $x+(-6)$

Solution to Exercise 11.6.22 (p. 660) $x-[-(-1)]$

Solution to Exercise 11.6.24 (p. 660) $3 x+11$

Solution to Exercise 11.6.26 (p. 660) $2 x-7=4$

Solution to Exercise 11.6.28 (p. 660) $14+2 x=6$

Solution to Exercise 11.6.30 (p. 660) $\frac{3}{5} x+8=50$

Solution to Exercise 11.6.32 (p. 660) $\frac{4}{3} x+12=5$

Solution to Exercise 11.6.34 (p. 661)

$8 x+5=x+26$

Solution to Exercise 11.6.36 (p. 661)

$\frac{x}{6}+9=1$

Solution to Exercise 11.6.38 (p. 661)

$\frac{x}{7}+2=17$

Solution to Exercise 11.6.40 (p. 661)

$x-2=10$

Solution to Exercise 11.6.42 (p. 661)

$x-3=2 x-6$

Solution to Exercise 11.6.44 (p. 661)

$5 x-12=x-2$

Solution to Exercise 11.6.46 (p. 661)

$6-x=x+4$

Solution to Exercise 11.6.48 (p. 661)

$9-x+1=3 x+8$

Solution to Exercise 11.6.50 (p. 661)

$2 x-7-4 x=-x-6$

Solution to Exercise 11.6.52 (p. 662)

$\frac{3}{4}$

Solution to Exercise 11.6.54 (p. 662)

$8 \frac{2}{3}$

Solution to Exercise 11.6.56 (p. 662)

$x=-8$

Solution to Exercise 11.7.1 (p. 665)

$-6$

Solution to Exercise 11.7.2 (p. 665)

6

Solution to Exercise 11.7.3 (p. 665)

17 and 18

Solution to Exercise 11.7.4 (p. 665)

$-8,-6$, and -4

Solution to Exercise 11.7.5 (p. 666)

3 inches, 5 inches, and 8 inches

Solution to Exercise 11.7.6 (p. 667)

24 
Solution to Exercise 11.7.8 (p. 667)

9

Solution to Exercise 11.7.10 (p. 667)

10

Solution to Exercise 11.7.12 (p. 668)

6

Solution to Exercise 11.7.14 (p. 668)

1

Solution to Exercise 11.7.16 (p. 668)

Length $=12$ inches, Width $=6$ inches

Solution to Exercise 11.7.18 (p. 669)

$15,16,17$

Solution to Exercise 11.7.20 (p. 669)

$-2,-1,0,1,2$

Solution to Exercise 11.7.22 (p. 670)

Length is 7 , width is 2

Solution to Exercise 11.7.24 (p. 670)

Length is 15 , width is 22

Solution to Exercise 11.7.26 (p. 670)

The perimeter is 20 inches. Other answers are possible. For example, perimeters such as 26,32 are possible.

Solution to Exercise 11.7.28 (p. 670)

15 meters

Solution to Exercise 11.7.30 (p. 670)

$n=0$

Solution to Exercise 11.7.32 (p. 670)

$n=1$

Solution to Exercise 11.7.34 (p. 671)

$-2,-1,0,1$

Solution to Exercise 11.7.36 (p. 671)

$-35,-33,-31$

Solution to Exercise 11.7.38 (p. 671)

...because the sum of any even number (in this case, 2) o even integers (consecutive or not) is even and, therefore, cannot be odd (in this case, 139)

Solution to Exercise 11.7.40 (p. 671)

$2,15,20$

Solution to Exercise 11.7.42 (p. 671)

6 feet tall

Solution to Exercise 11.7.44 (p. 671)

$46.3 \mathrm{cg}$

Solution to Exercise 11.9.1 (p. 674)

$6 a,-2 b, 5 c$

Solution to Exercise 11.9.3 (p. 674)

$7 m,-3 n$

Solution to Exercise 11.9.5 (p. 674)

$x, 2 n,-z$

Solution to Exercise 11.9.7 (p. 674)

$-y,-3 z$

Solution to Exercise 11.9.9 (p. 674)

$-4$

Solution to Exercise 11.9.11 (p. 674)

$k$ 
Solution to Exercise 11.9.13 (p. 674) 7

Solution to Exercise 11.9.15 (p. 674)

$-5$

Solution to Exercise 11.9.17 (p. 674)

$-27$

Solution to Exercise 11.9.19 (p. 674)

1

Solution to Exercise 11.9.21 (p. 675)

$-5$

Solution to Exercise 11.9.23 (p. 675)

0

Solution to Exercise 11.9.25 (p. 675)

5

Solution to Exercise 11.9.27 (p. 675)

18

Solution to Exercise 11.9.29 (p. 675)

20

Solution to Exercise 11.9.31 (p. 675)

11

Solution to Exercise 11.9.33 (p. 675)

$-1$

Solution to Exercise 11.9.35 (p. 675)

$-93$

Solution to Exercise 11.9.37 (p. 675)

45

Solution to Exercise 11.9.39 (p. 675)

55

Solution to Exercise 11.9.41 (p. 675)

10

Solution to Exercise 11.9.43 (p. 676)

$-6$

Solution to Exercise 11.9.45 (p. 676)

$-14$

Solution to Exercise 11.9.47 (p. 676)

$2 a+6$

Solution to Exercise 11.9.49 (p. 676)

$-4 n+4 m-3$

Solution to Exercise 11.9.51 (p. 676)

$-15 x+12 y$

Solution to Exercise 11.9.53 (p. 676)

$3 k+2 m-6 s$

Solution to Exercise 11.9.55 (p. 676) $38 h-7 k$

Solution to Exercise 11.9.57 (p. 676) $x=4$

Solution to Exercise 11.9.59 (p. 676) $x=-2$

Solution to Exercise 11.9.61 (p. 677) $x=5$ 
Solution to Exercise 11.9.63 (p. 677) $x=12$

Solution to Exercise 11.9.65 (p. 677) $x=-15$

Solution to Exercise 11.9.67 (p. 677) $x=-3$

Solution to Exercise 11.9.69 (p. 677) $x=1$

Solution to Exercise 11.9.71 (p. 677) $x=-27$

Solution to Exercise 11.9.73 (p. 677) $y=-\frac{7}{3}$

Solution to Exercise 11.9.75 (p. 677) $m=-\frac{8}{5}$

Solution to Exercise 11.9.77 (p. 677) $x=\frac{1}{15}$

Solution to Exercise 11.9.79 (p. 677) $s=1$

Solution to Exercise 11.9.81 (p. 677) $x=-3$

Solution to Exercise 11.9.83 (p. 677) $x=-4$

Solution to Exercise 11.9.85 (p. 678) $x=-\frac{1}{2}$

Solution to Exercise 11.9.87 (p. 678) $a=-5$

Solution to Exercise 11.9.89 (p. 678) $x=15$

Solution to Exercise 11.9.91 (p. 678) $x=5$

Solution to Exercise 11.9.93 (p. 678) $a=\frac{15}{2}$

Solution to Exercise 11.9.95 (p. 678) $x=\frac{8}{5}$

Solution to Exercise 11.9.97 (p. 678) $x=2$

Solution to Exercise 11.9.99 (p. 678) $x=5$

Solution to Exercise 11.9.101 (p. 678) $y=-12$

Solution to Exercise 11.9.103 (p. 678) $w=\frac{37}{4}$

Solution to Exercise 11.9.105 (p. 678) $x=5$

Solution to Exercise 11.9.107 (p. 679) $x=-24$

Solution to Exercise 11.9.109 (p. 679) $w=38$

Solution to Exercise 11.9.111 (p. 679) $x=\frac{39}{2}$ 
Solution to Exercise 11.9.113 (p. 679) $x=-\frac{35}{2}$

Solution to Exercise 11.9.115 (p. 679)

$y=-3$

Solution to Exercise 11.9.117 (p. 679) $x=\frac{6}{5}$

Solution to Exercise 11.9.119 (p. 679) $y=\frac{2}{3}$

Solution to Exercise 11.9.121 (p. 679) $m=-3$

Solution to Exercise 11.9.123 (p. 679) $a=-24$

Solution to Exercise 11.9.125 (p. 679) $x=\frac{1}{2}$

Solution to Exercise 11.9.127 (p. 679) $x=32$

Solution to Exercise 11.9.129 (p. 679) $x=-2$

Solution to Exercise 11.9.131 (p. 680) $x=4$

Solution to Exercise 11.9.133 (p. 680) $x=6$

Solution to Exercise 11.9.135 (p. 680) $x=\frac{-32}{3}$

Solution to Exercise 11.9.137 (p. 680) $y=\frac{130}{3}$

Solution to Exercise 11.9.139 (p. 680) $m=\frac{2}{13}$

Solution to Exercise 11.10.1 (p. 680) $5 x, 6 y, 3 z$

Solution to Exercise 11.10.2 (p. 680) $8 m,-2 n,-4$

Solution to Exercise 11.10.3 (p. 680) $-9$

Solution to Exercise 11.10.4 (p. 680) $-9$

Solution to Exercise 11.10.5 (p. 680) 7

Solution to Exercise 11.10.6 (p. 680)

$-15$

Solution to Exercise 11.10.7 (p. 680) 1

Solution to Exercise 11.10.8 (p. 681) 0

Solution to Exercise 11.10.9 (p. 681) $-5$

Solution to Exercise 11.10.10 (p. 681) $4 y+6$

Solution to Exercise 11.10.11 (p. 681) $8 a+b$ 
Solution to Exercise 11.10.12 (p. 681)

$13 x-y-13$

Solution to Exercise 11.10.13 (p. 681)

$x=8$

Solution to Exercise 11.10.14 (p. 681)

$y=8$

Solution to Exercise 11.10.15 (p. 681)

$m=-9$

Solution to Exercise 11.10.16 (p. 681)

$a=1$

Solution to Exercise 11.10.17 (p. 681)

$x=26$

Solution to Exercise 11.10.18 (p. 681)

$y=-4$

Solution to Exercise 11.10.19 (p. 681)

$m=4$

Solution to Exercise 11.10.20 (p. 681)

$y=-4$

Solution to Exercise 11.10.21 (p. 681)

$x=-8$

Solution to Exercise 11.10.22 (p. 681)

$y=\frac{-9}{5}$

Solution to Exercise 11.10.23 (p. 681)

$-14,-12,-10$

Solution to Exercise 11.10.24 (p. 681)

$l=13, w=6$

Solution to Exercise 11.10.25 (p. 681)

$6,-9,0,1$ 


\section{Index of Keywords and Terms}

Keywords are listed by the section with that keyword (page numbers are in parentheses). Keywords do not necessarily appear in the text of the page. They are merely associated with that section. Ex. apples, $\S 1.1$ (1) Terms are referenced by the page they appear on. Ex. apples, 1

" $\quad$ "invert the divisor and, 257

- . Denominate numbers, 420

A absolute value, $\S 10.4(588), 588$

Acknowledgments, $\S(7)$

add whole numbers, 30

addends, 30, 69

Adding, § 1.1(9), § 1.7(62), § 1.9(69),

$\S 1.10(74)$

addition, $\S 1.5(29), 30, \S 1.7(62), \S 1.8(68), 69$, $\S 1.9(69), \S 1.10(74), \S 5.1(295), \S 5.2(296)$, $\S 5.3(300), \S 5.4(306), \S 5.5(310), \S 5.6(314)$, $\S 5.7(318), \S 5.8(323), \S 5.9(323), \S 5.10(327)$, $\S 6.1(339), \S 6.2(340), \S 6.3(346), \S 6.4(350)$, $\S 6.5(354), \S 6.6(360), \S 6.7(371), \S 6.8(383)$, $\S 6.9(388), \S 6.10(394), \S 6.11(398)$,

$\S 6.12(399), \S 6.13(402), \S 10.5(592)$, $\S 11.3(638)$ addition/subtraction property of equality, 643 additive identity, $\S 1.7(62), 64,69,594$ algebra, §11.1(631), § 11.2(632), § 11.3(638), $\S 11.4(641), \S 11.5(649), \S 11.6(657)$, $\S 11.7(662), \S 11.8(672), \S 11.9(674)$, $\S 11.10(680)$

algebraic expression, 632

Answers to Practice Sets, 2

approximation, $\S 1.4(21)$

area, $\S 9.6(542), 542$

associative property, $\S 2.6(130)$

associative property of addition, $\S 1.7(62)$

B Bar, 162

base, $155, \S 7.7(448), 448$

base ten, 11,68

Base Ten Positional Number System, § 1.2(10)

borrow more than once, 49

borrowing, § 1.6(44), 48

Braces, 162

Brackets, 162

Burzynski, § (1), § 1.1(9), § 2.1(91), $\S 3.1(153), \S 3.2(154), \S 3.3(162), \S 3.4(172)$,
$\S 3.5(180), \S 3.6(184), \S 3.7(191), \S 3.8(193)$, $\S 3.9(198), \S 4.1(213), \S 4.2(214), \S 4.3(223)$, $\S 4.4(233), \S 4.5(244), \S 4.6(255), \S 4.7(261)$, $\S 4.8(268), \S 4.9(270), \S 4.10(276), \S 5.1(295)$, $\S 5.2(296), \S 5.3(300), \S 5.4(306), \S 5.5(310)$, $\S 5.6(314), \S 5.7(318), \S 5.8(323), \S 5.9(323)$, $\S 5.10(327), \S 6.1(339), \S 6.2(340), \S 6.3(346)$, $\S 6.4(350), \S 6.5(354), \S 6.6(360), \S 6.7(371)$, $\S 6.8(383), \S 6.9(388), \S 6.10(394), \S 6.11(398)$, $\S 6.12(399), \S 6.13(402), \S 7.1(419), \S 7.2(419)$, $\S 7.3(425), \S 7.4(431), \S 7.5(437), \S 7.6(444)$, $\S 7.7(448), \S 7.8(459), \S 7.9(460), \S 7.10(464)$, $\S 8.1(477), \S 8.2(477), \S 8.3(484), \S 8.4(487)$, $\S 8.5(493), \S 8.6(496), \S 8.7(496), \S 8.8(502)$, $\S 9.1(513), \S 9.2(514), \S 9.3(519), \S 9.4(524)$, $\S 9.5(532), \S 9.6(542), \S 9.7(557), \S 9.8(559)$, $\S 9.9(564), \S 10.1(577), \S 10.2(578)$, $\S 10.3(584), \S 10.4(588), \S 10.5(592)$, $\S 10.6(599), \S 10.7(604), \S 10.8(613)$, $\S 10.9(614), \S 10.10(618), \S 11.1(631)$, $\S 11.2(632), \S 11.3(638), \S 11.4(641)$, $\S 11.5(649), \S 11.6(657), \S 11.7(662)$, $\S 11.8(672), \S 11.9(674), \S 11.10(680)$

C calculator, $\S 1.6(44), \S 2.3(105)$

calculators, $\S 1.5(29)$

cancelling common factors, 235

carrying, 32, 93

circumference, $\S 9.5(532), 534$

cluster, 484

clustering, § 8.3(484)

coefficient, 633, 633

combining terms, $\S 11.3(638)$

commas, 11, 68

common factor, 180

commutative property, $\S 2.6(130)$

commutative property of addition, $\S 1.7(62)$

Comparison by division, 420

Comparison of two numbers by subtraction, 420

complex decimals, 347

complex fraction, 314 
complex fractions, $\S 5.6(314)$

composite number, 174

conditional equations, 641

constant, 578

conversion, $\S 6.3(346)$

coordinate, 578

counting numbers, 579

cross products, 233

cube root, 156, 156

cubed, 155

D decimal fraction, 341

decimal fractions, 341

decimal point, 341

decimals, § 6.1(339), § 6.2(340), 341,

$\S 6.3(346), \S 6.4(350), \S 6.5(354), \S 6.6(360)$,

$\S 6.7(371), \S 6.8(383), \S 6.9(388), \S 6.10(394)$,

$\S 6.11(398), \S 6.12(399), \S 6.13(402)$

denominate numbers, $\S 7.2(419), 524$

denominator, 215,515

diameter, § 9.5(532), 534

difference, 45

digits, 11, 68

distributive property, $\S 8.4(487), 488$

divide, $\S 2.3(105), \S 2.4(113), \S 2.5(126)$

dividend, 105, 136

divisible by 10,128

divisible by 2,126

divisible by 3,126

divisible by 4,126

divisible by 5,127

divisible by 6,127

divisible by 8,127

divisible by 9,128

division, $\S 2.3(105), 105, \S 2.4(113), \S 2.5(126)$,

$\S 2.7(136), 136, \S 2.8(137), \S 2.9(140)$,

$\S 4.1(213), \S 4.2(214), \S 4.3(223), \S 4.4(233)$,

$\S 4.5(244), \S 4.6(255), \S 4.7(261), \S 4.8(268)$,

$\S 4.9(270), \S 4.10(276), \S 6.1(339), \S 6.2(340)$,

$\S 6.3(346), \S 6.4(350), \S 6.5(354), \S 6.6(360)$,

$\S 6.7(371), \S 6.8(383), \S 6.9(388), \S 6.10(394)$,

$\S 6.11(398), \S 6.12(399), \S 6.13(402)$,

$\S 10.7(604)$

divisor, 105, 136

double-negative, $\S 10.3(584)$

E Elementary, § 1.2(10), § 1.3(15), § 1.4(21)

elementary algebra, $\S(1)$

elementary math, $\S 1.5(29), \S 1.6(44)$,

$\S 1.7(62), \S 1.8(68), \S 1.9(69), \S 2.2(92)$,

$\S 2.3(105), \S 2.5(126), \S 2.6(130), \S 2.7(136)$,

$\S 2.8(137), \S 2.9(140)$
Ellis, $\S(1), \S 1.1(9), \S 2.1(91), \S 3.1(153)$, $\S 3.2(154), \S 3.3(162), \S 3.4(172), \S 3.5(180)$, $\S 3.6(184), \S 3.7(191), \S 3.8(193), \S 3.9(198)$, $\S 4.1(213), \S 4.2(214), \S 4.3(223), \S 4.4(233)$, $\S 4.5(244), \S 4.6(255), \S 4.7(261), \S 4.8(268)$, $\S 4.9(270), \S 4.10(276), \S 5.1(295), \S 5.2(296)$, $\S 5.3(300), \S 5.4(306), \S 5.5(310), \S 5.6(314)$, $\S 5.7(318), \S 5.8(323), \S 5.9(323), \S 5.10(327)$, $\S 6.1(339), \S 6.2(340), \S 6.3(346), \S 6.4(350)$, $\S 6.5(354), \S 6.6(360), \S 6.7(371), \S 6.8(383)$, $\S 6.9(388), \S 6.10(394), \S 6.11(398)$, $\S 6.12(399), \S 6.13(402), \S 7.1(419), \S 7.2(419)$, $\S 7.3(425), \S 7.4(431), \S 7.5(437), \S 7.6(444)$, $\S 7.7(448), \S 7.8(459), \S 7.9(460), \S 7.10(464)$, $\S 8.1(477), \S 8.2(477), \S 8.3(484), \S 8.4(487)$, $\S 8.5(493), \S 8.6(496), \S 8.7(496), \S 8.8(502)$, $\S 9.1(513), \S 9.2(514), \S 9.3(519), \S 9.4(524)$, $\S 9.5(532), \S 9.6(542), \S 9.7(557), \S 9.8(559)$, $\S 9.9(564), \S 10.1(577), \S 10.2(578)$,

$\S 10.3(584), \S 10.4(588), \S 10.5(592)$, $\S 10.6(599), \S 10.7(604), \S 10.8(613)$, $\S 10.9(614), \S 10.10(618), \S 11.1(631)$, $\S 11.2(632), \S 11.3(638), \S 11.4(641)$, $\S 11.5(649), \S 11.6(657), \S 11.7(662)$, $\S 11.8(672), \S 11.9(674), \S 11.10(680)$ equal symbol, 30 equation solving, 643 equations, $\S 11.1(631), \S 11.2(632)$, $\S 11.3(638), \S 11.4(641), \S 11.5(649)$, $\S 11.6(657), \S 11.7(662), \S 11.8(672)$, $\S 11.9(674), \S 11.10(680)$

equivalent fractions, $\S 4.4(233), 233$

estimation, $\S 8.1(477), \S 8.2(477), 478$, $\S 8.3(484), \S 8.4(487), \S 8.5(493), \S 8.6(496)$, $\S 8.7(496), \S 8.8(502)$

exact divisions, 383

exam, $\S 1.10(74)$

Exercise Supplement, 2

exercises, § 1.9(69), § 1.10(74)

Exercises for Review, 2

exponent, 154, 154, 155

exponents, § 3.1(153), § 3.2(154), § 3.3(162), $\S 3.4(172), \S 3.5(180), \S 3.6(184), \S 3.7(191)$, $\S 3.8(193), \S 3.9(198)$

Exponential notation, 154 expressions, § 11.1(631), § 11.2(632), 632, $\S 11.3(638), \S 11.4(641), \S 11.5(649)$, $\S 11.6(657), \S 11.7(662), \S 11.8(672)$, $\S 11.9(674), \S 11.10(680)$

F factor, 172, § 4.7(261), § 7.3(425) factorization, § 3.1(153), § 3.2(154), § 3.3(162), 
$\S 3.4(172), \S 3.5(180), \S 3.6(184), \S 3.7(191)$, $\S 3.8(193), \S 3.9(198)$

factors, 93, 136, 172, § 11.2(632), 632

facts, $\S 2.5(126)$

first partial product, 95

five-step method, § 7.4(431)

formula, 535

fourth root, 156

fraction, 215

fraction bar, 215

fractions, $\S 4.1(213), \S 4.2(214), \S 4.3(223)$, $\S 4.4(233), \S 4.5(244), \S 4.6(255), \S 4.7(261)$, $\S 4.8(268), \S 4.9(270), \S 4.10(276), \S 5.1(295)$, $\S 5.2(296), \S 5.3(300), \S 5.4(306), \S 5.5(310)$, $\S 5.6(314), \S 5.7(318), \S 5.8(323), \S 5.9(323)$, $\S 5.10(327), \S 8.5(493), 579$

fractions of one percent, $\S 7.6(444)$

functions, § 11.2(632)

Fundamentals, § (1), § (7), § 1.1(9), § 2.1(91), $\S 2.2(92), \S 3.1(153), \S 3.2(154), \S 3.3(162)$, $\S 3.4(172), \S 3.5(180), \S 3.6(184), \S 3.7(191)$, $\S 3.8(193), \S 3.9(198), \S 4.1(213), \S 4.2(214)$, $\S 4.3(223), \S 4.4(233), \S 4.5(244), \S 4.6(255)$, $\S 4.7(261), \S 4.8(268), \S 4.9(270), \S 4.10(276)$, $\S 5.1(295), \S 5.2(296), \S 5.3(300), \S 5.4(306)$, $\S 5.5(310), \S 5.6(314), \S 5.7(318), \S 5.8(323)$, $\S 5.9(323), \S 5.10(327), \S 6.1(339), \S 6.2(340)$, $\S 6.3(346), \S 6.4(350), \S 6.5(354), \S 6.6(360)$, $\S 6.7(371), \S 6.8(383), \S 6.9(388), \S 6.10(394)$, $\S 6.11(398), \S 6.12(399), \S 6.13(402)$,

$\S 7.1(419), \S 7.2(419), \S 7.3(425), \S 7.4(431)$, $\S 7.5(437), \S 7.6(444), \S 7.7(448), \S 7.8(459)$, $\S 7.9(460), \S 7.10(464), \S 8.1(477), \S 8.2(477)$, $\S 8.3(484), \S 8.4(487), \S 8.5(493), \S 8.6(496)$, $\S 8.7(496), \S 8.8(502), \S 9.1(513), \S 9.2(514)$, $\S 9.3(519), \S 9.4(524), \S 9.5(532), \S 9.6(542)$, $\S 9.7(557), \S 9.8(559), \S 9.9(564), \S 10.1(577)$, $\S 10.2(578), \S 10.3(584), \S 10.4(588)$, $\S 10.5(592), \S 10.6(599), \S 10.7(604)$, $\S 10.8(613), \S 10.9(614), \S 10.10(618)$, $\S 11.1(631), \S 11.2(632), \S 11.3(638)$, $\S 11.4(641), \S 11.5(649), \S 11.6(657)$, $\S 11.7(662), \S 11.8(672), \S 11.9(674)$, $\S 11.10(680)$

fundamentals of mathematics, $\S 2.4(113)$, $\S 2.5(126), \S 2.6(130), \S 2.7(136), \S 2.8(137)$, $\S 2.9(140)$

G GCF, 181

geometry, § 9.1(513), § 9.2(514), § 9.3(519), $\S 9.4(524), \S 9.5(532), \S 9.6(542), \S 9.7(557)$, $\S 9.8(559), \S 9.9(564)$ graph, 578

Graphing, 68

Graphing Whole Numbers, $\S 1.2(10)$

greatest common factor, $\S 3.5(180), 181$

greatest common factor (GCF), 181

grouping symbols, $\S 5.5(310)$

H Hindu-Arabic, 68

Hindu-Arabic Numeration System, § 1.2(10), 11

hyphen, 218

I improper fraction, $\S 4.3(223)$

indeterminant, 108

index, 156

integers, 579

K k-12, § (1)

key concepts, $\S 1.8(68)$

L LCM, 186, 186

least common multiple, $\S 3.6(184), 186,186$

like denominate numbers, 420

like denominators, $\S 5.2(296)$

like terms, $\S 11.3(638), 638$

line, 13

lowest terms, $\S 4.4(233)$

M Mass, 521

math, § 1.2(10), § 1.3(15), § 1.4(21)

Mathematics, § (1), § (7), § 1.1(9), § 1.7(62), $\S 2.1(91), \S 2.2(92), \S 2.3(105), \S 3.1(153)$, $\S 3.2(154), \S 3.3(162), \S 3.4(172), \S 3.5(180)$, $\S 3.6(184), \S 3.7(191), \S 3.8(193), \S 3.9(198)$, $\S 4.1(213), \S 4.2(214), \S 4.3(223), \S 4.4(233)$, $\S 4.5(244), \S 4.6(255), \S 4.7(261), \S 4.8(268)$, $\S 4.9(270), \S 4.10(276), \S 5.1(295), \S 5.2(296)$, $\S 5.3(300), \S 5.4(306), \S 5.5(310), \S 5.6(314)$, $\S 5.7(318), \S 5.8(323), \S 5.9(323), \S 5.10(327)$, $\S 6.1(339), \S 6.2(340), \S 6.3(346), \S 6.4(350)$, $\S 6.5(354), \S 6.6(360), \S 6.7(371), \S 6.8(383)$, $\S 6.9(388), \S 6.10(394), \S 6.11(398)$, $\S 6.12(399), \S 6.13(402), \S 7.1(419), \S 7.2(419)$, $\S 7.3(425), \S 7.4(431), \S 7.5(437), \S 7.6(444)$, $\S 7.7(448), \S 7.8(459), \S 7.9(460), \S 7.10(464)$, $\S 8.1(477), \S 8.2(477), \S 8.3(484), \S 8.4(487)$, $\S 8.5(493), \S 8.6(496), \S 8.7(496), \S 8.8(502)$, $\S 9.1(513), \S 9.2(514), \S 9.3(519), \S 9.4(524)$, $\S 9.5(532), \S 9.6(542), \S 9.7(557), \S 9.8(559)$, $\S 9.9(564), \S 10.1(577), \S 10.2(578)$, $\S 10.3(584), \S 10.4(588), \S 10.5(592)$, $\S 10.6(599), \S 10.7(604), \S 10.8(613)$, $\S 10.9(614), \S 10.10(618), \S 11.1(631)$, 
$\S 11.2(632), \S 11.3(638), \S 11.4(641)$, $\S 11.5(649), \S 11.6(657), \S 11.7(662)$, $\S 11.8(672), \S 11.9(674), \S 11.10(680)$ measurement, § 9.1(513), § 9.2(514), 514, $\S 9.3(519), \S 9.4(524), \S 9.5(532), \S 9.6(542)$, $\S 9.7(557), \S 9.8(559), \S 9.9(564)$

metric prefixes, 519

metric system, § 9.3(519)

minuend, 45,48

minus symbol, 45

mixed numbers, § 4.3(223), 226, § 5.4(306)

multiples, 184

multiplicand, 136

multiplication, $\S 2.2(92), 92, \S 2.6(130)$, $\S 2.7(136), 136, \S 2.8(137), \S 2.9(140)$, $\S 4.1(213), \S 4.2(214), \S 4.3(223), \S 4.4(233)$, $\S 4.5(244), \S 4.6(255), \S 4.7(261), \S 4.8(268)$, $\S 4.9(270), \S 4.10(276), \S 6.1(339), \S 6.2(340)$, $\S 6.3(346), \S 6.4(350), \S 6.5(354), \S 6.6(360)$, $\S 6.7(371), \S 6.8(383), \S 6.9(388), \S 6.10(394)$, $\S 6.11(398), \S 6.12(399), \S 6.13(402)$, $\S 10.7(604)$ multiplication statement, 262 multiplicative identity, § 2.6(130), 132, 136 multiplier, 92, 136

multiply.", 257

multiplicand, 92

$\mathbf{N}$ natural, 579

negative, $\S 10.3(584), 584,584$

negative numbers, 579

non-terminating division, 383

nonterminating divisions, $\S 6.1(339)$, $\S 6.2(340), \S 6.3(346), \S 6.4(350), \S 6.5(354)$, $\S 6.6(360), \S 6.7(371), \S 6.8(383), \S 6.9(388)$, $\S 6.10(394), \S 6.11(398), \S 6.12(399)$,

$\S 6.13(402)$

number, 10, 10, 68

number line, 13, 68

Numbers, $\S 1.2(10), \S 1.4(21)$

numeral, 10, 10, 68

Numerals, § 1.2(10)

numerator, 215,515

numerical coefficient, 633

numerical evaluation, § 11.2(632), 634

numerical expression, 632

O Objectives, 2

Opposites, 585

order of numbers, $\S 5.5(310)$

order of operations, $\S 5.7(318)$

ordered number system, 310 origin, 13,579

P Parentheses, 162

Parentheses in addition, 69

percent, § 7.5(437), 438, § 7.7(448), 448

percentage, $\S 7.7(448), 448$

percents, 438

perimeter, $\S 9.5(532), 532$

period, 68

periods, 11

pi, § 9.5(532)

plus symbol, 30

polygon, 532

positional, 11

positive, 584

positive fraction, 215

positive improper fractions, 224

positive mixed number, 224

positive number, 584

positive numbers, $579, \S 10.3(584)$

positive proper fractions, 223

power, 155

Practice Sets, 1

preface, $\S(1)$

prime factorization, 175

prime number, 174

product, 93, 136, 172

product statements, $\S 4.7(261)$

products, $\S 4.7(261)$

Proficiency Exam, 2

proper fraction, $\S 4.3(223)$

properties, § 1.8(68), § 2.6(130)

proportion, $425, \S 7.4(431)$

proportions, $\S 7.3(425)$

pure numbers, $\S 7.2(419), 420$

Q quotient, 105, 136

$\mathbf{R}$ radical, 156

radical sign, 156, 156

radicand, 156

radius, § 9.5(532), 535

raising a fraction to higher terms, 238

rate, 421,425

rates, $\S 7.1(419), \S 7.2(419), \S 7.3(425)$, $\S 7.4(431), \S 7.5(437), \S 7.6(444), \S 7.7(448)$, $\S 7.8(459), \S 7.9(460), \S 7.10(464)$

ratio, $421,425,438$

rational numbers, 579

ratios, § 7.1(419), §7.2(419), §7.3(425), $\S 7.4(431), \S 7.5(437), \S 7.6(444), \S 7.7(448)$, $\S 7.8(459), \S 7.9(460), \S 7.10(464)$ 
reading, § 1.3(15)

reading strategy, 662

real number, 579

real number line, 578

real numbers, $\S 10.3(584)$

reciprocal, § 4.6(255)

reciprocals, 255

reduced, 235

reduced to lowest terms, 235

reducing a fraction, 235

relatively prime, 235,235

remainder, $\S 2.4(113)$

repeating decimal, 384

roots, § 3.1(153), §3.2(154), §3.3(162),

$\S 3.4(172), \S 3.5(180), \S 3.6(184), \S 3.7(191)$, $\S 3.8(193), \S 3.9(198)$

round a whole number, 22

rounding, § 1.4(21), 21, 69, § 8.2(477),

$\S 8.5(493)$

S Sample Sets, 1

second partial product, 95

Section Exercises, 2

Section Overview, 1

sign, 584

signed numbers, $\S 10.1(577), \S 10.2(578)$,

$\S 10.3(584), \S 10.4(588), \S 10.5(592)$,

$\S 10.6(599), \S 10.7(604), \S 10.8(613)$,

$\S 10.9(614), \S 10.10(618)$

simple fraction, 314

simple fractions, $\S 5.6(314)$

simplified, 524, 638

solutions, 641

solved, 641

square root, 156, 156

squared, 155

standard unit of measure, 514

Subtracting, § 1.1(9)

subtraction, § 1.6(44), 45, § 1.8(68), 69,

$\S 1.9(69), \S 1.10(74), \S 5.1(295), \S 5.2(296)$,
$\S 5.3(300), \S 5.4(306), \S 5.5(310), \S 5.6(314)$, $\S 5.7(318), \S 5.8(323), \S 5.9(323), \S 5.10(327)$, $\S 6.1(339), \S 6.2(340), \S 6.3(346), \S 6.4(350)$, $\S 6.5(354), \S 6.6(360), \S 6.7(371), \S 6.8(383)$, $\S 6.9(388), \S 6.10(394), \S 6.11(398)$, $\S 6.12(399), \S 6.13(402), \S 10.6(599)$, $\S 11.3(638)$

subtrahend, 45,48

sum, 30, 69

summary, § 2.7(136)

Summary of Key Concepts, 2

symbols, § 11.6(657)

$\mathbf{T}$ terminating division, 383

terms, $30, \S 11.2(632), 632$

test, $\S 2.9(140)$

total product, 95

$\mathbf{U}$ unit fraction, 515

United States System, § 9.2(514)

unlike denominate numbers, 420

unlike denominators, $\S 5.3(300)$

use parentheses, 63

$\mathrm{V}$ value of a digit, 12

variable, 578

volume, $\S 9.6(542), 547$

W weight, 521

whole number, 68

Whole Numbers, $\S 1.1(9), \S 1.2(10), 12$, $\S 1.3(15), \S 1.4(21), \S 1.5(29), \S 1.6(44)$, $\S 1.7(62), \S 1.8(68), \S 1.9(69), \S 1.10(74)$, $\S 2.2(92), \S 2.3(105), \S 2.4(113), \S 2.5(126)$, $\S 2.6(130), \S 2.7(136), \S 2.8(137), \S 2.9(140)$, $\S 3.1(153), \S 3.2(154), \S 3.3(162), \S 3.4(172)$, $\S 3.5(180), \S 3.6(184), \S 3.7(191), \S 3.8(193)$, $\S 3.9(198), 579$

word problems, $\S 11.6(657)$

writing, $\S 1.3(15)$ 


\section{Attributions}

Collection: Fundamentals of Mathematics

Edited by: Denny Burzynski, Wade Ellis

URL: http://cnx.org/content/col10615/1.4/

License: http://creativecommons.org/licenses/by/2.0/

Module: "Fundamentals of Mathematics: Preface"

Used here as: "Preface"

By: Denny Burzynski, Wade Ellis

URL: http://cnx.org/content/m18884/1.4/

Pages: $1-5$

Copyright: Denny Burzynski, Wade Ellis

License: http://creativecommons.org/licenses/by/2.0/

Module: "Fundamentals of Mathematics: Acknowledgements"

Used here as: "Acknowledgements"

By: Denny Burzynski, Wade Ellis

URL: http://cnx.org/content/m34775/1.2/

Pages: 7-8

Copyright: Denny Burzynski, Wade Ellis

License: http://creativecommons.org/licenses/by/3.0/

Module: "Addition and Subtraction of Whole Numbers: Objectives"

Used here as: "Objectives"

By: Denny Burzynski, Wade Ellis

URL: http://cnx.org/content/m18885/1.4/

Page: 9

Copyright: Denny Burzynski, Wade Ellis

License: http://creativecommons.org/licenses/by/2.0/

Module: "Addition and Subtraction of Whole Numbers: Whole Numbers"

Used here as: "Whole Numbers"

By: Denny Burzynski, Wade Ellis

URL: http://cnx.org/content/m34795/1.5/

Pages: $10-15$

Copyright: Denny Burzynski, Wade Ellis

License: http://creativecommons.org/licenses/by/3.0/

Module: "Addition and Subtraction of Whole Numbers: Reading and Writing Whole Numbers"

Used here as: "Reading and Writing Whole Numbers"

By: Denny Burzynski, Wade Ellis

URL: http://cnx.org/content/m34778/1.5/

Pages: $15-21$

Copyright: Denny Burzynski, Wade Ellis

License: http://creativecommons.org/licenses/by/3.0/

Module: "Addition and Subtraction of Whole Numbers: Rounding Whole Numbers"

Used here as: "Rounding Whole Numbers"

By: Wade Ellis, Denny Burzynski

URL: http://cnx.org/content/m34780/1.3/

Pages: 21-29

Copyright: Wade Ellis, Denny Burzynski

License: http://creativecommons.org/licenses/by/3.0/ 
Module: "Addition and Subtraction of Whole Numbers: Addition of Whole Numbers" Used here as: "Addition of Whole Numbers"

By: Denny Burzynski, Wade Ellis

URL: http://cnx.org/content/m34786/1.5/

Pages: 29-44

Copyright: Denny Burzynski, Wade Ellis

License: http://creativecommons.org/licenses/by/3.0/

Module: "Addition and Subtraction of Whole Numbers: Subtraction of Whole Numbers"

Used here as: "Subtraction of Whole Numbers"

By: Wade Ellis, Denny Burzynski

URL: http://cnx.org/content/m34784/1.5/

Pages: 44-62

Copyright: Wade Ellis, Denny Burzynski

License: http://creativecommons.org/licenses/by/3.0/

Module: "Addition and Subtraction of Whole Numbers: Properties of Addition"

Used here as: "Properties of Addition"

By: Wade Ellis, Denny Burzynski

URL: http://cnx.org/content/m34802/1.5/

Pages: $62-67$

Copyright: Wade Ellis, Denny Burzynski

License: http://creativecommons.org/licenses/by/3.0/

Module: "Addition and Subtraction of Whole Numbers: Summary of Key Concepts"

Used here as: "Summary of Key Concepts"

By: Wade Ellis, Denny Burzynski

URL: http://cnx.org/content/m34798/1.3/

Pages: $68-69$

Copyright: Wade Ellis, Denny Burzynski

License: http://creativecommons.org/licenses/by/3.0/

Module: "Addition and Subtraction of Whole Numbers: Exercise Supplement"

Used here as: "Exercise Supplement"

By: Denny Burzynski, Wade Ellis

URL: http://cnx.org/content/m34800/1.5/

Pages: $69-74$

Copyright: Denny Burzynski, Wade Ellis

License: http://creativecommons.org/licenses/by/3.0/

Module: "Addition and Subtraction of Whole Numbers: Proficiency Exam"

Used here as: "Proficiency Exam"

By: Wade Ellis, Denny Burzynski

URL: http://cnx.org/content/m34805/1.4/

Pages: $74-75$

Copyright: Wade Ellis, Denny Burzynski

License: http://creativecommons.org/licenses/by/3.0/ 
Module: "Multiplication and Division of Whole Numbers: Objectives"

Used here as: "Objectives"

By: Denny Burzynski, Wade Ellis

URL: http://cnx.org/content/m18888/1.4/

Page: 91

Copyright: Denny Burzynski, Wade Ellis

License: http://creativecommons.org/licenses/by/2.0/

Module: "Multiplication and Division of Whole Numbers: Multiplication of Whole Numbers"

Used here as: "Multiplication of Whole Numbers"

By: Wade Ellis, Denny Burzynski

URL: http://cnx.org/content/m34863/1.2/

Pages: 92-105

Copyright: Wade Ellis, Denny Burzynski

License: http://creativecommons.org/licenses/by/3.0/

Module: "Multiplication and Division of Whole Numbers: Concepts of Division of Whole Numbers"

Used here as: "Concepts of Division of Whole Numbers"

By: Wade Ellis, Denny Burzynski

URL: http://cnx.org/content/m34864/1.3/

Pages: 105-113

Copyright: Wade Ellis, Denny Burzynski

License: http://creativecommons.org/licenses/by/3.0/

Module: "Multiplication and Division of Whole Numbers: Division of Whole Numbers"

Used here as: "Division of Whole Numbers "

By: Wade Ellis, Denny Burzynski

URL: http://cnx.org/content/m34865/1.2/

Pages: $113-126$

Copyright: Wade Ellis, Denny Burzynski

License: http://creativecommons.org/licenses/by/3.0/

Module: "Multiplication and Division of Whole Numbers: Some Interesting Facts about Division"

Used here as: "Some Interesting Facts about Division "

By: Wade Ellis, Denny Burzynski

URL: http://cnx.org/content/m34866/1.2/

Pages: 126-130

Copyright: Wade Ellis, Denny Burzynski

License: http://creativecommons.org/licenses/by/3.0/

Module: "Multiplication and Division of Whole Numbers: Properties of Multiplication"

Used here as: "Properties of Multiplication "

By: Wade Ellis, Denny Burzynski

URL: http://cnx.org/content/m34867/1.2/

Pages: 130-135

Copyright: Wade Ellis, Denny Burzynski

License: http://creativecommons.org/licenses/by/3.0/ 
Module: "Multiplication and Division of Whole Numbers: Summary of Key Concepts"

Used here as: "Summary of Key Concepts "

By: Wade Ellis, Denny Burzynski

URL: http://cnx.org/content/m34868/1.2/

Pages: 136-137

Copyright: Wade Ellis, Denny Burzynski

License: http://creativecommons.org/licenses/by/3.0/

Module: "Multiplication and Division of Whole Numbers: Exercise Supplement"

Used here as: "Exercise Supplement "

By: Wade Ellis, Denny Burzynski

URL: http://cnx.org/content/m34869/1.2/

Pages: $137-140$

Copyright: Wade Ellis, Denny Burzynski

License: http://creativecommons.org/licenses/by/3.0/

Module: "Multiplication and Division of Whole Numbers: Proficiency Exam"

Used here as: "Proficiency Exam "

By: Wade Ellis, Denny Burzynski

URL: http://cnx.org/content/m34870/1.2/

Pages: 140-141

Copyright: Wade Ellis, Denny Burzynski

License: http://creativecommons.org/licenses/by/3.0/

Module: "Exponents, Roots, and Factorizations of Whole Numbers: Objectives"

Used here as: "Objectives"

By: Denny Burzynski, Wade Ellis

URL: http://cnx.org/content/m18890/1.4/

Page: 153

Copyright: Denny Burzynski, Wade Ellis

License: http://creativecommons.org/licenses/by/2.0/

Module: "Exponents, Roots, and Factorization of Whole Numbers: Exponents and Roots"

Used here as: "Exponents and Roots "

By: Wade Ellis, Denny Burzynski

URL: http://cnx.org/content/m34871/1.2/

Pages: $154-162$

Copyright: Wade Ellis, Denny Burzynski

License: http://creativecommons.org/licenses/by/3.0/

Module: "Exponents, Roots, Factorization of Whole Numbers: Grouping Symbols and the Order of Operations"

Used here as: "Grouping Symbols and the Order of Operations "

By: Wade Ellis, Denny Burzynski

URL: http://cnx.org/content/m34872/1.2/

Pages: 162-172

Copyright: Wade Ellis, Denny Burzynski

License: http://creativecommons.org/licenses/by/3.0/ 
Module: "Exponents, Roots, Factorization of Whole Numbers: Prime Factorization of Natural Numbers" Used here as: "Prime Factorization of Natural Numbers"

By: Wade Ellis, Denny Burzynski

URL: http://cnx.org/content/m34873/1.2/

Pages: $172-180$

Copyright: Wade Ellis, Denny Burzynski

License: http://creativecommons.org/licenses/by/3.0/

Module: "Exponents, Roots, and Factorizations of Whole Numbers: The Greatest Common Factor"

Used here as: "The Greatest Common Factor "

By: Wade Ellis, Denny Burzynski

URL: http://cnx.org/content/m34874/1.2/

Pages: $180-184$

Copyright: Wade Ellis, Denny Burzynski

License: http://creativecommons.org/licenses/by/3.0/

Module: "Exponents, Roots, Factorization of Whole Numbers: The Least Common Multiple"

Used here as: "The Least Common Multiple"

By: Wade Ellis, Denny Burzynski

URL: http://cnx.org/content/m34876/1.2/

Pages: 184-190

Copyright: Wade Ellis, Denny Burzynski

License: http://creativecommons.org/licenses/by/3.0/

Module: "Exponents, Roots, Factorization of Whole Numbers: Summary of Key Concepts"

Used here as: "Summary of Key Concepts"

By: Wade Ellis, Denny Burzynski

URL: http://cnx.org/content/m34877/1.2/

Pages: 191-193

Copyright: Wade Ellis, Denny Burzynski

License: http://creativecommons.org/licenses/by/3.0/

Module: "Exponents, Roots, Factorization of Whole Numbers: Exercise Supplement"

Used here as: "Exercise Supplement "

By: Wade Ellis, Denny Burzynski

URL: http://cnx.org/content/m34878/1.2/

Pages: 193-198

Copyright: Wade Ellis, Denny Burzynski

License: http://creativecommons.org/licenses/by/3.0/

Module: "Exponents, Roots, Factorization of Whole Numbers: Proficiency Exam"

Used here as: "Proficiency Exam "

By: Wade Ellis, Denny Burzynski

URL: http://cnx.org/content/m34879/1.2/

Pages: 198-199

Copyright: Wade Ellis, Denny Burzynski

License: http://creativecommons.org/licenses/by/3.0/ 
Module: "Introduction to Fractions and Multiplication and Division of Fractions: Objectives"

Used here as: "Objectives "

By: Denny Burzynski, Wade Ellis

URL: http://cnx.org/content/m18892/1.3/

Page: 213

Copyright: Denny Burzynski, Wade Ellis

License: http://creativecommons.org/licenses/by/2.0/

Module: "Introduction to Fractions and Multiplication and Division of Fractions: Fractions of Whole Numbers"

Used here as: "Fractions of Whole Numbers "

By: Wade Ellis, Denny Burzynski

URL: http://cnx.org/content/m34908/1.2/

Pages: 214-223

Copyright: Wade Ellis, Denny Burzynski

License: http://creativecommons.org/licenses/by/3.0/

Module: "Introduction to Fractions and Multiplication and Division of Fractions: Proper Fractions, Improper Fractions, and Mixed Numbers"

Used here as: "Proper Fractions, Improper Fractions, and Mixed Numbers"

By: Wade Ellis, Denny Burzynski

URL: http://cnx.org/content/m34912/1.2/

Pages: 223-233

Copyright: Wade Ellis, Denny Burzynski

License: http://creativecommons.org/licenses/by/3.0/

Module: "Introduction to Fractions and Multiplication and Division of Fractions: Equivalent Fractions, Reducing Fractions to Lowest Terms, and Raising Fractions to Higher Terms"

Used here as: "Equivalent Fractions, Reducing Fractions to Lowest Terms, and Raising Fractions to Higher Terms "

By: Wade Ellis, Denny Burzynski

URL: http://cnx.org/content/m34927/1.3/

Pages: 233-244

Copyright: Wade Ellis, Denny Burzynski

License: http://creativecommons.org/licenses/by/3.0/

Module: "Introduction to Fractions and Multiplication and Division of Fractions: Multiplication of Fractions"

Used here as: "Multiplication of Fractions"

By: Wade Ellis, Denny Burzynski

URL: http://cnx.org/content/m34928/1.2/

Pages: 244-255

Copyright: Wade Ellis, Denny Burzynski

License: http://creativecommons.org/licenses/by/3.0/

Module: "Introduction to Fractions and Multiplication and Division of Fractions: Division of Fractions"

Used here as: "Division of Fractions"

By: Wade Ellis, Denny Burzynski

URL: http://cnx.org/content/m34929/1.2/

Pages: 255-261

Copyright: Wade Ellis, Denny Burzynski

License: http://creativecommons.org/licenses/by/3.0/ 
Module: "Introduction to Fractions and Multiplication and Division of Fractions: Applications Involving Fractions"

Used here as: "Applications Involving Fractions "

By: Wade Ellis, Denny Burzynski

URL: http://cnx.org/content/m34930/1.2/

Pages: 261-267

Copyright: Wade Ellis, Denny Burzynski

License: http://creativecommons.org/licenses/by/3.0/

Module: "Introduction to Fractions and Multiplication and Division of Fractions: Summary of Key Concepts"

Used here as: "Summary of Key Concepts "

By: Wade Ellis, Denny Burzynski

URL: http://cnx.org/content/m34931/1.2/

Pages: 268-270

Copyright: Wade Ellis, Denny Burzynski

License: http://creativecommons.org/licenses/by/3.0/

Module: "Introduction to Fractions and Multiplication and Division of Fractions: Exercise Supplement"

Used here as: "Exercise Supplement "

By: Wade Ellis, Denny Burzynski

URL: http://cnx.org/content/m34932/1.2/

Pages: $270-276$

Copyright: Wade Ellis, Denny Burzynski

License: http://creativecommons.org/licenses/by/3.0/

Module: "Introduction to Fractions and Multiplication and Division of Fractions: Proficiency Exam"

Used here as: "Proficiency Exam"

By: Wade Ellis, Denny Burzynski

URL: http://cnx.org/content/m34933/1.2/

Pages: $276-277$

Copyright: Wade Ellis, Denny Burzynski

License: http://creativecommons.org/licenses/by/3.0/

Module: "Addition and Subtraction of Fractions, Comparing Fractions, and Complex Fractions: Objectives" Used here as: "Objectives"

By: Denny Burzynski, Wade Ellis

URL: http://cnx.org/content/m18893/1.3/

Page: 295

Copyright: Denny Burzynski, Wade Ellis

License: http://creativecommons.org/licenses/by/2.0/

Module: "Addition and Subtraction of Fractions, Comparing Fractions, and Complex Fractions: Addition and Subtractions of Fractions with Like Denominators"

Used here as: "Addition and Subtraction of Fractions with Like Denominators"

By: Wade Ellis, Denny Burzynski

URL: http://cnx.org/content/m34934/1.2/

Pages: 296-300

Copyright: Wade Ellis, Denny Burzynski

License: http://creativecommons.org/licenses/by/3.0/ 
Module: "Addition and Subtraction of Fractions, Comparing Fractions, and Complex Fractions: Addition and Subtraction of Fractions with Unlike Denominators"

Used here as: "Addition and Subtraction of Fractions with Unlike Denominators"

By: Wade Ellis, Denny Burzynski

URL: http://cnx.org/content/m34935/1.2/

Pages: $300-306$

Copyright: Wade Ellis, Denny Burzynski

License: http://creativecommons.org/licenses/by/3.0/

Module: "Addition and Subtraction of Fractions, Comparing Fractions, and Complex Fractions: Addition and Subtraction of Mixed Numbers"

Used here as: "Addition and Subtraction of Mixed Numbers"

By: Wade Ellis, Denny Burzynski

URL: http://cnx.org/content/m34936/1.2/

Pages: 306-310

Copyright: Wade Ellis, Denny Burzynski

License: http://creativecommons.org/licenses/by/3.0/

Module: "Addition and Subtraction of Fractions, Comparing Fractions, and Complex Fractions: Comparing Fractions"

Used here as: "Comparing Fractions"

By: Wade Ellis, Denny Burzynski

URL: http://cnx.org/content/m34937/1.3/

Pages: $310-314$

Copyright: Wade Ellis, Denny Burzynski

License: http://creativecommons.org/licenses/by/3.0/

Module: "Addition and Subtraction of Fractions, Comparing Fractions, and Complex Fractions: Complex Fractions"

Used here as: "Complex Fractions "

By: Wade Ellis, Denny Burzynski

URL: http://cnx.org/content/m34941/1.2/

Pages: 314-317

Copyright: Wade Ellis, Denny Burzynski

License: http://creativecommons.org/licenses/by/3.0/

Module: "Addition and Subtraction of Fractions, Comparing Fractions, and Complex Fractions: Combinations of Operations with Fractions"

Used here as: "Combinations of Operations with Fractions "

By: Wade Ellis, Denny Burzynski

URL: http://cnx.org/content/m34942/1.3/

Pages: 318-322

Copyright: Wade Ellis, Denny Burzynski

License: http://creativecommons.org/licenses/by/3.0/

Module: "Addition and Subtraction of Fractions, Comparing Fractions, and Complex Fractions: Summary of Key Concepts"

Used here as: "Summary of Key Concepts "

By: Wade Ellis, Denny Burzynski

URL: http://cnx.org/content/m34943/1.2/

Page: 323

Copyright: Wade Ellis, Denny Burzynski

License: http://creativecommons.org/licenses/by/3.0/ 
Module: "Addition and Subtraction of Fractions, Comparing Fractions, and Complex Fractions: Exercise Supplement"

Used here as: "Exercise Supplement "

By: Wade Ellis, Denny Burzynski

URL: http://cnx.org/content/m34944/1.2/

Pages: $323-327$

Copyright: Wade Ellis, Denny Burzynski

License: http://creativecommons.org/licenses/by/3.0/

Module: "Addition and Subtraction of Fractions, Comparing Fractions, and Complex Fractions: Proficiency Exam"

Used here as: "Proficiency Exam "

By: Wade Ellis, Denny Burzynski

URL: http://cnx.org/content/m34948/1.2/

Pages: $327-328$

Copyright: Wade Ellis, Denny Burzynski

License: http://creativecommons.org/licenses/by/3.0/

Module: "Decimals: Objectives"

Used here as: "Objectives"

By: Denny Burzynski, Wade Ellis

URL: http://cnx.org/content/m18894/1.3/

Pages: 339-340

Copyright: Denny Burzynski, Wade Ellis

License: http://creativecommons.org/licenses/by/2.0/

Module: "Decimals: Reading and Writing Decimals"

Used here as: "Reading and Writing Decimals"

By: Wade Ellis, Denny Burzynski

URL: http://cnx.org/content/m34957/1.2/

Pages: $340-346$

Copyright: Wade Ellis, Denny Burzynski

License: http://creativecommons.org/licenses/by/3.0/

Module: "Decimals: Converting a Decimal to a Fraction"

Used here as: "Converting a Decimal to a Fraction"

By: Wade Ellis, Denny Burzynski

URL: http://cnx.org/content/m34958/1.2/

Pages: 346-350

Copyright: Wade Ellis, Denny Burzynski

License: http://creativecommons.org/licenses/by/3.0/

Module: "Decimals: Rounding Decimals"

Used here as: "Rounding Decimals"

By: Wade Ellis, Denny Burzynski

URL: http://cnx.org/content/m34959/1.2/

Pages: $350-354$

Copyright: Wade Ellis, Denny Burzynski

License: http://creativecommons.org/licenses/by/3.0/ 
Module: "Decimals: Addition and Subtraction of Decimals"

Used here as: "Addition and Subtraction of Decimals"

By: Wade Ellis, Denny Burzynski

URL: http://cnx.org/content/m34960/1.5/

Pages: 354-360

Copyright: Wade Ellis, Denny Burzynski

License: http://creativecommons.org/licenses/by/3.0/

Module: "Decimals: Multiplication of Decimals"

Used here as: "Multiplication of Decimals"

By: Wade Ellis, Denny Burzynski

URL: http://cnx.org/content/m34963/1.2/

Pages: $360-370$

Copyright: Wade Ellis, Denny Burzynski

License: http://creativecommons.org/licenses/by/3.0/

Module: "Decimals: Division of Decimals"

Used here as: "Division of Decimals"

By: Wade Ellis, Denny Burzynski

URL: http://cnx.org/content/m34968/1.2/

Pages: 371-383

Copyright: Wade Ellis, Denny Burzynski

License: http://creativecommons.org/licenses/by/3.0/

Module: "Decimals: Nonterminating Divisions"

Used here as: "Nonterminating Divisions"

By: Wade Ellis, Denny Burzynski

URL: http://cnx.org/content/m34969/1.2/

Pages: $383-388$

Copyright: Wade Ellis, Denny Burzynski

License: http://creativecommons.org/licenses/by/3.0/

Module: "Decimals: Converting a Fraction to a Decimal"

Used here as: "Converting a Fraction to a Decimal"

By: Wade Ellis, Denny Burzynski

URL: http://cnx.org/content/m34970/1.2/

Pages: 388-394

Copyright: Wade Ellis, Denny Burzynski

License: http://creativecommons.org/licenses/by/3.0/

Module: "Decimals: Combinations of Operations with Decimals and Fractions"

Used here as: "Combinations of Operations with Decimals and Fractions"

By: Wade Ellis, Denny Burzynski

URL: http://cnx.org/content/m34971/1.2/

Pages: 394-397

Copyright: Wade Ellis, Denny Burzynski

License: http://creativecommons.org/licenses/by/3.0/ 
Module: "Decimals: Summary of Key Concepts"

Used here as: "Summary of Key Concepts"

By: Wade Ellis, Denny Burzynski

URL: http://cnx.org/content/m34972/1.2/

Pages: $398-399$

Copyright: Wade Ellis, Denny Burzynski

License: http://creativecommons.org/licenses/by/3.0/

Module: "Decimals: Exercise Supplement"

Used here as: "Exercise Supplement"

By: Wade Ellis, Denny Burzynski

URL: http://cnx.org/content/m34978/1.2/

Pages: 399-402

Copyright: Wade Ellis, Denny Burzynski

License: http://creativecommons.org/licenses/by/3.0/

Module: "Decimals: Proficiency Exam"

Used here as: "Proficiency Exam"

By: Wade Ellis, Denny Burzynski

URL: http://cnx.org/content/m34979/1.2/

Pages: 402-403

Copyright: Wade Ellis, Denny Burzynski

License: http://creativecommons.org/licenses/by/3.0/

Module: "Ratios and Rates: Objectives"

Used here as: "Objectives"

By: Denny Burzynski, Wade Ellis

URL: http://cnx.org/content/m18895/1.3/

Page: 419

Copyright: Denny Burzynski, Wade Ellis

License: http://creativecommons.org/licenses/by/2.0/

Module: "Ratios and Rates: Ratios and Rates"

Used here as: "Ratios and Rates"

By: Wade Ellis, Denny Burzynski

URL: http://cnx.org/content/m34980/1.2/

Pages: $419-424$

Copyright: Wade Ellis, Denny Burzynski

License: http://creativecommons.org/licenses/by/3.0/

Module: "Ratios and Rates: Proportions"

Used here as: "Proportions"

By: Wade Ellis, Denny Burzynski

URL: http://cnx.org/content/m34981/1.2/

Pages: 425-431

Copyright: Wade Ellis, Denny Burzynski

License: http://creativecommons.org/licenses/by/3.0/ 
Module: "Ratios and Rates: Applications of Proportions"

Used here as: "Applications of Proportions"

By: Wade Ellis, Denny Burzynski

URL: http://cnx.org/content/m34982/1.2/

Pages: 431-437

Copyright: Wade Ellis, Denny Burzynski

License: http://creativecommons.org/licenses/by/3.0/

Module: "Ratios and Rates: Percent"

Used here as: "Percent"

By: Wade Ellis, Denny Burzynski

URL: http://cnx.org/content/m34983/1.2/

Pages: 437-444

Copyright: Wade Ellis, Denny Burzynski

License: http://creativecommons.org/licenses/by/3.0/

Module: "Ratios and Rates: Fractions of One Percent"

Used here as: "Fractions of One Percent"

By: Wade Ellis, Denny Burzynski

URL: http://cnx.org/content/m34997/1.2/

Pages: 444-447

Copyright: Wade Ellis, Denny Burzynski

License: http://creativecommons.org/licenses/by/3.0/

Module: "Ratios and Rates: Applications of Percents"

Used here as: "Applications of Percents"

By: Wade Ellis, Denny Burzynski

URL: http://cnx.org/content/m35007/1.2/

Pages: $448-458$

Copyright: Wade Ellis, Denny Burzynski

License: http://creativecommons.org/licenses/by/3.0/

Module: "Ratios and Rates: Summary of Key Concepts"

Used here as: "Summary of Key Concepts"

By: Wade Ellis, Denny Burzynski

URL: http://cnx.org/content/m35008/1.2/

Pages: 459-460

Copyright: Wade Ellis, Denny Burzynski

License: http://creativecommons.org/licenses/by/3.0/

Module: "Ratios and Rates: Exercise Supplement"

Used here as: "Exercise Supplement"

By: Wade Ellis, Denny Burzynski

URL: http://cnx.org/content/m35009/1.2/

Pages: $460-464$

Copyright: Wade Ellis, Denny Burzynski

License: http://creativecommons.org/licenses/by/3.0/ 
Module: "Ratios and Rates: Proficiency Exam"

Used here as: "Proficiency Exam"

By: Wade Ellis, Denny Burzynski

URL: http://cnx.org/content/m35010/1.2/

Pages: $464-465$

Copyright: Wade Ellis, Denny Burzynski

License: http://creativecommons.org/licenses/by/3.0/

Module: "Techniques of Estimation: Objectives"

Used here as: "Objectives"

By: Denny Burzynski, Wade Ellis

URL: http://cnx.org/content/m18896/1.3/

Page: 477

Copyright: Denny Burzynski, Wade Ellis

License: http://creativecommons.org/licenses/by/2.0/

Module: "Techniques of Estimation: Estimation by Rounding"

Used here as: "Estimation by Rounding"

By: Wade Ellis, Denny Burzynski

URL: http://cnx.org/content/m35011/1.2/

Pages: 477-484

Copyright: Wade Ellis, Denny Burzynski

License: http://creativecommons.org/licenses/by/3.0/

Module: "Techniques of Estimation: Estimation by Clustering"

Used here as: "Estimation by Clustering"

By: Wade Ellis, Denny Burzynski

URL: http://cnx.org/content/m35012/1.2/

Pages: $484-487$

Copyright: Wade Ellis, Denny Burzynski

License: http://creativecommons.org/licenses/by/3.0/

Module: "Techniques of Estimation: Mental Arithmetic- Using the Distibutive Property"

Used here as: "Mental Arithmetic-Using the Distributive Property"

By: Wade Ellis, Denny Burzynski

URL: http://cnx.org/content/m35013/1.2/

Pages: 487-493

Copyright: Wade Ellis, Denny Burzynski

License: http://creativecommons.org/licenses/by/3.0/

Module: "Techniques of Estimation: Estimation by Rounding Fractions"

Used here as: "Estimation by Rounding Fractions"

By: Wade Ellis, Denny Burzynski

URL: http://cnx.org/content/m35014/1.2/

Pages: $493-495$

Copyright: Wade Ellis, Denny Burzynski

License: http://creativecommons.org/licenses/by/3.0/ 
Module: "Techniques of Estimation: Summary of Key Concepts"

Used here as: "Summary of Key Concepts"

By: Wade Ellis, Denny Burzynski

URL: http://cnx.org/content/m35015/1.2/

Page: 496

Copyright: Wade Ellis, Denny Burzynski

License: http://creativecommons.org/licenses/by/3.0/

Module: "Techniques of Estimation: Exercise Supplement"

Used here as: "Exercise Supplement"

By: Wade Ellis, Denny Burzynski

URL: http://cnx.org/content/m35016/1.2/

Pages: 496-502

Copyright: Wade Ellis, Denny Burzynski

License: http://creativecommons.org/licenses/by/3.0/

Module: "Techniques of Estimation: Proficiency Exam"

Used here as: "Proficiency Exam"

By: Wade Ellis, Denny Burzynski

URL: http://cnx.org/content/m35017/1.2/

Pages: 502-504

Copyright: Wade Ellis, Denny Burzynski

License: http://creativecommons.org/licenses/by/3.0/

Module: "Measurement and Geometry: Objectives"

Used here as: "Objectives"

By: Denny Burzynski, Wade Ellis

URL: http://cnx.org/content/m18897/1.3/

Page: 513

Copyright: Denny Burzynski, Wade Ellis

License: http://creativecommons.org/licenses/by/2.0/

Module: "Measurement and Geometry: Measurement and the United States System"

Used here as: "Measurement and the United States System"

By: Wade Ellis, Denny Burzynski

URL: http://cnx.org/content/m35018/1.2/

Pages: 514-518

Copyright: Wade Ellis, Denny Burzynski

License: http://creativecommons.org/licenses/by/3.0/

Module: "Measurement and Geometry: The Metric System of Measurement"

Used here as: "The Metric System of Measurement"

By: Wade Ellis, Denny Burzynski

URL: http://cnx.org/content/m35019/1.2/

Pages: 519-524

Copyright: Wade Ellis, Denny Burzynski

License: http://creativecommons.org/licenses/by/3.0/ 
Module: "Measurement and Geometry: Simplification of Denominate Numbers"

Used here as: "Simplification of Denominate Numbers"

By: Wade Ellis, Denny Burzynski

URL: http://cnx.org/content/m35021/1.2/

Pages: 524-531

Copyright: Wade Ellis, Denny Burzynski

License: http://creativecommons.org/licenses/by/3.0/

Module: "Measurement and Geometry: Perimeter and Circumference of Geometric Figures"

Used here as: "Perimeter and Circumference of Geometric Figures"

By: Wade Ellis, Denny Burzynski

URL: http://cnx.org/content/m35022/1.2/

Pages: 532-542

Copyright: Wade Ellis, Denny Burzynski

License: http://creativecommons.org/licenses/by/3.0/

Module: "Measurement and Geometry: Area and Volume of Geometric Figures and Objects"

Used here as: "Area and Volume of Geometric Figures and Objects"

By: Wade Ellis, Denny Burzynski

URL: http://cnx.org/content/m35023/1.2/

Pages: 542-556

Copyright: Wade Ellis, Denny Burzynski

License: http://creativecommons.org/licenses/by/3.0/

Module: "Measurement and Geometry: Summary of Key Concepts"

Used here as: "Summary of Key Concepts"

By: Denny Burzynski, Wade Ellis

URL: http://cnx.org/content/m35024/1.2/

Pages: $557-558$

Copyright: Denny Burzynski, Wade Ellis

License: http://creativecommons.org/licenses/by/3.0/

Module: "Measurement and Geometry: Exercise Supplement"

Used here as: "Exercise Supplement"

By: Wade Ellis, Denny Burzynski

URL: http://cnx.org/content/m35025/1.2/

Pages: 559-564

Copyright: Wade Ellis, Denny Burzynski

License: http://creativecommons.org/licenses/by/3.0/

Module: "Measurement and Geometry: Proficiency Exam"

Used here as: "Proficiency Exam"

By: Wade Ellis, Denny Burzynski

URL: http://cnx.org/content/m35026/1.2/

Pages: 564-568

Copyright: Wade Ellis, Denny Burzynski

License: http://creativecommons.org/licenses/by/3.0/ 
Module: "Signed Numbers: Objectives"

Used here as: "Objectives"

By: Denny Burzynski, Wade Ellis

URL: http://cnx.org/content/m18898/1.3/

Page: 577

Copyright: Denny Burzynski, Wade Ellis

License: http://creativecommons.org/licenses/by/2.0/

Module: "Signed Numbers: Variables, Constants, and Real Numbers"

Used here as: "Variables, Constants, and Real Numbers "

By: Wade Ellis, Denny Burzynski

URL: http://cnx.org/content/m35027/1.2/

Pages: 578-583

Copyright: Wade Ellis, Denny Burzynski

License: http://creativecommons.org/licenses/by/3.0/

Module: "Signed Numbers: Signed Numbers"

Used here as: "Signed Numbers "

By: Wade Ellis, Denny Burzynski

URL: http://cnx.org/content/m35029/1.3/

Pages: 584-588

Copyright: Wade Ellis, Denny Burzynski

License: http://creativecommons.org/licenses/by/3.0/

Module: "Signed Numbers: Absolute Value"

Used here as: "Absolute Value"

By: Wade Ellis, Denny Burzynski

URL: http://cnx.org/content/m35030/1.3/

Pages: 588-592

Copyright: Wade Ellis, Denny Burzynski

License: http://creativecommons.org/licenses/by/3.0/

Module: "Signed Numbers: Addition of Signed Numbers"

Used here as: "Addition of Signed Numbers"

By: Wade Ellis, Denny Burzynski

URL: http://cnx.org/content/m35031/1.2/

Pages: 592-599

Copyright: Wade Ellis, Denny Burzynski

License: http://creativecommons.org/licenses/by/3.0/

Module: "Signed Numbers: Subtraction of Signed Numbers"

Used here as: "Subtraction of Signed Numbers"

By: Wade Ellis, Denny Burzynski

URL: http://cnx.org/content/m35032/1.2/

Pages: 599-604

Copyright: Wade Ellis, Denny Burzynski

License: http://creativecommons.org/licenses/by/3.0/ 
Module: "Signed Numbers: Multiplication and Division of Signed Numbers"

Used here as: "Multiplication and Division of Signed Numbers"

By: Wade Ellis, Denny Burzynski

URL: http://cnx.org/content/m35033/1.2/

Pages: 604-612

Copyright: Wade Ellis, Denny Burzynski

License: http://creativecommons.org/licenses/by/3.0/

Module: "Signed Numbers: Summary of Key Concepts"

Used here as: "Summary of Key Concepts"

By: Wade Ellis, Denny Burzynski

URL: http://cnx.org/content/m35034/1.2/

Pages: $613-614$

Copyright: Wade Ellis, Denny Burzynski

License: http://creativecommons.org/licenses/by/3.0/

Module: "Signed Numbers: Exercise Supplement"

Used here as: "Exercise Supplement "

By: Wade Ellis, Denny Burzynski

URL: http://cnx.org/content/m35035/1.2/

Pages: 614-618

Copyright: Wade Ellis, Denny Burzynski

License: http://creativecommons.org/licenses/by/3.0/

Module: "Signed Numbers: Proficiency Exam"

Used here as: "Proficiency Exam"

By: Wade Ellis, Denny Burzynski

URL: http://cnx.org/content/m35036/1.2/

Pages: 618-619

Copyright: Wade Ellis, Denny Burzynski

License: http://creativecommons.org/licenses/by/3.0/

Module: "Algebraic Expressions and Equations: Objectives"

Used here as: "Objectives"

By: Denny Burzynski, Wade Ellis

URL: http://cnx.org/content/m18899/1.3/

Page: 631

Copyright: Denny Burzynski, Wade Ellis

License: http://creativecommons.org/licenses/by/2.0/

Module: "Algebraic Expressions and Equations: Algebraic Expressions"

Used here as: "Algebraic Expressions"

By: Wade Ellis, Denny Burzynski

URL: http://cnx.org/content/m35038/1.2/

Pages: 632-638

Copyright: Wade Ellis, Denny Burzynski

License: http://creativecommons.org/licenses/by/3.0/ 
Module: "Algebraic Expressions and Equations: Combining Like Terms Using Addition and Subtraction" Used here as: "Combining Like Terms Using Addition and Subtraction"

By: Wade Ellis, Denny Burzynski

URL: http://cnx.org/content/m35039/1.2/

Pages: 638-641

Copyright: Wade Ellis, Denny Burzynski

License: http://creativecommons.org/licenses/by/3.0/

Module: "Algebraic Expressions and Equations: Solving Equations of the Form $\mathrm{x}+\mathrm{a}=\mathrm{b}$ and $\mathrm{x}-\mathrm{a}=\mathrm{b}$ "

Used here as: "Solving Equations of the Form $\mathrm{x}+\mathrm{a}=\mathrm{b}$ and $\mathrm{x}-\mathrm{a}=\mathrm{b}$ "

By: Wade Ellis, Denny Burzynski

URL: http://cnx.org/content/m35044/1.3/

Pages: $641-648$

Copyright: Wade Ellis, Denny Burzynski

License: http://creativecommons.org/licenses/by/3.0/

Module: "Algebraic Expressions and Equations: Solving Equations of the Form $\mathrm{ax}=\mathrm{b}$ and $\mathrm{x} / \mathrm{a}=\mathrm{b}$ "

Used here as: "Solving Equations of the Form $\mathrm{ax}=\mathrm{b}$ and $\mathrm{x} / \mathrm{a}=\mathrm{b}$ "

By: Wade Ellis, Denny Burzynski

URL: http://cnx.org/content/m35045/1.3/

Pages: 649-657

Copyright: Wade Ellis, Denny Burzynski

License: http://creativecommons.org/licenses/by/3.0/

Module: "Algebraic Expressions and Equations: Applications I: Translating Words to Mathematical Symbols"

Used here as: "Applications I: Translating Words to Mathematical Symbols"

By: Wade Ellis, Denny Burzynski

URL: http://cnx.org/content/m35046/1.2/

Pages: $657-662$

Copyright: Wade Ellis, Denny Burzynski

License: http://creativecommons.org/licenses/by/3.0/

Module: "Algebraic Expressions and Equations: Applications II: Solving Problems"

Used here as: "Applications II: Solving Problems"

By: Wade Ellis, Denny Burzynski

URL: http://cnx.org/content/m35047/1.2/

Pages: 662-671

Copyright: Wade Ellis, Denny Burzynski

License: http://creativecommons.org/licenses/by/3.0/

Module: "Algebraic Expressions and Equations: Summary of Key Concepts"

Used here as: "Summary of Key Concepts"

By: Wade Ellis, Denny Burzynski

URL: http://cnx.org/content/m35049/1.2/

Pages: 672-673

Copyright: Wade Ellis, Denny Burzynski

License: http://creativecommons.org/licenses/by/3.0/ 
Module: "Algebraic Expressions and Equations: Exercise Supplement"

Used here as: "Exercise Supplement "

By: Wade Ellis, Denny Burzynski

URL: http://cnx.org/content/m35050/1.2/

Pages: $674-680$

Copyright: Wade Ellis, Denny Burzynski

License: http://creativecommons.org/licenses/by/3.0/

Module: "Algebraic Expressions and Equations: Proficiency Exam"

Used here as: "Proficiency Exam"

By: Wade Ellis, Denny Burzynski

URL: http://cnx.org/content/m35052/1.2/

Pages: 680-681

Copyright: Wade Ellis, Denny Burzynski

License: http://creativecommons.org/licenses/by/3.0/ 


\title{
Fundamentals of Mathematics
}

Fundamentals of Mathematics is a work text that covers the traditional topics studied in a modern prealgebra course, as well as topics of estimation, elementary analytic geometry, and introductory algebra. It is intended for students who have had a previous course in prealgebra, wish to meet the prerequisite of a higher level course such as elementary algebra, and need to review fundamental mathematical concepts and techniques.

\begin{abstract}
About Connexions
Since 1999, Connexions has been pioneering a global system where anyone can create course materials and make them fully accessible and easily reusable free of charge. We are a Web-based authoring, teaching and learning environment open to anyone interested in education, including students, teachers, professors and lifelong learners. We connect ideas and facilitate educational communities.

Connexions's modular, interactive courses are in use worldwide by universities, community colleges, K-12 schools, distance learners, and lifelong learners. Connexions materials are in many languages, including English, Spanish, Chinese, Japanese, Italian, Vietnamese, French, Portuguese, and Thai. Connexions is part of an exciting new information distribution system that allows for Print on Demand Books. Connexions has partnered with innovative on-demand publisher QOOP to accelerate the delivery of printed course materials and textbooks into classrooms worldwide at lower prices than traditional academic publishers.
\end{abstract}

